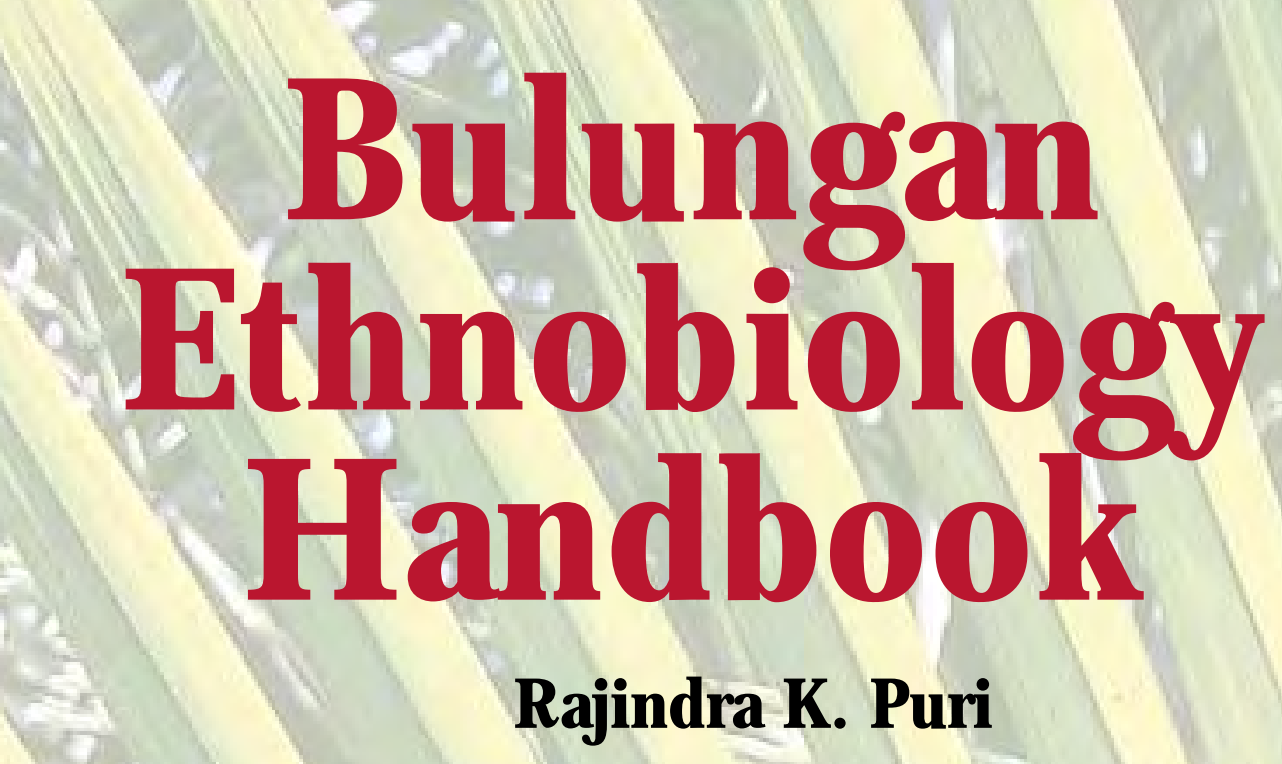

स|||||||

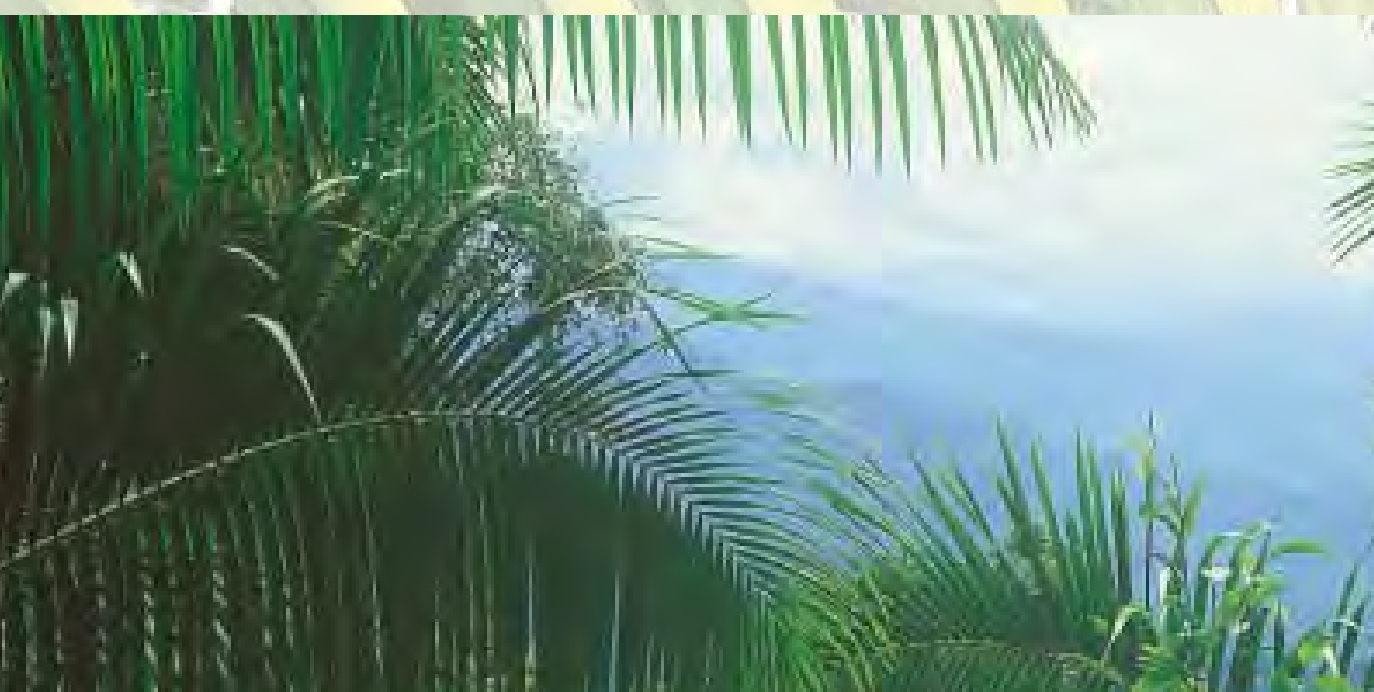
sexesery

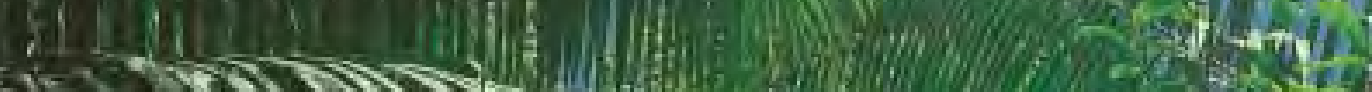




\section{THE BULUNGAN ETHNOBIOLOGY HANDBOOK}

A FIELD MANUAL FOR BIOLOGICAL AND SOCIAL SCIENCE RESEARCH ON THE KNOWLEDGE AND USE OF PLANTS AND ANIMALS AMONG 18 INDIGENOUS GROUPS IN NORTHERN EAST KaLIMANTAN, INDONESIA

Rajindra K. Puri 
C2001 by Center for International Forestry Research

All rights reserved. Published 2001

Printed by SMK Grafika Desa Putera, Indonesia

Front cover photo: R.K. Puri.

Eugeissona utilis, hill sago, growing on a ridge overlooking the Lurah valley in Kayan Mentarang National Park.

Back cover photos: Francis $\mathrm{Ng}$ and R.K. Puri.

Metroxylon sagu, Kenyah fishermen, Bahau river forest, children at Long Peliran, Penan hunter with hornbill and Dimocarpus longan fruit for market.

Dividers: LBN-LIPI 1978: 15 and Payne et al. 1985: Pl. 51, CKaren Phillipps

\section{ISBN 979-8764-45-5}

Puri, R.K. 2001. Bulungan ethnobiology handbook. Center for International Forestry Research, Bogor, Indonesia. 310p.

Center for International Forestry Research

Office address: Jalan CIFOR, Situ Gede, Sindang Barang, Bogor 16680, Indonesia Mailing address: P.O. Box 6596 JKPWB, Jakarta 10065, Indonesia

Tel: +62 (251) 622622; Fax: +62 (251) 622100

E-mail: cifor@cgiar.org

Website: http://www.cifor.cgiar.org 


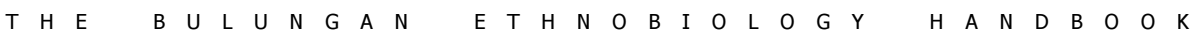

\section{Contents}

Acknowledgements $\quad$ iv

List of drawing references and copyrights $\quad \mathrm{V}$

Introduction $\quad \mathrm{xV}$

Objectives $\quad \mathrm{xv}$

How to use the handbook xvi

Documenting regional ethnobiology $\quad \mathrm{xx}$

Environment and people of the Bulungan area xxiv

Notes on linguistics and orthography $\quad x x v$

References cited xxvii

1. Plant profiles 1

2. Animal profiles 165

3. Environmental glossary 241

4. Indexes 255

4.1 Scientific names for plants 255

4.2 Scientific names for animals 258

4.3 Vernacular names for plants 260

4.4 Vernacular names for animals 292 


\section{ACKNOWLEDGEMENTS}

This project was funded by CIFOR and the East-West Center, while PROSEA provided a substantial number of botanical drawings. Dr. Eva Wollenberg, at CIFOR, originated the idea of a regional vernacular dictionary, which has since grown into this handbook thanks to her sustained support. The research team in East Kalimantan included the author and three local assistants, Kasim Amat, Sapu Billa and Lalung Bisa. The team is grateful for logistical assistance in the field from Yayasan WWF Indonesia's Project Kayan Mentarang, Bilung Njau and Taang Bawan at WWF's post in Long Pujungan, Made Sudana and staff at WWF's Lalut Birai Forest Station, Maman and Iwan at the Hotel Handayani in Malinau, and Awang Iluk and Alek from Respen Sembuak. The team also thanks all the residents of the 25 villages who so generously hosted us and participated in the survey. The orthography for vernacular terms and the introductory section on linguistics were edited by Antonia Soriente. Plant specimens were identified by Ismail at the Herbarium Bogoriense, and Ambriansyah and Zainal Arifin at Herbarium Wanariset. Additional voucher specimens that were borrowed from WWF Indonesia were originally collected and identified by Dr. J. Andrew McDonald and colleagues at the Arnold Arboretum. Dr. Kade Sidiyasa, Dr. Herwasono Soedjito, Dr. Kuswata Kartawinata and Dr. Johan van Valkenburg reviewed the botanical data. Ms. Asung Uluk of CIFOR field-checked parts of the manuscript. Many thanks to Dr. J.S. Siemonsma, Dr. Johan van Valkenburg and Dr. Leo Oyen at PROSEA, Dr. Bas van Helvoort at MOFEC-Tropenbos Kalimantan, Dr. M. Nesbitt at RBG Kew, and Mr. C. W. Lut and Mrs. J. R. Kruyer at the National Herbarium of the Netherlands, for providing most of the botanical illustrations. Faunal illustrations and photographs were provided by Karen Phillipps, Dr. Robert F. Inger, Dr. Robert Steubing, Drs. P. Hans Hazebroek, Stephen von Peltz, C. L. Chan, W. M. Poon, Dr. Ike Rachmatika, Andreas S. Brahm, Dr. Bernard Sellato, Museum National d'Histoire Naturelle, Cambridge Univ. Press, Australian Gov. Pub. Office, and the British Natural History Museum. Photographs not reprinted from other sources were taken by Anggoro Santoso or the author. Anggoro Santoso, Gideon Suharyanto, and Dina J. Hubudin at CIFOR, skillfully constructed the indexes, and completed the page layout and graphics. Dr. Jefferson Fox at EWC, and Dr. Carol Colfer and Dr. Kuswata Kartawinata at CIFOR, supported the editing of the manuscript. The author thanks all these participants and several anonymous reviewers for their efforts, but retains responsibility for the final work. 


\section{LIST OF DRAWING REFERENCES AND COPYRIGHTS}

\begin{tabular}{|c|c|c|}
\hline $\begin{array}{l}\text { Page } \\
\text { No. }\end{array}$ & Genus Species & $\begin{array}{l}\text { Drawing Reference } \\
\text { and Copyright }\end{array}$ \\
\hline 1 & Gendarussa vulgaris Nees. & PROSEA Vol. 12: 330 \\
\hline 2 & Amaranthus hybridus $\mathrm{L}$. & Sastrapradja, S. et al. 1977b: 8 \\
\hline 3 & Celosia cristata L. & Ochse 1980: 29 \\
\hline 4 & Buchanania sessifolia BI. & $\begin{array}{l}\text { Kessler \& Sidiyasa 1994: } 245 \\
\text { (CTropenbos Foundation }\end{array}$ \\
\hline 5 & Mangifera quadrafida Jack. & A. Santoso \\
\hline 6 & Mangifera odorata Griff. & PROSEA Vol. 5(2): 336 \\
\hline 7 & Mangifera pajang Kostermans & Herwasono Soedjito \\
\hline 8 & Semecarpus glaucus Engl. & A. Santoso \\
\hline 9 & Annona muricata $\mathrm{L}$. & PROSEA Vol. 2: 75 \\
\hline 10 & Eryngium foetidum $\mathrm{L}$. & PROSEA Vol. 13: 122 \\
\hline 11 & Acorus calamus $\mathrm{L}$. & PROSEA Vol. 12: 84 \\
\hline 12 & Amydrium medium (Z. \& M.) Nichols. & Koorders Vol 3: 172 \\
\hline 13 & Colocasia esculentum (L.) Schott & PROSEA Vol. 9: 70 \\
\hline 14 & Holochlamis beccarii (Engl.) Engl. & Koorders Vol 3: 182 \\
\hline 15 & Homalomena cordata Schott. & A. Santoso \\
\hline 16 & Agathis borneensis Warb. & $\begin{array}{l}\text { Soepadmo \& Wong 1995: } 31 \\
\text { CFRIM, Sabah Forestry Dept., } \\
\text { Sarawak Forestry Dept. }\end{array}$ \\
\hline 17 & Ageratum conyzoides $\mathrm{L}$. & PROSEA Vol. 12: 93 \\
\hline 18 & Blumea balsamifera (L.) DC. & PROSEA Vol. 19: 69 \\
\hline 19 & Synedrella nodiflora (L.) Gaertn. & Backer \& van Slooten 1924: 227 \\
\hline 20 & Basella alba L. & PROSEA Vol. 8: 94 \\
\hline 21 & Bixa orellana $\mathrm{L}$. & PROSEA Vol. 3: 50 \\
\hline 22 & Ceiba pentandra (L.) Gaertn. & Ochse 1980: 80 \\
\hline 23 & Durio kutejensis (Hassk.) Becc. & Valkenburg 1997: 75 \\
\hline 24 & Durio oxleyanus Griff. & $\begin{array}{l}\text { Kessler \& Sidiyasa 1994: } 264 \\
\text { CTropenbos Foundation }\end{array}$ \\
\hline 25 & Durio zibethinus Murr. & PROSEA Vol. 2: 157 \\
\hline 26 & Ananas comosus (L.) Merr. & PROSEA Vol. 2: 66 \\
\hline 27 & Canarium odontophyllum Miq. & Cockburn 1976: Fig. 7 \\
\hline 28 & Dacryodes rostrata (Blume) H.J. Lam & $\begin{array}{l}\text { Kessler \& Sidiyasa 1994: } 270 \\
\text { CTropenbos Foundation }\end{array}$ \\
\hline 29 & Koompassia excelsa (Becc.) Taubert & Kraemer 1951: 127 \\
\hline 30 & Carica papaya L. (2 varieties) & PROSEA Vol. 2: 108 \\
\hline 31 & Ipomoea aquatica Forsskal & PROSEA Vol. 8: 182 \\
\hline 32 & Ipomoea batatas (L.) L. & PROSEA Vol. 9: 102 \\
\hline
\end{tabular}




\begin{tabular}{ll}
\hline $\begin{array}{l}\text { Page } \\
\text { No. Genus Species }\end{array}$ & $\begin{array}{l}\text { Drawing Reference } \\
\text { and Copyright }\end{array}$
\end{tabular}

\begin{tabular}{|c|c|c|}
\hline 33 & Merremia peltata (L.) Merr. & A. Santoso \\
\hline 34 & Kalanchoe pinnata L. & A. Santoso \\
\hline 35 & Cucumis sativus L. & PROSEA Vol. 8: 158 \\
\hline 36 & Cucurbita moschata (Duch.) Poir. & PROSEA Vol. 8: 162 \\
\hline 37 & Diplazium esculentum (Retz.) Swartz. & Ochse 1980: 599 \\
\hline 38 & Dipterocarpus oblongifolius Blume & Kraemer 1951: 279 \\
\hline 39 & Dryobalanops lanceolata Burck & PROSEA Vol. 5(1): 192 \\
\hline 40 & Shorea macrophylla (de Vriese) Ashton & LBN 3-SDE 36: 103 \\
\hline 41 & Diospyros borneensis Hiern & $\begin{array}{l}\text { Kessler \& Sidiyasa 1994: } 333 \\
\text { (C)Tropenbos Foundation }\end{array}$ \\
\hline 42 & Baccaurea bracteata M.A. & A. Santoso \\
\hline 43 & Baccaurea lanceolata (Miq.) M.A. & A. Santoso \\
\hline 44 & Baccaurea macrocarpa (Miq.) M.A. & $\begin{array}{l}\text { Kessler \& Sidiyasa 1994: } 338 \\
\text { (C)Tropenbos Foundation }\end{array}$ \\
\hline 45 & Baccaurea motleyana M.A. & Valkenburg 1997: 70 \\
\hline 46 & Baccaurea parviflora (M.A.) M.A. & Kochummen 1997: 438 \\
\hline 47 & Baccaurea pyriformis Gage & Valkenburg 1997: 71 \\
\hline 48 & Hevea brasiliensis (Willd. ex A. Juss) M.A. & PROSEA Vol. 5(2): 262 \\
\hline 49 & Macaranga triloba M.A. & LBN 3-SDE 36 \\
\hline 50 & Manihot esculenta Crantz & PROSEA Vol. 9: 109 \\
\hline 51 & Phyllanthus urinaria L. & Basu 1918: plate 859 \\
\hline 52 & Sauropus androgynus (L.) Merr. & Ochse 1927: 69 \\
\hline 53 & Sumbaviopsis albicans (BL.) J.J.S. & Flora Sinicae $44(2): 4$ \\
\hline 54 & Albizia chinensis (Osbeck) Merr. & PROSEA Vol. 11: 64 \\
\hline 55 & Bauhinia semibifida Roxb. & A. Santoso \\
\hline 56 & Leucaena leucocephala Bth. & PROSEA Vol. 11: 177 \\
\hline 57 & Milletia sericea Wight. et Arn. & Ochse 1981: 395 \\
\hline 58 & Parkia speciosa Hassk. & PROSEA Vol. 8: 223 \\
\hline 59 & Senna alata (L.) Roxb. & A. Santoso \\
\hline 60 & Vigna unguiculata (L.) Walp. & PROSEA Vol. 1: 77 \\
\hline 61 & Castanopsis argentea (Blume) A. DC. & PROSEA Vol. 5(2): 113 \\
\hline 62 & Lithocarpus conocarpus (Oudem.) Rehd. & Koorders \& Valeton 1913: Fig. 40 \\
\hline 63 & Lithocarpus coopertus (Blanco) Rehd. & $\begin{array}{l}\text { Kessler \& Sidiyasa 1994: } 355 \\
\text { (CTropenbos Foundation }\end{array}$ \\
\hline 64 & $\begin{array}{l}\text { Lithocarpus nieuwenhuisii (v. Seem.) } \\
\text { A. Camus }\end{array}$ & Cockburn 1976: Fig. 10 \\
\hline 65 & Quercus argentata Korth. & $\begin{array}{l}\text { Kessler \& Sidiyasa 1994: } 356 \\
\text { (CTropenbos Foundation }\end{array}$ \\
\hline
\end{tabular}




\begin{tabular}{|c|c|c|}
\hline $\begin{array}{l}\text { Page } \\
\text { No. }\end{array}$ & Genus Species & $\begin{array}{l}\text { Drawing Reference } \\
\text { and Copyright }\end{array}$ \\
\hline 66 & Quercus gemelliflora BI. & PROSEA Vol. 5(2): 414 \\
\hline 67 & Pangium edule Reinw. & Ochse 1927: 70 \\
\hline 68 & Dicranopteris linearis (Burm.) Underw. & A. Santoso \\
\hline 69 & Cymbopogon nardus (L.) Randle & R. K. Puri \\
\hline 70 & Imperata cylindrica (L.) Beauv. & PROSEA Vol. 12: 310 \\
\hline 71 & Oryza sativa $\mathrm{L}$. & PROSEA Vol. 10: 108 \\
\hline 72 & Saccharum officinarum L. & PROSEA Vol. 9: 144 \\
\hline 73 & Setaria palmifolia (Willd.) Stapf. & A. Santoso \\
\hline 74 & Garcinia forbesii King & Corner \& Watanabe 1969: 188 \\
\hline 75 & Garcinia lateriflora BI. & Ochse 1927: 93 \\
\hline 76 & Garcinia mangostana L. & PROSEA Vol. 2: 177 \\
\hline 77 & Garcinia parvifolia (Miq.) Miq. & $\begin{array}{l}\text { Kessler \& Sidiyasa 1994: } 360 \\
\text { (CTropenbos Foundation }\end{array}$ \\
\hline 78 & Eleutherine americana (Aubl.) Merr. & R. K. Puri \\
\hline 79 & Orthosiphon aristatus (Blume) Miq. & PROSEA Vol. 12: 369 \\
\hline 80 & Cinnamomum burmanni Nees ex BI. & PROSEA Vol. 13: 96 \\
\hline 81 & Cinnamomum javanicum Bl. (2 varieties) & A. Santoso \\
\hline 82 & Eusideroxylon zwageri Teijsm. \& Binn & PROSEA Vol. 5(1): 212 \\
\hline 83 & Litsea garciae Vidal & Kanehira 1936: 218 \\
\hline 84 & Alium tuberosum Rottler ex Sprengel & PROSEA Vol. 8: 81 \\
\hline 85 & Elmerrillia mollis Dandy. & Meijer 1974: 202 \\
\hline 86 & Abelmoschus manihot Medik & PROSEA Vol. 8: 60 \\
\hline 87 & Melastoma affine D. Don. & A. Santoso \\
\hline 88 & Memecylon paniculatum Jack & Bremer 1983: 13 \\
\hline 89 & Lancium domesticum Correa & PROSEA Vol. 2: 186 \\
\hline 90 & Melia azedarach L. & PROSEA Vol. 11: 187 \\
\hline 91 & Pycnarrhena cauliflora & ICONES, NHN \\
\hline 92 & Antiaris toxicaria Leschen & PROSEA Vol. 12: 128 \\
\hline 93 & Artocarpus altilis (Z.) Fosberg & PROSEA Vol. 2: 83 \\
\hline 94 & Artocarpus elasticus Reinw. ex BI. & Koorders Vol. 4: 472 \\
\hline 95 & Artocarpus heterophyllus Lam & PROSEA Vol. 2: 86 \\
\hline 96 & Artocarpus integer (Thunb.) Merr. & PROSEA Vol. 2: 91 \\
\hline 97 & Artocarpus lanceifolius Roxb. & Valkenburg 1997: 79 \\
\hline 98 & Artocarpus nitidus Trec & Ahern 1901: 35 \\
\hline 99 & Artocarpus odoratissimus Blanco & PROSEA Vol. 2: 94 \\
\hline 100 & Artocarpus rigidus Blume & PROSEA Vol. 5(2): 69 \\
\hline 101 & Ficus fistulosa Reinw. & Ochse 1931: 495 \\
\hline 102 & Ficus glomerata Roxb. & Ochse 1931: 498 \\
\hline
\end{tabular}




\begin{tabular}{ll}
\hline $\begin{array}{l}\text { Page } \\
\text { No. }\end{array}$ & $\begin{array}{l}\text { Drawing Reference } \\
\text { and Copyright }\end{array}$ \\
\hline
\end{tabular}

\begin{tabular}{|c|c|c|}
\hline 103 & Ficus xylophylla Wall. ex Miq. & A. Santoso \\
\hline 104 & Musa paradisiaca L. & PROSEA Vol. 2: 225 \\
\hline 105 & Knema sp. & PROSEA Vol. 5(3): 318 \\
\hline 106 & Psidium guajava L. & PROSEA Vol. 2: 266 \\
\hline 107 & Syzygium sp. & PROSEA Vol. 2: 297 \\
\hline 108 & Tristaniopsis whiteana (Griff.) Wil. \& Wat. & PROSEA Vol. 5(3): 567 \\
\hline 109 & Averrhoa carambola L. & PROSEA Vol. 2: 96 \\
\hline 110 & Areca catechu L. & Ochse 1931: 551 \\
\hline 111 & Arenga brevipes Becc. & LBN-LIPI 1978: 15 \\
\hline 112 & Arenga undulatifolia Becc. & A. Santoso \\
\hline 113 & Calamus caesius BI. & PROSEA Vol. 6: 44 \\
\hline 114 & Calamus ornatus Blume & PROSEA Vol. 6: 59 \\
\hline 115 & Caryota mitis Lour. & PROSEA Vol. 5(3): 141 \\
\hline 116 & Caryota no Becc. & A. Santoso \\
\hline 117 & Cocos nucifera L. & Koorders Vol. 2: 160 \\
\hline 118 & Eugeissona utilis Becc. & LBN-LIPI 1978: 109 \\
\hline 119 & Licuala valida Becc. & LBN-LIPI 1978: 49 \\
\hline 120 & Oncosperma horridum (Griff.) Scheffer & LBN-LIPI 1978: 17 \\
\hline 121 & Salacca affinis Griff. & A. Santoso \\
\hline 122 & Salacca zalacca (Gaertner) Voss & PROSEA Vol. 2: 281 \\
\hline 123 & Pandanus sp. & A. Santoso \\
\hline 124 & Passiflora foetida $\mathrm{L}$. & Ochse 1927: 195 \\
\hline 125 & Piper betle L. (Variety 1) & A. Santoso \\
\hline 126 & Piper betle L. (Variety 2) & A. Santoso \\
\hline 127 & Piper nigrum L. & PROSEA Vol. 13: 190 \\
\hline 128 & $\begin{array}{l}\text { Dendrocalamus asper (Schult.F.) } \\
\text { Backer ex Heyne }\end{array}$ & PROSEA Vol. 7: 81 \\
\hline 129 & Schizostachyum brachycladum Kurz & PROSEA Vol. 7: 132 \\
\hline 130 & Schizostachyum latifolium Gamble & PROSEA Vol. 7: 138 \\
\hline 131 & Xanthophyllum obscurum Bennett & $\begin{array}{l}\text { Kessler \& Sidiyasa 1994: } 406 \\
\text { (C)Tropenbos Foundation }\end{array}$ \\
\hline 132 & Prunus arborea (BI.) Kalkman & PROSEA Vol. 5(3): 475 \\
\hline 133 & $\begin{array}{l}\text { Anthocephalus chinensis (Lamk) Rich } \\
\text { ex. Walp. ( } 2 \text { varieties) }\end{array}$ & PROSEA Vol. 5(1): 107 \\
\hline 134 & Anthocephalus sp. & A. Santoso \\
\hline 135 & Coffea robusta L. & PROSEA Vol 16: 69 \\
\hline 136 & Citrus hystrix DC. & Ochse 1927: 226 \\
\hline 137 & Citrus maxima (Burm.) Merr. & PROSEA Vol. 2: 128 \\
\hline
\end{tabular}




\begin{tabular}{|c|c|c|}
\hline $\begin{array}{l}\text { Page } \\
\text { No. }\end{array}$ & Genus Species & $\begin{array}{l}\text { Drawing Reference } \\
\text { and Copyright }\end{array}$ \\
\hline 138 & Citrus reticulata Blanco & PROSEA Vol. 2: 135 \\
\hline 139 & $\begin{array}{l}\text { Dimocarpus longan Lour. subsp. } \\
\text { malesianus var. malesianus Leenh. } \\
\text { (jilen) }\end{array}$ & PROSEA Vol. 2: 146 \\
\hline 140 & $\begin{array}{l}\text { Dimocarpus longan Lour. subsp. } \\
\text { malesianus var. malesianus Leenh. (sau) }\end{array}$ & Valkenburg 1997: 72, 202 \\
\hline 141 & $\begin{array}{l}\text { Nephelium cuspidatum BI. var. } \\
\text { eriopetalum (Miq.) Leenh. }\end{array}$ & $\begin{array}{l}\text { Kessler and Sidiyasa 1994: } 422 \\
\text { CTropenbos Foundation }\end{array}$ \\
\hline 142 & Nephelium juglandifolium BI. & A. Santoso \\
\hline 143 & Nephelium lappaceum L. & Ochse 1927: 249 \\
\hline 144 & Nephelium maingayi Hiern. & PROSEA Vol. 2. 233 \\
\hline 145 & Nephelium meduseum Leenh. & $\begin{array}{l}\text { Soepadmo and Wong 1995: } 346 \\
\text { CFRIM, Sabah Forestry Dept., Sarawak } \\
\text { Forestry Dept. }\end{array}$ \\
\hline 146 & $\begin{array}{l}\text { Nephelium ramboutan-ake (Labill.) } \\
\text { Leenh. }\end{array}$ & $\begin{array}{l}\text { Soepadmo and Wong 1995: } 346 \\
\text { OFRIM, Sabah Forestry Dept., Sarawak } \\
\text { Forestry Dept. }\end{array}$ \\
\hline 147 & $\begin{array}{l}\text { Palaquium quercifolium (De Vriese) } \\
\text { Burck. }\end{array}$ & $\begin{array}{l}\text { Kessler \& Sidiyasa 1994: } 429 \\
\text { CTropenbos Foundation }\end{array}$ \\
\hline 148 & Selaginella plana (Desv.) Hieron & A. Santoso \\
\hline 149 & $\begin{array}{l}\text { Capsicum annuum L. var. abbreviata } \\
\text { Fingerhuth. }\end{array}$ & Ochse 1980: 668 \\
\hline 150 & Capsicum frutescens $\mathrm{L}$. & Ochse 1927: 171 \\
\hline 151 & Nicotiana tabacum $\mathrm{L}$. & PROSEA Vol. 16: 95 \\
\hline 152 & Solanum melongena $\mathrm{L}$. & PROSEA Vol. 8: 256 \\
\hline 153 & Solanum torvum Swartz. & PROSEA Vol. 8: 259 \\
\hline 154 & Aquilaria beccariana van Tigh. & Corner \& Watanabe 1969: 494 \\
\hline 155 & Aquilaria malaccensis Lamk. & PROSEA Vol. 19: 65 \\
\hline 156 & $\begin{array}{l}\text { Gonystylus macrophyllus (Miq.) } \\
\text { Airy Shaw }\end{array}$ & $\begin{array}{l}\text { FM I Vol. } 4 \text { (4): } 360 \\
\text { (CFlora Malesiana }\end{array}$ \\
\hline 157 & Poikilospermum suaveolens (BI.) Merr. & A. Santoso \\
\hline 158 & Callicarpa longifolia Lam. & A. Santoso \\
\hline 159 & Vitex pinnata L. & PROSEA Vol. 5(2): 508 \\
\hline 160 & Alpinia galanga (I.) Willd. & PROSEA Vol. 13: 66 \\
\hline 161 & Curcuma aeruginosa Roxb. & Sastrapradja et al. 1977b: 32 \\
\hline 162 & Curcuma domestica Val. & PROSEA Vol. 13: 113 \\
\hline 163 & Etlingera elatior (Jack) R. M. Smith & PROSEA Vol. 13: 124 \\
\hline 164 & Kaemferia galanga L. & PROSEA Vol. 12: 334 \\
\hline
\end{tabular}




\begin{tabular}{|c|c|c|}
\hline $\begin{array}{l}\text { Page } \\
\text { No. }\end{array}$ & Genus Species & $\begin{array}{l}\text { Drawing Reference } \\
\text { and Copyright }\end{array}$ \\
\hline 165 & Cynocephalus variegatus & Payne et al. 1985: PI. 19, OKaren Phillipps \\
\hline 166 & Pteropus vampyrus & Payne et al. 1985: PI. 8, OKaren Phillipps \\
\hline 167 & Nycticebus coucang & Payne et al. 1985: PI. 19, OKaren Phillipps \\
\hline 168 & Tarsius bancanus & Payne et al. 1985: PI. 19, OKaren Phillipps \\
\hline 169 & Presbytis rubicunda & Payne et al. 1985: PI. 20, OKaren Phillipps \\
\hline 170 & Presbytis frontata & Payne et al. 1985: PI. 20, CKaren Phillipps \\
\hline 171 & Presbytis hosei & Payne et al. 1985: PI. 20, OKaren Phillipps \\
\hline 172 & Nasalis larvatus & Payne et al. 1985: PI. 21, OKaren Phillipps \\
\hline 173 & Macaca fascicularis & Payne et al. 1985: PI. 21, CKaren Phillipps \\
\hline 174 & Macaca nemestrina & Payne et al. 1985: PI. 21, OKaren Phillipps \\
\hline 175 & Hylobates muelleri & Payne et al. 1985: PI. 22, OKaren Phillipps \\
\hline 176 & Pongo pygmaeus & Payne et al. 1985: PI. 22, CKaren Phillipps \\
\hline 177 & Manis javanica & Payne et al. 1985: PI. 19, CKaren Phillipps \\
\hline 178 & Ratufa affinis & Payne et al. 1985: PI. 23, OKaren Phillipps \\
\hline 179 & Rattus tiomanicus & Payne et al. 1985: PI. 35, CKaren Phillipps \\
\hline 180 & Rattus rattus & Payne et al. 1985: PI. 35, OKaren Phillipps \\
\hline 181 & Trichys fasciculata & Payne et al. 1985: PI. 42, CKaren Phillipps \\
\hline 182 & Hystrix brachyura & Payne et al. 1985: PI. 42, OKaren Phillipps \\
\hline 183 & Canis familiaris & Eva Wollenberg \\
\hline 184 & Ursus malayanus & Payne et al. 1985: PI. 43, CKaren Phillipps \\
\hline 185 & Mustela nudipes & Payne et al. 1985: PI. 44, CKaren Phillipps \\
\hline 186 & Aonyx cinerea & Payne et al. 1985: PI. 45, CKaren Phillipps \\
\hline 187 & Paradoxurus hermaphroditus & Payne et al. 1985: PI. 47, CKaren Phillipps \\
\hline 188 & Paguma larvata & Payne et al. 1985: PI. 47, CKaren Phillipps \\
\hline 189 & Arctictis binturong & Payne et al. 1985: PI. 46, OKaren Phillipps \\
\hline 190 & Herpestes brachyurus & Payne et al. 1985: PI. 49, CKaren Phillipps \\
\hline 191 & Pardofelis nebulosa & Payne et al. 1985: PI. 50, CKaren Phillipps \\
\hline 192 & Pardofelis marmorata & Payne et al. 1985: PI. 50, CKaren Phillipps \\
\hline 193 & Prionailurus bengalensis & Payne et al. 1985: PI. 50, OKaren Phillipps \\
\hline 194 & Felis catus & Eva Wollenberg \\
\hline 195 & Dicerorhinus sumatrensis & Payne et al. 1985: PI. 51, OKaren Phillipps \\
\hline 196 & Sus barbatus & Payne et al. 1985: PI. 51, CKaren Phillipps \\
\hline 197 & Sus domesticus & Payne et al. 1985: PI. 60, CKaren Phillipps \\
\hline 198 & Tragulus javanicus & Payne et al. 1985: PI. 52, CKaren Phillipps \\
\hline 199 & Tragulus napu & Payne et al. 1985: PI. 52, OKaren Phillipps \\
\hline 200 & Muntiacus muntjak & Payne et al. 1985: PI. 53, CKaren Phillipps \\
\hline 201 & Muntiacus atherodes & Payne et al. 1985: PI. 53, OKaren Phillipps \\
\hline 202 & Cervus unicolor & Payne et al. 1985: PI. 54, @Karen Phillipps \\
\hline
\end{tabular}




\begin{tabular}{|c|c|c|}
\hline $\begin{array}{l}\text { Page } \\
\text { No. }\end{array}$ & Genus Species & $\begin{array}{l}\text { Drawing Reference } \\
\text { and Copyright }\end{array}$ \\
\hline 203 & Bos javanicus & Payne et al. 1985: PI. 64, CKaren Phillipps \\
\hline 204 & Bos taurus & Payne et al. 1985: PI. 60, CKaren Phillipps \\
\hline 205 & Capra hircus & Payne et al. 1985: PI. 60, CKaren Phillipps \\
\hline 206 & Haliastur indus & $\begin{array}{l}\text { MacKinnon and Phillipps 1993: PI. } 9 \\
\text { @John MacKinnon and Karen Phillipps }\end{array}$ \\
\hline 206 & Lophura bulweri & $\begin{array}{l}\text { MacKinnon and Phillipps 1993: PI. } 16 \\
\text { CJohn MacKinnon and Karen Phillipps }\end{array}$ \\
\hline 207 & Gallus sp. & $\begin{array}{l}\text { MacKinnon and Phillipps 1993: PI. } 17 \\
\text { CJohn MacKinnon and Karen Phillipps }\end{array}$ \\
\hline 207 & Argusianus argus & $\begin{array}{l}\text { MacKinnon and Phillipps 1993: PI. } 15 \\
\text { CJohn MacKinnon and Karen Phillipps }\end{array}$ \\
\hline 208 & Lophura ignita & $\begin{array}{l}\text { MacKinnon and Phillipps 1993: PI. } 16 \\
\text { CJohn MacKinnon and Karen Phillipps }\end{array}$ \\
\hline 208 & Treron curvirostra & $\begin{array}{l}\text { MacKinnon and Phillipps 1993: PI. } 31 \\
\text { CJohn MacKinnon and Karen Phillipps }\end{array}$ \\
\hline 209 & Loriculus galgulus & $\begin{array}{l}\text { MacKinnon and Phillipps 1993: PI. } 35 \\
\text { @John MacKinnon and Karen Phillipps }\end{array}$ \\
\hline 209 & $\begin{array}{l}\text { Collocalia fuciphaga } \\
\text { subsp. vestita }\end{array}$ & $\begin{array}{l}\text { MacKinnon and Phillipps 1993: PI. } 42 \\
\text { CJohn MacKinnon and Karen Phillipps }\end{array}$ \\
\hline 210 & Harpactes duvaucelii & $\begin{array}{l}\text { MacKinnon and Phillipps 1993: PI. } 43 \\
\text { CJohn MacKinnon and Karen Phillipps }\end{array}$ \\
\hline 210 & Pelargopsis capensis & $\begin{array}{l}\text { MacKinnon and Phillipps 1993: PI. } 44 \\
\text { CJohn MacKinnon and Karen Phillipps }\end{array}$ \\
\hline 211 & Buceros rhinoceros & $\begin{array}{l}\text { MacKinnon and Phillipps 1993: PI. } 47 \\
\text { CJohn MacKinnon and Karen Phillipps }\end{array}$ \\
\hline 211 & Buceros vigil & $\begin{array}{l}\text { MacKinnon and Phillipps 1993: PI. } 47 \\
\text { CJohn MacKinnon and Karen Phillipps }\end{array}$ \\
\hline 212 & Anorrhinus galeritus & $\begin{array}{l}\text { MacKinnon and Phillipps 1993: PI. } 47 \\
\text { CJohn MacKinnon and Karen Phillipps }\end{array}$ \\
\hline 212 & Anthracoceros malayanus & $\begin{array}{l}\text { MacKinnon and Phillipps 1993: PI. } 47 \\
\text { CJohn MacKinnon and Karen Phillipps }\end{array}$ \\
\hline 213 & Aceros corrugatus & $\begin{array}{l}\text { MacKinnon and Phillipps 1993: PI. } 47 \\
\text { CJohn MacKinnon and Karen Phillipps }\end{array}$ \\
\hline 213 & Sasia abnormis & $\begin{array}{l}\text { MacKinnon and Phillipps 1993: PI. } 50 \\
\text { CJohn MacKinnon and Karen Phillipps }\end{array}$ \\
\hline 214 & Blythipicus rubiginosus & $\begin{array}{l}\text { MacKinnon and Phillipps 1993: PI. } 50 \\
\text { (C)John MacKinnon and Karen Phillipps }\end{array}$ \\
\hline
\end{tabular}




\begin{tabular}{|c|c|c|}
\hline $\begin{array}{l}\text { Page } \\
\text { No. }\end{array}$ & Genus Species & $\begin{array}{l}\text { Drawing Reference } \\
\text { and Copyright }\end{array}$ \\
\hline 214 & Pycnonotus zeylanicus & $\begin{array}{l}\text { MacKinnon and Phillipps 1993: PI. } 58 \\
\text { @John MacKinnon and Karen Phillipps }\end{array}$ \\
\hline 215 & Platylophus galericulatus & $\begin{array}{l}\text { MacKinnon and Phillipps 1993: PI. } 63 \\
\text { CJohn MacKinnon and Karen Phillipps }\end{array}$ \\
\hline 215 & Copsychus malabaricus & $\begin{array}{l}\text { MacKinnon and Phillipps 1993: PI. } 70 \\
\text { CJohn MacKinnon and Karen Phillipps }\end{array}$ \\
\hline 216 & Gracula religiosa & $\begin{array}{l}\text { MacKinnon and Phillipps 1993: PI. } 82 \\
\text { CJohn MacKinnon and Karen Phillipps }\end{array}$ \\
\hline 216 & Arachnothera longirostra & $\begin{array}{l}\text { MacKinnon and Phillipps 1993: PI. } 84 \\
\text { CJohn MacKinnon and Karen Phillipps }\end{array}$ \\
\hline 217 & Lonchura malacca & $\begin{array}{l}\text { MacKinnon and Phillipps 1993: PI. } 87 \\
\text { CJohn MacKinnon and Karen Phillipps }\end{array}$ \\
\hline 217 & Crocodylus porosus & Glasby et al. 1993: 326, @Commonwealth of Australia \\
\hline 218 & Varanus salvator & Bennett 1998: 243 \\
\hline 218 & Varanus rudicollis & Inger and Tan 1996: Fig. 24, OR.F. Inger \\
\hline 219 & Varanus dumerilii & Bennett 1998: 103 \\
\hline 219 & Gonocephalus doriae & Inger and Tan 1996: Fig. 22, OR.F. Inger \\
\hline 220 & Gonocephalus liogaster & Inger and Tan 1996: Fig. 48b, CR.B. Stuebing \\
\hline 220 & Mabuya rudis & Inger and Tan 1996: Fig. 56, CC.L. Chan \\
\hline 221 & Python curtus & Inger and Tan 1996: Fig. 95, CS. Von Peltz \\
\hline 221 & Python reticulatus & Tweedie and Harrison 1954: 44 \\
\hline 222 & Ophiophagus hannah & Inger and Tan 1996: Fig. 30, @S. Von Peltz \\
\hline 222 & Tropidolaemus wagleri & Inger and Tan 1996: Fig. 29, CP. Hans Hazebroek \\
\hline 223 & Ahaetulla prasina & Inger and Tan 1996: Fig. 90, @C.L. Chan \\
\hline 223 & Maticora bivirgata & Inger and Tan 1996: Fig. 91, OR.F. Inger \\
\hline 224 & Dogania subplana & Inger and Tan 1996: Fig. 19, OR.F. Inger \\
\hline 224 & Cyclemys dentata & Inger and Tan 1996: Fig. 52, OW.M. Poon \\
\hline 225 & Notochelys platynota & Inger and Tan 1996: Fig. 104, OW.M. Poon \\
\hline 225 & Rhacophorus reinwardti & Inger and Tan 1996: Fig. 15, OR.F. Inger \\
\hline 226 & Rhacophorus nigropalmatus & Inger and Tan 1996: Fig. 60, CR.F. Inger \\
\hline 226 & Polypedates macrotis & Inger and Tan 1996: Fig. 62, OR.F. Inger \\
\hline 227 & Kaloula baleata & Glasby et al. 1993, (C) Commonwealth of Australia \\
\hline 227 & Rana ingeri & Inger and Tan 1996: Fig. 75, OR.F. Inger \\
\hline 228 & Hemibagrus wyckii & Kottelar et al. 1993: PI. 32 \\
\hline 228 & Barbodes balleroides & Kottelar et al. 1993: PI. 8 \\
\hline 229 & Lobocheilos sp. & Kottelar et al. 1993: PI. 10 \\
\hline 229 & Tor tambra & Ike Rachmatika 1999 \\
\hline 230 & Pangasius sp. & Roberts and Vidthayanon 1991: 124 \\
\hline
\end{tabular}




\begin{tabular}{lll}
\hline $\begin{array}{l}\text { Page } \\
\text { No. }\end{array}$ & Genus Species & $\begin{array}{l}\text { Drawing Reference } \\
\text { and Copyright }\end{array}$ \\
\hline 230 & & Holthuis 1979: PI. 2. \\
231 & Macrobrachium sp. & Farfante and Kensley 1997: Titlepage \\
231 & Cipangopaludina sp. & Tweedie and Harrison 1954: 204 \\
232 & & Pinhey 1974: 7 \\
232 & & British Natural History Museum \\
233 & & Tweedie and Harrison 1954: 147 \\
233 & Mantis sp. & Little 1963: 137 \\
234 & & Pinhey 1974: 8 \\
234 & Phyllium sp. & Imms 1957: 337 \\
235 & Gerris remigis & Tweedie and Harrison 1954: 150 \\
235 & Tibicen sp. & Little 1963: 137 \\
236 & Lucanus sp. & Imms 1957: 784 \\
236 & Rhynchophorus ferrugineus & Tweedie and Harrison 1954: 141 \\
237 & Tenebroides sp. & Imms 1957: 798 \\
237 & Apis dorsata & Butler 1962: 66 \\
238 & & Imms 1957: 734 \\
238 & & British Natural History Museum \\
239 & & Imms 1957: 563 \\
239 & Attacus atlas & Imms 1957: 552 \\
240 & Anopheles sp. & Pinhey 1974: 15 \\
240 & Musca domestica & Pinhey 1974: 15 \\
\hline & & \\
\hline
\end{tabular}




\section{INTRODUCTION}

\section{Objectives}

This handbook presents both biological and ethnobiological data for 164 plant species and 111 animal taxa found in the rainforests, mountains, streams and cultivated areas of the Bulungan area in northern East Kalimantan, Indonesian Borneo (see Figure 1). While the actual number of plants and animals found in this area may be orders of magnitude greater, this book profiles those taxa most likely to be encountered in villages, markets, or in discussion with residents, traders, and local government officials. These taxa have been and mostly likely will continue to be prime subjects for scientific investigation, economic development, conservation initiatives, and government policy.

Figure 1. Map of the surveyed villages in the Bulungan area, East Kalimantan, Indonesia

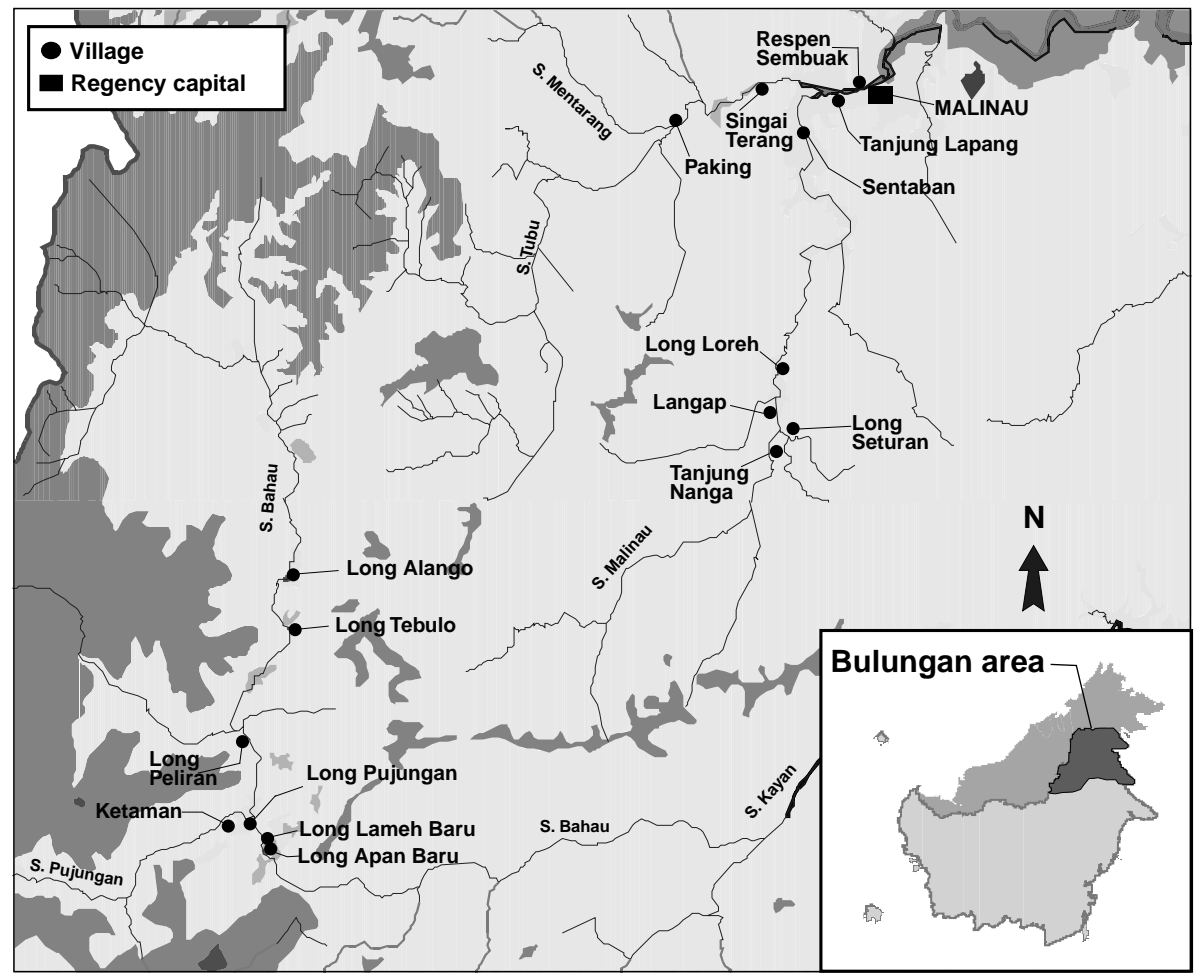


Intended as a resource for researchers working in the Bulungan area, including the Kayan Mentarang National Park (KM) and the Bulungan Research Forest (BRF), this handbook serves as both a field guide to common plants and animals, and a dictionary of their vernacular terms from 18 different linguistic/cultural groups. Also included is a vernacular glossary of related environmental vocabulary, such as terms for inclusive categories of plants and animals, geological, hydrological, and ecological features, and descriptive adjectives that often appear in the local names of plants and animals. A second aim in producing this book, and the dictionary in particular, is to facilitate communication about familiar flora and fauna among residents of the Bulungan. Indeed, many residents were quite excited to have their knowledge of plants and animals recorded for future generations as well as to promote cross-cultural communication. Finally, this book provides the basis for future analyses of regional patterns of resource use, ethnobiological classification, and comparative and historical linguistics. The author does not intend to present the complete ethnobiology of any of the surveyed groups, and thus one should be careful about using this material for comparative studies outside the data set presented here. The reasons for this result from the methodology used to collect the data.

\section{How to use this handbook}

The handbook is divided into several sections containing a variety of formats and types of data. The first two sections present profiles of 164 plants and 111 animal taxa. Each profile page consists of an entry with a photograh or illustration and both biological and ethnobiological data. In some profiles, data for some categories were not available, or found to be questionable, and therefore excluded. The absence of data should not be taken as evidence that a vernacular name or local use does not exist, rather, among the limited number of informants interviewed no information was forthcoming. The rationale for choosing the survey methods is explained in the section Documenting REGIONAL ETHNOBIOLOGY.

\section{Biological data}

Biological data include scientific names in the first line and common English and then Indonesian names in the second line. Next to a photograph or drawing of the taxa are descriptions of morphology (DESCRIPTION), habitat preference (HABITAT), geographical origin (ORIGINS), general uses (USES), and current protection status. Where uses were specific to one group, the code for that group is given next to the use. Most of this data has been abstracted from the published literature (references). Full citations for this literature is in References Cited. Profiles 
for most of the invertebrates are not of particular species, but instead of more inclusive categories at the rank of family or order, such as 'ants' or 'grasshoppers'.

\section{Ethnobiological data: Vernacular names}

Ethnobiological data for each profile are contained in a table below the biological data. The table contains information collected from 18 linguistic groups, identified by a three-letter language code (CODE). For each language, all the elicited vernacular names for each taxon are listed and can be assumed to be synonyms of names in use. The author does not claim to have elicited the "correct" vernacular name for any of the taxa investigated, and one should question the validity of such claims, especially in an area such as the Bulungan, where languages and isolects are closely related, where there is high intergenerational variability in vocabulary, and where people speak many languages. Researchers will have to determine which term is used among the people they stay with, and space is provided for revisions at the bottom of each profile. The meaning of some of these names can be translated by consulting the ENVIRONMENTAL GLOSSARY. For help in pronouncing the names please refer to NOTES ON LINGUISTICS AND ORTHOGRAPHY.

\section{Ethnobiological data: Uses and ecological information}

For the plant and mammal profiles, the table of local names also includes informants' responses to questions about uses (USE), common habitats and local abundance ( $\mathrm{AAB}-\mathrm{AB})$. The use categories are explained in Table 1; multiple uses were recorded. Where informants differed over habitat preferences and local abundance, all responses are included. No attempt was made to quantify informant variability. Readers should be clear that informants' responses might provide supporting or contradictory information to that derived from the scientific literature. Some taxa are well known, but seldom used today, so informants were also asked how recently the taxon in question had been used in their household (Recent Use or RU). A " $\mathrm{v}$ " indicates that a taxon was used by at least one informant's household during the past year. An " $\mathrm{x}$ " indicates that it was not used. Blank indicates no information.

\section{Icons}

A set of icons on the outer margins of each page summarizes the data presented for that taxon (Figure 2). The top icon identifies the lifeform of the taxon. The uses, habitat, and abundance icons, on the lower half of the page, represent an assessment of the ethnobiological data from all of the surveyed villages in the Bulungan area, and not the conditions found in any one location. 
Table 1. Explanation of plant and animal use categories

\section{PLANT USE CATEGORIES}

\begin{tabular}{|c|c|c|}
\hline 1. & Food & Plant parts consumed raw or cooked for food. \\
\hline 2. & Medicine & Plant parts used in a medicine or medical treatment. \\
\hline 3. & Heavy construction & Timber for house posts, beams, floors, walls, and roof shingles. \\
\hline 4. & Light construction & $\begin{array}{l}\text { Timber, poles, leafy roofing material for swidden field huts and } \\
\text { temporary forest shelters. }\end{array}$ \\
\hline 5. & Boat construction & Wood and resins used for wooden canoes, but not accessories. \\
\hline 6. & Household tools & $\begin{array}{l}\text { Paddles, punting poles, blowpipes, spear shafts, furniture, } \\
\text { handles for axes and farming tools, tools for processing rice and } \\
\text { sago palms; parts for storage of food. }\end{array}$ \\
\hline 7. & Firewood/fuel & Wood and resins burned for cooking, light or heat. \\
\hline 8. & Cordage/basketry: & $\begin{array}{l}\text { Vines, cane, bark or other material used as rope to tie and bind, } \\
\text { or used in the manufacture of baskets, backpacks, and mats. }\end{array}$ \\
\hline 9. & Cloth/dye & $\begin{array}{l}\text { Plant parts used to make traditional cloth or dyes for both cloth } \\
\text { and other materials. }\end{array}$ \\
\hline 10. & Hunting function & $\begin{array}{l}\text { Plants providing fish poison, dart poison, gums to entrap, } \\
\text { or bait for either terrestrial or aquatic animals; includes honey } \\
\text { trees. }\end{array}$ \\
\hline 11. & Sold & Plants or plant parts that are exchanged for cash. \\
\hline
\end{tabular}

\section{ANIMAL USE CATEGORIES}

\begin{tabular}{|l|l|l|l|}
\hline 1. & Food & Animal parts cooked for food, including cooking fat. \\
\hline 2. & Medicine & $\begin{array}{l}\text { Animal parts used in a medicine or medical treatment. } \\
\text { Animal parts used for tool handles, mats, packs, needles, } \\
\text { and rope. }\end{array}$ \\
\hline 3. & Tool & $\begin{array}{l}\text { Animal parts used in traditional loincloth, hats, skirts, and vests. } \\
\text { Animal parts used in traditional religious ceremonies, rites, } \\
\text { costumes; includes charms for good fortune and protection }\end{array}$ \\
\hline 4. & Cloth & Ritual & $\begin{array}{l}\text { Animals and animal parts used as trophies, jewellery, and } \\
\text { ornamentation. }\end{array}$ \\
\hline 6. & Decoration & Animals and animal parts used to hunt terrestrial or aquatic \\
\hline 7. & Bait & animals. \\
\hline 8. & Sold & Animals or animal parts that are exchanged for cash. \\
\hline 9. & Pet & $\begin{array}{l}\text { Wild and domesticated animals that are kept for amusement or } \\
\text { companionship, including those raised for eventual consump- }\end{array}$ \\
\hline tion or sale.
\end{tabular}


Figure 2. Icons used in this handbook

\section{PLANT}

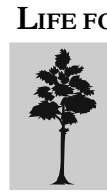

1. Tree

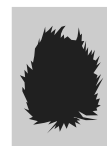

2. Shrub

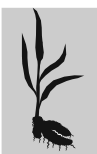

3. Herb

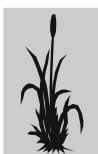

4. Grass

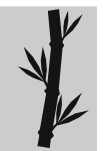

5. Bamboo

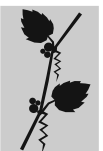

6. Vinelliana

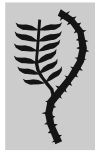

7.Climbing palms

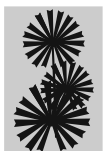

8. Tree \& fan palms

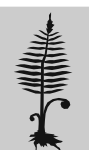

9. Fern

UsES

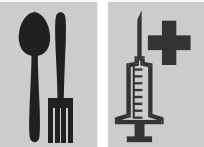

1.

2.

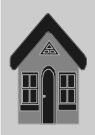

3. Heavy
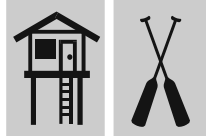

5. Boat

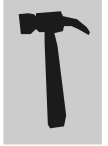

6.

struction struction struction hold tools

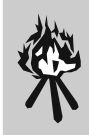

7. Fire-

wood /fuel

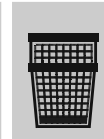

8.

Cordage/ basketry

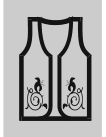

9.

Cloth/ Hunting

dye function

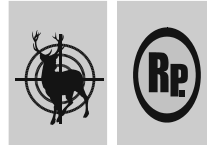

11.

Sold

\section{ANIMAL}

\section{LIFE FORM}

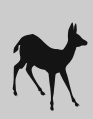

1. Mammal

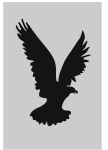

2. Bird

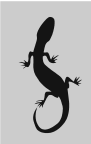

3. Reptile

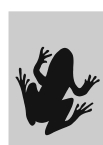

4. Amphibian

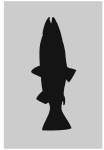

5. Fish

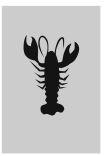

6. Arthropod (non-insect)

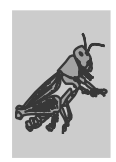

7. Insect

UsES

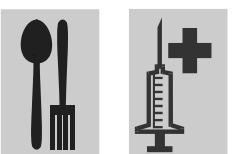

1. Food

2. Medicine

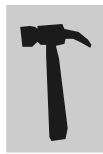

3. Tool

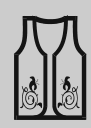

4. Cloth

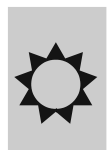

5. Ritual

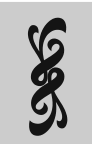

6. Decoration

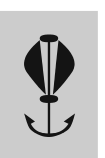

7. Bait

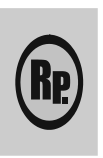

8. Sold

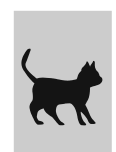

9. Pet

\section{HABITAT}

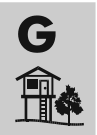

1.

Garden

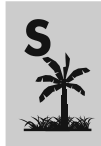

2. Swidden field

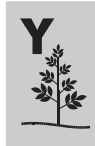

3. Young secondary

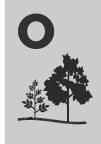

4. Old secondary

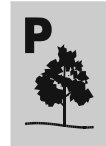

5. Primary forest

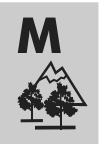

6. Mountain forest

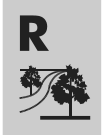

7. Riverine forest

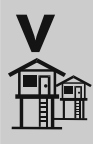

8. Village land

\section{ABUNDANCE}

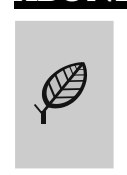

1. Rare

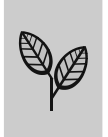

2. Few

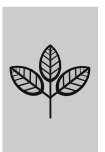

3. Average

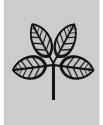

4. Very abundant

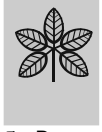

5. Dense

xix 


\section{Environmental glossary}

This section contains word lists for 120 environment-related terms, including all 18 language groups and Bahasa Indonesia. Included here are terms for features of the landscape (topographical, geological, hydrological and ecological), terms for more inclusive categories in animal and plant taxonomies, terms for plant and animal parts, and adjectives commonly found in plant or animal names. Blank spaces do not mean that a term does not exist for that language, rather, among the informants interviewed, no term has yet been discovered.

\section{Indexes}

The last section of the book is a series of indexes to scientific and vernacular names for the profiled plants and animals. The indexes allow researchers to discover whether a vernacular name is specific to a language and species, or refers to different scientific taxa within the same language or in other languages.

\section{Documenting regional ethnobiology}

The methods used to collect the information for this book derive from standard survey and interview techniques used in anthropology and ethnobiology (e.g., Bernard 1994; Martin 1995), and were in part inspired by Brent Berlin's vision of the comparative ethnobiologist hauling a portable museum of specimens, photographs, and other paraphernalia from village to village across the Amazon Basin (Berlin 1992: 267). Thus the principal methodology adopted here was to use a common set of stimuli to elicit names, uses and other information from a sample of residents representing all the large linguistic/cultural groups across the Bulungan area. The two critical decisions concerned which plants and animals to sample and who to interview.

It was not possible to sample all habitats and all taxonomic groups. Instead, the study concentrated on cultivated areas and forests close to villages, and those plants and animals with some documented salience for people in Bulungan. Published and unpublished materials from previous research in the area were reviewed to construct an initial list of plants and animals. Previous research by the author among several of these groups (Penan Benalui, Kenyah Badeng, Punan Tubu') provided the initial materials and data to design and conduct the research and assess its results (Puri 1997a, 1998a). Lists compiled by de Beer and McDermott (1996), Chin (1985), Kaskija (1991), Saccheri and Walker (1991), 
TAD (1981), and Wollenberg et al. (1999) were also consulted. Museum specimens, photos, and pictures of plants and animals on an initial list were collected.

Duplicate voucher specimens collected by staff at the Lalut Birai Forest Station in Kayan Mentarang National Park were borrowed. Pictures, especially of fruit, flowers or other colorful parts of identified specimens were included to aid in identification. Additional botanical specimens were collected or photographed, and the survey questionnaire pre-tested in several villages in the Pujungan and Malinau districts.

The plants thus chosen for this handbook are common, wild and cultivated species found in human settlements, cultivated areas, and nearby secondary and primary forest. There are species from all habitat types and most common lifeforms (see Figure 2). The profiled animals are those most commonly hunted, collected for sale, or recognized as important in some way by residents. However, there is a bias toward terrestrial animals and especially mammals, which are endangered because of their importance in local diets and their high market value. Fish species are undoubtedly as important as mammals for most people in Bulungan, but are under-represented in this study. The biological and ethnobiological literature for fish and fishing in Kalimantan was too poor to provide photographs or drawings, scientific identifications, and background information on more than a few common species. In fact, for all domains, the sample presented here is tiny compared to what might be encountered in even a few hectares of Bornean mature forest (see Table 2).

Table 2. Representativeness of the Bornean flora and fauna surveyed in this handbook

\begin{tabular}{|l|c|c|}
\hline & $\begin{array}{c}\text { Estimated Species } \\
\text { Richness in Borneo }\end{array}$ & $\begin{array}{c}\text { Species in this } \\
\text { Handbook }\end{array}$ \\
\hline Plants & $10,000-15,000$ & 164 \\
\hline Mammals & 222 & 41 \\
\hline Birds & 420 & 23 \\
\hline Lizards (Crocodiles incl.) & 74 & 7 \\
\hline Snakes (Land/Freshwater) & 104 & 6 \\
\hline Turtles (Land/Freshwater) & 13 & 3 \\
\hline Amphibians & 100 & 5 \\
\hline Fish (Freshwater) & 394 & 5 \\
\hline Arthropods (non-insects) & Not available & 5 families \\
\hline Insects & $600+$ families & 16 families \\
\hline
\end{tabular}

(Sources: MacKinnon et al. 1996: 47, Inger and Tan 1996.) 
Interviewing and fieldwork were conducted intermittently between February and August 1998, in three districts of the Bulungan (see Figure 1): Pujungan (in the mountainous western and inland side of the BRF), Malinau (on the southeastern and more coastal lowland side of the BRF) and Mentarang (a lowland area encompassing most of the BRF). The 18 language groups chosen for the survey are a sample of the total number of local languages spoken in the interior of this part of East Kalimantan (see Table 3). Priority was given to groups inhabiting current or future research areas surrounding the Bulungan Research Forest (BRF). Excluded were some populous linguistic groups such as the Kayan, the Berusu, the Tidung, most of the Kerayan languages, and several Kenyah subgroups, including the Leppo' Tau, Uma' Kulit, Uma' Baka and Uma' Jalan. Arabic, Chinese, Javanese, Buginese, Banjarese and languages from the Tana Toraja in Sulawesi are also spoken in the Bulungan area but are not included here.

Table 3. Villages in Bulungan Regency surveyed for this handbook

\begin{tabular}{|c|c|c|c|c|c|c|}
\hline $\begin{array}{l}\text { ID } \\
\text { No. }\end{array}$ & $\begin{array}{l}\text { Lang. } \\
\text { Code }\end{array}$ & Language & $\begin{array}{l}\text { Original } \\
\text { Village }\end{array}$ & $\begin{array}{l}\text { Original } \\
\text { District }\end{array}$ & $\begin{array}{l}\text { Current } \\
\text { Village }\end{array}$ & $\begin{array}{l}\text { Current } \\
\text { District }\end{array}$ \\
\hline 1 & PUT & Punan Tubu' & Menabur Besar & Mentarang & Respen Sembuak & Malinau \\
\hline 1 & PUT & Punan Tubu' & Bila Bekayuk & Mentarang & Long Loreh & Malinau \\
\hline 2 & PUB & Punan Beketan & Long Lakeh & Malinau & Long Loreh & Malinau \\
\hline 2 & PUB & Punan Beketan & Pelancau & Malinau & Long Loreh & Malinau \\
\hline 3 & PUD & Punan Derian & Sungai Rian & Malinau & Long Seturan & Malinau \\
\hline 4 & PUM & Punan Mentarang & Long Iman & Mentarang & Paking & Mentarang \\
\hline 5 & ALS & Abai & Sentaban & Malinau & Sentaban & Malinau \\
\hline 6 & LDY & Lun Daye & Long Sulit & Mentarang & Singai Terang & Mentarang \\
\hline 6 & LDY & Lun Daye & Long Gafit & Mentarang & Singai Terang & Mentarang \\
\hline 7 & LIT & Lengilu' & Lg. Sepayang & Kerayan & Tanjung Lapang & Malinau \\
\hline 8 & BRU & Berau & Long Bila & Malinau & Sentaban & Malinau \\
\hline 9 & MRP & Merap & Sengayan & Malinau & Long Loreh & Malinau \\
\hline 9 & MRP & Merap & Langap & Malinau & Langap & Malinau \\
\hline 10 & PTN & Pua' & Tanjung Nanga & Malinau & Tanjung Nanga & Malinau \\
\hline 11 & KLK & Leppo' Ké & Long Lat & Pujungan & Long Loreh & Malinau \\
\hline 11 & KLK & Leppo' Ké & Long Lat & Pujungan & Long Tebulo & Pujungan \\
\hline 12 & KLM & Leppo' Ma'ut & Long Alango & Pujungan & Long Alango & Pujungan \\
\hline 13 & KLO & Uma' Long & Long Sa'an & Pujungan & Long Pujungan & Pujungan \\
\hline 14 & KLA & Uma' Lasan & Long Pujungan & Pujungan & Long Pujungan & Pujungan \\
\hline 15 & KAL & Uma' Alim & Ketaman & Pujungan & Ketaman & Pujungan \\
\hline 16 & KBD & Uma' Badeng & Long Peliran & Pujungan & Long Peliran & Pujungan \\
\hline 17 & KBK & Uma' Bakung & Long Apan & Pujungan & Long Apan Baru & Pujungan \\
\hline 18 & PEB & Penan Benalui & Long Lameh & Pujungan & Long Apan Baru & Pujungan \\
\hline
\end{tabular}


Even with such a small sample of the region's biota, the survey questionnaire was still too time consuming to interview many informants separately, so it was decided to conduct interviews with groups of men and women (usually no more than three or four). Throughout the Bulungan area, residents expressed concerns that the "correct" answers and pronunciations, as far as could be known, be recorded for posterity. Rather than attempt to survey all age sets, only elders considered by the local residents to be experts in various domains were consulted. The only drawback to this approach was that many of these elders no longer hunt or travel far from their villages, making them questionable sources for information on present day use of these taxa. As it turns out, interviews with elders were seldom uninterrupted by curious spectators adding or correcting information about current use or recent hunting captures.

The groups were separated, one being interviewed about animals and the other about plants. The informants provided all known vernacular names, uses (by plant and animal part), habitat preferences, local assessment of abundance and distribution, and self-reports on how recently the item had been used. At least one group was asked to provide basic environment-related vocabulary. After a lunch break, the groups switched topics from plants to animals or vice versa. Depending on the informants, each interview session could last from three to six hours. Assuming all the arrangements for the interviews were made the night before, the two groups could be interviewed in one day. This was rarely achieved, however, and it usually took two to three days to finish interviews in one village.

One weakness in this survey method was the limited sample of informants (albeit elders of both sexes) that could be interviewed for each language group. As a result, there is a good probability that some synonyms were missed, and that some of those terms elicited are not widely known or scarcely used anymore. A second weakness was not being able to observe the daily use of resources in the surveyed villages in order to corroborate informants' reports. Both of these weaknesses are consequences of the rapid and extensive nature of the survey method. For additional information, recent studies of resource use practices in this part of the Bulungan area can be found in de Beer and McDermott (1996), Kaskija (1995), Puri (1997a, 1998a), Sorensen and Morris (1997), and Wollenberg et al. (1999). 


\section{Environment and people of the Bulungan area}

The Bulungan area, at roughly 64000 sq. km with a 1996 population of 288499 (Badan Pusat Statistik 1997), stretches from the islands of Tarakan, Bunyu, Nunukan, and Sebatik in the Straits of Makassar, more than $200 \mathrm{~km}$ to the mountainous spine that separates Sarawak from East Kalimantan. To the north is the mountainous border with Sabah, while the southern border follows the mountains separating the Kayan River valley from the Berau River and upper Mahakam River valleys (Figure 1). This area encompasses three major river systems, the Kayan, Sesayap, and Sembakung, that flow from the mountainous interior, converge and empty into the muddy delta surrounding the above mentioned islands. This coastal area of mangrove, peat swamps, and heath forests gives way to lowland plain with tropical dipterocarp forest and cultivated lands. Roughly 50 $\mathrm{km}$ from the coast, the steep hills and mountains rise up to between $1000 \mathrm{~m}$ 1,500 $\mathrm{m}$ with several peaks above $2000 \mathrm{~m}$. The mountains have a mix of moist to wet hill dipterocarp and oak-laurel forests, with some montane forests on the isolated peaks. Cultivated areas, including gardens, orchards, and rice swiddens, and old fallow and secondary forests are abundant along large rivers, on mountain plateaux, and wherever there is relatively level ground.

The area's main towns are located in the lowlands close to the coast: Tanjung Selor on the Kayan River, and Malinau on the Sesayap River. Ships and ferries link these towns with the populous island centres of trade and industry, Tarakan and Nunukan. Tanjung Selor and Malinau are gateway towns to the interior of Bulungan, and were in fact the seats of sultanates that controlled the trade in forest products from the interior until the 20th century (Magenda 1991; Peluso 1983; Warren 1981). Even today their strategic importance has not been lost, as they remain centres of trade and seats of local government. Both of these towns can receive moderately large ships carrying oil, large machinery, vehicles, and other goods and materials. Upriver travel is primarily by small river taxis and speedboats. River travel and trade with the far interior is dependent on regular flooding which enable the large longboats to pass over the many rapids. With average travel conditions most inhabited areas can be reached in three to four days from Tanjung Selor or Malinau. Prolonged drought periods can result in these interior areas being nearly cut off from the coast.

Only in the last few years has road construction reached Bulungan. An unpaved road now runs from Tanjung Selor to Tanjung Redeb (on the Berau River) and on to Samarinda, the provincial capital of East Kalimantan. Another road, 
requiring the construction of bridges over the Kayan and Malinau Rivers, will link Tanjung Selor, Malinau, and Lumbis (close to the border with Sabah). There are former logging roads that are being transformed into public roads, such as the one linking the upriver towns of the Malinau and mining and logging camps with the urban and administrative centre in the town of Malinau. Logging roads extend all the way up the Kayan River to the inland towns of Long Peso and Long Pujungan. The villages on the two high altitude plateaux of the area, the Apo Kayan and the Kerayan, are each internally linked by walking paths, but remain cut off from the eastern lowlands. Small aircraft now service these areas, but the flight costs are so high that it is cheaper for residents to walk into neighbouring Sarawak to buy supplies and trade forest products.

The indigenous Dayak groups of Bulungan practice agriculture and use forest resources in a wide variety of ways, including the cultivation of irrigated rice fields, mixed crop swiddens, tuber gardens and small plantations of such cash crops as banana, coffee, cacao, and cinnamon. They manage and harvest forest-grown fruit trees, sago palms, rattan, and aromatic woods for trade. Food, materials, and trade items are also obtained by fishing, hunting, trapping, and gathering (Puri 1997a). In a survey of five villages, Wollenberg et al. (1999) conservatively estimate between 139 and 214 wild and cultivated taxa per village were harvested and used in a one-year period. In a biodiversity assessment survey with Punan Tubu', 330 plant taxa were reported to have uses (Puri 1998a). The diversity of subsistence practices is found not only on a regional scale, but even within single communities, where people often combine or switch between different forms of resource use. The population in the interior is decreasing as families and even whole communities move down-river toward the larger towns on the coast such as Malinau and Tanjung Selor. The primary reasons for the migration appear to be a desire to be near cheaper and larger markets, higher education, better healthcare, and a livelier social atmosphere.

\section{Notes on linguistics and orthography}

The ethnobiological data was collected from informants representing 18 different indigenous ethnic groups of the Bulungan area. However, these groups do not represent 18 distinct languages. There are seven subgroups of the Dayak Kenyah language. The Pua' language appears to be a Kenyah-Kayan variant, as it shares elements of both groups. The Punan of the Malinau, Tubu', and Mentarang can be considered subgroups of the same Punan language. Abai, Merap, Berau, 
Lengilu', Lun Daye, and Penan Benalui are distinct enough to warrant status as separate languages, although all share vocabulary with past and present neighboring groups. A long history of migration, intermarriage, and political and economic alliance building have led to constant interaction among these groups with the result that words have been borrowed and languages have come to be shared and even hybridized in some cases. For instance, Merap shows evidence of Ngorek, Kayan, Kenyah, and Punan Tubu' influences. The Ngorek people inhabited the Pujungan district before the arrival of the Kayan in the $18^{\text {th }}$ century. Their language is now extinct in East Kalimantan, although its descendants are recognized in the Hueng Bau language of the Kayan River and the Murik language of the Baram River in Sarawak.

All of the languages surveyed for this book belong to the Western branch of the Malayo Polynesian language family known as Austronesian. In particular they belong to the Kayan-Kenyah group in Central Borneo, and share similarities in phonology, vocabulary, and grammar. However, each subgroup or language contains characteristic phonemes that often identify and distinguish it from others. Some of these phonemes are difficult to write without resorting to the complicated diacritical marks of the International Phonetic Alphabet. Since this book is meant to be used by scientists and local people in the field, rather than as a source for study in regional or historical linguistics, some linguistic accuracy has been sacrificed in favor of a user-friendly format. Vernacular terms are written in the wellknown orthography used to write Malay dialects, such as Bahasa Indonesia, the national language. A few vowels have varying pronunciation across the region, but most terms are written as spoken using the orthography presented below. This makes it possible for local people and others not trained in linguistics to read and speak the terms presented here, although non-native speakers will have to learn local variants in pronunciation. The following letter characters are used to represent phonemes seldom encountered by an English speaker:

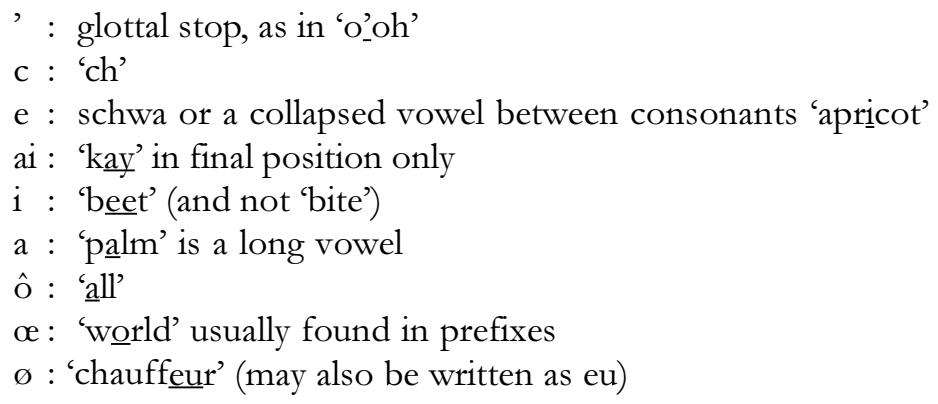




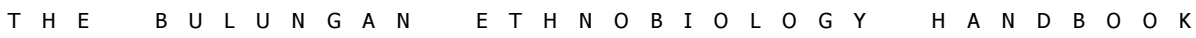

\section{References cited}

Ahern, G.P. 1901. Compilation of notes on the most important timber tree species of the Philippine Islands. Forestry Bureau, Manila, Philippines.

Airy-Shaw, H.K. 1953. Thymelaeaceae-Gonystyloideae. Flora Malesiana Series 1, Volume 4(4): 349-365. Rijksherbarium/Hortus Botanicus, Leiden University, Leiden, The Netherlands.

Ashton, P.S. 1982. Dipterocarpaceae. Flora Malesiana Series I, Volume 9(2). Rijksherbarium/Hortus Botanicus, Leiden University, Leiden, The Netherlands.

Backer, C.A. and van den Brink, R.C. Bakhuizen. 1963-68. Flora of Java (Spermatophytes only). Three Volumes. Groningen, O. Noordhoff.

Backer, C.A. and van Slooten, D.F. 1924. Geillustreerd Handboek der Javaansche Theeonkruiden. Drukkerijen Ruygrok \& Co, Batavia.

Badan Pusat Statistik. 1997. Kabupaten Bulungan dalam angka tahun 1996. Kantor Statistik Kabupaten Bulungan. Tanjung Selor, Kalimantan Timur.

Basu, B.D., 1918. Indian Medicinal Plants. Bahadurganj, Allahabad: S.N. Basu. The Panini Office.

Bennett, D. 1998. Monitor Lizards: Natural history, biology and husbandry. $2^{\text {nd }}$ ed. Frankfurt am Main: Edition Chimaira.

Berlin, B. 1992. Ethnobiological classification systems. Princeton University Press, Princeton.

Bernard, H.R. 1994. Research methods in anthropology. Sage, Thousand Oaks, California, USA.

Bremer, K. 1983. Taxonomy of Memecylon (Melastomataceae) in Borneo. Opera Botanica 69: 1-47.

Burkill, I.H. 1966. A dictionary of the economic products of the Malay Peninsular. $2^{\text {nd }}$ ed. 2 Volumes. Minister of Agriculture and Cooperatives, Kuala Lumpur, Malaysia.

Butler, C. G. 1962 (1954). The world of the honeybee. Revised edition. London: Collins.

Chin, H.F. and Yong, H.S. 1980. Malaysian fruits in color. Tropical Press Sdn. Bhd, Kuala Lumpur, Malaysia.

Chin, S.C. 1985. Agriculture and resource utilization in a lowland rainforest Kenyah community. Sarawak Museum Journal. Special Monograph No. 4. 
Clutton-Brock, J. 1999. A natural history of domesticated mammals. $2^{\text {nd }}$ ed. The Natural History Museum and Cambridge University Press, Cambridge, UK.

Cockburn, P.F. 1976. Trees of Sabah: Volume one. Sabah Forest Record No. 10. Borneo Literature Bureau for Forest Department Sabah, Malaysia.

Cockburn, P.F. 1981. Trees of Sabah: Volume two. Sabah Forest Record No. 10. Dewan Bahasa dan Pustaka Cawangan Sarawak for Forest Department Sabah.

Corbet, G. B. and Hill, J. E. 1992. The mammals of the Indomalayan region: a systematic review. Oxford: Oxford University Press.

Corner, E.J.H. 1988. Wayside trees of Malaya. $3^{\text {rd }}$ ed. Two Volumes. The Malaysian Nature Society. United Selangor Press, Kuala Lumpur, Malaysia.

Corner, E.J.H. and Watanabe, K. 1969. Illustrated guide to tropical plants. Hirokawa Publishing Co., Tokyo, Japan.

Cranbrook, Earl of, and Edwards, D.S. 1994. A tropical rainforest: the nature of biodiversity in Borneo at Belalong, Brunei. Sun Tree Publishing and the Royal Geographical Society, Singapore.

Davison, G.W.H. and Chew Yen Fook. 1996. A photographic guide to birds of Borneo. New Holland, London, UK.

de Beer, J.H. and McDermott, M.J. 1996. The economic value of non-timber forest products in Southeast Asia. 2nd ed. IUCN, Amsterdam, The Netherlands.

de Guzman, C.C. and Siemonsma, J.S. (eds.) 1999. Plant resources of SouthEast Asia No 13. Spices. Backhuys Publishers, Leiden, The Netherlands.

de Padua, L.S., Bunyapraphatsara, N. and Lemmens, R.H.M.J. (eds.) 1999. Plant resources of South-East Asia No. 12(1). Medicinal and poisonous plants 1. Backhuys Publishers, Leiden, The Netherlands.

Ding Hou, 1978. Anacardiaceae. In: van Steenis, C.G.G.J. (general editor), Flora Malesiana. Series I, Volume 8 part 3: 301-577. Alphen aan den Rijn, the Netherlands: Sijthoff \& Noordhoff International Publishers.

Dransfield, J. and Manokaran, N. (eds.) 1994. Plant resources of South-East Asia No. 6. Rattans. Backhuys Publishers, Leiden, The Netherlands.

Dransfield, S. 1992. The bamboos of Sabah. Sabah Forest Records, No. 14. Forest Department, Sabah, Malaysia.

Dransfield, S. and Widjaja, E.A. (eds.) 1995. Plant resources of South-East Asia No. 7. Bamboos. Backhuys Publishers, Leiden, The Netherlands. 
Elzinga, R.J. 1997. Fundamentals of entomology. $4^{\text {th }}$ ed. Prentice-Hall, Upper Saddle River, New Jersey.

Ernst, C.H. and Barbour, R.W. 1989. Turtles of the world. Smithsonian Institution Press, Washington, DC.

Farfante, I.P. and Kensley, B. 1997. Penaeoid and sergestoid shrimps and prawns of the world. Memoires du Museum National d'Histoire Naturelle, No. 175. Paris.

Faridah Hanum, I. and van der Maesen, L.J.G. (eds.) 1997. Plant resources of South-East Asia No. 11. Auxiliary plants. Backhuys Publishers, Leiden, The Netherlands.

Flach, M. and Rumawas, F. (eds.) 1996. Plant resources of South-East Asia No. 9. Plants yielding non-seed carbohydrates. Backhuys Publishers, Leiden, The Netherlands.

Flora Sinicae, see Kiu 1996.

FM I Vol. 4(4), see Airy-Shaw, H. K. 1953.

FM I Vol. 7(2), see Soepadmo 1972.

FM I Vol. 8(3), see Ding Hou 1978.

FM I Vol. 9(2), see Ashton, P.S. 1982.

FOJ, see Backer, C.A. 1963-68.

Francis, C.M. 1984. Pocket guide to the birds of Borneo. The Sabah Society, Kota Kinabalu, Sabah, Malaysia.

Glasby, C.J., Ross, G.J.B. and Beesley, P.L. (eds.) 1993. Fauna of Australia. Vol. 2A Amphibia and Reptilia. Australian Government Publishing Service, Canberra.

Grubben, G.J.H. and Partohardjono, S. (eds.) 1996. Plant resources of SouthEast Asia No. 10. Cereals. Backhuys Publishers, Leiden, The Netherlands.

Heyne, K. 1927. De nuttige planten van Nederlands-Indie [The useful plants of the Dutch East Indies]. 2nd ed. 3 volumes. Departement van Landbouw, Nijverheid en Handel in Nedelandsch-Indie, 's-Gravenhage.

Ho, Pham-hoang. 1992. Cayco Vietnam (an illustrated flora of Vietnam). Mekong Printing, Santa Ana, California, USA.

Holthuis, L.B. 1979. Cavericolous and terrestrial decapod Crustacea from Northern Sarawak, Borneo. Zoologische Verhandelingen (Leiden) No. 171: $1-47$.

Imms, A. D. 1957 (1925). A General textbook of Entomology. Ninth edition. London: Methuen \& Co. Ltd. 
Inger, R.F. and Chin, P.K. 1962. The fresh-water fishes of North Borneo. Fieldiana: Zoology 45: 1-268.

Inger, R.F. and Tan Fui Lian. 1996. The natural history of amphibians and reptiles in Sabah. Natural History Publications (Borneo) Sdn. Bhd, Kota Kinabalu, Sabah, Malaysia.

Kanehira, R. 1936. Formosan trees indigenous to the island. Department of Forestry, Government Research Institute, Taihoku, Formosa, Taiwan.

Kaskija, L. 1991. Animals and plants in bahasa Punan Malinau. Manuscript. Anthropology Department, University of Uppsala, Sweden.

Kaskija, L. 1995. Punan Malinau: the persistence of an unstable culture. Masters thesis, Anthropology Department, University of Uppsala, Sweden.

Kessler, P.J.A. and Sidiyasa, K. 1994. Trees of Balikpapan-Samarinda area, East Kalimantan, Indonesia: a manual to 280 selected species. Tropenbos Series No. 7. Tropenbos Foundation, Wageningen, The Netherlands.

King, B.F., Dickinson E.C., and Woodcock, M.W. 1975. A field guide to the birds of Southeast Asia. Collins, London, UK.

Kiu Huashing, (ed.) 1996. Euphorbiaceae (2). Flora Reipublicae Popularis Sinicae. Volume 44 (2). Science Press.

Kochummen, K.M. 1997. Tree Flora of Pasoh Forest. Malayan Forest Records No. 44. Forest Research Institute Malaysia, Kuala Lumpur.

Koorders, S.H. 1921-1924. Exkursionsflora von Java. Atlas. 4 volumes. Jena: Verlag von Gustav Fischer.

Koorders, S.H. and Valeton, T. 1913. Atlas der Baumarten von Java. Volume 1. Buch- und Steindruckerei von Fa. P. W. M. Trap, Leiden, The Netherlands.

Kottelat, M., Whitten, A.J. and Kartikasari, N. 1993. Freshwater fishes of western Indonesia and Sulawesi. Periplus Editions, Singapore.

Kraemer, J.H. 1951. Trees of the Western Pacific Region. West Lafeyette, Indiana, USA

Lembaga Biologi Nasional (LBN)-LIPI. 1978. Palem Indonesia. Proyek Sumber Daya Ekonomi No. 54. LBN-LIPI, Bogor, Indonesia.

Leaman, D. J., Yusuf, R., and Sangat-Roemantyo, H. 1991. Kenyah Dayak Forest Medicines. Jakarta: World Wide Fund for Nature-Indonesia Programme.

Lemmens, R.H.M.J. and Wulijarni-Soetjipto, N. (eds.) 1992. Plant resources of South-East Asia No. 3. Dye and tannin-producing plants. Backhuys Publishers, Leiden, The Netherlands. 
Lemmens, R.H.M.J., Soerianegara I. and Wong, W.C. (eds.) 1995. Plant resources of South-East Asia No. 5(2). Timber Trees: Minor commercial timbers. Backhuys Publishers, Leiden, The Netherlands.

Little, V. A. 1963. General and applied entomology. Second Edition. New York: Harper and Row.

MacKinnon, J.R. and Phillipps, K. 1993. A field guide to the birds of Borneo, Sumatra, Java, and Bali, the Greater Sunda Islands. Oxford University Press, Oxford, UK.

MacKinnon, K., Gusti, H., Halim, H. and Mangalik A. 1996. The ecology of Kalimantan. The Ecology of Indonesia Series, Volume III. Periplus Editions, Singapore.

Maesen, L.J.G. van der and Somaatmadja, S. (eds.) 1992. Plant resources of South-East Asia No. 1. Pulses. Backhuys Publishers, Leiden, The Netherlands.

Magenda, B. 1991. East Kalimantan: the decline of a commercial aristocracy. Monograph Series No. 70. Cornell Modern Indonesia Project, Ithaca, New York, USA

Mannetje, L. and Jones, R.M. (eds.) 1992. Plant resources of South-East Asia No. 4. Forages. Backhuys Publishers, Leiden, The Netherlands.

Martin, G.J. 1995. Manual of ethnobotany methods. People and Plants Initiative, WWF/KEW/UNESCO, London, UK.

Medway, L. 1977. Mammals of Borneo. Monographs of the Malaysian Branch of the Royal Asiatic Society No.7. MBRAS, Kuala Lumpur, Malaysia.

Meijer, W. 1974. Field guide to trees of West Malaysia. Self-published.

Newman, M.F., Burgess, P. F., and Whitmore, T. C. 1996. Borneo island light hardwoods: Anisoptera, Parashorea, Shorea (Red, White and Yellow Meranti). Manuals of Dipterocarps for Foresters. Royal Botanic Garden Edinburgh and Center for International Forestry Research, Jakarta.

Ng, F.S.P. (ed.) 1978. Tree flora of Malaya: a manual for foresters. Volume 3. Longman, Kuala Lumpur, Malaysia.

Ng, F.S.P. (ed.) 1989. Tree flora of Malaya: a manual for foresters. Volume 4. Longman, Kuala Lumpur, Malaysia.

Ng, P.K.L. 1988. The freshwater crabs of Peninsular Malaysia and Singapore. The Department of Zoology, National University of Singapore, Singapore.

O’Brien, T.G., (ed.) 1998. Bulungan biodiversity survey. Preliminary report prepared for the Center for International Forestry Research (CIFOR), Bogor, Indonesia. 
Ochse, J.J. 1927. Indische Vruchten. Volkslectuur-Weltevreden.

Ochse, J.J. and Bakhuizen van den Brink, R.C. 1980. Vegetables of the Dutch East Indies. Third English Edition. Asher \& Co. (Translation of 'Indische Groenten', 1931. Buitenzorg.), Amsterdam, The Netherlands.

Oyen, L.P.A. and Nguyen Xuan Dung, (eds.) 1999. Plant resources of SouthEast Asia No. 19. Essential-oil plants. Backhuys Publishers, Leiden, The Netherlands.

Payne, J.B., Francis, C.M. and Phillips, K. 1985. A field guide to the mammals of Borneo. Kuala Lumpur, Malaysia, Sabah Society \& World Wildlife Fund Malaysia.

Peluso, N.L. 1983. Markets and merchants: the forest products trade of East Kalimantan in historical perspective. Masters Thesis, Cornell University, Ithaca, New York.

Pinhey, E. C. G. 1974. A Guide to Insects of Africa. London: Hamilyn.

PROSEA Vol. 1, see Maesen and Somaatmadja, 1992.

PROSEA Vol. 2, see Verheij and Coronel, 1992.

PROSEA Vol. 3, see Lemmens and Wulijarni-Soetjipto, 1992.

PROSEA Vol. 4, see Mannetje and Jones, 1992.

PROSEA Vol. 5(1), see Soerianegara and Lemmons, 1994.

PROSEA Vol. 5(2), see Lemmens et al., 1995.

PROSEA Vol. 5(3), see Sosef et al., 1998.

PROSEA Vol. 6, see Dransfield, J. and Manokaran, 1994.

PROSEA Vol. 7, see Dransfield, S. and Widjaja, 1995.

PROSEA Vol. 8, see Siesmonsma and Piluek, 1994.

PROSEA Vol. 9, see Flach and Rumawas, 1996.

PROSEA Vol. 10, see Grubben and Partohardjono, 1996.

PROSEA Vol. 11, see Faridah Hanum and van der Maesen, 1997.

PROSEA Vol. 12(1), see de Padua et al., 1999.

PROSEA Vol. 13, see de Guzman and Siemonsma, 1999.

PROSEA Vol. 19, see Oyen and Nguyen, 1999.

Puri, R.K. 1997a. Hunting knowledge of the Penan Benalui of East Kalimantan, Indonesia. Ph.D. Dissertation, Department of Anthropology, University of Hawaii, Honolulu. 
Puri, R.K. 1997b. Penan Benalui knowledge and use of treepalms. In: Sorensen, K.W. and Morris, B. (eds.) People and Plants of Kayan Mentarang, 194226. WWF-IP/UNESCO, London, UK

Puri, R.K. 1998a. The biodiversity and ethnoecology of the Punan Tubu': a preliminary study. Consultancy report prepared for the Center for International Forestry Research and Wildlife Conservation Society, Bogor, Indonesia.

Puri, R.K. 1998b. An emerging NTFP market and its future prospects: the case of the fruit 'mata kucing' (Dimocarpus longan) in East Kalimantan. Manuscript. Center for International Forestry Research, Bogor, Indonesia.

Roberts, T.R. and Vidthayanon, C. 1991. Systematic revision of the Asian catfish family Pangasiidae, with biological observations and descriptions of three new species. Proceedings of the Academy of Natural Sciences of Philadelphia Vol. 143: 97-143.

Saccheri, I. and Walker. D. 1991. Subsistence and environment of a highland Kenyah community, Sarawak Museum Journal Vol. 42 (N.S.) No. 63: 193249.

Sastrapradja, S. et al. 1977a. Sayur-sayuran. Proyek Sumber Daya Ekonomi No. 39. Lembaga Biologi Nasional - LIPI, Bogor, Indonesia. (English Translation: Soetjipto, N.W. and Lubis, S.H.A. 1981. Vegetables. IBPGR Secretariat, Rome, Italy.)

Sastrapradja, S. et al. 1977b. Ubi-ubian. Proyek Sumber Daya Ekonomi No. 40. Lembaga Biologi Nasional - LIPI, Bogor, Indonesia. (English Translation: Prana, M.S. and S. Danimihardja. 1981. Root and Tuber Crops. IBPGR Secretariat, Rome, Italy.)

Sastrapradja, S. et al. 1977c. Buah-buahan. Proyek Sumber Daya Ekonomi No. 41. Lembaga Biologi Nasional - LIPI, Bogor, Indonesia.(English Translation: Rifai, M.A. and I. Lubis. 1980. Fruits. IBPGR Secretariat, Rome, Italy.)

Siemonsma, J.S. and Piluek, K. (eds.) 1994. Plant resources of South-East Asia No. 8. Vegetables. Backhuys Publishers, Leiden, The Netherlands.

Smythies, B.E. 1981. The birds of Borneo. $3^{\text {rd }}$ ed. Sabah Society \& Malay Nature Society, Kota Kinabalu, Malaysia.

Soepadmo, E., Wong, K. M., and Saw, L. G. (eds.) 2000. Tree Flora of Sabah and Sarawak. Volume 2. Kuala Lumpur: Forest Research Institute Malaysia.

Soepadmo, E. 1972. Fagaceae. Flora Malesiana Series I, Volume 7 (2). Rijksherbarium/Hortus Botanicus, Leiden University, Leiden, The Netherlands. 
Soepadmo, E. and Wong, K.M. (eds.) 1995. Tree flora of Sabah and Sarawak. Volume I. Sabah Forestry Dept., Forest Research Institute, Sarawak Forestry Dept, Kuala Lumpur, Malaysia.

Soerianegara, I. and Lemmens, R.H.M.J. (eds.) 1994. Plant resources of SouthEast Asia No. 5(1). Timber Trees: Major commercial timbers. Backhuys Publishers, Leiden, The Netherlands.

Sorensen, K.W. and Morris, B. (eds.) 1997. People and Plants of Kayan Mentarang. WWF-IP/UNESCO, London, UK.

Sosef, M.S.M., Hong, L.T. and Soerianegara, I. (eds.) 1998. Plant resources of South-East Asia No. 5(3). Timber trees: Lesser-known timbers. Backhuys Publishers, Leiden, The Netherlands.

Suyanto, A., Yoneda, M., Maryanto, I., Maharada-tunkamsi, and Sugardjito, J. 1998. Checklist of the mammals of Indonesia. LIPI and JICA, Bogor, Indonesia.

TFM Vol. 1, see Whitmore, 1983.

TFM Vol. 2, see Whitmore, 1983.

TFM Vol. 3, see Ng, 1978.

TFM Vol. 4, see Ng, 1989.

TFSS Vol. 1, see Soepadmo and Wong, 1995.

TFSS Vol. 2, see Soepadmo et al., 2000.

Transmigration Area Development (TAD). 1981. Forest for food, Phase 1. TAD - Materielen 11, TAD, Samarinda, Indonesia.

Tweedie, M.W.F. and Harrison, J.L. 1954. Malayan animal life. Longmans, Green and Co., London, UK.

Valkenburg, J.L.C.H. van. 1997. Non-timber forest products of East Kalimantan: Potentials for sustainable forest use. Tropenbos Series No. 16. Tropenbos Foundation, Wageningen, The Netherlands.

Verheij, E.W.M. and Coronel, R.E. (eds.) 1992. Plant resources of SouthEast Asia No. 2. Edible fruits and nuts. Backhuys Publishers, Leiden, The Netherlands.

Warren, J.F. 1981. The Sulu Zone 1768-1898. Singapore University Press, Singapore.

Whitmore, T.C. (ed.) 1983. Tree flora of Malaya: a manual for foresters. Volumes 1 and 2. $2^{\text {nd }}$ ed. Forest Department, Malayan Forest Records, Longman, Kuala Lumpur, Malaysia. 
Whitmore, T.C. 1985. Palms of Malaya. $3^{\text {rd }}$ ed. Oxford University Press, Oxford, UK.

Wollenberg, E., Nawir, A.S., Uluk, A. and Pramono, H. 1999. Income is not enough: The effect of economic incentives on forest conservation. Draft Report. Center for International Forestry Research (CIFOR), Bogor, Indonesia.

Yasuma, S. 1994. An Invitation to the Mammals of East Kalimantan. Tropical Rainforest Research Center (PUSREHUT), Universitas Mulawarman. Special Publication No. 3. Samarinda, East Kalimantan, Indonesia.

Zug, G.R. 1993. Herpetology. Academic Press, Inc., San Diego, USA. 


\section{Gendarussa vulgaris Nees. (Acanthaceae)}

\section{GENDARUSSA. GANDARUSA}

\section{DESCRIPTION}

Shrubby herb, propagated by cuttings; young stems violet, old ones light brown; leaves opposite, lanceolate, thin, glabrous, violet nerves, 5-20 x 1-3.5 $\mathrm{cm}$; inflorescence $3-12 \mathrm{~cm}$ long, flowers violet

\section{HABITAT}

Wild varieties in wastelands, edges, fallows; cultivated varieties in home and swidden gardens

\section{ORIGINS}

Possibly China

\section{USES}

Leaves for medicine; ornamental plant; fish poison (KLM)

\section{REFERENCES}

Burkill 1966: 1082; FOJ Vol 2: 589;

PROSEA Vol 12(1): 330

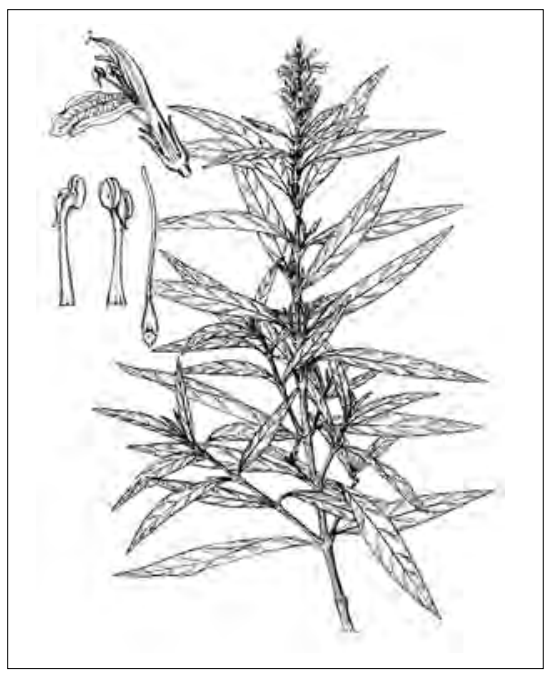

\begin{tabular}{|c|c|c|c|c|c|}
\hline \# & Code & Local Name & Use & RU & Hab.-Ab. \\
\hline 1 & PUT & uru' seniom, uru' entuh & 2 & $\mathrm{v}$ & $\mathrm{R}$ \\
\hline 2 & PUB & puli urip, uru urip & 2 & $x$ & G-3, R \\
\hline 3 & PUD & uru senom & 2 & $x$ & G-3, R \\
\hline 4 & PUM & uru nduh & 2 & $x$ & G-1 \\
\hline 5 & ALS & tunob penganan & & $x$ & ALL \\
\hline 6 & LDY & tunob bata, tuneb bata & 2 & v & ALL \\
\hline 7 & LIT & petuneb, petunap & 2 & v & $\mathrm{S}-2, \mathrm{Y}-2$ \\
\hline 8 & BRU & bungsie & & $x$ & ALL \\
\hline 9 & MRP & luru wai, luroi wey, lurui wey & 2 & $x$ & $\mathrm{R}$ \\
\hline 10 & PTN & uro' bengai, kelanjabi'i & & $x$ & $\mathrm{R}$ \\
\hline 11 & KLK & parang dakau, lan lami & & $x$ & $\mathrm{R}$ \\
\hline 12 & KLM & tubo sanit, balang lakau & 10 & $x$ & $\mathrm{R}$ \\
\hline 13 & KLO & tobo saniek & & $x$ & $\mathrm{R}$ \\
\hline 14 & KLA & tuba sanit & & $x$ & $\mathrm{R}$ \\
\hline 15 & KAL & udu fenen & & & \\
\hline 16 & KBD & kebelong & & $x$ & $\mathrm{R}$ \\
\hline 17 & KBK & & & & \\
\hline 18 & PEB & ureu tengolai, polu asa' & 2 & $x$ & $\mathrm{G}-2, \mathrm{~S}-2$ \\
\hline
\end{tabular}

Notes : 


\section{DESCRIPTION}

Very variable, annual, erect, unarmed herb to 1.5 $\mathrm{m}$, leaves ovate acuminate with clearly defined veins, flowers are on erect panicle, flowers throughout the year

\section{HABITAT}

Wild varieties in wastelands, edges, fallows; cultivated varieties in home and swidden gardens

\section{ORIGINS}

America

\section{USES}

Green leafy variety eaten as spinach, red variety as ornament; medicine; sold locally

\section{REFERENCES}

Sastrapradja et al. 1977b: 8; Ochse 1980: 20
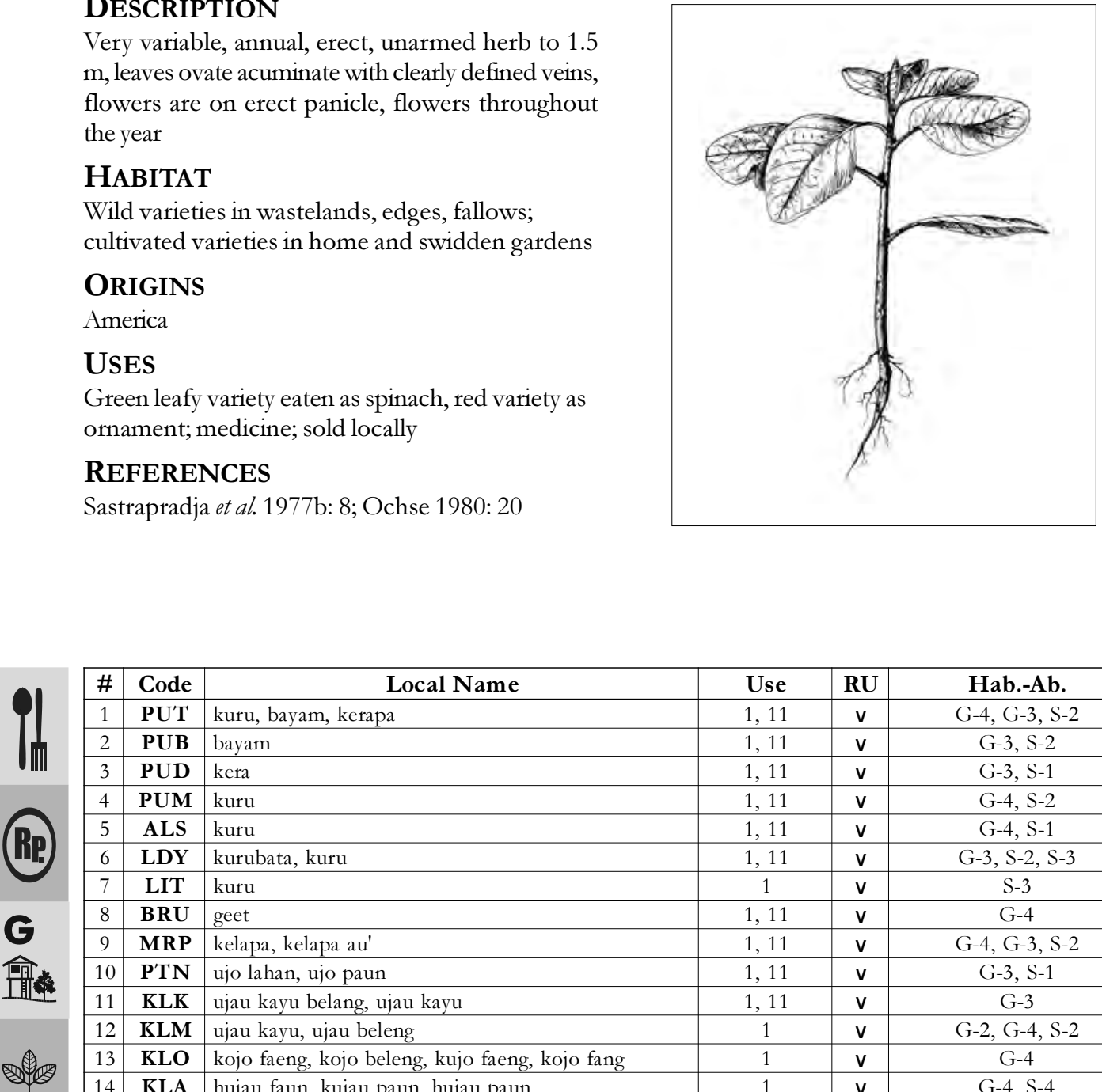

\begin{tabular}{|c|c|l|c|c|c|}
\hline$\#$ & Code & \multicolumn{1}{|c|}{ Local Name } & Use & RU & Hab.-Ab. \\
\hline 1 & PUT & kuru, bayam, kerapa & 1,11 & $\mathbf{v}$ & G-4, G-3, S-2 \\
\hline 2 & PUB & bayam & 1,11 & v & G-3, S-2 \\
\hline 3 & PUD & kera & 1,11 & $\mathbf{v}$ & G-3, S-1 \\
\hline 4 & PUM & kuru & 1,11 & $\mathbf{v}$ & G-4, S-2 \\
\hline 5 & ALS & kuru & 1,11 & $\mathbf{v}$ & G-4, S-1 \\
\hline 6 & LDY & kurubata, kuru & 1,11 & $\mathbf{v}$ & G-3, S-2, S-3 \\
\hline 7 & LIT & kuru & 1,11 & $\mathbf{v}$ & v \\
\hline 8 & BRU & geet & 1,11 & $\mathbf{v}$ & G-4, G-3, S-2 \\
\hline 9 & MRP & kelapa, kelapa au' & 1,11 & $\mathbf{v}$ & G-3, S-1 \\
\hline 10 & PTN & ujo lahan, ujo paun & 1,11 & $\mathbf{v}$ & G-3 \\
\hline 11 & KLK & ujau kayu belang, ujau kayu & 1 & $\mathbf{v}$ & G-2, G-4, S-2 \\
\hline 12 & KLM & ujau kayu, ujau beleng & 1 & $\mathbf{v}$ & G-4 \\
\hline 13 & KLO & kojo faeng, kojo beleng, kujo faeng, kojo fang & 1 & $\mathbf{v}$ & G-4, S-4 \\
\hline 14 & KLA & hujau faun, kujau paun, hujau paun & & & \\
\hline 15 & KAL & ujau faun bileng & 1 & $\mathbf{v}$ & G-3 \\
\hline 16 & KBD & ujau bayam, ozau bayam & & & \\
\hline 17 & KBK & ujo lan & 1 & $\mathbf{v}$ & G-3, S-2 \\
\hline 18 & PEB & bayam & & \\
\hline
\end{tabular}

Notes : 


\section{Celosia cristata L. (Amaranthaceae)}

\section{RED SPINACH, COCK'S COMB. BAYAM MERAH}

\section{DESCRIPTION}

Variable, annual, erect shrub-herb, to $1.5 \mathrm{~m}$; ovate leaves with red spot; cock's-comb-like panicle, flaming red spikes

\section{HABITAT}

Cultivated in homegardens; occasionally wild

\section{ORIGINS}

Asia

\section{USES}

Ornament; boiled leaves for vegetable; dried flowers for medicine

\section{REFERENCES}

Burkill 1966: 512; Ochse 1980: 28

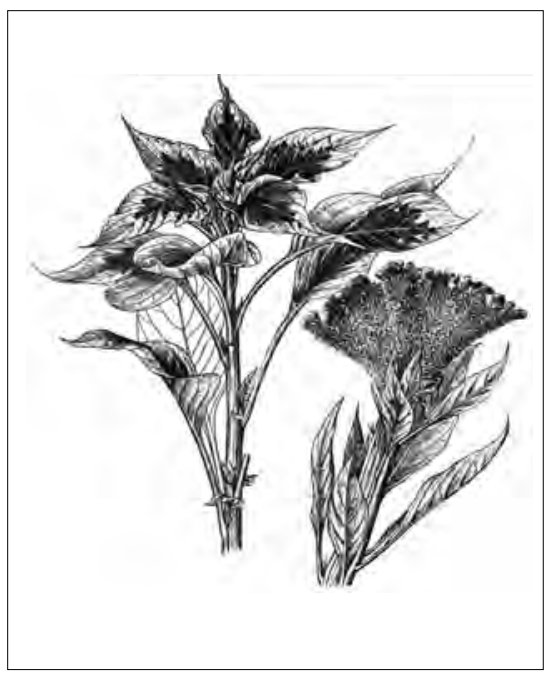

\begin{tabular}{|c|c|c|c|c|c|}
\hline \# & Code & Local Name & Use & RU & Hab.-Ab. \\
\hline 1 & PUT & tunyung, kerapa serou, tunyung serou & 1 & $\mathrm{v}$ & G-1, G-2, S-1 \\
\hline 2 & PUB & & & & \\
\hline 3 & PUD & ketunyung & 1 & $x$ & G-2 \\
\hline 4 & PUM & tunyung & 1 & V & G-3, S-1 \\
\hline 5 & ALS & tunyung & 1 & $x$ & G-2, S-1 \\
\hline 6 & LDY & busak tunung & 1 & V & G-2, S-2, Y-3 \\
\hline 7 & LIT & tunung, busak tung, busak tunung & 1 & $\mathrm{v}$ & S-2 \\
\hline 8 & BRU & geet & 1 & V & G-3, S-1 \\
\hline 9 & MRP & kelapa toi, kelapa tui, kelapa luru & 1 & $\mathrm{v}$ & G-2, G-3 \\
\hline 10 & PTN & ujo apa', ujo apa & 1,2 & v & G-2, G-3 \\
\hline 11 & KLK & ujau kayo alo, pangung & 1 & $x$ & G-2 \\
\hline 12 & KLM & panggong, ujau kayu & 1 & V & G-2, S-2, S-3 \\
\hline 13 & KLO & kojo ave', kujo ave' & 1 & $x$ & $\mathrm{G}-2$ \\
\hline 14 & KLA & hujau feliran, hujau peliran, kujau peleran, peleran & 1 & $\mathrm{x}$ & $\mathrm{G}-4, \mathrm{~S}-4$ \\
\hline 15 & KAL & kojo peleren & & & \\
\hline 16 & KBD & ujau, ojau lasan & 1 & v & G-2 \\
\hline 17 & KBK & ujo semangga' & & & \\
\hline 18 & PEB & bunga pagung poti, uru pagung & 1 & $\mathrm{x}$ & G-3 \\
\hline
\end{tabular}

Notes : 


\section{Buchanania sessifolia B1. (Anacardiaceae)}

\section{SPARROW'S MANGO. OTAKUDANG}

\section{DESCRIPTION}

Tree, up to $42 \mathrm{~m}$, occasionally buttressed; leaves obovate-oblong, 7.5-31 cm long, 4-10.5 cm wide; flowers white or whitish yellow, panicles $4.5-35 \mathrm{~cm}$ long; fruit a red drupe, heart-shaped, 10-13 mm long

\section{HABITAT}

Lowland secondary forests; dry land

\section{ORIGINS}

Southeast Asia

\section{USES}

Bark may be used for light building; wood not durable; sour fruit may be eaten and used in medicine

\section{REFERENCES}

Burkill 1966: 382; Kessler and Sidiyasa 1994: 39
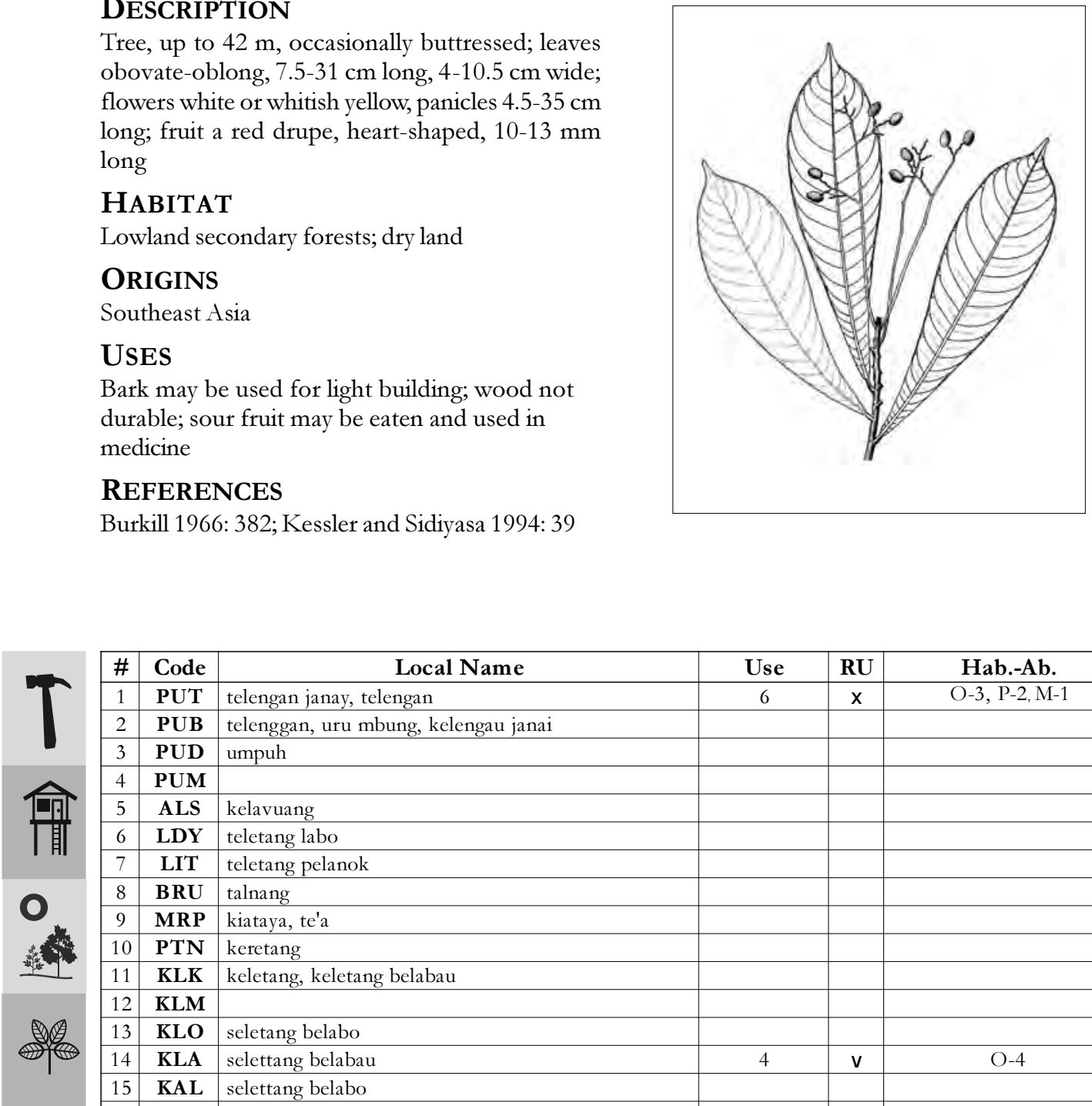

\begin{tabular}{|c|c|l|c|c|c|}
\hline$\#$ & Code & \multicolumn{1}{|c|}{ Local Name } & Use & RU & Hab.-Ab. \\
\hline 1 & PUT & telengan janay, telengan & 6 & X & O-3, P-2, M-1 \\
\hline 2 & PUB & telenggan, uru mbung, kelengau janai & & & \\
\hline 3 & PUD & umpuh & & & \\
\hline 4 & PUM & & & & \\
\hline 5 & ALS & kelavuang & & & \\
\hline 6 & LDY & teletang labo & & & \\
\hline 7 & LIT & teletang pelanok & & & \\
\hline 8 & BRU & talnang & & & \\
\hline 9 & MRP & kiataya, te'a & & & \\
\hline 10 & PTN & keretang & & & \\
\hline 11 & KLK & keletang, keletang belabau & & & \\
\hline 12 & KLM & & & & \\
\hline 13 & KLO & seletang belabo & 4 & $\mathbf{V}$ & \\
\hline 14 & KLA & selettang belabau & & & \\
\hline 15 & KAL & selettang belabo & & & \\
\hline 16 & KBD & & 7 & $\mathbf{X}$ & \\
\hline 17 & KBK & keletang iut, kelapu & & Y-4, O-4 \\
\hline 18 & PEB & kelotang & & & \\
\hline
\end{tabular}

Notes : 


\section{Mangifera quadrifida Jack. (Anacardiaceae)}

\section{SWEET-ACID MANGO. ASEM KUMBANG, ASAM RAWA}

\section{DESCRIPTION}

Tree, 10-35 m, 20-90 cm dbh, sometimes buttresses; leaves elliptic $16-30 \mathrm{~cm} \times 3-9 \mathrm{~cm}$; flowers white to greenish white, panicles to $25 \mathrm{~cm}$ long; fruit green ellipsoid 8-10 × 5-7 cm, yellow fibrous flesh

\section{HABITAT}

Lowland forests; riverine or wet areas; cultivated in gardens

\section{ORIGINS}

Malaysia and western Indonesia

\section{USES}

Fruit eaten, sold locally

\section{REFERENCES}

PROSEA Vol. 2: 203; FM I Vol. 8(3): 429

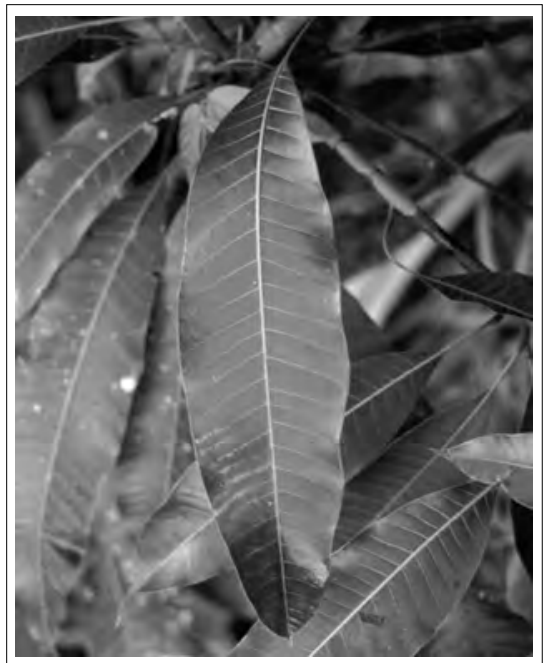

\begin{tabular}{|c|c|l|c|c|c|}
\hline \# & Code & \multicolumn{1}{|c|}{ Local Name } & Use & RU & Hab.-Ab. \\
\hline 1 & PUT & mempelom, pelom & $1,6,7,11$ & $\mathbf{v}$ & G-2, G-3 \\
\hline 2 & PUB & pelom, timu' & 1 & $\mathbf{v}$ & G-2, G-3 \\
\hline 3 & PUD & bua' pelom, mempelam & 1,11 & $\mathbf{v}$ & G-2, G-3 \\
\hline 4 & PUM & bua mempelom & 1,11 & $\mathbf{v}$ & G-3 \\
\hline 5 & ALS & bua' mepolom & 1,7 & $\mathbf{v}$ & G-3 \\
\hline 6 & LDY & kunyi, mampalam & $1,6,11$ & $\mathbf{v}$ & G-2, G-3 \\
\hline 7 & LIT & lipit, lam lifit, lamlipit & 1,11 & $\mathbf{v}$ & G-2, G-3 \\
\hline 8 & BRU & wi mempom & $1,7,11$ & $\mathbf{v}$ & G-2 \\
\hline 9 & MRP & bua pelam, pelam & 1,11 & $\mathbf{v}$ & G-2, G-3 \\
\hline 10 & PTN & bua mangga, mangga timai & 1 & $\mathbf{v}$ & G-2 \\
\hline 11 & KLK & mangga & 1,11 & $\mathbf{v}$ & G-2 \\
\hline 12 & KLM & alim bio, mangga & 1,11 & $\mathbf{v}$ & G-2, G-3 \\
\hline 13 & KLO & alieng alo', aleng alo' & $\mathbf{X}$ & G-3 \\
\hline 14 & KLA & mangga, mangga kenya, mangga rumit & & $\mathbf{X}$ & G-3 \\
\hline 15 & KAL & alim beleng & $1,7,11$ & $\mathbf{v}$ & G-3 \\
\hline 16 & KBD & mangga, mangga timai & & & \\
\hline 17 & KBK & mangga & 1 & $\mathbf{v}$ & G-3, Y-2 \\
\hline 18 & PEB & pangin alo & & \\
\hline
\end{tabular}

Notes : 


\section{Mangifera odorata Griff. (Anacardiaceae)}

\section{KUWINI. BEMBEM, KUWENI}

\section{DESCRIPTION}

Tree, 15-35 m, bark grey, smooth or fissured; leaves elliptic-lanceolate $9-35 \mathrm{~cm}$ long, $3.5-10 \mathrm{~cm}$ wide; flowers yellowish white becoming red, panicles 12$50 \mathrm{~cm}$ long; fruit dark green when ripe, flesh yellow, sweet, fibrous, $10-13 \mathrm{~cm}$ long

\section{HABITAT}

Only found in cultivation, may be hybrid

\section{ORIGINS}

Unknown

\section{USES}

Fruit eaten, sold locally; timber for firewood

\section{REFERENCES}

Kessler and Sidiyasa 1994: 42;

PROSEA Vol. 2: 218;

PROSEA Vol. 5(2): 336
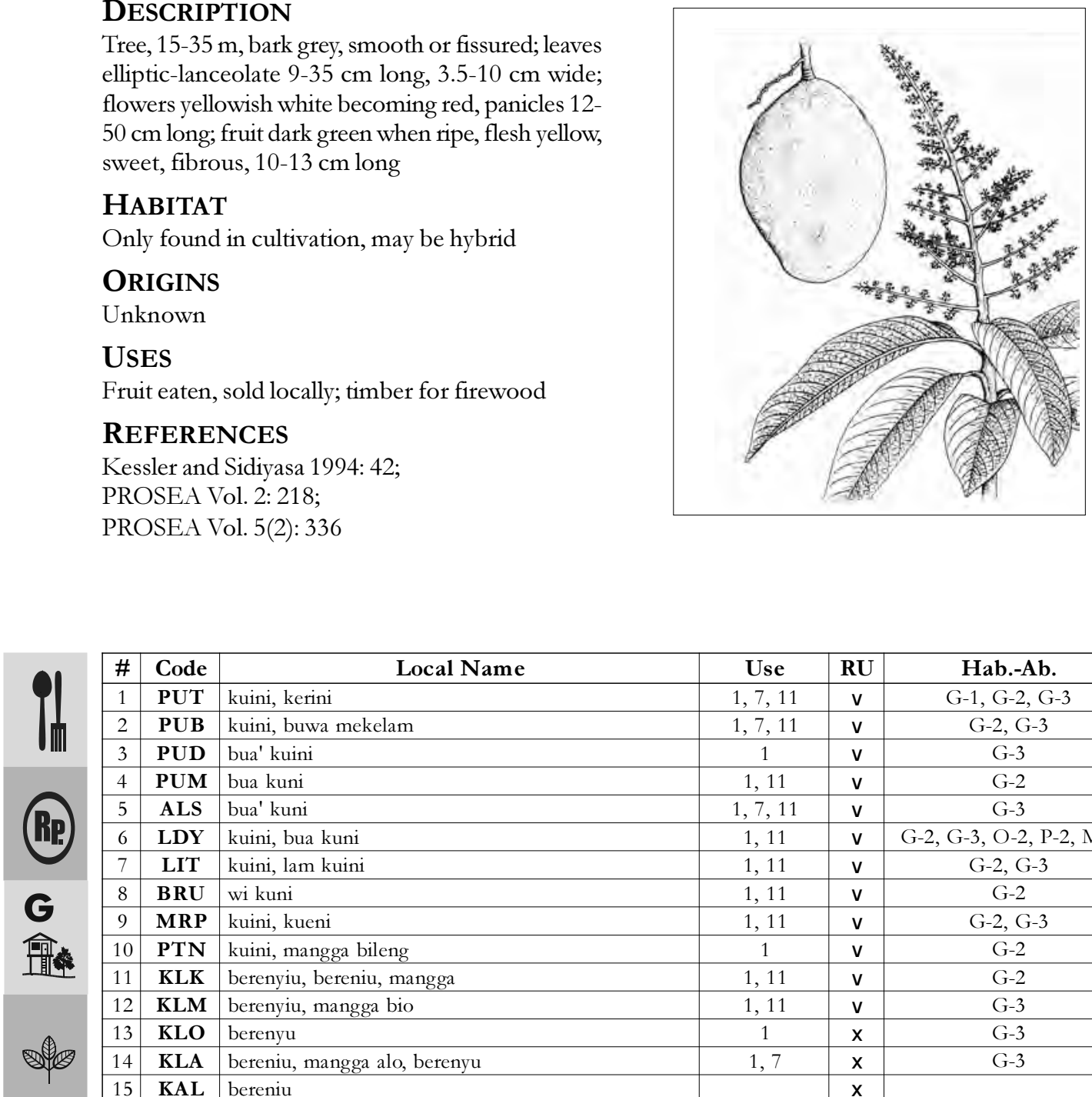

\begin{tabular}{|c|c|l|c|c|c|}
\hline$\#$ & Code & \multicolumn{1}{|c|}{ Local Name } & Use & RU & Hab.-Ab. \\
\hline 1 & PUT & kuini, kerini & $1,7,11$ & $\mathbf{v}$ & G-1, G-2, G-3 \\
\hline 2 & PUB & kuini, buwa mekelam & $1,7,11$ & $\mathbf{v}$ & G-2, G-3 \\
\hline 3 & PUD & bua' kuini & 1 & $\mathbf{v}$ & G-3 \\
\hline 4 & PUM & bua kuni & 1,11 & $\mathbf{v}$ & G-2 \\
\hline 5 & ALS & bua' kuni & $1,7,11$ & $\mathbf{v}$ & G-3 \\
\hline 6 & LDY & kuini, bua kuni & 1,11 & $\mathbf{v}$ & G-2, G-3, O-2, P-2, M-1 \\
\hline 7 & LIT & kuini, lam kuini & 1,11 & $\mathbf{v}$ & G-2, G-3 \\
\hline 8 & BRU & wi kuni & 1,11 & $\mathbf{v}$ & G-2 \\
\hline 9 & MRP & kuini, kueni & 1 & $\mathbf{v}$ & G-2, G-3 \\
\hline 10 & PTN & kuini, mangga bileng & 1,11 & $\mathbf{v}$ & G-2 \\
\hline 11 & KLK & berenyiu, bereniu, mangga & 1,11 & $\mathbf{v}$ & G-2 \\
\hline 12 & KLM & berenyiu, mangga bio & 1 & $\mathbf{X}$ & G-3 \\
\hline 13 & KLO & berenyu & 1,7 & $\mathbf{X}$ & G-3 \\
\hline 14 & KLA & bereniu, mangga alo, berenyu & & $\mathbf{x}$ & \\
\hline 15 & KAL & bereniu & $1,6,7,11$ & $\mathbf{v}$ & G-3, G-4 \\
\hline 16 & KBD & berengeu, mangga lata & & & \\
\hline 17 & KBK & belenyu & 1,11 & $\mathbf{v}$ & G-4, Y-2, O-2 \\
\hline 18 & PEB & maga timai, pangin alo jau & &
\end{tabular}

Notes : 


\section{Mangifera pajang Kostermans (Anacardiaceae)}

\section{SHERBERT MANGO. BAMBANGAN, ASEM PAYANG}

\section{DESCRIPTION}

Tree, 15-33 m, bark brown or grey, smooth or fissured; leaves elliptic-oblong $28-45 \mathrm{~cm} \mathrm{x}$ $10-15 \mathrm{~cm}$, prominent nerves; flower panicles 30 $\mathrm{cm}$, purple and pinkish white; fruit a thick-skinned brown globular drupe, up to $20 \mathrm{~cm} \mathrm{~d}$, bright yellow flesh

\section{HABITAT}

Lowland and hill primary forests; cultivated in village fruit gardens

\section{ORIGINS}

Borneo endemic

\section{USES}

Fruit eaten, sold locally; hunting location when fruiting

\section{REFERENCES}

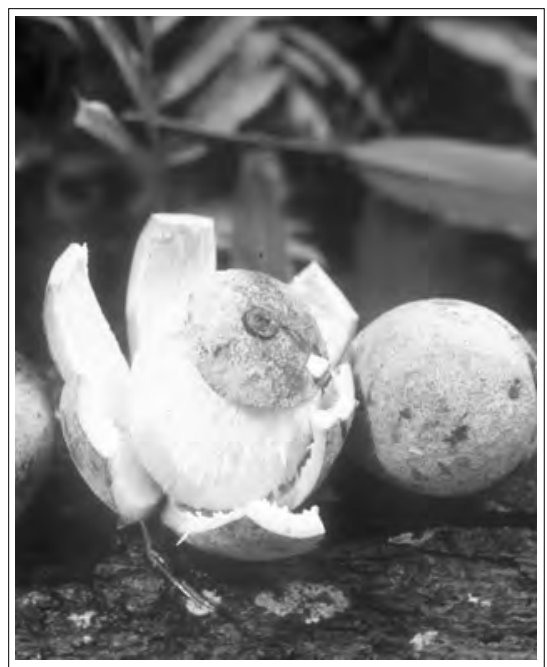

PROSEA Vol. 2: 209; Valkenburg 1997: 91

\begin{tabular}{|c|c|l|c|c|c|}
\hline$\#$ & Code & Local Name & Use & RU & Hab.-Ab. \\
\hline 1 & PUT & pangin & $1,6,10,11$ & $\mathbf{v}$ & G-2, P-3 \\
\hline 2 & PUB & pangin & 1 & $\mathbf{v}$ & G-2 \\
\hline 3 & PUD & pangin & 1,11 & $\mathbf{v}$ & G-3, P-4, M-1 \\
\hline 4 & PUM & pangin & 1 & $\mathbf{v}$ & G-2 \\
\hline 5 & ALS & bua' mangan & 1 & $\mathbf{v}$ & G-3, O-2, P-3 \\
\hline 6 & LDY & lampayang & 1,11 & $\mathbf{v}$ & G-3, Y-2, O-3, P-4 \\
\hline 7 & LIT & lampayang & 1,10 & $\mathbf{v}$ & G-2, P-2, P-3 \\
\hline 8 & BRU & wi pem, wi pangin, wi yan ca' & 1,10 & $\mathbf{v}$ & G-2 \\
\hline 9 & MRP & pangaing, pangaeng, pangeng & 1,10 & $\mathbf{v}$ & G-1, G-2, O-2, P-3, M-3 \\
\hline 10 & PTN & pangin & 1 & $\mathbf{v}$ & G-2, P-3 \\
\hline 11 & KLK & alim & 1,11 & $\mathbf{v}$ & G-2, P-2 \\
\hline 12 & KLM & alim & 1 & $\mathbf{v}$ & G-2/G-3 \\
\hline 13 & KLO & alieng, aling & 1,7 & $\mathbf{x}$ & G-2 \\
\hline 14 & KLA & alim lan, alim & & & G-3, P-3 \\
\hline 15 & KAL & alim lata & 1 & $\mathbf{v}$ & G-2, Y-2, O-2 \\
\hline 16 & KBD & alim, alim pawen & & & \\
\hline 17 & KBK & alim & 1 & $\mathbf{v}$ & G-3, O-3, P-2 \\
\hline 18 & PEB & pangin & & \\
\hline
\end{tabular}

Notes : 


\section{Semecarpus glaucus Engl. (Anacardiaceae)}

\section{RENGAS. RENGAS}

\section{DESCRIPTION}

Small tree, black sap; leaves glaucous beneath, prominent reticulate veins underneath; fruit fleshy, oblong seated on a fleshy body made from the base of the flower

\section{HABITAT}

Lowland and hill primary forests

\section{ORIGINS}

Borneo

\section{USES}

Poisonous sap; only base of fruit may be eaten; timber for furniture

\section{REFERENCES}

Corner 1988: 131; TFM Vol. 4: 50;

FM I Vol. 8(3): 513
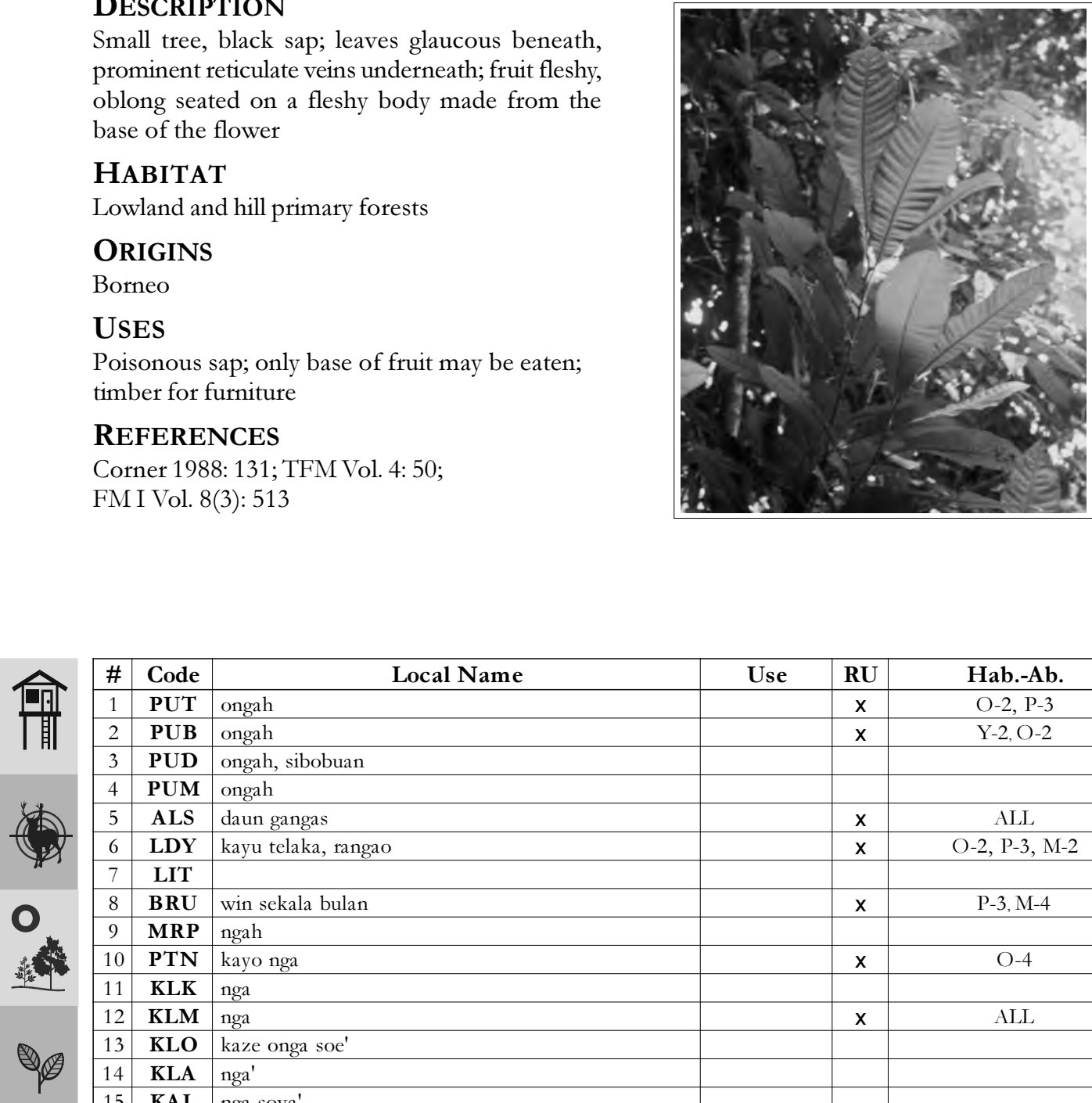

\begin{tabular}{|c|c|l|c|c|c|}
\hline$\#$ & Code & \multicolumn{1}{|c|}{ Local Name } & Use & RU & Hab.-Ab. \\
\hline 1 & PUT & ongah & & $\mathbf{x}$ & O-2, P-3 \\
\hline 2 & PUB & ongah & & $\mathbf{x}$ & Y-2, O-2 \\
\hline 3 & PUD & ongah, sibobuan & & & \\
\hline 4 & PUM & ongah & & $\mathbf{x}$ & ALL \\
\hline 5 & ALS & daun gangas & & $\mathbf{x}$ & O-2, P-3, M-2 \\
\hline 6 & LDY & kayu telaka, rangao & & & \\
\hline 7 & LIT & X & P-3, M-4 \\
\hline 8 & BRU & win sekala bulan & & & \\
\hline 9 & MRP & ngah & & X & \\
\hline 10 & PTN & kayo nga & & \\
\hline 11 & KLK & nga & & $\mathbf{x}$ & \\
\hline 12 & KLM & nga & & & \\
\hline 13 & KLO & kaze onga soe' & & & \\
\hline 14 & KLA & nga' & & & \\
\hline 15 & KAL & nga sova' & & & \\
\hline 16 & KBD & nga & & & \\
\hline 17 & KBK & nga & & & \\
\hline 18 & PEB & ngeu, nge & & \\
\hline
\end{tabular}

Notes : 


\section{DESCRIPTION}

Shrub or small tree 3-10 m, branched from base; leaves oblong 8-16 × 3-7 cm; flowers regular, greenish white, strong smelling; fruit a dark green collective berry, ovoid 10-20 x 15-35 cm, covered with spines $6 \mathrm{~mm}$ long, fleshy, juicy white pulp

\section{HABITAT}

Cultivated in homegardens

\section{ORIGINS}

America

\section{USES}

Fruit eaten, for medicine, sold locally

\section{REFERENCES}

PROSEA Vol. 2: 75; Ochse 1980: 47

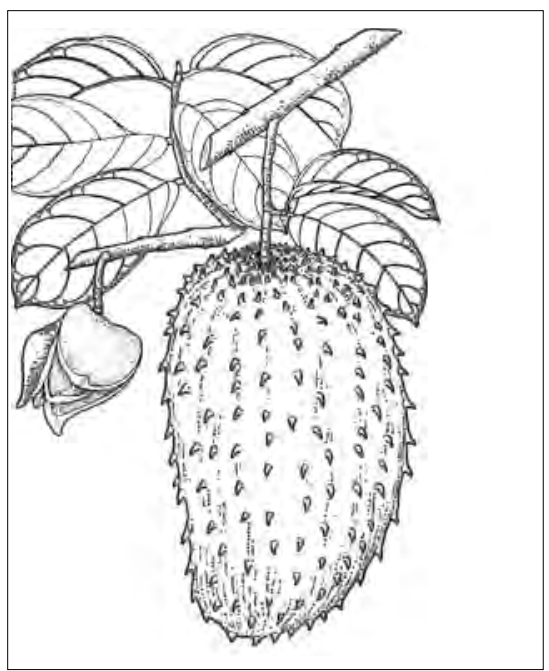

\begin{tabular}{|c|c|l|c|c|c|}
\hline \# & Code & \multicolumn{1}{|c|}{ Local Name } & Use & RU & Hab.-Ab. \\
\hline 1 & PUT & mangga belanda, doco belanda & 1,2 & $\mathbf{v}$ & G-2, G-3 \\
\hline 2 & PUB & docou belanda, tungen belanda & 1,7 & $\mathbf{v}$ & G-2 \\
\hline 3 & PUD & doso belanda & 1 & $\mathbf{v}$ & G-2 \\
\hline 4 & PUM & doso belanda' & 1 & $\mathbf{v}$ & G-2 \\
\hline 5 & ALS & lambun belanda & 1,2 & $\mathbf{v}$ & G-3 \\
\hline 6 & LDY & datu belanda, datu belada & 1,2 & $\mathbf{v}$ & G-2, G-3, S-1, S-2, Y-1 \\
\hline 7 & LIT & datu belada, datu belanda, data' belenda' & 1 & $\mathbf{v}$ & G-2, G-3 \\
\hline 8 & BRU & wi atau belanda & 1,2 & $\mathbf{v}$ & G-3 \\
\hline 9 & MRP & yang ada', yang belada, yang belanda & $1,2,11$ & $\mathbf{v}$ & G-2 \\
\hline 10 & PTN & bua belenda, belada & 1,2 & $\mathbf{v}$ & G-2 \\
\hline 11 & KLK & dian belenta, dian belenda & 1,2 & $\mathbf{X}$ & G-3 \\
\hline 12 & KLM & belenta, belenda & 1,2 & $\mathbf{v}$ & G-2 \\
\hline 13 & KLO & belente' & 1 & $\mathbf{v}$ & G-4 \\
\hline 14 & KLA & belada', berede & 1 & $\mathbf{X}$ & G-2 \\
\hline 15 & KAL & belenta' & 1 & $\mathbf{X}$ & G-2 \\
\hline 16 & KBD & belenta, beleda & 1 & $\mathbf{v}$ & G-2, G-4 \\
\hline 17 & KBK & dian bida & 1 & $\mathbf{v}$ & G-2 \\
\hline 18 & PEB & paken beleda, paken alo' & G-3, S-2 \\
\hline
\end{tabular}

Notes : 


\section{Eryngium foetidum L. (Apiaceae)}

\section{JAVA CORIANDER, FITWEED. KETUMBAR JAWA}

\section{DESCRIPTION}

Herb, 10-80 cm, perennial, foul smelling glabrous, branching tap root; leaves in radial rosette when young, sharply serrate $5-25 \times 1-4 \mathrm{~cm}$; flowers minute, greenish white on $1-2 \mathrm{~cm}$ spike at apex; fruit small, ovoid and warty

\section{HABITAT}

Sunny, wet, fertile lands, edges of forest and cultivated areas, waste lands

\section{ORIGINS}

America

\section{USES}

Leaves boiled eaten as vegetable, Chinese use as coriander substitute; medicine, poison antidote

\section{REFERENCES}

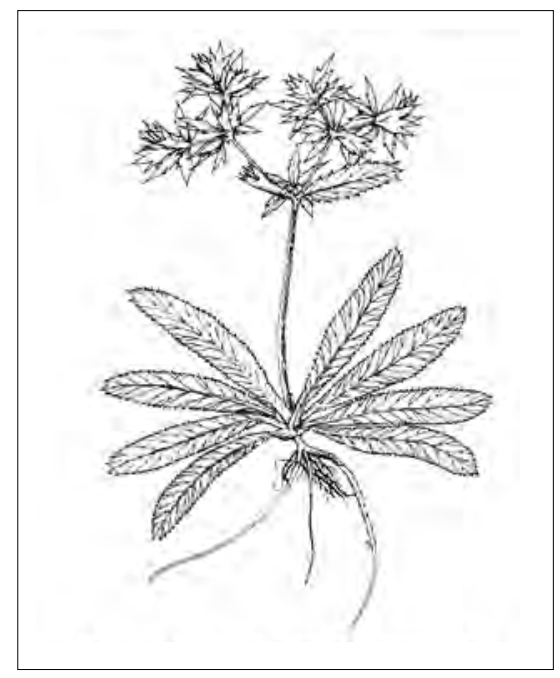

Burkill 1966: 959; Ochse 1980: 712;

PROSEA Vol. 13: 122

\begin{tabular}{|c|c|c|c|c|c|}
\hline \# & Code & Local Name & Use & $\mathbf{R U}$ & Hab.-Ab. \\
\hline 1 & PUT & sup dayak, sup & 1,2 & $\mathrm{v}$ & G-2 \\
\hline 2 & PUB & puli otuh & 2 & $x$ & G-1 \\
\hline 3 & PUD & & & & \\
\hline 4 & PUM & cup & 1,2 & $\mathrm{x}$ & G-3, S-4 \\
\hline 5 & ALS & daun sup & 1 & v & G-2 \\
\hline 6 & LDY & sup jendal, sup & 1 & v & G-2, G-3 \\
\hline 7 & LIT & sop la'ar, udu la'al & 1 & v & $\mathrm{G}-4$ \\
\hline 8 & BRU & uen kalata & 1,2 & v & G-2 \\
\hline 9 & MRP & rou payau, rau payau, ro mpaling payau & 1 & v & $\mathrm{G}-2, \mathrm{G}-3$ \\
\hline 10 & PTN & uleng, dun uleng & 1 & v & G-2 \\
\hline 11 & KLK & tampan & & $x$ & \\
\hline 12 & KLM & sup dayak, sup & 1 & v & G-2 \\
\hline 13 & KLO & sup & & & \\
\hline 14 & KLA & sup & & & \\
\hline 15 & KAL & sup & & & \\
\hline 16 & KBD & sup tana & & & \\
\hline 17 & KBK & sop kenya & & & \\
\hline 18 & PEB & ureu pelaing dek & & $x$ & G-3, Y-2 \\
\hline \multicolumn{6}{|c|}{ Notes: } \\
\hline
\end{tabular}




\section{Acorus calamus L. (Araceae) \\ SWEET FLAG. JERANGO}

\section{DESCRIPTION}

Herb, with creeping rhizome that gives rise to tufts of narrow lanceolate leaves

\section{HABITAT}

Marsh or riverine

\section{ORIGINS}

Asia (probably India)

\section{USES}

Rhizome for medicine (poultices for fever, rheumatism, soreness); ginger substitute

\section{REFERENCES}

Burkill 1966: 34; PROSEA Vol. 12(1): 81

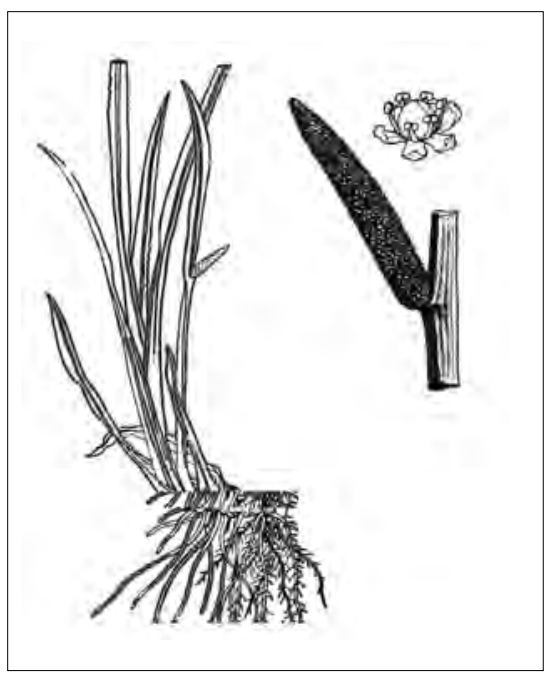

\begin{tabular}{|c|c|c|c|c|c|}
\hline \# & Code & Local Name & Use & RU & Hab.-Ab. \\
\hline 1 & PUT & kerenga & 2,6 & $x$ & G-1, G-2, G-3 \\
\hline 2 & PUB & kerenga & 1,2 & $\mathrm{v}$ & G-1, G-2 \\
\hline 3 & PUD & kerenga & 2 & $x$ & G-1, G-2 \\
\hline 4 & PUM & kerenga & 2 & $x$ & G-4 \\
\hline 5 & ALS & boro & 2 & $x$ & G-3 \\
\hline 6 & LDY & kereboro, kariboro & 2 & $\mathrm{v}$ & G-2, G-3 \\
\hline 7 & LIT & keburau & 2 & v & G-2, G-3 \\
\hline 8 & BRU & bua weu & 2 & v & G-3 \\
\hline 9 & MRP & kanga, kenga, kerenga & 2 & $\mathrm{v}$ & G-1, G-2 \\
\hline 10 & PTN & kerenga & 2 & v & G-1 \\
\hline 11 & KLK & kerenga & 2 & v & G-1 \\
\hline 12 & KLM & terenga & 2 & v & G-2 \\
\hline 13 & KLO & & & & \\
\hline 14 & KLA & & & & \\
\hline 15 & KAL & & & & \\
\hline 16 & KBD & telenga & & & \\
\hline 17 & KBK & & & & \\
\hline 18 & PEB & ureu tuban & 2 & v & O-2, P-4, M-3 \\
\hline
\end{tabular}

Notes : 


\section{Amydrium medium (Z. \& M.) Nichols. (Araceae)}

\section{(NOENGLISH/INDONESIAN NAME)}

\section{DESCRIPTION}

Herb, to $10 \mathrm{~m}$ long, creeping with adhesive roots, slender stem; leaves ovate with deeply cordate base, $17-42 \times 16-32 \mathrm{~cm}$, adult leaves pinnatifid with 2-10 openings on either side of mid rib; petiole $10-37 \mathrm{~cm}$, grooved; fruit a berry ovoid, white, $7 \mathrm{~mm}$

\section{HABITAT}

Lowland primary and secondary forest; limestone cliffs

\section{ORIGINS}

Unknown

\section{USES}

Leafy vegetable, medicine

\section{REFERENCES}

FOJ Vol. III: 105; Koorders Vol. 3: 172
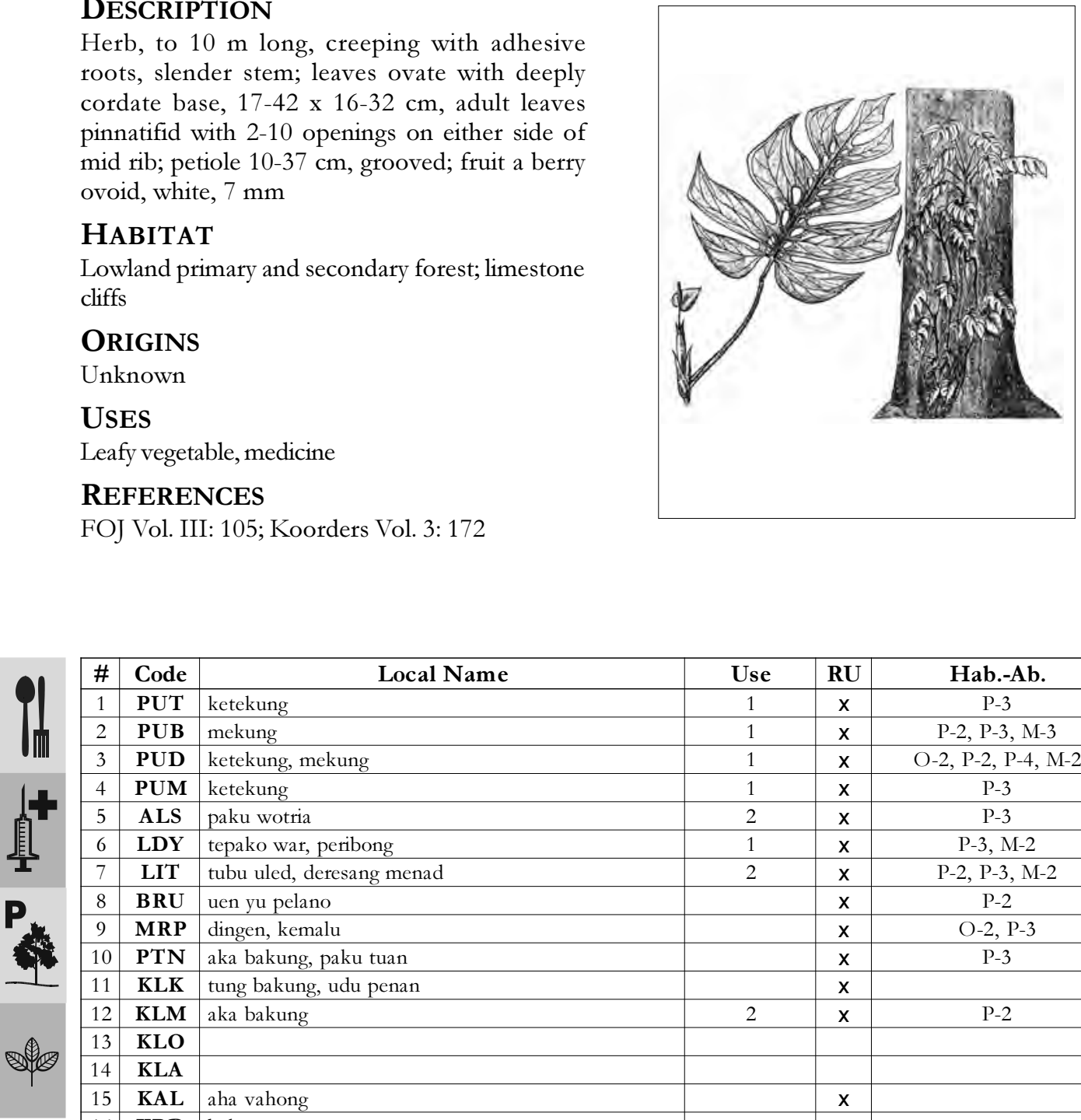

\begin{tabular}{|c|c|l|c|c|c|}
\hline$\#$ & Code & \multicolumn{1}{|c|}{ Local Name } & Use & RU & Hab.-Ab. \\
\hline 1 & PUT & ketekung & 1 & $\mathbf{X}$ & P-3 \\
\hline 2 & PUB & mekung & 1 & $\mathbf{X}$ & P-2, P-3, M-3 \\
\hline 3 & PUD & ketekung, mekung & 1 & $\mathbf{X}$ & O-2, P-2, P-4, M-2 \\
\hline 4 & PUM & ketekung & 1 & $\mathbf{X}$ & P-3 \\
\hline 5 & ALS & paku wotria & 2 & $\mathbf{X}$ & P-3 \\
\hline 6 & LDY & tepako war, peribong & 1 & $\mathbf{X}$ & P-3, M-2 \\
\hline 7 & LIT & tubu uled, deresang menad & 2 & $\mathbf{X}$ & P-2, P-3, M-2 \\
\hline 8 & BRU & uen yu pelano & & $\mathbf{X}$ & P-2 \\
\hline 9 & MRP & dingen, kemalu & & $\mathbf{X}$ & O-2, P-3 \\
\hline 10 & PTN & aka bakung, paku tuan & & $\mathbf{X}$ & P-3 \\
\hline 11 & KLK & tung bakung, udu penan & & $\mathbf{X}$ & \\
\hline 12 & KLM & aka bakung & 2 & $\mathbf{X}$ & P-2 \\
\hline 13 & KLO & & & & \\
\hline 14 & KLA & & & $\mathbf{X}$ & \\
\hline 15 & KAL & aha vahong & & $\mathbf{X}$ & \\
\hline 16 & KBD & bakung & & & \\
\hline 17 & KBK & & & $\mathbf{X}$ & P-4, M-3 \\
\hline 18 & PEB & tuban penako, tongat jau & & & \\
\hline
\end{tabular}

Notes : 


\section{Colocasia esculentum (L.) Schott (Araceae)}

\section{TARO, KARO. KELADI}

\section{DESCRIPTION}

Very variable herb, 30-150 cm, perennial, stem thick and swollen below surface as rootstock; vegetative reproduction; leaves large obliquely erect $2-60$ × 7-50 cm; long petioled, $40-150 \mathrm{~cm}$ long

\section{HABITAT}

Cultivated in wetter areas: irrigated fields, wet rice fields, riverine, marshes

\section{ORIGINS}

India and Southeast Asia

\section{USES}

Tubers and leaves eaten, famine food; medicine, poison antidote; wrapping food

\section{REFERENCES}

Burkill 1966: 647; Ochse 1980: 51

PROSEA Vol. 9: 69

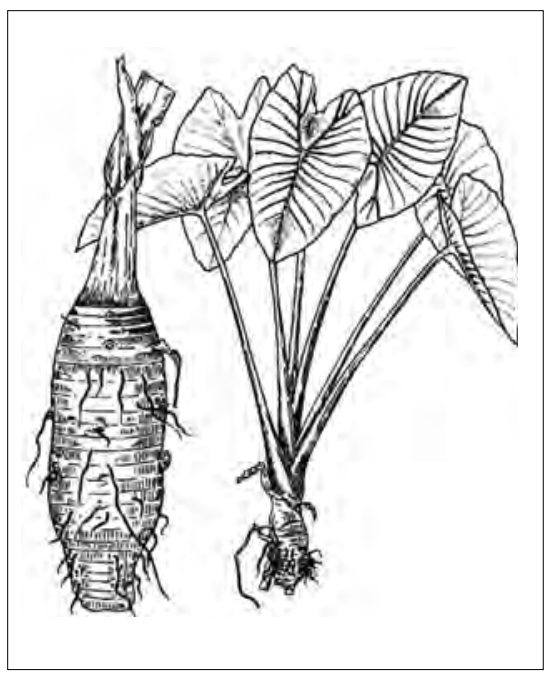

\begin{tabular}{|r|l|l|r|c|c|}
\hline$\#$ & Code & Local Name & Use & RU & Hab.-Ab. \\
\hline 1 & PUT & luai & 1,2 & $\mathbf{v}$ & G-3 \\
\hline 2 & PUB & lo'ai & 1,2 & $\mathbf{v}$ & G-3 \\
\hline 3 & PUD & kelu'ai & 1,2 & $\mathbf{v}$ & G-3 \\
\hline 4 & PUM & lo'ai & 1,2 & $\mathbf{v}$ & G-3 \\
\hline 5 & ALS & malau & 1,2 & $\mathbf{v}$ & G-3, S-2 \\
\hline 6 & LDY & ofa' & 1,2 & $\mathbf{v}$ & G-4, S-2 \\
\hline 7 & LIT & upa' & 1,2 & $\mathbf{v}$ & G-4, S-2 \\
\hline 8 & BRU & fua' & 1,2 & $\mathbf{v}$ & G-4, S-2 \\
\hline 9 & MRP & u'ae, kluoie & 1,2 & $\mathbf{v}$ & G-4, S-2 \\
\hline 10 & PTN & lu'ae & 1,2 & $\mathbf{v}$ & G-4, S-2 \\
\hline 11 & KLK & upa' kayan & 1,2 & $\mathbf{v}$ & G-4, S-2 \\
\hline 12 & KLM & opa' & 1,2 & $\mathbf{v}$ & G-4, S-2 \\
\hline 13 & KLO & ofa & 1,2 & $\mathbf{v}$ & G-4, S-2 \\
\hline 14 & KLA & ufa & 1,2 & $\mathbf{v}$ & G-4, S-2 \\
\hline 15 & KAL & ofa & 1,2 & $\mathbf{v}$ & G-4, S-2 \\
\hline 16 & KBD & opa' & 1,2 & $\mathbf{v}$ & G-4, S-2 \\
\hline 17 & KBK & lo'ae & 1,2 & $\mathbf{v}$ & G-4, S-2 \\
\hline 18 & PEB & opa' & 1,2 & $\mathbf{v}$ & G-2 \\
\hline
\end{tabular}

Notes : 


\section{Holochlamis beccarii (Engl.) Engl. (Araceae)}

\section{(NOENGLISH/INDONESIAN NAME)}

\section{DESCRIPTION}

Herb, to $4 \mathrm{~m}$, climbing, perennial; green fleshy stem; petioles c. $20-40 \mathrm{~cm}$ long by $1 \mathrm{~cm}$ diameter; leaves elliptic-oblong to oval-lanceolate, to 42-50 x 10-12 $\mathrm{cm}$, acute apex, cuneate base; midrib stiff brown sunken above c. 2-3 mm; 27-29 pairs sec. veins

\section{HABITAT}

Lowland primary and secondary forest

\section{ORIGINS}

India and Southeast Asia

\section{USES}

Medicine; air-roots used for weaving baskets

\section{REFERENCES}

Burkill 1966: 2014; Koorders Vol. 3: 182
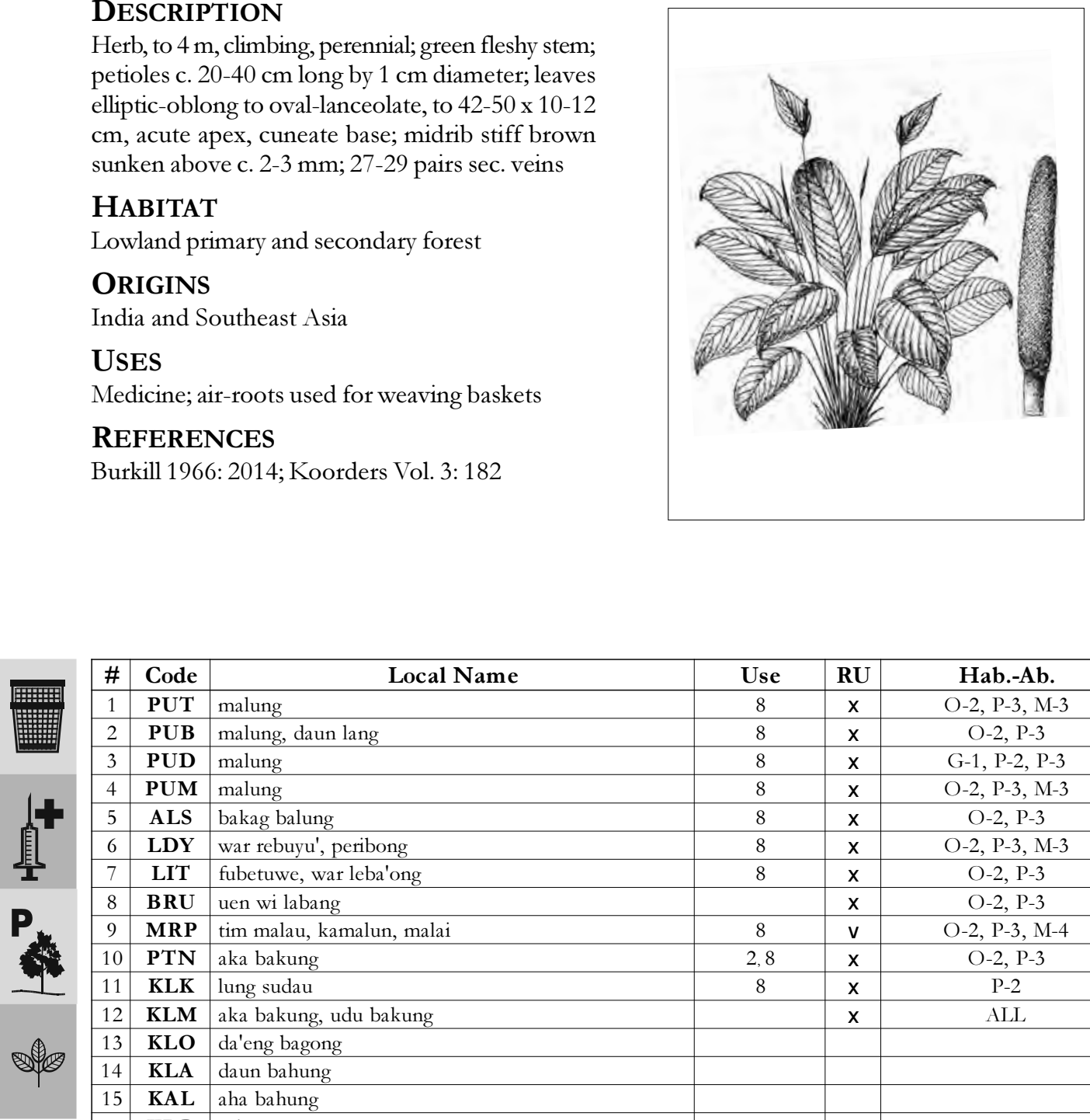

\begin{tabular}{|c|c|l|c|c|c|}
\hline$\#$ & Code & \multicolumn{1}{|c|}{ Local Name } & Use & RU & Hab.-Ab. \\
\hline 1 & PUT & malung & 8 & $\mathbf{X}$ & O-2, P-3, M-3 \\
\hline 2 & PUB & malung, daun lang & 8 & $\mathbf{X}$ & O-2, P-3 \\
\hline 3 & PUD & malung & 8 & $\mathbf{X}$ & G-1, P-2, P-3 \\
\hline 4 & PUM & malung & 8 & $\mathbf{X}$ & O-2, P-3, M-3 \\
\hline 5 & ALS & bakag balung & 8 & $\mathbf{X}$ & O-2, P-3 \\
\hline 6 & LDY & war rebuyu', peribong & 8 & $\mathbf{X}$ & O-2, P-3, M-3 \\
\hline 7 & LIT & fubetuwe, war leba'ong & 8 & $\mathbf{X}$ & O-2, P-3 \\
\hline 8 & BRU & uen wi labang & & $\mathbf{X}$ & O-2, P-3 \\
\hline 9 & MRP & tim malau, kamalun, malai & 8 & $\mathbf{V}$ & O-2, P-3, M-4 \\
\hline 10 & PTN & aka bakung & 2,8 & $\mathbf{X}$ & O-2, P-3 \\
\hline 11 & KLK & lung sudau & 8 & $\mathbf{X}$ & P-2 \\
\hline 12 & KLM & aka bakung, udu bakung & & $\mathbf{X}$ & ALL \\
\hline 13 & KLO & da'eng bagong & & & \\
\hline 14 & KLA & daun bahung & & & \\
\hline 15 & KAL & aha bahung & & & \\
\hline 16 & KBD & tuban & & & \\
\hline 17 & KBK & aka bakung & 8 & $\mathbf{X}$ & O-3, P-4 \\
\hline 18 & PEB & tuban soli, tuban & & \\
\hline
\end{tabular}

Notes : 


\section{Homalomena cordata Schott. (Araceae)}

\section{(NOENGLISH NAME).KELEMOYANG}

\section{DESCRIPTION}

Herb, erect $50-100 \mathrm{~cm}$, with aromatic rhizomes; leaves long petioled, heart-shaped, green to sordid purple, 7-10 primary nerves; flower preduncle thin, widening at top, $6-22 \mathrm{~cm}$ tall, with red or green; fruits oblong to ovoid, yellow, $1.5-4 \mathrm{~mm}$

\section{HABITAT}

Lowland and hill primary forests; shady riverine areas

\section{ORIGINS}

Asia

\section{USES}

Leaves for wrapping food; rhizome for medicine for people, and dogs (PEB)

\section{REFERENCES}

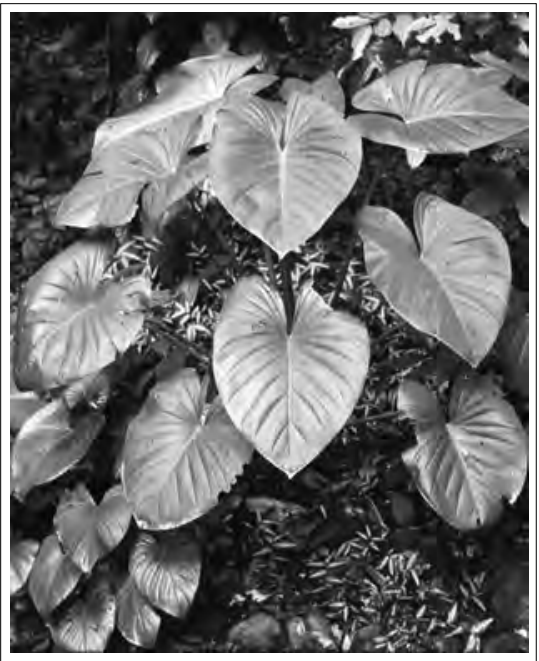

Burkill 1966: 1204; FOJ Vol. III: 115

\begin{tabular}{|c|c|c|c|c|c|}
\hline \# & Code & Local Name & Use & RU & Hab.-Ab. \\
\hline 1 & PUT & lung, lung bulah & 2,6 & $\mathrm{x}$ & $\mathrm{O}-2, \mathrm{P}-3$ \\
\hline 2 & PUB & lung bulah, lung bula & 2 & $x$ & $\mathrm{O}-2, \mathrm{P}-3$ \\
\hline 3 & PUD & lung & 2,6 & $x$ & $\mathrm{O}-2, \mathrm{P}-3$ \\
\hline 4 & PUM & lung & 2 & $x$ & $\mathrm{O}-3, \mathrm{P}-3, \mathrm{M}-3$ \\
\hline 5 & ALS & daun atu & 2 & $x$ & $\mathrm{O}-2, \mathrm{P}-3$ \\
\hline 6 & LDY & duli sia', duli & 2 & $x$ & $\mathrm{O}-1, \mathrm{O}-3, \mathrm{P}-2, \mathrm{P}-3, \mathrm{M}-3$ \\
\hline 7 & LIT & duli & 2 & $x$ & $\mathrm{Y}-2, \mathrm{O}-2, \mathrm{P}-2, \mathrm{P}-3$ \\
\hline 8 & BRU & uen ilae & 2 & $x$ & P-3 \\
\hline 9 & MRP & tim lauu, laung, lau & 2 & $x$ & $\mathrm{O}-2, \mathrm{P}-3, \mathrm{M}-3, \mathrm{M}-4$ \\
\hline 10 & PTN & lung, lung asit & 2 & v & $\mathrm{Y}-2, \mathrm{O}-3, \mathrm{P}-3$ \\
\hline 11 & KLK & lung adek & 2 & $x$ & P-3 \\
\hline 12 & KLM & lung bala, lung adek & 2 & $x$ & ALL \\
\hline 13 & KLO & long futih & & & \\
\hline 14 & KLA & lung & & & \\
\hline 15 & KAL & lung beleng & & & \\
\hline 16 & KBD & lung bala & & & \\
\hline 17 & KBK & lung & & & \\
\hline 18 & PEB & lisong, lesong & 2 & $\mathrm{v}$ & $\mathrm{Y}-2, \mathrm{O}-3, \mathrm{P}-4$ \\
\hline
\end{tabular}

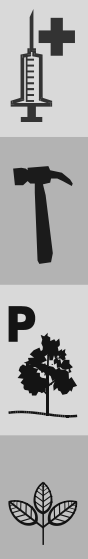

Notes : 


\section{Agatbis borneensis Warb. (Araucariaceae)}

\section{MOUNTAIN AGATHIS. KAYU DAMAR DAGING}

\section{DESCRIPTION}

Huge tree, to $55 \mathrm{~m}, 1.5 \mathrm{~m}$ dbh; bark grey to reddish brown, peeling in large thin flakes dippled with clear white resin known as 'copal; leaves opposite, ovate $6-12 \times 2-3.5 \mathrm{~cm}$ with many parallel veins; mature seed cones oval 6-8 x 5-6 cm

\section{HABITAT}

Lowland and hill primary forest; prefers peaks, ridges

\section{ORIGINS}

Oceania, Asia

\section{USES}

Timber for furniture, light construction; copal resin (or damar) sold for pitch production

\section{REFERENCES}

Kessler and Sidiyasa 1994: 55;

PROSEA Vol. 5(1): 79

Soepadmo and Wong 1995: 31

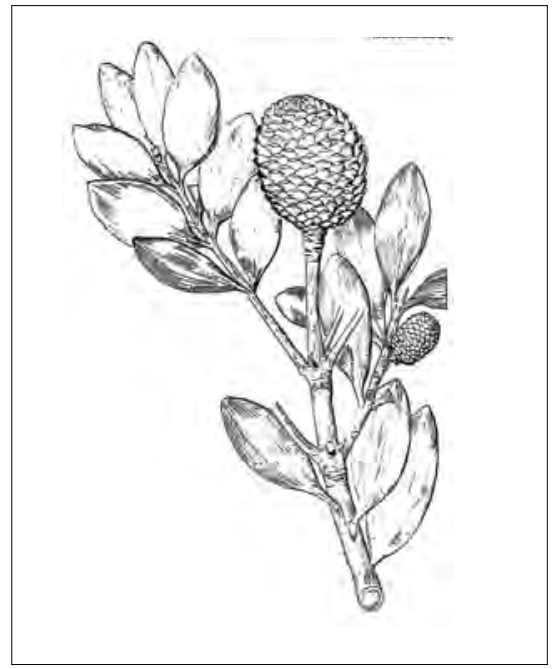

\begin{tabular}{|c|c|c|c|c|c|}
\hline \# & Code & Local Name & Use & RU & Hab.-Ab. \\
\hline 1 & PUT & tumu' & 6,11 & $x$ & P-2, M-2 \\
\hline 2 & PUB & tumu & 6,11 & $x$ & P-2, M-2 \\
\hline 3 & PUD & tumu' & 6,11 & $x$ & P-2, M-2 \\
\hline 4 & PUM & tumu & 6,11 & $x$ & P-2, M-2 \\
\hline 5 & ALS & tumu & 6,11 & $x$ & P-2, M-2 \\
\hline 6 & LDY & tumu & 6,11 & $x$ & P-2, M-2 \\
\hline 7 & LIT & tumu & 6,11 & $x$ & P-2, M-2 \\
\hline 8 & BRU & wi mo & 6,11 & $x$ & P-2, M-2 \\
\hline 9 & MRP & tumo', tomo' & 6,11 & $x$ & P-2, M-2 \\
\hline 10 & PTN & tumu & 6,11 & $x$ & P-2, M-2 \\
\hline 11 & KLK & tumu & 6,11 & $x$ & P-2, M-2 \\
\hline 12 & KLM & tumu & 6,11 & $x$ & P-2, M-2 \\
\hline 13 & KLO & tome & 6,11 & $x$ & P-2, M-2 \\
\hline 14 & KLA & tumu & 6,11 & $x$ & P-2, M-2 \\
\hline 15 & KAL & tumu & 6,11 & $x$ & P-2, M-2 \\
\hline 16 & KBD & tumu & 6,11 & $x$ & P-2, M-2 \\
\hline 17 & KBK & tumo' & 6,11 & $x$ & P-2, M-2 \\
\hline 18 & PEB & tumu & 6,11 & $x$ & P-2, M-2 \\
\hline
\end{tabular}

Notes : 


\section{BILLY-GOAT WEED. RUMPUT TAHI AYAM}

\section{DESCRIPTION}

Herb, to $1.2 \mathrm{~m}$, erect, annual; stems with rather long, crispy hairs; leaves ovate, triangular ovate, $2-10 \times 0.5-5 \mathrm{~cm}$, with hairs above, glandular below; flower white or violet

\section{HABITAT}

Cultivated in village lands; wild in waste places, fields, road sides

\section{ORIGINS}

Tropical America

\section{USES}

Leaves eaten, medicine, sold locally

\section{REFERENCES}

Burkill 1966: 71; FOJ Vol. III: 377;

PROSEA Vol. 12(1): 88

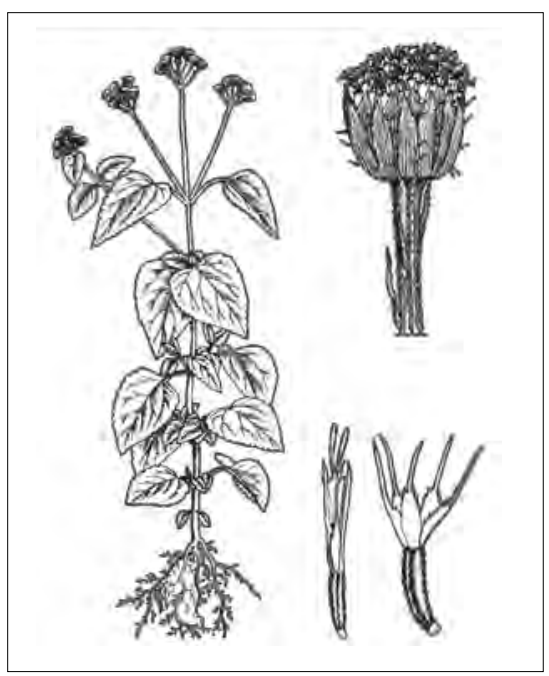

\begin{tabular}{|c|c|l|c|c|c|}
\hline \# & Code & \multicolumn{1}{|c|}{ Local Name } & Use & RU & Hab.-Ab. \\
\hline 1 & PUT & saga amik, belung & $1,2,11$ & $\mathbf{v}$ & S-3, Y-3, P-3, P-4 \\
\hline 2 & PUB & belung & 1 & $\mathbf{v}$ & O-2, P-3 \\
\hline 3 & PUD & belung, bua belung & 1 & $\mathbf{v}$ & G-2, P-3, P-4 \\
\hline 4 & PUM & belung & 1 & $\mathbf{v}$ & G-1, P-4 \\
\hline 5 & ALS & udu amek & 2 & $\mathbf{x}$ & ALL \\
\hline 6 & LDY & keling, bua karing & 1,11 & $\mathbf{v}$ & G-2, P-3 \\
\hline 7 & LIT & keling & 1 & $\mathbf{v}$ & G-2, P-3, M-2, M-4 \\
\hline 8 & BRU & dau meak & 2 & $\mathbf{v}$ & S-3 \\
\hline 9 & MRP & belau, melau, melawe & 1 & $\mathbf{v}$ & P-3 \\
\hline 10 & PTN & keling & 1,11 & $\mathbf{v}$ & G-1, P-3, M-2 \\
\hline 11 & KLK & udu pute, bua' melung, bua' keling & 1 & $\mathbf{v}$ & P-3, M-2 \\
\hline 12 & KLM & udu puti, bua keling & 1 & $\mathbf{x}$ & ALL \\
\hline 13 & KLO & udu rieng & 1 & $\mathbf{X}$ & G-2 \\
\hline 14 & KLA & kelieng, udu hading & $\mathbf{x}$ & G-2 \\
\hline 15 & KAL & udu futie & 1 & $\mathbf{v}$ & G-2, P-3 \\
\hline 16 & KBD & pelawok, pelawek & & & \\
\hline 17 & KBK & & 2 & $\mathbf{v}$ & G-3, S-2, Y-2, O-2 \\
\hline 18 & PEB & ureu lopek, daun aut & & \\
\hline
\end{tabular}
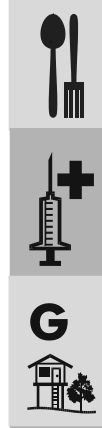

Notes : 


\section{Blumea balsamifera (L.) DC. (Asteraceae)}

NGAI CAMPHOR-PLANT. EEMBUNG

\section{DESCRIPTION}

Sub-shrubby herb, to $4 \mathrm{~m}$; aromatic, camphor smell; flower stalk and leaf undersides felty; leaves ovaloblong, dentate at apex, $8-40 \times 2-20 \mathrm{~cm}$; flowers yellow or violet; fruit an achene, c. $1 \mathrm{~mm}$ long with white hairs, angular, 5-10 ribbed

\section{HABITAT}

Wild in cultivated and waste areas; poor soils lead to slower growth and more camphor production

\section{ORIGINS}

South and Southeast Asia

\section{USES}

Leaves for medicine; camphor distilled and sold; lampoil

\section{REFERENCES}

Burkill 1966: 337; FOJ Vol. II: 387;

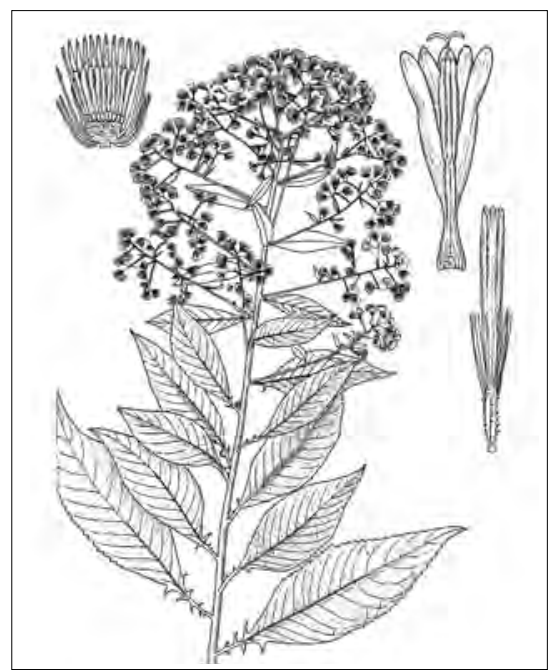

PROSEA Vol. 12(1): 158

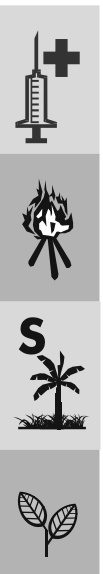

\begin{tabular}{|c|l|l|c|c|c|}
\hline$\#$ & Code & \multicolumn{1}{|c|}{ Local Name } & Use & RU & Hab.-Ab. \\
\hline 1 & PUT & uru umpung & 2 & $\mathbf{X}$ & G-1, Y-3, O-2 \\
\hline 2 & PUB & uru umpung & 2 & $\mathbf{X}$ & Y-3 \\
\hline 3 & PUD & uru umpung & 2 & $\mathbf{X}$ & S-2, Y-2, Y-3 \\
\hline 4 & PUM & umbu & 2 & $\mathbf{X}$ & Y-3, O-1 \\
\hline 5 & ALS & tiowo & 2 & $\mathbf{X}$ & S-2, Y-3 \\
\hline 6 & LDY & kayu ifung, atap ba' & 2,7 & $\mathbf{V}$ & G-2, S-2, O-3 \\
\hline 7 & LIT & & & & \\
\hline 8 & BRU & uen peng & 2 & $\mathbf{X}$ & S-3, Y-2 \\
\hline 9 & MRP & ro mpu & & $\mathbf{X}$ & S-2, Y-3 \\
\hline 10 & PTN & uru' mbong & 2,6 & $\mathbf{X}$ & S-2, Y-3, O-4 \\
\hline 11 & KLK & mbung i'ut & 2 & & \\
\hline 12 & KLM & mpung, mbong & & $\mathbf{X}$ & ALL \\
\hline 13 & KLO & da'eng mpung & & & \\
\hline 14 & KLA & mpung & & & \\
\hline 15 & KAL & da'un mpung & & & \\
\hline 16 & KBD & empung & & & \\
\hline 17 & KBK & mpung & 7 & $\mathbf{V}$ & O-4, P-3 \\
\hline 18 & PEB & ureu asi, uru asi & & \\
\hline
\end{tabular}

Notes : 


\section{Synedrella nodiflora (L.) Gaertn. (Asteraceae)}

\section{PIG'S GRASS. RUMPUT BABI}

\section{DESCRIPTION}

Herb, 8-90 cm, annual, erect repeatedly forked; leaves elliptic or ovate, $1.5-12.5$ x $0.5-9 \mathrm{~cm}$, suddenly narrowed to petiole, serrate margin, acute apex; flowers yellow; fruit achenes black or dark brown, 4-5 $\mathrm{mm}$ long, ribbed

\section{HABITAT}

Common weed of cultivated areas, waste lands

\section{ORIGINS}

America

\section{USES}

Leaves cooked as vegetable, medicine

\section{REFERENCES}

Burkill 1966: 2154; FOJ Vol II: 410

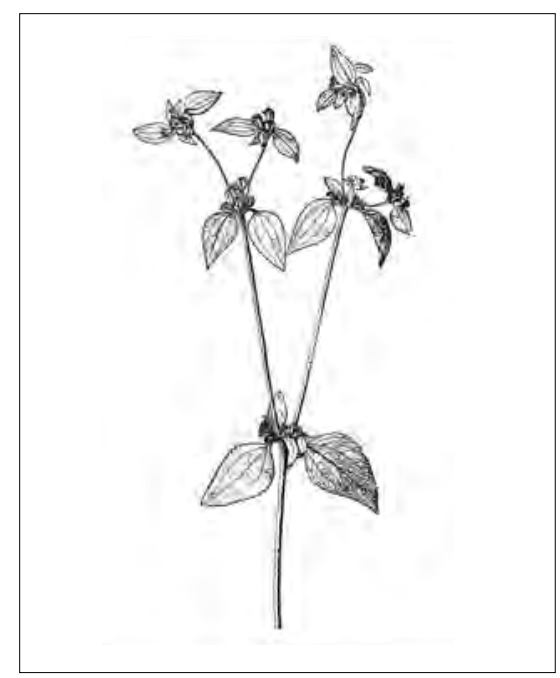

\begin{tabular}{|c|c|c|c|c|c|}
\hline \# & Code & Local Name & Use & RU & Hab.-Ab. \\
\hline 1 & PUT & belung & 1 & $\mathrm{x}$ & \\
\hline 2 & PUB & pulifan, uru ta'i & 2 & $x$ & $\mathrm{Y}-2$ \\
\hline 3 & PUD & uru teh & & $x$ & $\mathrm{~S}-2, \mathrm{Y}-2$ \\
\hline 4 & PUM & saga' amik & 2 & $x$ & $\mathrm{Y}-3$ \\
\hline 5 & ALS & bua' bundung & 1 & v & P-3 \\
\hline 6 & LDY & udu amek, fira' urat & 2 & v & $\mathrm{S}-2, \mathrm{Y}-2, \mathrm{Y}-3, \mathrm{O}-2$ \\
\hline 7 & LIT & udu mek & 2 & v & $\mathrm{Y}-2, \mathrm{Y}-3$ \\
\hline 8 & BRU & wi dau leng & 1 & $x$ & \\
\hline 9 & MRP & rau kedaye, ro kadaye & 2 & $x$ & $\mathrm{Y}-2, \mathrm{Y}-3$ \\
\hline 10 & PTN & uro' kading, uro' taei & 2 & $x$ & $\mathrm{~S}-2, \mathrm{Y}-3$ \\
\hline 11 & KLK & udu pute', udu pute & & $x$ & $\mathrm{Y}-2, \mathrm{O}-3$ \\
\hline 12 & KLM & keleng, udu pute & 1 & $x$ & ALL \\
\hline 13 & KLO & beva' kelieng & & $x$ & Y-3 \\
\hline 14 & KLA & heleng & & $x$ & $\mathrm{Y}-2$ \\
\hline 15 & KAL & udu kadieng & & & \\
\hline 16 & KBD & udu kading & 2 & $x$ & G-4, Y-3, O-3 \\
\hline 17 & KBK & bua' keling & & & \\
\hline 18 & PEB & perawek, perawok & 1 & $\mathrm{v}$ & G-2, P-4, M-3 \\
\hline
\end{tabular}

Notes : 


\section{Basella alba L. (Basellaceae)}

\section{CEYLON SPINACH. GANDOLA, GENJEROT}

\section{DESCRIPTION}

Creeping herb, perennial, 2-10 $\mathrm{m}$ long, stem-leaves dark red, purple, or yellowish green; leaves ovate or lanceolate $2-12$ x 1-10 cm; flowers axillary on peduncle $1-10 \mathrm{~cm}$ long, dark red or yellowish green; fruits globose, dark purple, juicy, 5-7 mm

\section{HABITAT}

Cultivated in homegardens

\section{ORIGINS}

Tropical Asia

\section{USES}

Young shoots boiled eaten as vegetable, pot herb, medicine; powdered fruit as ink, cosmetic and food dye

\section{REFERENCES}

Burkill 1966: 310; Ochse 1980: 75;

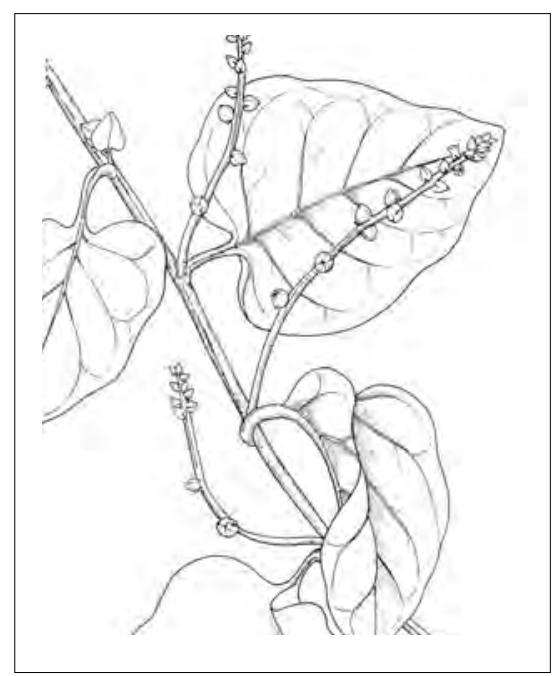

PROSEA Vol. 8: 93

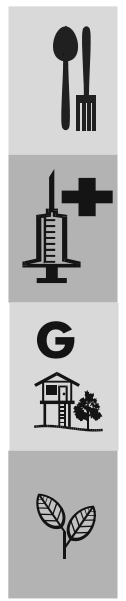

\begin{tabular}{|c|c|l|c|c|c|}
\hline$\#$ & Code & Local Name & Use & RU & Hab.-Ab. \\
\hline 1 & PUT & luruh, loruh & 1 & $\mathbf{V}$ & G-2, G-3, S-2 \\
\hline 2 & PUB & lenggang, luruh & 1 & $\mathbf{V}$ & G-1 \\
\hline 3 & PUD & leperu, luruh & 1 & $\mathbf{V}$ & G-2 \\
\hline 4 & PUM & luruh & 1 & $\mathbf{X}$ & Y-1, P-3 \\
\hline 5 & ALS & tengilung & 1 & $\mathbf{V}$ & G-3 \\
\hline 6 & LDY & war denudur, dinudur & 1 & $\mathbf{V}$ & G-2, G-3, S-1, S-3 \\
\hline 7 & LIT & dindur, denudor, linodor & 1 & $\mathbf{X}$ & G-3 \\
\hline 8 & BRU & uen neder & 1 & $\mathbf{V}$ & G-3, S-2, P-2 \\
\hline 9 & MRP & lurou, lura'u, luru & 1,2 & $\mathbf{V}$ & G-2, G-3, S-1 \\
\hline 10 & PTN & luro, ujo luro & 1 & $\mathbf{V}$ & G-2 \\
\hline 11 & KLK & ludo & 1 & $\mathbf{V}$ & G-2 \\
\hline 12 & KLM & lodo aka & & & \\
\hline 13 & KLO & lodo agha & & & \\
\hline 14 & KLA & ludo aha & & & \\
\hline 15 & KAL & lodo aha & & & \\
\hline 16 & KBD & lodo aka & & & \\
\hline 17 & KBK & ludo aka & 1 & $\mathbf{v}$ & G-4 \\
\hline 18 & PEB & ludo, kujau lanya & & \\
\hline
\end{tabular}

Notes : 


\section{Bixa orellana L. (Bixaceae)}

\section{ANATTO, LIPSTICK TREE. KESUMBA}

\section{DESCRIPTION}

Shrub, small tree, 2-6 m; bark brown, smooth; leaves spirally arranged, ovate 7.5-24 x 4-16 cm, dark green above, grey green below; flower white, pink or purple 4-6 cm; fruit bristled, ovoid capsule, 2 valves, 2-4 x 2-3.5 cm, green, brown or red, many seeds

\section{HABITAT}

Cultivated in gardens, waste lands

\section{ORIGINS}

Tropical America

\section{USES}

Pigment in food, cosmetics; basketry dye sold locally; timber for light construction (PEB)

\section{REFERENCES}

Burkill 1966: 333; PROSEA Vol. 3: 50

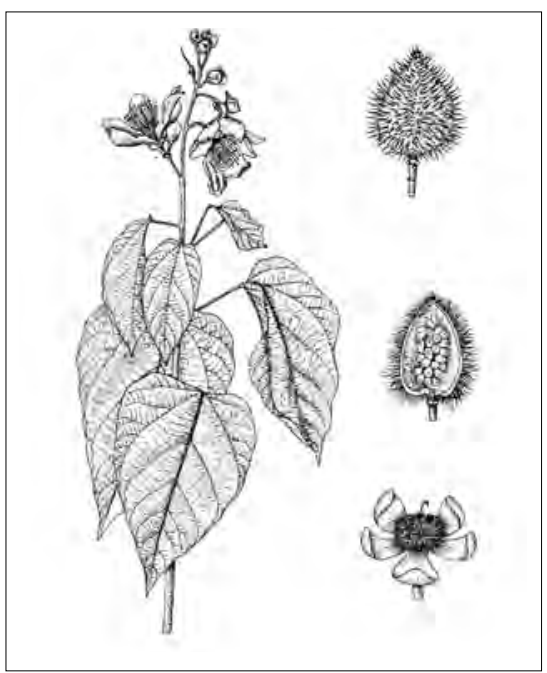

\begin{tabular}{|r|c|l|c|c|c|}
\hline \# & Code & \multicolumn{1}{|c|}{ Local Name } & Use & RU & Hab.-Ab. \\
\hline 1 & PUT & pang & 6 & $\mathbf{X}$ & G-1, G-2 \\
\hline 2 & PUB & piang, kelanga & $1,6,11$ & $\mathbf{V}$ & G-1, O-2, P-3 \\
\hline 3 & PUD & kayu pang, pang & 6 & $\mathbf{X}$ & G-1 \\
\hline 4 & PUM & pang & & $\mathbf{X}$ & G-1 \\
\hline 5 & ALS & bua' gifang & 6 & $\mathbf{X}$ & G-2 \\
\hline 6 & LDY & bua sia'ol & & $\mathbf{X}$ & G-1, G-3 \\
\hline 7 & LIT & udu ipang & 6 & $\mathbf{X}$ & G-2 \\
\hline 8 & BRU & wi pang & 6,9 & $\mathbf{V}$ & G-2, Y-1, O-1 \\
\hline 9 & MRP & pai, paeh, pae & 6 & $\mathbf{X}$ & G-1 \\
\hline 10 & PTN & bua piling, pileng & 6 & $\mathbf{X}$ & G-1 \\
\hline 11 & KLK & bala suwa, kayu bala suwa' & 6 & $\mathbf{X}$ & G-1, G-2 \\
\hline 12 & KLM & kadang sua, balasua & & $\mathbf{X}$ & G-2 \\
\hline 13 & KLO & kadeng sevu & & $\mathbf{X}$ & G-2 \\
\hline 14 & KLA & hadeng & & & \\
\hline 15 & KAL & tong kadeng & 6,9 & $\mathbf{V}$ & G-2 \\
\hline 16 & KBD & kela'a suwa, kela'a suwa' & & & \\
\hline 17 & KBK & kayu kadeng sua' & 4,6 & $\mathbf{X}$ & G-3 \\
\hline 18 & PEB & semuk & & \\
\hline
\end{tabular}

Notes : 


\section{DESCRIPTION}

Tree, 7-30 m, tapering trunk with sharp prickles; tiers of lateral branches; leaves crowded at tips of branches, 5-9 leaflets; flowers 5 petals, white; fruit a green oblong capsule $7-14 \times 3-4 \mathrm{~cm}$, with 5 cells, white floss fibres within, many seeds

\section{HABITAT}

Cutlivated in gardens, edge of pastures

\section{ORIGINS}

America

\section{USES}

Floss (fibres) for stuffing; tree for fences, shade; fruit eaten, medicine; sold locally

\section{REFERENCES}

Burkill 1966: 507; Ochse et al. 1980: 79

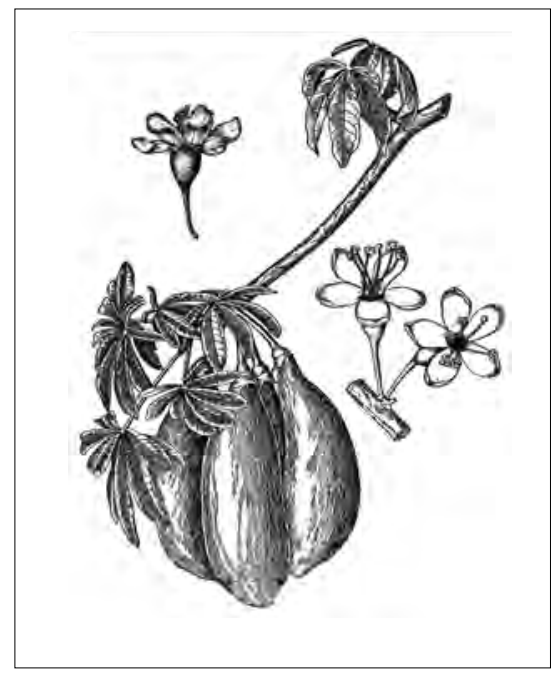

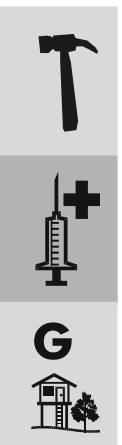

2)

\begin{tabular}{|r|c|l|c|c|c|}
\hline \# & Code & \multicolumn{1}{|c|}{ Local Name } & Use & RU & Hab.-Ab. \\
\hline 1 & PUT & kapuk, kayu legen, bua buro & $6,2,11$ & $\mathbf{X}$ & G-2, G-3 \\
\hline 2 & PUB & bua bure', kayu buro' & 6 & $\mathbf{X}$ & G-2, G-3 \\
\hline 3 & PUD & bua buro & $1,2,6$ & $\mathbf{X}$ & G-2, G-4 \\
\hline 4 & PUM & kapuk & 6 & $\mathbf{X}$ & G-3 \\
\hline 5 & ALS & bua' kavok & 6 & $\mathbf{X}$ & \\
\hline 6 & LDY & kayu kafuk, bua kafuk & 6,2 & $\mathbf{X}$ & G-3, G-4 \\
\hline 7 & LIT & gapuk & 6,11 & $\mathbf{X}$ & G-3 \\
\hline 8 & BRU & uen kapas & 6 & $\mathbf{X}$ & G-3 \\
\hline 9 & MRP & waberuwa, kayo wamperwa, bua merewa & 6 & $\mathbf{X}$ & G-3 \\
\hline 10 & PTN & bua bura & 6,2 & $\mathbf{X}$ & G-3 \\
\hline 11 & KLK & bua' bura, bua' bura' & 6 & $\mathbf{X}$ & G-2 \\
\hline 12 & KLM & bua' bura & 7 & $\mathbf{V}$ & G-2, G-3 \\
\hline 13 & KLO & beva' bora & 6 & $\mathbf{X}$ & G-3, P-3 \\
\hline 14 & KLA & beva' bura, beva bura & & & G-3 \\
\hline 15 & KAL & beva' bura' & 6,8 & $\mathbf{X}$ & G-2, G-4 \\
\hline 16 & KBD & bora', bora & & & \\
\hline 17 & KBK & bua' bura & 6 & $\mathbf{v}$ & G-3, O-2 \\
\hline 18 & PEB & bura, daun bura' & 6 & $\mathbf{6}$ \\
\hline
\end{tabular}

Notes : 


\section{Durio kutejensis (Hassk.) Becc. (Bombacaceae)}

\section{KUTAI DURIAN. LAI}

\section{DESCRIPTION}

Tree, $24 \mathrm{~m}$; fruit yellow brown ovoid to ellipsoid, pentangular, up to $20 \times 13 \mathrm{~cm}$, slighly curved, broadly pyramidal, soft spines up to $1.5 \mathrm{~cm}$; aril white to orange, fleshy, sweet, fragrant; seeds glossy brown

\section{HABITAT}

Cultivated in home and swidden gardens

\section{ORIGINS}

\section{Borneo}

\section{USES}

Fruit eaten, sold locally; timber for tools, light construction

\section{REFERENCES}

Kessler and Sidiyasa 1994: 60; PROSEA Vol. 2: 330; Valkenburg 1997: 73

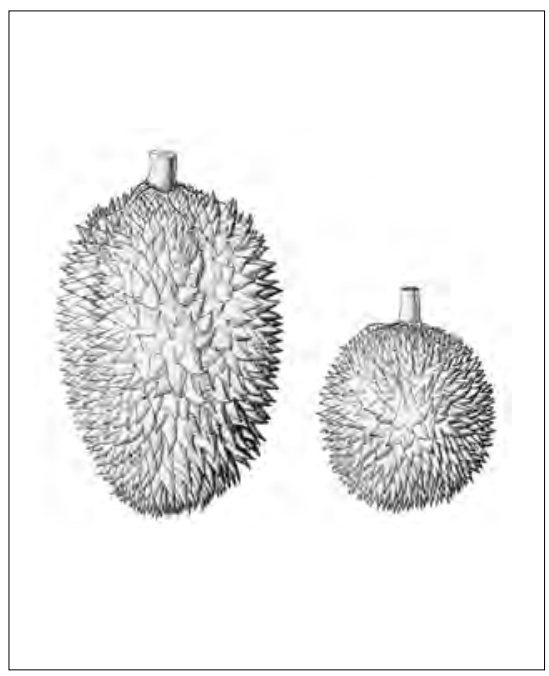

\begin{tabular}{|c|c|l|c|c|c|}
\hline$\#$ & Code & \multicolumn{1}{|c|}{ Local Name } & Use & RU & Hab.-Ab. \\
\hline 1 & PUT & paken & 1,11 & $\mathbf{v}$ & G-2, G-3, O-3, P-4, P-3, M-3 \\
\hline 2 & PUB & paken & 1,11 & $\mathbf{v}$ & G-3, Y-2, O-2, P-3, P-4 \\
\hline 3 & PUD & paken & 1,11 & $\mathbf{v}$ & G-2, G-3, P-2, P-4 \\
\hline 4 & PUM & paken & 1,11 & $\mathbf{v}$ & G-3, O-2, P-3, M-4 \\
\hline 5 & ALS & ruwas bongo & $1,7,11$ & $\mathbf{v}$ & G-3, O-2, P-3, M-2 \\
\hline 6 & LDY & ruwat, ruat & 1,11 & $\mathbf{v}$ & G-3, O-2, P-3 \\
\hline 7 & LIT & ruwat, bua ruat & 1,11 & $\mathbf{v}$ & G-4, Y-4, O-4, P-4 \\
\hline 8 & BRU & wi paken & 1,11 & $\mathbf{v}$ & G-3, O-2, P-3 \\
\hline 9 & MRP & pakaing, pakeng, pekeing & 1,11 & $\mathbf{v}$ & G-3, G-4, P-3, P-4, M-4 \\
\hline 10 & PTN & dian bela & 1,11 & $\mathbf{v}$ & G-3, P-4 \\
\hline 11 & KLK & pakin, bua paken & 1,11 & $\mathbf{v}$ & G-3, P-3 \\
\hline 12 & KLM & paken & 1,11 & $\mathbf{v}$ & G-3 \\
\hline 13 & KLO & lezing bala & 1,11 & $\mathbf{v}$ & G-3 \\
\hline 14 & KLA & dizan bala & 1,11 & $\mathbf{v}$ & G-3 \\
\hline 15 & KAL & dezin bala & 1,11 & $\mathbf{v}$ & G-3 \\
\hline 16 & KBD & dian bela', dian lei, dian bala & 1,11 & $\mathbf{v}$ & G-3 \\
\hline 17 & KBK & dian bala & $1,5,6,11$ & $\mathbf{v}$ & G-3, O-2, P-4, M-4 \\
\hline 18 & PEB & lai & $\mathbf{v}$ & G-3 \\
\hline
\end{tabular}

Notes : 


\section{Durio oxleyanus Griff. (Bombacaceae)}

\section{LEAF DURIAN. KERANTUNGAN}

\section{DESCRIPTION}

Tree, $35-45 \mathrm{~m}$, buttresses to $3 \mathrm{~m}$; brown bark deeply fissured; leaves elliptic 7-20 x 3-7.5 cm, lower surface velvety with grey hairs; fruit grey green globular $15-20 \mathrm{~cm}$, spines to $4 \mathrm{~cm}$; aril creamy to dark yellow, not fragrant

\section{HABITAT}

Lowland and hill primary forest; moist places; cultivated in village fruit gardens

\section{ORIGINS}

Malaysia and western Indonesia

\section{USES}

Fruit eaten, sold locally; timber for light construction; hunting location when fruiting

\section{REFERENCES}

Burkill 1966: 886; Kessler and Sidiyasa 1994: 61;

Valkenburg 1997: 197

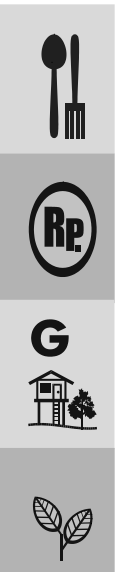

\begin{tabular}{|c|c|c|c|c|c|}
\hline \# & Code & Local Name & Use & RU & Hab.-Ab. \\
\hline 1 & PUT & tungen & $1,6,10,11$ & $\mathrm{v}$ & $\mathrm{G}-1, \mathrm{G}-2, \mathrm{O}-2, \mathrm{P}-3$ \\
\hline 2 & PUB & tungen & 1,10 & v & G-1, P-3, M-2 \\
\hline 3 & PUD & tungen & 1 & v & G-1, P-3, P-4 \\
\hline 4 & PUM & tungen & $1,10,11$ & v & G-2, P-4 \\
\hline 5 & ALS & bua' ketungon & $1,6,10$ & v & G-2, P-3 \\
\hline 6 & LDY & kayu keritungen, kari tungon & $1,8,11$ & v & G-2, P-3, M-2, M-4 \\
\hline 7 & LIT & reten, keretungen & 1,11 & v & G-2, P-3 \\
\hline 8 & BRU & wi nyan un & 1,10 & $\mathrm{v}$ & G-1 \\
\hline 9 & MRP & yaeng laung, yang laung & 1,10 & v & G-2, P-3 \\
\hline 10 & PTN & dian daun & 1 & v & G-1 \\
\hline 11 & KLK & dian kalang, dian da'un & 1 & v & G-2 \\
\hline 12 & KLM & dian daun & 1 & $x$ & ALL \\
\hline 13 & KLO & lezing da'eng & 1 & $\mathrm{v}$ & G-2 \\
\hline 14 & KLA & dizan bala, dizan daun & 1,10 & $x$ & G-3, P-3 \\
\hline 15 & KAL & dezin da'un & 1,11 & $\mathrm{v}$ & G-2 \\
\hline 16 & KBD & dian bala, dian belang & 1,10 & v & $\mathrm{G}-2, \mathrm{P}-3, \mathrm{P}-4$ \\
\hline 17 & KBK & dian daun & 1 & v & G-2 \\
\hline 18 & PEB & tungen & $1,4,6,11$ & v & P-4, M-4 \\
\hline
\end{tabular}

Notes : 


\section{Durio zibetbinus Murr. (Bombacaceae)}

\section{DURIAN. DURIAN}

\section{DESCRIPTION}

Tree, $35 \mathrm{~m}$, buttresses steep; bark dark red brown 2 cm thick; leaves elliptic 10-15 x 3-4.5 cm, lower surface silvery or golden scales; fruit green to yellow globular to ellipsoid to $25 \mathrm{~cm}$ long, sharp spines $1 \mathrm{~cm}$; aril thick, white or yellow, fragrant

\section{HABITAT}

Cultivated in fruit gardens, homegardens; possibly wild in primary forests

\section{ORIGINS}

Malaysia and western Indonesia

\section{USES}

Fruit eaten, sold locally; timber for furniture; firewood

\section{REFERENCES}

Kessler and Sidiyasa 1994: 62; Ochse 1980: 82; PROSEA Vol. 2: 157; Valkenburg 1997: 75

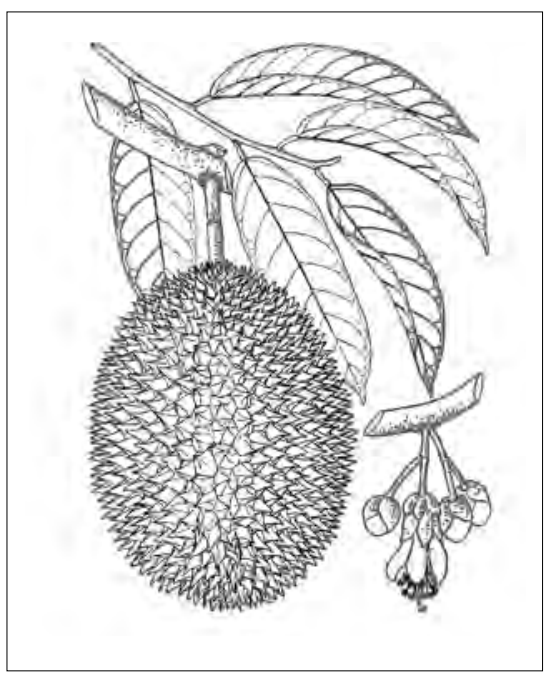

\begin{tabular}{|c|c|l|c|c|c|}
\hline$\#$ & Code & \multicolumn{1}{|c|}{ Local Name } & Use & RU & Hab.-Ab. \\
\hline 1 & PUT & docou & $1,6,7,11$ & $\mathbf{v}$ & G-3, Y-3, O-2, P-2 \\
\hline 2 & PUB & docou & $1,6,11$ & $\mathbf{v}$ & G-3, P-2, P-3 \\
\hline 3 & PUD & dosou, doso & 1,11 & $\mathbf{v}$ & G-2, P-4 \\
\hline 4 & PUM & tefela & 1,7 & $\mathbf{v}$ & P-4, M-3 \\
\hline 5 & ALS & bua lambun & $1,5,6,7,11$ & $\mathbf{v}$ & G-4 \\
\hline 6 & LDY & kayu datu, derian & $1,2,6,11$ & $\mathbf{v}$ & G-3, O-3, P-4 \\
\hline 7 & LIT & datu, derian, durian merah & 1,11 & $\mathbf{v}$ & G-4, O-4, P-2, P-3 \\
\hline 8 & BRU & wi atau & $1,6,11$ & $\mathbf{v}$ & G-3, P-3, M-2 \\
\hline 9 & MRP & yang, yaeng, yan kale & $1,6,11$ & $\mathbf{v}$ & G-4, Y-3, O-3, P-3, M-2 \\
\hline 10 & PTN & dian puti, dian kalang & $1,6,11$ & $\mathbf{v}$ & G-3, P-3, M-3 \\
\hline 11 & KLK & dian, dian kalang, dian surun & $1,6,11$ & $\mathbf{x}$ & G-4, P-3 \\
\hline 12 & KLM & dian kalang, pakin & 1,11 & $\mathbf{v}$ & G-2, G-3 \\
\hline 13 & KLO & lezing kalang, lezing talang & 1,11 & $\mathbf{v}$ & G-2, G-3 \\
\hline 14 & KLA & dizan da'un, dian kalang & 1,11 & $\mathbf{v}$ & G-3 \\
\hline 15 & KAL & dezin kalang & 1,11 & $\mathbf{v}$ & G-3 \\
\hline 16 & KBD & dian bileng, dian beleng & 1,11 & $\mathbf{v}$ & G-3 \\
\hline 17 & KBK & dian putei & 1,11 & $\mathbf{v}$ & G-3 \\
\hline 18 & PEB & paken & $1,5,6,11$ & $\mathbf{v}$ & G-3, O-2, P-4, M-4 \\
\hline
\end{tabular}

Notes : 


\section{DESCRIPTION}

Herb, 50-150 cm, leaves sword-shaped to $1 \mathrm{~m}$ by 5 $8 \mathrm{~cm}$ wide, margin spiny, arranged in close spiral; flowers red purple; prickly fruit with spiny crown of leaves; flesh pale to yellow, usually seedless

\section{HABITAT}

Cultivated in homegardens, plantations

\section{ORIGINS}

Tropical America

\section{USES}

Fruit eaten, sold locally

\section{REFERENCES}

Burkill 1966: 149; Ochse 1980: 84;

PROSEA Vol. 2: 66

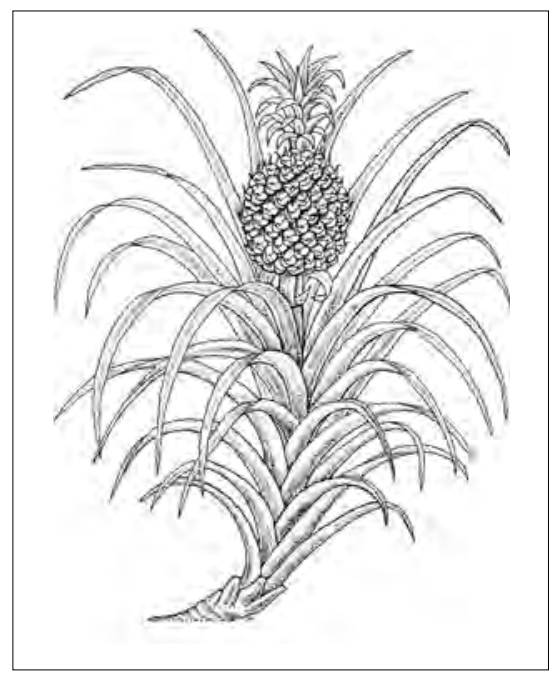

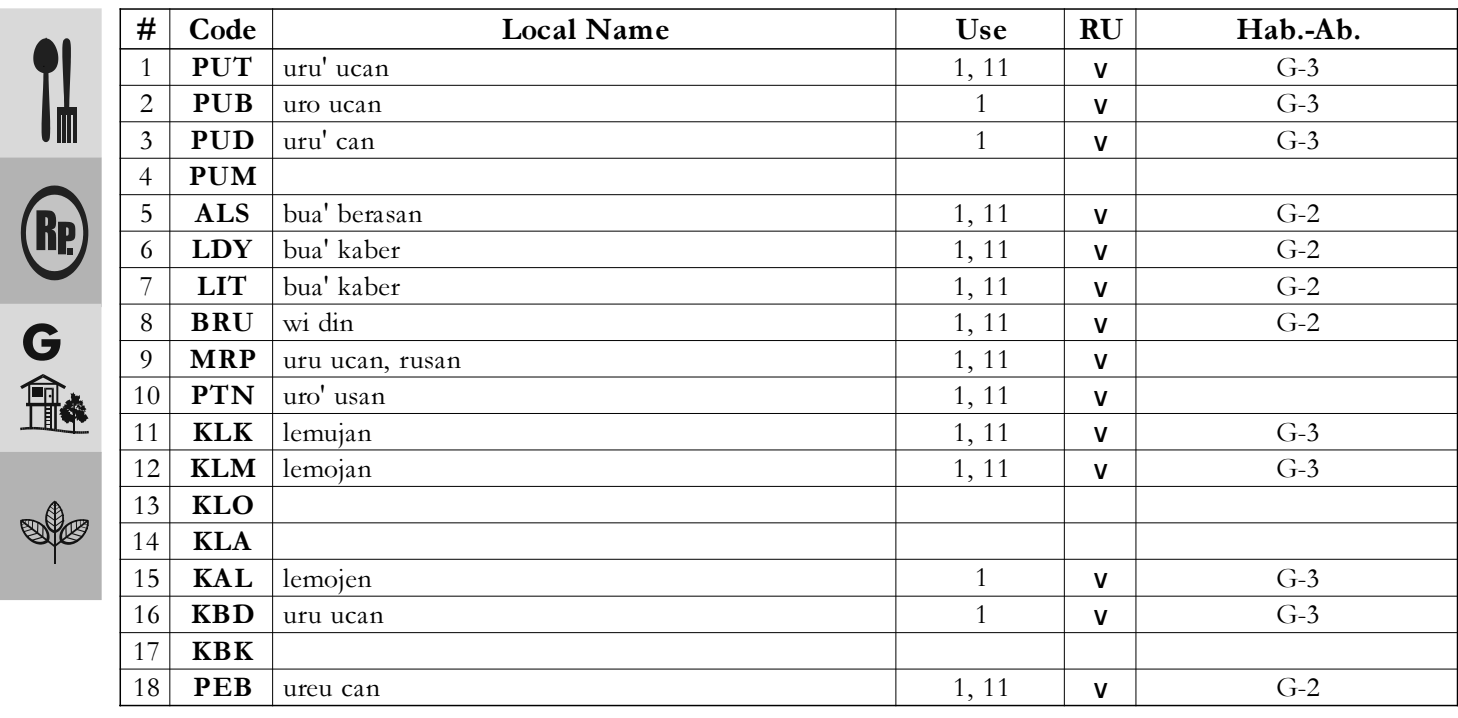

Notes : 


\section{Canarium odontopbyllum Miq. (Burseraceae)}

\section{DANAU MAJANG, KENARI}

\section{DESCRIPTION}

Tree, to $35 \mathrm{~m}$, buttresses; bark smooth grey, inner bark exuding white resin; leaves with 7-17 dentate leaflets, hairy below and on midrib above; flowers creamy white; fruit ovoid to ellipsoid, 25$35 \times 17-20 \mathrm{~mm}$, blue black

\section{HABITAT}

Lowland primary forest; cultivated in village fruit gardens

\section{ORIGINS}

Malaysia and western Indonesia

\section{USES}

Fruit eaten, sold locally; timber for firewood

\section{REFERENCES}

PROSEA Vol. 5(2): 103

Cockburn 1976: Fig. 7

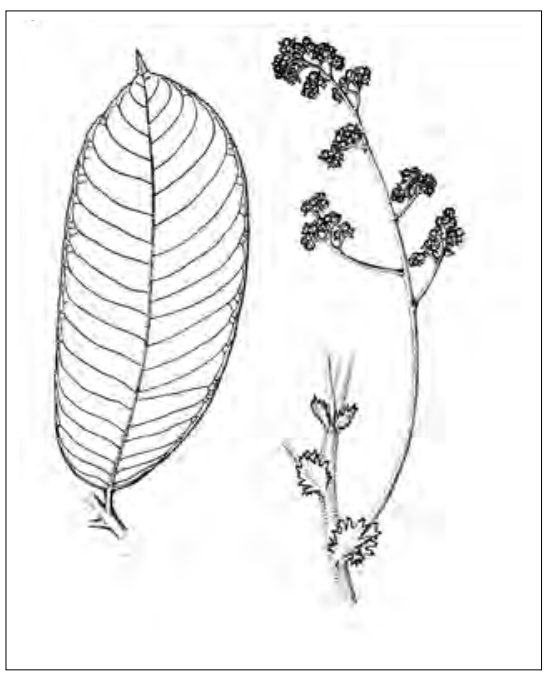

\begin{tabular}{|c|c|l|c|c|c|}
\hline$\#$ & Code & \multicolumn{1}{|c|}{ Local Name } & Use & RU & Hab.-Ab. \\
\hline 1 & PUT & defai & 1,11 & $\mathbf{v}$ & G-2, P-3, G-3, O-2, P-4 \\
\hline 2 & PUB & defai & $1,7,11$ & $\mathbf{v}$ & O-2, P-3, G-2, G-3, M-2 \\
\hline 3 & PUD & defai & 1,11 & $\mathbf{v}$ & G-2, O-2, P-2, P-4, Y-2 \\
\hline 4 & PUM & defai & $1,2,11$ & $\mathbf{v}$ & G-3, M-4, O-2, P-3 \\
\hline 5 & ALS & bua' fasan & 1,11 & $\mathbf{v}$ & G-3, O-2, P-3 \\
\hline 6 & LDY & kayu pasan, bua pasan & 1,11 & $\mathbf{v}$ & G-3, M-2, O-2, P-4, O-3, P-3, Y-2 \\
\hline 7 & LIT & pasan, desan & 1,11 & $\mathbf{v}$ & G-3, M-2, M-3, O-2, P-2, P-4, Y-2 \\
\hline 8 & BRU & wi kabe & $1,2,7,11$ & $\mathbf{v}$ & G-3, P-3 \\
\hline 9 & MRP & bua labai, labae & 1,11 & $\mathbf{v}$ & G-3, O-2, P-3, M-4, M-2 \\
\hline 10 & PTN & kerawe & $1,7,11$ & $\mathbf{v}$ & G-3, O-2, P-4 \\
\hline 11 & KLK & labai & 1,11 & $\mathbf{v}$ & G-2, P-3, M-2 \\
\hline 12 & KLM & kerawai & 1 & $\mathbf{v}$ & \\
\hline 13 & KLO & keravei & & & \\
\hline 14 & KLA & beva keravai & & & \\
\hline 15 & KAL & keravae & & & \\
\hline 16 & KBD & kerawai & & & \\
\hline 17 & KBK & keramo' rawai & 1 & $\mathbf{v}$ & O-2, P-4, M-4 \\
\hline 18 & PEB & davei & & & \\
\hline
\end{tabular}

Notes : 


\section{Dacryodes rostrata (Blume) H.J. Lam. (Burseraceae)}

\section{KERUT, KERAMUH BERPARUH}

\section{DESCRIPTION}

Tree, 10-25 m, buttressed; inner bark pinkish with clear to white exudate; leaves with 2-10 pairs of leaflets, ovate to oblong 3.5-20 x $1.5-10 \mathrm{~cm}$, parchment-like, drying reddish brown or dark brown; fruits oblong 1.75-3.5 x 0.75-1.75 cm, mauve black

\section{HABITAT}

Lowland and hill primary forest; planted in home and swidden gardens

\section{ORIGINS}

Malaysia and western Indonesia

\section{USES}

Fruit eaten, sold locally; timber for blowpipe, firewood; hunting location when fruiting

\section{REFERENCES}

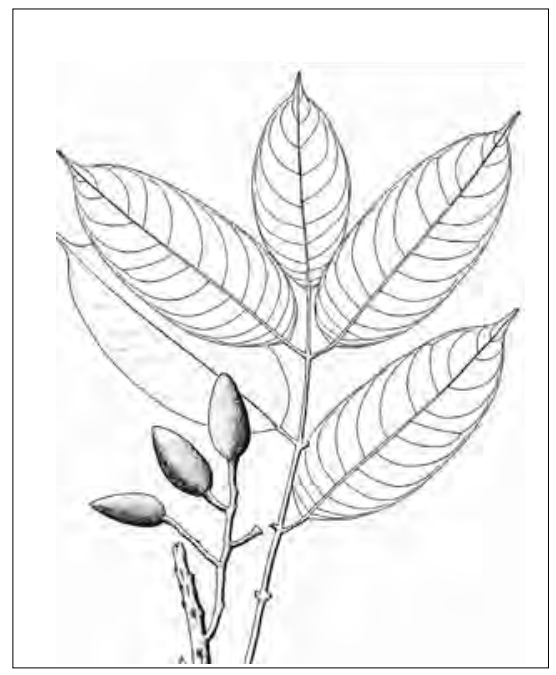

Kessler and Sidiyasa 1994: 67; PROSEA Vol. 2:

327; TFM Vol. 1: 143

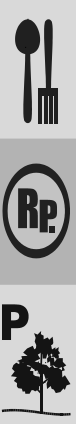

\begin{tabular}{|r|c|l|c|c|c|}
\hline \# & Code & \multicolumn{1}{|c|}{ Local Name } & Use & RU & Hab.-Ab. \\
\hline 1 & PUT & keramuh & $1,10,11$ & $\mathbf{v}$ & G-1, O-2, P-3, M-3 \\
\hline 2 & PUB & keramuh & 1,10 & $\mathbf{v}$ & P-3 \\
\hline 3 & PUD & keramuh & 1,11 & $\mathbf{v}$ & P-3 \\
\hline 4 & PUM & keramuh & 1,11 & $\mathbf{v}$ & P-3 \\
\hline 5 & ALS & bua' keramu & 1,11 & $\mathbf{v}$ & G-1, P-3 \\
\hline 6 & LDY & kayu kedamu, kedamu & 1,11 & $\mathbf{v}$ & G-1, S-2, P-3, M-3 \\
\hline 7 & LIT & rengurin, rengoren & 1,10 & $\mathbf{v}$ & P-3 \\
\hline 8 & BRU & wi karemou & 1,10 & $\mathbf{v}$ & G-1 \\
\hline 9 & MRP & buwa keramau, kelamo, lelamu & $1,10,11$ & $\mathbf{v}$ & G-2, P-3, M-2 \\
\hline 10 & PTN & keramo', keramo & $1,7,10$ & $\mathbf{v}$ & G-1, P-3 \\
\hline 11 & KLK & keramo', keramo & 1 & $\mathbf{v}$ & \\
\hline 12 & KLM & keramo, kalamu & 1,10 & $\mathbf{v}$ & ALL \\
\hline 13 & KLO & keramu, keramu & 1,10 & $\mathbf{x}$ & G-2, P-3, M-3 \\
\hline 14 & KLA & keramu & & & G-2, P-3 \\
\hline 15 & KAL & keramu & 1,10 & $\mathbf{v}$ & G-2, O-3, P-4 \\
\hline 16 & KBD & keramo, keramu & & & \\
\hline 17 & KBK & keramo' & 1 & $\mathbf{v}$ & G-3, O-2, P-4, M-4 \\
\hline 18 & PEB & kerameu, keramu & & \\
\hline
\end{tabular}

Notes : 


\section{TUALANG. MANGARIS, KEMPAS MADU}

\section{DESCRIPTION}

Giant tree, to $85 \mathrm{~m}$, buttresses, few large branches; bark smooth, shiny purplish grey tinged with fluorescent green; leaflets 7-13, elliptic, $3.5 \times 1.5 \mathrm{~cm}$; Fruit a flat elliptical pod surrounded by broad, veined and brittle wing $8-12 \times 2-3.5 \mathrm{~cm}$

\section{HABITAT}

Lowland primary forest; riverine, lower slopes

\section{ORIGINS}

Malaysia and western Indonesia

\section{USES}

Timber for furniture, light construction; honey tree that supports hives of Apis cerana; honey sold locally and to traders

\section{REFERENCES}

Kessler and Sidiyasa 1994: 73; PROSEA Vol.

5(1): 270; TFM Vol. 1: 264

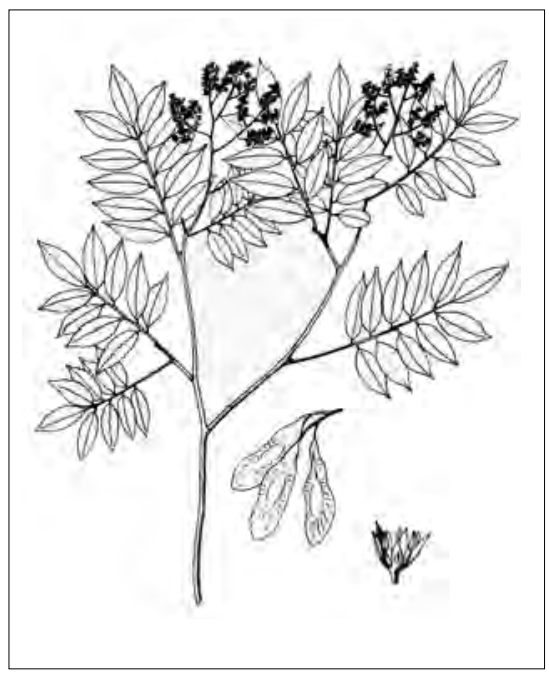

\begin{tabular}{|c|c|c|c|c|c|}
\hline \# & Code & Local Name & Use & RU & Hab.-Ab. \\
\hline 1 & PUT & laloh & $4,6,10,11$ & $\mathrm{v}$ & $\mathrm{R}-3, \mathrm{P}-2, \mathrm{M}-2$ \\
\hline 2 & PUB & tanyit & $4,6,10,11$ & $\mathrm{v}$ & $\mathrm{R}-3, \mathrm{P}-2, \mathrm{M}-2$ \\
\hline 3 & PUD & laloh & $4,6,10,11$ & $\mathrm{v}$ & $\mathrm{R}-3, \mathrm{P}-2, \mathrm{M}-2$ \\
\hline 4 & PUM & laloh & $4,6,10,11$ & v & $\mathrm{R}-3, \mathrm{P}-2, \mathrm{M}-2$ \\
\hline 5 & ALS & magis & 10,11 & $x$ & $\mathrm{R}-3, \mathrm{P}-2$ \\
\hline 6 & LDY & tanit & 10,11 & $x$ & $\mathrm{R}-3, \mathrm{P}-2$ \\
\hline 7 & LIT & tanit & 10,11 & $x$ & R-3, P-2 \\
\hline 8 & BRU & bingai & 10,11 & $x$ & R-3, P-2 \\
\hline 9 & MRP & tenyit, tanyi' & 10,11 & $x$ & $\mathrm{R}-3, \mathrm{P}-2$ \\
\hline 10 & PTN & tanyit & 10,11 & $x$ & R-3, P-2 \\
\hline 11 & KLK & tanyit & 10,11 & $x$ & R-2, P-2, M-1 \\
\hline 12 & KLM & tanyit & 10,11 & $x$ & $\mathrm{R}-2, \mathrm{P}-2, \mathrm{M}-1$ \\
\hline 13 & KLO & tanyi' & 10,11 & $\mathrm{v}$ & R-2, P-2 \\
\hline 14 & KLA & tanyit & 10,11 & $x$ & $\mathrm{R}-2, \mathrm{P}-2$ \\
\hline 15 & KAL & tanyit & 10,11 & $\mathrm{v}$ & $\mathrm{R}-2, \mathrm{P}-2$ \\
\hline 16 & KBD & tanyit & 10,11 & $x$ & $\mathrm{R}-2, \mathrm{P}-2$ \\
\hline 17 & KBK & tanyit & 10,11 & $x$ & $\mathrm{R}-2, \mathrm{P}-2$ \\
\hline 18 & PEB & tanyit & 10,11 & $\mathrm{v}$ & $\mathrm{R}-2, \mathrm{P}-2, \mathrm{M}-2$ \\
\hline
\end{tabular}

Notes : 


\section{Carica papaya L. (Caricaceae)}

\section{Papaya. Kates}

\section{DESCRIPTION}

Tree-like herb, 2-10 $\mathrm{m}$, white latex in all parts; leaves spirally arranged at apex, larger blade $50-75 \mathrm{~cm}$ diameter, palmate 7-11 lobes; flowers unisexualdioeceous; fruit a fleshy berry, ovoid, grooved, 7 $30 \mathrm{~cm}$ long, yellow orange; 2 varieties in Bulungan: small and large leaf

\section{HABITAT}

Cultivated in home and swidden gardens, plantations

\section{ORIGINS}

America (Portugese brought in 1600s)

\section{USES}

Fruit, flower and leaves eaten; leaves for tenderizing meat, medicine; fruit sold locally

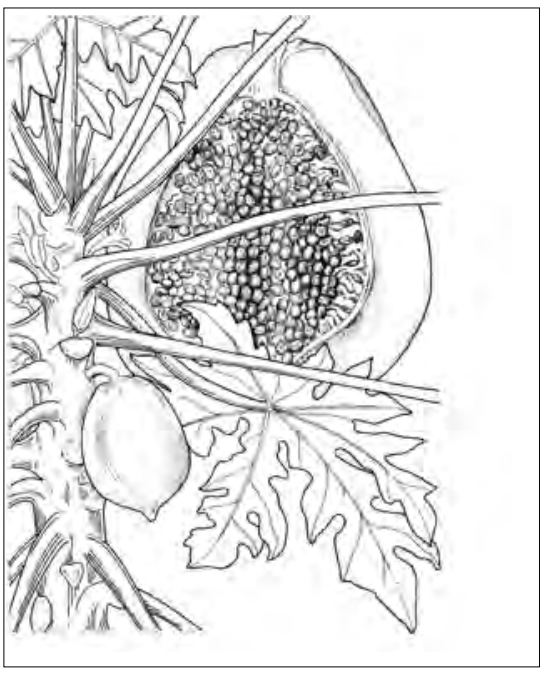

\section{REFERENCES}

Burkill 1966: 464; Ochse 1980: 98;

PROSEA Vol. 2: 108

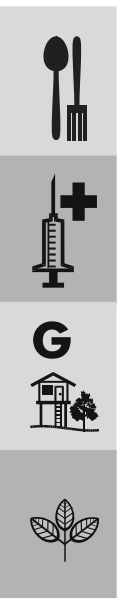

\begin{tabular}{|c|c|l|c|c|c|}
\hline$\#$ & Code & \multicolumn{1}{|c|}{ Local Name } & Use & RU & Hab.-Ab. \\
\hline 1 & PUT & mujan, mujan ayo doun, mujan panjang & $1,2,11$ & $\mathbf{v}$ & G-3, G-2 \\
\hline 2 & PUB & mujan & $1,2,11$ & $\mathbf{v}$ & G-3 \\
\hline 3 & PUD & mujan & $1,2,11$ & $\mathbf{v}$ & G-3, S-1 \\
\hline 4 & PUM & mujan & $1,2,11$ & $\mathbf{v}$ & G-3, S-2 \\
\hline 5 & ALS & padas & $1,2,11$ & $\mathbf{v}$ & G-4, Y-2 \\
\hline 6 & LDY & bua sesila', sesila & $1,2,11$ & $\mathbf{v}$ & G-3, G-4, S-2, S-3, Y-1 \\
\hline 7 & LIT & bua ila', bua sesila, bua sosila' & $1,2,11$ & $\mathbf{v}$ & G-4 \\
\hline 8 & BRU & wi yan & $1,2,11$ & $\mathbf{v}$ & G-3, S-1 \\
\hline 9 & MRP & jang, zaen, jan & $1,2,11$ & $\mathbf{v}$ & G-3, G-2 \\
\hline 10 & PTN & manjan & $1,2,11$ & $\mathbf{v}$ & G-3, S-2 \\
\hline 11 & KLK & manjan & $1,2,11$ & $\mathbf{v}$ & G-3, S-2 \\
\hline 12 & KLM & manjan & $1,2,11$ & $\mathbf{v}$ & G-3, G-2 \\
\hline 13 & KLO & facen, faceny, facen dadu & $1,2,11$ & $\mathbf{v}$ & G-3, S-3 \\
\hline 14 & KLA & macan, tung macan & $1,2,11$ & $\mathbf{v}$ & G-3, S-3 \\
\hline 15 & KAL & mancin & $1,2,11$ & $\mathbf{v}$ & G-3, S-3 \\
\hline 16 & KBD & manjan & $1,2,11$ & $\mathbf{v}$ & G-3, S-3 \\
\hline 17 & KBK & manjan & $1,2,11$ & $\mathbf{v}$ & G-3, S-3 \\
\hline 18 & PEB & bodung & $1,2,11$ & $\mathbf{v}$ & G-3, S-3 \\
\hline
\end{tabular}

Notes : 


\section{WATER SPINACH. KANGKUNG}

\section{DESCRIPTION}

Herb, creeping or floating, perennial; leaves alternate, long petioled, triangular, $2.5-15 \mathrm{~cm} \times 0.5-10 \mathrm{~cm}$; flowers funnel form $4-7.5 \mathrm{~cm}$ long, magenta throat; fruit brown ovoid capsule 7-9 $\mathrm{mm}$ diameter, 2-4 seeds

\section{HABITAT}

Cultivated in garden and fishponds

\section{ORIGINS}

Asia

\section{USES}

Young stems and leaves eaten, sold locally; fodder

\section{REFERENCES}

Ochse 1980: 159; PROSEA Vol. 8: 181

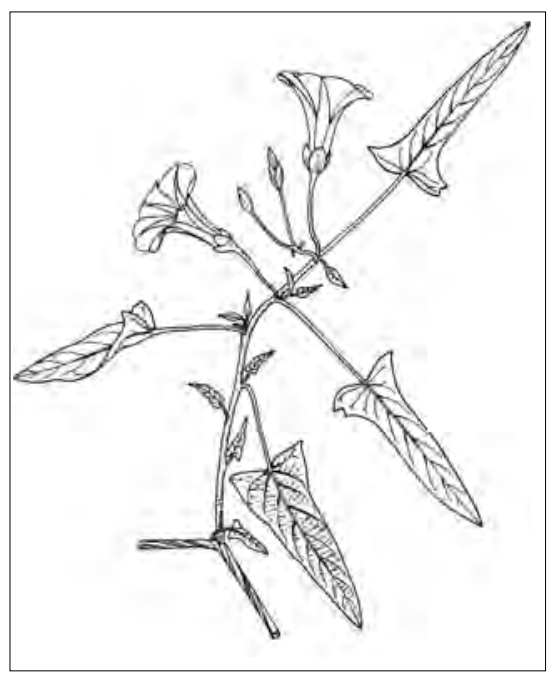

\begin{tabular}{|r|l|l|c|c|c|}
\hline \# & Code & \multicolumn{1}{|c|}{ Local Name } & Use & RU & Hab.-Ab. \\
\hline 1 & PUT & ubi oka, kangkung & 1,11 & $\mathbf{v}$ & G-3 \\
\hline 2 & PUB & kangkung & 1,11 & $\mathbf{v}$ & G-3 \\
\hline 3 & PUD & kangkung & 1,11 & $\mathbf{v}$ & G-2 \\
\hline 4 & PUM & ubi oka & 1 & $\mathbf{v}$ & G-3, S-1 \\
\hline 5 & ALS & daun kangkung & 1,11 & $\mathbf{v}$ & G-2 \\
\hline 6 & LDY & war kangkung, kangkung & 1,11 & $\mathbf{v}$ & G-3 \\
\hline 7 & LIT & kangkung, kangkung buda, kai leung & 1 & $\mathbf{v}$ & G-2, S-1 \\
\hline 8 & BRU & bai gual & 1,11 & $\mathbf{v}$ & G-3 \\
\hline 9 & MRP & boee, bua nyenggung, boe janggoe & 1,11 & $\mathbf{v}$ & G-3 \\
\hline 10 & PTN & kangkung, ube aka & 1,11 & $\mathbf{v}$ & G-2 \\
\hline 11 & KLK & kangkung bileng, kangkung bala & 1,11 & $\mathbf{v}$ & G-2 \\
\hline 12 & KLM & kangkung & 1,11 & $\mathbf{v}$ & G-3 \\
\hline 13 & KLO & ubi agha & 1,11 & $\mathbf{v}$ & G-3 \\
\hline 14 & KLA & ubi aha & 1,11 & $\mathbf{v}$ & G-3 \\
\hline 15 & KAL & pangkung & 1,11 & $\mathbf{v}$ & G-3 \\
\hline 16 & KBD & kangkung & 1,11 & $\mathbf{v}$ & G-4 \\
\hline 17 & KBK & kangkung & 1 & $\mathbf{v}$ & G-4, S-3 \\
\hline 18 & PEB & ludo, kangkung & & \\
\hline
\end{tabular}

Notes : 


\section{t Ipomoea batatas (L.) L. (Convolvulaceae) SWEET POTATO. UBI JALAR}

\section{DESCRIPTION}

Herb, creeping, perennial, 1-8 m long; stems hollow; roots variable; leaves spiral, grooved petiole, ovate 4-15 x 4-12 cm, entire or palmately lobed; flower funnel-form white or lavender with purple throat; fruit a brown capsule 5-8 mm, 1-4 seeds

\section{HABITAT}

Cultivated in home gardens

\section{ORIGINS}

Unknown

\section{USES}

Roots and young shoots eaten, for medicine

\section{REFERENCES}

Ochse 1980: 155; PROSEA Vol. 9: 102

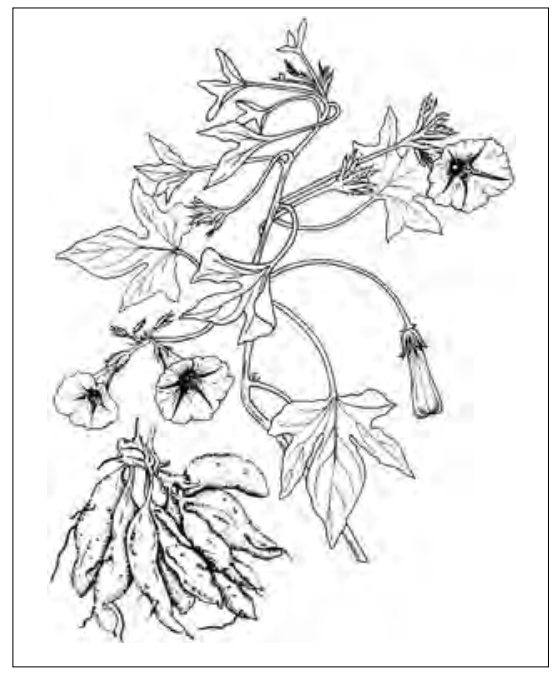

\begin{tabular}{|c|c|c|c|c|c|}
\hline \# & Code & Local Name & Use & RU & Hab.-Ab. \\
\hline 1 & PUT & ubi oka & 1 & $\mathrm{v}$ & G-3, S-1 \\
\hline 2 & PUB & ubi oka & 1 & v & G-3 \\
\hline 3 & PUD & ubi oka & 1 & v & G-3 \\
\hline 4 & PUM & ubi oka & 1 & v & G-3 \\
\hline 5 & ALS & abai & 1 & $\mathrm{v}$ & G-3 \\
\hline 6 & LDY & ubi war, kangkung & 1 & v & G-3, S-2 \\
\hline 7 & LIT & ubi war, ubi uwar & 1 & $\mathrm{v}$ & G-1, G-4 \\
\hline 8 & BRU & uen baigual & 1 & $\mathrm{v}$ & G-3 \\
\hline 9 & MRP & buwei, boie, boie kah & 1 & v & G-3, G-2 \\
\hline 10 & PTN & ube aka & 1,2 & $\mathrm{v}$ & G-2, G-3, S-2 \\
\hline 11 & KLK & ubi wak, ubi uwak & 1 & v & G-3, S-1 \\
\hline 12 & KLM & ubi wak & 1 & $\mathrm{v}$ & $\mathrm{G}-2, \mathrm{G}-3$ \\
\hline 13 & KLO & ubi agha, ubi aka & 1 & v & G-3 \\
\hline 14 & KLA & ubi aha & 1,2 & v & G-4 \\
\hline 15 & KAL & ubi aha & 1 & v & G-3 \\
\hline 16 & KBD & ubi lan & 1 & v & G-3 \\
\hline 17 & KBK & ubi aka & 1 & v & G-3 \\
\hline 18 & PEB & uvei lakeu poti, uvei lakeu & 1 & $\mathrm{v}$ & G-4 \\
\hline
\end{tabular}

Notes : 


\section{Merremia peltata (L.) Merr. (Convolvulaceae)}

\section{ULAN VINE. AKAR ULAN, KANGKUNG BUKIT}

\section{DESCRIPTION}

Woody climber, 5-10 m long, perennial, twinning with large subterranean tuber; small leaves broadly ovate-orbicular; large leaves peltate, $6-30 \mathrm{~cm}$ each way; flowers funnel form, $5-7 \mathrm{~cm}$ long, yellow to white

\section{HABITAT}

Lowland secondary forests

\section{ORIGINS}

Southeast Asia

\section{USES}

Leaves for wrapping; roots eaten; stem sap for medicine

\section{REFERENCES}

Burkill 1966: 1480; FOJ Vol II: 489

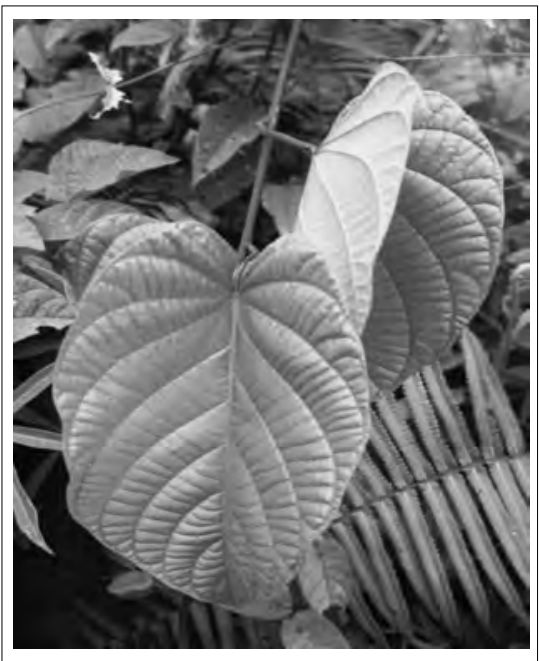

\begin{tabular}{|c|c|l|c|c|c|}
\hline \# & Code & \multicolumn{1}{|c|}{ Local Name } & Use & RU & Hab.-Ab. \\
\hline 1 & PUT & oka belang & 2,6 & $\mathbf{X}$ & S-2, Y-3, O-3, P-1 \\
\hline 2 & PUB & oka belang & 6 & $\mathbf{X}$ & O-1, S-3, Y-3 \\
\hline 3 & PUD & oka belang & 6 & $\mathbf{X}$ & O-3, S-3, Y-3, P-2 \\
\hline 4 & PUM & oka belang & 2 & $\mathbf{X}$ & Y-2, O-3 \\
\hline 5 & ALS & bakag belalan & 6 & $\mathbf{X}$ & Y-3, O-3, P-1 \\
\hline 6 & LDY & war sebeburuk, bengunyung & 2,6 & $\mathbf{v}$ & S-3, Y-3, O-3, P-2 \\
\hline 7 & LIT & war lapulu, war lepulu, war lepuluh & 2 & $\mathbf{v}$ & S-2, Y-4, O-3, P-2 \\
\hline 8 & BRU & dau guwal & & $\mathbf{X}$ & S-2, Y-3 \\
\hline 9 & MRP & aka balaya, kabelalai, kabalai & & $\mathbf{X}$ & S-2, Y-3, O-2 \\
\hline 10 & PTN & aka aceng, aka acang bilen & 6 & $\mathbf{X}$ & S-3, Y-4, O-3, P-1 \\
\hline 11 & KLK & acang, aceng lata' & 6 & $\mathbf{V}$ & Y-3, O-4 \\
\hline 12 & KLM & & & & \\
\hline 13 & KLO & & & & \\
\hline 14 & KLA & & & $\mathbf{X}$ & \\
\hline 15 & KAL & aha ceng & & $\mathbf{X}$ & \\
\hline 16 & KBD & layo & & $\mathbf{X}$ & \\
\hline 17 & KBK & aka pulut & & $\mathbf{X}$ & \\
\hline 18 & PEB & lakeu buken & & \\
\hline
\end{tabular}

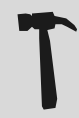

Notes : 


\section{AIR PLANT. COCOR BEBEK}

\section{DESCRIPTION}

Succulent herb, 30-200 cm; erect, stems quadrangular; sprouts vegetatively from leaf; leaves fleshy, oval-oblong, crenate, $5-20 \times 2.5-15 \mathrm{~cm}$; flowers purple

\section{HABITAT}

Cultivated in home gardens to $1000 \mathrm{~m}$; stony localities, under hedges

\section{ORIGINS}

Africa

\section{USES}

Leaves for medicine

\section{REFERENCES}

Burkill 1966: 1297; FOJ Vol. I: 202;

PROSEA Vol. 12 (1): 166

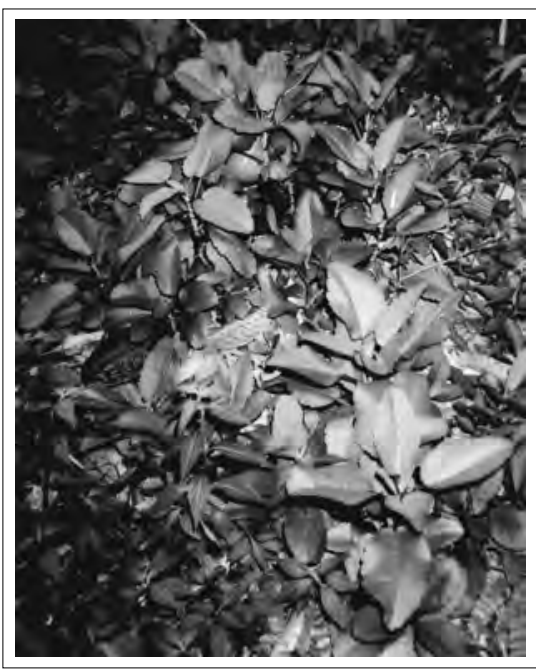

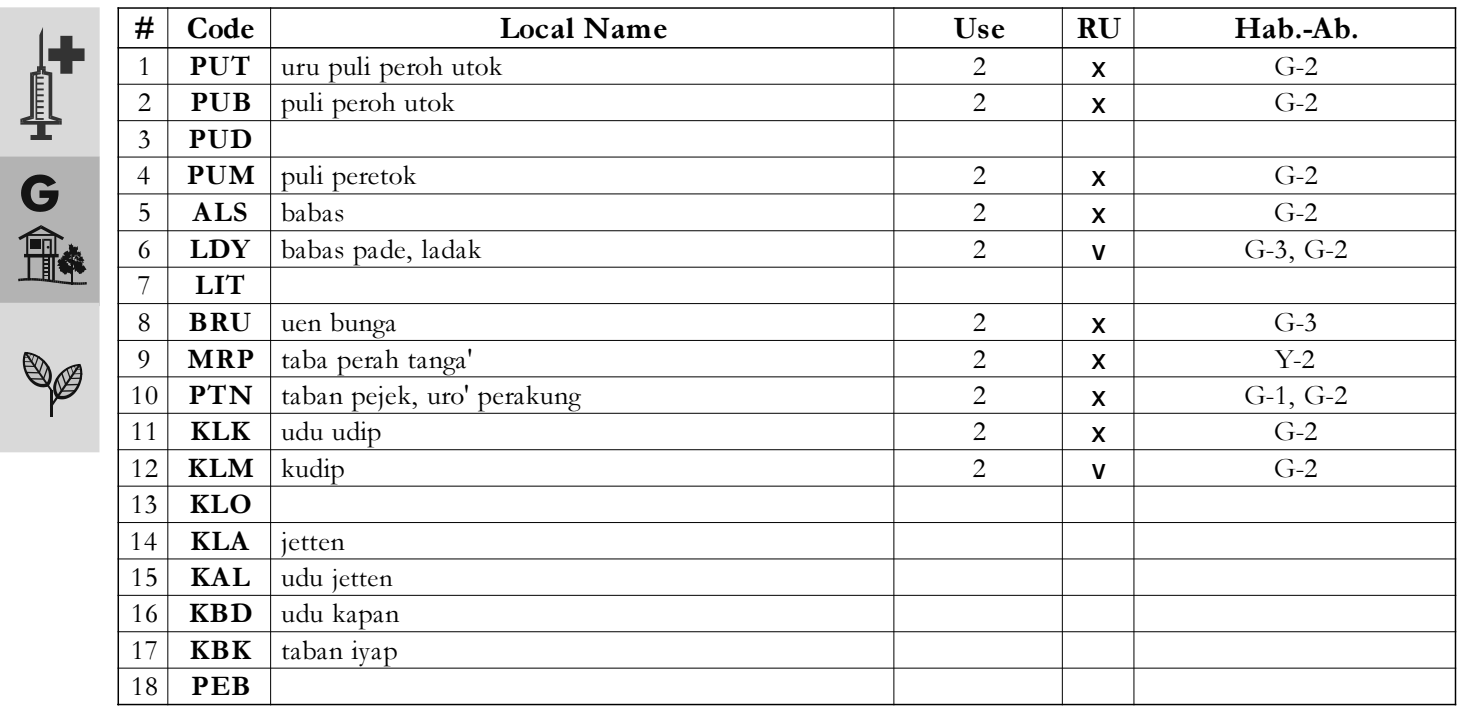

Notes : 


\section{Cucumis sativus L. (Cucurbitaceae)}

\section{CuCuMBer. KeTimun}

\section{DESCRIPTION}

Herb, climbing, annual, to $5 \mathrm{~m}$ long; stem stiff bristly hairs; leaves alternate, triangular-ovate 7 $20 \times 7-15 \mathrm{~cm}, 3-7$ lobed deeply cordate at base; flowers axillary, yellow, $2.5-4 \mathrm{~cm} \mathrm{~d}$; fruit pendulous, variable size, with warts when immature

\section{HABITAT}

Cultivated in home and swidden gardens

\section{ORIGINS}

Unknown

\section{USES}

Fruits eaten fresh or cooked, thirst quencher, medicine

\section{REFERENCES}

Ochse 1980: 193; PROSEA Vol. 8: 157;

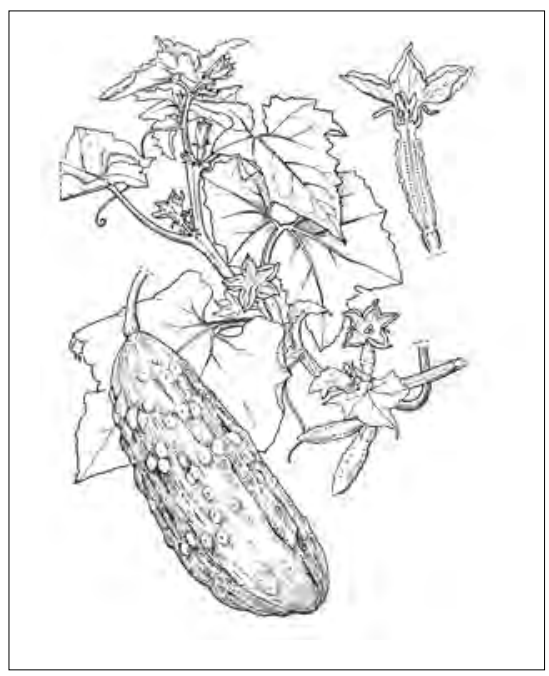

FOJ Vol. I: 301

\begin{tabular}{|r|l|l|c|c|c|}
\hline$\#$ & Code & Local Name & Use & RU & Hab.-Ab. \\
\hline 1 & PUT & ketinon & $1,2,11$ & $\mathbf{v}$ & G-2, S-3 \\
\hline 2 & PUB & ketinong & 1 & $\mathbf{v}$ & G-2, S-3 \\
\hline 3 & PUD & ketinun & 1 & $\mathbf{v}$ & G-2, S-3 \\
\hline 4 & PUM & ketinon & 1 & $\mathbf{v}$ & G-2, S-3 \\
\hline 5 & ALS & ndimun & 1,11 & $\mathbf{v}$ & G-2, S-3 \\
\hline 6 & LDY & bua timon & 1,11 & $\mathbf{v}$ & G-2, S-3 \\
\hline 7 & LIT & bua' timon & 1 & $\mathbf{v}$ & G-2, S-3 \\
\hline 8 & BRU & wi mun & 1 & $\mathbf{v}$ & G-2, S-3 \\
\hline 9 & MRP & taenim, tinue & 1 & $\mathbf{v}$ & G-2, S-3 \\
\hline 10 & PTN & tera' & 1,11 & $\mathbf{v}$ & G-2, S-3 \\
\hline 11 & KLK & timun & 1,11 & $\mathbf{v}$ & G-2, S-3 \\
\hline 12 & KLM & timun & 1,2 & $\mathbf{v}$ & G-2, S-3 \\
\hline 13 & KLO & timung & 1,2 & $\mathbf{v}$ & G-2, S-3 \\
\hline 14 & KLA & timon & 1,2 & $\mathbf{v}$ & G-2, S-3 \\
\hline 15 & KAL & timun & 1,2 & $\mathbf{v}$ & G-2, S-3 \\
\hline 16 & KBD & timun & $1,2,11$ & $\mathbf{v}$ & G-2, S-4 \\
\hline 17 & KBK & timon tegan & 1,2 & $\mathbf{v}$ & G-2, S-3 \\
\hline 18 & PEB & timun & & \\
\hline
\end{tabular}

Notes : 


\section{Cucurbita moschata (Duch.) Poir. (Cucurbitaceae) CROOKNECK PUMPKIN. LABU}

\section{DESCRIPTION}

Herb, climbing, annual, stem hard and angular, tendrils; leaves softly hairy, large shallowly lobed; flowers large, showy, lemon yellow to orange; fruit stalk hard, grooved, enlarged at fruit; fruit gobular, grooved to $40 \mathrm{~cm} \mathrm{~d}$, orange flesh

\section{HABITAT}

Cultivated in home and swidden gardens

\section{ORIGINS}

Central America

\section{USES}

Fruits, leaves and flowers cooked as vegetables; seeds for snacks, medicine; sold locally

\section{REFERENCES}

Ochse 1980: 194; PROSEA Vol. 8: 160

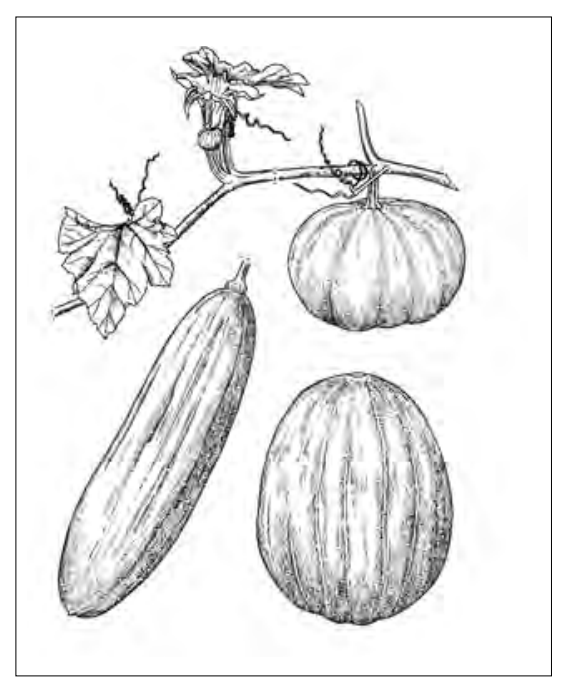

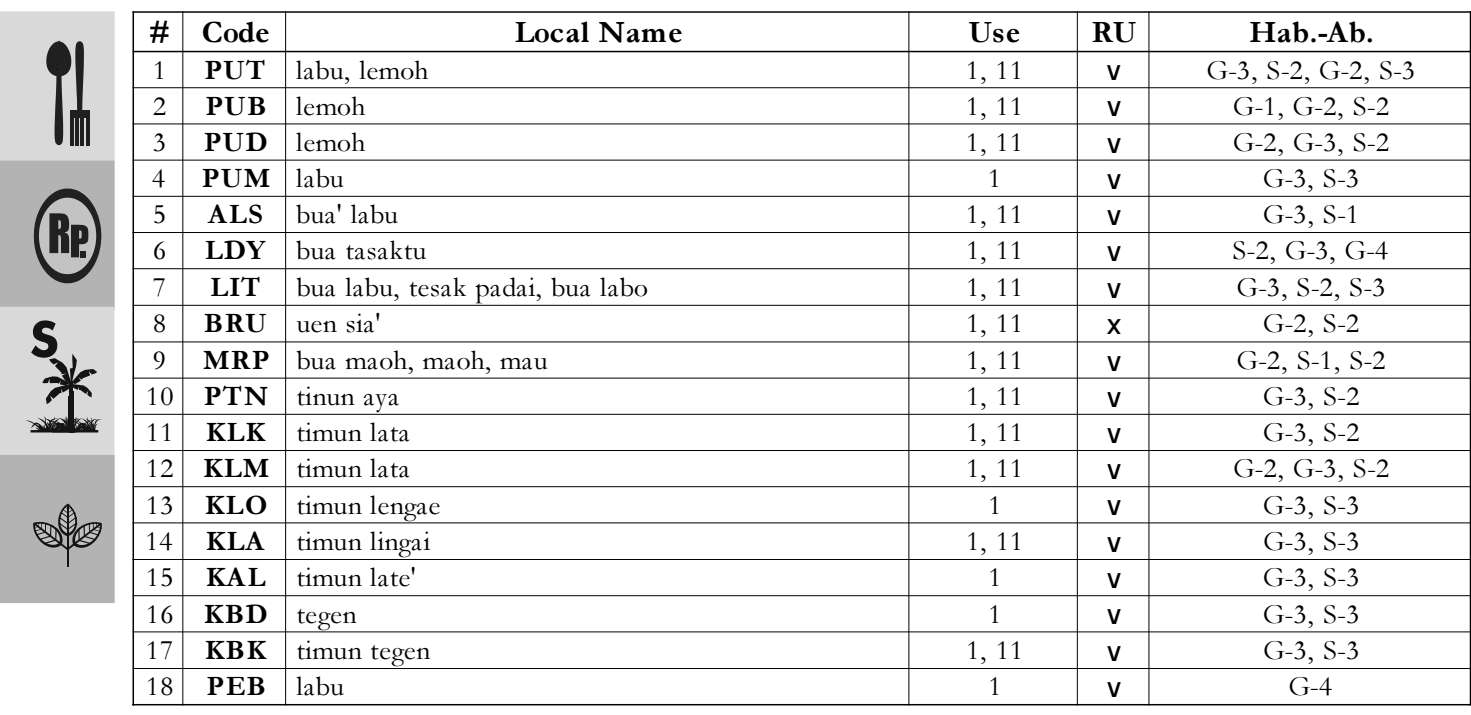

Notes : 


\section{TRUE FERN. PAKU BENAR}

\section{DESCRIPTION}

Fern, 0.5-2.5 m, rhizome 10-50 x 2-3 cm, black horsehair-like roots; leaves spiral, long petiole, obliquely erect, $25-260 \times 15-100 \mathrm{~cm}$, bipinnate, dark green; leaflets subsessile, oblong or linear, 7$15 \times 1.5-2.5 \mathrm{~cm}$; sori brown on underside veins

\section{HABITAT}

Wild in moist, swampy areas, riverine

\section{ORIGINS}

Tropical Asia and Pacific

\section{USES}

Young leaves eaten raw, cooked, sold locally; medicine

\section{REFERENCES}

Burkill 1966: 848; Ochse 1980: 600

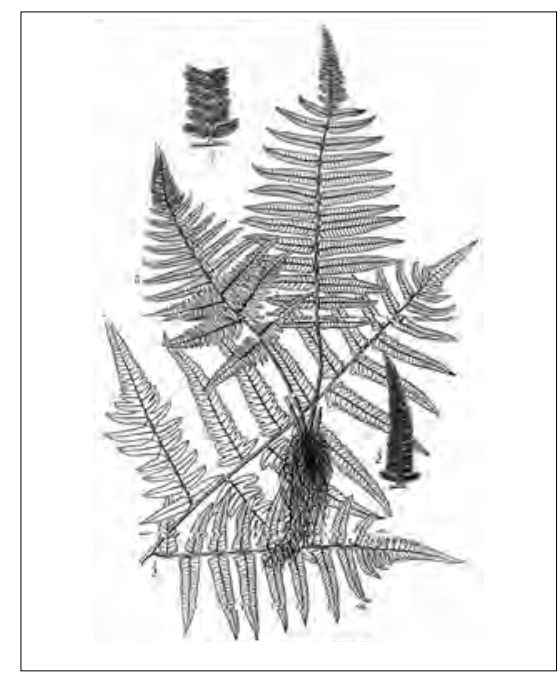

\begin{tabular}{|r|c|l|c|c|c|}
\hline \# & Code & \multicolumn{1}{|c|}{ Local Name } & Use & RU & Hab.-Ab. \\
\hline 1 & PUT & kujau & 1,11 & $\mathbf{v}$ & R-3 \\
\hline 2 & PUB & kujau, kujau paku & $1,2,11$ & $\mathbf{v}$ & R-3 \\
\hline 3 & PUD & kujau & 1 & $\mathbf{v}$ & R-3 \\
\hline 4 & PUM & kujau & 1,11 & $\mathbf{v}$ & R-3 \\
\hline 5 & ALS & kujau & 1,11 & $\mathbf{v}$ & R-3 \\
\hline 6 & LDY & kusau & $1,2,11$ & $\mathbf{v}$ & R-3 \\
\hline 7 & LIT & peku' fa', pau fa, pau & 1,11 & $\mathbf{v}$ & R-3 \\
\hline 8 & BRU & pako & 1,11 & $\mathbf{X}$ & R-3 \\
\hline 9 & MRP & pakau, pako, peko san & 1,11 & $\mathbf{v}$ & R-3 \\
\hline 10 & PTN & pako sen, paku atuk & 1 & $\mathbf{v}$ & R-3 \\
\hline 11 & KLK & paku uway, paku bai & 1,11 & $\mathbf{v}$ & R-3 \\
\hline 12 & KLM & paku bai & 1,11 & $\mathbf{v}$ & ALL \\
\hline 13 & KLO & faghe, fako lemo', fake lemek, fage lemo, fage leme' & $\mathbf{v}$ & R-3 \\
\hline 14 & KLA & pahu lema, fahu, pahu lemak, pahu lemek, pahu leme' & 1 & $\mathbf{v}$ & G-4, R \\
\hline 15 & KAL & paku hatok & 1,11 & $\mathbf{v}$ & G-2, Y-2, R \\
\hline 16 & KBD & paku, paku danum & 1 & $\mathbf{v}$ & R-3 \\
\hline 17 & KBK & paku damun & 1,11 & $\mathbf{v}$ & G-3, R-3 \\
\hline 18 & PEB & pakeu bei, pako & 1 & $\mathbf{v}$ & G-2, S-2, R-3 \\
\hline
\end{tabular}

Notes : 


\section{Dipterocarpus oblongifolius Blume (Dipterocarpaceae)}

\section{KERUING NERAM. KERUING NERAM}

\section{DESCRIPTION}

Tree, to $35 \mathrm{~m}$, big buttresses on bank, bole twisted, leaning over river; bark pale grey; leaves narrowly elliptical to lanceolate $14-18$ x $4-7 \mathrm{~cm}$, old ones scarlet; fruits red, narrow, oblong with 2 wings $10-12 \times 1.5 \mathrm{~cm}$, and 3 wings $10 \times 3 \mathrm{~mm}$

\section{HABITAT}

Gregariously on banks of inland rivers

\section{ORIGINS}

Malay Peninsula and Borneo

\section{USES}

Wood for building; fruit eaten; shade tree; site to ambush migrating pigs as they cross rivers

(Pujungan district villages)

\section{REFERENCES}

Burkill 1966: 858; Corner 1988: 237; PROSEA Vol.

5(1): 183; FM I Vol. 9(2): 317

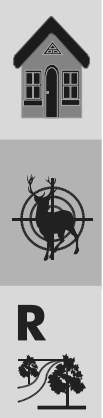

\begin{tabular}{|c|c|l|c|c|c|}
\hline$\#$ & Code & Local Name & Use & RU & Hab.-Ab. \\
\hline 1 & PUT & piri & $3,6,10$ & $\mathbf{x}$ & R-1 \\
\hline 2 & PUB & bua ilat & & & \\
\hline 3 & PUD & tepudau & $3,6,10$ & $\mathbf{x}$ & $\mathrm{R}-1$ \\
\hline 4 & PUM & & & & \\
\hline 5 & ALS & & & & \\
\hline 6 & LDY & & & & \\
\hline 7 & LIT & & & & \\
\hline 8 & BRU & & & & \\
\hline 9 & MRP & laran ngui & $3,6,10$ & $\mathbf{x}$ & \\
\hline 10 & PTN & laran & $3,6,10$ & $\mathbf{V}$ & $\mathrm{R}-1$ \\
\hline 11 & KLK & laran & $3,6,10$ & $\mathbf{v}$ & $\mathrm{R}-3$ \\
\hline 12 & KLM & laran & $3,6,10$ & $\mathbf{v}$ & $\mathrm{R}-3$ \\
\hline 13 & KLO & laran & $3,6,10$ & $\mathbf{v}$ & $\mathrm{R}-3$ \\
\hline 14 & KLA & laran & $3,6,10$ & $\mathbf{v}$ & $\mathrm{R}-3$ \\
\hline 15 & KAL & laran & $3,6,10$ & $\mathbf{v}$ & $\mathrm{R}-3$ \\
\hline 16 & KBD & laran & $3,6,10$ & $\mathbf{v}$ & $\mathrm{R}-3$ \\
\hline 17 & KBK & laran & $1,3,6,10$ & $\mathbf{v}$ & $\mathrm{R}-3$ \\
\hline 18 & PEB & laran & & $\mathrm{R}-3$ \\
\hline
\end{tabular}

Notes : 


\section{Dryobalanops lanceolata Burck (Dipterocarpaceae) SABAH KAPUR. KAPUR TANDUK}

\section{DESCRIPTION}

Giant tree, to $75 \mathrm{~m}$; branchless for $35 \mathrm{~m}$, buttresses to $4 \mathrm{~m}$; straight bole, bark slate grey, aromatic; leaves lanceolate $7-10 \times 2-3.5 \mathrm{~cm}$, glabrous; fruit 5 brown calyx lobes, $9 \times 2 \mathrm{~cm}$, fruit $5 \times 20 \mathrm{~mm}$

\section{HABITAT}

Lowland and hill primary forests

\section{ORIGINS}

Borneo endemic

\section{USES}

Timber for building, locally sold to traders for export; camphor; site for ambushing pigs when fruiting

\section{REFERENCES}

PROSEA Vol. 5(1): 191; FM I Vol. 9(2): 374

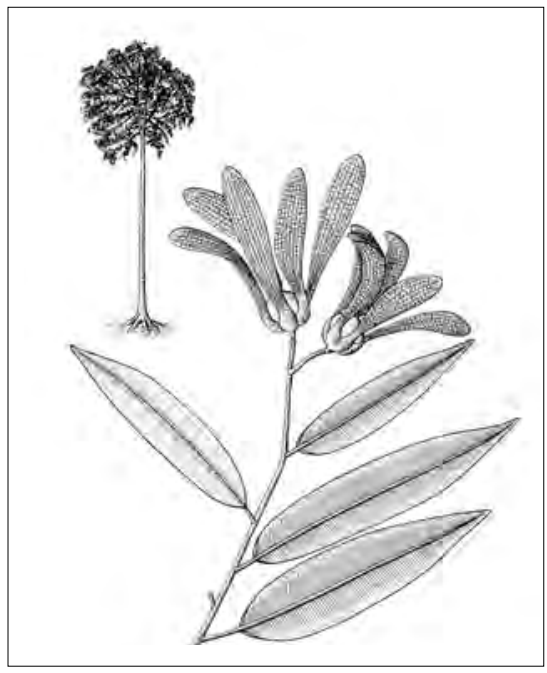

\begin{tabular}{|c|c|c|c|c|c|}
\hline$\#$ & Code & Local Name & Use & RU & Hab.-Ab. \\
\hline 1 & PUT & betiting & $3,4,6,10$ & $\mathrm{v}$ & P-2, M-2 \\
\hline 2 & PUB & ngei & $3,4,6,10$ & v & P-2, M-2 \\
\hline 3 & PUD & betiting & $3,4,6,10$ & v & P-2, M-2 \\
\hline 4 & PUM & & & & \\
\hline 5 & ALS & kuyung & $3,4,6,10$ & $x$ & P-1, M-2 \\
\hline 6 & LDY & kuyung & $3,4,6,10$ & $x$ & P-1, M-2 \\
\hline 7 & LIT & kuyung & $3,4,6,10$ & $x$ & P-1, M-2 \\
\hline 8 & BRU & & & & \\
\hline 9 & MRP & ngai & $3,4,6,10,11$ & $\mathrm{v}$ & P-1, M-2 \\
\hline 10 & P'TN & kapun & $3,4,6,10,11$ & v & P-1, M-2 \\
\hline 11 & KLK & kapun & $3,4,6,10,11$ & v & P-2, M-2 \\
\hline 12 & KLM & kapun & $3,4,6,10,11$ & v & P-3, M-2 \\
\hline 13 & KLO & kafung & $3,4,6,10,11$ & $\mathrm{v}$ & P-3, M-2 \\
\hline 14 & KLA & hapun & $3,4,6,10,11$ & v & P-3, M-2 \\
\hline 15 & KAL & kafun & $3,4,6,10,11$ & $\mathrm{v}$ & P-3, M-2 \\
\hline 16 & KBD & kapun & $3,4,6,10,11$ & v & P-3, M-2 \\
\hline 17 & KBK & kapun & $3,4,6,10,11$ & v & P-3, M-2 \\
\hline 18 & PEB & kapun & $3,4,6,7,10$ & $x$ & P-3, M-2 \\
\hline
\end{tabular}

Notes : 


\section{Shorea macropbylla (de Vriese) Ashton (Dipterocarpaceae)}

\section{ILLIPE NUT TREE. TENGKAWANG}

\section{DESCRIPTION}

Tree, to $50 \mathrm{~m}$, early branching, butts. to $2 \mathrm{~m}$; bark smooth greenish grey to brown; leaves ellipticoblong 19-48 x 6-22 cm, hairy midrib above, 1318 pairs of nerves above; flowers pale pink; fruit calyx 3 long wings, $6-10 \times 3-4 \mathrm{~cm}, 2$ short wings

\section{HABITAT}

Lowland forests below $600 \mathrm{~m}$; riverine, clay-rich, periodically inundated land; planted in village fruit gardens

\section{ORIGINS}

Borneo endemic

\section{USES}

Timber for building; nuts sold to traders for export; site for ambushing pigs when fruiting

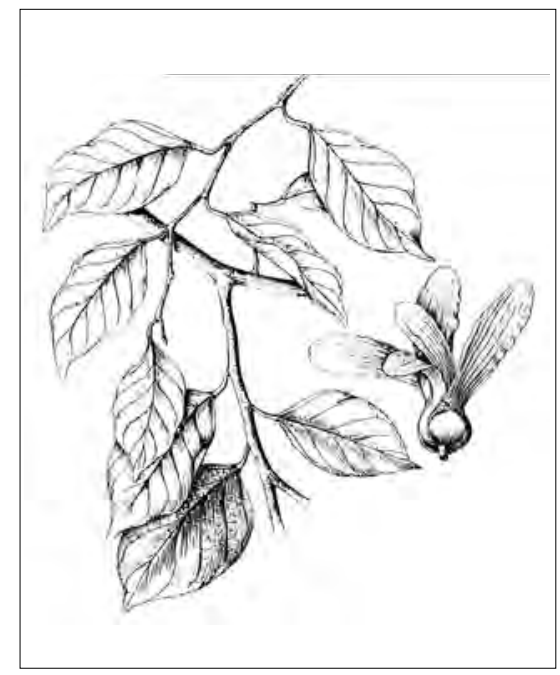

\section{REFERENCES}

Newman et al. 1996: 163; PROSEA Vol. 5(1):

397; FM I Vol. 9(2): 523

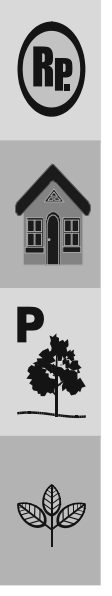

\begin{tabular}{|c|l|l|c|c|c|}
\hline$\#$ & Code & \multicolumn{1}{|c|}{ Local Name } & Use & RU & Hab.-Ab. \\
\hline 1 & PUT & afang, awang tufeng & $3,6,10,11$ & $\mathbf{v}$ & G-2, P-3, M-2 \\
\hline 2 & PUB & afang tufung, avang tuvung & $7,10,11$ & $\mathbf{v}$ & P-3 \\
\hline 3 & PUD & avang, afang & 10,11 & $\mathbf{v}$ & P-4 \\
\hline 4 & PUM & afang tufung & 6,11 & $\mathbf{v}$ & G-2 \\
\hline 5 & ALS & daun abang & $4,5,11$ & $\mathbf{v}$ & G-2, O-2, P-3 \\
\hline 6 & LDY & kayu tengkawang, tengkawang & $6,9,10,11$ & $\mathbf{v}$ & G-2, O-2, P-4, M-4 \\
\hline 7 & LIT & tengkawang & 10,11 & $\mathbf{v}$ & P-4 \\
\hline 8 & BRU & wi abang & 5,11 & $\mathbf{v}$ & G-3, P-4 \\
\hline 9 & MRP & bua baya, baee', bae tokue & $1,10,11$ & $\mathbf{v}$ & G-2, P-4, M-3 \\
\hline 10 & PTN & unjung abang, ambang aya' & $3,11,10$ & $\mathbf{v}$ & G-2, P-3 \\
\hline 11 & KLK & abang, a' bang & $7,10,11$ & $\mathbf{v}$ & P-3 \\
\hline 12 & KLM & abang, abang & 10,11 & $\mathbf{v}$ & P-3 \\
\hline 13 & KLO & abang, beva' abang & 10 & $\mathbf{X}$ & P-3 \\
\hline 14 & KLA & apang & 10 & $\mathbf{x}$ & P-3 \\
\hline 15 & KAL & ampang & 10 & $\mathbf{x}$ & P-3 \\
\hline 16 & KBD & abang & 10 & $\mathbf{x}$ & P-3 \\
\hline 17 & KBK & kayu ambang & $5,10,11$ & $\mathbf{x}$ & G-2, O-2, P-4, M-3 \\
\hline 18 & PEB & abang & & & P-3 \\
\hline
\end{tabular}

Notes : 
Diospyros borneensis Hiern (Ebenaceae)

BORNEAN EBONY. KAYU ARANG

\section{DESCRIPTION}

Tree, to $25 \mathrm{~m}$, bark brown to black, hard, brittle, fissured, scaly, no exudate; leaves broadly ovate to elliptic $15-20$ x 7-10 cm, sec. veins 8-12 looping at margin; flowers in axillary clusters, dioeceous, 5-merous; fruit a berry with 1 or more seeds

\section{HABITAT}

Lowland and hill primary and secondary forests

\section{ORIGINS}

Malaysia and western Indonesia

\section{USES}

Timber for boats, house posts, furniture; sold locally

\section{REFERENCES}

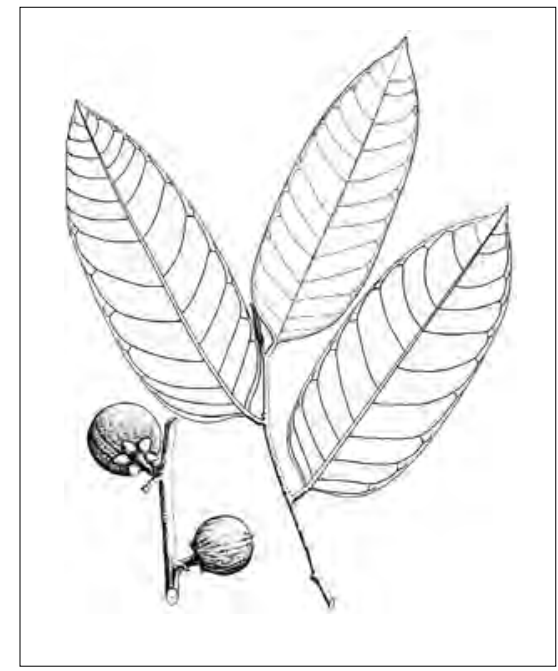

Kessler and Sidiyasa 1994: 116

\begin{tabular}{|c|c|l|c|c|c|}
\hline$\#$ & Code & Local Name & Use & RU & Hab.-Ab. \\
\hline 1 & PUT & kelen & 5,6 & $\mathbf{X}$ & P-3, M-2 \\
\hline 2 & PUB & pa'dom & & & \\
\hline 3 & PUD & pa'dom, akap & 5,6 & $\mathbf{X}$ & P-3, M-2 \\
\hline 4 & PUM & & & & \\
\hline 5 & ALS & & & & \\
\hline 6 & LDY & & & & \\
\hline 7 & LIT & & & & \\
\hline 8 & BRU & & 5,6 & $\mathbf{X}$ & \\
\hline 9 & MRP & tiyem, kayu mak & & & \\
\hline 10 & PTN & & $5,6,11$ & $\mathbf{X}$ & \\
\hline 11 & KLK & kayu saleng & $5,6,11$ & $\mathbf{X}$ & P-2, M-2 \\
\hline 12 & KLM & kayu saleng & $5,6,11$ & $\mathbf{X}$ & P-2, M-2 \\
\hline 13 & KLO & ghazu saleng & $5,6,11$ & $\mathbf{X}$ & P-2, M-2 \\
\hline 14 & KLA & avang buang & $5,6,11$ & $\mathbf{X}$ & P-2, M-2 \\
\hline 15 & KAL & kazu saleng & $5,6,11$ & $\mathbf{V}$ & P-2, M-2 \\
\hline 16 & KBD & kayu saleng & $5,6,11$ & $\mathbf{x}$ & P-2, M-2 \\
\hline 17 & KBK & ambang buang & $5,6,11$ & $\mathbf{V}$ & P-2, M-2 \\
\hline 18 & PEB & kelen & P-2, M-2 \\
\hline
\end{tabular}

Notes : 


\section{Baccaurea bracteata M.A. (Euphorbiaceae)}

\section{MONKEY'S TAMPOI. PANGAL, RAMBAI HUTAN}

\section{DESCRIPTION}

Tree, to $20 \mathrm{~m}$; sometimes with stilt roots; leaves to $17 \times 9 \mathrm{~cm}$, underside with raised brown dots; fruit a globose, trilocular capsule, $1.5-2 \mathrm{~cm} \mathrm{~d}$, dark red; seeds in orange yellow jacket

\section{HABITAT}

Lowland, kerangas forests, along rivers in swampy areas; secondary forest on poor sandy soils

\section{ORIGINS}

Malaysia and western Indonesia

\section{USES}

Fruit eaten; timber for building, firewood

\section{REFERENCES}

Kessler and Sidiyasa 1994: 123; PROSEA Vol. 2: 319; TFM Vol. 2: 67
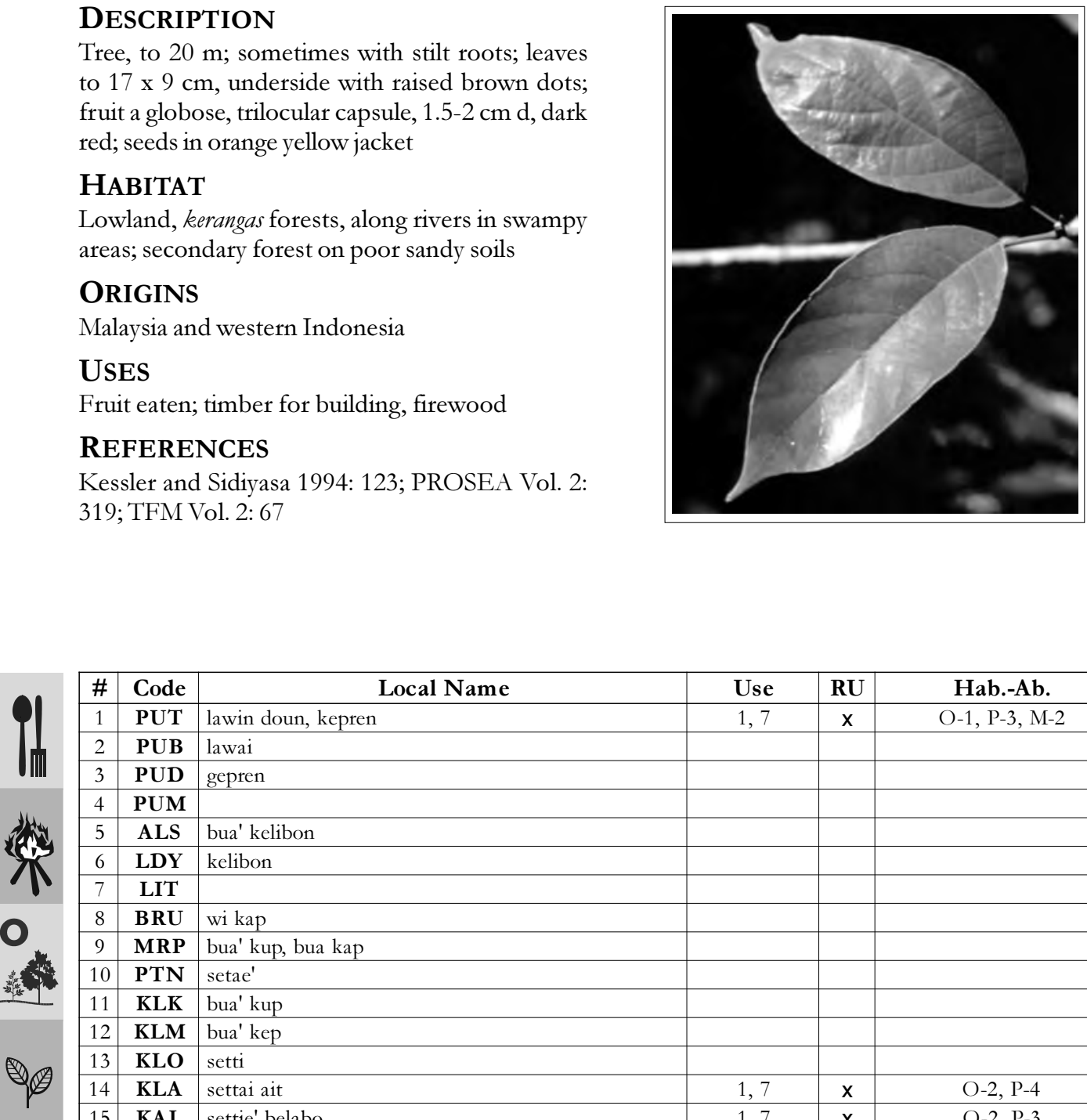

\begin{tabular}{|c|c|l|c|c|c|}
\hline$\#$ & Code & \multicolumn{1}{|c|}{ Local Name } & Use & RU & Hab.-Ab. \\
\hline 1 & PUT & lawin doun, kepren & 1,7 & X & O-1, P-3, M-2 \\
\hline 2 & PUB & lawai & & & \\
\hline 3 & PUD & gepren & & & \\
\hline 4 & PUM & & & \\
\hline 5 & ALS & bua' kelibon & & & \\
\hline 6 & LDY & kelibon & & & \\
\hline 7 & LIT & & & & \\
\hline 8 & BRU & wi kap & & & \\
\hline 9 & MRP & bua' kup, bua kap & & & \\
\hline 10 & PTN & setae' & & & \\
\hline 11 & KLK & bua' kup & & & \\
\hline 12 & KLM & bua' kep & & & \\
\hline 13 & KLO & setti & 1,7 & $\mathbf{x}$ & O-2, P-4 \\
\hline 14 & KLA & settai ait & 1,7 & $\mathbf{x}$ & O-2, P-3 \\
\hline 15 & KAL & settie' belabo & & & \\
\hline 16 & KBD & & 1 & $\mathbf{x}$ & O-4, P-4, M-2 \\
\hline 17 & KBK & lembang belabau, lembang bila' & 1,7 & $\mathbf{v}$ & O-3, P-3 \\
\hline 18 & PEB & lawin da'un & & \\
\hline
\end{tabular}

Notes : 


\section{Baccaurea lanceolata (Miq.) M.A. (Euphorbiaceae)}

\section{GREEN RAMBAI. RAMBAI UTAN,LEMPASU}

\section{DESCRIPTION}

Tree, to $14 \mathrm{~m}$; leaves spirally arranged, ovate-elliptic, $7-35 \times 3-15 \mathrm{~cm}$, drying clear grey green; fruits on clustered racemes, $18 \mathrm{~cm}$, on branches and trunk, grey green drying brown and knobbly ovoid 25 $\mathrm{mm}$ long; seeds in translucent jacket

\section{HABITAT}

Lowland primary and secondary forests to $300 \mathrm{~m}$

\section{ORIGINS}

Malaysia and western Indonesia

\section{USES}

Fruit eaten, medicine; timber

\section{REFERENCES}

PROSEA Vol. 2: 319; TFM Vol. 2: 65

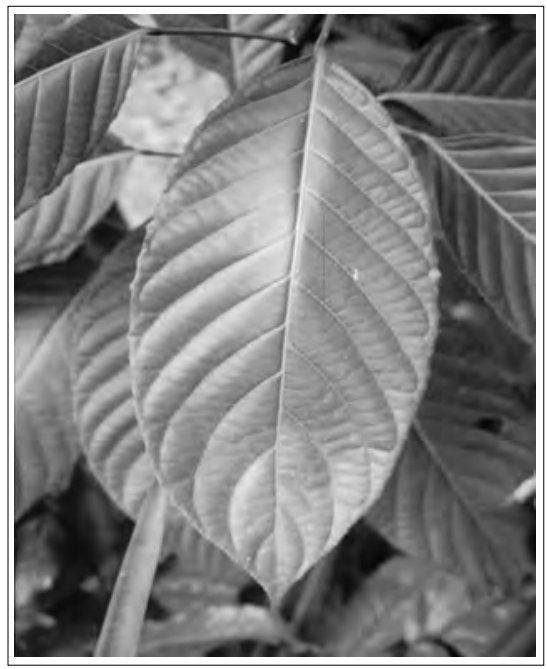

\begin{tabular}{|c|c|c|c|c|c|}
\hline \# & Code & Local Name & Use & RU & Hab.-Ab. \\
\hline 1 & PUT & lepo', lepau & 1,2 & V & $\mathrm{Y}-2, \mathrm{P}-3$ \\
\hline 2 & PUB & lepau & 1 & $x$ & $\mathrm{O}-2, \mathrm{P}-3$ \\
\hline 3 & PUD & lepau & 1 & $x$ & $\mathrm{O}-2, \mathrm{P}-3, \mathrm{M}-1$ \\
\hline 4 & PUM & lepou & 1,2 & $x$ & $\mathrm{O}-2, \mathrm{P}-4$ \\
\hline 5 & ALS & bua' lembasu & 1 & $x$ & $\mathrm{O}-3, \mathrm{P}-2$ \\
\hline 6 & LDY & kayu lifau, lifau & 1,2 & $x$ & $\mathrm{O}-1, \mathrm{O}-2, \mathrm{P}-3, \mathrm{M}-2$ \\
\hline 7 & LIT & lepau, bua lepau, kelepa'ou & 1,2 & V & $\mathrm{Y}-2, \mathrm{O}-3, \mathrm{P}-2, \mathrm{P}-4$ \\
\hline 8 & BRU & wi apau' & 1 & $x$ & $\mathrm{Y}-2, \mathrm{O}-2, \mathrm{P}-3$ \\
\hline 9 & MRP & tim lopohou, pohou & 1 & $x$ & $\mathrm{O}-2, \mathrm{P}-3, \mathrm{P}-2, \mathrm{M}-3$ \\
\hline 10 & PTN & lefuhu, lepuhu & 1 & $x$ & $\mathrm{O}-2, \mathrm{P}-3$ \\
\hline 11 & KLK & lepesso, lepesso', kelepesso & 1,2 & $x$ & $\mathrm{Y}-2, \mathrm{O}-2$ \\
\hline 12 & KLM & kelepessu, kelepesso & 1 & $x$ & ALL \\
\hline 13 & KLO & lepessu & & & \\
\hline 14 & KLA & lepessue, lepessu & & & \\
\hline 15 & KAL & kelepessua' & & & \\
\hline 16 & KBD & kelepesso & & & \\
\hline 17 & KBK & kalapesso, kelepesso' & & & \\
\hline 18 & PEB & leposu, lepesu & 1 & v & $\mathrm{O}-2, \mathrm{P}-4$ \\
\hline
\end{tabular}

Notes : 


\section{Baccaurea macrocarpa (Miq.) M.A. (Euphorbiaceae)}

\section{GREATER TAMPOI. TAMPOI BULAN, KAPUL}

\section{DESCRIPTION}

Tree, 10-15 m; steep buttresses; bark brown smooth to flaky; leaves spirally arranged, ovate, $15-30 \times 6-11 \mathrm{~cm}$, fruit on branches and trunk, oblongoid capsule $7 \mathrm{~cm}$ diameter, orange brown to grey; 4-8 seeds in pulpy cream white jacket

\section{HABITAT}

Lowland primary and secondary forest to $900 \mathrm{~m}$

\section{ORIGINS}

Malaysia and western Indonesia

\section{USES}

Fruit eaten, sold locally; timber, firewood

\section{REFERENCES}

Kessler and Sidiyasa 1994: 123;

PROSEA Vol. 2: 319
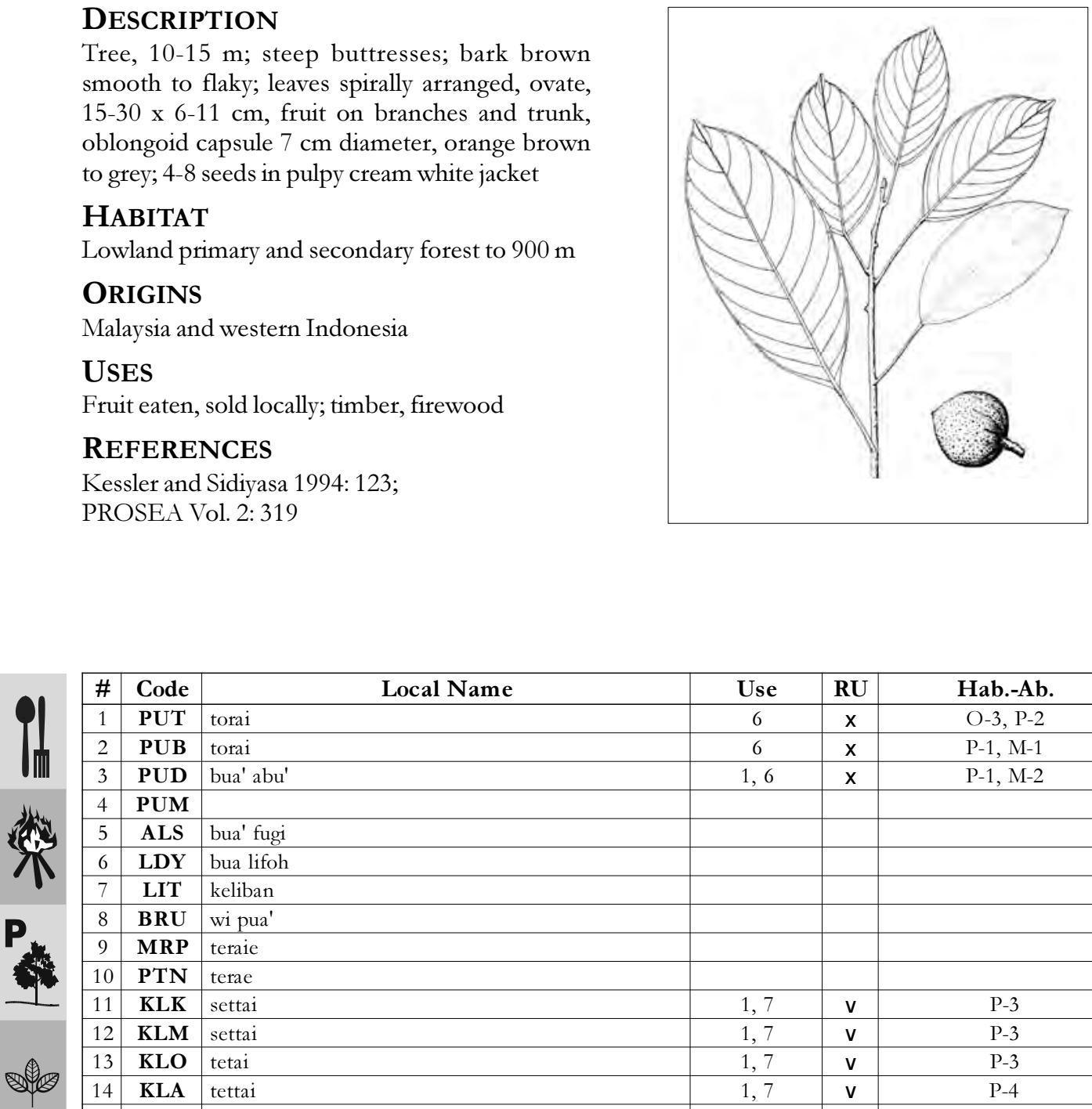

\begin{tabular}{|c|c|l|c|c|c|}
\hline$\#$ & Code & \multicolumn{1}{|c|}{ Local Name } & Use & RU & Hab.-Ab. \\
\hline 1 & PUT & torai & 6 & $\mathbf{X}$ & O-3, P-2 \\
\hline 2 & PUB & torai & 6 & $\mathbf{X}$ & P-1, M-1 \\
\hline 3 & PUD & bua' abu' & 1,6 & $\mathbf{X}$ & P-1, M-2 \\
\hline 4 & PUM & & & & \\
\hline 5 & ALS & bua' fugi & & & \\
\hline 6 & LDY & bua lifoh & & & \\
\hline 7 & LIT & keliban & & & \\
\hline 8 & BRU & wi pua' & & & \\
\hline 9 & MRP & teraie & & & \\
\hline 10 & PTN & terae & & & \\
\hline 11 & KLK & settai & 1,7 & $\mathbf{V}$ & \\
\hline 12 & KLM & settai & 1,7 & $\mathbf{v}$ & P-3 \\
\hline 13 & KLO & tetai & 1,7 & $\mathbf{v}$ & P-3 \\
\hline 14 & KLA & tettai & 1,7 & $\mathbf{v}$ & P-3 \\
\hline 15 & KAL & tettai & 1,7 & $\mathbf{v}$ & P-4 \\
\hline 16 & KBD & settai & 1,7 & $\mathbf{x}$ & G-2, S-2, P-3, M-3 \\
\hline 17 & KBK & settai bura' & 1,7 & $\mathbf{v}$ & O-2, P-3, M-2 \\
\hline 18 & PEB & melanggan & & & \\
\hline
\end{tabular}

Notes : 
Baccaurea motleyana M.A. (Euphorbiaceae)

RAMBAI. RAMBAI

\section{DESCRIPTION}

Tree, 15-20 m; steep buttresses; bark brown scaly; leaves spirally arranged, obovate, $15-25$ x 6-12 cm, underside velvety; flowers ochre brown on branches; fruit globular 2-celled capsule, $2.5 \mathrm{~cm}$ diameter, buff colored; seeds in blue or pink jacket

\section{HABITAT}

Cultivated in village fruit gardens

\section{ORIGINS}

Sumatra

\section{USES}

Fruit eaten, sold locally

\section{REFERENCES}

Kessler and Sidiyasa 1994: 123; PROSEA Vol. 2:

98; TFM Vol. 2: 66; FOJ I: 454

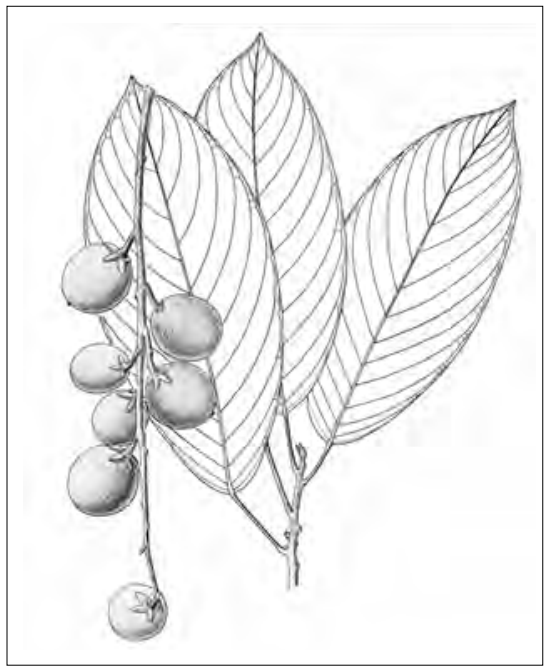

\begin{tabular}{|c|c|c|c|c|c|}
\hline \# & Code & Local Name & Use & RU & Hab.-Ab. \\
\hline 1 & PUT & rambai, kuni & 1 & $x$ & G-2 \\
\hline 2 & PUB & lepo' & 1 & $x$ & G-2 \\
\hline 3 & PUD & & & & \\
\hline 4 & PUM & & & & \\
\hline 5 & ALS & & & & \\
\hline 6 & LDY & sekoyun & 1 & $x$ & G-1 \\
\hline 7 & LIT & & & & \\
\hline 8 & BRU & wi sia' & 1 & $x$ & G-1 \\
\hline 9 & MRP & pahae' & & & \\
\hline 10 & PTN & lepu'u & 1 & $x$ & G-1 \\
\hline 11 & KLK & & & & \\
\hline 12 & KLM & rambai & 1 & $x$ & G-1 \\
\hline 13 & KLO & & & & \\
\hline 14 & KLA & & & & \\
\hline 15 & KAL & & & & \\
\hline 16 & KBD & & & & \\
\hline 17 & KBK & & & & \\
\hline 18 & PEB & & & & \\
\hline
\end{tabular}

Notes : 


\section{Baccaurea parviflora (M.A.) M.A. (Euphorbiaceae)}

\section{(NOENGLISH NAME).SETAMBON}

\section{DESCRIPTION}

Tree, to $6 \mathrm{~m}$; leaves elliptic, to $20 \times 9 \mathrm{~cm}$; flowers on trunks or at ground level; fruit a berry-like capsule, to $2.5 \mathrm{x} 1 \mathrm{~cm}$, angled, sometimes winged, purplish black; seed with fleshy jacket

\section{HABITAT}

Lowland primary and disturbed forest, to $450 \mathrm{~m}$

\section{ORIGINS}

Southeast Asia

\section{USES}

Fruit eaten fresh or cooked; timber for tools, light construction; firewood

\section{REFERENCES}

PROSEA Vol. 2: 320; TFM Vol. 2: 64

Kochummen 1997: 224
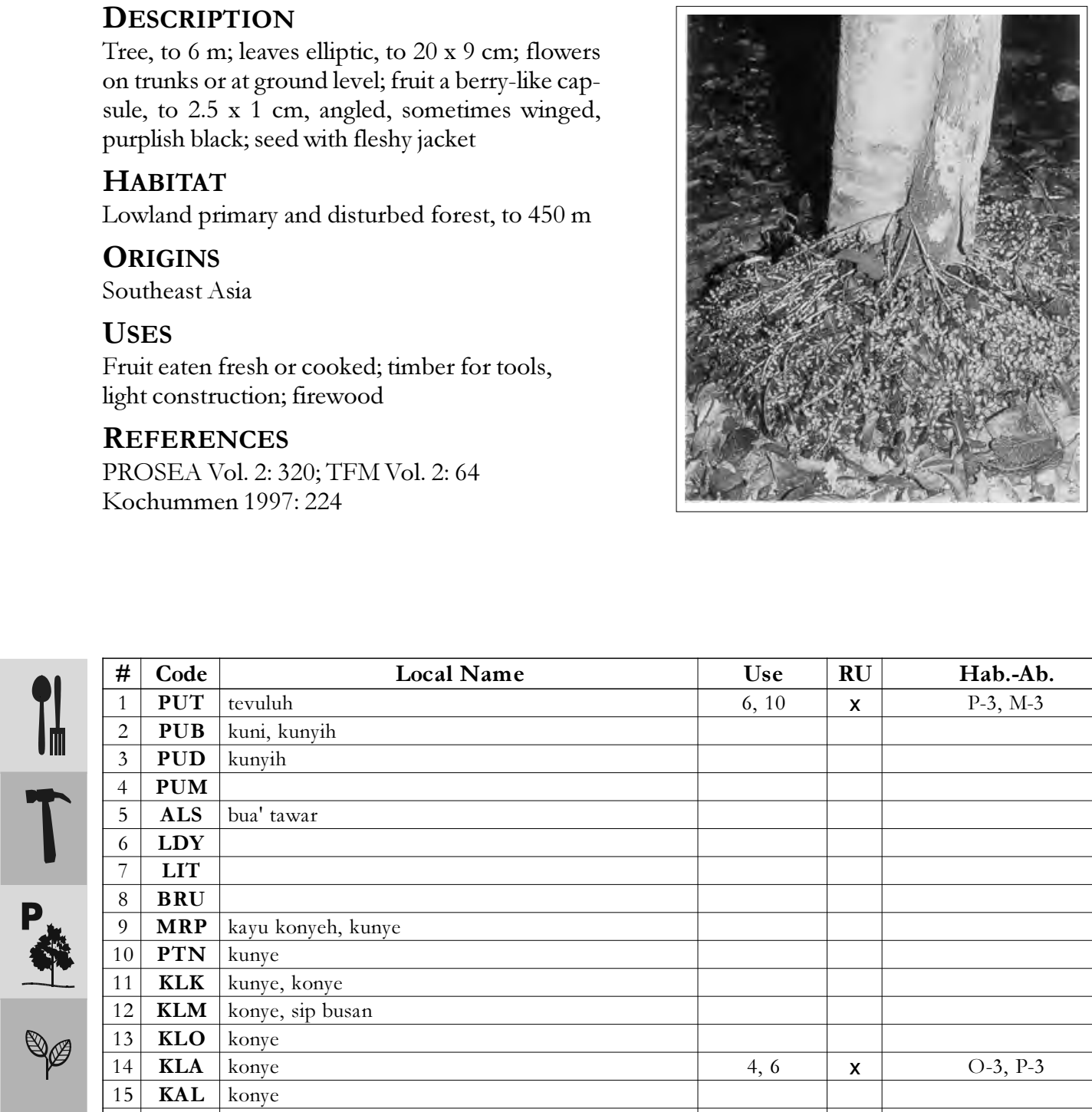

\begin{tabular}{|c|c|l|c|c|c|}
\hline$\#$ & Code & \multicolumn{1}{|c|}{ Local Name } & Use & RU & Hab.-Ab. \\
\hline 1 & PUT & tevuluh & 6,10 & $\mathbf{x}$ & P-3, M-3 \\
\hline 2 & PUB & kuni, kunyih & & & \\
\hline 3 & PUD & kunyih & & & \\
\hline 4 & PUM & & & \\
\hline 5 & ALS & bua' tawar & & & \\
\hline 6 & LDY & & & \\
\hline 7 & LIT & & & \\
\hline 8 & BRU & & & \\
\hline 9 & MRP & kayu konyeh, kunye & & & \\
\hline 10 & PTN & kunye & & & \\
\hline 11 & KLK & kunye, konye & & & \\
\hline 12 & KLM & konye, sip busan & & & \\
\hline 13 & KLO & konye & & & \\
\hline 14 & KLA & konye & 4,6 & $\mathbf{x}$ & O-3, P-3 \\
\hline 15 & KAL & konye & & & \\
\hline 16 & KBD & konye & $1,4,6$ & $\mathbf{v}$ & O-3, P-2 \\
\hline 17 & KBK & sip busan & 1,7 & $\mathbf{x}$ & O-4, P-1, M-2 \\
\hline 18 & PEB & konye & 1,7 & $\mathbf{v}$ & P-2, M-2 \\
\hline
\end{tabular}

Notes : 
Baccaurea pyriformis Gage (Euphorbiaceae)

\section{FIG TAMPOI. TAMPOI TUNGGAU}

\section{DESCRIPTION}

Tree, to $20 \mathrm{~m}$; twigs and leaf stalks finely velvety; leaves elliptic-ovate, to 13 × $6 \mathrm{~cm}$, drying blackish above; fruits a globose, fig or pear-shaped capsule, $2.5 \mathrm{~cm}$, drying dark, dull brown, wall thick, smooth surface; seeds in orange red fleshy jacket

\section{HABITAT}

Lowland forests, dry land

\section{ORIGINS}

Malaysia and western Indonesia

\section{USES}

Fruit eaten

\section{REFERENCES}

PROSEA Vol. 2: 320; TFM Vol. 2: 67

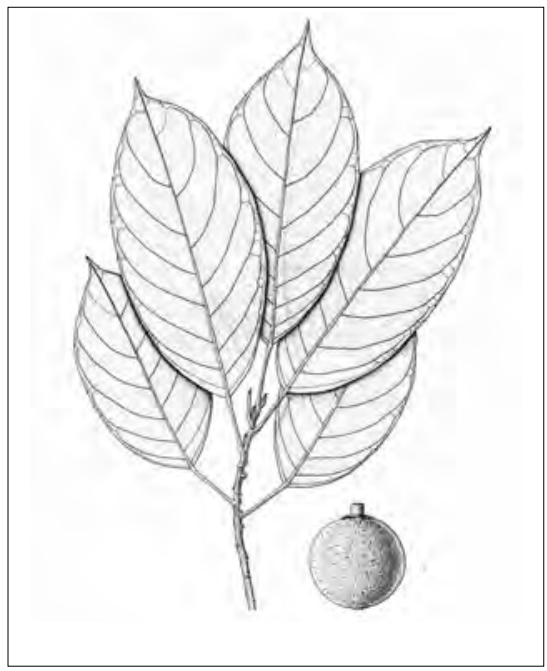

\begin{tabular}{|c|c|c|c|c|c|}
\hline \# & Code & Local Name & Use & RU & Hab.-Ab. \\
\hline 1 & PUT & gilepren, gelepren & 1 & $x$ & $\mathrm{O}-2, \mathrm{P}-3$ \\
\hline 2 & PUB & lawai lepren & 1 & $x$ & $\mathrm{O}-2, \mathrm{P}-3$ \\
\hline 3 & PUD & & & & \\
\hline 4 & PUM & & & & \\
\hline 5 & ALS & & & & \\
\hline 6 & LDY & bua olop & 1 & $x$ & P-3 \\
\hline 7 & LIT & bua' keliben & 1 & $x$ & P-3 \\
\hline 8 & BRU & wi pua' abang & 1 & $x$ & P-3 \\
\hline 9 & MRP & & & & \\
\hline 10 & PTN & bua it & 1 & $x$ & $\mathrm{P}-3$ \\
\hline 11 & KLK & bua' kep & & & \\
\hline 12 & KLM & bua' kep & 1 & $x$ & $\mathrm{O}-3, \mathrm{P}-3$ \\
\hline 13 & KLO & & & & \\
\hline 14 & KLA & & & & \\
\hline 15 & KAL & & & & \\
\hline 16 & KBD & & & & \\
\hline 17 & KBK & & & & \\
\hline 18 & PEB & & & & \\
\hline
\end{tabular}

Notes : 


\section{Hevea brasiliensis (Willd. ex A. Juss.) M.A. (Euphorbiaceae)}

\section{PARA RUBBER TREE. KaYU KaRET, KayU GETAH}

\section{DESCRIPTION}

Tree, $10-20 \mathrm{~m}$, with copious latex in all parts; leaves trifoliate, spirally arranged; leaflets, elliptic-oblong, 5-35 x 2.5-12.5 cm; flowers small yellow, fragrant; fruit a large 3-lobed capsule, 3-5 cm diameter, exploding, one pale brown seed per lobe

\section{HABITAT}

Cultivated in homegardens and plantations to $600 \mathrm{~m}$

\section{ORIGINS}

Brazil

\section{USES}

Latex is sold; seeds detoxified and eaten; timber for building; latex used to trap birds

\section{REFERENCES}

Burkill 1966: 1178; Corner 1988: 292;

Ochse 1980: 276; FOJ 1:479;

PROSEA Vol. 5(2): 260

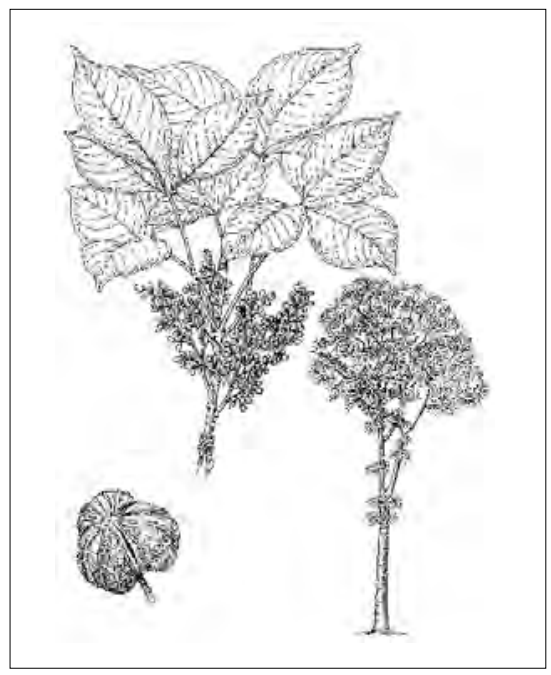

\section{(B.).
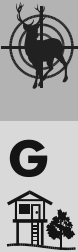

\begin{tabular}{|c|c|c|c|c|c|}
\hline \# & Code & Local Name & Use & RU & Hab.-Ab. \\
\hline 1 & PUT & karet & 11 & $x$ & G-2 \\
\hline 2 & PUB & karet & 7,11 & $x$ & G-2 \\
\hline 3 & PUD & karet & 7 & $x$ & G-2 \\
\hline 4 & PUM & karet & $1,7,11$ & $x$ & G-2 \\
\hline 5 & ALS & ula' & 6,10 & $x$ & $\mathrm{G}-1$ \\
\hline 6 & LDY & kayu para', fara & $7,10,11$ & $x$ & G-3, G-4, P-4 \\
\hline 7 & LIT & para, para' & 10,11 & $x$ & G-3 \\
\hline 8 & BRU & uen mud & 10 & $x$ & G-1, P-3 \\
\hline 9 & MRP & kayo tepei, kata'ang & 6,10 & $x$ & G-2 \\
\hline 10 & PTN & pulut & 1,10 & $x$ & G-2, O-3, P-4 \\
\hline 11 & KLK & ketipai baing, kayu pulut & 7 & v & $\mathrm{G}-3$ \\
\hline 12 & KLM & pulut, pulut & 10 & $x$ & G-3 \\
\hline 13 & KLO & tegei fei & & $\mathrm{x}$ & G-2 \\
\hline 14 & KLA & kettifai, pulut & 10,11 & $x$ & G-2 \\
\hline 15 & KAL & ketifai avak & & & \\
\hline 16 & KBD & kayu pulut & 7,10 & $x$ & G-2 \\
\hline 17 & KBK & kayu ketipai & & & \\
\hline 18 & PEB & polep alo & 5,6 & $x$ & \\
\hline
\end{tabular}

Notes : 


\section{COMMONMAHANG. MAHANGDAMAR}

\section{DESCRIPTION}

Tree, to $13 \mathrm{~m}$; bark smooth grey, red sap; twigs hollow with ants; young leaves reddish purple, 3lobed, $10-30 \mathrm{~cm}$ wide, thin, finely toothed; flowers brownish crimson; fruit a leathery capsule 10-12.5 cm wide, reddish brown, with yellow patches

\section{HABITAT}

Secondary forests, gaps, very common

\section{ORIGINS}

Southeast Asia

\section{USES}

Leaves for wrapping, medicine; firewood

\section{REFERENCES}

Corner 1988: 304; TFM Vol. 2: 107

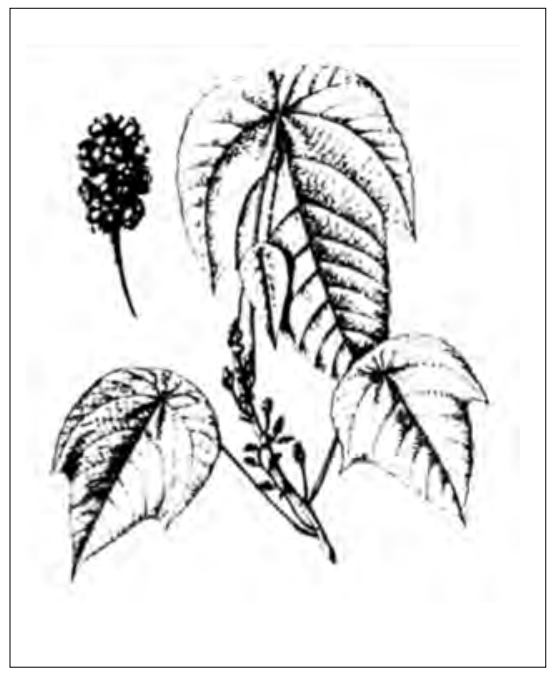

\begin{tabular}{|c|c|l|c|c|c|}
\hline$\#$ & Code & \multicolumn{1}{|c|}{ Local Name } & Use & RU & Hab.-Ab. \\
\hline 1 & PUT & lekeran, lekeran serou & 6 & $\mathbf{x}$ & Y-2, O-3, P-3 \\
\hline 2 & PUB & dun lekera, langkau serou, lekeran & 6 & $\mathbf{x}$ & Y-2, O-3 \\
\hline 3 & PUD & lekeran, lekeran bau & 6 & $\mathbf{x}$ & Y-2, O-3, P-2 \\
\hline 4 & PUM & doun lekeran & 6 & $\mathbf{X}$ & O-3, P-4, M-2 \\
\hline 5 & ALS & daun sedaman & 6,7 & $\mathbf{x}$ & O-2, P-3, M-2 \\
\hline 6 & LDY & binasing, don benasing & 6,7 & $\mathbf{x}$ & Y-2, O-2, O-3, P-4 \\
\hline 7 & LIT & binua, benua & 6 & $\mathbf{x}$ & Y-3, O-3, P-3 \\
\hline 8 & BRU & kayau bua & 6 & $\mathbf{x}$ & Y-3, P-3 \\
\hline 9 & MRP & tim la', ngela' & 1 & $\mathbf{x}$ & Y-2, O-3, P-2, M-3 \\
\hline 10 & PTN & doun ngela', ngela po & 6,7 & $\mathbf{x}$ & Y-2, O-3, O-4, P-3 \\
\hline 11 & KLK & kayu pedek, pedek bileng & 6,7 & $\mathbf{x}$ & Y-2, O-2 \\
\hline 12 & KLM & bedek, benua & & $\mathbf{X}$ & ALL \\
\hline 13 & KLO & da'eng benava & & & \\
\hline 14 & KLA & beneva' & & & \\
\hline 15 & KAL & da'un beneva' & & & \\
\hline 16 & KBD & benua', benua beleng & & & \\
\hline 17 & KBK & daun luko punan & 6 & $\mathbf{v}$ & O-3, P-4, M-3 \\
\hline 18 & PEB & menuang poti, menuang pute & & \\
\hline
\end{tabular}

Notes : 


\section{Manibot esculenta Crantz (Euphorbiaceae)}

\section{CASSAVA,MANIOC,TAPIOCA. UBI KAYU, SINGKONG}

\section{DESCRIPTION}

Shrub, 2-7 m; not or sparingly branched, thick stems, young stems green with violet, thick starchy roots; leaves alternate, long stalked, palmate 3-9 partite, $5-20 \mathrm{~cm} \times 2-6 \mathrm{~cm}$, petiole yellowish green or red; fruit ovoid-globose

\section{HABITAT}

Cultivated in gardens

\section{ORIGINS}

Tropical America

\section{USES}

Leaves eaten, medicine; roots eaten, alchohol, fodder, important famine food

\section{REFERENCES}

Burkill 1966: 1434; Ochse 1980: 280

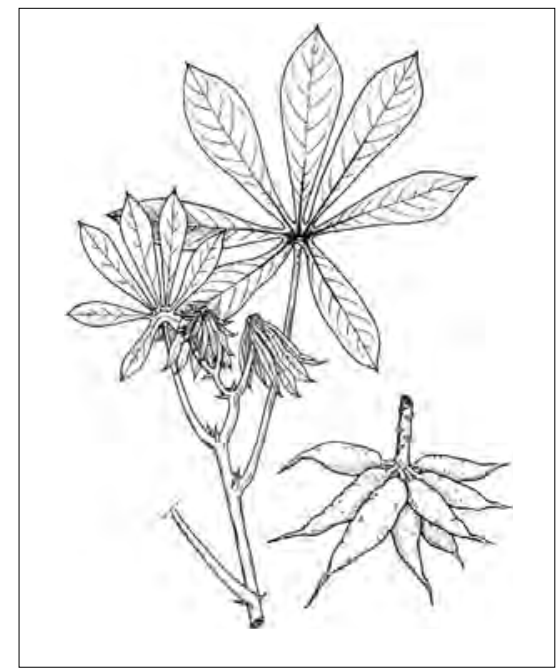

PROSEA Vol. 9: 107

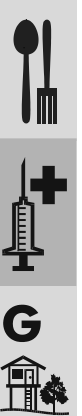

\begin{tabular}{|c|c|c|c|c|c|}
\hline \# & Code & Local Name & Use & RU & Hab.-Ab. \\
\hline 1 & PUT & ubi kayu, ubi & 1,2 & $\mathrm{v}$ & G-4, S-4 \\
\hline 2 & PUB & ubi kayu & 1,2 & v & G-4, S-4 \\
\hline 3 & PUD & ubi kayu & 1,2 & v & $G-4, S-4$ \\
\hline 4 & PUM & ubi kayu & 1,2 & v & G-4, S-4 \\
\hline 5 & ALS & belui & 1,2 & v & G-4, S-4 \\
\hline 6 & LDY & ubi kayu & 1,2 & $\mathrm{v}$ & G-4, S-4 \\
\hline 7 & LIT & ubi kayu & 1,2 & v & G-4, S-4 \\
\hline 8 & BRU & bwai kayau & 1,2 & v & G-4, S-4 \\
\hline 9 & MRP & buae kayau & 1,2 & $\mathrm{v}$ & G-4, S-4 \\
\hline 10 & PTN & ube kayo & 1,2 & v & G-4, S-4 \\
\hline 11 & KLK & ubi kayu & 1,2 & v & G-4, S-4 \\
\hline 12 & KLM & ubi kayu & 1,2 & $\mathrm{v}$ & G-4, S-4 \\
\hline 13 & KLO & ubi ghazu & 1,2 & $\mathrm{v}$ & $\mathrm{G}-4, \mathrm{~S}-4$ \\
\hline 14 & KLA & ubi hazu & 1,2 & v & $G-4, S-4$ \\
\hline 15 & KAL & ubi hazu & 1 & v & G-4, S-4 \\
\hline 16 & KBD & ubi kayu & 1,2 & v & G-4, S-4 \\
\hline 17 & KBK & ubi kayu & 1 & $\mathrm{v}$ & G-4, S-4 \\
\hline 18 & PEB & uvei kayo & 1,2 & $\mathrm{v}$ & G-4, S-4 \\
\hline
\end{tabular}

Notes : 


\section{CHAMBER BITTER. DUKONG ANAK, MENIRAN}

\section{DESCRIPTION}

Herb, 5-80 cm, stem angular suffused with red; higher twigs densely crowded; leaves oval-oblong, 5-20 × 1- $8 \mathrm{~mm}$; male flowers yellow white, female flowers red; fruit warty, $3 \mathrm{~mm}$, top side of bent branches (as a child is carried piggy-back)

\section{HABITAT}

Cultivated in home gardens; wild in fields, roadsides, waste areas, to $1500 \mathrm{~m}$

\section{ORIGINS}

Tropics

\section{USES}

Medicine

\section{REFERENCES}

Burkill 1966: 1749; FOJ Vol. 1: 469;

Basu 1918: plate 859

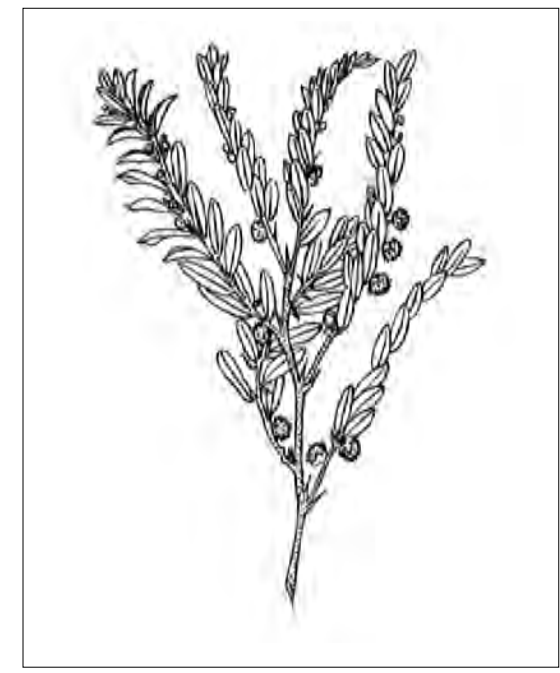

\begin{tabular}{|c|c|l|r|c|c|}
\hline$\#$ & Code & Local Name & Use & RU & Hab.-Ab. \\
\hline 1 & PUT & lampin anak & 2 & $\mathbf{x}$ & Y-2 \\
\hline 2 & PUB & & & & \\
\hline 3 & PUD & uru kiten & & $\mathbf{x}$ & ALL \\
\hline 4 & PUM & & 2 & $\mathbf{x}$ & A-2 \\
\hline 5 & ALS & petiga & 2 & $\mathbf{x}$ & S-2, Y-2 \\
\hline 6 & LDY & udu mabebua & 2 & $\mathbf{v}$ & G-4 \\
\hline 7 & LIT & udu maboang, udu mabanak, mabe anak & & & \\
\hline 8 & BRU & & & $\mathbf{x}$ & G-1, P-3 \\
\hline 9 & MRP & rau naeh, ro bae naia & & $\mathbf{x}$ & ALL \\
\hline 10 & PTN & uro' hae & 2 & $\mathbf{x}$ & S-2 \\
\hline 11 & KLK & udu takak, udu tukak & 2 & $\mathbf{x}$ & ALL \\
\hline 12 & KLM & udu kak & & $\mathbf{x}$ & ALL \\
\hline 13 & KLO & udu ali & & & \\
\hline 14 & KLA & & & & \\
\hline 15 & KAL & & & $\mathbf{x}$ & ALL \\
\hline 16 & KBD & i'ing & & $\mathbf{x}$ & Y-2, O-3 \\
\hline 17 & KBK & & & \multicolumn{2}{|c|}{} \\
\hline 18 & PEB & ureu nep & & & \\
\hline
\end{tabular}

Notes : 


\section{Sauropus androgynus (L.) Merr. (Euphorbiaceae)}

\section{(NO ENGLISH NAME). CEKOP MANIS, KATUK}

\section{DESCRIPTION}

Shrub, 2-3.5 m, propogated by cuttings; leaves hanging from lateral branches, biseriate, alternate, ovate or oblong, 2-7.5 x 1-3 cm; flowers red; fruits on red calyx, depressed globose, 6 angular, dull white or slightly tinged with rose, $1.2 \times 1.7 \mathrm{~cm}$

\section{HABITAT}

Cultivated; wild in and around villages

\section{ORIGINS}

India to western Indonesia

\section{USES}

Fencing; leaves and fruit eaten, locally sold; a food colour, medicine

\section{REFERENCES}

Burkill 1966: 2002; Ochse 1980: 290;

PROSEA Vol. 8: 244

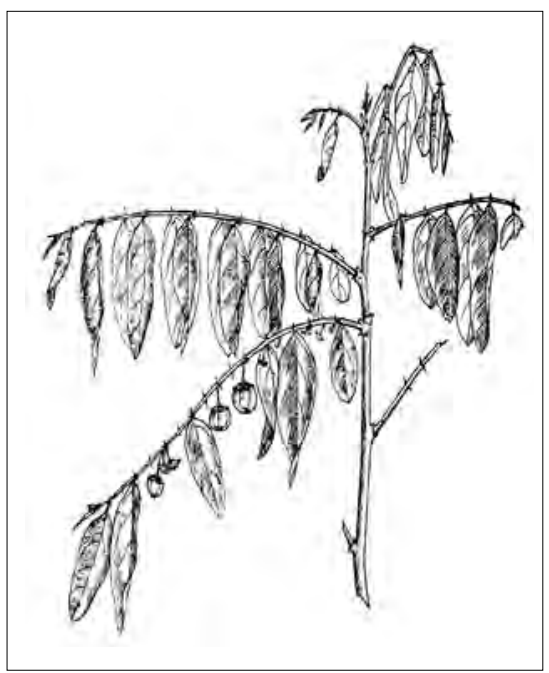

\begin{tabular}{|c|c|c|c|c|c|}
\hline \# & Code & Local Name & Use & $\mathbf{R U}$ & Hab.-Ab. \\
\hline 1 & PUT & cangkuk manis, talu keliyen, talu & 1,11 & v & G-3 \\
\hline 2 & PUB & cankuk manis, cankuk mis & 1,11 & v & G-3 \\
\hline 3 & PUD & doun talau, talu & 1 & v & G-3 \\
\hline 4 & PUM & lekoi sina & 1,11 & v & G-3 \\
\hline 5 & ALS & sanggu & 1,11 & v & G-3 \\
\hline 6 & LDY & cangkuk manis, sangkuk manis & 1,11 & $\mathrm{v}$ & G-4, S-3, Y-1 \\
\hline 7 & LIT & cangkuk manis, cangkok manis & 1,11 & v & $\mathrm{G}-4$ \\
\hline 8 & BRU & kayau men & 1,11 & v & G-3 \\
\hline 9 & MRP & talau & 1,11 & v & G-3 \\
\hline 10 & PTN & ujo balau, ujo balo & 1,11 & v & G-3 \\
\hline 11 & KLK & ujau kayu belang, ujau kayu alo & 1,11 & v & G-3 \\
\hline 12 & KLM & ujau alo & 1,11 & v & G-3 \\
\hline 13 & KLO & kojo balo, ojo balo & 1,2 & v & G-3 \\
\hline 14 & KLA & hujau balau, kujau balau & 1 & v & G-4 \\
\hline 15 & KAL & kojau balau & & & \\
\hline 16 & KBD & kozau balau, ozau balau & 1 & v & G-3 \\
\hline 17 & KBK & ujau balau & & & \\
\hline 18 & PEB & ujau balau, kujau balau & 1 & $\mathrm{v}$ & $G-3, S-3$ \\
\hline
\end{tabular}

Notes : 


\section{(NO ENGSISH NAME). PAYANG SALAP.}

\section{DESCRIPTION}

Tree, to $10 \mathrm{~m}$; leaf stalk to $6 \mathrm{~cm}$, kneed both ends; leaf blade ovate $15-23 \times 7-13 \mathrm{~cm}$, underside pale straw brown with dense felt of hairs; fruit a globose, 3 lobed capsule $3.3 \times 1.5 \mathrm{~cm}$, golden brown

\section{HABITAT}

Lowland and hill primary forests to $1000 \mathrm{~m}$; planted in village fruit gardens

\section{ORIGINS}

Southeast Asia

\section{USES}

Nuts eaten, medicine

\section{REFERENCES}

TFM: Vol. 2: 132

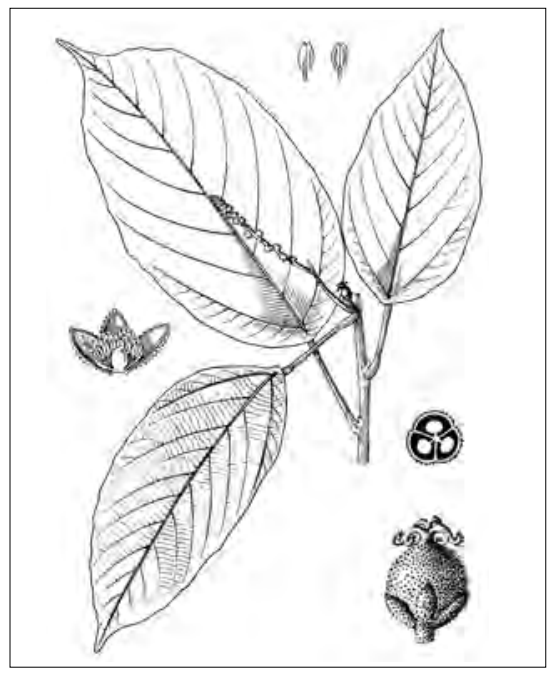

\begin{tabular}{|r|c|l|r|r|c|}
\hline$\#$ & Code & \multicolumn{1}{|c|}{ Local Name } & Use & RU & Hab.-Ab. \\
\hline 1 & PUT & & & & \\
\hline 2 & PUB & piang & & & \\
\hline 3 & PUD & piang salap & & & V \\
\hline 4 & PUM & & 1 & $\mathbf{V}$ & G-1 \\
\hline 5 & ALS & kemasar & & & \\
\hline 6 & LDY & & & & \\
\hline 7 & LIT & & 1 & $\mathbf{x}$ & \\
\hline 8 & BRU & payang awah & 1 & $\mathbf{x}$ & G-2 \\
\hline 9 & MRP & paye haar, paye halu & 1 & $\mathbf{v}$ & G-2 \\
\hline 10 & PTN & payang halap & 1 & $\mathbf{x}$ & G-2 \\
\hline 11 & KLK & payang salap, salap & 1 & $\mathbf{v}$ & G-3 \\
\hline 12 & KLM & salap & 1 & $\mathbf{v}$ & G-2 \\
\hline 13 & KLO & pazang sala', sala, lazayang sala, da'eng sala' & 1 & $\mathbf{x}$ & G-4 \\
\hline 14 & KLA & salap & & $\mathbf{x}$ & \\
\hline 15 & KAL & salap & 1 & $\mathbf{v}$ & G-2 \\
\hline 16 & KBD & salap & & $\mathbf{x}$ & \\
\hline 17 & KBK & kayu payang salap & 1 & $\mathbf{x}$ & G-3, S-2, Y-2, O-2 \\
\hline 18 & PEB & salap, payang salap & & \\
\hline
\end{tabular}

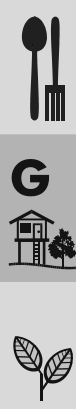

Notes : 


\section{Albizia chinensis (Osbeck) Merr. (Fabaceae)}

\section{SILKTREE. SENGON BATAI}

\section{DESCRIPTION}

Tree, to $30 \mathrm{~m}$; big spreading surface roots; bark grey smooth; leaves twice pinnate, leaflets tiny; flowers small white; pods large, thin, flat, splitting with numerous seeds

\section{HABITAT}

Lowland primary and secondary forests, riverine, cultivated in villages; sleeping tree for long-tail macaques (Macaca fascicularis)

\section{ORIGINS}

Tropical Asia

\section{USES}

Bark for fish poison; medicine; shade tree for tea; hunting location

\section{REFERENCES}

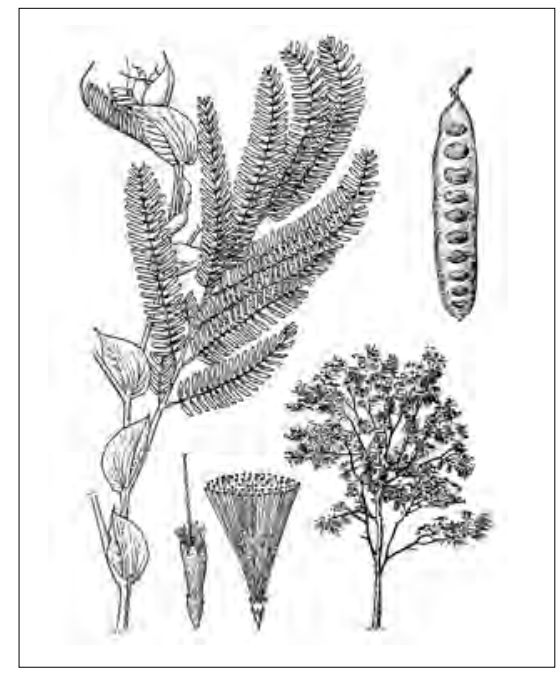

Burkill 1966: 84; Corner 1988: 451; Leaman et al. 1991: 22; PROSEA Vol. 11: 64

\begin{tabular}{|c|c|c|c|c|c|c|}
\hline \multirow{4}{*}{$((4)$} & \# & Code & Local Name & Use & RU & Hab.-Ab. \\
\hline & 1 & PUT & puluh & 10 & $x$ & R-3 \\
\hline & 2 & PUB & puluh & 10 & $x$ & $\mathrm{R}-3$ \\
\hline & 3 & PUD & puluh & 10 & $x$ & $\mathrm{R}-3$ \\
\hline \multirow{8}{*}{$\begin{array}{l}R \\
9 \sqrt{6}\end{array}$} & 4 & PUM & puluh & 10 & $x$ & $\mathrm{R}-3$ \\
\hline & 5 & ALS & & & & \\
\hline & 6 & LDY & & & & \\
\hline & 7 & LIT & & & & \\
\hline & 8 & BRU & & & & \\
\hline & 9 & MRP & & & & \\
\hline & 10 & PTN & peta' & 10 & $x$ & R-3 \\
\hline & 11 & KLK & nep & 10 & $x$ & $\mathrm{R}-3$ \\
\hline \multirow{7}{*}{ \& } & 12 & KLM & & & & \\
\hline & 13 & KLO & nep & 10 & $x$ & $\mathrm{R}-3$ \\
\hline & 14 & KLA & nep & 10 & $x$ & $\mathrm{R}-3$ \\
\hline & 15 & KAL & nep & 10 & $x$ & $\mathrm{R}-3$ \\
\hline & 16 & KBD & nep & 10 & $\mathrm{v}$ & R-3 \\
\hline & 17 & KBK & nep & 10 & $x$ & R-3 \\
\hline & 18 & PEB & nep & 2,10 & v & $\mathrm{R}-3$ \\
\hline
\end{tabular}

Notes : 


\section{Baubinia semibifida Roxb. (Fabaceae) \\ BUTTERFLY VINE. RUMPUT KUPU-KUPU}

\section{DESCRIPTION}

Vine, with tendrils, leaves bilobed (1/4 to $2 / 5)$ as in a butterfly wing, 11-13 nerved; distinctly dialated tubular hypanthium, c. $10 \mathrm{~mm}$; flowers white to yellow or green; pods flat smooth oblong, $10-20 \times 3-4 \mathrm{~cm}$, seeds c. 6 flat

\section{HABITAT}

Lowland and hill primary forest to $2000 \mathrm{~m}$; forest margins

\section{ORIGINS}

Malaysia and western Indonesia

\section{USES}

Leaves eaten; roots for medicine; cordage; sold locally

\section{REFERENCES}

FM I Vol. 12: 490

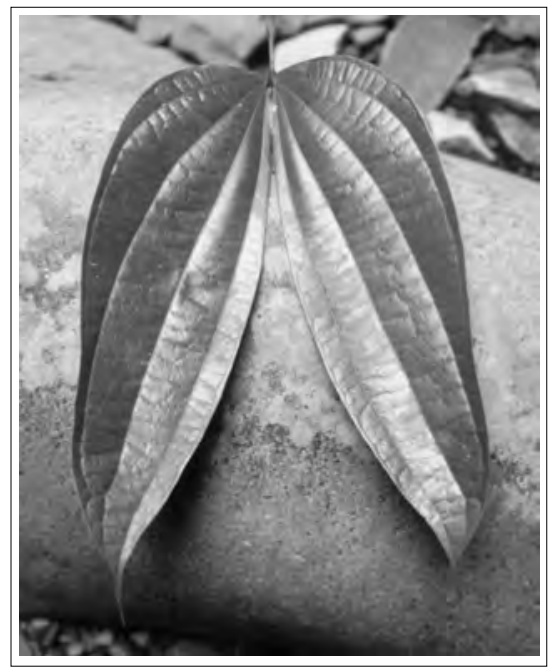

\begin{tabular}{|c|c|l|c|c|c|}
\hline$\#$ & Code & \multicolumn{1}{|c|}{ Local Name } & Use & RU & Hab.-Ab. \\
\hline 1 & PUT & buwa lepau, kapiu, lepeu & $1,8,11$ & $\mathbf{V}$ & P-4 \\
\hline 2 & PUB & lapiu, oka lapiu & $1,8,11$ & $\mathbf{V}$ & P-4 \\
\hline 3 & PUD & rapiu & 1,11 & $\mathbf{V}$ & P-4 \\
\hline 4 & PUM & bua lapiu & $1,8,11$ & $\mathbf{v}$ & P-3 \\
\hline 5 & ALS & bakag laviu & $1,8,11$ & $\mathbf{V}$ & P-3 \\
\hline 6 & LDY & war leviyu, war ayep & $1,8,11$ & $\mathbf{V}$ & P-3 \\
\hline 7 & LIT & leviu, war ayap, lepiyu & 1,11 & $\mathbf{V}$ & P-4 \\
\hline 8 & BRU & uen ayap & 1,11 & $\mathbf{V}$ & P-3 \\
\hline 9 & MRP & kah lepeu, kalepeu, lapiu & $1,8,11$ & $\mathbf{V}$ & P-3 \\
\hline 10 & PTN & aka anak bela, kelulo & 1,11 & $\mathbf{V}$ & P-3 \\
\hline 11 & KLK & te ayang & & & \\
\hline 12 & KLM & & & & \\
\hline 13 & KLO & & & & \\
\hline 14 & KLA & & & $\mathbf{X}$ & \\
\hline 15 & KAL & aha fa'bet & & $\mathbf{X}$ & \\
\hline 16 & KBD & lepeu & & $\mathbf{X}$ & \\
\hline 17 & KBK & lengkong & 6 & $\mathbf{X}$ & O-2, P-4 \\
\hline 18 & PEB & lakeu kabava', lakeu kebava & & \\
\hline
\end{tabular}

Notes : 


\section{Leucaena leucocephala Bth (Lam.) De Wit (Fabaceae)}

\section{WILD TAMARIND. PETAI CINA, LAMTORO}

\section{DESCRIPTION}

Tree, bush, to $10 \mathrm{~m}$; bark light grey; leaf-stalks 10-18 cm long, 4-8 pairs side stalks; leaflets tiny feathery, pale green white underneath; flowers cream white; pods $12.5-17.5 \times 1.25-1.50 \mathrm{~cm}$, thick edges, transluscent, drying dirty brown, in bundles

\section{HABITAT}

Wild and planted in villages, waste places

\section{ORIGINS}

America

\section{USES}

Shade tree for coffee; young pods eaten as vegetable, raw seeds eaten; sold locally; hunting location

\section{REFERENCES}

Corner 1988: 456; FM I Vol. 11(1): 182

PROSEA Vol. 11: 175

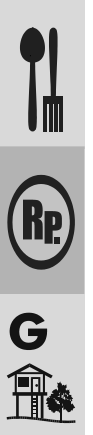

\begin{tabular}{|c|c|l|c|c|c|}
\hline$\#$ & Code & \multicolumn{1}{|c|}{ Local Name } & Use & RU & Hab.-Ab. \\
\hline 1 & PUT & peta, peta cina & 1 & $\mathbf{x}$ & G-4 \\
\hline 2 & PUB & peta, peta haru & 1,11 & $\mathbf{v}$ & G-2, P-3 \\
\hline 3 & PUD & peta, lengenun & 1,11 & $\mathbf{v}$ & O-3, P-4 \\
\hline 4 & PUM & peta & 1 & $\mathbf{v}$ & G-3 \\
\hline 5 & ALS & bua' fata' & $1,7,11$ & $\mathbf{v}$ & G-3, P-3 \\
\hline 6 & LDY & patar, bua patar & $1,10,11$ & $\mathbf{v}$ & G-3 \\
\hline 7 & LIT & potar, petar cina, petar & $1,10,11$ & $\mathbf{v}$ & G-3, P-3 \\
\hline 8 & BRU & wi tal & $1,7,11$ & $\mathbf{v}$ & G-3 \\
\hline 9 & MRP & petah, peta rau, tah hamang & $1,11,10$ & $\mathbf{v}$ & G-2 \\
\hline 10 & PTN & peta alo', peta time & 1 & $\mathbf{X}$ & G-3 \\
\hline 11 & KLK & beta' alo, kayu beta' & 1,11 & $\mathbf{v}$ & G-2 \\
\hline 12 & KLM & beta, beta alu & $1,10,11$ & $\mathbf{v}$ & G-2 \\
\hline 13 & KLO & beta' & $1,7,10$ & $\mathbf{x}$ & G-2, P-2 \\
\hline 14 & KLA & beta' & 1,7 & $\mathbf{X}$ & G-2, P-3 \\
\hline 15 & KAL & beta' late' & & & \\
\hline 16 & KBD & beta', beta', beta padai & 1,10 & $\mathbf{v}$ & G-2, P-3 \\
\hline 17 & KBK & beta & & & \\
\hline 18 & PEB & bete' alo, bete & $1,7,11$ & $\mathbf{v}$ & G-3, O-2, P-4 \\
\hline
\end{tabular}

Notes : 


\section{Milletia sericea (Vent.) Wight. et Arn. (Fabaceae)}

\section{(NO ENGLISH NAME). AKAR MAMBU, AKAR TUBA}

\section{DESCRIPTION}

Climbing shrub, 10-30 m long; leaves alternate, short stalked, odd-pinnate, leaflets 5-9, oblong to obovate, $7-25 \times 3.5-10 \mathrm{~cm}$, silvery scales underneath; flowers violet within; pods turgid, rusty $5-7.5 \mathrm{~cm}$ long, seeds $1-2$, rarely 3

\section{HABITAT}

Lowland and hill primary forests; shady riverine or wet areas

\section{ORIGINS}

Malaysia and western Indonesia

\section{USES}

Young leaves and stem tips eaten; medicine, fish poison; timber for firewood

\section{REFERENCES}

Burkill 1966: 1496; Ochse 1980: 396

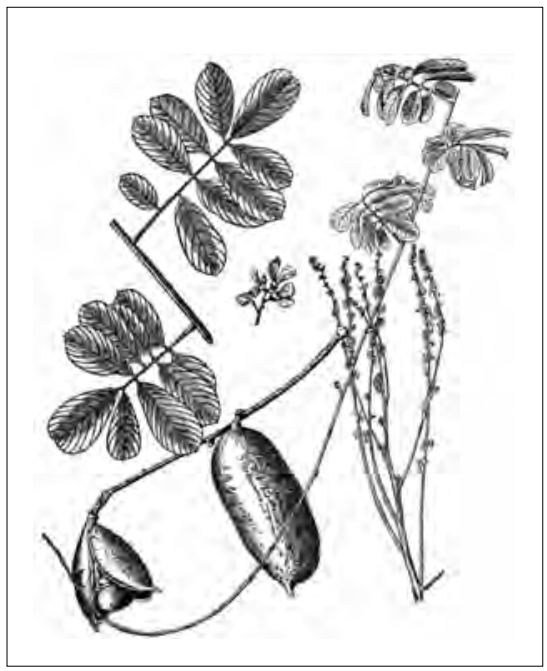

\begin{tabular}{|r|c|l|c|c|c|}
\hline$\#$ & Code & Local Name & Use & RU & Hab.-Ab. \\
\hline 1 & PUT & & & & \\
\hline 2 & PUB & & & & \\
\hline 3 & PUD & kelungau apan & & $\mathbf{X}$ & P-3 \\
\hline 4 & PUM & & & $\mathbf{X}$ & ALL \\
\hline 5 & ALS & pembarang nyabuk & & & \\
\hline 6 & LDY & & & & \\
\hline 7 & LIT & & & $\mathbf{X}$ & \\
\hline 8 & BRU & kayau salanga & & $\mathbf{X}$ & P-3 \\
\hline 9 & MRP & nyou & & $\mathbf{V}$ & Y-4, O-4, P-4 \\
\hline 10 & PTN & kayo buyo & & $\mathbf{X}$ & ALL \\
\hline 11 & KLK & & & & \\
\hline 12 & KLM & balut & & & \\
\hline 13 & KLO & & & & \\
\hline 14 & KLA & & 7 & $\mathbf{X}$ & O-3, P-3 \\
\hline 15 & KAL & & & & \\
\hline 16 & KBD & balut & 7 & $\mathbf{V}$ & O-4, P-3 \\
\hline 17 & KBK & & & & \\
\hline 18 & PEB & nyeu tuvah, nye tuva & & & \\
\hline
\end{tabular}

Notes : 


\section{Parkia speciosa Hassk. (Fabaceae)}

\section{Petai. Petai hutan}

\section{DESCRIPTION}

Tree, to $30 \mathrm{~m}$, buttresses; bark reddish brown smooth; leaves alternate bipinnate, side stalks 10 18 pairs; leaflets tiny 20 to 35 pairs, blade blunt with prominant midrib; pod twisted, $30-40 \times 3.5-$ $5 \mathrm{~cm} ; 12-18$ seeds oblique horizontal across pod, bitter

\section{HABITAT}

Lowland and hill primary and secondary forests to $1000 \mathrm{~m}$; riverine; planted in village fruit gardens

\section{ORIGINS}

Malaysia and western Indonesia

\section{USES}

Seeds eaten, sold locally; used for medicine (LDY, LIT)

\section{REFERENCES}

Burkill 1966: 1700; Ochse 1980: 404; PROSEA Vol.

8: 222; TFM Vol. 1: 281; Valkenburg 1997: 82
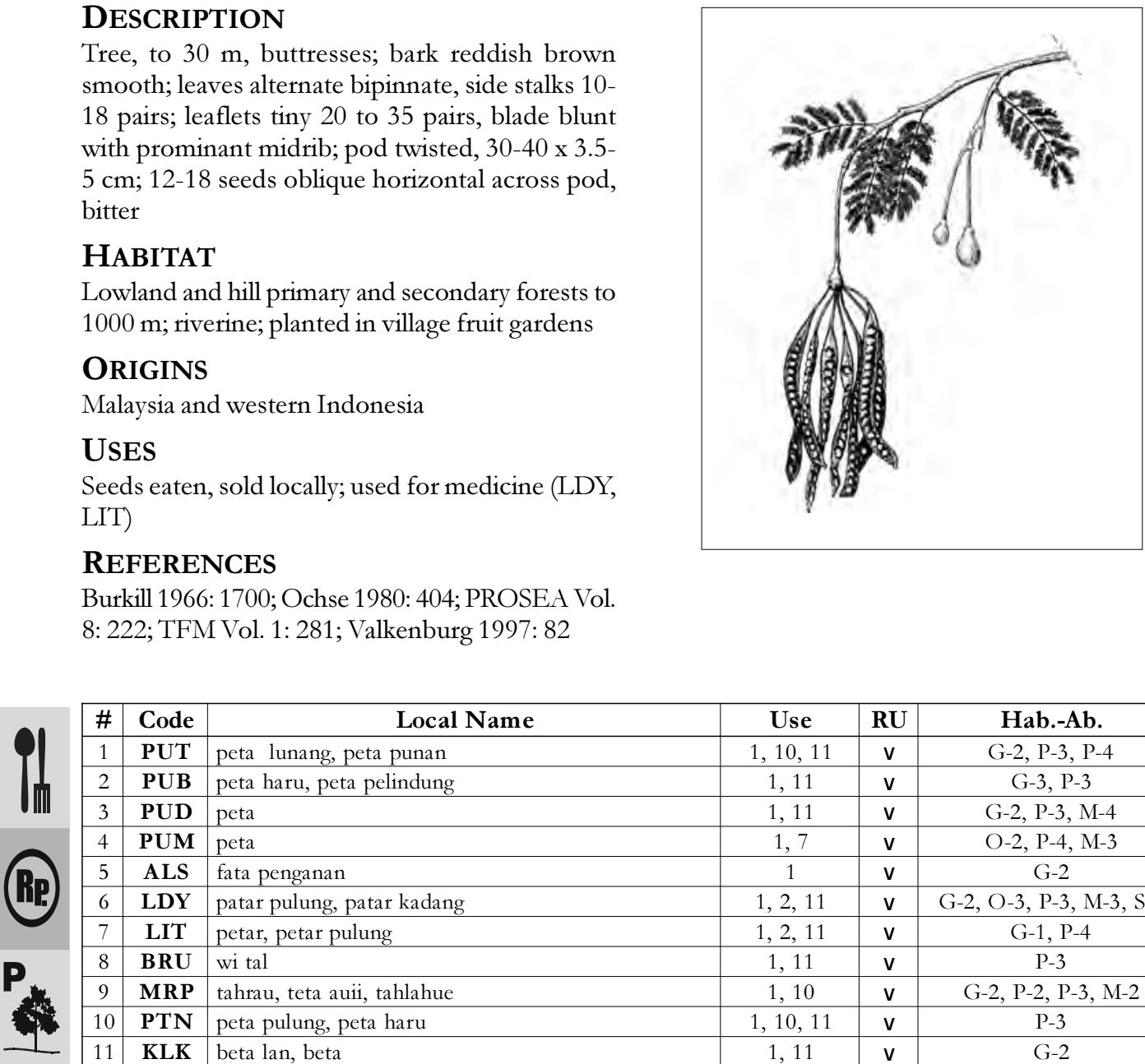

\begin{tabular}{|c|l|l|c|c|c|}
\hline \# & Code & \multicolumn{1}{|c|}{ Local Name } & Use & RU & Hab.-Ab. \\
\hline 1 & PUT & peta lunang, peta punan & $1,10,11$ & $\mathbf{v}$ & G-2, P-3, P-4 \\
\hline 2 & PUB & peta haru, peta pelindung & 1,11 & $\mathbf{v}$ & G-3, P-3 \\
\hline 3 & PUD & peta & 1,11 & $\mathbf{v}$ & G-2, P-3, M-4 \\
\hline 4 & PUM & peta & 1,7 & $\mathbf{v}$ & O-2, P-4, M-3 \\
\hline 5 & ALS & fata penganan & 1 & $\mathbf{v}$ & G-2 \\
\hline 6 & LDY & patar pulung, patar kadang & $1,2,11$ & $\mathbf{v}$ & G-2, O-3, P-3, M-3, S-2 \\
\hline 7 & LIT & petar, petar pulung & $1,2,11$ & $\mathbf{v}$ & G-1, P-4 \\
\hline 8 & BRU & wi tal & 1,11 & $\mathbf{v}$ & P-3 \\
\hline 9 & MRP & tahrau, teta auii, tahlahue & 1,10 & $\mathbf{v}$ & G-2, P-2, P-3, M-2 \\
\hline 10 & PTN & peta pulung, peta haru & $1,10,11$ & $\mathbf{v}$ & P-3 \\
\hline 11 & KLK & beta lan, beta & 1,11 & $\mathbf{v}$ & G-2 \\
\hline 12 & KLM & beta alo, beta kenya & 1,11 & $\mathbf{v}$ & G-2 \\
\hline 13 & KLO & beta & 1,11 & $\mathbf{v}$ & P-3 \\
\hline 14 & KLA & beta & 1,11 & $\mathbf{v}$ & G-1, P-3 \\
\hline 15 & KAL & beta' avak & 1,11 & $\mathbf{v}$ & P-3 \\
\hline 16 & KBD & beta', beta & 1,11 & $\mathbf{v}$ & P-3 \\
\hline 17 & KBK & beta alo & $1,7,11$ & $\mathbf{v}$ & G-3, O-2, P-4, M-4 \\
\hline 18 & PEB & poteu ba', bete alo' & & P-3 \\
\hline
\end{tabular}

Notes : 
Senna alata L. Roxb. (Fabaceae)

SEVEN GOLDEN CANDLESTICKS. GELENGGANG

\section{DESCRIPTION}

A shrub, 1-5 m; woody, perennial; leaves pinnate to $75 \mathrm{~cm}$; leaflets 9 pairs, sessile, opposite, oblong to obovate, $3.5-15 \times 2.5-9 \mathrm{~cm}$, flowers yellow; pods black with longitudinal broadwing, $12.5-18 \mathrm{~cm} \mathrm{x} 2$. $5 \mathrm{~cm}$; seeds $50-70$

\section{HABITAT}

Cultivated in homegardens; wild in meadows, waste lands, fallow rice fields

\section{ORIGINS}

Tropical Asia

\section{USES}

Leaves for medicine

\section{REFERENCES}

Burkill 1966: 479; FOJ Vol. I: 540

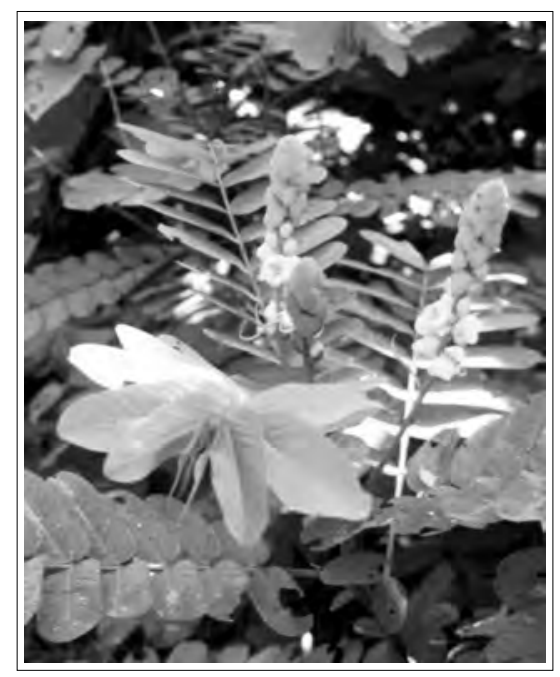

\begin{tabular}{|c|c|l|c|c|c|}
\hline$\#$ & Code & \multicolumn{1}{|c|}{ Local Name } & Use & RU & Hab.-Ab. \\
\hline 1 & PUT & kesama & 2 & $\mathrm{X}$ & $\mathrm{Y}-2$ \\
\hline 2 & PUB & kecama & 2 & $\mathrm{X}$ & $\mathrm{Y}-2$ \\
\hline 3 & PUD & kecama & 2 & $\mathrm{X}$ & $\mathrm{Y}-2$ \\
\hline 4 & PUM & kesama & 2 & $\mathrm{X}$ & $\mathrm{R}$ \\
\hline 5 & ALS & saga' kais & 2 & $\mathrm{X}$ & $\mathrm{ALL}$ \\
\hline 6 & LDY & saga & & $\mathrm{X}$ & $\mathrm{Y}-3, \mathrm{R}$ \\
\hline 7 & LIT & & 2 & $\mathrm{X}$ & $\mathrm{Y}-2$, O-2 \\
\hline 8 & BRU & uen dau pai & 2 & $\mathrm{X}$ & $\mathrm{R}$ \\
\hline 9 & MRP & ro korua & 2 & $\mathrm{X}$ & $\mathrm{R}$ \\
\hline 10 & PTN & kap netam, kop dom & 2 & $\mathrm{X}$ & $\mathrm{Y}-2$ \\
\hline 11 & KLK & udu kip & 2 & $\mathrm{X}$ & $\mathrm{ALL}$ \\
\hline 12 & KLM & udu kip & 2 & $\mathrm{X}$ & $\mathrm{ALL}$ \\
\hline 13 & KLO & da'eng tabing & 2 & $\mathrm{X}$ & $\mathrm{ALL}$ \\
\hline 14 & KLA & tung taban punan & 2 & $\mathrm{X}$ & $\mathrm{Y}-2$ \\
\hline 15 & KAL & udu kep & 2 & $\mathrm{X}$ & $\mathrm{R}, \mathrm{Y}-2$ \\
\hline 16 & KBD & udu kop & 2 & $\mathrm{~V}$ & $\mathrm{R}$ \\
\hline 17 & KBK & udu kep & & \\
\hline 18 & PEB & ureu kep, uru kep & $\mathrm{Y}-4$, O-2 \\
\hline
\end{tabular}

Notes : 


\section{1) Vigna unguiculata (L.)Walp. (Fabaceae) YARD-LONG BEAN. KACANG PANJANG}

\section{DESCRIPTION}

Herb, climbing, annual, 2-4 m long; leaves alternate, trifoliate, ovate 7-13.5 x 4-9.5 cm, petiole 5$25 \mathrm{~cm}$; flowers yellowish, or pale blue; pods green 30-120 cm long, 10-30 seeds

\section{HABITAT}

Cultivated in gardens

\section{ORIGINS}

Southern China

\section{USES}

Young leaves, shoots, pods and seeds eaten, sold locally; medicine

\section{REFERENCES}

Burkill 1966: 2271; Ochse 1980: 435; PROSEA

Vol. 8: 274; PROSEA Vol. 1: 77
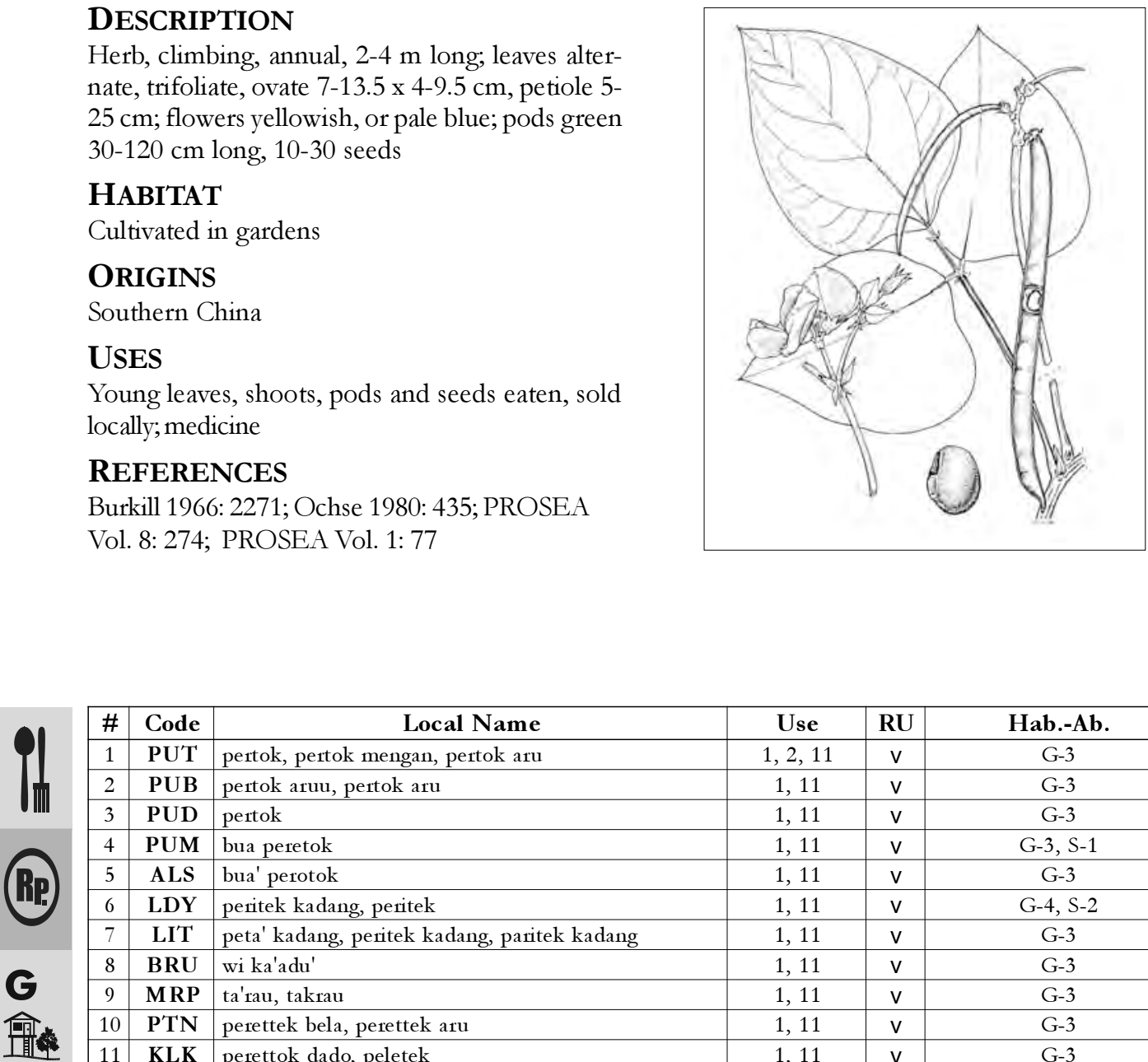

\begin{tabular}{|c|c|c|c|c|c|}
\hline \# & Code & Local Name & Use & RU & Hab.-Ab. \\
\hline 1 & PU'T & pertok, pertok mengan, pertok aru & $1,2,11$ & $\mathrm{v}$ & G-3 \\
\hline 2 & PUB & pertok aruu, pertok aru & 1,11 & $\mathrm{v}$ & G-3 \\
\hline 3 & PUD & pertok & 1,11 & $\mathrm{v}$ & G-3 \\
\hline 4 & PUM & bua peretok & 1,11 & $\mathrm{v}$ & G-3, S-1 \\
\hline 5 & ALS & bua' perotok & 1,11 & $\mathrm{v}$ & G-3 \\
\hline 6 & LDY & peritek kadang, peritek & 1,11 & $\mathrm{v}$ & G-4, S-2 \\
\hline 7 & LIT & peta' kadang, peritek kadang, paritek kadang & 1,11 & $\mathrm{v}$ & G-3 \\
\hline 8 & BRU & wi ka'adu' & 1,11 & $\mathrm{v}$ & G-3 \\
\hline 9 & MRP & ta'rau, takrau & 1,11 & $\mathrm{v}$ & G-3 \\
\hline 10 & PTN & perettek bela, perettek aru & 1,11 & $\mathrm{v}$ & G-3 \\
\hline 11 & KLK & perettok dado, peletek & 1,11 & $\mathrm{v}$ & G-3 \\
\hline 12 & KLM & perettek dado & 1,11 & $\mathrm{v}$ & G-3 \\
\hline 13 & KLO & ferettek & 1,11 & $\mathrm{v}$ & G-3 \\
\hline 14 & KLA & perettek & 1,11 & $\mathrm{v}$ & G-3 \\
\hline 15 & KAL & perettek & 1,11 & $\mathrm{v}$ & G-3 \\
\hline 16 & KBD & pertok & 1,11 & $\mathrm{v}$ & G-3 \\
\hline 17 & KBK & perettek & 1,11 & $\mathrm{v}$ & G-3 \\
\hline 18 & PEB & perotek baleu, perotek & 1,11 & $\mathrm{v}$ & G-4, S-3 \\
\hline
\end{tabular}

Notes : 
Castanopsis argented (Blume) A. DC. (Fagaceae)

\section{MALAYAN CHESTNUT. SANINTEN, BERANGAN}

\section{DESCRIPTION}

Tree, to $30 \mathrm{~m}$; bark dark grey fissured; leaves elliptic to ovate, $10-18 \times 5-8 \mathrm{~cm}$, drying greenish above, silvery brown below, with silvery hairs or scales; fruit enclosed by spiny cupule, 3-4 cm diameter, spines $1-1.5 \mathrm{~cm}$; 3 seeds, $2-2.5 \mathrm{~cm}$ diameter

\section{HABITAT}

Primary or old secondary forests to $1400 \mathrm{~m}$; dry fertile soil

\section{ORIGINS}

Sumatra, Java

\section{USES}

Timber; tanin; black dye from bark; fruits edible, sold locally; site for ambushing pigs when fruiting

\section{REFERENCES}

Corner 1988: 329; Kessler and Sidiyasa 1994: 137;

PROSEA Vol. 5(2): 112; FM I Vol. 7(2): 331

\begin{tabular}{|c|c|l|c|c|c|}
\hline$\#$ & Code & \multicolumn{1}{|c|}{ Local Name } & Use & RU & Hab.-Ab. \\
\hline 1 & PUT & bangan & 1,10 & X & P-2, M-2 \\
\hline 2 & PUB & movang & & & \\
\hline 3 & PUD & bangan & & & \\
\hline 4 & PUM & bangan & & & \\
\hline 5 & ALS & tegerangan lambun & & & \\
\hline 6 & LDY & bua keberangan & & & \\
\hline 7 & LIT & bua' berangan & & & \\
\hline 8 & BRU & wi belangan & & & \\
\hline 9 & MRP & mengang, mangang & & & \\
\hline 10 & PTN & bangan & 1,10 & $\mathrm{X}$ & \\
\hline 11 & KLK & bangan, bangan mbui buan & & & \\
\hline 12 & KLM & bangan & & & \\
\hline 13 & KLO & bangainy & & \\
\hline 14 & KLA & bangan silu & & \\
\hline 15 & KAL & bangen & 1,10 & $\mathrm{~V}$ & \\
\hline 16 & KBD & bangan soet & & P-2, M-1 \\
\hline 17 & KBK & bangan & $1,10,11$ & $\mathrm{~V}$ & \\
\hline 18 & PEB & bangan & & \\
\hline
\end{tabular}

Notes : 


\section{Litbocarpus conocarpus (Oudem.) Rehd. (Fagaceae)}

\section{SINGAPORE OAK. PASANG IJANG}

\section{DESCRIPTION}

Tree, to $45 \mathrm{~m}$; bark grey brown; leaves ellipticalobovate 8-12 x 3-4 cm, dark glossy green, upcurled sides, hairy and silvery beneath, petiole $0.5-1 \mathrm{~cm}$; acorn cup 1.6-2.0 cm with minute scale tips; nut ovoid-conical, $2 \mathrm{~cm}$ tall, mostly free from the cup

\section{HABITAT}

Lowland and hill primary forests to $1800 \mathrm{~m}$

\section{ORIGINS}

Malaysia and western Indonesia

\section{USES}

Site for ambushing pigs when fruiting; timber for construction, firewood

\section{REFERENCES}

Corner 1988: 331-340; PROSEA Vol. 5(2): 291;

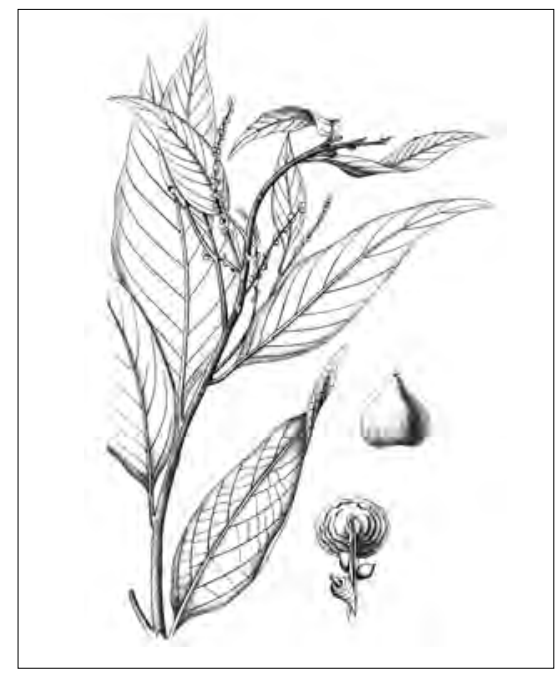

FM I Vol. 7(2): 349

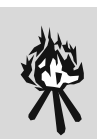

\begin{tabular}{|c|c|l|c|c|c|}
\hline$\#$ & Code & \multicolumn{1}{|c|}{ Local Name } & Use & RU & Hab.-Ab. \\
\hline 1 & PUT & tekalet piling, tekalet livoh & 7,10 & X & P-3, M-3 \\
\hline 2 & PUB & tekalet & & & \\
\hline 3 & PUD & tekalet buwa' & & & \\
\hline 4 & PUM & & & & \\
\hline 5 & ALS & tekolod bua' & & & \\
\hline 6 & LDY & saled fadei & & & \\
\hline 7 & LIT & bua' salid padai & & & \\
\hline 8 & BRU & wi salet to'u & & & \\
\hline 9 & MRP & kelan, kalan & & & \\
\hline 10 & P'N & palan & & & \\
\hline 11 & KLK & palan tekalet, palan timai & & & \\
\hline 12 & KLM & palan, palan tekalet & & & \\
\hline 13 & KLO & tegalet jelae & 6,7 & $\mathrm{X}$ & P-4, M-4 \\
\hline 14 & KLA & talet & & & \\
\hline 15 & KAL & tehalet pa'an & 7,10 & $\mathrm{X}$ & P-3, M-3 \\
\hline 16 & KBD & tekalet & $3,7,10$ & $\mathrm{x}$ & $\mathrm{P}-4, \mathrm{M}-4$ \\
\hline 17 & KBK & tekalet & 7,10 & $\mathrm{~V}$ & $\mathrm{O}-2, \mathrm{P}-3, \mathrm{M}-4$ \\
\hline 18 & PEB & tekalet paya & & & \\
\hline
\end{tabular}

Notes : 


\section{Litbocarpus coopertus (Blanco) Rehd. (Fagaceae)}

\section{HIDDEN OAK. BARUSANG}

\section{DESCRIPTION}

Tree, to $40 \mathrm{~m}$, buttresses $2 \mathrm{~m}$ high; bark grey brown; inner bark c. $2 \mathrm{~cm}$ thick, deep red granular; leaves elliptic, $7-15 \times 3-5 \mathrm{~cm}$, grey brown thinly leathery; acorn cup $2.0 \mathrm{~cm}$ diameter, covered by reflexed spines almost enclosing nut, $2 \mathrm{~cm}$ tall

\section{HABITAT}

Lowland and hill primary forests to $1800 \mathrm{~m}$

\section{ORIGINS}

Malay Peninsula, Borneo, Philippines

\section{USES}

Timber for house construction; hunting location when fruiting

\section{REFERENCES}

Kessler and Sidiyasa 1994: 140; PROSEA Vol.

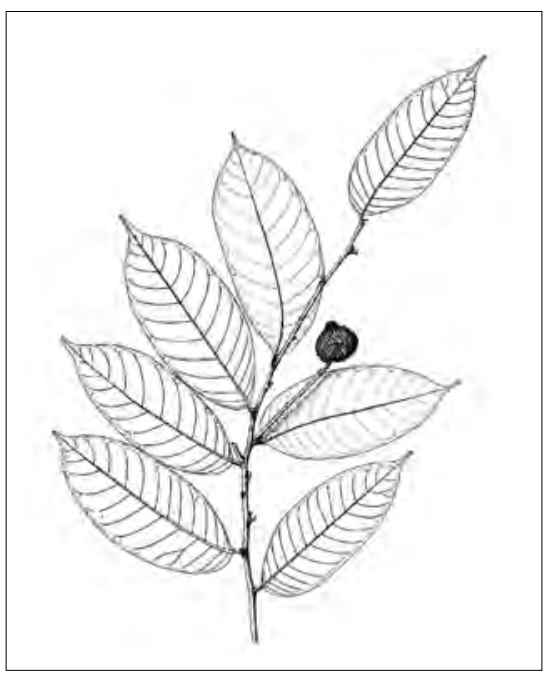

5(2): 291; TFM Vol. 1: 217; FM I Vol. 7(2): 335

\begin{tabular}{|c|c|c|c|c|c|}
\hline \# & Code & Local Name & Use & RU & Hab.-Ab. \\
\hline 1 & PU'T & & & & \\
\hline 2 & PUB & tekalet balun & 10 & $x$ & P-2, M-3 \\
\hline 3 & PUD & tekalet & 10 & $x$ & P-2, M-3 \\
\hline 4 & PUM & & & & \\
\hline 5 & ALS & & & & \\
\hline 6 & LDY & & & & \\
\hline 7 & LIT & okam & & & \\
\hline 8 & BRU & wi salet & & & \\
\hline 9 & MRP & mengang mabai & & & \\
\hline 10 & P'TN & so'et & & & \\
\hline 11 & KLK & bangan soet & & & \\
\hline 12 & KLM & & & & \\
\hline 13 & KLO & tegalet so'o & & & \\
\hline 14 & KLA & tahalet & & & \\
\hline 15 & KAL & se'dat & 10 & $x$ & P-2, M-3 \\
\hline 16 & KBD & sekkut & 4,10 & $\mathrm{v}$ & P-2, M-3 \\
\hline 17 & KBK & segut & & & \\
\hline 18 & PEB & segut & $3,4,10$ & $\mathrm{v}$ & P-2, M-3 \\
\hline
\end{tabular}

Notes : 


\section{Lithocarpus nieuwenbuisii (v. Seem.) A.Camus (Fagaceae)}

\section{CLEMENTI'S OAK. MEMPENING}

\section{DESCRIPTION}

Tree, to $25 \mathrm{~m}$, buttresses or stilt roots; bark scaly greyish brown; leaves elliptical, 14-20 x 4-6 cm; acorn cup 2- $2.5 \mathrm{~cm}$ wide, top shaped with distinct flat rings; half-covered nut, ovoid-cylindrical, pointed, tomentose

\section{HABITAT}

Lowland primary forest, peat-swamp forest, heath forest; riverine

\section{ORIGINS}

Malay Peninsula, Borneo, West Java

\section{USES}

Timber for house building, firewood; hunting location when fruiting

\section{REFERENCES}

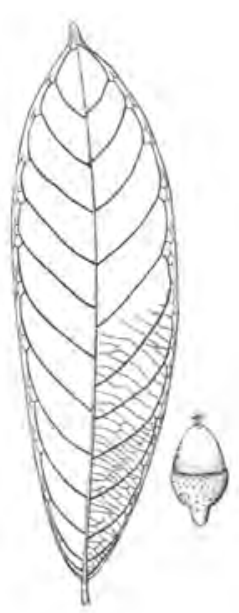

Kessler and Sidiyasa 1994: 140; PROSEA Vol. 5(2):

291; TFM Vol. 1: 217; FM Vol. 7(2): 389

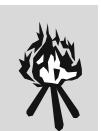

\begin{tabular}{|r|r|l|c|c|c|}
\hline$\#$ & Code & \multicolumn{1}{|c|}{ Local Name } & Use & RU & Hab.-Ab. \\
\hline 1 & PUT & tekalet baco, tekalet malun, jemit, piling & 7,10 & $\mathrm{X}$ & $\mathrm{P}-3, \mathrm{M}-3$ \\
\hline 2 & PUB & balet, tekalet bua & & & \\
\hline 3 & PUD & tekalet bowang & & & \\
\hline 4 & PUM & & & & \\
\hline 5 & ALS & guakat, tekolod langun & & & \\
\hline 6 & LDY & ronop, saled kubung, saled taket dat & & & \\
\hline 7 & LIT & sukem, pegawi, salid urak & & & \\
\hline 8 & BRU & wi salet, salet langong, salet ingial & & & \\
\hline 9 & MRP & kelan, kalan & & & \\
\hline 10 & PTN & palan & & & \\
\hline 11 & KLK & palan, palan suwa', palan soet, palan jekkau, palan gerau & & & \\
\hline 12 & KLM & tekalet, palan, palan soa', palan solau, palan teliwau & & & \\
\hline 13 & KLO & nyelaivai, tegalet asieng & & \\
\hline 14 & KLA & palan, tahalet, talet abu & 5,7 & $\mathrm{X}$ & \\
\hline 15 & KAL & tehalet & & & \\
\hline 16 & KBD & tekalet asing & 7,10 & $\mathrm{~V}$ & \\
\hline 17 & KBK & palan & $3,7,10$ & $\mathrm{X}$ & \\
\hline 18 & PEB & tekalet batu munin, tekalet tasing & 7,10 & $\mathrm{~V}$ & \\
\hline
\end{tabular}

\section{Notes :}




\section{Quercus argentata Korth. (Fagaceae) \\ SILVER OAK. MEMPENING}

\section{DESCRIPTION}

Tree, to $40 \mathrm{~m}$, buttresses $2 \mathrm{~m}$ high; bark smooth pale grey; leaves elliptical-oblong, 6-22 x 3-7 cm, midrib sunken above, silvery below; acorn cup to $3 \mathrm{~cm}$ diameter, 6-9 slightly toothed flanges; nut half covered, dull brown, $2 \times 2.5 \mathrm{~cm}$

\section{HABITAT}

Lowland and mountain forests to $2700 \mathrm{~m}$; wet areas

\section{ORIGINS}

Malay Peninsula, Borneo, West Java

\section{USES}

Timber for houses, boats, firewood; hunting location when fruiting

\section{REFERENCES}

Kessler and Sidiyasa 1994: 142; PROSEA Vol. 5(2): 413; TFM Vol. 1: 228; FM I Vol. 7(2): 389

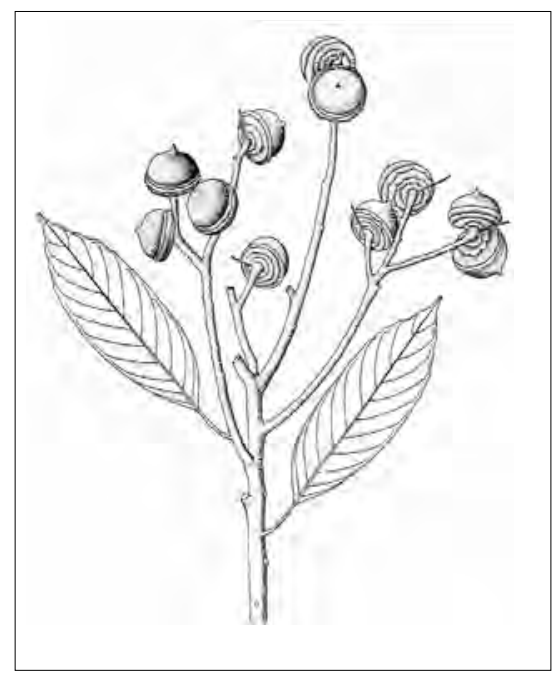

\begin{tabular}{|c|c|l|c|c|c|}
\hline$\#$ & Code & \multicolumn{1}{|c|}{ Local Name } & Use & RU & Hab.-Ab. \\
\hline 1 & PUT & tekalet & 7,10 & $\mathrm{X}$ & $\mathrm{P}-3, \mathrm{M}-3$ \\
\hline 2 & PUB & movang, tekalet paan & & & \\
\hline 3 & PUD & tekalet & & & \\
\hline 4 & PUM & & & \\
\hline 5 & ALS & tekolod kusop & & & \\
\hline 6 & LDY & saled & & & \\
\hline 7 & LIT & bua' salid & & & \\
\hline 8 & BRU & wi salet adia' & & & \\
\hline 9 & MRP & klan u, kalan & & & \\
\hline 10 & PTN & kayo nyeliwai & $3,5,10,11$ & $\mathrm{~V}$ & \\
\hline 11 & KLK & nyeliwae, nyeliwai bala & $3,5,10,11$ & $\mathrm{~V}$ & \\
\hline 12 & KLM & nyeliwai, nyelaiwai pa'an & $3,5,10,11$ & $\mathrm{~V}$ & \\
\hline 13 & KLO & tegalet & $3,5,6,7$ & $\mathrm{~V}$ & \\
\hline 14 & KLA & nyelivai & 3,3 & $\mathrm{P}-3, \mathrm{M}-3$ \\
\hline 15 & KAL & jelaevai & $3,5,10$ & $\mathrm{~V}$ & \\
\hline 16 & KBD & nyalaiwae & $3,4,4$ \\
\hline 17 & KBK & manyiliwai balah, nyiliwai pute & $3,5,10,11$ & $\mathrm{~V}$ & $\mathrm{P}-3, \mathrm{M}-2$ \\
\hline 18 & PEB & jelevai & 7,10 & $\mathrm{~V}$ & $\mathrm{P}-4, \mathrm{M}-4$ \\
\hline
\end{tabular}

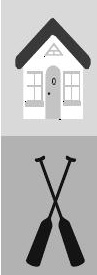

Notes : 


\section{Quercus gemelliflora Bl. (Fagaceae)}

\section{TWIN-FLOWERED OAK. MEMPENING, PASANG JAMBE}

\section{DESCRIPTION}

Tree, to $30 \mathrm{~m}$, buttresses $2 \mathrm{~m}$ high; bark smooth greyish brown; leaves elliptical, $5-15 \times 2-5.5 \mathrm{~cm}$, midrib raised above, margin toothed in upper half; cup 1.5-2.5 cm diameter, 6-7 flanges; nut, 2-5.5 x 1$2 \mathrm{~cm}$, cup enclosing whole nut when immature

\section{HABITAT}

Lowland and mountain forests to $2150 \mathrm{~m}$; along streams

\section{ORIGINS}

Malaysia and Western Indonesia

\section{USES}

Timber for house and boat construction, firewood; hunting location when fruiting

\section{REFERENCES}

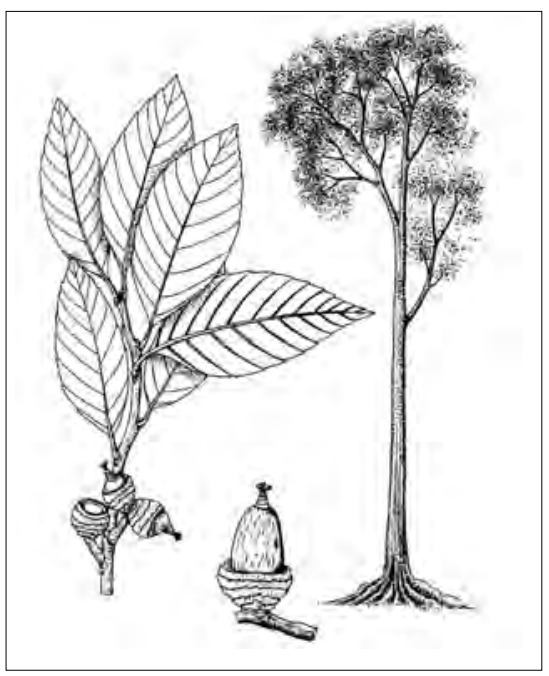

PROSEA Vol. 5(2): 414; TFM Vol. 1: 229

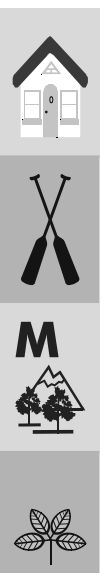

\begin{tabular}{|c|c|l|c|c|c|}
\hline$\#$ & Code & \multicolumn{1}{|c|}{ Local Name } & Use & RU & Hab.-Ab. \\
\hline 1 & PUT & tekalet, tekalet bang, ngong & 7,10 & X & P-3, M-3 \\
\hline 2 & PUB & tekalet balun & & & \\
\hline 3 & PUD & tekalet kuyut, malun & & & \\
\hline 4 & PUM & & & & \\
\hline 5 & ALS & tekolod fungoh & & & \\
\hline 6 & LDY & saled silo, dapu & & & \\
\hline 7 & LIT & bua' salid & & & \\
\hline 8 & BRU & wi salet adia', lemanau & & & \\
\hline 9 & MRP & klan nya', kalan & & & \\
\hline 10 & PTN & kayo nyeliwai & $3,5,10,11$ & $\mathrm{~V}$ & \\
\hline 11 & KLK & palan belesae, nyeliwai bileng & $3,5,10,11$ & $\mathrm{~V}$ & \\
\hline 12 & KLM & nyeliwai, nyelaiwai & $3,5,10,11$ & $\mathrm{~V}$ & \\
\hline 13 & KLO & nyelaivai bileng & $3,5,6,7$ & $\mathrm{X}$ & \\
\hline 14 & KLA & nyelivai & 3,4 & $\mathrm{P}-4,4$ \\
\hline 15 & KAL & jelaevai & $3,4,7,10$ & $\mathrm{~V}$ & $\mathrm{P}-4, \mathrm{M}-4$ \\
\hline 16 & KBD & nyalaiwae & $3,5,10,11$ & $\mathrm{P}-4, \mathrm{M}-4$ \\
\hline 17 & KBK & manyiliwae pute, nyiliwai bala & 7,10 & $\mathrm{~V}$ & \\
\hline 18 & PEB & tekalet siyek & & $\mathrm{P}-4, \mathrm{M}-4$ \\
\hline
\end{tabular}

Notes : 


\section{PAYANG. PANGI, KEPAYANG}

\section{DESCRIPTION}

Tree, $20-40 \mathrm{~m}$, buttresses, bark brownish grey, smooth; leaves ovate, $20-60 \times 15-50 \mathrm{~cm}$, shiny dark green above, greyish green below, clustered at branch ends; fruit ovoid, brown, $10-25 \mathrm{~cm}$ diameter, dense rusty hairs, triangular grey white seeds, $5 \mathrm{~cm}$ wide

\section{HABITAT}

Lowland primary and secondary forest to $1000 \mathrm{~m}$; dryland, cultivated in village fruit gardens

\section{ORIGINS}

Island Southeast Asia, Oceania

\section{USES}

Poisonous tree; seeds eaten as relish; leaves for preserving meat; fish poison

\section{REFERENCES}

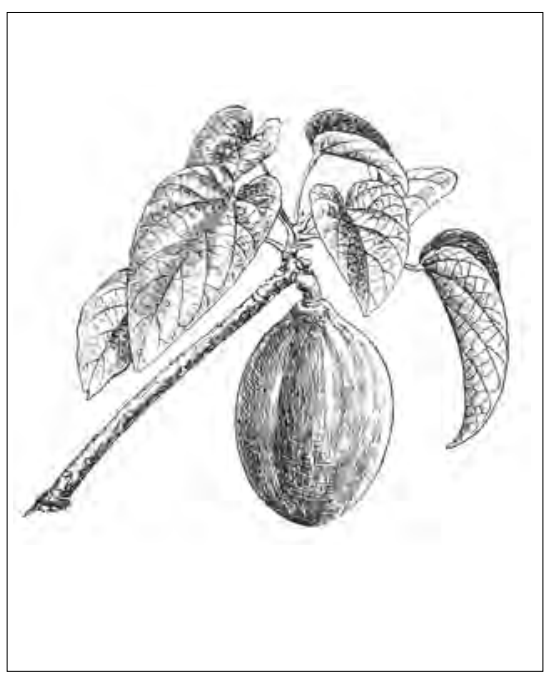

Burkill 1966: 1680; Corner 1988: 347;

Ochse 1980: 293; TFM Vol. 2: 155

\begin{tabular}{|c|c|c|c|c|c|}
\hline \# & Code & Local Name & Use & RU & Hab.-Ab. \\
\hline 1 & PUT & piang & 1,10 & $\mathrm{v}$ & G-1, O-2, P-3 \\
\hline 2 & PUB & piang, piang kayu & 1,10 & $\mathrm{v}$ & G-2, O-2, P-3 \\
\hline 3 & PUD & piang, lekeran & $1,6,10$ & $\mathrm{v}$ & $\mathrm{G}-2, \mathrm{O}-2, \mathrm{P}-3$ \\
\hline 4 & PUM & piang & 1,10 & $\mathrm{v}$ & G-1, P-3 \\
\hline 5 & ALS & bua' kuwom & 1,10 & $\mathrm{v}$ & G-2, P-3 \\
\hline 6 & LDY & bua payang, payang & $1,2,10$ & $\mathrm{v}$ & G-1, G-3, O-2, P-2, P-3 \\
\hline 7 & LIT & payang & 1,10 & $\mathrm{v}$ & $\mathrm{O}-2, \mathrm{P}-3$ \\
\hline 8 & BRU & wi payang & 1,10 & $\mathrm{v}$ & G-2, P-3 \\
\hline 9 & MRP & paye bela, pai bela, pai mla & 1,10 & $\mathrm{v}$ & G-2, P-3 \\
\hline 10 & P'TN & payang kayo & 1,10 & $\mathrm{v}$ & P-3, G-1 \\
\hline 11 & KLK & payang kayu & 1 & & G-2 \\
\hline 12 & KLM & payang kayu & 1 & $\mathrm{v}$ & G-2, P-3 \\
\hline 13 & KLO & fazang, fazeng kaze & 1 & v & G-3 \\
\hline 14 & KLA & pazang kazu, tong fazang & 1,10 & $x$ & G-3, P-3 \\
\hline 15 & KAL & pazang kazu & & & \\
\hline 16 & KBD & payang kayu & 1,10 & $\mathrm{v}$ & G-2, P-3 \\
\hline 17 & KBK & payang kayu & & & \\
\hline 18 & PEB & payang, payang kayo & 6 & $x$ & P-3, M-4 \\
\hline
\end{tabular}

Notes : 


\section{Dicranopteris linearis (Burm.) Underw. (Gleicheniaceae)}

\section{RATTAN FERN. PAKIS ANDAM, RESAM}

\section{DESCRIPTION}

Fern, 1-4 m long, crawling, dense; branches forking, shiny brown, hard and hollow; leaves pinnate, branching at right angles, leaflets linear, blunt, light green

\section{HABITAT}

Thick beds on landslips, forest gaps, infertile soils, old swiddens

\section{ORIGINS}

Tropics

\section{USES}

Tough stems for matting, ropes, or writing pens; leaves for medicine; hunting location

\section{REFERENCES}

Burkill 1966: 1089; Heyne Vol. 1: 83
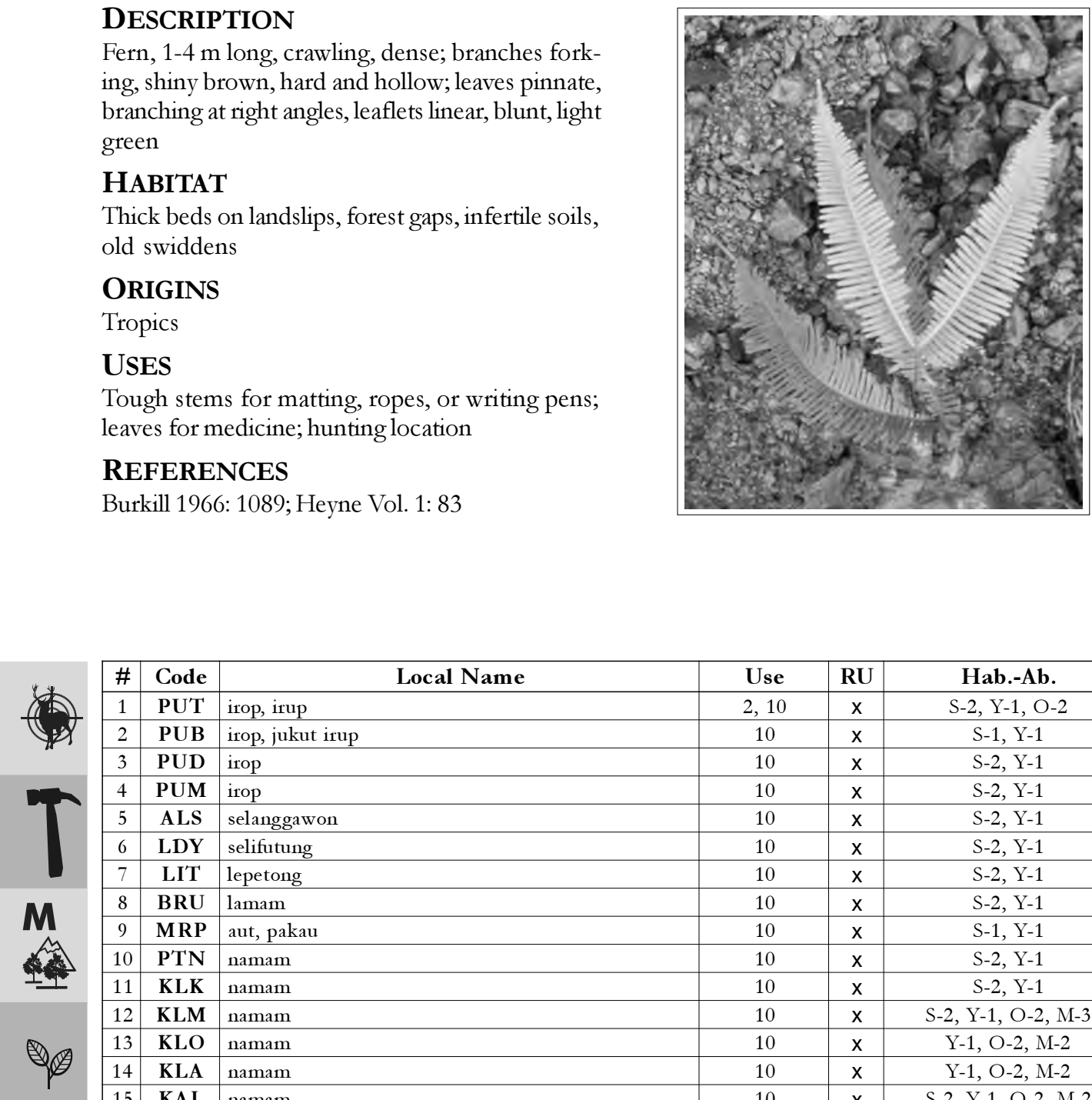

\begin{tabular}{|c|c|c|c|c|c|}
\hline$\#$ & Code & Local Name & Use & RU & Hab.-Ab. \\
\hline 1 & PUT & irop, irup & 2,10 & $\mathrm{x}$ & $\mathrm{S}-2, \mathrm{Y}-1, \mathrm{O}-2$ \\
\hline 2 & PUB & irop, jukut irup & 10 & $x$ & S-1, Y-1 \\
\hline 3 & PUD & irop & 10 & $x$ & S-2, Y-1 \\
\hline 4 & PUM & irop & 10 & $\mathrm{x}$ & S-2, Y-1 \\
\hline 5 & ALS & selanggawon & 10 & $x$ & S-2, Y-1 \\
\hline 6 & LDY & selifutung & 10 & $x$ & S-2, Y-1 \\
\hline 7 & LIT & lepetong & 10 & $x$ & S-2, Y-1 \\
\hline 8 & BRU & lamam & 10 & $\mathrm{x}$ & S-2, Y-1 \\
\hline 9 & MRP & aut, pakau & 10 & $x$ & S-1, Y-1 \\
\hline 10 & P'TN & namam & 10 & $x$ & S-2, Y-1 \\
\hline 11 & KLK & namam & 10 & $x$ & S-2, Y-1 \\
\hline 12 & KLM & namam & 10 & $x$ & $\mathrm{~S}-2, \mathrm{Y}-1, \mathrm{O}-2, \mathrm{M}-3$ \\
\hline 13 & KLO & namam & 10 & $x$ & $\mathrm{Y}-1, \mathrm{O}-2, \mathrm{M}-2$ \\
\hline 14 & KLA & namam & 10 & $x$ & $\mathrm{Y}-1, \mathrm{O}-2, \mathrm{M}-2$ \\
\hline 15 & KAL & namam & 10 & $x$ & $\mathrm{~S}-2, \mathrm{Y}-1, \mathrm{O}-2, \mathrm{M}-2$ \\
\hline 16 & KBD & namam & 10 & $x$ & $\mathrm{~S}-2, \mathrm{Y}-1, \mathrm{O}-2, \mathrm{M}-3$ \\
\hline 17 & KBK & namam & 10 & $x$ & $\mathrm{Y}-1, \mathrm{O}-2, \mathrm{M}-2$ \\
\hline 18 & PEB & namam & 10 & v & $\mathrm{S}-3, \mathrm{O}-3, \mathrm{M}-3$ \\
\hline
\end{tabular}

Notes : 
Cymbopogon nardus (L.) Randle (Gramineae)

\section{Citronella GRASS. SEREH WANGI}

\section{DESCRIPTION}

Grass, perennial, bunching; leaves drooping in all directions, linear, acute, fragrant, 50-100 x 0.5-1.5 $\mathrm{cm}$, leaf sheaths tinged with purple

\section{HABITAT}

Cultivated in homegardens

\section{ORIGINS}

India, arrived in mid 1800s

\section{USES}

Heart of leaf shoots eaten as spice; sold locally; oil extracted for medicine and perfume

\section{REFERENCES}

Burkill 1966: 737; Ochse 1980: 299

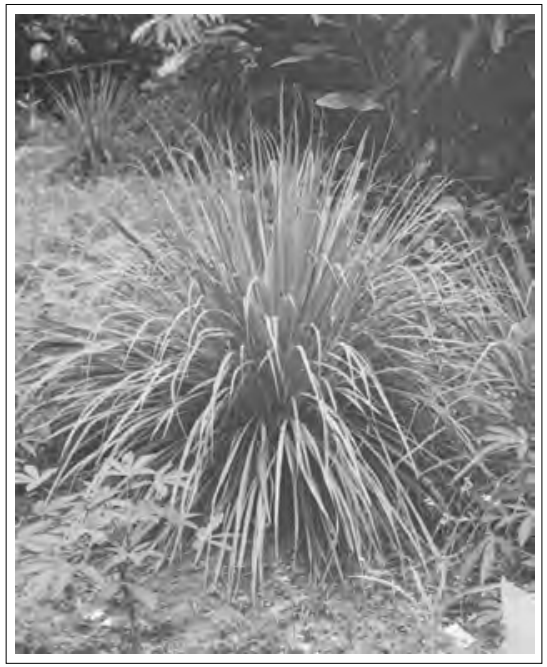

\begin{tabular}{|c|c|c|c|c|c|}
\hline \# & Code & Local Name & Use & RU & Hab.-Ab. \\
\hline 1 & PUT & bawing tufuh & 1,2 & $\mathrm{~V}$ & G-3 \\
\hline 2 & PUB & bawing & 1,2 & $\mathrm{v}$ & G-2, G-3 \\
\hline 3 & PUD & bawing & 1,2 & $\mathrm{~V}$ & G-2 \\
\hline 4 & PUM & bawing tufu & $1,2,10$ & $\mathrm{v}$ & G-3 \\
\hline 5 & ALS & segumau & $1,2,11$ & v & G-3 \\
\hline 6 & LDY & segumau, gesumau & $1,2,11$ & v & G-3, G-4, S-1 \\
\hline 7 & LI'T & segumau & 1,2 & $\mathrm{v}$ & G-3, G-4 \\
\hline 8 & BRU & bawang pade & $1,2,11$ & v & G-4, S-1 \\
\hline 9 & MRP & mawaie, mawai ngai, mawai tupui & $1,2,11$ & $\mathrm{v}$ & G-2, G-3, G-4 \\
\hline 10 & P'TN & ping bawing, uro' bawing & 1,2 & v & G-2, G-3, S-1 \\
\hline 11 & KLK & ping bawing & 1 & v & G-3, S-1 \\
\hline 12 & KLM & ping baweng, ping bawing & $1,2,11$ & $\mathrm{~V}$ & G-2, G-3 \\
\hline 13 & KLO & peng bavieng, peng baveng, ping baving, pieng bavieng & $1,2,3$ & $\mathrm{~V}$ & G-3 \\
\hline 14 & KLA & baving, pieng bavieng, ping baving, serai, ping baveng & 1,2 & $\mathrm{~V}$ & G-3 \\
\hline 15 & KAL & bavieng balang la'a & & & \\
\hline 16 & KBD & ping ba'o & 1,2 & $\mathrm{~V}$ & G-3 \\
\hline 17 & KBK & ping bawing & & & \\
\hline 18 & PEB & ping bawing, ai podeng & 1 & $\mathrm{v}$ & G-4, S-3 \\
\hline
\end{tabular}

Notes : 


\section{Imperata cylindrica (L.) Beauv. (Gramineae) COGON GRASS. LALANG,ALANG-ALANG}

\section{DESCRIPTION}

Grass, perennial, aggressively rhizomatous, white branched rhizome, more than $1 \mathrm{~m}$; leaves lanceolate, drooping, sharp edged, 10-180 x 0.5-2.5 $\mathrm{cm}$

\section{HABITAT}

Wild, in dense fields in infertile fallows, waste spaces, fire adapted

\section{ORIGINS}

Tropics

\section{USES}

Runners for medicine, beer; leaves for thatch; fields burned to attract prey, hunting ground REFERENCES

Burkill 1966: 1249; PROSEA Vol. 4: 140
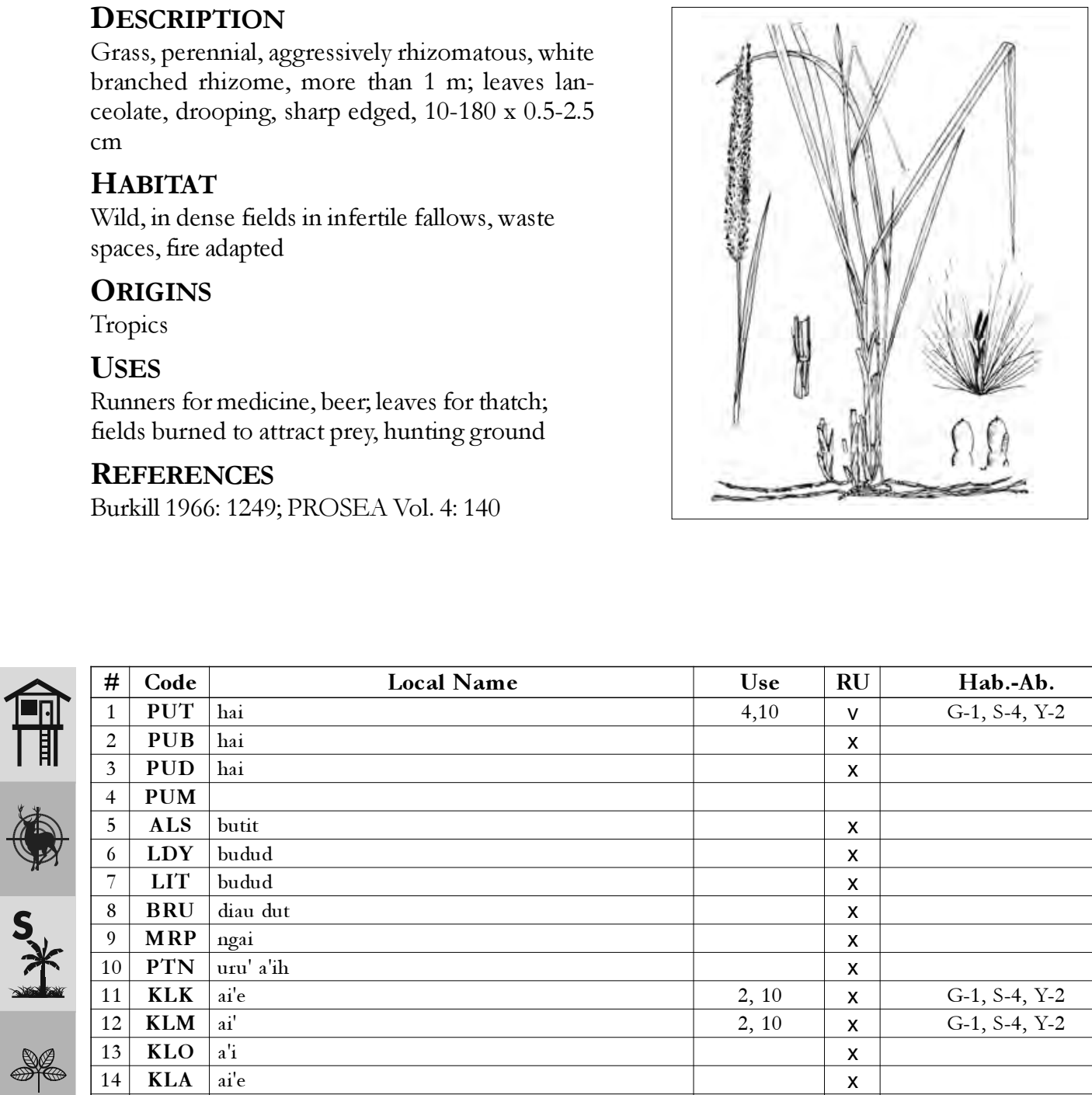

\begin{tabular}{|c|c|c|c|c|c|}
\hline$\#$ & Code & Local Name & Use & RU & Hab.-Ab. \\
\hline 1 & PUT & hai & 4,10 & $\mathrm{v}$ & G-1, S-4, Y-2 \\
\hline 2 & PUB & hai & & $x$ & \\
\hline 3 & PUD & hai & & $x$ & \\
\hline 4 & PUM & & & & \\
\hline 5 & ALS & butit & & $x$ & \\
\hline 6 & LDY & budud & & $x$ & \\
\hline 7 & LIT & budud & & $x$ & \\
\hline 8 & BRU & diau dut & & $x$ & \\
\hline 9 & MRP & ngai & & $x$ & \\
\hline 10 & P'TN & uru' a'ih & & $x$ & \\
\hline 11 & KLK & ai'e & 2,10 & $x$ & G-1, S-4, Y-2 \\
\hline 12 & KLM & ai' & 2,10 & $x$ & G-1, S-4, Y-2 \\
\hline 13 & KLO & $a^{\prime} i$ & & $x$ & \\
\hline 14 & KLA & ai'e & & $x$ & \\
\hline 15 & KAL & ai'e & & $x$ & \\
\hline 16 & KBD & ai & 4,10 & $\mathrm{v}$ & G-1, S-4, Y-2 \\
\hline 17 & KBK & & & & \\
\hline 18 & PEB & a'ut & 4,10 & $\mathrm{v}$ & G-1, S-4, Y-2 \\
\hline
\end{tabular}

Notes : 


\section{Oryza sativa L. (Gramineae) \\ RICE. PADI}

\section{DESCRIPTION}

Grass, annual, 300-400 varieties in Malaysia and Indonesia

\section{HABITAT}

Cultivated in irrigated and rainfed fields

\section{ORIGINS}

Indochina

\section{USES}

Primary starch staple in Asia; medicine; alchohol; fodder; barter currency and sold locally; used in traditional agricultural and religious rituals, modern Christian rituals and feasts.

\section{REFERENCES}

Burkill 1966: 1620; PROSEA Vol. 10: 106

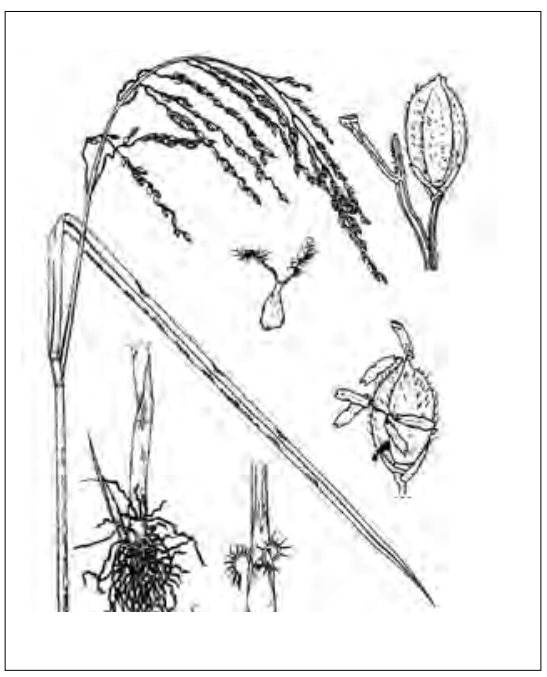

\begin{tabular}{|c|c|c|c|c|c|}
\hline \# & Code & Local Name & Use & RU & Hab.-Ab. \\
\hline 1 & $\begin{array}{l}\text { PUT } \\
\end{array}$ & parei & $1,2,11$ & $\mathrm{~V}$ & S-5 \\
\hline 2 & PUB & parei & $1,2,11$ & v & S-5 \\
\hline 3 & PUD & parae & $1,2,11$ & $\mathrm{v}$ & S-5 \\
\hline 4 & PUM & parei & $1,2,11$ & v & S-5 \\
\hline 5 & ALS & bilod & $1,2,11$ & $\mathrm{v}$ & S-5 \\
\hline 6 & LDY & fadei & $1,2,11$ & $\mathrm{v}$ & S-5 \\
\hline 7 & LIT & padai & $1,2,11$ & v & S-5 \\
\hline 8 & BRU & padei & $1,2,11$ & $\mathrm{v}$ & S-5 \\
\hline 9 & M RP & parai & $1,2,11$ & v & S-5 \\
\hline 10 & PTN & parei & $1,2,11$ & $\mathrm{v}$ & S-5 \\
\hline 11 & KLK & padei & $1,2,11$ & $\mathrm{v}$ & S-5 \\
\hline 12 & KLM & padai & $1,2,11$ & $\mathrm{v}$ & S-5 \\
\hline 13 & KLO & fadei & $1,2,11$ & $\mathrm{v}$ & S-5 \\
\hline 14 & KLA & padai & $1,2,11$ & $\mathrm{v}$ & S-5 \\
\hline 15 & KAL & padi & $1,2,11$ & $\mathrm{v}$ & S-5 \\
\hline 16 & KBD & padei & $1,2,11$ & $\mathrm{v}$ & S-5 \\
\hline 17 & KBK & parei & $1,2,11$ & $\mathrm{v}$ & S-5 \\
\hline 18 & PEB & fadai & 1,2 & $\mathrm{v}$ & S-5 \\
\hline
\end{tabular}

Notes : 
 \\ Saccharum officinarum L. (Gramineae) \\ SugarCANE.TEBu}

\section{DESCRIPTION}

Grass, perennial, 1-6 m, adventitious root system; stem robust, $2-5 \mathrm{~cm}$ diameter divided into $10-40$ swollen internodes; leaves from nodes, alternate in two rows on either side of the stem, linear 70-200 x 3-7 cm; flower on terminal panicle $25-50 \mathrm{~cm}$ long

\section{HABITAT}

Cultivated in home and swidden gardens, plantations; sandy soils

\section{ORIGINS}

New Guinea

\section{USES}

Cane eaten raw; sap for alchohol, medicine; leaves for thatch

\section{REFERENCES}

Burkill 1966: 1959; PROSEA Vol. 9: 143

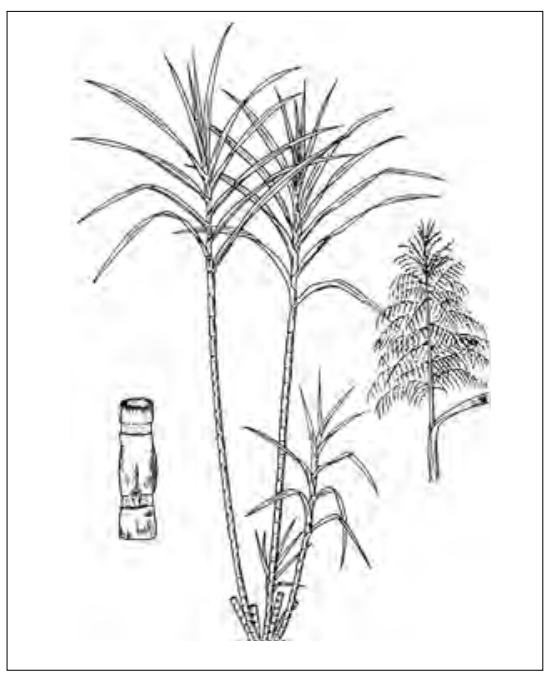

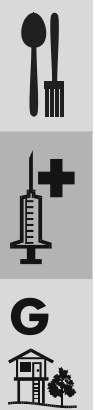

\begin{tabular}{|c|c|c|c|c|c|}
\hline$\#$ & Code & Local Name & Use & RU & Hab.-Ab. \\
\hline 1 & PUT & tovuh, tovu' & $1,2,4$ & $\mathrm{v}$ & G-3, S-2 \\
\hline 2 & PUB & tovuh & 1,2 & $\mathrm{v}$ & G-3, S-2 \\
\hline 3 & PUD & tovu' & 1,2 & $\mathrm{v}$ & G-3, S-2 \\
\hline 4 & PUM & tovuh & 1,2 & $\mathrm{v}$ & G-3, S-2 \\
\hline 5 & ALS & tabu & 1 & $\mathrm{v}$ & G-3, S-2 \\
\hline 6 & LDY & tapuh & 1 & $\mathrm{v}$ & G-3, S-2 \\
\hline 7 & LIT & tefu & 1 & $\mathrm{v}$ & G-3, S-2 \\
\hline 8 & BRU & pi'au & 1 & $\mathrm{v}$ & G-3, S-2 \\
\hline 9 & MRP & te' bau, tebau & 1 & $\mathrm{v}$ & G-3, S-2 \\
\hline 10 & P'TN & tebo' & 1 & $\mathrm{v}$ & G-3, S-2 \\
\hline 11 & KLK & teppu & 1,2 & v & G-3, S-2 \\
\hline 12 & KLM & teppu & 1,2 & $\mathrm{v}$ & G-3, S-2 \\
\hline 13 & KLO & tefe' & 1,2 & $\mathrm{v}$ & G-3, S-2 \\
\hline 14 & KLA & teppu & 1,2 & $\mathrm{v}$ & G-3, S-2 \\
\hline 15 & KAL & teppu & 1,2 & $\mathrm{v}$ & G-3, S-2 \\
\hline 16 & KBD & teppu & 1,2 & $\mathrm{v}$ & G-3, S-2 \\
\hline 17 & KBK & teppu & 1,2 & $\mathrm{v}$ & G-3, S-2 \\
\hline 18 & PEB & tebu & 1,2 & $\mathrm{v}$ & G-1, S-1 \\
\hline
\end{tabular}

Notes : 
Setaria palmifolia (Willd.) Stapf. (Gramineae)

PALM LEAF SETARIA. LACHANG

\section{DESCRIPTION}

Grass, perennial, tussocky, $0.75-3 \mathrm{~m}$; culms erect or tillering; leaves broadly lanceolate, apex acute, 15$70 \times 1-10 \mathrm{~cm}$, edges scaborous, longitudinally plaited; flower on terminal panicle, $15-60 \mathrm{~cm}$ long

\section{HABITAT}

Lowland and hill primary forests to $1000 \mathrm{~m}$; planted in villages

\section{ORIGINS}

Asia

\section{USES}

Shoots eaten raw, cooked, sold locally; leaves for medicine, natural pesticide

\section{REFERENCES}

Burkill 1966: 2035; Ochse 1980: 335

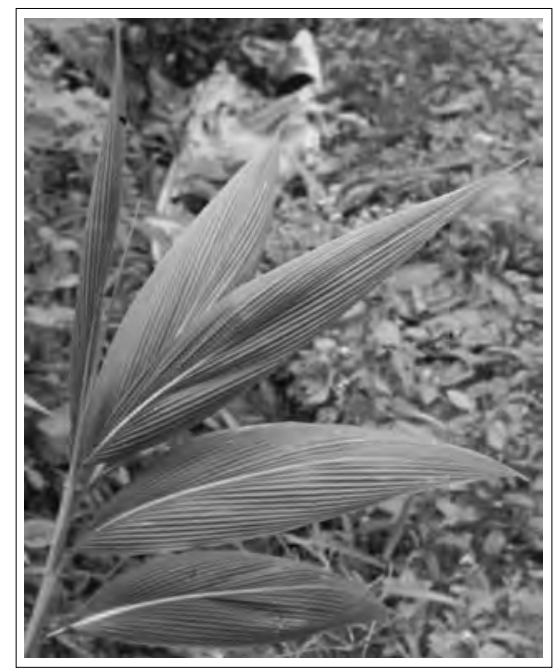

\begin{tabular}{|c|c|c|c|c|c|}
\hline$\#$ & Code & Local Name & Use & RU & Hab.-Ab. \\
\hline 1 & PUT & ingga & 1 & $\mathrm{x}$ & $\mathrm{Y}-2, \mathrm{Y}-3$ \\
\hline 2 & PUB & ingga & 1 & $x$ & $\mathrm{Y}-2, \mathrm{Y}-3, \mathrm{O}-2$ \\
\hline 3 & PUD & ingga & 1 & $x$ & $\mathrm{~S}-2, \mathrm{Y}-3, \mathrm{O}-2$ \\
\hline 4 & PUM & ingga & 1 & $x$ & \\
\hline 5 & ALS & abang & 1 & $x$ & Y-3 \\
\hline 6 & LDY & abang & 1 & $x$ & $\mathrm{Y}-2$ \\
\hline 7 & LIT & abang bulu & 1 & $x$ & $\mathrm{R}$ \\
\hline 8 & BRU & abi'ang & 1 & $x$ & ALL \\
\hline 9 & MRP & mawai tepui, teh kei, ngkieh & 1 & $\mathrm{v}$ & G-3, Y-3, O-3 \\
\hline 10 & P'TN & ingga & 1 & $x$ & S-2, Y-3 \\
\hline 11 & KLK & sengka, ujau senggah, ubut senggah & 1 & $\mathrm{x}$ & $\mathrm{Y}-2$ \\
\hline 12 & KLM & sengka, sengga & 1 & $\mathrm{v}$ & G-2, Y-3 \\
\hline 13 & KLO & sengko, sengku & 1 & $\mathrm{v}$ & $\mathrm{Y}-2, \mathrm{O}-2$ \\
\hline 14 & KLA & sengka & 1 & $\mathrm{v}$ & G-2, S-2 \\
\hline 15 & KAL & sengko & & & \\
\hline 16 & KBD & sengka, sengga & 1,2 & $\mathrm{v}$ & G-2 \\
\hline 17 & KBK & sengga' & & & \\
\hline 18 & PEB & ujau sega, uru ping & 1 & $\mathrm{v}$ & G-3, S-3, Y-4, O-2 \\
\hline
\end{tabular}

Notes : 


\section{Garcinia forbesii King (Guttiferae)}

\section{ROSE KANDIS. KANDIS ROSA, MANGGIS HUTAN}

\section{DESCRIPTION}

Tree, to $18 \mathrm{~m}$; yellow exudate; leaves elliptic with many veins, $7.5-17.5 \times 3-8 \mathrm{~cm}$; flowers $1-2 \mathrm{~cm}$ wide with rose red or pinkish yellow petals; fruits applelike, but flattened, $2.5-3 \mathrm{~cm}$ diameter, rose red to purple, with watery flesh

\section{HABITAT}

Lowland and hill primary forests to $1700 \mathrm{~m}$

\section{ORIGINS}

Malaysia and western Indonesia

\section{USES}

Fruit eaten, timber for firewood

\section{REFERENCES}

Burkill 1966: 1066; Corner 1988: 355;

TFM Vol. 2: 211

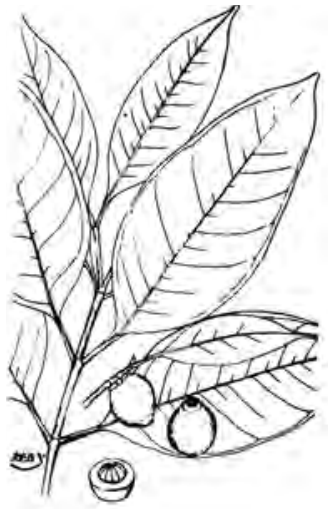

\begin{tabular}{|c|c|c|c|c|c|}
\hline \# & Code & Local Name & Use & RU & Hab.-Ab. \\
\hline 1 & PUT & riu, mowan & $1,6,7,10$ & $x$ & $\mathrm{O}-3, \mathrm{P}-3$ \\
\hline 2 & PUB & riu, belung & & $x$ & \\
\hline 3 & PUD & bunoh & & $x$ & \\
\hline 4 & PUM & & & & \\
\hline 5 & ALS & bua' tate & & $x$ & \\
\hline 6 & LDY & itan & & $x$ & \\
\hline 7 & LIT & bua' renako' & & $x$ & \\
\hline 8 & BRU & wi akau & & $\mathrm{x}$ & \\
\hline 9 & MRP & bua' tetoh telato & & $\mathrm{x}$ & \\
\hline 10 & P'TN & ariyu' & & $x$ & \\
\hline 11 & KLK & adiu, bua' adiu & 1 & $x$ & Y-3, O-2 \\
\hline 12 & KLM & adiu, adiu ma' & 1 & $\mathrm{x}$ & $\mathrm{Y}-3, \mathrm{O}-2$ \\
\hline 13 & KLO & ati kitung & 1 & $x$ & $\mathrm{Y}-3, \mathrm{O}-2$ \\
\hline 14 & KLA & kitung belavao & 1 & $\mathrm{x}$ & $\mathrm{Y}-4, \mathrm{O}-4$ \\
\hline 15 & KAL & adiu & 1 & $\mathrm{x}$ & $\mathrm{Y}-3, \mathrm{O}-2$ \\
\hline 16 & KBD & adiu & 1 & $x$ & $\mathrm{Y}-3, \mathrm{O}-2$ \\
\hline 17 & KBK & adiu & 1,7 & $\mathrm{x}$ & $\mathrm{Y}-3, \mathrm{O}-3, \mathrm{P}-3, \mathrm{M}-1$ \\
\hline 18 & PEB & adiu $\sin$ & 1,7 & $\mathrm{v}$ & $\mathrm{O}-3, \mathrm{P}-2$ \\
\hline
\end{tabular}

\section{Notes :}




\section{Garcinia lateriflora B1. (Guttiferae)}

\section{(NO ENGLISH NAME). MANGGIS HUTAN}

\section{DESCRIPTION}

Tree, to $15 \mathrm{~m}$, yellow exudate; leaves, shining dark green, elliptic to lanceolate, $14-24$ x $5-10.5 \mathrm{~cm}$, thin sec. nerves 14 or less, petiole $1-2 \mathrm{~cm}$; flowers fleshy light yellow; fruit rounded $2-3.5 \mathrm{~cm}$ diameter, seeds $2-3$

\section{HABITAT}

Lowland and hill primary forest to $2000 \mathrm{~m}$; forest margins

\section{ORIGINS}

Malaysia and western Indonesia

\section{USES}

Leaves used as poison; fruit eaten; timber for firewood

\section{REFERENCES}

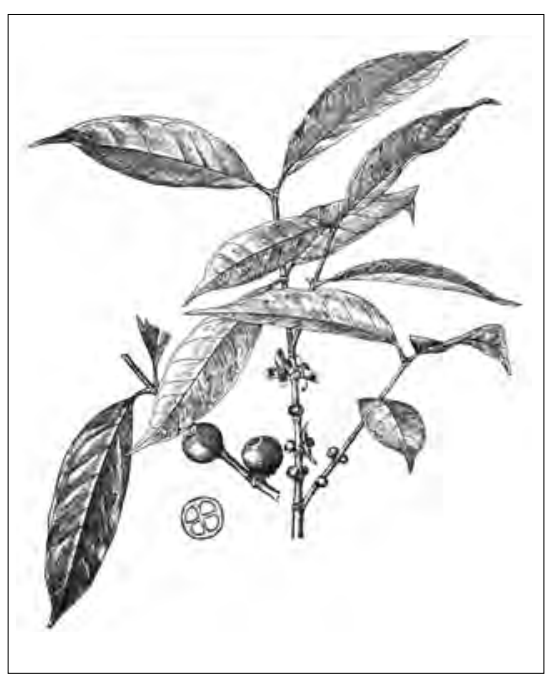

FOJ Vol. I: 387; Ochse 1927: 92

\begin{tabular}{|c|c|c|c|c|c|}
\hline$\#$ & Code & Local Name & Use & RU & Hab.-Ab. \\
\hline 1 & PUT & kendih, riu & 1,7 & $x$ & $\mathrm{O}-3, \mathrm{P}-2, \mathrm{M}-2$ \\
\hline 2 & PUB & munah, riu & & $x$ & \\
\hline 3 & PUD & kitung & & $x$ & \\
\hline 4 & PUM & & & & \\
\hline 5 & ALS & bua' beriaku & & $x$ & \\
\hline 6 & LDY & & & & \\
\hline 7 & LIT & bua' renako' & & $x$ & \\
\hline 8 & BRU & wi sei & & $x$ & \\
\hline 9 & MRP & bua rai & & $x$ & \\
\hline 10 & P'TN & ariyu' & & $x$ & \\
\hline 11 & KLK & pitong batu, bua' eti' & 1,7 & v & $\mathrm{G}-1, \mathrm{Y}-2, \mathrm{O}-3$ \\
\hline 12 & KLM & adiu, adiu batu & 1,7 & $\mathrm{v}$ & $\mathrm{G}-1, \mathrm{Y}-2, \mathrm{O}-3$ \\
\hline 13 & KLO & ati beranuk & 1,7 & $\mathrm{v}$ & $\mathrm{G}-1, \mathrm{Y}-2, \mathrm{O}-3$ \\
\hline 14 & KLA & adiu & 1,7 & v & $\mathrm{G}-1, \mathrm{Y}-4, \mathrm{O}-4$ \\
\hline 15 & KAL & adiu & 1,7 & $\mathrm{v}$ & G-1, Y-3, O-4 \\
\hline 16 & KBD & adiu & 1,7 & $\mathrm{v}$ & G-1, Y-3, O-4 \\
\hline 17 & KBK & adiu & 1,7 & v & $\mathrm{Y}-1, \mathrm{O}-4, \mathrm{P}-2, \mathrm{M}-1$ \\
\hline 18 & PEB & adiu & 1,7 & v & $\mathrm{Y}-2, \mathrm{O}-4, \mathrm{P}-2$ \\
\hline
\end{tabular}

Notes : 


\section{Garcinia mangostana L. (Guttiferae)}

\section{MANGOSTEEN.MANGGIS}

\section{DESCRIPTION}

Tree, to $5 \mathrm{~m}$, dense glossy green crown, yellow exudate; leaves ovate $6.5-15.5 \times 3.5-7 \mathrm{~cm}$, fine secondary nerves parallel, $2 \mathrm{~mm}$ apart; flowers 5 $\mathrm{cm}$ wide, petals yellow flushed pink; fruit purple, to $5 \mathrm{~cm}$ diameter, 4-7 wedge-shaped, pulpy, white lobes

\section{HABITAT}

Cultivated in villages

\section{ORIGINS}

Indochina

\section{USES}

Fruit eaten, sold locally; rind for medicine; wood for tools, firewood; hunting location when fruiting

\section{REFERENCES}

Burkill 1966: 1069; Corner 1988: 357;

PROSEA Vol. 2: 177; TFM Vol. 2: 215

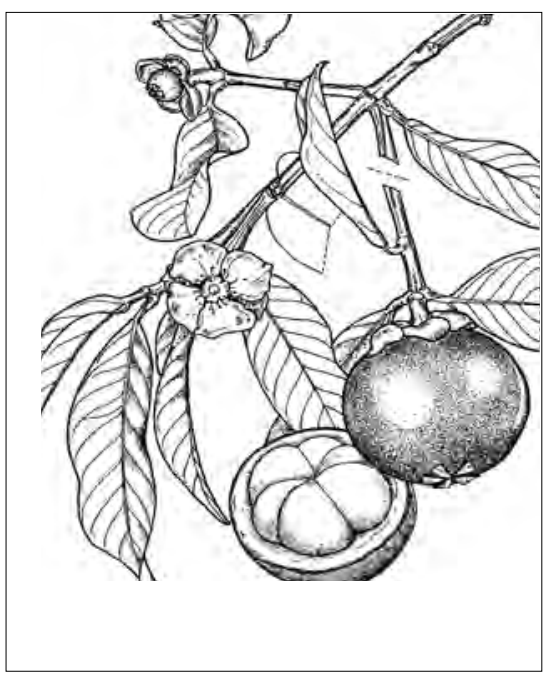

\section{in}

\begin{tabular}{|c|c|c|c|c|c|}
\hline \# & Code & Local Name & Use & RU & Hab.-Ab. \\
\hline 1 & $\begin{array}{l}\text { PUT } \\
\end{array}$ & buno, bunoh & 1,10 & $\mathrm{x}$ & G-2, P-3, M-1 \\
\hline 2 & PUB & bunoh & 1 & $\mathrm{x}$ & G-2, P-3, M-1 \\
\hline 3 & PUD & malung & 1 & $\mathrm{x}$ & G-2, P-3, M-1 \\
\hline 4 & PUM & & & & \\
\hline 5 & ALS & fadafalu & & $x$ & \\
\hline 6 & LDY & kitong & & $\mathrm{x}$ & \\
\hline 7 & LIT & kitung & & $\mathrm{x}$ & \\
\hline 8 & BRU & teung & & $\mathrm{x}$ & \\
\hline 9 & MRP & bua tiu', tiue & & $\mathrm{x}$ & \\
\hline 10 & PTN & kitong & & $\mathrm{x}$ & \\
\hline 11 & KLK & bitong & 1,11 & $\mathrm{v}$ & G-2, S-1 \\
\hline 12 & KLM & petung & 1,11 & $\mathrm{v}$ & G-2, S-1 \\
\hline 13 & KLO & kitung & 1,11 & $\mathrm{v}$ & G-2, S-1 \\
\hline 14 & KLA & bua vaitia' & $1,4,6$ & $\mathrm{x}$ & $\mathrm{G}-3, \mathrm{Y}-4, \mathrm{O}-4, \mathrm{P}-2$ \\
\hline 15 & KAL & kitung & $1,7,11$ & $\mathrm{v}$ & G-2 \\
\hline 16 & KBD & pitung & $1,7,11$ & $\mathrm{v}$ & G-3 \\
\hline 17 & KBK & pitung & $1,7,11$ & $\mathrm{v}$ & G-1, S-1, Y-1, P-2 \\
\hline 18 & PEB & karot & 1,7 & $\mathrm{v}$ & G-1, Y-1 \\
\hline
\end{tabular}

Notes : 


\section{Garcinia parvifolia (Miq.) Miq. (Guttiferae)}

\section{WILD YELLOW KANDIS. KANDIS}

\section{DESCRIPTION}

Tree, $15-30 \mathrm{~m}$; bark brown, yellow exudate; leaves elliptic, $7-10 \times 2.5-3.5 \mathrm{~cm}$, tipped, sec. veins faint; fruits roundish or elongate, not grooved, $2 \mathrm{~cm}$, diameter, yellow brown, thin skin, watery, 1-8 small seeds

\section{HABITAT}

Lowland primary and secondary forest

\section{ORIGINS}

Malaysia and western Indonesia

\section{USES}

Fruit eaten, for medicine; hunting location when fruiting

\section{REFERENCES}

Burkill 1966: 1073; Corner 1988: 358; Kessler and Sidiyasa 1994; TFM Vol. 2: 219

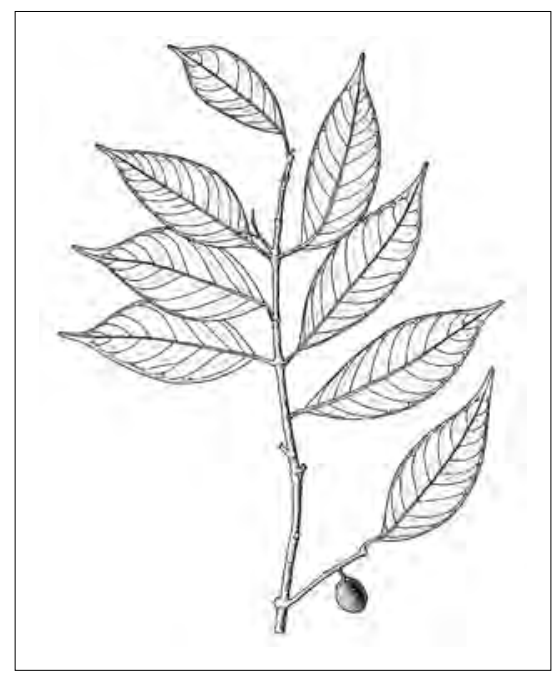

\begin{tabular}{|c|c|c|c|c|c|}
\hline \# & Code & Local Name & Use & RU & Hab.-Ab. \\
\hline 1 & PU'T & kendih & & $x$ & \\
\hline 2 & PUB & & & & \\
\hline 3 & PUD & & & & \\
\hline 4 & PUM & & & & \\
\hline 5 & ALS & & & & \\
\hline 6 & LDY & & & & \\
\hline 7 & LIT & & & & \\
\hline 8 & BRU & & & & \\
\hline 9 & MRP & & & & \\
\hline 10 & PTN & bua ite & 1,2 & $x$ & $\mathrm{O}-3, \mathrm{P}-3$ \\
\hline 11 & KLK & bua' itei, kayu ite, kayu bua' lana & 2 & $x$ & $\mathrm{O}-3, \mathrm{P}-3$ \\
\hline 12 & KLM & bua' ite & 2 & $x$ & $\mathrm{O}-3, \mathrm{P}-3$ \\
\hline 13 & KLO & va iti & & $x$ & \\
\hline 14 & KLA & beva' itie & & $x$ & \\
\hline 15 & KAL & beva itie & 1 & $\mathrm{v}$ & $\mathrm{O}-3, \mathrm{P}-3$ \\
\hline 16 & KBD & bua iti & 1,10 & $\mathrm{v}$ & $\mathrm{O}-3, \mathrm{P}-3$ \\
\hline 17 & KBK & bua' ite & & $x$ & \\
\hline 18 & PEB & juwi & 1,10 & $\mathrm{v}$ & $\mathrm{O}-3, \mathrm{P}-3$ \\
\hline
\end{tabular}

Notes : 


\section{Eleutherine americana (Aubl.) Merr. (Iridaceae) ELEUTHERINE. BAWANG}

\section{DESCRIPTION}

Herb, $25-50 \mathrm{~cm}$, stem erect or drooping; subterranean, elongated ovoid, red bulb; leaves radial, lanceolate, glabrous $25-60 \times 1-2.5 \mathrm{~cm}$; flowers opening in evening for two hours then closing for ever, white and yellow

\section{HABITAT}

Wild on roadsides, waste areas, mixed with plantations, $600-1500 \mathrm{~m}$; cultivated in home gardens

\section{ORIGINS}

South America

\section{USES}

Tubers for food, medicine

\section{REFERENCES}

Burkill 1966: 933; FOJ Vol. 1: 150

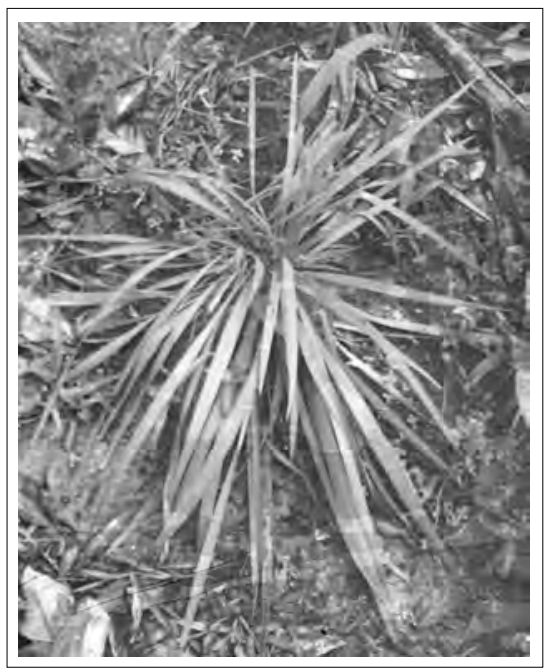

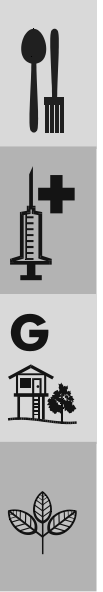

\begin{tabular}{|c|c|c|c|c|c|}
\hline \# & Code & Local Name & Use & RU & Hab.-Ab. \\
\hline 1 & PUT & bawang lufa & 1,2 & $\mathrm{v}$ & G-3, P-2 \\
\hline 2 & PUB & bawang lufa & & & \\
\hline 3 & PUD & bang mengan & 1,2 & $\mathrm{v}$ & G-3, S-2 \\
\hline 4 & PUM & bang mengan & 1,2 & $\mathrm{v}$ & G-3 \\
\hline 5 & ALS & bawing ria' & 1,2 & $\mathrm{v}$ & G-3, S-1 \\
\hline 6 & LDY & bawang sia', bawang mesia' & 1,2 & v & G-3, S-2 \\
\hline 7 & LIT & bawang sia' & 1,2 & $x$ & G-2 \\
\hline 8 & BRU & bawang nial & 1 & $\mathrm{v}$ & G-3 \\
\hline 9 & MRP & bawang lahui, kuhung bela & 1,2 & $\mathrm{v}$ & G-3, P-2, M-2 \\
\hline 10 & P'TN & uro' lumba & 1,2 & $\mathrm{v}$ & G-3 \\
\hline 11 & KLK & bawang lempa & 1,2 & $x$ & G-3 \\
\hline 12 & KLM & bawang lempa, bawang lemba & 1,2 & $\mathrm{v}$ & G-3 \\
\hline 13 & KLO & bavang lempa, bavang lempa', udu lo lempa & 1,2 & $\mathrm{x}$ & G-2 \\
\hline 14 & KLA & bavang lepa', udo lo lempa, bawang lempa, bavang lempa & 1,2 & $\mathrm{v}$ & G-3 \\
\hline 15 & KAL & bawang lempa & & & \\
\hline 16 & KBD & bawang lempa & 1 & $\mathrm{v}$ & G-2 \\
\hline 17 & KBK & tika lempa & & & \\
\hline 18 & PEB & bawang lova' baleu, tabat dek mama & 1 & $\mathrm{x}$ & G-3 \\
\hline
\end{tabular}

Notes : 


\section{Ortbosiphon aristatus (Blume) Miq. (Labiatae)}

\section{JAVA TEA. KUMIS KUCING}

\section{DESCRIPTION}

Herb, 0.4-2 m, erect perennial, stem rectangular, hairy; leaves glandular dots, ovateoblong-lanceolate, $1-10 \times 0.75-5 \mathrm{~cm}$, petiole to $3 \mathrm{~cm}$; flower racemes 7-29 cm tall, lanceolate, violet and white; nutlets dark brown 1.75-2.0 $\mathrm{mm}$ long

\section{HABITAT}

Wild in secondary forests to $900 \mathrm{~m}$; waste spaces, wet and shady areas; cultivated in gardens

\section{ORIGINS}

Asia

\section{USES}

Ornamental plant; leaves for medicine

\section{REFERENCES}

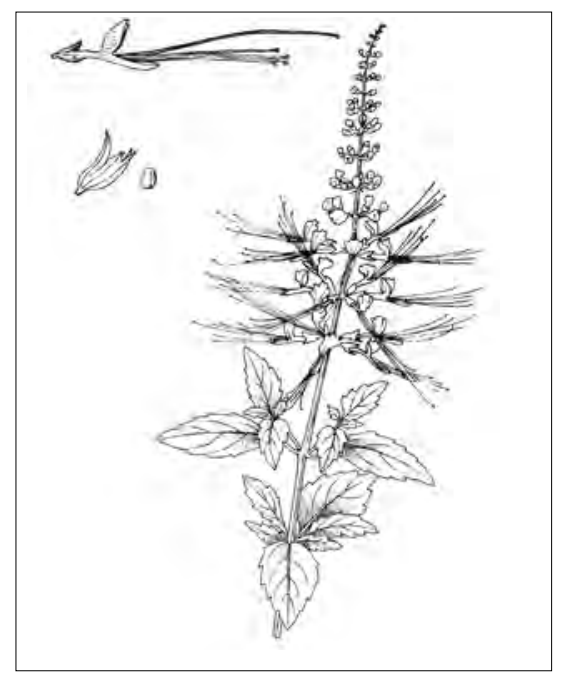

Burkill 1966: 1619; FOJ Vol. 2: 640;

PROSEA Vol. 12(1): 368

\begin{tabular}{|c|c|c|c|c|c|}
\hline \# & Code & Local Name & Use & RU & Hab.-Ab. \\
\hline 1 & PUT & kumis kucing, kumis kusing & 2 & $x$ & G-3 \\
\hline 2 & PUB & kumis kucing, kumis kusing & 2 & $\mathrm{x}$ & G-3 \\
\hline 3 & PUD & kumis kucing & 2 & $x$ & $\mathrm{~S}-2$ \\
\hline 4 & PUM & kumis kucing & 2 & $x$ & G-2 \\
\hline 5 & ALS & janggu kucing & 2 & $\mathrm{x}$ & G-2 \\
\hline 6 & LDY & bulu tang usi', kumis kucin & 2 & $\mathrm{v}$ & G-2, S-1 \\
\hline 7 & LIT & kumis kucing, bulu ta'ang using & 2 & v & G-3 \\
\hline 8 & BRU & bua et lamagua & 2 & $\mathrm{v}$ & G-2 \\
\hline 9 & MRP & raungau, kumis kucing, ro muhungau & 2 & $\mathrm{v}$ & G-2 \\
\hline 10 & PTN & bulo basingo', uro' muhusing & 2 & $\mathrm{v}$ & G-2 \\
\hline 11 & KLK & bulu pa'sing & 2 & $\mathbf{v}$ & G-2 \\
\hline 12 & KLM & kumis kucing & 2 & $\mathbf{v}$ & G-2 \\
\hline 13 & KLO & kumis kucing, bulu pasieng, bule pa' sieng & 2 & $x$ & $\mathrm{G}-3, \mathrm{O}-2$ \\
\hline 14 & KLA & kumis kucing & 2 & $x$ & G-2, Y-3 \\
\hline 15 & KAL & bulu pa' sieng & & & \\
\hline 16 & KBD & bulu pangeu & 2 & $\mathrm{x}$ & G-2 \\
\hline 17 & KBK & kumis kucing & & & \\
\hline 18 & PEB & ureu rapeh, uru lape & 2 & $\mathrm{x}$ & G-2 \\
\hline
\end{tabular}

Notes : 


\section{Cinnamomum burmanni Nees ex Bl. (Lauraceae)}

\section{INDONESIAN CASSIA. KAYU MANIS}

\section{DESCRIPTION}

Tree, 5-15 m, bark cinnamon fragrance; leaves opposite, elliptic to ovate, 3-nerved, petiole 0.5$1.5 \mathrm{~cm}$ long; dull greyish green pubescent below, young leaves red; fruit a berry $1 \mathrm{~cm}$ diameter

\section{HABITAT}

Lowland and hill primary and secondary forest, 700-2400 m; cultivated in Java and Sumatra

\section{ORIGINS}

Malaysia and western Indonesia

\section{USES}

Leaves for medicine; bark ground with coffee; sold to traders for export as 'Korintji cinnamon' in US

\section{REFERENCES}

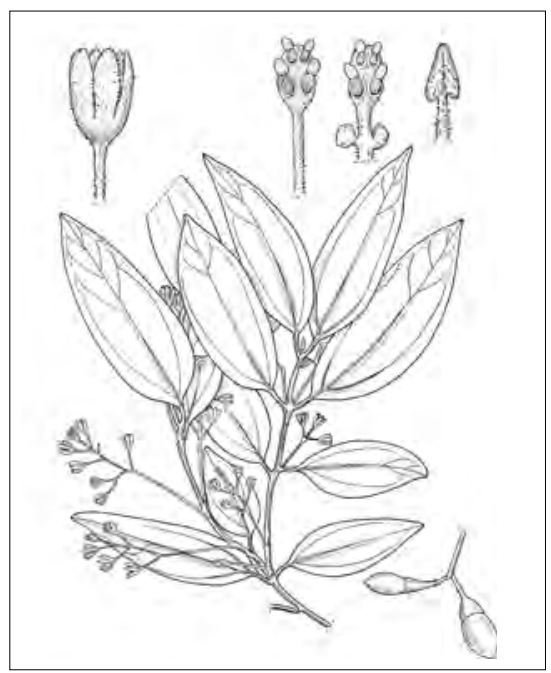

FOJ Vol. I: 121; PROSEA Vol. 13: 94

\begin{tabular}{|c|c|c|c|c|c|}
\hline$\#$ & Code & Local Name & Use & RU & Hab.-Ab. \\
\hline 1 & PUT & kayu manis, tamban lung tufuh & $1,2,11$ & v & G-2, P-3, P-4, M-3 \\
\hline 2 & PUB & kayu mi, kayu mih & 1,11 & $\mathbf{v}$ & G-2 \\
\hline 3 & PUD & kayu manis & $1,11,2$ & v & $\mathrm{Y}-2, \mathrm{O}-3, \mathrm{P}-3$ \\
\hline 4 & PUM & kefowan icit doun & 1,2 & $\mathrm{x}$ & P-3 \\
\hline 5 & ALS & taun linus & 1,11 & v & G-2, P-3, M-2 \\
\hline 6 & LDY & kayu fesi' & 1,11 & $\mathrm{v}$ & G-2, P-4 \\
\hline 7 & LIT & kayu manis, kayu pesei & $1,2,11$ & v & G-2 \\
\hline 8 & BRU & kayau me & 1,11 & v & P-3, M-4 \\
\hline 9 & MRP & kayau maih, kayo maeh, ting kayo mai & $1,2,11$ & v & G-2, G-1 \\
\hline 10 & PTN & kayo me & 1,11 & v & G-1, P-3 \\
\hline 11 & KLK & kayu me, kayu mei & 1,11 & v & G-2 \\
\hline 12 & KLM & kayu me & 1,11 & v & G-3, P-3 \\
\hline 13 & KLO & kaze me' & 1,2 & v & G-3 \\
\hline 14 & KLA & kazu mei & 1 & v & G-4 \\
\hline 15 & KAL & kazu mei' & 1 & $x$ & G-3 \\
\hline 16 & KBD & kayu mee & 1 & v & G-3 \\
\hline 17 & KBK & kayu me & 1 & $x$ & G-1 \\
\hline 18 & PEB & kayo mi & 1 & $x$ & G-4, S-2 \\
\hline
\end{tabular}

Notes : 
Cinnamomum javanicum Bl. (Lauraceae)

JAVAN CINNAMON. MEDANG WANGI

\section{DESCRIPTION}

Tree, to $21 \mathrm{~m}$, buttresses to $1 \mathrm{~m}$; bark smooth grey; leaves opposite, 13-40 x 3-15 cm, velvety hairy below, 3 main veins prominent above, tertiary veins prominent below; flowers velvety hairy; fruits ovoid, $1.5 \times 1 \mathrm{~cm}$; two varieties distinguished as (1) or (2)

\section{HABITAT}

Lowland and hill forest to $1100 \mathrm{~m}$

\section{ORIGINS}

Malaysia and western Indonesia

\section{USES}

Timber for building, firewood; bark, leaves for medicine

\section{REFERENCES}

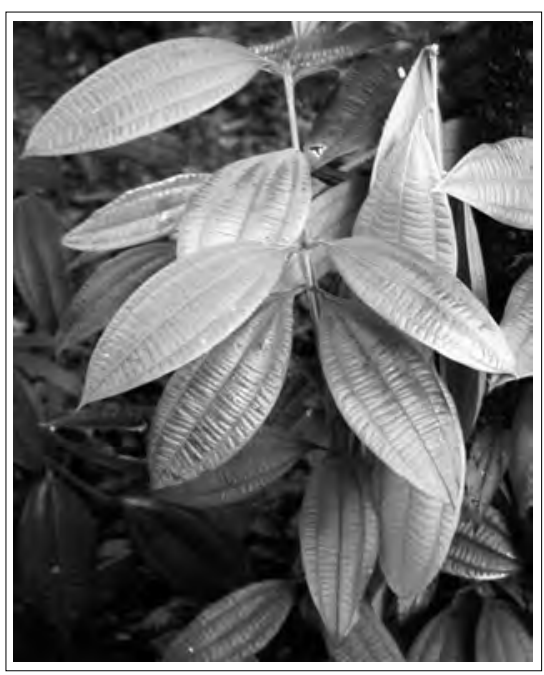

Corner 1988: 378; PROSEA Vol. 5(2): 136;

TFM Vol. 4: 126; PROSEA Vol. 13: 94

\begin{tabular}{|c|c|c|c|c|c|}
\hline \# & Code & Local Name & Use & RU & Hab.-Ab. \\
\hline 1 & PUT & kefowan (1), kevoan (1), tamban lung (2) & 1,2 & $\mathrm{x}$ & $\mathrm{P}-3, \mathrm{M}-3$ \\
\hline 2 & PUB & kevoan (1), ninga (2) & 2,7 & $x$ & P-2, M-3 \\
\hline 3 & PUD & kevoan (1) & 2 & $x$ & P-3 \\
\hline 4 & PUM & & & & \\
\hline 5 & ALS & felunak (1), sanggir (2) & 2 & $x$ & P-2, M-2 \\
\hline 6 & LDY & $\operatorname{tabar}(1,2)$ & 2 & $x$ & $\mathrm{O}-3, \mathrm{P}-3, \mathrm{M}-1$ \\
\hline 7 & LIT & & & & \\
\hline 8 & BRU & kayau tabil (1) & 2 & $\mathrm{x}$ & $\mathrm{O}-3, \mathrm{P}-3$ \\
\hline 9 & MRP & tamban laue (1), tele (2) & 2 & $x$ & $\mathrm{Y}-1, \mathrm{O}-1, \mathrm{P}-2$ \\
\hline 10 & PTN & kewo (1) & 2 & $x$ & $\mathrm{Y}-1, \mathrm{O}-1, \mathrm{P}-3$ \\
\hline 11 & KLK & kayu kelasa (1), kelembun (2) & 2 & $x$ & P-3 \\
\hline 12 & KLM & & & & \\
\hline 13 & KLO & lemoting mbe' (1), lemoting (2) & 2,6 & $\mathrm{x}$ & P-3 \\
\hline 14 & KLA & kayu lung (1), daun sevuhau (2) & $2,5,6,8$ & v & $\mathrm{O}-3, \mathrm{P}-4$ \\
\hline 15 & KAL & kaze long (1) & 2 & v & $\mathrm{O}-1, \mathrm{O}-3, \mathrm{P}-4$ \\
\hline 16 & KBD & sebuau (2) & 2 & $x$ & \\
\hline 17 & KBK & & & & \\
\hline 18 & PEB & sa'it (1), kebuau (2) & $2,6,7,8$ & $\mathrm{x}$ & $\mathrm{P}-4, \mathrm{M}-2$ \\
\hline
\end{tabular}

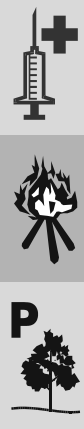

Notes : 


\section{DESCRIPTION}

Tree, to $40 \mathrm{~m}$; buttresses small rounded; bark smooth, slightly flaky, reddish brown; leaves spiral, ovate-oblong, 20-30 × 6-9 cm, acute tip; flowers greenish, yellow or purplish, 3-3.5 mm long; fruit a black drupe, 7-16 x 5-9 cm, one large seed

\section{HABITAT}

Lowland primary and old secondary forests to $500 \mathrm{~m}$; well drained soils

\section{ORIGINS}

Western Indonesia, Philippines

\section{USES}

Timber for heavy construction, posts, shingles, sold locally; fruit for medicine

\section{REFERENCES}

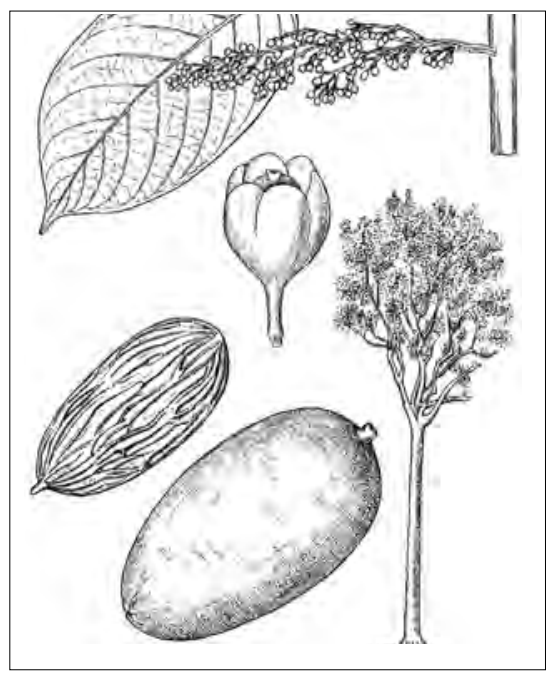

Kessler and Sidiyasa 1994: 156; PROSEA Vol.

5(1): 211

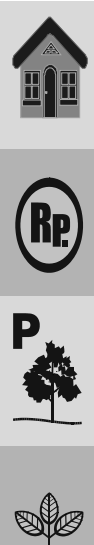

\begin{tabular}{|c|c|l|c|c|c|}
\hline$\#$ & Code & Local Name & Use & RU & Hab.-Ab. \\
\hline 1 & PUT & kaci & $3,6,11$ & $\mathbf{X}$ & P-3, M-3 \\
\hline 2 & PUB & kaci, kacih & 3,6 & $\mathbf{X}$ & P-4, M-2 \\
\hline 3 & PUD & kaci, kacih & 3,6 & $\mathbf{X}$ & P-3, M-2 \\
\hline 4 & PUM & kaci & 3,6 & $\mathbf{X}$ & P-3, M-4 \\
\hline 5 & ALS & tun tagas & $3,6,11$ & $\mathbf{x}$ & P-3 \\
\hline 6 & LDY & kayu tagas, tagas & $3,6,11$ & $\mathbf{x}$ & O-2, P-4, M-3 \\
\hline 7 & LIT & tagas & & & \\
\hline 8 & BRU & uen kasi & 3,6 & $\mathbf{X}$ & P-4, M-2 \\
\hline 9 & MRP & kaceh, keci & 3,6 & $\mathbf{X}$ & P-3, M-4 \\
\hline 10 & PTN & daun belien, belien & 3,6 & $\mathbf{X}$ & P-3 \\
\hline 11 & KLK & belien & & & \\
\hline 12 & KLM & balien, beleien & 6 & $\mathbf{X}$ & ALL \\
\hline 13 & KLO & belayen, belien & & & \\
\hline 14 & KLA & beli'en, belien & & & \\
\hline 15 & KAL & beleien & & & \\
\hline 16 & KBD & beleyan, beleyen & & & \\
\hline 17 & KBK & belien & & & \\
\hline 18 & PEB & teu & 5,6 & $\mathbf{X}$ & P-4, M-4 \\
\hline
\end{tabular}

Notes : 


\section{Litsea garciae Vidal (Lauraceae) \\ LAURAL. MALAI, KANGKALA}

\section{DESCRIPTION}

Tree, to $35 \mathrm{~m}$, bark smooth, greyish white; leaves spiral, oblong-lanceolate, $12-50 \times 5-18 \mathrm{~cm}$; fruits a depressed globose berry, $2.5 \times 4.5 \mathrm{~cm}$ diameter, pink to purple black

\section{HABITAT}

Lowland primary and secondary forests, planted in villages

\section{ORIGINS}

Borneo endemic

\section{USES}

Fruit eaten raw or cooked; seed oil for candles and soap; timber for building; site for ambushing pigs when fruiting

\section{REFERENCES}

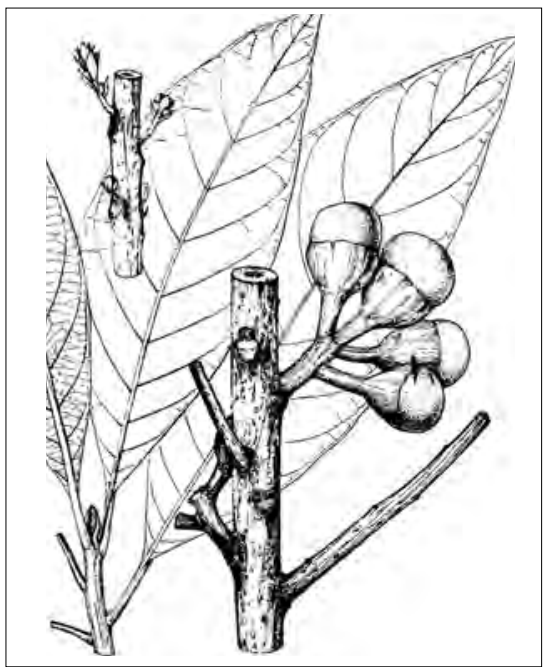

Kessler and Sidiyasa 1994: 158; PROSEA Vol. 2:

344

\begin{tabular}{|c|c|c|c|c|c|}
\hline$\#$ & Code & Local Name & Use & RU & Hab.-Ab. \\
\hline 1 & PUT & kelimah & $1,10,11$ & v & G-2, P-3 \\
\hline 2 & PUB & kelimah & 1,10 & v & $\mathrm{G}-2, \mathrm{O}-3, \mathrm{P}-3$ \\
\hline 3 & PUD & kelimah & $1,10,11$ & v & $\mathrm{G}-3, \mathrm{O}-3, \mathrm{P}-3$ \\
\hline 4 & PUM & kelimah & 1,10 & v & $\mathrm{G}-2, \mathrm{O}-3, \mathrm{P}-4$ \\
\hline 5 & ALS & bua' vengolobon & $1,2,10$ & v & $\mathrm{G}-3, \mathrm{O}-2, \mathrm{P}-3$ \\
\hline 6 & LDY & kayu talal, bua talal & 1,10 & v & $\mathrm{G}-3, \mathrm{O}-4, \mathrm{P}-4$ \\
\hline 7 & LIT & telal, bua' telau & 1,11 & v & G-2, P-3 \\
\hline 8 & BRU & wi lahal & 1,10 & v & G-3 \\
\hline 9 & MRP & kelimie, kelime & 1,10 & v & G-2, P-3 \\
\hline 10 & PTN & kelima & 1,10 & v & G-2, P-4 \\
\hline 11 & KLK & mali & 1 & v & G-2 \\
\hline 12 & KLM & mali & 1,10 & v & G-2 \\
\hline 13 & KLO & mali & 1 & $x$ & $\mathrm{G}-3, \mathrm{P}-3$ \\
\hline 14 & KLA & beva' mali, mali & $1,2,10$ & $x$ & $\mathrm{G}-2, \mathrm{O}-2, \mathrm{P}-2$ \\
\hline 15 & KAL & mali & & & \\
\hline 16 & KBD & mali & $1,7,10$ & $x$ & $\mathrm{G}-2, \mathrm{O}-3, \mathrm{P}-3$ \\
\hline 17 & КВ K & kayu mali & & & \\
\hline 18 & PEB & malei, mali & 1 & v & $\mathrm{G}-3, \mathrm{O}-3, \mathrm{P}-4, \mathrm{M}-4$ \\
\hline
\end{tabular}

Notes : 


\section{Allium tuberosum Rottler ex Sprengel. (Liliaceae)}

Chinese CHIVES. KUCAI

\section{DESCRIPTION}

Herb, 20-30 cm, tussocky, robust rhizome, small white bulbs, leaves linear rounded apex, grasslike, dense white dots, $15-30$ x 0.3-0.6 cm; flowers on pedicels, petals white with greenish or reddish streak, elliptic; fruits obovoid green, $0.25 \mathrm{~cm} \mathrm{~d}$

\section{HABITAT}

Cultivated in homegardens, well drained fertile soils

\section{ORIGINS}

\section{China}

\section{USES}

Leaves used for flavoring, like leeks, sold locally; seeds for medicine

\section{REFERENCES}

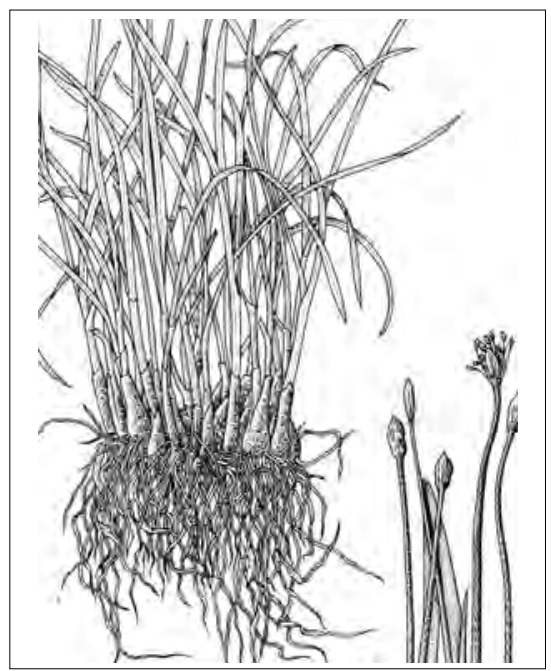

Burkill 1966: 102; Ochse 1980: 450;

PROSEA Vol. 8: 80

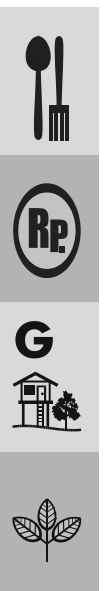

\begin{tabular}{|c|c|l|c|c|c|}
\hline$\#$ & Code & \multicolumn{1}{|c|}{ Local Name } & Use & RU & Hab.-Ab. \\
\hline 1 & PUT & bawang kucai, bawang kelien, bawang & $1,7,11$ & $\mathbf{v}$ & G-3 \\
\hline 2 & PUB & bawang lo, bawang & 1,11 & $\mathbf{v}$ & G-3 \\
\hline 3 & PUD & bawang serou, uru luh & 1 & $\mathbf{v}$ & G-3 \\
\hline 4 & PUM & bawang kucai & 1,11 & $\mathbf{v}$ & G-2 \\
\hline 5 & ALS & bawang kucai & 1 & $\mathbf{v}$ & G-2 \\
\hline 6 & LDY & don kusai, bawang buda & 1,11 & $\mathbf{v}$ & G-3 \\
\hline 7 & LIT & kucai, kusai & 1,11 & $\mathbf{v}$ & G-3 \\
\hline 8 & BRU & kucai & 1,11 & $\mathbf{v}$ & G-3 \\
\hline 9 & MRP & bawang lahui, mawai, ro mpau & 1,11 & $\mathbf{v}$ & G-3 \\
\hline 10 & PTN & uro' lau', uro' lo & 1 & $\mathbf{v}$ & G-3 \\
\hline 11 & KLK & bawang pute, bawang po' & 1,11 & $\mathbf{v}$ & G-3 \\
\hline 12 & KLM & bawang kenya & 1,11 & $\mathbf{v}$ & G-3 \\
\hline 13 & KLO & udu lu & 1 & $\mathbf{v}$ & G-3 \\
\hline 14 & KLA & udu lo & 1 & $\mathbf{v}$ & G-3 \\
\hline 15 & KAL & udu lo & 1 & $\mathbf{v}$ & G-3 \\
\hline 16 & KBD & udu lo & 1 & $\mathbf{v}$ & G-3 \\
\hline 17 & KBK & udu tika & 1 & $\mathbf{v}$ & G-3 \\
\hline 18 & PEB & ureu lo poti, uru lo & 1 & $\mathbf{v}$ & G-4, S-3 \\
\hline
\end{tabular}

Notes : 


\section{Elmerrillia mollis Dandy. (Magnoliaceae)}

\section{WAU BEECH. CHEMPAKA HUTAN ALUS}

\section{DESCRIPTION}

Tree, to $30 \mathrm{~m}$, buttresses $2 \mathrm{~m}$ high; bark smooth greyish brown; leaves elliptical, 5-15 x 2-5.5 cm, midrib raised above, margin toothed in upper half; cup 1.5-2.5 cm diam, 6-7 flanges; nut, 2-5.5 x 1-2 $\mathrm{cm}$, cup enclosing whole nut when immature

\section{HABITAT}

Lowland and mountain forests to $2150 \mathrm{~m}$; along streams

\section{ORIGINS}

Malaysia and western Indonesia

\section{USES}

Timber highly preferred for wooden canoes; timber for light construction; source of food for hunted prey

\section{REFERENCES}

PROSEA Vol. 5(2): 414; TFM Vol. 1: 229

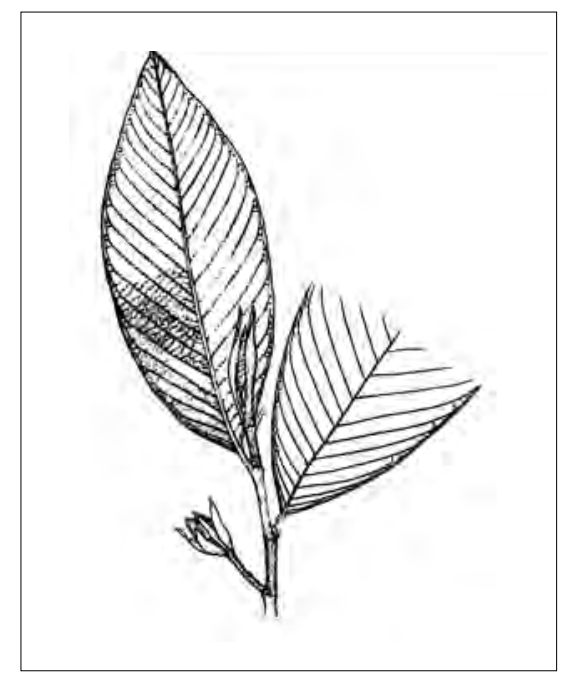

\begin{tabular}{|r|l|l|c|c|c|}
\hline$\#$ & Code & \multicolumn{1}{|c|}{ Local Name } & Use & RU & Hab.-Ab. \\
\hline 1 & PUT & arau & $4,5,6$ & $\mathbf{x}$ & O-2, P-3, M-3 \\
\hline 2 & PUB & arau & & & \\
\hline 3 & PUD & arau & $4,5,6$ & $\mathbf{v}$ & P-2 \\
\hline 4 & PUM & & & & \\
\hline 5 & ALS & amung & & & \\
\hline 6 & LDY & merabung & & & \\
\hline 7 & LIT & adau & & & \\
\hline 8 & BRU & beldieu & & & \\
\hline 9 & MRP & arau, ro & & & \\
\hline 10 & PTN & aroh & $4,5,6$ & $\mathbf{v}$ & P-2 \\
\hline 11 & KLK & adao & $4,5,6$ & $\mathbf{v}$ & P-3, M-2 \\
\hline 12 & KLM & a'dau & $4,5,6$ & $\mathbf{v}$ & P-3, M-2 \\
\hline 13 & KLO & ado & $4,5,6$ & $\mathbf{v}$ & P-3, M-3 \\
\hline 14 & KLA & adau & $4,5,6$ & $\mathbf{v}$ & O-2, P-3, M-3 \\
\hline 15 & KAL & adau & $4,5,6$ & $\mathbf{v}$ & O-2, P-3, M-3 \\
\hline 16 & KBD & adau & & & \\
\hline 17 & KBK & & $4,5,6$ & $\mathbf{v}$ & O-2, P-3, M-3 \\
\hline 18 & PEB & arau & & \\
\hline
\end{tabular}

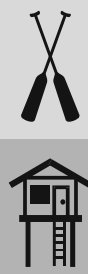

Notes : 


\section{Abelmoschus manibot (L.) Medik (Malvaceae)}

\section{SUNSET HIBISCUS. DEGI, GEDI}

\section{DESCRIPTION}

Shrub, perennial, 1-3 m, stems erect, woody, no prickly hairs; leaves simple, alternate, petiole 3-25 $\mathrm{cm}$ long, blade linear, lanceolate, cordate or deeply lobed; flowers bell-shaped, 3-8 cm diameter, pale yellow with dark brown or reddish central spot

\section{HABITAT}

Cultivated in homegardens; propagated by stem cuttings

\section{ORIGINS}

Indochina

\section{USES}

Leaves and stem tips eaten; ornamental plant; medicine

\section{REFERENCES}

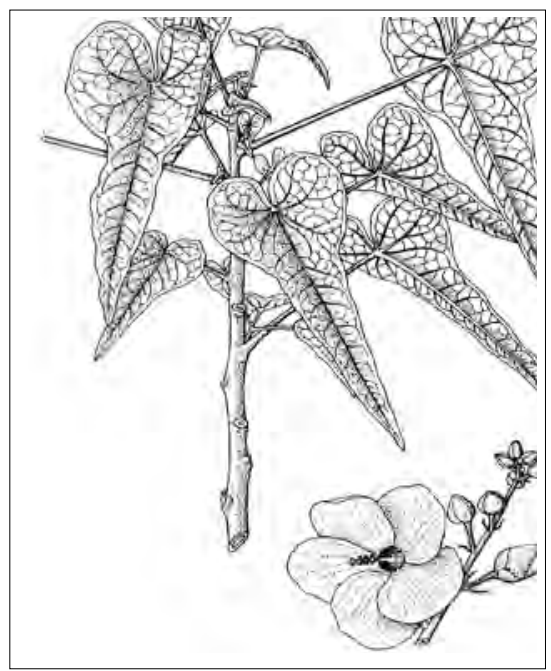

Ochse 1980: 464; PROSEA Vol. 8: 60

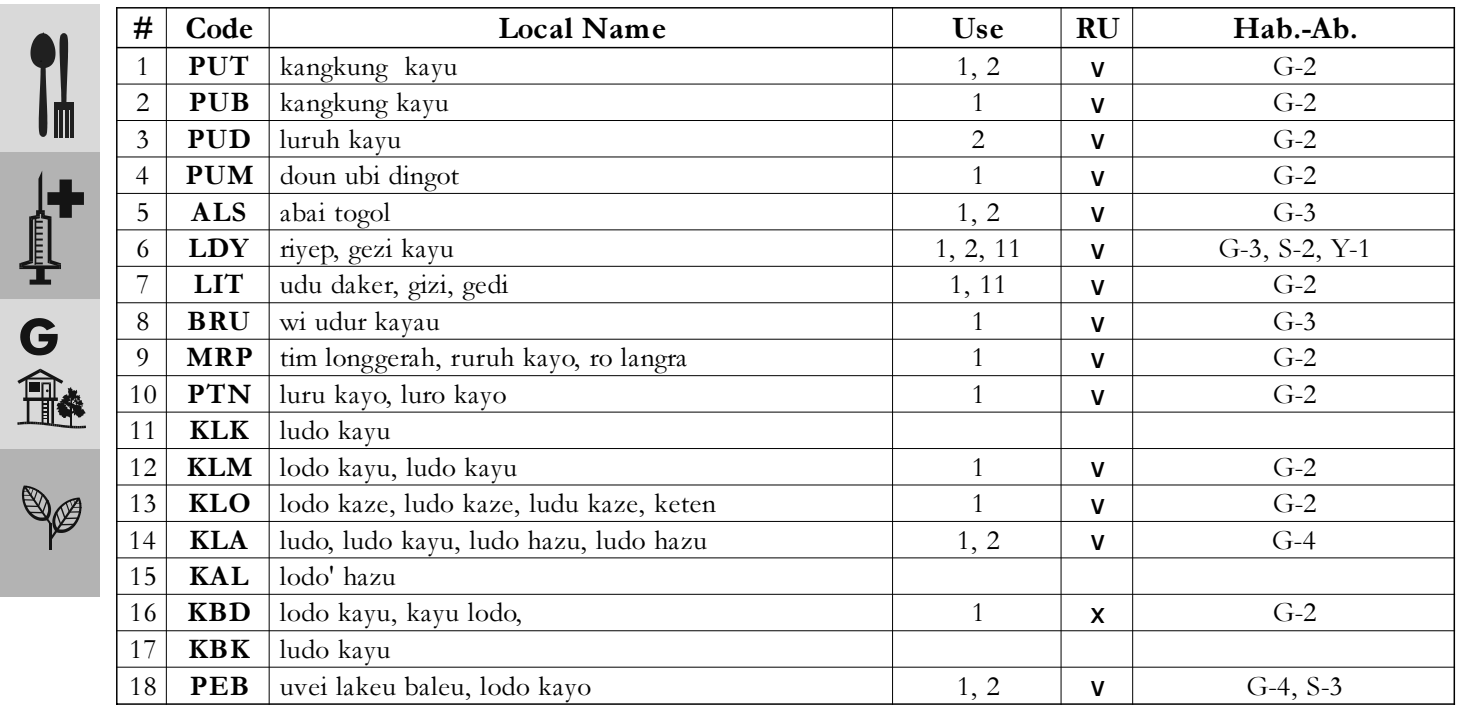

Notes : 


\section{Melastoma affine D. Don. (Melastomataceae) \\ INDIAN RHODONDENDRON. HARENDONG}

DESCRIPTION

Shrub, to $5 \mathrm{~m}$, woody perennial; leaves opposite, short petioled, ovate, base acute, 3-5 nerved, above rough, beneath softly hairy, $5-20$ x $1.5-8 \mathrm{~cm}$; flowers 5 petals purple, rose or white; fruit berries dark violet pulp within, $0.8-1 \mathrm{~cm}$ long

\section{HABITAT}

Wild in secondary forests, formerly cultivated areas, edges, wastes and fallows

\section{ORIGINS}

Asia, Pacific

\section{USES}

Leaves, roots for medicine; fruit, leaves for dye

\section{REFERENCES}

Burkill 1966: 1463; Ochse 1980: 485

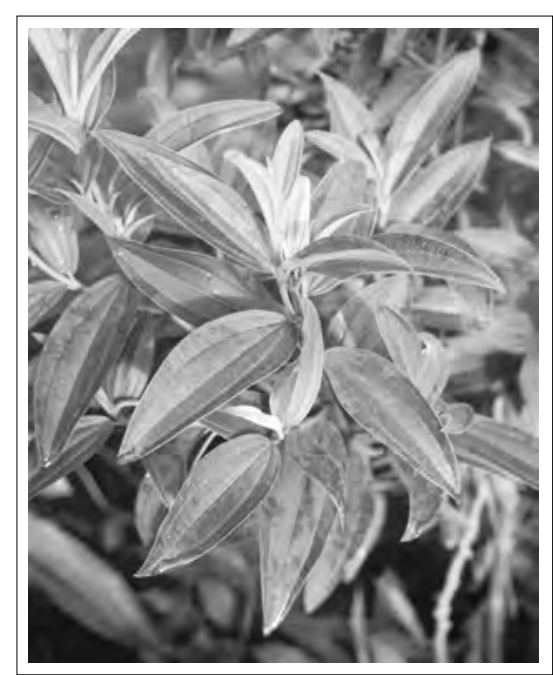

\begin{tabular}{|c|c|c|c|c|c|}
\hline \# & Code & Local Name & Use & RU & Hab.-Ab. \\
\hline 1 & PUT & kang karang, kang karan & 2,1 & $x$ & $\mathrm{~S}-3, \mathrm{Y}-3, \mathrm{O}-4$ \\
\hline 2 & PUB & kang karang & 1,2 & $\mathrm{x}$ & $\mathrm{Y}-3, \mathrm{O}-2$ \\
\hline 3 & PUD & kang karan & 2 & $\mathrm{x}$ & $\mathrm{S}-2, \mathrm{Y}-2, \mathrm{O}-3, \mathrm{P}-4$ \\
\hline 4 & PUM & kang karang & 1,2 & $\mathrm{x}$ & $\mathrm{Y}-2, \mathrm{O}-3$ \\
\hline 5 & ALS & taun ladit & 2 & $\mathrm{x}$ & $\mathrm{Y}-3, \mathrm{O}-2$ \\
\hline 6 & LDY & kayu sekali', sekale becuk & 1,2 & $\mathrm{x}$ & $\mathrm{S}-2, \mathrm{Y}-3, \mathrm{O}-2$ \\
\hline 7 & LIT & tekele, tekeley & 2,1 & $\mathrm{x}$ & $\mathrm{O}-4, \mathrm{Y}-3$ \\
\hline 8 & BRU & uen zit & 1,2 & $x$ & $\mathrm{Y}-3, \mathrm{O}-2$ \\
\hline 9 & MRP & rau nau, kayau nyau, ro nyau & 2 & $x$ & $\mathrm{~S}-2, \mathrm{Y}-3, \mathrm{O}-4$ \\
\hline 10 & PTN & kangkarang & 1,2 & $x$ & $\mathrm{~S}-3, \mathrm{Y}-2$ \\
\hline 11 & KLK & jela mutin & 1,2 & $x$ & $\mathrm{~S}-4, \mathrm{Y}-3, \mathrm{O}-2$ \\
\hline 12 & KLM & jelemetin, keremutin & 1,2 & $\mathrm{x}$ & ALL \\
\hline 13 & KLO & lemuting & 1,2 & $x$ & $\mathrm{~S}-1, \mathrm{Y}-3, \mathrm{O}-3$ \\
\hline 14 & KLA & lemutim & 1,2 & $x$ & $\mathrm{~S}-2, \mathrm{Y}-2 \mathrm{O}-2$ \\
\hline 15 & KAL & jala mutim & 1,2 & $\mathrm{x}$ & $\mathrm{S}-1, \mathrm{Y}-3, \mathrm{O}-3$ \\
\hline 16 & KBD & jelemutin & 1,2 & $x$ & S-1, Y-3 \\
\hline 17 & KBK & usit kang & 1,2 & $x$ & $\mathrm{~S}-1, \mathrm{Y}-3$ \\
\hline 18 & PEB & nyerutin & 1,2 & v & $\mathrm{Y}-4$ \\
\hline
\end{tabular}

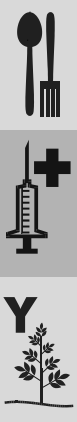

Notes : 


\section{Memecylon paniculatum Jack. (Melastomataceae)}

\section{(NO ENGLISH NAME). KAYU TULANG}

\section{DESCRIPTION}

Tree, to $20 \mathrm{~m}$, ramrod straight; bark smooth, finely fissured, thin, grey to brown; leaves elliptic 3-9 $\mathrm{x} 1$ $4 \mathrm{~cm}$, venation invisible; fruit greenish white becoming blue

\section{HABITAT}

Hill primary forests, above $500 \mathrm{~m}$, ridge tops

\section{ORIGINS}

Borneo

\section{USES}

Saplings used for punting poles and spear shafts, timber for building

\section{REFERENCES}

Burkill 1966: 1474; Corner 1988: 487;

PROSEA Vol. 5(3): 371

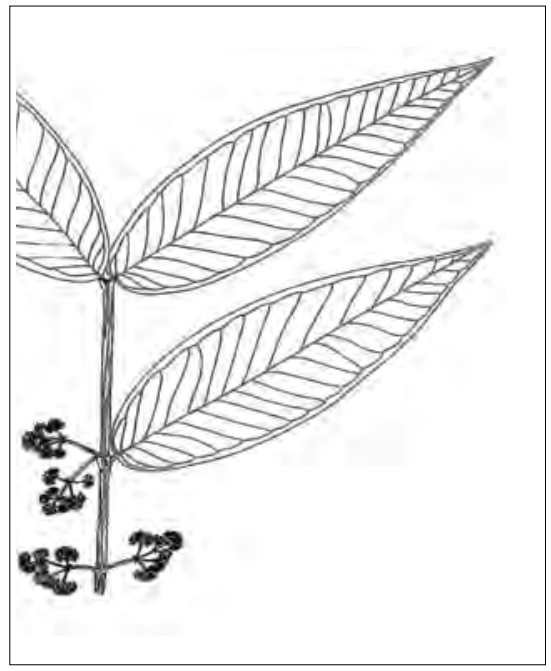

\begin{tabular}{|c|c|c|c|c|c|}
\hline \# & Code & Local Name & Use & RU & Hab.-Ab. \\
\hline 1 & PUT & temah, kema' & 6 & $x$ & P-3, M-3 \\
\hline 2 & PUB & tema & & & \\
\hline 3 & PUD & tema & 4,6 & v & P-3, M-2 \\
\hline 4 & PUM & & & & \\
\hline 5 & ALS & liasab & & & \\
\hline 6 & LDY & sinarar & & & \\
\hline 7 & LIT & uber & & & \\
\hline 8 & BRU & tamah & & & \\
\hline 9 & MRP & tama & 4,6 & v & P-3, M-2 \\
\hline 10 & PTN & tema & & & \\
\hline 11 & KLK & tema, tema bala & $3,4,6$ & $\mathrm{v}$ & P-3, M-2 \\
\hline 12 & KLM & tema, tema bala & $3,4,6$ & v & P-3, M-2 \\
\hline 13 & KLO & temaring & $3,4,6$ & v & P-3, M-2 \\
\hline 14 & KLA & lu & $3,4,6$ & v & $\mathrm{P}-4, \mathrm{M}-2$ \\
\hline 15 & KAL & temma & $3,4,6$ & $\mathrm{v}$ & P-3, M-2 \\
\hline 16 & KBD & temma & 4,6 & v & P-3, M-3 \\
\hline 17 & KB K & jangin lapan & 4,6 & v & $\mathrm{O}-4, \mathrm{P}-5, \mathrm{M}-5$ \\
\hline 18 & PEB & teme bale & 4,6 & v & $\mathrm{P}-3, \mathrm{M}-3$ \\
\hline
\end{tabular}

Notes : 


\section{Lansium domesticum Correa (Meliaceae) \\ LANGSAT, DUKU. LANGSAT, DUKU}

\section{DESCRIPTION}

Tree, to $30 \mathrm{~m}$, fluted buttresses; bark mottled grey orange, milky sap; leaves alternate, odd-pinnate, 30-50 cm long; leaflets alternate, 6-9, elliptic to oblong 9-21 x 5-10 cm; flowers white to yellow; fruit yellow globose $2-4 \mathrm{~cm}$, white aril, 1-5 seeds

\section{HABITAT}

Wild in lowland and hill forests, cultivated in villages

\section{ORIGINS}

Malaysia and western Indonesia

\section{USES}

Fruit eaten, sold locally; fruit peel, seeds, bark for medicine

\section{REFERENCES}

Burkill 1966: 1335; Corner 1988: 501;

PROSEA Vol. 2: 186

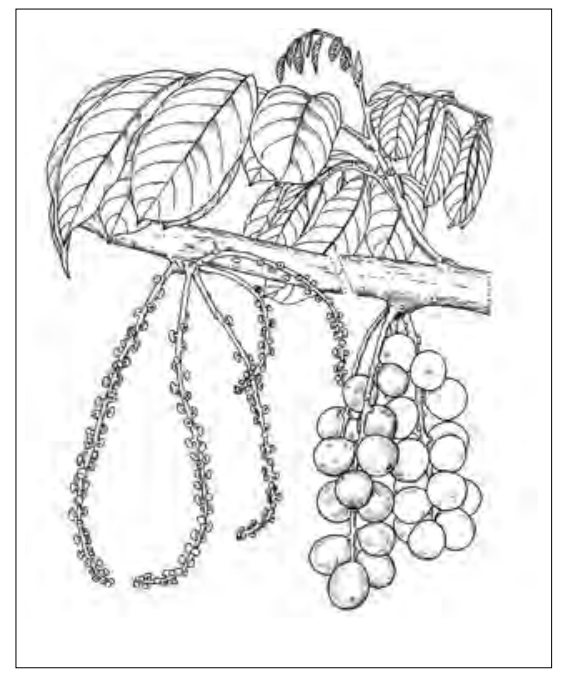

\begin{tabular}{|c|c|l|c|c|c|}
\hline$\#$ & Code & Local Name & Use & RU & Hab.-Ab. \\
\hline 1 & PUT & lengot & $1,2,7,11$ & $\mathbf{v}$ & G-3, O-2, P-4, M-3 \\
\hline 2 & PUB & lengot & $1,2,11$ & $\mathbf{v}$ & G-3, O-3 \\
\hline 3 & PUD & lengot & $1,2,11$ & $\mathbf{v}$ & G-3, P-3 \\
\hline 4 & PUM & lengot & $1,2,11$ & $\mathbf{v}$ & G-3, O-3, P-4, M-3 \\
\hline 5 & ALS & bua' lanjat & $1,2,11$ & $\mathbf{v}$ & G-3 \\
\hline 6 & LDY & kayu lingat, bua lingat & $1,2,11$ & $\mathbf{v}$ & G-3, P-2 \\
\hline 7 & LIT & bua lengat, lengat, lingut & $1,2,7,11$ & $\mathbf{v}$ & G-4, P-2, S-2, P-4 \\
\hline 8 & BRU & wi langat & 1,11 & $\mathbf{x}$ & G-3, P-3 \\
\hline 9 & MRP & bua ngahat, ngahat, langahat & $1,2,11$ & $\mathbf{v}$ & G-3, P-3 \\
\hline 10 & PTN & ngelahat, ngelahet & $1,2,11$ & $\mathbf{v}$ & G-4, P-3 \\
\hline 11 & KLK & langset & $1,2,11$ & $\mathbf{v}$ & G-3, P-3 \\
\hline 12 & KLM & langset & $1,2,11$ & $\mathbf{v}$ & G-3 \\
\hline 13 & KLO & lungsit, lengsit & $1,2,11$ & $\mathbf{v}$ & G-3 S-2, Y-2 \\
\hline 14 & KLA & lungset, langset & $1,2,11$ & $\mathbf{v}$ & G-3 S-2, Y-2 \\
\hline 15 & KAL & lengesit & $1,2,11$ & $\mathbf{v}$ & G-3 S-3, Y-3 \\
\hline 16 & KBD & bua leset, leset & $1,2,11$ & $\mathbf{v}$ & G-3 S-3, Y-3 \\
\hline 17 & KBK & langsat & $1,2,11$ & $\mathbf{v}$ & G-3 S-3, Y-3 \\
\hline 18 & PEB & lasiu & $1,7,11$ & $\mathbf{v}$ & G-4, M-3, Y-3, P-4 \\
\hline
\end{tabular}

Notes : 


\section{Melia azedarach L. (Meliaceae)}

\section{PeRsian lilaC. MINDI}

\section{DESCRIPTION}

Tree, to $20 \mathrm{~m}$, conical crown; buds and young shoots brownish; leaves $17.5-50 \mathrm{~cm}$ long, doubly pinnate; leaflets lanceolate-elliptic, 3-7.5 x 1-2 cm, light green strongly toothed; flowers lilac or purple, $1-2 \mathrm{~cm}$ wide; fruit $1 \mathrm{~cm}$ round, yellow brown

\section{HABITAT}

Cultivated in villages

\section{ORIGINS}

South Asia

\section{USES}

Leaves, roots for medicine; fruit for fish poison, insecticide, alcohol; timber for building; ornamental plant, leaves eaten

\section{REFERENCES}

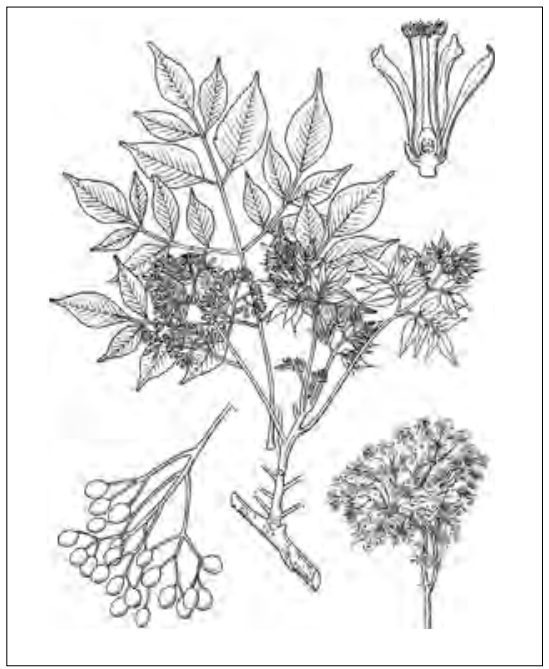

Burkill 1966: 1465; Corner 1988: 502;

PROSEA Vol. 2: 187

\begin{tabular}{|c|c|c|c|c|c|c|}
\hline & \# & Code & Local Name & Use & RU & Hab.-Ab. \\
\hline & 1 & PUT & doun teh, bawing kayu & 6 & $\mathrm{x}$ & G-1 \\
\hline & 2 & PUB & & & & \\
\hline & 3 & PUD & & & & \\
\hline \multirow{8}{*}{$\begin{array}{l}\mathbf{C} \\
\text { 侖期 }\end{array}$} & 4 & PUM & bawing kayu tufuh & & $x$ & G-1 \\
\hline & 5 & ALS & sembulu & 6 & $\mathrm{x}$ & G-1 \\
\hline & 6 & LDY & kayu sambulu, bunga & & $x$ & G-1, G-2 \\
\hline & 7 & LIT & da'on teh & & & \\
\hline & 8 & BRU & wi bunga & 6 & $x$ & G-1 \\
\hline & 9 & MRP & bunga & & & \\
\hline & 10 & PTN & te time & 1 & $x$ & G-1 \\
\hline & 11 & KLK & te kayu & & $x$ & G-1 \\
\hline \multirow{7}{*}{$Q$} & 12 & KLM & kayu te & 2 & v & G-1, G-2 \\
\hline & 13 & KLO & tee' & & $x$ & G-2 \\
\hline & 14 & KLA & te' $^{\prime}$ & 1 & v & G-2 \\
\hline & 15 & KAL & kazu te' & & & \\
\hline & 16 & KBD & kayu te', te & 1 & v & G-2 \\
\hline & 17 & KBK & kayu te & & & \\
\hline & 18 & PEB & kayo te, kayo bunga & 3 & $x$ & G-2 \\
\hline
\end{tabular}

Notes : 
Pycnarrbena cauliflora (Menispermaceae)

(NOENGLISH/INDONESIANNAME)

\section{DESCRIPTION}

Vine, 1-5 m, woody, leaves thin coracious, elliptic-lanceolate to oval-oblong, 21-25 x 7-12 cm, short apical tip, cuneate base; 5-7 pairs sec. nerves, sunken above, prominent below, petiole $4 \mathrm{~cm}$

\section{HABITAT}

Lowland and hill primary and secondary forests

\section{ORIGINS}

Borneo

\section{USES}

Leaves for spice; medicine, antidote for snake venom; sold locally

\section{REFERENCES}

FOJ Vol. I: 153; FM I Vol. 10: 172

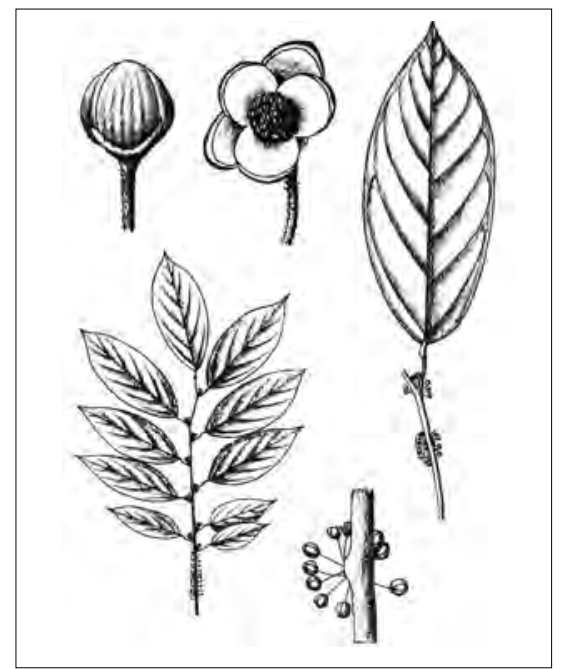

\begin{tabular}{|c|c|l|c|c|c|}
\hline \# & Code & Local Name & Use & RU & Hab.-Ab. \\
\hline 1 & PUT & lekoi & 1,11 & $\mathbf{v}$ & G-1, M-4, O-3, P-3, Y-2 \\
\hline 2 & PUB & lekoi & 1,11 & $\mathbf{v}$ & O-2, P-3, M-2 \\
\hline 3 & PUD & lekoi & 1,11 & $\mathbf{v}$ & P-2 \\
\hline 4 & PUM & lekoi & 1,11 & $\mathbf{x}$ & G-2, O-2, P-3, M-3 \\
\hline 5 & ALS & daun afa, pangapi umus & 1 & $\mathbf{v}$ & O-2, P-3 \\
\hline 6 & LDY & war afa, apa & 1,11 & $\mathbf{v}$ & G-1, O-3, P-3 \\
\hline 7 & LIT & daun pa, daun pa' & 1,11 & $\mathbf{v}$ & Y-2, O-3, P-3, M-3 \\
\hline 8 & BRU & uen longdau & 1,11 & $\mathbf{v}$ & O-2, P-3 \\
\hline 9 & MRP & kaie, kai, kae & 1,11 & $\mathbf{v}$ & O-2, M-2, P-3 \\
\hline 10 & PTN & beke & 1,11 & $\mathbf{v}$ & O-3, P-3 \\
\hline 11 & KLK & bekkai lan & 1,11 & $\mathbf{v}$ & P-3 \\
\hline 12 & KLM & bekkai kenya, bekkai & 1,11 & $\mathbf{v}$ & P-3 \\
\hline 13 & KLO & bekkae & $1,2,11$ & $\mathbf{v}$ & P-3, M-1 \\
\hline 14 & KLA & bekkai kenya & 1,11 & $\mathbf{v}$ & P-3, M-1 \\
\hline 15 & KAL & bekkai & 1,11 & $\mathbf{v}$ & P-3, M-3 \\
\hline 16 & KBD & bekkai & $1,2,11$ & $\mathbf{v}$ & P-3, M-2 \\
\hline 17 & KBK & bekkai kenya & 1,11 & $\mathbf{v}$ & P-2, M-2 \\
\hline 18 & PEB & mekei & 1 & $\mathbf{x}$ & P-4, M-4 \\
\hline
\end{tabular}

Notes : 


\section{Antiaris toxicaria Leschen (Moraceae)}

UPAS TREE. UPAS

\section{DESCRIPTION}

Tree, to $50 \mathrm{~m}$, bark smooth greyish white; latex creamy white turning brown and granular on contact to air; leaves oblong elliptic, $7.5-20 \times 4-9 \mathrm{~cm}$, rounded heart-shaped base, short petiole; fruit 1 $\mathrm{cm}$ wide, pear-shaped, velvety, crimson then black

\section{HABITAT}

Lowland primary forests

\section{ORIGINS}

Old World Tropics

\section{USES}

Latex for poison for blowpipe darts

\section{REFERENCES}

Burkill 1966: 175; Corner 1988: 510;

PROSEA Vol. 12(1): 126

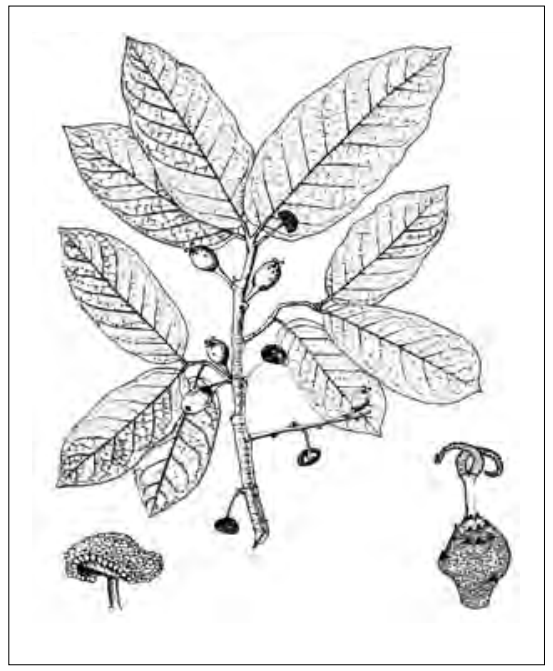

\begin{tabular}{|c|c|c|c|c|c|}
\hline \# & Code & Local Name & Use & RU & Hab.-Ab. \\
\hline 1 & PUT & tacom & 10 & $x$ & P-2, M-2 \\
\hline 2 & PUB & tacom & 10 & v & P-2, M-2 \\
\hline 3 & PUD & tajom & 10 & v & P-2, M-2 \\
\hline 4 & PUM & tacom & 10 & v & P-2, M-2 \\
\hline 5 & ALS & valig & 10 & $x$ & P-2, M-2 \\
\hline 6 & LDY & farir & 10 & $x$ & P-2, M-2 \\
\hline 7 & LIT & kayu parel & 10 & $x$ & P-2, M-2 \\
\hline 8 & BRU & kayu pagel & 10 & $x$ & P-2, M-2 \\
\hline 9 & MRP & pieu, pio & 10 & $x$ & P-2, M-2 \\
\hline 10 & PTN & halu & 10 & $x$ & P-2, M-2 \\
\hline 11 & KLK & kayu ipo' (salo) & 10 & v & P-1, M-1, M-2 \\
\hline 12 & KLM & salu' & 10 & v & P-1, M-1 \\
\hline 13 & KLO & & & & \\
\hline 14 & KLA & & & & \\
\hline 15 & KAL & salue & 10 & v & P-1, M-1 \\
\hline 16 & KBD & salo & 10 & v & P-1, M-1 \\
\hline 17 & KBK & salo & 10 & $x$ & P-1, M-1 \\
\hline 18 & PEB & tajem & 10 & v & P-1, M-1 \\
\hline
\end{tabular}

Notes : 


\section{Artocarpus altilis Z. Fosberg (Moraceae) \\ BREADFRUIT. SUKUN}

\section{DESCRIPTION}

Tree, to $30 \mathrm{~m}$, copious white sap everywhere; twigs thick, sparsely hairy; buds $10-30 \mathrm{~cm}$ long, covered by conical stipules; leaves very large, $30-60 \mathrm{~cm}$, deeply cut into pointed lobes; fruits $12.5-30 \mathrm{~cm}$ thick, smooth or prickly; often seedless

\section{HABITAT}

Cultivated to $1500 \mathrm{~m}$; cultivars adapted to varying conditions

\section{ORIGINS}

Island Southeast Asia to Pacific Islands

\section{USES}

Cooked fruit eaten, sold locally; bark for cloth, cordage and basketry; latex for birdlime; timber for boats; medicine

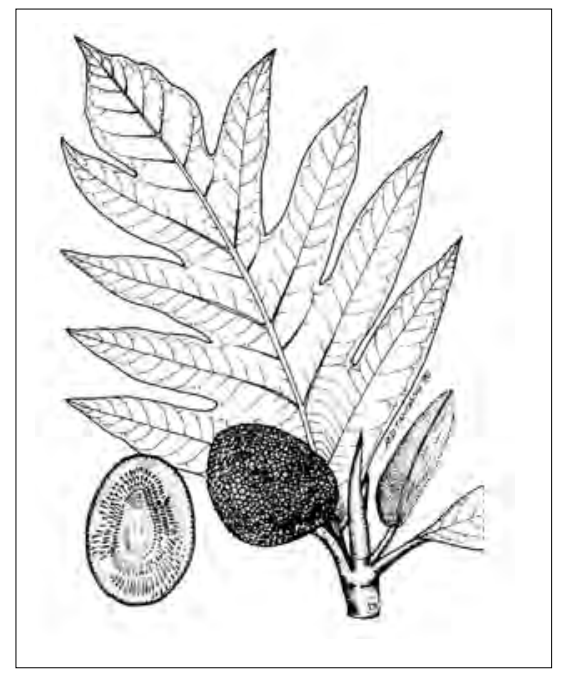

\section{REFERENCES}

Corner 1988: 518; PROSEA Vol. 2: 83

\begin{tabular}{|c|c|c|c|c|c|}
\hline \# & Code & Local Name & Use & RU & Hab.-Ab. \\
\hline 1 & PUT & kumut, irah, tenga'u & $1,8,9,10,11$ & v & G-2, S-1 \\
\hline 2 & PUB & tenga'u, tepuwing & $1,8,9,10,11$ & v & G-2, S-1 \\
\hline 3 & PUD & tenga' u, kumut & $1,8,9,10,11$ & v & G-2, S-1 \\
\hline 4 & PUM & tengo'hu & $1,8,9,10,11$ & v & G-2, S-1 \\
\hline 5 & ALS & talun & $1,8,9,10,11$ & v & G-2, S-1 \\
\hline 6 & LDY & kayu talun & $1,8,9,10,11$ & v & $\mathrm{G}-2, \mathrm{~S}-1$ \\
\hline 7 & LIT & talun & $1,8,9,10,11$ & v & G-2, S-1 \\
\hline 8 & BRU & kayau talon & $1,8,9,10,11$ & v & G-2, S-1 \\
\hline 9 & MRP & tai muk, tai mug, tai & $1,8,9,10,11$ & v & G-2, S-1 \\
\hline 10 & PTN & dun kumut, kumut & $1,8,9,10,11$ & v & G-2, S-1 \\
\hline 11 & KLK & talun, temai & $1,8,10$ & v & G-2, S-1 \\
\hline 12 & KLM & talun & $1,8,10$ & v & G-2, S-1 \\
\hline 13 & KLO & taleng & $1,8,10$ & v & G-2, S-1 \\
\hline 14 & KLA & talun & $1,8,10$ & v & G-2, S-1 \\
\hline 15 & KAL & talun & $1,8,10$ & v & G-2, S-1 \\
\hline 16 & KBD & talun & $1,8,10$ & v & $\mathrm{G}-2, \mathrm{~S}-1$ \\
\hline 17 & KB K & kayu talun & $1,8,10$ & $\mathrm{v}$ & G-2, S-1 \\
\hline 18 & PEB & talei, tali & $1,8,10$ & $\mathrm{v}$ & G-2, S-1 \\
\hline
\end{tabular}

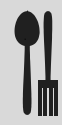

Notes : 


\section{Artocarpus elasticus Reinw. ex B1. (Moraceae)}

\section{TERAP. TERAP}

\section{DESCRIPTION}

Tree, to $45 \mathrm{~m}$; buttresses in old trees; leaves of saplings deeply cut into lobes, $60-180 \mathrm{~cm}$ long; adult leaves elliptic, stiff, leathery, 22.5-55 x 12.5$30 \mathrm{~cm}$; fruit yellow then brownish, 15-17.5 x 9-10 $\mathrm{cm}$, shaggy woolly, recurved spines; white pulp

\section{HABITAT}

Wild in lowland primary and secondary forests

\section{ORIGINS}

Malaysia and western Indonesia

\section{USES}

Bark for cloth, linings, walls, twine; sap for birdlime; fruit eaten

\section{REFERENCES}

Corner 1988: 516
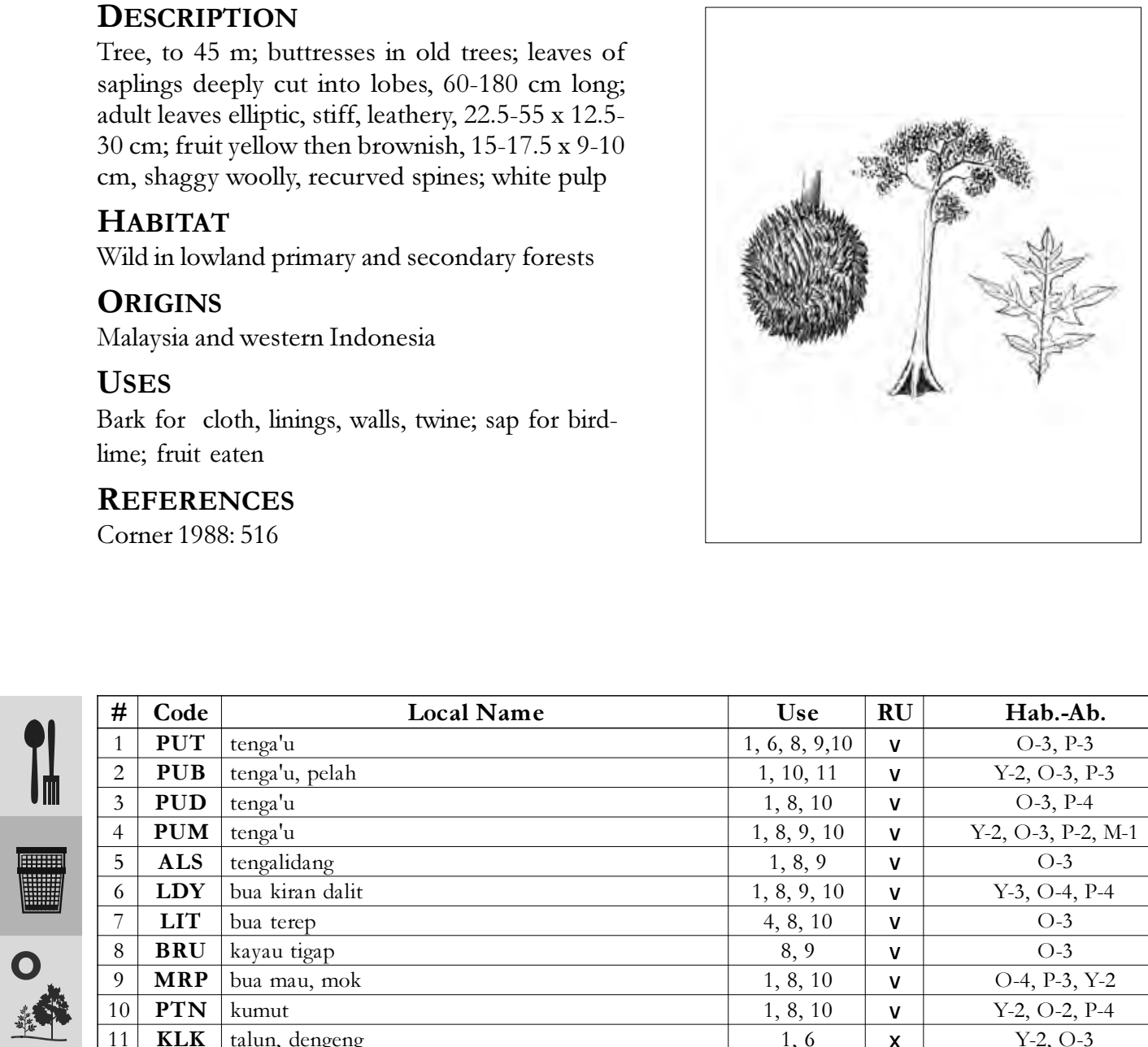

\begin{tabular}{|c|c|l|c|c|c|}
\hline$\#$ & Code & Local Name & Use & RU & Hab.-Ab. \\
\hline 1 & PUT & tenga'u & $1,6,8,9,10$ & $\mathbf{v}$ & O-3, P-3 \\
\hline 2 & PUB & tenga'u, pelah & $1,10,11$ & $\mathbf{v}$ & Y-2, O-3, P-3 \\
\hline 3 & PUD & tenga'u & $1,8,10$ & $\mathbf{v}$ & O-3, P-4 \\
\hline 4 & PUM & tenga'u & $1,8,9,10$ & $\mathbf{v}$ & Y-2, O-3, P-2, M-1 \\
\hline 5 & ALS & tengalidang & $1,8,9$ & $\mathbf{v}$ & O-3 \\
\hline 6 & LDY & bua kiran dalit & $1,8,9,10$ & $\mathbf{v}$ & Y-3, O-4, P-4 \\
\hline 7 & LIT & bua terep & $4,8,10$ & $\mathbf{v}$ & O-3 \\
\hline 8 & BRU & kayau tigap & 8,9 & $\mathbf{v}$ & O-3 \\
\hline 9 & MRP & bua mau, mok & $1,8,10$ & $\mathbf{v}$ & O-4, P-3, Y-2 \\
\hline 10 & PTN & kumut & $1,8,10$ & $\mathbf{v}$ & Y-2, O-2, P-4 \\
\hline 11 & KLK & talun, dengeng & 1,6 & $\mathbf{X}$ & Y-2, O-3 \\
\hline 12 & KLM & talun, talun & $1,8,9$ & $\mathbf{v}$ & ALL \\
\hline 13 & KLO & kaleng & 1,10 & $\mathbf{X}$ & Y-3, O-3 \\
\hline 14 & KLA & kian & 1,10 & $\mathbf{x}$ & Y-3, P-3 \\
\hline 15 & KAL & talun & 1,10 & $\mathbf{v}$ & Y-3, O-3, P-3 \\
\hline 16 & KBD & talun & $1,8,9,10$ & $\mathbf{v}$ & Y-3, O-3 \\
\hline 17 & KBK & talun & $1,8,9,10$ & $\mathbf{v}$ & Y-2, O-3, P-3 \\
\hline 18 & PEB & burai & $1,4,6$ & $\mathbf{v}$ & G-2, O-4, P-2 \\
\hline
\end{tabular}

Notes : 


\section{Artocarpus beteropbyllus Lam (Moraceae)}

\section{JACKFRUIT.NANGKA}

\section{DESCRIPTION}

Tree, to $30 \mathrm{~m}$; twigs, leaves glabrous; sapling leaves with lobes; leaves elliptic to obovate, tapered to stalk, thin leathery, $5-25 \times 3.5-12 \mathrm{~cm}$, shiny dark green above; fruit from trunk, $30-100 \mathrm{~cm} \times 25-50$ $\mathrm{cm}$, yellow, sharp warts, waxy-firm yellow pulp

\section{HABITAT}

Cultivated to $1000 \mathrm{~m}$

\section{ORIGINS}

India

\section{USES}

Fruit eaten, sold locally; bark for cordage, dye; timber for light construction; medicine

\section{REFERENCES}

Corner 1988: 517; PROSEA Vol. 2: 87

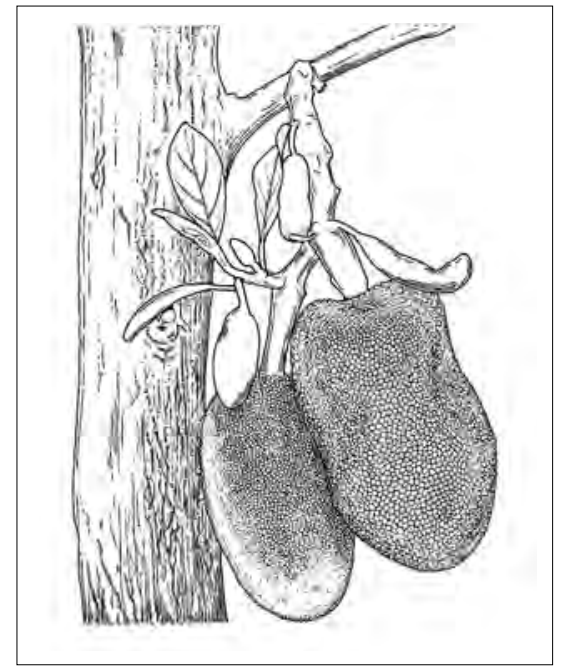

\begin{tabular}{|c|c|l|c|c|c|}
\hline \# & Code & Local Name & Use & RU & Hab.-Ab. \\
\hline 1 & PUT & mangga, mangka & $1,3,4,11$ & $\mathbf{v}$ & G-3, S-3 \\
\hline 2 & PUB & mangka & $1,3,4,11$ & $\mathbf{v}$ & G-3, S-3 \\
\hline 3 & PUD & mangga & $1,3,4,11$ & $\mathbf{v}$ & G-3, S-3 \\
\hline 4 & PUM & mangga & $1,3,4,11$ & $\mathbf{v}$ & G-3, S-3 \\
\hline 5 & ALS & bua' mangga & $1,3,4,11$ & $\mathbf{v}$ & G-3, S-3, Y-1 \\
\hline 6 & LDY & bua maka & $1,3,4,11$ & $\mathbf{v}$ & G-3, S-3, Y-1 \\
\hline 7 & LIT & bua' baduk & $1,3,4,11$ & $\mathbf{v}$ & G-3, S-3 \\
\hline 8 & BRU & wi manggah & $1,3,4,11$ & $\mathbf{v}$ & G-3, S-3 \\
\hline 9 & MRP & temangga & $1,3,4,11$ & $\mathbf{v}$ & G-3, S-3 \\
\hline 10 & PTN & baduk & $1,3,4,11$ & $\mathbf{v}$ & G-3, S-3 \\
\hline 11 & KLK & baduk & $1,3,4$ & $\mathbf{v}$ & G-2, S-2 \\
\hline 12 & KLM & badok & $1,3,4$ & $\mathbf{v}$ & G-2, S-2 \\
\hline 13 & KLO & baduk & $1,3,4$ & $\mathbf{v}$ & G-2, S-2 \\
\hline 14 & KLA & baduk & $1,3,4$ & $\mathbf{v}$ & G-2, S-2 \\
\hline 15 & KAL & baduk & $1,3,4$ & $\mathbf{v}$ & G-2, S-2 \\
\hline 16 & KBD & baduk & $1,3,4$ & $\mathbf{v}$ & G-2, S-2 \\
\hline 17 & KBK & baduk & $1,3,4$ & $\mathbf{v}$ & G-4, S-3 \\
\hline 18 & PEB & baduk & $1,3,4$ & $\mathbf{v}$ & G-1, S-2, Y-1 \\
\hline
\end{tabular}

Notes : 


\section{Artocarpus integer (Thunb.) Merr. (Moraceae)}

CHEMPEDAK. CEMPEDAK

\section{DESCRIPTION}

Tree, to $20 \mathrm{~m}$, grey brown bark; twigs, leaves with brown wiry hairs; leaves obovate-elliptic, 5-25 x 2.5-12 cm, dull, light green; fruits from trunk, cylindrical 20-35 x 10-15 cm, ochre to yellow brown, flat warts; pulp custardy-slimy, strong smell

\section{HABITAT}

Lowland and hill primary and secondary forests to $1300 \mathrm{~m}$; cultivated in villages

\section{ORIGINS}

Southeast Asia

\section{USES}

Fruit eaten raw or cooked, sold locally; seeds cooked; young leaves as vegetable; bark for cordage; sap for bird-lime; timber for light construction

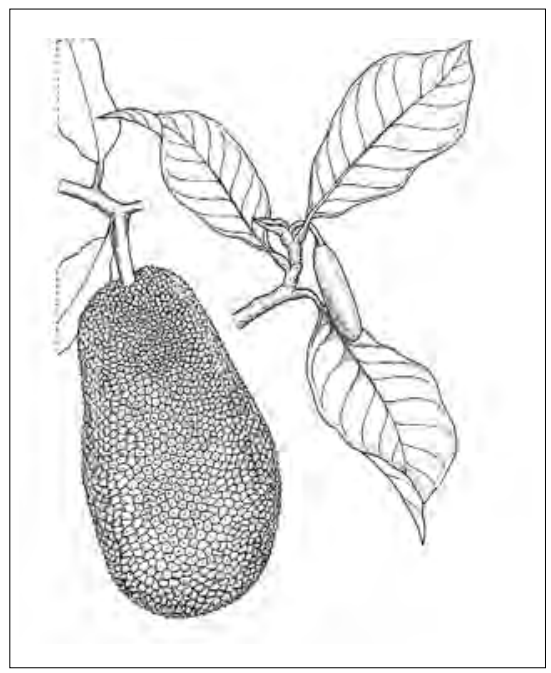

\section{REFERENCES}

Corner 1988: 518; PROSEA Vol. 2: 91

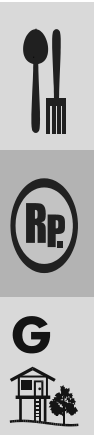

\begin{tabular}{|c|c|l|c|c|c|}
\hline$\#$ & Code & Local Name & Use & RU & Hab.-Ab. \\
\hline 1 & PUT & ka'ang & $1,6,11$ & $\mathbf{v}$ & G-3, P-4 \\
\hline 2 & PUB & ka'ang & $1,10,11$ & $\mathbf{v}$ & G-2, P-3 \\
\hline 3 & PUD & ka'ang & 1,11 & $\mathbf{v}$ & G-2, P-4 \\
\hline 4 & PUM & ka'ang & $1,6,11$ & $\mathbf{v}$ & G-2, O-2, P-4, M-3 \\
\hline 5 & ALS & bua' felutan & 1,11 & $\mathbf{v}$ & G-3, P-3 \\
\hline 6 & LDY & kayu belunu, belunu & $1,6,11$ & $\mathbf{v}$ & G-2, P-4, M-3 \\
\hline 7 & LIT & belunu, beluno & $1,10,11$ & $\mathbf{v}$ & G-3, P-3 \\
\hline 8 & BRU & wi balunu & 1,11 & $\mathbf{v}$ & G-2 \\
\hline 9 & MRP & lakang & 1,11 & $\mathbf{v}$ & G-3, P-4 \\
\hline 10 & PTN & nakan & $1,6,11$ & $\mathbf{v}$ & P-4 \\
\hline 11 & KLK & nakan & $1,6,11$ & $\mathbf{v}$ & P-3 \\
\hline 12 & KLM & nakan & $1,2,11$ & $\mathbf{v}$ & G-2, P-3 \\
\hline 13 & KLO & naghieng, navieng & 1,11 & $\mathbf{v}$ & P-3, M-3 \\
\hline 14 & KLA & nahan & 1,11 & $\mathbf{v}$ & P-3, M-3 \\
\hline 15 & KAL & nahin & 1,11 & $\mathbf{v}$ & G-3, P-3, M-3 \\
\hline 16 & KBD & nakan & 1 & $\mathbf{v}$ & O-2, P-4, M-4 \\
\hline 17 & KBK & nakan & 1,11 & $\mathbf{v}$ & G-2, P-4, M-3 \\
\hline 18 & PEB & paduk & 1 & $\mathbf{v}$ & O-2, P-4, M-4 \\
\hline
\end{tabular}

Notes : 


\section{Artocarpus lanceifolius Roxb. (Moraceae) (NO ENGLISH NAME). KELEDANG}

\section{DESCRIPTION}

Tree, to $36 \mathrm{~m}$, short buttresses, bark grey to black, smooth; twigs, leaves glabrous; leaves elliptic, 12.535 x 5-20 cm, shiny, sapling leaves deeply lobed; fruits $11 \times 10 \mathrm{~cm}$ olive brown, finely velvety minute brown hairs; pulp fleshy orange

\section{HABITAT}

Lowland and hill primary and secondary forests

\section{ORIGINS}

Malaysia and western Indonesia

\section{USES}

Fruit eaten, sold locally; timber for constuction; latex for birdlime, for medicine; hunting location when fruiting; bark for cordage

\section{REFERENCES}

Corner 1988: 519; TFM Vol. 3: 129;

Valkenburg 1997: 78

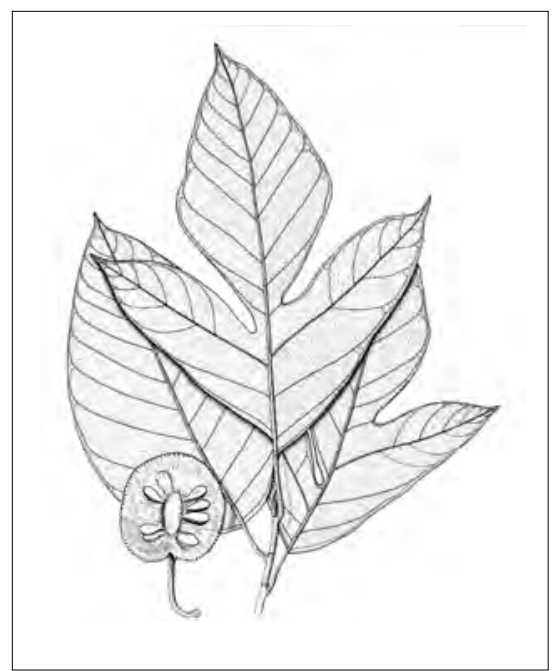

\begin{tabular}{|c|c|l|c|c|c|}
\hline \# & Code & \multicolumn{1}{|c|}{ Local Name } & Use & RU & Hab.-Ab. \\
\hline 1 & PUT & ulai & $1,2,5,10$ & $\mathbf{v}$ & O-2, P-3, M-2 \\
\hline 2 & PUB & ulai & $1,10,11$ & $\mathbf{v}$ & O-2, P-3 \\
\hline 3 & PUD & ulai & 1,10 & $\mathbf{v}$ & G-2, P-3 \\
\hline 4 & PUM & ulai & $1,2,10$ & $\mathbf{X}$ & O-2, P-2 \\
\hline 5 & ALS & bua' takulidang & 1,5 & $\mathbf{v}$ & G-2, P-3 \\
\hline 6 & LDY & takalidang, tekelidang & $1,9,10$ & $\mathbf{v}$ & G-1, O-3, P-3 \\
\hline 7 & LIT & kelidang & 1,10 & $\mathbf{v}$ & O-2, P-3 \\
\hline 8 & BRU & wi keledieng & 1 & $\mathbf{v}$ & G-1, P-3 \\
\hline 9 & MRP & bua kelereie, bua loei'i, kelere & 1,10 & $\mathbf{v}$ & P-3, M-3 \\
\hline 10 & PTN & basut & 1,10 & $\mathbf{v}$ & O-2 \\
\hline 11 & KLK & basut & 1,10 & $\mathbf{v}$ & P-3 \\
\hline 12 & KLM & pudu, ukuk & 1 & $\mathbf{v}$ & ALL \\
\hline 13 & KLO & ta' & 1 & $\mathbf{v}$ & P-3, M-3 \\
\hline 14 & KLA & basut & 1 & $\mathbf{v}$ & P-3, M-3 \\
\hline 15 & KAL & basut & 1 & $\mathbf{v}$ & P-3, M-3 \\
\hline 16 & KBD & tap, basut & 1 & $\mathbf{v}$ & P-3, M-3 \\
\hline 17 & KBK & pong ubi & 1,10 & $\mathbf{v}$ & P-3, M-3 \\
\hline 18 & PEB & kelirang, bungeu & & & M-3 \\
\hline
\end{tabular}

Notes : 


\section{Artocarpus nitidus Trec. (Moraceae)}

\section{SHINY TAMPANG. TAMPANG

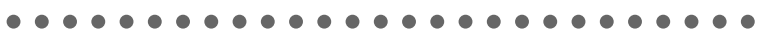

\section{DESCRIPTION}

Tree, to $35 \mathrm{~m}$, short buttresses; bark red brown, white exudate; leaves elliptic to obovate-oblong, $3.5-23 \times 1.5-9 \mathrm{~cm}$, shiny, leathery, short stalk 0.5$1 \mathrm{~cm}$; fruit shiny, smooth green turning orange pink with bright pink flesh, 2-6 cm diameter

\section{HABITAT}

Lowland primary and secondary forests; riverine and hill sides; cultivated in villages

\section{ORIGINS}

Malaysia, western Indonesia, Indochina

\section{USES}

Fruit eaten, sold locally; bark and roots added to betel

\section{REFERENCES}

Corner 1988: 520; Kessler and Sidiyasa 1994: 175;

PROSEA Vol. 2: 79; TFM Vol. 3: 131
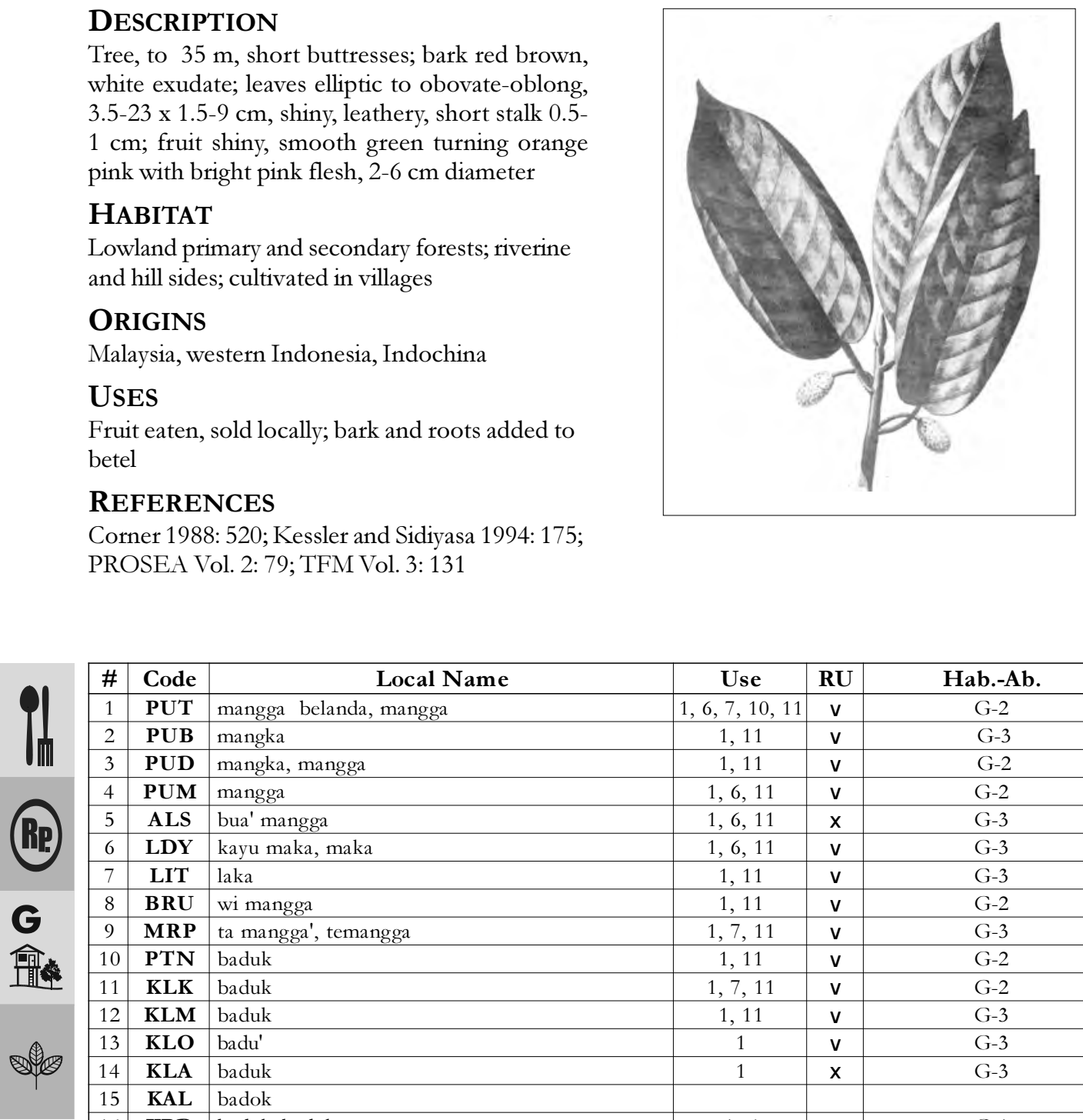

\begin{tabular}{|c|c|l|c|c|c|}
\hline$\#$ & Code & \multicolumn{1}{|c|}{ Local Name } & Use & RU & Hab.-Ab. \\
\hline 1 & PUT & mangga belanda, mangga & $1,6,7,10,11$ & $\mathbf{v}$ & G-2 \\
\hline 2 & PUB & mangka & 1,11 & $\mathbf{v}$ & G-3 \\
\hline 3 & PUD & mangka, mangga & 1,11 & $\mathbf{v}$ & G-2 \\
\hline 4 & PUM & mangga & $1,6,11$ & $\mathbf{v}$ & G-2 \\
\hline 5 & ALS & bua' mangga & $1,6,11$ & $\mathbf{X}$ & G-3 \\
\hline 6 & LDY & kayu maka, maka & $1,6,11$ & $\mathbf{v}$ & G-3 \\
\hline 7 & LIT & laka & 1,11 & $\mathbf{v}$ & G-3 \\
\hline 8 & BRU & wi mangga & 1,11 & $\mathbf{v}$ & G-2 \\
\hline 9 & MRP & ta mangga', temangga & $1,7,11$ & $\mathbf{v}$ & G-3 \\
\hline 10 & PTN & baduk & 1,11 & $\mathbf{v}$ & G-2 \\
\hline 11 & KLK & baduk & $1,7,11$ & $\mathbf{v}$ & G-2 \\
\hline 12 & KLM & baduk & 1,11 & $\mathbf{v}$ & G-3 \\
\hline 13 & KLO & badu' & 1 & $\mathbf{v}$ & G-3 \\
\hline 14 & KLA & baduk & 1 & $\mathbf{X}$ & G-3 \\
\hline 15 & KAL & badok & & & G-1 \\
\hline 16 & KBD & badok, baduk & 1,6 & $\mathbf{X}$ & \\
\hline 17 & KBK & baduk & & & $\mathbf{v}$ \\
\hline 18 & PEB & maduk & 1 & G-3, Y-2, O-3 \\
\hline
\end{tabular}

Notes : 


\section{Artocarpus odoratissimus Blanco (Moraceae)}

\section{MARANG. TERAP}

\section{DESCRIPTION}

Tree, to $25 \mathrm{~m}$, low buttresses; twigs yellow red hairs; leaves hairy, broadly elliptic-obovate, 16-50 x 11-28 $\mathrm{cm}$, margin slightly crenate, blunt tip; flowers in leaf axils; fruits globose to $16 \times 13 \mathrm{~cm}$, green yellow, stiff hairs $1 \mathrm{~cm}$ long; flesh white, juicy

\section{HABITAT}

Secondary forests to $1000 \mathrm{~m}$; cultivated in villages

\section{ORIGINS}

Borneo, Philippines

\section{USES}

Fruit eaten raw or cooked, sold locally; seeds cooked and eaten; latex for birdlime, for medicine; hunting location when fruiting

\section{REFERENCES}

PROSEA Vol. 2: 94

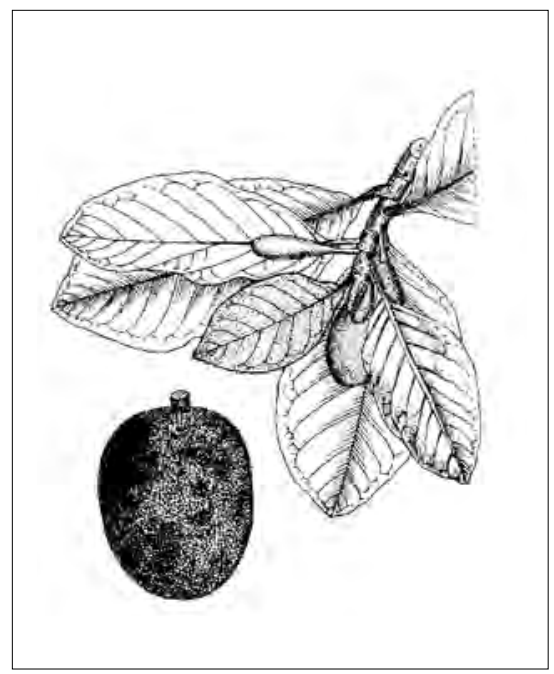

\begin{tabular}{|c|c|l|c|c|c|}
\hline$\#$ & Code & Local Name & Use & RU & Hab.-Ab. \\
\hline 1 & PUT & pelah & $1,10,11$ & $\mathbf{v}$ & G-3, Y-2, O-2, P-3 \\
\hline 2 & PUB & pelah & $1,10,11$ & $\mathbf{v}$ & G-3, P-2 \\
\hline 3 & PUD & pelah & $1,10,11$ & $\mathbf{v}$ & G-3, P-3 \\
\hline 4 & PUM & pelah & 1,11 & $\mathbf{v}$ & G-3, O-2, P-3, M-1 \\
\hline 5 & ALS & bua' kian & $1,7,11$ & $\mathbf{v}$ & G-3, P-3 \\
\hline 6 & LDY & kiran & 1,11 & $\mathbf{v}$ & G-4, O-2, P-2, M-1 \\
\hline 7 & LIT & kiran & $1,10,11$ & $\mathbf{v}$ & G-3, P-2 \\
\hline 8 & BRU & wi kien & 1,11 & $\mathbf{v}$ & G-3, O-2, P-2 \\
\hline 9 & MRP & kiang & $1,10,11$ & $\mathbf{v}$ & G-3, P-3, M-3 \\
\hline 10 & PTN & kian & 1 & $\mathbf{v}$ & G-3, O-2, P-1 \\
\hline 11 & KLK & kian & 1,11 & $\mathbf{v}$ & G-3, P-2 \\
\hline 12 & KLM & kian & $1,10,11$ & $\mathbf{v}$ & G-3 \\
\hline 13 & KLO & da'eng kegheng & 1 & $\mathbf{x}$ & G-3, P-2 \\
\hline 14 & KLA & kian, beva hian & 1 & $\mathbf{v}$ & G-3, O-3, P-3 \\
\hline 15 & KAL & keien & 1 & $\mathbf{v}$ & G-3 \\
\hline 16 & KBD & kian & $1,7,10$ & $\mathbf{v}$ & G-3 \\
\hline 17 & KBK & kian & 1 & $\mathbf{v}$ & G-3 \\
\hline 18 & PEB & basut & $1,2,11$ & $\mathbf{v}$ & G-4, S-2, Y-2, O-2, P-3 \\
\hline
\end{tabular}

Notes : 


\section{Artocarpus rigidus Blume (Moraceae)}

\section{MONKEY JACKFRUIT. TEMPUNAI, PUSSAR}

\section{DESCRIPTION}

Tree, to $35 \mathrm{~m}$, buttresses; bark grey brown, rough; twigs, leaf stalk, underside of leaves harshly hairy; leaves obovate-elliptic, 9-32 x 5-15 cm, blunt tip, stiff, shiny dark green; fruit round, orange, $7-13 \mathrm{~cm}$ diameter, stiff spines 7-9 $\mathrm{mm}$ long; pulp waxy orange

\section{HABITAT}

Lowland and hill forests to $1000 \mathrm{~m}$; cultivated in villages

\section{ORIGINS}

Malaysia, western Indonesia, Indochina

\section{USES}

Fruit eaten; timber for light construction; latex for medicine; hunting location when fruiting

\section{REFERENCES}

Corner 1988: 521; Kessler and Sidiyasa 1994: 175;

PROSEA Vol. 2: 79; TFM Vol. 3: 131
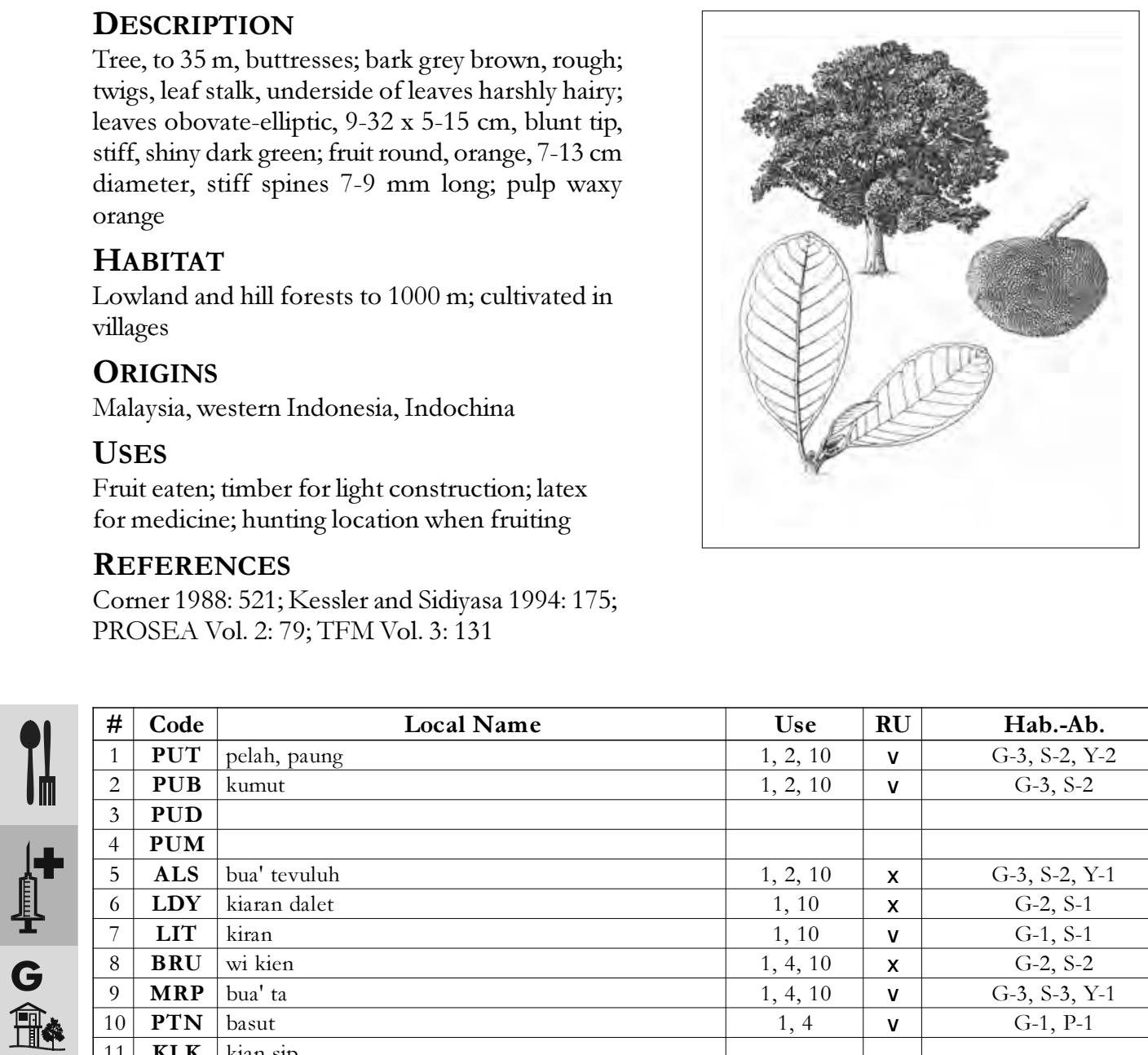

\begin{tabular}{|c|c|l|c|c|c|}
\hline$\#$ & Code & Local Name & Use & RU & Hab.-Ab. \\
\hline 1 & PUT & pelah, paung & $1,2,10$ & $\mathbf{V}$ & G-3, S-2, Y-2 \\
\hline 2 & PUB & kumut & $1,2,10$ & $\mathbf{V}$ & G-3, S-2 \\
\hline 3 & PUD & & & & \\
\hline 4 & PUM & & $1,2,10$ & $\mathbf{X}$ & G-3, S-2, Y-1 \\
\hline 5 & ALS & bua' tevuluh & 1,10 & $\mathbf{X}$ & G-2, S-1 \\
\hline 6 & LDY & kiaran dalet & 1,10 & $\mathbf{v}$ & G-1, S-1 \\
\hline 7 & LIT & kiran & $1,4,10$ & $\mathbf{X}$ & G-2, S-2 \\
\hline 8 & BRU & wi kien & $1,4,10$ & $\mathbf{V}$ & G-3, S-3, Y-1 \\
\hline 9 & MRP & bua' ta & 1,4 & $\mathbf{V}$ & G-1, P-1 \\
\hline 10 & PTN & basut & & & \\
\hline 11 & KLK & kian sip & 1 & $\mathbf{X}$ & G-1, P-2 \\
\hline 12 & KLM & kian bala & & & \\
\hline 13 & KLO & & & & \\
\hline 14 & KLA & & 1 & $\mathbf{X}$ & G-1, P-2 \\
\hline 15 & KAL & kian & & & \\
\hline 16 & KBD & & & & \\
\hline 17 & KBK & & & \\
\hline 18 & PEB & & & \\
\hline
\end{tabular}

Notes : 


\section{Ficus fistulosa Reinw. (Moraceae)}

\section{COMMON YELLOW STEM-FIG. LOLO, BENIING}

\section{DESCRIPTION}

Tree, 5-10 m, stout, no aerial roots, yellow exudate; leaves elliptic-obovate 7-33 x 2-15 cm, shallowly serrate, base narrowed or rounded, apex acute, stalk 1-6 cm long; figs on stems and branches, globular $1.5-3 \mathrm{~cm}$ diameter, green with white speckles

\section{HABITAT}

Primary and secondary forests to $2100 \mathrm{~m}$; open forest, edges, hedges and thickets

\section{ORIGINS}

India, southern China, Malaysia, western Indonesia

\section{USES}

Young fruit, shoots eaten raw; medicine; fruit for fishing bait; firewood

\section{REFERENCES}

Burkill 1966: 1025; Ochse 1980: 495; PROSEA

Vol. 8: 291; TFM Vol. 3: 146

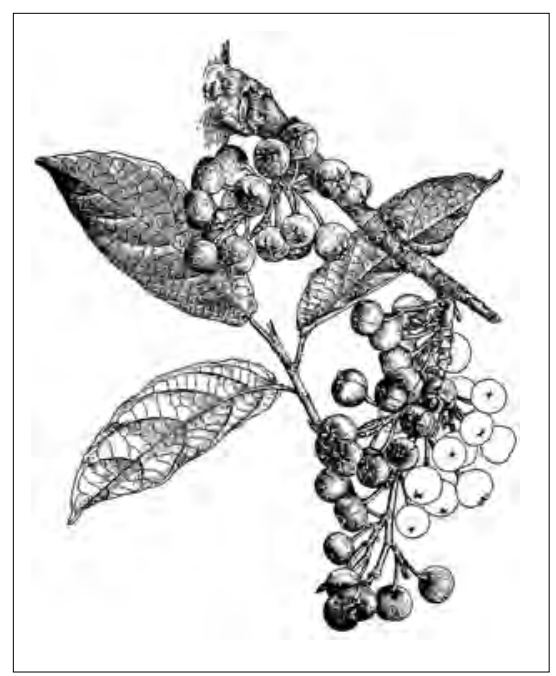

\begin{tabular}{|c|c|l|c|c|c|}
\hline$\#$ & Code & \multicolumn{1}{|c|}{ Local Name } & Use & RU & Hab.-Ab. \\
\hline 1 & PUT & abung ti fung & 10 & $\mathbf{X}$ & Y-2, O-3 \\
\hline 2 & PUB & abung ti & 1 & $\mathbf{X}$ & Y-2, O-3, P-2, \\
\hline 3 & PUD & entang awat & & $\mathbf{X}$ & Y-2, S-2 \\
\hline 4 & PUM & & & & \\
\hline 5 & ALS & taun njaubou & 7 & $\mathbf{X}$ & Y-2, O-2 \\
\hline 6 & LDY & kayu uk, don amel & & $\mathbf{X}$ & Y-2, O-3 \\
\hline 7 & LIT & & 7 & $\mathbf{X}$ & Y-3, O-3 \\
\hline 8 & BRU & uen belania & & $\mathbf{X}$ & Y-3, O-4 \\
\hline 9 & MRP & mawe bie' & 7 & $\mathbf{X}$ & Y-3, O-3, P-1 \\
\hline 10 & PTN & abung unjing & 2 & $\mathbf{X}$ & Y-2, O-3, P-4 \\
\hline 11 & KLK & abung selarang & 1 & $\mathbf{X}$ & ALL \\
\hline 12 & KLM & abung bala & & & \\
\hline 13 & KLO & abung buing & & & \\
\hline 14 & KLA & abung buin & & & \\
\hline 15 & KAL & & & & \\
\hline 16 & KBD & tung abung & & & \\
\hline 17 & KBK & abung putei & 1,7 & $\mathbf{V}$ & Y-2, O-3, P-4 \\
\hline 18 & PEB & pan batang, pan & & \\
\hline
\end{tabular}

Notes : 


\section{Ficus glomerata Roxb. (Moraceae)}

\section{Cluster FIG. ELO, LOA, ARAH}

\section{DESCRIPTION}

Tree, 20-30 m, buttressed, deciduous; leaves elliptic 6-15 x 1.8-7.5 cm, glaucous below; fruit in big clusters on branching leafless twigs on stems, branches; fig pear-shaped, short stalked, $2.5-5 \mathrm{~cm}$, rose red when ripe, streaked

\section{HABITAT}

Lowland riverine open forests; cultivated in India

\section{ORIGINS}

Malaysia and western Indonesia

\section{USES}

Fruits and shoots eaten; leaves eaten and for medicine, fodder and mulch; latex for industry; shade tree; host for lac insects

\section{REFERENCES}

Corner 1988: 684; Ochse 1980: 498; PROSEA

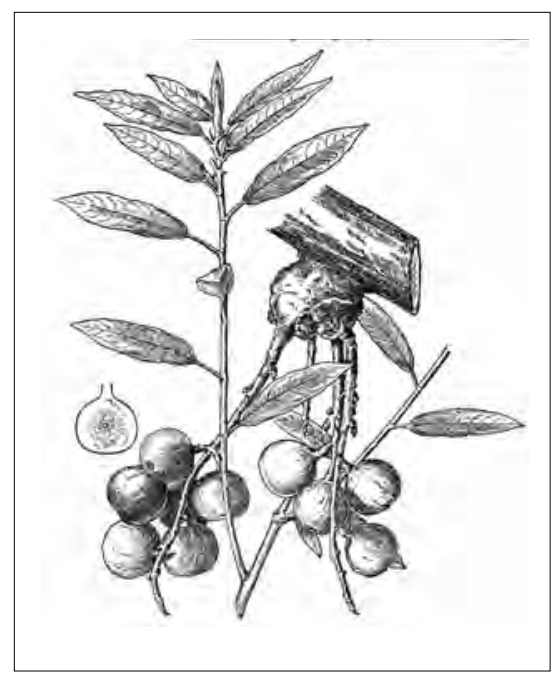

Vol. 2: 335; TFM Vol. 3: 154

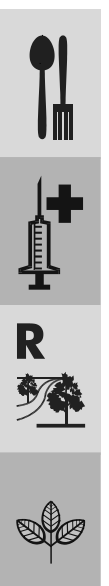

\begin{tabular}{|c|c|c|c|c|c|}
\hline \# & Code & Local Name & Use & RU & Hab.-Ab. \\
\hline 1 & PUT & nungu, abung ha & 1,2 & $x$ & $\mathrm{R}$ \\
\hline 2 & PUB & abung ha' & 1 & $x$ & $\mathrm{R}$ \\
\hline 3 & PUD & nungu & 1,2 & $x$ & $\mathrm{R}$ \\
\hline 4 & PUM & nungu & 1,2 & $x$ & $\mathrm{R}$ \\
\hline 5 & ALS & bua nungu & $1,2,10$ & $x$ & $\mathrm{R}$ \\
\hline 6 & LDY & bua geremita & $1,2,10$ & $\mathrm{x}$ & ALL \\
\hline 7 & LIT & gita & 1,10 & $x$ & $\mathrm{R}$ \\
\hline 8 & BRU & wi nungu & $1,2,10$ & $x$ & $\mathrm{R}$ \\
\hline 9 & MRP & bunga & $1,2,10$ & $x$ & $\mathrm{R}$ \\
\hline 10 & PTN & abung san, abung atuk & 1,2 & v & \\
\hline 11 & KLK & abung a & 2,10 & $x$ & $\mathrm{R}$ \\
\hline 12 & KLM & abung a & 1 & v & ALL \\
\hline 13 & KLO & abung a & 1 & $\mathrm{v}$ & $\mathrm{R}$ \\
\hline 14 & KLA & abung a, abung a' & 1 & $\mathrm{v}$ & $\mathrm{R}$ \\
\hline 15 & KAL & abung a & & & \\
\hline 16 & KBD & abung a' & 1,10 & $x$ & $\mathrm{R}$ \\
\hline 17 & KBK & abung a & & & \\
\hline 18 & PEB & abung a', abung acu & $1,2,10$ & $\mathrm{v}$ & $\mathrm{R}$ \\
\hline
\end{tabular}

\section{Notes :}




\section{Ficus xylopbylla Wall. ex Miq. (Moraceae)}

\section{BROAD-LEAF STRANGLING FIG. ARA DAUN LEBAR}

\section{DESCRIPTION}

Tree, to $40 \mathrm{~m}$, starting life epiphytically; wood soft red fawn color; leaves thick leathery obovate 15-30 x 6-15 cm, apex blunt, stalk $1.25-3 \mathrm{~cm}$ long; figs oblong sessile $2.5-5 \mathrm{~cm}$ long, ripening orange then purple red

\section{HABITAT}

Lowland primary forest

\section{ORIGINS}

Malaysia, western Indonesia, Indochina

\section{USES}

Timber for building, firewood; hunting site when fruiting

\section{REFERENCES}

Burkill 1966: 1033; TFM Vol. 3: 162

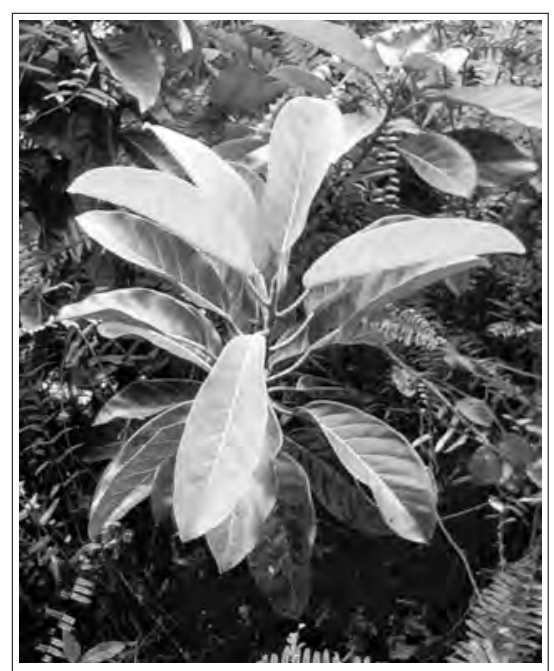

\begin{tabular}{|c|c|l|c|c|c|}
\hline$\#$ & Code & \multicolumn{1}{|c|}{ Local Name } & Use & RU & Hab.-Ab. \\
\hline 1 & PUT & lunuk & 10 & $\mathbf{X}$ & P-2, M-2 \\
\hline 2 & PUB & lunuk & & & \\
\hline 3 & PUD & lunuk & & & \\
\hline 4 & PUM & & & & \\
\hline 5 & ALS & lunuk & & & \\
\hline 6 & LDY & lunok & & & \\
\hline 7 & LIT & bungan & & & \\
\hline 8 & BRU & noe' & & & \\
\hline 9 & MRP & lunoh, lunue & & & \\
\hline 10 & PTN & lunok aya' & 10 & $\mathbf{X}$ & P-2, M-2 \\
\hline 11 & KLK & lunok, lunuk nyambung & 10 & $\mathbf{X}$ & P-2, M-2 \\
\hline 12 & KLM & lunok temengang, lunok membat & & & \\
\hline 13 & KLO & feng adieng & 10 & $\mathbf{X}$ & P-2 \\
\hline 14 & KLA & lunok temengang & & & \\
\hline 15 & KAL & lunok late' & 10 & $\mathbf{x}$ & P-3, M-2 \\
\hline 16 & KBD & lunuk & & & \\
\hline 17 & KBK & lunuk & 7,10 & $\mathbf{x}$ & P-3, M-2 \\
\hline 18 & PEB & lonok & & \\
\hline
\end{tabular}

Notes : 


\section{Musa paradisiaca L. (Musaceae)}

\section{BANANA, PLANTAIN. PISANG}

\section{DESCRIPTION}

Herb, 2-9 m, perennial, underground stem (corm) with short rhizomes that produce aerial shoots 20 $50 \mathrm{~cm}$ diameter; leaves long petiole, lanceolate 150$400 \times 70-100 \mathrm{~cm}$; inflorescences terminal, nodding, long stemmed, red bracts; fruit a seedless berry

\section{HABITAT}

Cultivated in village gardens and plantations

\section{ORIGINS}

Malaysia and western Indonesia

\section{USES}

Fruit, flower eaten, sold locally; leaves for wrapping; stem for fiber, water, storage

\section{REFERENCES}

Burkill 1966: 1536; Ochse 1980: 515;

PROSEA Vol. 2: 225
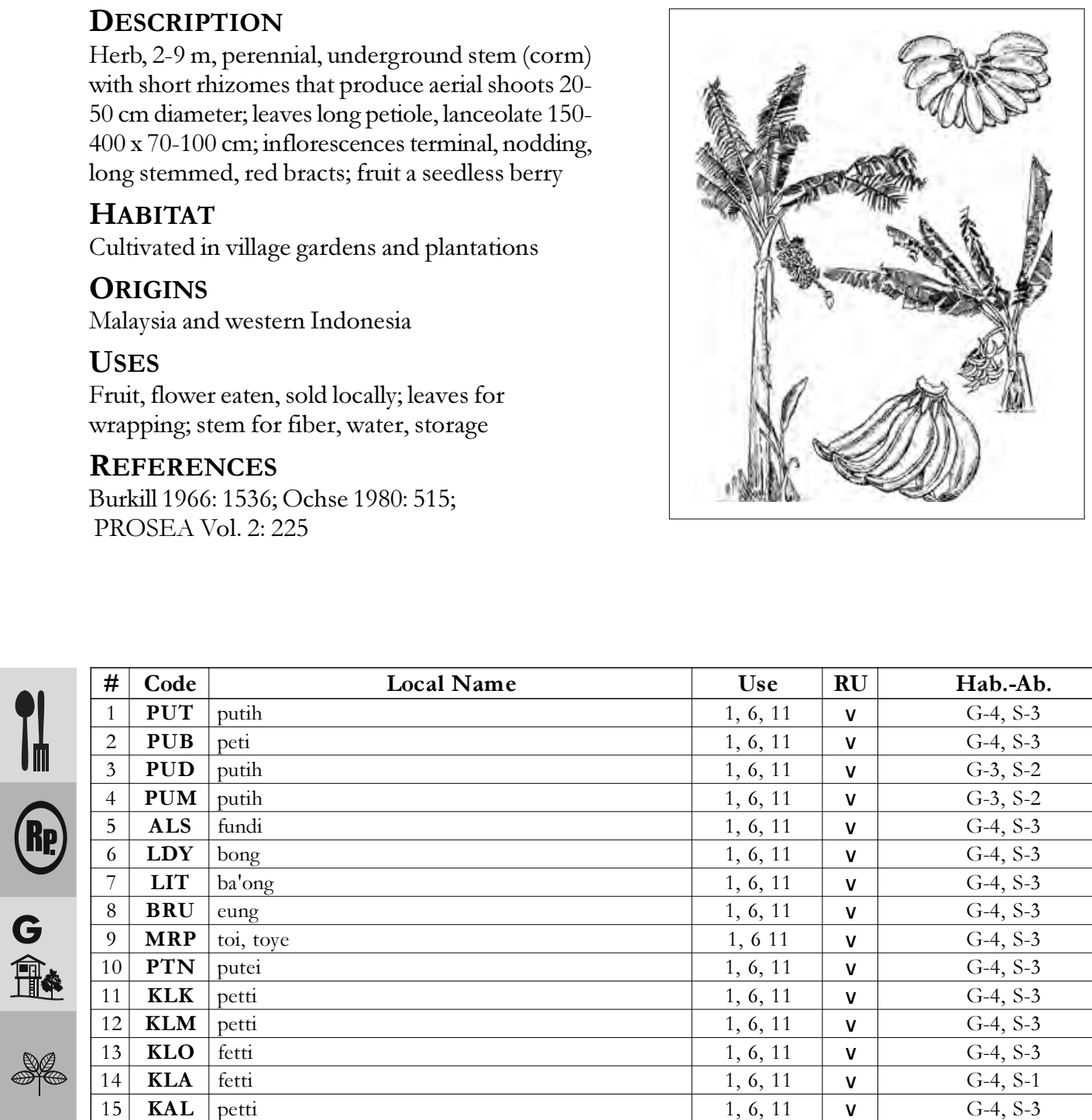

\begin{tabular}{|c|c|l|c|c|c|}
\hline$\#$ & Code & Local Name & Use & RU & Hab.-Ab. \\
\hline 1 & PUT & putih & $1,6,11$ & $\mathbf{v}$ & G-4, S-3 \\
\hline 2 & PUB & peti & $1,6,11$ & $\mathbf{v}$ & G-4, S-3 \\
\hline 3 & PUD & putih & $1,6,11$ & $\mathbf{v}$ & G-3, S-2 \\
\hline 4 & PUM & putih & $1,6,11$ & $\mathbf{v}$ & G-3, S-2 \\
\hline 5 & ALS & fundi & $1,6,11$ & $\mathbf{v}$ & G-4, S-3 \\
\hline 6 & LDY & bong & $1,6,11$ & $\mathbf{v}$ & G-4, S-3 \\
\hline 7 & LIT & ba'ong & $1,6,11$ & $\mathbf{v}$ & G-4, S-3 \\
\hline 8 & BRU & eung & $1,6,11$ & $\mathbf{v}$ & G-4, S-3 \\
\hline 9 & MRP & toi, toye & 1,611 & $\mathbf{v}$ & G-4, S-3 \\
\hline 10 & PTN & putei & $1,6,11$ & $\mathbf{v}$ & G-4, S-3 \\
\hline 11 & KLK & petti & $1,6,11$ & $\mathbf{v}$ & G-4, S-3 \\
\hline 12 & KLM & petti & $1,6,11$ & $\mathbf{v}$ & G-4, S-3 \\
\hline 13 & KLO & fetti & $1,6,11$ & $\mathbf{v}$ & G-4, S-3 \\
\hline 14 & KLA & fetti & $1,6,11$ & $\mathbf{v}$ & G-4, S-1 \\
\hline 15 & KAL & petti & $1,6,11$ & $\mathbf{v}$ & G-4, S-3 \\
\hline 16 & KBD & petti susu & $1,6,11$ & $\mathbf{v}$ & G-4, S-3 \\
\hline 17 & KBK & petti & $1,6,11$ & $\mathbf{v}$ & G-4, S-4 \\
\hline 18 & PEB & balak & $1,6,11$ & $\mathbf{v}$ & G-4, S-1 \\
\hline
\end{tabular}

Notes : 


\section{Knema sp. (Myristicaceae) \\ WILD NUTMEG. PENDARAHAN,DARA-DARA}

\section{DESCRIPTION}

Tree, $16 \mathrm{~m}, 35 \mathrm{~cm}$ dbh; bole straight, red exudate, bark brown black; twigs flakey; leaves lanceolate 2227 x 5-7 cm, sunken midrib, shiny brown above glabrous below, grooved petiole $1-2 \mathrm{~cm}, 20-22 \mathrm{sec}$. veins; fruit green and white globose $3.5 \times 2.5 \mathrm{~cm}$

\section{HABITAT}

Lowland primary forest

\section{ORIGINS}

Borneo

\section{USES}

Timber for building, firewood

\section{REFERENCES}

Kessler and Sidiyasa 1994: 181; TFM Vol. 1: 330

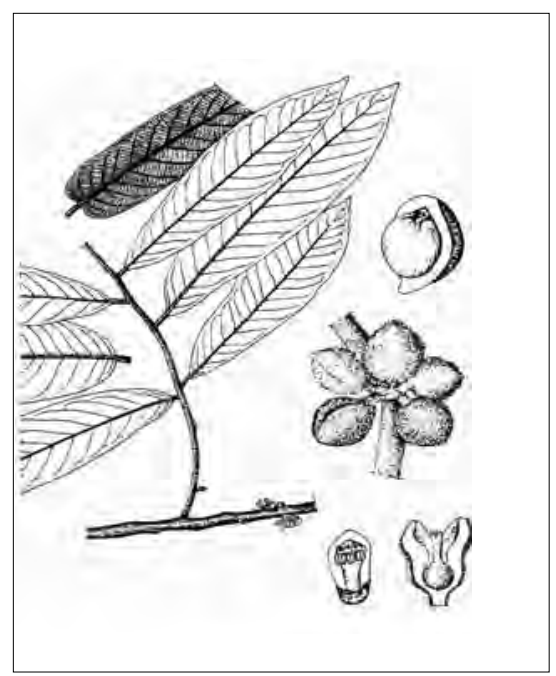

\begin{tabular}{|c|c|l|c|c|c|}
\hline$\#$ & Code & Local Name & Use & RU & Hab.-Ab. \\
\hline 1 & PUT & lera' & 7 & $\mathbf{X}$ & P-3, M-3 \\
\hline 2 & PUB & lerada & & & \\
\hline 3 & PUD & lera' & 7 & $\mathbf{X}$ & P-3, M-3 \\
\hline 4 & PUM & lera' & & $\mathbf{X}$ & P-3, M-3 \\
\hline 5 & ALS & lavak & & & \\
\hline 6 & LDY & seme dara & & & \\
\hline 7 & LIT & berdara' & & & \\
\hline 8 & BRU & kayau pialon & 7 & $\mathbf{X}$ & P-3, M-3 \\
\hline 9 & MRP & lao, alao & & & \\
\hline 10 & PTN & nyera' & & & \\
\hline 11 & KLK & nyera & & & \\
\hline 12 & KLM & nyeda & & & \\
\hline 13 & KLO & nyera & 7 & $\mathbf{X}$ & P-3, M-3 \\
\hline 14 & KLA & nyera & & & \\
\hline 15 & KAL & nyera & 3,7 & $\mathbf{x}$ & P-3, M-3 \\
\hline 16 & KBD & nyera & 3,7 & $\mathbf{x}$ & Y-1, O-2, P-4, M-4 \\
\hline 17 & KBK & nyera', nyera & 3,7 & $\mathbf{x}$ & P-3, M-3 \\
\hline 18 & PEB & nyere bup & & & \\
\hline
\end{tabular}

Notes : 


\section{Psidium guajava L. (Myrtaceae)}

\section{GUAVA. JAMBU BIJI}

\section{DESCRIPTION}

Tree, to $10 \mathrm{~m}$; branching from the base; bark smooth, green to red brown peeling; leaves opposite, petiole 3-10 mm, blade elliptical 5-15 x 3-7 cm; flowers white $3 \mathrm{~cm}$ diameter; fruit oblong $4-12 \mathrm{~cm}$ long green to yellow, flesh pink, red, or white

\section{HABITAT}

Cultivated in gardens, orchards, wild in secondary forests to $1500 \mathrm{~m}$.

\section{ORIGINS}

Tropical America

\section{USES}

Fruit eaten, sold locally; leaves for cooking, medicine, dye; timber for building

\section{REFERENCES}

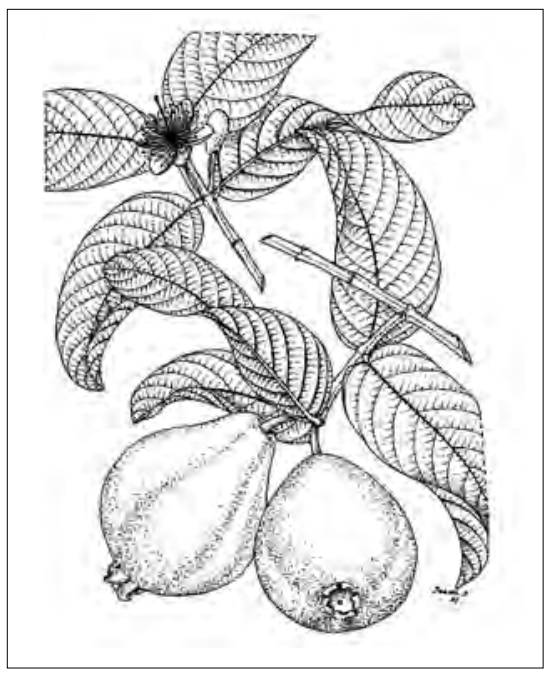

PROSEA Vol. 2: 266

\begin{tabular}{|c|c|c|c|c|c|}
\hline \# & Code & Local Name & Use & RU & Hab.-Ab. \\
\hline 1 & PUT & jambu, libun & 1,2 & v & G-2 \\
\hline 2 & PUB & jampu' & 1,2 & $x$ & G-2, Y-2 \\
\hline 3 & PUD & libun & 1,2 & $x$ & G-3 \\
\hline 4 & PUM & jambu & 1,2 & $x$ & G-2 \\
\hline 5 & ALS & jambu & 1,2 & $x$ & G-3 \\
\hline 6 & LDY & jambu & $1,2,11$ & $\mathrm{v}$ & G-2, S-3 \\
\hline 7 & LIT & kelibun, bua kelibun, bua libun & 1,2 & v & G-2 \\
\hline 8 & BRU & wi libo & 1,2 & $x$ & G-2 \\
\hline 9 & MRP & bing, bim, biung & 1,2 & v & G-2 \\
\hline 10 & PTN & limbun & 1,2 & $x$ & G-2 \\
\hline 11 & KLK & libun & 1,2 & $x$ & G-2 \\
\hline 12 & KLM & lebun, libun & 1,2 & v & G-2 \\
\hline 13 & KLO & livong, livung, liveng & 1,2 & v & G-3 \\
\hline 14 & KLA & livun & 1,2 & v & G-3 \\
\hline 15 & KAL & livun & & & \\
\hline 16 & KBD & nyibun & 1,2 & v & G-2 \\
\hline 17 & KBK & nyibun & & & \\
\hline 18 & PEB & nyibun, bue libu & 1 & $\mathrm{v}$ & G-4, S-2 \\
\hline
\end{tabular}

Notes : 


\section{Syzygium sp. (Myrtaceae) \\ (NO ENGLISH NAME). JAMBU}

\section{DESCRIPTION}

Tree, $1-5 \mathrm{~m}$, shrubby; leaves opposite, petiole 5 $\mathrm{mm}$ long, blade lanceolate-linear, $13-19 \times 2-3 \mathrm{~cm}$, acuminate tip and base; drying dull dark brown above, light brown or reddish brown below, more than 20 pairs sec. veins

\section{HABITAT}

Lowland primary and secondary forests, riverine

\section{ORIGINS}

Borneo

\section{USES}

Fruit for fishing bait; timber for light construction, firewood

\section{REFERENCES}

Corner 1988: 574; TFM Vol. 3: 182; PROSEA Vol 2: 297

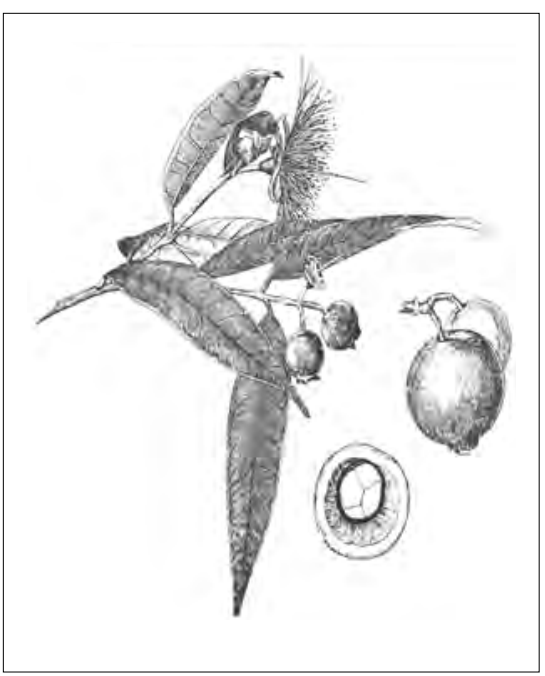

\begin{tabular}{|c|c|c|c|c|c|}
\hline \# & Code & Local Name & Use & RU & Hab.-Ab. \\
\hline 1 & PUT & ufo bulah, ufo ungei & 7,10 & $x$ & $\mathrm{O}-2, \mathrm{P}-3, \mathrm{M}-2$ \\
\hline 2 & PUB & ufo ungei & 1,7 & $x$ & $\mathrm{R}$ \\
\hline 3 & PUD & ufo ungei & 7 & $x$ & P-3 \\
\hline 4 & PUM & ufo ungei & 7 & $x$ & $\mathrm{R}$ \\
\hline 5 & ALS & pembarang, gematak & 10 & $x$ & ALL \\
\hline 6 & LDY & kayu tematak & 7 & $x$ & $\mathrm{R}$ \\
\hline 7 & LIT & uber & 7 & $x$ & $\mathrm{R}$ \\
\hline 8 & BRU & laang bal & 7,10 & $x$ & $\mathrm{R}$ \\
\hline 9 & MRP & boah & 10 & $x$ & $\mathrm{R}$ \\
\hline 10 & PTN & uba & 6,10 & $x$ & $\mathrm{Y}-2$ \\
\hline 11 & KLK & uba & 7 & $x$ & $\mathrm{R}$ \\
\hline 12 & KLM & uba, uba temen & 7 & $x$ & ALL \\
\hline 13 & KLO & obo mempei da'eng & & & \\
\hline 14 & KLA & uba & & & \\
\hline 15 & KAL & obo bai'i & & & \\
\hline 16 & KBD & oba bai & 7,10 & $x$ & $\mathrm{R}-3$ \\
\hline 17 & KBK & uba & & & \\
\hline 18 & PEB & uveu, uveu beu & 7,10 & $\mathrm{v}$ & $\mathrm{O}-4, \mathrm{P}-3$ \\
\hline
\end{tabular}

Notes : 


\section{Tristaniopsis whiteana (Griff.) Wil. \& Wat. (Myrtaceae)}

\section{(NO ENGLISH NAME). KAYU MULU, PELAWAN}

\section{DESCRIPTION}

Tree, to $25 \mathrm{~m}$; bark orange brown to pinkish grey, peeling off in large, scroll-like pieces which accumulate at base; leaves spirally arranged, thinly leathery obovate $7.5-20$ x 2.5-5.5 cm; flowers white with reddish calyx; fruit capsule $2.5 \mathrm{~cm}$

\section{HABITAT}

Lowland secondary forests; riversides, hillsides, ridges, sandy or stony soils

\section{ORIGINS}

Malaysia, western Indonesia, New Guinea

\section{USES}

Timber for firewood, tools and light construction

\section{REFERENCES}

Burkill 1966: 2226; Kessler and Sidiyasa 1994:

186; TFM Vol. 3: 253; PROSEA Vol. 5(3): 567

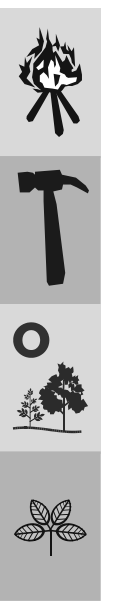

\begin{tabular}{|c|c|l|c|c|c|}
\hline \# & Code & Local Name & Use & RU & Hab.-Ab. \\
\hline 1 & PUT & belevan & 6,7 & $\mathbf{v}$ & O-3, P-2, M-1 \\
\hline 2 & PUB & belevan & 7 & $\mathbf{v}$ & O-2, P-2, M-2 \\
\hline 3 & PUD & belevan & 7 & $\mathbf{x}$ & O-1, P-1 \\
\hline 4 & PUM & & & & \\
\hline 5 & ALS & telemakas & 7 & $\mathbf{x}$ & O-4 \\
\hline 6 & LDY & belaban & 7 & $\mathbf{x}$ & O-3, P-1 \\
\hline 7 & LIT & belaban & 7 & $\mathbf{x}$ & S-1, O-2, P-3 \\
\hline 8 & BRU & bleben & 7 & $\mathbf{x}$ & O-3, P-2 \\
\hline 9 & MRP & blaban & 7 & $\mathbf{v}$ & O-2, P-2 \\
\hline 10 & PTN & blaban & 6,7 & $\mathbf{v}$ & O-3, P-2 \\
\hline 11 & KLK & bela'ban & 6,7 & $\mathbf{v}$ & O-4, P-2, M-1 \\
\hline 12 & KLM & bela'ban & 6,7 & $\mathbf{x}$ & P-3 \\
\hline 13 & KLO & belabeng & 6,7 & $\mathbf{v}$ & O-3, P-4, M-2 \\
\hline 14 & KLA & bela' ban & 6,7 & $\mathbf{x}$ & O-4, P-4, M-1 \\
\hline 15 & KAL & belaben & 6,7 & $\mathbf{v}$ & O-3, P-3 \\
\hline 16 & KBD & belaban & 6,7 & $\mathbf{v}$ & O-3, P-3 \\
\hline 17 & KBK & belaban & 6,7 & $\mathbf{x}$ & O-3, P-3 \\
\hline 18 & PEB & belavan totong & 6,7 & $\mathbf{v}$ & O-4, P-4, M-2 \\
\hline
\end{tabular}

Notes : 


\section{Averrboa carambola L. (Oxalidaceae)}

\section{STAR FRUIT. BELIMBING MANIS}

\section{DESCRIPTION}

Tree, to $15 \mathrm{~m}$, many branches; leaves compound with 2-5 pairs of ovate leaflets; flowers light red with purple heart; fruits yellow, smooth, shiny, waxy, 5 deep ridges, $5-17.5 \mathrm{~cm}$ long, yellow flesh

\section{HABITAT}

Cultivated in villages, orchards

\section{ORIGINS}

Unknown

\section{USES}

Fruit eaten, sold locally; timber for firewood, light construction

\section{REFERENCES}

Chin and Yong 1980: 53; PROSEA Vol. 2: 96

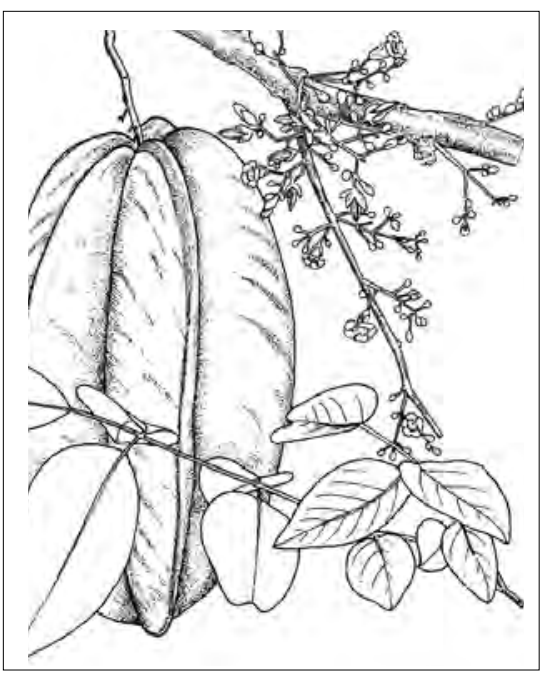

\begin{tabular}{|c|c|l|c|c|c|}
\hline \# & Code & \multicolumn{1}{|c|}{ Local Name } & Use & RU & Hab.-Ab. \\
\hline 1 & PUT & belimbing, pa & 1,7 & $\mathbf{V}$ & G-2 \\
\hline 2 & PUB & belimbing & 1 & $\mathbf{V}$ & G-2 \\
\hline 3 & PUD & pia & 1 & $\mathbf{V}$ & G-3 \\
\hline 4 & PUM & belimbing & 1 & $\mathbf{X}$ & G-3 \\
\hline 5 & ALS & bua' belimbing & 1 & $\mathbf{V}$ & G-2 \\
\hline 6 & LDY & kayu belimbing, belimbing & 1 & $\mathbf{X}$ & G-3 \\
\hline 7 & LIT & belimbing & 1,11 & $\mathbf{V}$ & G-2 \\
\hline 8 & BRU & wi belimbing & 1 & $\mathbf{X}$ & G-2 \\
\hline 9 & MRP & piea, pea, pi'ia & 1,6 & $\mathbf{V}$ & G-2 \\
\hline 10 & PTN & pia & 1 & $\mathbf{X}$ & G-2 \\
\hline 11 & KLK & pia', pi'a, pia & 1 & $\mathbf{X}$ & G-2 \\
\hline 12 & KLM & pe'a & 1 & $\mathbf{V}$ & G-2 \\
\hline 13 & KLO & feia & 1,7 & $\mathbf{X}$ & G-3 \\
\hline 14 & KLA & fi'a, pi'a & 1 & $\mathbf{X}$ & G-3 \\
\hline 15 & KAL & pe'a & & & \\
\hline 16 & KBD & pi'a & 1,6 & $\mathbf{X}$ & G-2 \\
\hline 17 & KBK & piah & & & \\
\hline 18 & PEB & pe'a', miu & 1 & $\mathbf{V}$ & G-3, S-3 \\
\hline
\end{tabular}

Notes : 


\section{Areca catechu L. (Palmae)}

\section{BETEL NUT, ARECA PALM. PINANG SIRIH

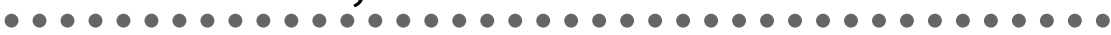

\section{DESCRIPTION}

Tree palm, 15-30 m; trunk straight cylindrical; leaves spirally arranged at top, petiole $100-150 \mathrm{~cm}$, leaf pinnae dark green $50-75 \mathrm{~cm}$ long linear to lanceolate, dentate top; flowers yellowish white, $5-7$ × 2-3 mm; fruit ovoid $3-6 \times 2-5 \mathrm{~cm}$, orange to red

\section{HABITAT}

Cultivated in villages

\section{ORIGINS}

Malaysia and western Indonesia

\section{USES}

Fruit chewed for narcotic effect, medicine, sold locally; cabbage eaten

\section{REFERENCES}

Burkill 1966: 225; LBN-LIPI 1978: 79;

Ochse 1980: 551; Whitmore 1985: 33
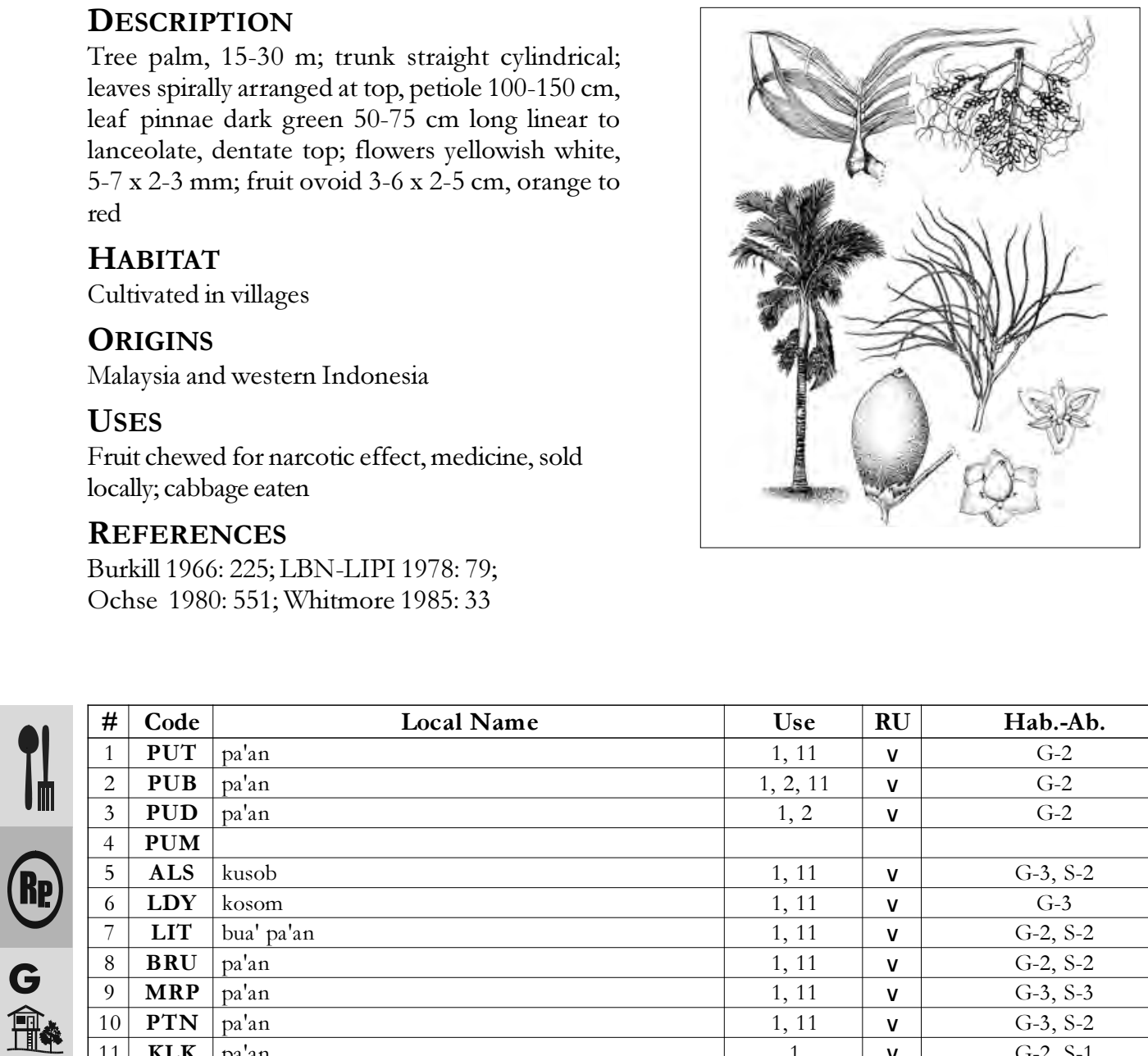

\begin{tabular}{|c|c|l|c|c|c|}
\hline \# & Code & Local Name & Use & RU & Hab.-Ab. \\
\hline 1 & PUT & pa'an & 1,11 & $\mathbf{v}$ & G-2 \\
\hline 2 & PUB & pa'an & $1,2,11$ & $\mathbf{v}$ & G-2 \\
\hline 3 & PUD & pa'an & 1,2 & $\mathbf{v}$ & G-2 \\
\hline 4 & PUM & & & & \\
\hline 5 & ALS & kusob & 1,11 & $\mathbf{v}$ & G-3, S-2 \\
\hline 6 & LDY & kosom & 1,11 & $\mathbf{v}$ & G-3 \\
\hline 7 & LIT & bua' pa'an & 1,11 & $\mathbf{v}$ & G-2, S-2 \\
\hline 8 & BRU & pa'an & 1,11 & $\mathbf{v}$ & G-2, S-2 \\
\hline 9 & MRP & pa'an & 1,11 & $\mathbf{v}$ & G-3, S-3 \\
\hline 10 & PTN & pa'an & 1,11 & $\mathbf{v}$ & G-3, S-2 \\
\hline 11 & KLK & pa'an & 1 & $\mathbf{v}$ & G-2, S-1 \\
\hline 12 & KLM & bua' pa'an & 1 & $\mathbf{v}$ & G-3 \\
\hline 13 & KLO & fa'an & 1 & $\mathbf{v}$ & G-3 \\
\hline 14 & KLA & pa'an & 1 & $\mathbf{v}$ & G-3 \\
\hline 15 & KAL & fa'an & 1 & $\mathbf{v}$ & G-3 \\
\hline 16 & KBD & pa'an & 1,2 & $\mathbf{v}$ & G-3, S-2 \\
\hline 17 & KBK & gahat & 1,11 & $\mathbf{x}$ & G-3 \\
\hline 18 & PEB & gat & 1,2 & $\mathbf{v}$ & G-3 \\
\hline
\end{tabular}

Notes : 


\section{Arenga brevipes Becc. (Palmae)}

\section{ARENGA.AREN}

\section{DESCRIPTION}

Tree palm, to $4 \mathrm{~m}$, single or multiple stems; leaves often flat, fishtail tip; leaflets in one plane, narrowly oblong, bitten ends, green above, light green to grey below; flowers purplish on pendulous spikes from stem; fruits round, $3-5 \mathrm{~cm}$ diameter, poisonous

\section{HABITAT}

Lowland and primary forest, hillsides usually above $700 \mathrm{~m}$

\section{ORIGINS}

Western Malaysia

\section{USES}

Pith for sago flour, leaf bud eaten, leaf stalks for light construction; hunting location

\section{REFERENCES}

LBN-LIPI 1978: 15; PROSEA Vol 9: 50

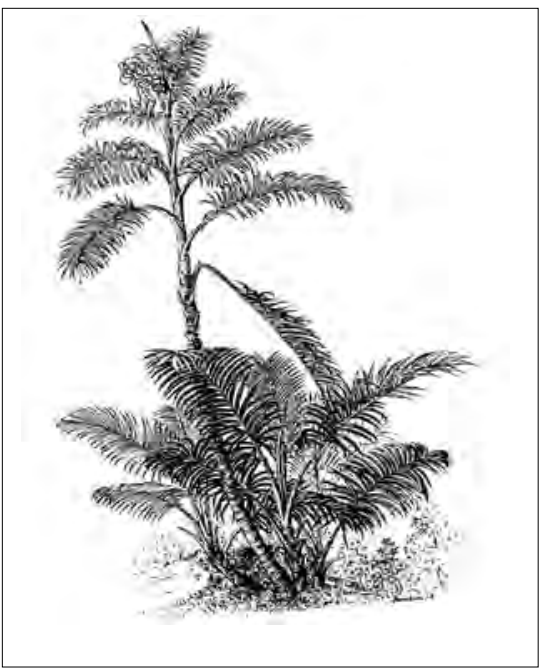

\begin{tabular}{|c|c|c|c|c|c|}
\hline \# & Code & Local Name & Use & RU & Hab.-Ab. \\
\hline 1 & PUT & fuh & & & \\
\hline 2 & PUB & noh & & & \\
\hline 3 & PUD & fuh & & & \\
\hline 4 & PUM & & & & \\
\hline 5 & ALS & tadan & & & \\
\hline 6 & LDY & tadan & & & \\
\hline 7 & LIT & & & & \\
\hline 8 & BRU & & & & \\
\hline 9 & MRP & labau & & & \\
\hline 10 & PTN & bu'o & & & \\
\hline 11 & KLK & bo'e & $1,4,10$ & $x$ & P-3, M-4 \\
\hline 12 & KLM & bo & $1,4,10$ & $x$ & P-3, M-4 \\
\hline 13 & KLO & $\mathrm{bu}$ & & & \\
\hline 14 & KLA & & & & \\
\hline 15 & KAL & buve & & & \\
\hline 16 & KBD & bo & $1,4,10$ & $x$ & P-2, M-3 \\
\hline 17 & KBK & baloh & & & \\
\hline 18 & PEB & bo & $1,4,10$ & $\mathrm{v}$ & P-2, M-3 \\
\hline
\end{tabular}

Notes : 


\section{Arenga undulatifolia Becc. (Palmae)}

\section{ARENGA. AREN GELORA

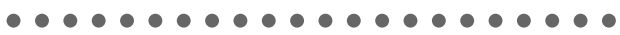

\section{DESCRIPTION}

Tree palm, to $10 \mathrm{~m}$; single to multiple stems; leaflet margins deeply toothed and undulating, dark green above, greyish below, fishtail tip; flowers and fruits on hanging spikes from stem; fruits green to black, round $3-5 \mathrm{~cm}$, poisonous

\section{HABITAT}

Lowland and hill secondary forest and old cultivated lands, riverine, steep banks, rocky thin soils

\section{ORIGINS}

Borneo endemic

\section{USES}

Pith for sago flour, highly preferred source of sago flour; leaf bud eaten; leaf stalks for hunting tools and light construction, blowpipe darts, plugs for darts; dried stems for firewood

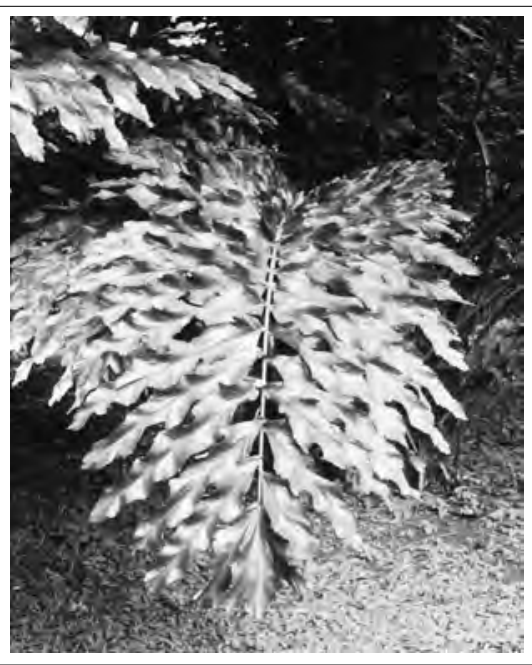

\section{REFERENCES}

LBN-LIPI 1978: 11; PROSEA Vol. 9: 50

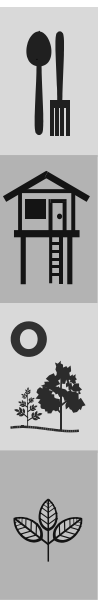

\begin{tabular}{|c|c|l|c|c|c|}
\hline$\#$ & Code & \multicolumn{1}{|c|}{ Local Name } & Use & RU & Hab.-Ab. \\
\hline 1 & PUT & jema, bia' & 1,4 & $\mathbf{x}$ & Y-2, O-3, P-3, M-2 \\
\hline 2 & PUB & jema & 1,4 & $\mathbf{x}$ & Y-2, O-3, P-4, M-4 \\
\hline 3 & PUD & jema & 1,4 & $\mathbf{v}$ & Y-2, O-3, P-4, M-1 \\
\hline 4 & PUM & jema & 1,4 & $\mathbf{x}$ & O-3, P-4 \\
\hline 5 & ALS & folod & 1,4 & $\mathbf{x}$ & O-3, P-3, M-2 \\
\hline 6 & LDY & pulod & 1,4 & $\mathbf{x}$ & O-3, P-4, M-4 \\
\hline 7 & LIT & pulud & 1,4 & $\mathbf{x}$ & Y-2, O-3, P-4, M-4 \\
\hline 8 & BRU & salang & 1,4 & $\mathbf{x}$ & O-3, P-4 \\
\hline 9 & MRP & talaya, talai, lehei & $1,4,7$ & $\mathbf{x}$ & O-2, P-3, M-2 \\
\hline 10 & PTN & talang & 1,4 & $\mathbf{x}$ & Y-2, O-4, P-3 \\
\hline 11 & KLK & talang & 1,4 & $\mathbf{x}$ & Y-2, O-3, P-4 \\
\hline 12 & KLM & talang & $1,4,7$ & $\mathbf{x}$ & O-3, P-3, M-2 \\
\hline 13 & KLO & talang & & $\mathbf{x}$ & O-3, P-3 \\
\hline 14 & KLA & talang & 1 & $\mathbf{x}$ & Y-2, O-3, P-3, M-3 \\
\hline 15 & KAL & talang & & & \\
\hline 16 & KBD & talang & $1,4,7,10$ & $\mathbf{x}$ & Y-3, O-3, P-3 \\
\hline 17 & KBK & talang & & & \\
\hline 18 & PEB & jaka & $1,4,10$ & $\mathbf{v}$ & Y-2, O-3, P-4, M-3 \\
\hline
\end{tabular}

Notes : 


\section{Calamus caesius B1. (Palmae)}

\section{GLAZED SILVER RATTAN. ROTAN SEGA \\ -}

\section{DESCRIPTION}

Rattan, high-climbing to $100 \mathrm{~m}$, prickly, clustering, dioecious; cane to $12 \mathrm{~mm}$ diameter; leaf $2 \mathrm{~m} ; 30$ leaflets alternate pairs, lanceolate $30 \times 5 \mathrm{~cm}$, green above, blue white below; inflorescence to $2 \mathrm{~m}$ long; fruits ovoid, $15 \times 10 \mathrm{~mm}$, green white scales

\section{HABITAT}

Lowland, hill forests to $800 \mathrm{~m}$; cultivated in gardens; dense stands of wild rattan in uplands attributed to cultivation (MRP, KBD, PEB)

\section{ORIGINS}

Southeast Asia

\section{USES}

Canes for basketry, mats, cordage, sold locally and to traders; highly preferred; fruit eaten, medicine

\section{REFERENCES}

Burkill 1966: 402; LBN-LIPI 1978: 97;

PROSEA Vol. 6: 43

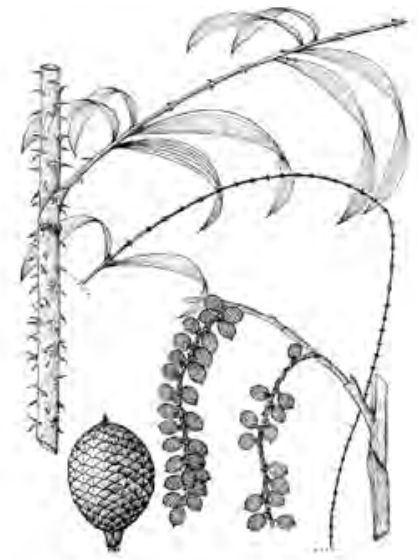

\begin{tabular}{|c|c|c|c|c|c|}
\hline \# & Code & Local Name & Use & RU & Hab.-Ab. \\
\hline 1 & PUT & wei ogoh & 8 & $\mathrm{x}$ & $\mathrm{G}-3, \mathrm{P}-4, \mathrm{M}-3$ \\
\hline 2 & PUB & wei ogoh & 8 & v & $\mathrm{G}-2, \mathrm{O}-2, \mathrm{P}-4, \mathrm{M}-4$ \\
\hline 3 & PUD & wei ogoh & 8,11 & v & G-2, P-4, M-2 \\
\hline 4 & PUM & wei ogoh & 8 & $x$ & G-2, P-3, M-4 \\
\hline 5 & ALS & awei sogoh & 2,8 & $x$ & G-2, P-3 \\
\hline 6 & LDY & uwe sugoh, uwe sogo & 8 & $x$ & G-3, O-2, P-3, M-4 \\
\hline 7 & LIT & wai itek & & & \\
\hline 8 & BRU & guwei ta' & 8 & $x$ & $\mathrm{O}-3, \mathrm{P}-3, \mathrm{M}-3$ \\
\hline 9 & MRP & ngwei ngga', ngue nga'; ngoe nga' & 8,11 & v & G-2, P-3, M-4 \\
\hline 10 & PTN & wei anggu, wei angga & 1,8 & $x$ & G-1, P-3 \\
\hline 11 & KLK & uwai sekka & 8,11 & v & G-2, P-3, M-2 \\
\hline 12 & KLM & uway sekka & 8,11 & $x$ & G-2, P-3, M-2 \\
\hline 13 & KLO & vei tevungen, uvei sekku & 8,11 & v & G-1, P-3, M-2 \\
\hline 14 & KLA & vai sekka & 8,11 & v & G-2, P-4, M-4 \\
\hline 15 & KAL & vei sekko & 8,11 & v & G-2, P-3, M-2 \\
\hline 16 & KBD & uwai sekka & 8,11 & v & G-2, O-3, P-3, M-2 \\
\hline 17 & KBK & uwai sekka & 8,11 & v & $\mathrm{O}-2, \mathrm{P}-3, \mathrm{M}-3$ \\
\hline 18 & PEB & lakeu linau & 8,11 & v & $\mathrm{O}-2, \mathrm{P}-4, \mathrm{M}-4$ \\
\hline
\end{tabular}

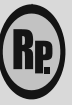

Notes : 


\section{Calamus ornatus Blume (Palmae)}

\section{STOUT RATTAN. ROTAN TEBAL}

\section{DESCRIPTION}

Rattan, climbing to $50 \mathrm{~m}$, prickly, massive clustering, dioecious; cane to $40 \mathrm{~mm}$ diameter; leaf stem to 10 $\mathrm{m}$; leaflets opposite, lanceolate $50-80 \times 5-8 \mathrm{~cm}$, prickly above; inflorescence to $8 \mathrm{~m}$ long; fruit ellipsoid, 3 x $2 \mathrm{~cm}, 15$ rows scales yellow brown to black

\section{HABITAT}

Lowland and hill primary and secondary forests to $1000 \mathrm{~m}$; prefers fertile or dry soils

\section{ORIGINS}

Malaysia, western Indonesia, Philippines

\section{USES}

Fruits and leafbud eaten, cane water; canes for furniture, cordage; sold locally as handicrafts; hunting location

\section{REFERENCES}

Burkill 1966: 404; PROSEA Vol. 6: 59

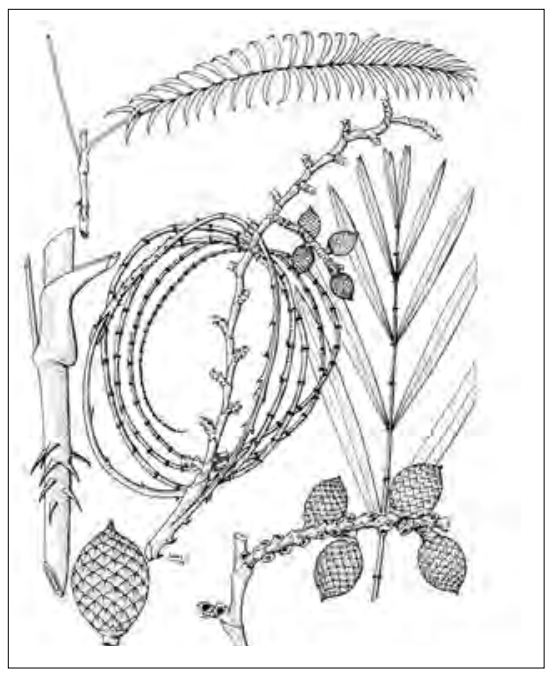

\begin{tabular}{|c|c|c|c|c|c|}
\hline \# & Code & Local Name & Use & RU & Hab.-Ab. \\
\hline 1 & PUT & wei tefungan, wei tevungan & $1,6,10,11$ & $\mathrm{x}$ & G-1, P-3, M-3 \\
\hline 2 & PUB & tevungan, wei tevungan & 1 & $x$ & $\mathrm{O}-2, \mathrm{P}-3, \mathrm{M}-2$ \\
\hline 3 & PUD & wei tevungan, tefungan & 1 & $x$ & P-3 \\
\hline 4 & PUM & tefungan & 1,6 & $x$ & $\mathrm{O}-2, \mathrm{P}-3, \mathrm{M}-4$ \\
\hline 5 & ALS & owoi lasun & $1,6,10$ & $x$ & P-3 \\
\hline 6 & LDY & uwe lasun & $1,6,10$ & $\mathbf{v}$ & $\mathrm{O}-2, \mathrm{P}-4$ \\
\hline 7 & LIT & wai lasun, lasun & 1,6 & $x$ & P-3, M-4 \\
\hline 8 & BRU & but lawai & 1,6 & $x$ & $\mathrm{O}-1, \mathrm{P}-3, \mathrm{M}-3$ \\
\hline 9 & MRP & nguwei lawan, ngoe lawan, lehung & 1,6 & $x$ & $\mathrm{O}-2, \mathrm{P}-3, \mathrm{M}-3$ \\
\hline 10 & PTN & tebungan, uwey tevungan & $1,6,10$ & $x$ & $\mathrm{O}-2, \mathrm{P}-3, \mathrm{M}-3$, \\
\hline 11 & KLK & wei tebungan, uwai tebungen & 1,6 & $x$ & P-2 \\
\hline 12 & KLM & uwey tebungan, uway tebungan & $1,6,10$ & $x$ & ALL \\
\hline 13 & KLO & vei tevungen & $1,6,8$ & v & $\mathrm{O}-1, \mathrm{P}-3, \mathrm{M}-2$ \\
\hline 14 & KLA & vai tevungen, vai tevungan & $1,6,8$ & v & P-3, M-4 \\
\hline 15 & KAL & vai tevungen & $1,6,8$ & $\mathbf{v}$ & $\mathrm{O}-2, \mathrm{P}-3, \mathrm{M}-3$ \\
\hline 16 & KBD & uwai tebungan, uwai tebongan & $1,6,8,11$ & v & $\mathrm{O}-2, \mathrm{P}-3, \mathrm{M}-3$ \\
\hline 17 & KBK & uwai tebungan & $1,6,8$ & $\mathrm{v}$ & $\mathrm{O}-3, \mathrm{P}-3, \mathrm{M}-3$ \\
\hline 18 & PEB & lakeu janan, janan & $1,6,8,11$ & $\mathrm{v}$ & $\mathrm{O}-3, \mathrm{P}-4$ \\
\hline
\end{tabular}

Notes : 


\section{Caryota mitis Lour. (Palmae)}

\section{FISHTAIL PALM. SARAI, RABOK, TUKAS}

\section{DESCRIPTION}

Tree palm, 4-12 m, clumped, 1-3 stems that die after fruiting; leaves twice pinnate 150 x $80 \mathrm{~cm}$; leaflets blunt jagged tip; monoecious; flowers in spiral on mass of hanging spikes, 36-72 cm long; fruits round $1.25 \mathrm{~cm}$ plum red, poisonous

\section{HABITAT}

Lowland secondary forest; cultivated in village gardens

\section{ORIGINS}

Tropical Asia

\section{USES}

Pith for sago flour, leaf bud eaten; leaves for thatch; ornamental plant

\section{REFERENCES}

Burkill 1966: 476; LBN-LIPI 1978: 105; Ochse 1980: 559; Whitmore 1985: 44; PROSEA Vol. 9: 66

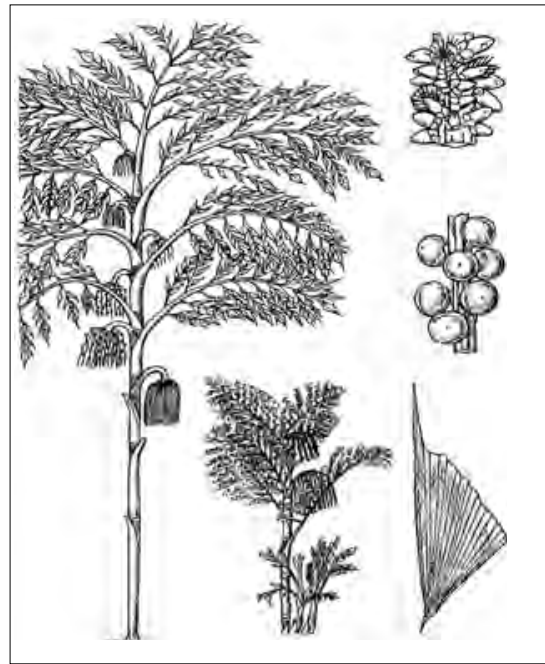

\begin{tabular}{|c|c|l|c|c|c|}
\hline$\#$ & Code & Local Name & Use & RU & Hab.-Ab. \\
\hline 1 & PUT & lelih & 1,4 & $\mathbf{x}$ & P-3 \\
\hline 2 & PUB & lelih & 1,4 & $\mathbf{x}$ & O-1, P-1 \\
\hline 3 & PUD & leli & 1,4 & $\mathbf{x}$ & O-1, P-1 \\
\hline 4 & PUM & lelih & 1,4 & $\mathbf{x}$ & O-2, P-2 \\
\hline 5 & ALS & dudug & 1 & $\mathbf{x}$ & G-1, S-2, O-3 \\
\hline 6 & LDY & liman labo & 1 & $\mathbf{x}$ & G-1, S-2, O-3 \\
\hline 7 & LIT & riman di'it & 1 & $\mathbf{x}$ & G-1, S-2, O-3 \\
\hline 8 & BRU & labiau & 1 & $\mathbf{x}$ & G-1, S-2, O-3 \\
\hline 9 & MRP & meheh, leheh & 1 & $\mathbf{x}$ & G-2, O-3, P-1 \\
\hline 10 & PTN & lisi & 1,4 & $\mathbf{x}$ & G-2, O-3, P-3 \\
\hline 11 & KLK & lisi & 1,4 & $\mathbf{x}$ & G-1, S-1, O-3, P-2 \\
\hline 12 & KLM & lisi & 1,4 & $\mathbf{x}$ & O-3, P-3 \\
\hline 13 & KLO & lisi & 1,4 & $\mathbf{x}$ & O-2, P-3, M-3 \\
\hline 14 & KLA & lisi & 1,4 & $\mathbf{x}$ & O-2, P-3, M-3 \\
\hline 15 & KAL & lisi & 1,4 & $\mathbf{x}$ & O-2, P-3, M-3 \\
\hline 16 & KBD & lesse & 1,4 & $\mathbf{x}$ & O-2, P-3, M-3 \\
\hline 17 & KBK & borung & 1,4 & $\mathbf{x}$ & O-2, P-4, M-4 \\
\hline 18 & PEB & lesi & 1,4 & $\mathbf{v}$ & O-2, P-3, M-3 \\
\hline
\end{tabular}

Notes : 
Caryota no Becc. (Palmae)

\section{KING FISHTAIL PALM. SARAI RAJA}

\section{DESCRIPTION}

Tree palm, to $25 \mathrm{~m}$, solitary, trunk bulging in middle; leaves terminal, twice pinnate 4-6 $\mathrm{m}$ long; leaflets sessile, fan-shaped, densely packed; monoecious; flowers in spiral on mass of hanging spikes from leaf axils; fruit round dark purple to black

\section{HABITAT}

Lowland and hill primary and secondary forests, ridges, planted in village lands

\section{ORIGINS}

Borneo

\section{USES}

Pith for sago flour, leaf bud eaten; leaves for thatch, cordage; ornamental plant

\section{REFERENCES}

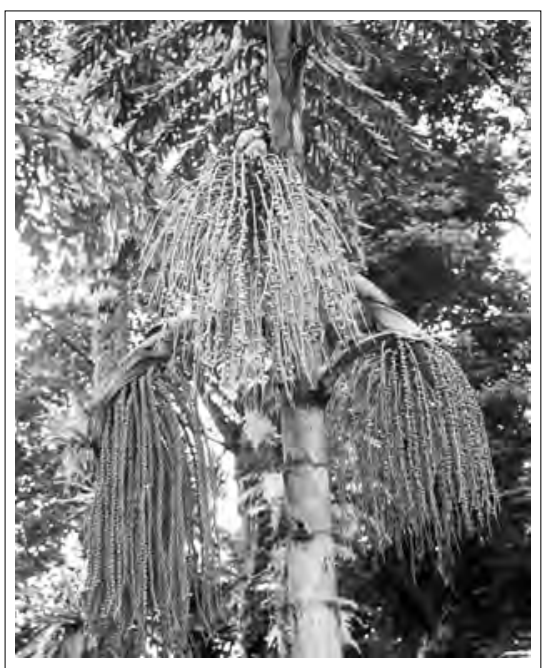

LBN-LIPI 1978: 109; PROSEA Vol. 9: 66

\begin{tabular}{|c|c|c|c|c|c|c|}
\hline & \# & Code & Local Name & Use & RU & Hab.-Ab. \\
\hline & 1 & PUT & iman & $1,4,8$ & $x$ & $\mathrm{G}-1, \mathrm{O}-2, \mathrm{P}-2$ \\
\hline & 2 & PUB & iman & $1,4,8$ & $x$ & G-1, O-2, P-2 \\
\hline & 3 & PUD & iman & $1,4,8$ & $x$ & $\mathrm{O}-1, \mathrm{P}-2$ \\
\hline & 4 & PUM & iman & $1,4,8$ & $x$ & $\mathrm{O}-1, \mathrm{P}-1, \mathrm{M}-2$ \\
\hline & 5 & ALS & buton & $1,4,8$ & $x$ & P-2, M-2 \\
\hline & 6 & LDY & liman & 1,8 & $x$ & $\mathrm{O}-2, \mathrm{M}-3$ \\
\hline & 7 & LIT & riman raye & 1,8 & $x$ & G-1, S-1, M-2 \\
\hline & 8 & BRU & iman & 1,8 & $x$ & G-1, S-1, M-2 \\
\hline & 9 & MRP & mien & 1,8 & $x$ & G-1, S-1, P-1, M-2 \\
\hline & 10 & PTN & iman & 1,8 & $x$ & G-2, S-2, P-3, M-2 \\
\hline & 11 & KLK & iman & 1,8 & $x$ & G-2, S-2, P-3 \\
\hline & 12 & KLM & eman & 1,8 & $x$ & G-1, S-1, M-3 \\
\hline & 13 & KLO & aimeng & 1,8 & $x$ & G-1, S-1, M-3 \\
\hline & 14 & KLA & iman & 1,8 & $x$ & $\mathrm{G}-2, \mathrm{~S}-1, \mathrm{O}-2, \mathrm{P}-3, \mathrm{M}-2$ \\
\hline & 15 & KAL & emen & 1,8 & $x$ & G-2, S-2, O-2, P-3 \\
\hline & 16 & KBD & iman & 1,8 & $x$ & G-2, S-2, O-2, P-3 \\
\hline & 17 & KBK & iman & 1,4 & $x$ & G-2, O-2, P-2, M-2 \\
\hline & 18 & PEB & iman & $1,4,8$ & $\mathrm{x}$ & $\mathrm{G}-2, \mathrm{O}-2, \mathrm{P}-2, \mathrm{M}-2$ \\
\hline
\end{tabular}

Notes : 
Cocos nucifera L. (Palmae)

COCONUT PALM. KELAPA

-

\section{DESCRIPTION}

Tree palm, 5-30 m, smooth grey trunk; terminal leaves to $7 \mathrm{~m}$ long, over 100 pairs leaflets, held flat in one plane; leaves falling; inflorescence from leaf axils, $1.5 \mathrm{~m}$ long with up to 40 spikes $60 \mathrm{~cm}$ long; fruit big hard nut with husk green; brown when ripe

\section{HABITAT}

Cultivated in villages, riverine, to $600 \mathrm{~m}$

\section{ORIGINS}

Unknown

\section{USES}

Fruit eaten, sold locally; leaves for thatch, cordage, basketry and handicrafts; timber for tools

\section{REFERENCES}

Burkill 1966: 604; Ochse 1980: 562;

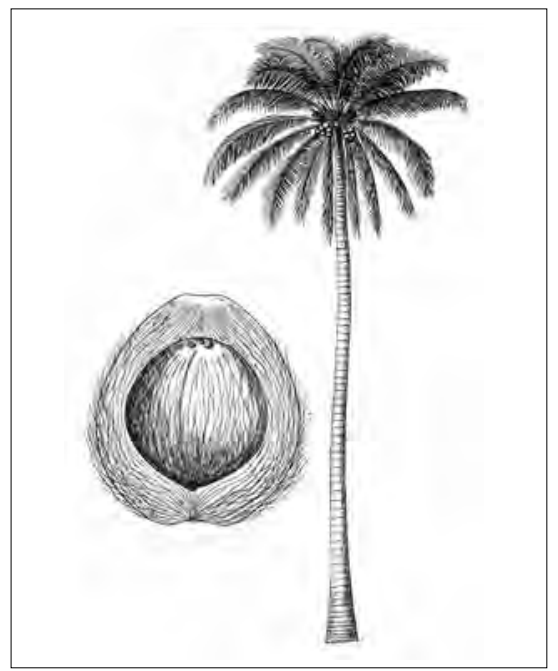

Whitmore 1973: 48

\begin{tabular}{|c|c|l|c|c|c|}
\hline$\#$ & Code & Local Name & Use & RU & Hab.-Ab. \\
\hline 1 & PUT & nyu & $1,4,6,8,11$ & $\mathbf{v}$ & G-4, S-4 \\
\hline 2 & PUB & nyo & $1,4,6,8,11$ & $\mathbf{v}$ & G-4, S-4 \\
\hline 3 & PUD & nyu' & $1,4,6,8,11$ & $\mathbf{v}$ & G-4, S-4 \\
\hline 4 & PUM & nyu & $1,4,6,8,11$ & $\mathbf{v}$ & G-4, S-4 \\
\hline 5 & ALS & fiasau & $1,4,6,8,11$ & $\mathbf{v}$ & G-4, S-4 \\
\hline 6 & LDY & butan & $1,4,6,8,11$ & $\mathbf{v}$ & G-4, S-4 \\
\hline 7 & LIT & bua' butan & $1,4,6,8,11$ & $\mathbf{v}$ & G-4, S-4 \\
\hline 8 & BRU & nyoh & $1,4,6,8,11$ & $\mathbf{v}$ & G-4, S-4 \\
\hline 9 & MRP & nyau & $1,4,6,8,11$ & $\mathbf{v}$ & G-4, S-4 \\
\hline 10 & PTN & nyoh & $1,4,6,8,11$ & $\mathbf{v}$ & G-4, S-4 \\
\hline 11 & KLK & nyo & $1,4,6,8,11$ & $\mathbf{v}$ & G-4, S-4 \\
\hline 12 & KLM & nyo & $1,4,6,8,11$ & $\mathbf{v}$ & G-4, S-4 \\
\hline 13 & KLO & nyo & $1,4,6,8,11$ & $\mathbf{v}$ & G-4, S-4 \\
\hline 14 & KLA & nyo & $1,4,6,8,11$ & $\mathbf{v}$ & G-4, S-4 \\
\hline 15 & KAL & nyo & $1,4,6,8,11$ & $\mathbf{v}$ & G-4, S-4 \\
\hline 16 & KBD & nyo' & $1,4,6,8,11$ & $\mathbf{v}$ & G-4, S-4 \\
\hline 17 & KBK & nyo & $1,4,6,8,11$ & $\mathbf{v}$ & G-4, S-4 \\
\hline 18 & PEB & nyo' & $1,4,6,8,11$ & $\mathbf{v}$ & G-4, S-4 \\
\hline
\end{tabular}

Notes : 


\section{Eugeissona utilis Becc. (Palmae)}

\section{BORNEAN HILL SAGO PALM. BERTAM, KAJATAO \\ ○}

\section{DESCRIPTION}

Tree palm, to $10 \mathrm{~m}$, clustering, spiny, hapaxanthic, polygamous palms, numerous stilt roots; leaves pinnate, spiny sheath, to $10 \mathrm{~m}$; inflorescence terminal, erect, $2 \mathrm{~m}$ long; flowers slender to $8-9 \mathrm{~cm}$ long; fruit ovoid, beaked, $10 \times 5 \mathrm{~cm}$, with brown scales

\section{HABITAT}

Lowland to hill primary forests, in dense clumps on ridge tops to $600 \mathrm{~m}$; planted in villages

\section{ORIGINS}

Borneo endemic

\section{USES}

Pith for sago flour; leaf bud eaten and for medicine; leaves for thatch; leafstalks for hunting tools and light construction; site for ambushing pigs

\section{REFERENCES}

LBN-LIPI 1978: 109; PROSEA Vol. 9: 175
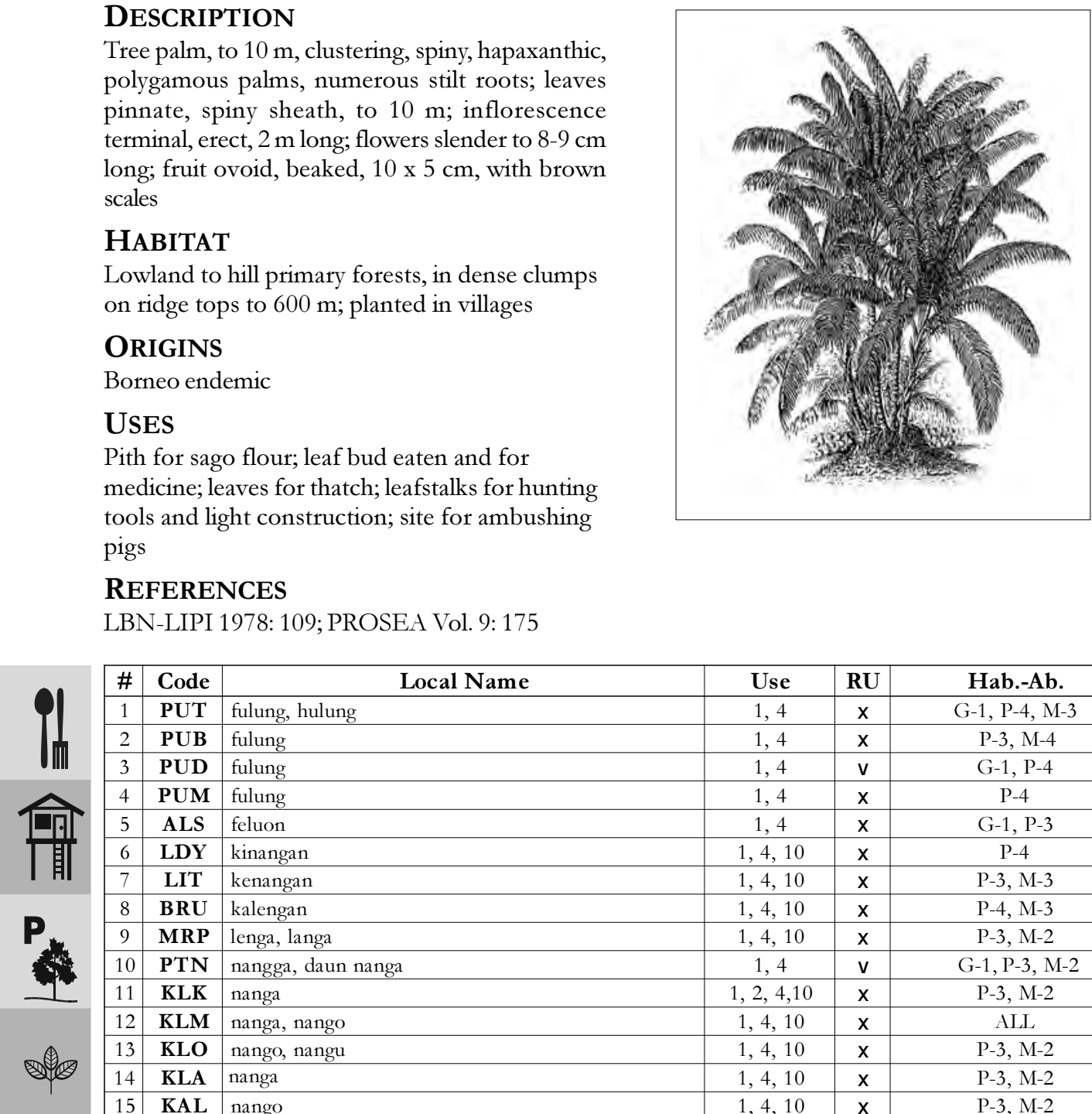

\begin{tabular}{|c|c|c|c|c|c|}
\hline$\#$ & Code & Local Name & Use & RU & Hab.-Ab. \\
\hline 1 & PUT & fulung, hulung & 1,4 & $x$ & G-1, P-4, M-3 \\
\hline 2 & PUB & fulung & 1,4 & $x$ & P-3, M-4 \\
\hline 3 & PUD & fulung & 1,4 & $\mathrm{v}$ & G-1, P-4 \\
\hline 4 & PUM & fulung & 1,4 & $x$ & P-4 \\
\hline 5 & ALS & feluon & 1,4 & $x$ & G-1, P-3 \\
\hline 6 & LDY & kinangan & $1,4,10$ & $x$ & P-4 \\
\hline 7 & LIT & kenangan & $1,4,10$ & $x$ & P-3, M-3 \\
\hline 8 & BRU & kalengan & $1,4,10$ & $x$ & P-4, M-3 \\
\hline 9 & MRP & lenga, langa & $1,4,10$ & $x$ & P-3, M-2 \\
\hline 10 & PTN & nangga, daun nanga & 1,4 & $\mathrm{v}$ & G-1, P-3, M-2 \\
\hline 11 & KLK & nanga & $1,2,4,10$ & $x$ & P-3, M-2 \\
\hline 12 & KLM & nanga, nango & $1,4,10$ & $x$ & ALL \\
\hline 13 & KLO & nango, nangu & $1,4,10$ & $x$ & P-3, M-2 \\
\hline 14 & KLA & nanga & $1,4,10$ & $x$ & P-3, M-2 \\
\hline 15 & KAL & nango & $1,4,10$ & $x$ & $\mathrm{P}-3, \mathrm{M}-2$ \\
\hline 16 & KBD & nanga & $1,2,4,10$ & v & P-3, M-2 \\
\hline 17 & KBK & nanga & $1,2,4,10$ & $x$ & P-3, M-2 \\
\hline 18 & PEB & nanga, uvut & $1,2,4,10$ & $\mathrm{v}$ & P-4, M-4 \\
\hline
\end{tabular}

Notes : 
Licuala valida Becc. (Palmae)

LICUALA PALM. PALAS BIRU

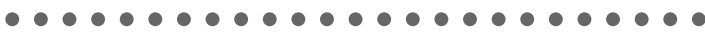

\section{DESCRIPTION}

Fan palm, to $2 \mathrm{~m}$, short stem, leaf stalk long slender with spines; round leaves split into 8-12 compound leaflets, wedge-shaped, with outer margin blunt, zig-zag; flowers on axillary panicle 1 $\mathrm{m}$ long; fruits yellowish brown

\section{HABITAT}

Lowland and hill primary forest to $900 \mathrm{~m}$; poor soils, ridge tops, slopes

\section{ORIGINS}

Borneo endemic

\section{USES}

Leaves for thatch, wrapping, and handicrafts (sun/ rain hats) sold locally; leafbud eaten

\section{REFERENCES}

LBN-LIPI 1978: 49; PROSEA Vol. 5(3): 329

\begin{tabular}{|c|c|c|c|c|c|}
\hline \# & Code & Local Name & Use & RU & Hab.-Ab. \\
\hline 1 & PUT & opou, poi, pojalai & $1,4,6$ & v & $\mathrm{Y}-2, \mathrm{O}-3, \mathrm{P}-4$ \\
\hline 2 & PUB & pou, ubu pou & 1,6 & $\mathrm{v}$ & $\mathrm{Y}-2, \mathrm{O}-3, \mathrm{P}-4$ \\
\hline 3 & PUD & poh pou & 4,6 & $\mathrm{v}$ & $\mathrm{Y}-2, \mathrm{O}-2$ \\
\hline 4 & PUM & doun opou & 4,6 & $\mathrm{v}$ & $\mathrm{O}-2, \mathrm{P}-3, \mathrm{M}-3$ \\
\hline 5 & ALS & daun silad & 1,6 & v & $\mathrm{O}-2, \mathrm{P}-3$ \\
\hline 6 & LDY & don ilad, donlilat & 1,6 & v & $\mathrm{Y}-2, \mathrm{O}-3, \mathrm{P}-4$ \\
\hline 7 & LIT & ilad, ilat, benasing & 4,6 & v & $\mathrm{Y}-2, \mathrm{O}-2, \mathrm{P}-3$ \\
\hline 8 & BRU & uen lit & $1,4,6$ & $\mathrm{v}$ & $\mathrm{O}-2, \mathrm{P}-3$ \\
\hline 9 & MRP & tim lea, hleae, lie & 4,6 & v & $\mathrm{Y}-2, \mathrm{O}-3, \mathrm{P}-2, \mathrm{M}-2$ \\
\hline 10 & PTN & sang & 1,6 & v & $\mathrm{Y}-2, \mathrm{O}-2, \mathrm{P}-3$ \\
\hline 11 & KLK & sang & 4,6 & $\mathrm{v}$ & $\mathrm{Y}-2, \mathrm{O}-2, \mathrm{P}-3$ \\
\hline 12 & KLM & sang, sang osan & 4,6 & $\mathrm{v}$ & ALL \\
\hline 13 & KLO & sang & 4,6 & $\mathrm{v}$ & P-3, M-3 \\
\hline 14 & KLA & sang & 4,6 & $\mathrm{v}$ & $\mathrm{P}-3, \mathrm{M}-3$ \\
\hline 15 & KAL & sang & 4,6 & v & $\mathrm{P}-3, \mathrm{M}-3$ \\
\hline 16 & KBD & sang & 4,6 & $\mathrm{v}$ & P-3, M-3 \\
\hline 17 & KBK & sang & 4,6 & $\mathrm{v}$ & P-3, M-3 \\
\hline 18 & PEB & silat & 4,6 & $\mathrm{v}$ & P-3, M-4 \\
\hline
\end{tabular}

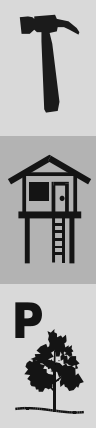

Notes : 


\section{Oncosperma borridum (Griff.) Scheffer (Palmae)}

\section{Prickly TREe PALM. Bayas, Bayeh, NIBUNG \\ ○}

\section{DESCRIPTION}

Tree palm, to $20 \mathrm{~m}$; clustering with basal suckers; 4-6 stems armed with massive downwards directed black spines; leaves finely feathery, leaf stalks spiny; leaflets numerous with long thread hanging from base; fruit round, $2 \mathrm{~cm}$, black

\section{HABITAT}

Lowland and hill primary forest to $900 \mathrm{~m}$

\section{ORIGINS}

Malaysia, western Indonesia, Philippines

\section{USES}

Leaf bud eaten; leaves for thatch

\section{REFERENCES}

LBN-LIPI 1978: 17; Whitmore 1985: 82

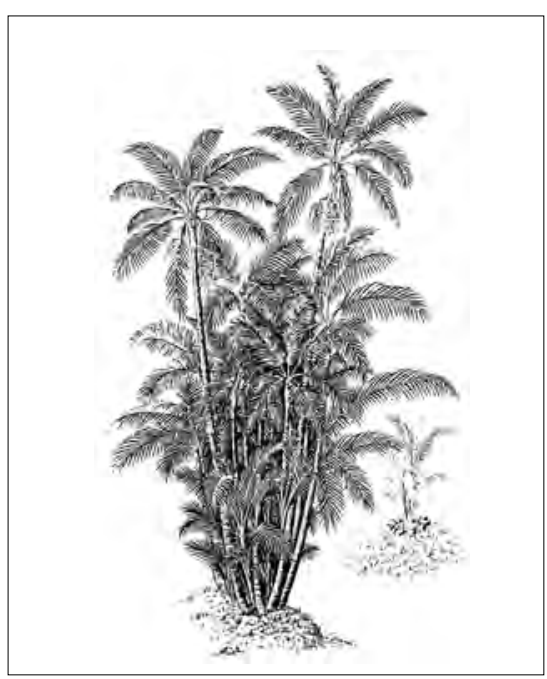

\begin{tabular}{|c|c|c|c|c|c|}
\hline$\#$ & Code & Local Name & Use & RU & Hab.-Ab. \\
\hline 1 & PUT & nyivung & 1 & $x$ & P-3, M-3 \\
\hline 2 & PUB & nyivung & & & \\
\hline 3 & PUD & nyivung, lerai (juv.) & 1 & $x$ & P-3, M-3 \\
\hline 4 & PUM & nyivung & & & \\
\hline 5 & ALS & lebungan & & & \\
\hline 6 & LDY & nyibung & & & \\
\hline 7 & LIT & nibung & & & \\
\hline 8 & BRU & ibeung & & & \\
\hline 9 & MRP & leybiu & 1 & $x$ & P-3, M-3 \\
\hline 10 & PTN & nyibung & & & \\
\hline 11 & KLK & nibung & 1,6 & $x$ & P-3, M-3 \\
\hline 12 & KLM & nyibung & 1,6 & $x$ & P-3, M-3 \\
\hline 13 & KLO & nibung & 1,6 & $\mathrm{x}$ & P-3, M-3 \\
\hline 14 & KLA & nibung & 1,6 & $x$ & P-3, M-3 \\
\hline 15 & KAL & nibung & 1,6 & $x$ & P-3, M-3 \\
\hline 16 & KBD & nibung & 1,6 & $x$ & P-3, M-3 \\
\hline 17 & KBK & nyibung & 1,6 & $x$ & P-4, M-4 \\
\hline 18 & PEB & leka' & 1,6 & v & P-4, M-4 \\
\hline
\end{tabular}

Notes : 


\section{Sallaca affinis (Griff.) (Palmae)}

\section{BEERAI PALM. KEPLAR

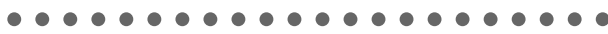

\section{DESCRIPTION}

Rattan, stemless, solitary or clustering, to $5 \mathrm{~m}$; leaf stems erect, armed; terminal leaflet with a single fold; fruits snakeskin-like, orange, 7-9 x 4-5 cm, sweet

\section{HABITAT}

Lowland primary and secondary forests to 600 $\mathrm{m}$; riverine, shaded areas; planted in village gardens

\section{ORIGINS}

Borneo endemic

\section{USES}

Fruit eaten; leafbud eaten and for medicine; leaf stems and blades for thatch

\section{REFERENCES}

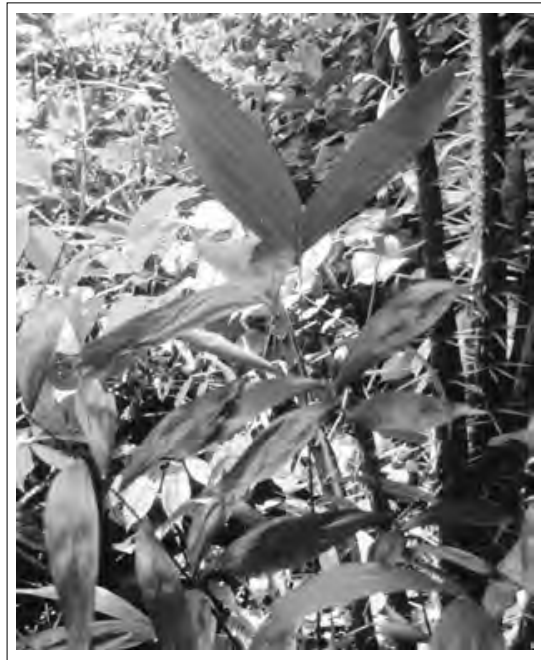

PROSEA Vol. 6: 109

\begin{tabular}{|c|c|c|c|c|c|}
\hline \# & Code & Local Name & Use & RU & Hab.-Ab. \\
\hline 1 & PUT & birai & 1,6 & $x$ & $\mathrm{Y}-2, \mathrm{O}-3, \mathrm{P}-4, \mathrm{M}-3$ \\
\hline 2 & PUB & birai & 1,6 & $x$ & $\mathrm{Y}-2, \mathrm{O}-3, \mathrm{P}-3, \mathrm{M}-1$ \\
\hline 3 & PUD & birai & 1,6 & $x$ & $\mathrm{Y}-3, \mathrm{O}-4, \mathrm{P}-2$ \\
\hline 4 & PUM & doun birai & $1,2,6$ & $x$ & $\mathrm{Y}-2, \mathrm{O}-3, \mathrm{P}-4, \mathrm{M}-4$ \\
\hline 5 & ALS & demuran & $1,2,6$ & $x$ & $\mathrm{Y}-2, \mathrm{O}-2, \mathrm{P}-3$ \\
\hline 6 & LDY & reburan, riburam & 1,6 & $x$ & $\mathrm{Y}-2, \mathrm{O}-3, \mathrm{P}-3, \mathrm{M}-2$ \\
\hline 7 & LIT & bua lukut, lukut & 1,6 & $\mathrm{x}$ & Y-3, O-4, P-3, \\
\hline 8 & BRU & uen lage & $1,2,6$ & $x$ & $\mathrm{Y}-2, \mathrm{O}-3, \mathrm{P}-3$ \\
\hline 9 & MRP & mberae, mbrae & 1,6 & $x$ & $\mathrm{Y}-2, \mathrm{O}-2, \mathrm{P}-3, \mathrm{M}-2$ \\
\hline 10 & PTN & bire & 1,6 & $x$ & $\mathrm{Y}-2, \mathrm{O}-3, \mathrm{P}-3, \mathrm{M}-2$ \\
\hline 11 & KLK & birai, birae & 1,6 & $x$ & $\mathrm{Y}-2, \mathrm{O}-3, \mathrm{P}-2$ \\
\hline 12 & KLM & birai, berai & $1,2,6$ & $x$ & ALL \\
\hline 13 & KLO & lemojeng & 1,6 & $x$ & $\mathrm{O}-2, \mathrm{P}-3, \mathrm{M}-3$ \\
\hline 14 & KLA & birai & 1,6 & $x$ & $\mathrm{O}-3, \mathrm{P}-3, \mathrm{M}-1$ \\
\hline 15 & KAL & berae & 1,6 & $x$ & $\mathrm{O}-3, \mathrm{P}-3, \mathrm{M}-3$ \\
\hline 16 & KBD & birai, berai & 1,6 & $\mathrm{v}$ & $\mathrm{O}-3, \mathrm{P}-3, \mathrm{M}-3$ \\
\hline 17 & KBK & birei & 1,6 & $x$ & $\mathrm{O}-3, \mathrm{P}-3, \mathrm{M}-3$ \\
\hline 18 & PEB & lemujan & 1,6 & v & $\mathrm{O}-3, \mathrm{P}-4$ \\
\hline
\end{tabular}

Notes : 
Salacca zalacca (Gaertner) Voss (Palmae)

\section{SALAK. SALAK}

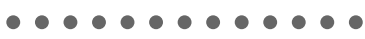

\section{DESCRIPTION}

Palm, 3-5 m, erect, almost stemless, very prickly, dioecious, compact clumps due to branching at base; leaves pinnate, 3-7 $\mathrm{m}$ long, armed with long thin black spines; leaflets $20-70 \mathrm{~cm} \times 2-7.5 \mathrm{~cm}$; fruit a round drupe $5-7 \times 5 \mathrm{~cm}$, yellow to brown scales

\section{HABITAT}

Cultivated in gardens, plantations

\section{ORIGINS}

Western Malaysia

\section{USES}

Fruit eaten, sold locally; leaves for thatch; hedge

\section{REFERENCES}

LBN-LIPI 1978: 103; Ochse 1980: 574;

PROSEA Vol. 2: 281; Whitmore 1985: 106

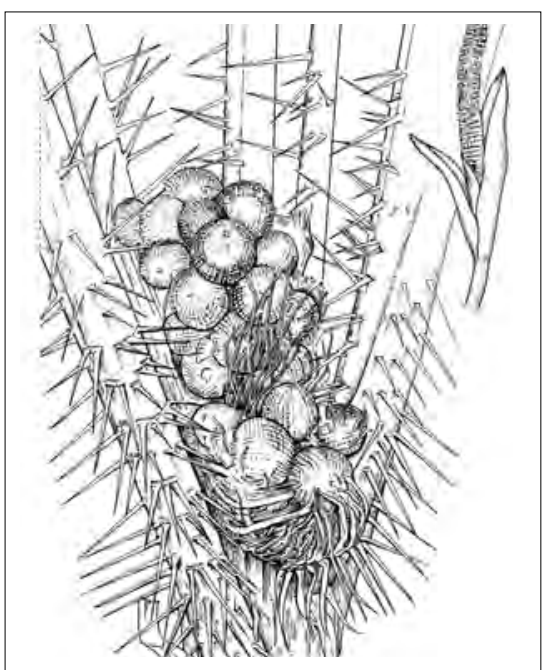

\begin{tabular}{|c|c|c|c|c|c|}
\hline \# & Code & Local Name & Use & RU & Hab.-Ab. \\
\hline 1 & PUT & salak & 1,11 & $x$ & G-3, S-3 \\
\hline 2 & PUB & lium & 1 & $x$ & G-1, S-1 \\
\hline 3 & PUD & lium & 1 & $x$ & G-1, S-1 \\
\hline 4 & PUM & & & & \\
\hline 5 & ALS & besiun & 1,11 & $x$ & $G-3, S-3$ \\
\hline 6 & LDY & besien & 1,11 & $x$ & $G-3, S-3$ \\
\hline 7 & LIT & besien & 1,11 & $x$ & G-3, S-3 \\
\hline 8 & BRU & wi yom & 1,11 & $x$ & G-2, S-2 \\
\hline 9 & MRP & salak & 1,11 & $x$ & G-3, S-3 \\
\hline 10 & PTN & lium & 1,11 & $x$ & G-3, S-3 \\
\hline 11 & KLK & salak & 1,11 & $x$ & G-2, S-2 \\
\hline 12 & KLM & salak & 1,11 & $x$ & G-2, S-2 \\
\hline 13 & KLO & sala' & 1,11 & $x$ & G-2, S-2 \\
\hline 14 & KLA & salak & 1,11 & $x$ & G-2, S-2 \\
\hline 15 & KAL & salak & 1,11 & $\mathrm{x}$ & G-2, S-2 \\
\hline 16 & KBD & salak & 1,11 & v & $G-2, S-2$ \\
\hline 17 & KBK & salak & 1,11 & $x$ & $\mathrm{G}-2, \mathrm{~S}-2$ \\
\hline 18 & PEB & sum & 1,11 & $x$ & G-2, S-2 \\
\hline
\end{tabular}

Notes : 
Pandanus sp. (Pandanaceae)

\section{Pandanus, SCREW PINE. MenKuANG, PANDAN}

\section{DESCRIPTION}

Tree, woody evergreen, to $5 \mathrm{~m}$, with prop roots; stem knobbly, branching often; leaves spiral, linear, $\mathrm{m}$-shaped in cross section, to $2 \mathrm{~m}$ long by $4 \mathrm{~cm}$ wide; fruit heads $10-20 \mathrm{~cm}$ long, blood red (at least two varieties)

\section{HABITAT}

Wild in secondary forests, abandoned villages; cultivated in villages

\section{ORIGINS}

Southeast Asia

\section{USES}

Leaves used for basketry, matting; sold locally as handicrafts; medicine

\section{REFERENCES}

Burkill 1966: 1672; PROSEA Vol 2: 240

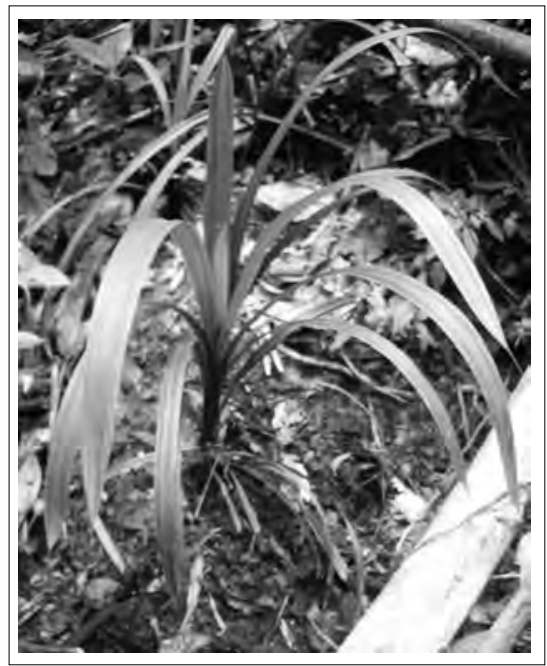

\begin{tabular}{|c|c|c|c|c|c|}
\hline \# & Code & Local Name & Use & RU & Hab.-Ab. \\
\hline 1 & PUT & $\mathrm{ra}^{\prime}, \mathrm{ra}$ & 8 & $\mathrm{v}$ & G-3, P-1 \\
\hline 2 & PUB & $\mathrm{ra}$ & 8 & v & G-2, P-1 \\
\hline 3 & PUD & $\mathrm{ra}$ & 8 & $x$ & G-2, P-1 \\
\hline 4 & PUM & $\mathrm{ra}$ & 8 & $x$ & $\mathrm{G}-2, \mathrm{P}-1$ \\
\hline 5 & ALS & pandan & 8 & $x$ & G-3, P-1 \\
\hline 6 & LDY & kaber fulung & 8 & $x$ & G-3, O-3, P-1 \\
\hline 7 & LIT & kaber & 8 & $x$ & G-3, O-3, P-1, M-2 \\
\hline 8 & BRU & uen la'ah & 8 & $x$ & G-3, O-3, P-1, M-2 \\
\hline 9 & MRP & la'a, la'ah & 8 & $x$ & G-3, O-3, P-1 \\
\hline 10 & PTN & la'a & 8 & $x$ & G-3, P-2 \\
\hline 11 & KLK & daa, da'a mba', da'a pandan & 2,8 & $\mathrm{v}$ & G-3, P-2 \\
\hline 12 & KLM & daa & 8 & $\mathrm{v}$ & $\mathrm{G}-3, \mathrm{P}-2$ \\
\hline 13 & KLO & da'a & 8 & $\mathrm{v}$ & $\mathrm{G}-3, \mathrm{P}-2$ \\
\hline 14 & KLA & da'a & 8 & v & G-3, P-2 \\
\hline 15 & KAL & da'a & 8 & $\mathrm{v}$ & G-3, P-2 \\
\hline 16 & KBD & da'a & 8,11 & v & $G-3, P-2$ \\
\hline 17 & KBK & da'a & 8 & $\mathrm{v}$ & G-4, P-1 \\
\hline 18 & PEB & reu & 8,11 & v & $\mathrm{G}-4, \mathrm{P}-2$ \\
\hline
\end{tabular}

Notes : 


\section{Passiflora foetida L. (Passifloraceae)}

\section{STINKING PASSION VINE.TIMUN DENDANG}

\section{DESCRIPTION}

Herb, leaves, 3-lobed, 4-5 cm long, foul smelling when crushed; flowers whitish purplish $2.5-5 \mathrm{~cm}$ diameter with 3 pale green mosslike leaves outside the sepals; fruit a yellow to orange round berry, 2 $\mathrm{cm}$

\section{HABITAT}

Wild weed in wasteland, roadsides; climbs fences and hedges; cultivated in gardens

\section{ORIGINS}

Tropical America

\section{USES}

Only ripe fruit is eaten; hedge plant, ground cover; medicine; leaves poisonous (cyanogenic)

\section{REFERENCES}

Burkill 1966: 1705; PROSEA Vol. 2: 350;

Chin and Yong 1980: 133
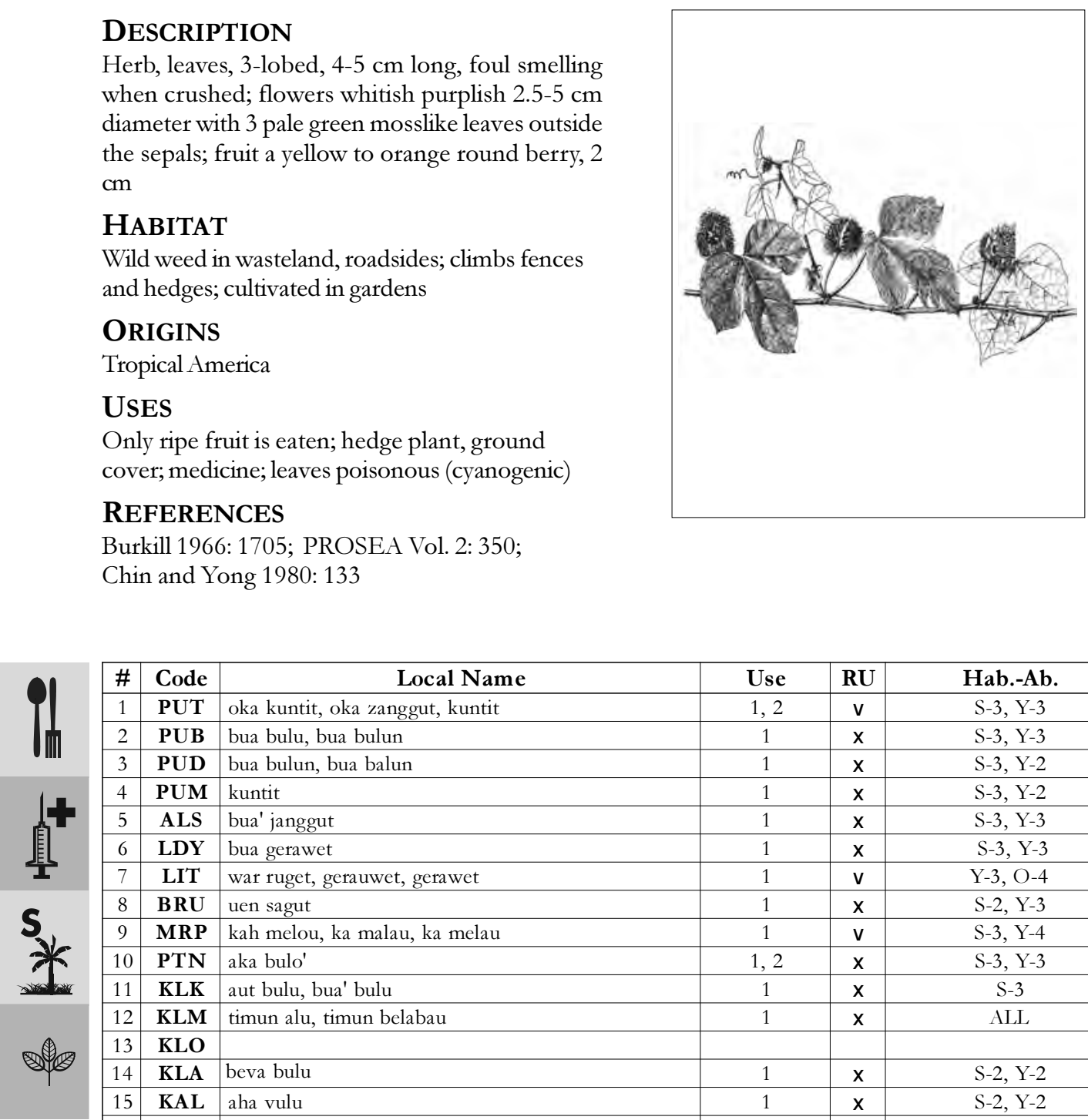

\begin{tabular}{|c|c|l|c|c|c|}
\hline$\#$ & Code & \multicolumn{1}{|c|}{ Local Name } & Use & RU & Hab.-Ab. \\
\hline 1 & PUT & oka kuntit, oka zanggut, kuntit & 1,2 & $\mathbf{v}$ & S-3, Y-3 \\
\hline 2 & PUB & bua bulu, bua bulun & 1 & $\mathbf{X}$ & S-3, Y-3 \\
\hline 3 & PUD & bua bulun, bua balun & 1 & $\mathbf{X}$ & S-3, Y-2 \\
\hline 4 & PUM & kuntit & 1 & $\mathbf{X}$ & S-3, Y-2 \\
\hline 5 & ALS & bua' janggut & 1 & $\mathbf{X}$ & S-3, Y-3 \\
\hline 6 & LDY & bua gerawet & 1 & $\mathbf{x}$ & S-3, Y-3 \\
\hline 7 & LIT & war ruget, gerauwet, gerawet & 1 & $\mathbf{v}$ & Y-3, O-4 \\
\hline 8 & BRU & uen sagut & 1 & $\mathbf{x}$ & S-2, Y-3 \\
\hline 9 & MRP & kah melou, ka malau, ka melau & 1 & $\mathbf{v}$ & S-3, Y-4 \\
\hline 10 & PTN & aka bulo' & 1,2 & $\mathbf{x}$ & S-3, Y-3 \\
\hline 11 & KLK & aut bulu, bua' bulu & 1 & $\mathbf{X}$ & S-3 \\
\hline 12 & KLM & timun alu, timun belabau & 1 & $\mathbf{X}$ & ALL \\
\hline 13 & KLO & & 1 & $\mathbf{x}$ & S-2, Y-2 \\
\hline 14 & KLA & beva bulu & 1 & $\mathbf{x}$ & S-2, Y-2 \\
\hline 15 & KAL & aha vulu & 1,2 & $\mathbf{x}$ & S-2, Y-2 \\
\hline 16 & KBD & peretta & 1 & $\mathbf{x}$ & S-2, Y-2 \\
\hline 17 & KBK & aka bulu & 1,2 & $\mathbf{v}$ & G-3, Y-4 \\
\hline 18 & PEB & pereta & & \\
\hline
\end{tabular}

Notes : 


\section{Piper betle L. (Piperaceae) (Variety 1)}

\section{BETEL PEPPER 1.DAUN SURUH, SIRIH \\ ○}

\section{DESCRIPTION}

Vine, 5-15 m, woody climber; leaves ovate or ovateoblong, with cordate, obliquely rounded base; 5 $18 \times 2.5-10 \mathrm{~cm}$, green to yellowish green; berry with rounded smooth apex, but sunken in densely hoary green body, 1-1.5 cm thick; this variety distinguished by a larger ovate leaf, often yellowish

\section{HABITAT}

Cultivated in homegardens; wild in secondary forests, brushwoods, to $700 \mathrm{~m}$

\section{ORIGINS}

Malaysia and western Indonesia

\section{USES}

Leaves chewed for narcotic or medicinal effect, with Areca catechu; sold locally

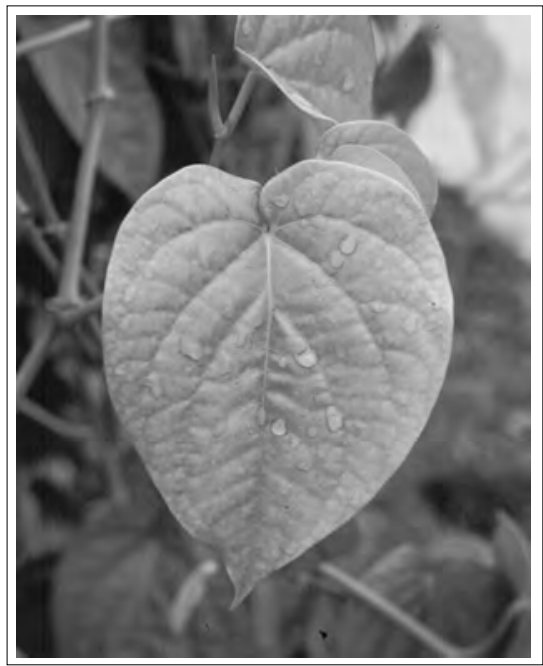

\section{REFERENCES}

Burkill 1966: 1767; FOJ Vol. 1: 173

\begin{tabular}{|c|c|c|c|c|c|}
\hline \# & Code & Local Name & Use & RU & Hab.-Ab. \\
\hline 1 & PUT & bangi, bangi'in, bangi laung & 1,2 & v & $\mathrm{G}-2, \mathrm{~S}-1$ \\
\hline 2 & PUB & bangi & 1,2 & v & G-2, S-1 \\
\hline 3 & PUD & bangi laung, bangi & 1,2 & $x$ & G-2, S-1 \\
\hline 4 & PUM & bangi & $1,2,11$ & v & G-2, S-1 \\
\hline 5 & ALS & buyu & 1,2 & $\mathrm{v}$ & G-2, S-1 \\
\hline 6 & LDY & buyu & 1,2 & v & G-3, S-2, Y-3 \\
\hline 7 & LIT & buyu, buyu ada', daun buyu & 1,2 & $x$ & $\mathrm{G}-2$ \\
\hline 8 & BRU & uen yu' & 1,2 & $\mathrm{x}$ & G-2 \\
\hline 9 & MRP & mangai, mangaei & 1,2 & v & G-3, Y-1 \\
\hline 10 & PTN & unga & 1,2 & v & G-2, S-1 \\
\hline 11 & KLK & unga & 1,2 & $x$ & G-2 \\
\hline 12 & KLM & onga & 1,2 & $\mathrm{v}$ & G-2 \\
\hline 13 & KLO & onga & 1,2 & $x$ & G-3 \\
\hline 14 & KLA & unga, aunga & 1,2 & $x$ & G-2 \\
\hline 15 & KAL & da'un onga & & & \\
\hline 16 & KBD & onga & 1,2 & $x$ & G-2 \\
\hline 17 & KBK & zien & & & \\
\hline 18 & PEB & onga & $1,2,11$ & v & G-3, S-2 \\
\hline
\end{tabular}

Notes : 


\section{Piper betle L. (Piperaceae) (Variety 2)}

\section{BETEL PEPPER 2. DAUNSURUH, SIRIH}

\section{DESCRIPTION}

Vine, 5-15 m, woody climber; leaves ovate or ovateoblong, with cordate, obliquely rounded base; 5 $18 \times 2.5-10 \mathrm{~cm}$; berry with rounded smooth apex, but sunken in densely hoary green body, $1-1.5 \mathrm{~cm}$ thick; this variety distinguished by a smaller, oblong leaf

\section{HABITAT}

Cultivated in homegardens; wild in secondary forests, brushwoods, to $700 \mathrm{~m}$

\section{ORIGINS}

Malaysia and western Indonesia

\section{USES}

Leaves chewed for narcotic or medicinal effect, with Areca catechu; sold locally

\section{REFERENCES}

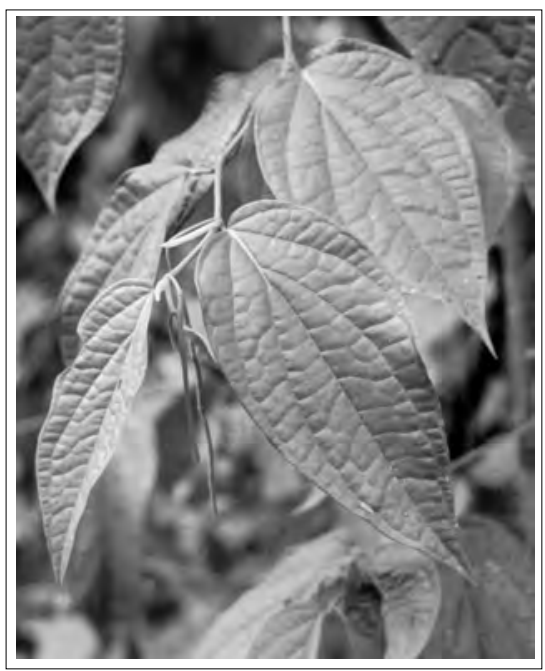

Burkill 1966: 1767; FOJ Vol. 1: 173

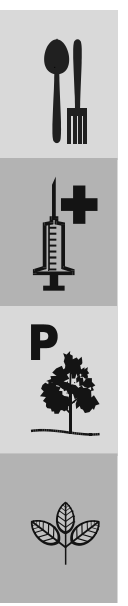

\begin{tabular}{|c|c|c|c|c|c|}
\hline \# & Code & Local Name & Use & RU & Hab.-Ab. \\
\hline 1 & PUT & bangi tano & 1 & $x$ & $\mathrm{Y}-3, \mathrm{O}-3, \mathrm{P}-2$ \\
\hline 2 & PUB & bangi tano & 1 & v & $\mathrm{Y}-2, \mathrm{O}-2$ \\
\hline 3 & PUD & bangi tano & 1 & v & $\mathrm{Y}-3, \mathrm{O}-4, \mathrm{P}-2$ \\
\hline 4 & PUM & pacan & 1 & $x$ & P-4 \\
\hline 5 & ALS & madat & 1,2 & v & $\mathrm{Y}-3, \mathrm{O}-3$ \\
\hline 6 & LDY & war madat, don adat & 2 & $x$ & $\mathrm{~S}-3, \mathrm{Y}-4, \mathrm{O}-3, \mathrm{P}-3$ \\
\hline 7 & LIT & buyu tana, buyu & $1,2,11$ & $\mathrm{x}$ & G-2, P-3 \\
\hline 8 & BRU & uen biyu tana' & 1 & $\mathrm{x}$ & $\mathrm{Y}-3, \mathrm{P}-3$ \\
\hline 9 & MRP & mangai tana, mangi tana & 2 & $x$ & Y-3, P-3 \\
\hline 10 & PTN & uro' bata ingen, unga kelaru & 2 & $\mathrm{x}$ & $\mathrm{Y}-2, \mathrm{O}-3, \mathrm{P}-2$ \\
\hline 11 & KLK & unga tana, onga kelaro & 2 & $x$ & $\mathrm{Y}-3, \mathrm{O}-4, \mathrm{P}-1$ \\
\hline 12 & KLM & onga bali, onga tana' & & $\mathrm{x}$ & $\mathrm{O}-3, \mathrm{P}-3$ \\
\hline 13 & KLO & onga bali, onga sanit, unga fali, onga fulung, onga & 1,2 & $\mathrm{x}$ & $\mathrm{Y}-3, \mathrm{P}-3$ \\
\hline 14 & KLA & onga bali, unga bali, & & $\mathrm{x}$ & $\mathrm{Y}-4, \mathrm{O}-4, \mathrm{P}-3$ \\
\hline 15 & KAL & honga vali & & & \\
\hline 16 & KBD & onga bali & & $\mathrm{x}$ & $\mathrm{Y}-3, \mathrm{O}-3$ \\
\hline 17 & KBK & kelaru' & & & \\
\hline 18 & PEB & onga balai, uru lengat & 2 & v & $\mathrm{G}-2, \mathrm{Y}-2$ \\
\hline
\end{tabular}

\section{Notes :}


Piper nigrum L. (Piperaceae)

\section{BLACK PEPPER, WHITE PEPPER. LADA, MERICA}

\section{DESCRIPTION}

Herb, climbing, adhesive roots; stems light green with nodes; leaves broadly ovate-elliptic, 5-18 x 2$12 \mathrm{~cm}$, dark green above, whitish green below, 5-7 nerved; petiole furrowed; fruit spikes $5-20 \mathrm{~cm}$ long, berries red when ripe, drying black, 3-6 mm

\section{HABITAT}

Cultivated in homegardens, intensively in plantations

\section{ORIGINS}

India

\section{USES}

Fruit for spice, medicine, sold locally and to traders for export

\section{REFERENCES}

Burkill 1966: 1776; Ochse 1980: 588;

PROSEA Vol 13: 109

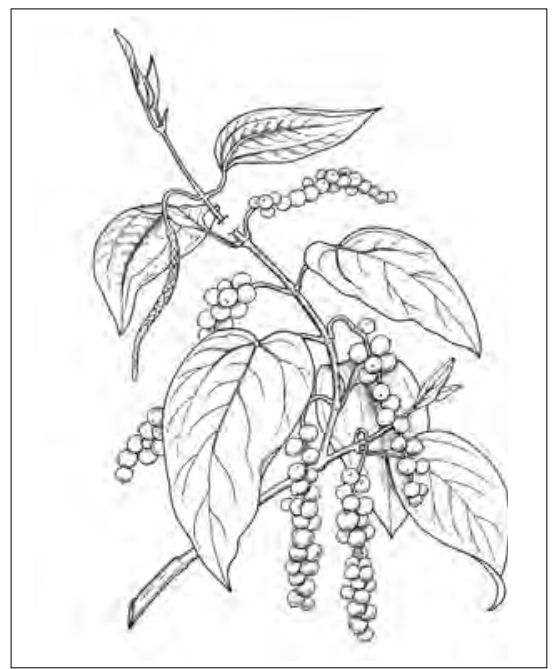

\begin{tabular}{|c|c|c|c|c|c|}
\hline \# & Code & Local Name & Use & RU & Hab.-Ab. \\
\hline 1 & PUT & sahang, cahang & 1,11 & $\mathrm{v}$ & G-3 \\
\hline 2 & PUB & cahang & $1,2,11$ & $\mathrm{v}$ & G-2 \\
\hline 3 & PUD & sahang & 1,11 & v & G-3 \\
\hline 4 & PUM & cahang & 1,11 & v & G-3 \\
\hline 5 & ALS & sahang & $1,2,11$ & v & G-3 \\
\hline 6 & LDY & sahang & 1,11 & $\mathrm{v}$ & $\mathrm{G}-3, \mathrm{~S}-2$ \\
\hline 7 & LIT & sahang, cahang & $1,2,11$ & v & G-3 \\
\hline 8 & BRU & wi sahang & 1,11 & v & G-3 \\
\hline 9 & MRP & cahang & $1,2,11$ & v & G-3 \\
\hline 10 & PTN & sahang & $1,2,11$ & v & G-3 \\
\hline 11 & KLK & ca'ang, lada & 1,11 & v & G-3 \\
\hline 12 & KLM & lada & $1,2,11$ & v & G-3 \\
\hline 13 & KLO & saghang, sakang & 1,2 & $\mathrm{v}$ & G-3 \\
\hline 14 & KLA & cahang, sahang & 1 & v & G-4 \\
\hline 15 & KAL & sahang & & & \\
\hline 16 & KBD & cahang & 1,2 & v & G-3 \\
\hline 17 & KBK & sahang & & & \\
\hline 18 & PEB & cahang, bue sahang & 1 & $x$ & $G-4, S-2$ \\
\hline
\end{tabular}

Notes : 


\section{Dendrocalamus asper (Schult.F.) Backer ex Heyne (Poaceae) GIANT BAMBOO. BAMBU BETUNG \\ ○。००}

\section{DESCRIPTION}

Bamboo, to $30 \mathrm{~m}$, densely tufted; branches at midculm nodes; culms $8-20 \mathrm{~cm}$ diameter, wall 11 $36 \mathrm{~mm}$ thick, internodes $10-20$ to $30-50 \mathrm{~cm}$ long; fine golden hairs when young; culm sheaths dark brown hairs; leaf blades $30 \times 2.5 \mathrm{~cm}$, petiole $4 \mathrm{~mm}$ long, hairy below

\section{HABITAT}

Cultivated in villages, swidden fields, riverine, to $1500 \mathrm{~m}$

\section{ORIGINS}

Southeast Asia

\section{USES}

Culms for building material, containers; shoots eaten

\section{REFERENCES}

PROSEA Vol. 7: 80

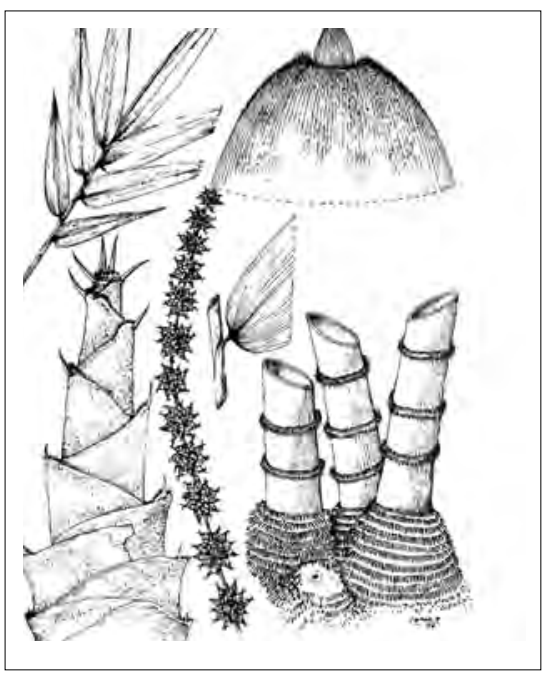

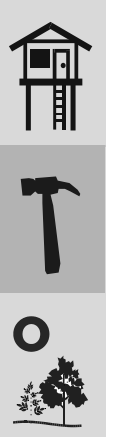

2)

\begin{tabular}{|c|c|l|c|c|c|}
\hline$\#$ & Code & Local Name & Use & RU & Hab.-Ab. \\
\hline 1 & PUT & bulu betung & $1,4,6$ & $\mathbf{v}$ & O-3, R \\
\hline 2 & PUB & bulu betung & $1,4,6$ & $\mathbf{v}$ & O-3, R \\
\hline 3 & PUD & bulu betung & $1,4,6$ & $\mathbf{v}$ & O-3, R \\
\hline 4 & PUM & & & \\
\hline 5 & ALS & bulu' batong & $1,4,6$ & $\mathbf{v}$ & G-2, S-2, O-3, R \\
\hline 6 & LDY & bulu' batung & $1,4,6$ & $\mathbf{v}$ & G-2, S-2, O-3, R \\
\hline 7 & LIT & bulu beletung & $1,4,6$ & $\mathbf{v}$ & G-2, S-2, O-3, R \\
\hline 8 & BRU & liu tong & $1,4,6$ & $\mathbf{v}$ & G-2, S-2, O-3, R \\
\hline 9 & MRP & blo tau, mblo & $1,4,6$ & $\mathbf{v}$ & O-3, R \\
\hline 10 & PTN & bulu latong & $1,4,6$ & $\mathbf{v}$ & S-2, O-3, R \\
\hline 11 & KLK & bulo latung & $1,4,6$ & $\mathbf{v}$ & S-2, O-3, R \\
\hline 12 & KLM & latong & $1,4,6$ & $\mathbf{v}$ & G-2, S-2, O-3, R \\
\hline 13 & KLO & bulu latung & $1,4,6$ & $\mathbf{v}$ & G-2, S-2, O-3, R \\
\hline 14 & KLA & bulue latung & $1,4,6$ & $\mathbf{v}$ & G-2, S-2, O-3, R \\
\hline 15 & KAL & bulue latong & $1,4,6$ & $\mathbf{v}$ & G-2, S-2, O-3, R \\
\hline 16 & KBD & bolo latung & $1,4,6$ & $\mathbf{v}$ & G-2, S-2, O-3, R \\
\hline 17 & KBK & bolo latung & $1,4,6$ & $\mathbf{v}$ & G-2, S-2, O-3, R \\
\hline 18 & PEB & leupek tup & $1,4,6$ & $\mathbf{v}$ & G-2, S-2, O-3, R \\
\hline
\end{tabular}

Notes : 
Schizostachyum bracbycladum Kurz (Poaceae)

\section{BAMBOO. BAMBU LEMANG

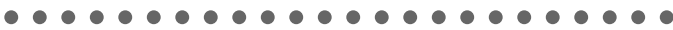

\section{DESCRIPTION}

Bamboo, 7-15 m; culm 7-10 cm diameter, wall 3-5 $\mathrm{mm}$ thick, green, bluish green, or golden yellow with narrow green stripes; internodes $30-58 \mathrm{~cm}$ long, nodes not swollen, with tuft of 25-30 slender branches; leaves lanceolate, $26-30$ x 3.5-6 cm, hairy below

\section{HABITAT}

Wild in secondary or disturbed forests to $600 \mathrm{~m}$; cultivated in villages

\section{ORIGINS}

Southeast Asia

\section{USES}

Culms for cooking rice, building materials, containers, handicrafts, sold locally; shoots eaten

\section{REFERENCES}

PROSEA Vol. 7: 132

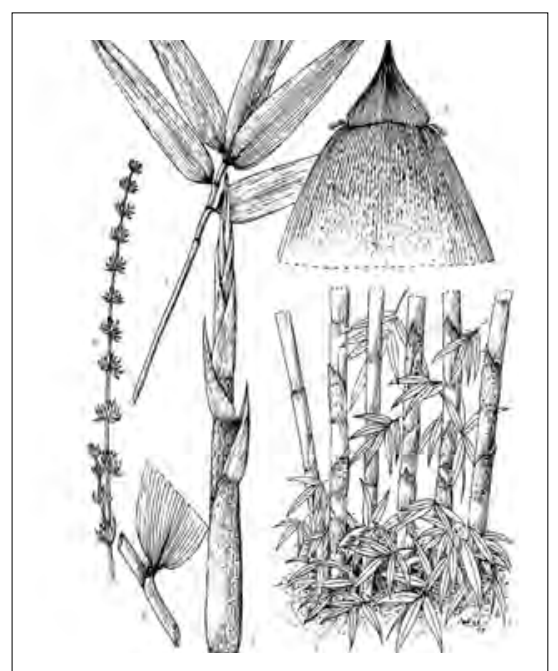

\begin{tabular}{|c|c|l|c|c|c|}
\hline$\#$ & Code & Local Name & Use & RU & Hab.-Ab. \\
\hline 1 & PUT & bulu pering & $4,6,11$ & $\mathbf{v}$ & G-2, S-1,Y-2, O-2 \\
\hline 2 & PUB & bulo' ring & & & \\
\hline 3 & PUD & & & \\
\hline 4 & PUM & & & \\
\hline 5 & ALS & & & \\
\hline 6 & LDY & & & \\
\hline 7 & LIT & bulu telang & $1,4,6$ & $\mathbf{v}$ & G-2, S-2, O-3 \\
\hline 8 & BRU & & & & \\
\hline 9 & MRP & blo' rai'e & & & \\
\hline 10 & PTN & & & & \\
\hline 11 & KLK & bulo lung & $4,6,11$ & $\mathbf{v}$ & G-2, S-2, O-3 \\
\hline 12 & KLM & bulu' lung & $4,6,11$ & $\mathbf{v}$ & G-2, S-2, O-3 \\
\hline 13 & KLO & bulu lung & $4,6,11$ & $\mathbf{v}$ & G-2, S-2, O-3 \\
\hline 14 & KLA & bulue lung & 4,6 & $\mathbf{v}$ & G-2, S-2, O-3 \\
\hline 15 & KAL & & & & \\
\hline 16 & KBD & bolo lung & $1,4,6$ & $\mathbf{v}$ & G-2, O-3, R \\
\hline 17 & KBK & bolo lung & $1,4,6,11$ & $\mathbf{v}$ & G-2, O-3, R \\
\hline 18 & PEB & leupek & $1,4,6,11$ & $\mathbf{v}$ & Y-2, O-3, R \\
\hline
\end{tabular}

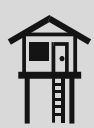

Notes : 


\section{y \\ Schizostachyum latifolium Gamble (Poaceae) \\ BAMBOO. BAMBU}

\section{DESCRIPTION}

Bamboo, 3-6 m, long pendulous tips; 1-2.5 cm d, wall c. 3-4 mm thick, light green, internodes 35-80 cm long; branches many from midculm up; culm sheath 10-16 x 4-5 cm, light green; leaves $12-30 \mathrm{x}$ $2.5-5.5 \mathrm{~cm}$, light green, glabrous

\section{HABITAT}

Lowland and hill primary forest to $1000 \mathrm{~m}$; riverine, forest margins, wastelands

\section{ORIGINS}

Borneo

\section{USES}

Culms for baskets, mats, containers, blowpipes, light construction; sold locally

\section{REFERENCES}

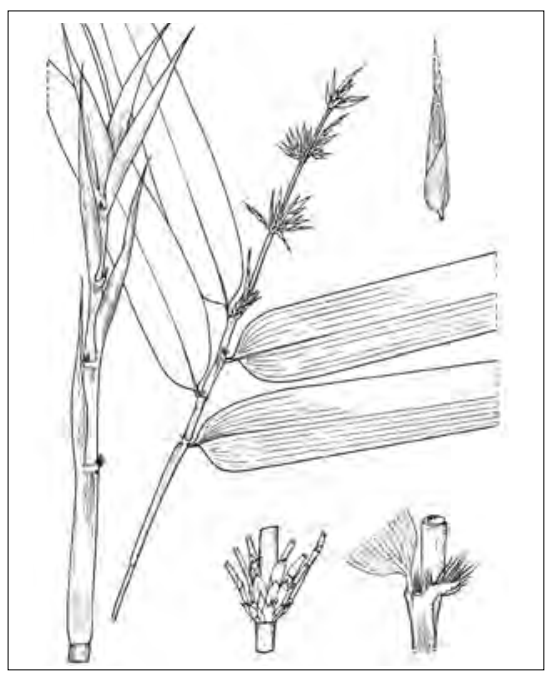

PROSEA Vol. 7: 137

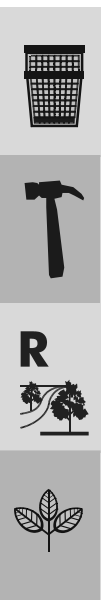

\begin{tabular}{|r|c|l|c|c|c|}
\hline$\#$ & Code & \multicolumn{1}{|c|}{ Local Name } & Use & RU & Hab.-Ab. \\
\hline 1 & PUT & bulu la'ung & $4,6,8,10,11$ & $\mathbf{v}$ & O-3, P-3, R \\
\hline 2 & PUB & bulo' kana & & & \\
\hline 3 & PUD & & & \\
\hline 4 & PUM & & & \\
\hline 5 & ALS & & & \\
\hline 6 & LDY & & & \\
\hline 7 & LIT & & & \\
\hline 8 & BRU & & & \\
\hline 9 & MRP & blo' nganu' & & & \\
\hline 10 & PTN & bulu lan & & $\mathbf{v}$ & Y-1, O-3, P-3, R \\
\hline 11 & KLK & bulo lan & $4,6,8,8,11$ & $\mathbf{v}$ & Y-1, O-3, P-3, R \\
\hline 12 & KLM & bulu' lan & $4,6,8,11$ & $\mathbf{v}$ & Y-1, O-3, P-3, R \\
\hline 13 & KLO & bulu tue & $4,6,8,11$ & $\mathbf{v}$ & Y-1, O-3, P-3, R \\
\hline 14 & KLA & bulue buvan & $4,6,8,11$ & $\mathbf{v}$ & Y-1, O-3, P-3, R \\
\hline 15 & KAL & bulue tu'u & $4,6,8,11$ & $\mathbf{v}$ & Y-1, O-3, P-3, R \\
\hline 16 & KBD & bolo lan & $4,6,8,11$ & $\mathbf{v}$ & Y-1, O-3, P-3, R \\
\hline 17 & KBK & bolo lan & $4,6,8$ & $\mathbf{v}$ & Y-1, O-3, P-3, R \\
\hline 18 & PEB & leupek le & $4,6,8,11$ & $\mathbf{v}$ & Y-1, O-3, P-3, R \\
\hline
\end{tabular}

Notes : 
Xanthopbyllum obscurum A.W. Benn. (Polygalaceae)

\section{(NOENGLISH NAME). KAYU BATU}

\section{DESCRIPTION}

Tree, to $40 \mathrm{~m}$, columnar; bark grey brown, finely cracked; leaves simple, alternate, elliptic to ovate 4.511 x $1.5-4.5 \mathrm{~cm}$, secondary nerves $3-4$ pairs only, drying shiny brown; flower petals pink or white $1-1.5 \mathrm{~cm}$ long; fruit round, pimply, $5.75-6.75 \mathrm{~cm}$.

\section{HABITAT}

Lowland and hill primary forests; cultivated in village fruit gardens

\section{ORIGINS}

Malaysia and western Indonesia

\section{USES}

Fruit eaten, sold locally; hunting location when fruiting

\section{REFERENCES}

TFM Vol. 1: 363; PROSEA Vol. 5(3): 585

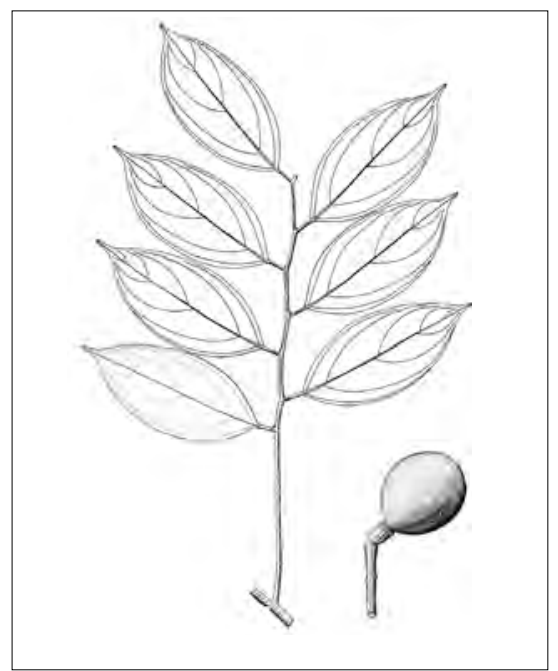

\begin{tabular}{|c|c|c|c|c|c|}
\hline \# & Code & Local Name & Use & RU & Hab.-Ab. \\
\hline 1 & PUT & mesalin, mesalin ulih & 1,10 & $\mathrm{v}$ & G-1, P-4 \\
\hline 2 & PUB & mesalin & 1 & v & G-1, P-3 \\
\hline 3 & PUD & mesalin & 1,10 & V & G-1 \\
\hline 4 & PUM & mesalin & 1,10 & V & G-1, P-3 \\
\hline 5 & ALS & bua' tembasak & 1 & v & G-2, P-3 \\
\hline 6 & LDY & tefasak & 1,11 & v & P-3 \\
\hline 7 & LIT & tepasak & 1 & v & P-3 \\
\hline 8 & BRU & wi beleau & 1,10 & v & P-3 \\
\hline 9 & MRP & banyaling, menyalin, nyaling & $1,10,11$ & v & P-3 \\
\hline 10 & PTN & bua menyalin, bejalin & 1,10 & v & G-2, P-3, M-2 \\
\hline 11 & KLK & mezalin, mejalin & 1 & v & $\mathrm{G}-2$ \\
\hline 12 & KLM & majalin & 1 & v & G-2 \\
\hline 13 & KLO & bezalin & 1 & $x$ & G-2 \\
\hline 14 & KLA & bezalin & 1 & $x$ & $G-2, P-2$ \\
\hline 15 & KAL & bejalin & 1 & $x$ & G-2 \\
\hline 16 & KBD & mezalin, menyalin & 1,10 & $x$ & $\mathrm{P}-3$ \\
\hline 17 & KBK & pejalin & 1 & $x$ & G-2 \\
\hline 18 & PEB & nyemalin & 1 & $\mathrm{v}$ & G-2, P-4, M-3 \\
\hline
\end{tabular}

Notes : 


\section{Prunus arborea (B1.) Kalkman (Rosaceae)}

\section{CuRRanT LAURAL. MEDANG}

\section{DESCRIPTION}

Tree, to $30 \mathrm{~m}$, bark smooth dark brown, twigs velvety, bark smell of bitter almonds; leaves 5-9 pairs side veins, glands at leaf stalk, elliptic, 3-25 x $1.5-13 \mathrm{~cm}$; flowers bunched stalks, yellow white petals; fruit a bilobed drupe, $11 \times 17 \mathrm{~mm}$, pink red, pulpy

\section{HABITAT}

Lowland and hill primary and secondary forests

\section{ORIGINS}

Tropical Asia

\section{USES}

Bark stripped for light and heavy construction, boats and storage containers

\section{REFERENCES}

FOJ Vol. I: 153; FM I Vol. 10: 172;

PROSEA Vol. 5(3): 475

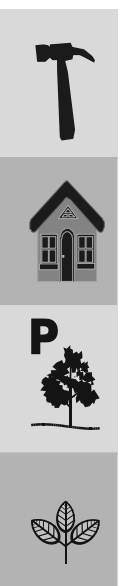

\begin{tabular}{|c|c|l|c|c|c|}
\hline$\#$ & Code & Local Name & Use & RU & Hab.-Ab. \\
\hline 1 & PUT & betolih, betoli & $4,6,10$ & $\mathbf{x}$ & O-2, P-2, M-2 \\
\hline 2 & PUB & betoli & $3,4,6$ & $\mathbf{v}$ & O-2, P-2 \\
\hline 3 & PUD & betoli & $3,4,6$ & $\mathbf{V}$ & O-2, P-2 \\
\hline 4 & PUM & & & & \\
\hline 5 & ALS & tengalonos & $3,4,6$ & $\mathbf{x}$ & O-3, P-2 \\
\hline 6 & LDY & gelanit & $3,4,6$ & $\mathbf{x}$ & O-3, P-2 \\
\hline 7 & LIT & galanad & $3,4,6$ & $\mathbf{x}$ & O-3, P-2 \\
\hline 8 & BRU & kayau lamana & $3,4,6$ & $\mathbf{x}$ & O-3, P-2 \\
\hline 9 & MRP & enggai, wig & $3,4,6$ & $\mathbf{x}$ & O-3, P-2 \\
\hline 10 & PTN & beteli & $3,4,6$ & $\mathbf{x}$ & O-3, P-2 \\
\hline 11 & KLK & beteli & $3,4,5,6$ & $\mathbf{x}$ & O-3, P-3 \\
\hline 12 & KLM & beteli & $3,4,5,6$ & $\mathbf{x}$ & O-3, P-3 \\
\hline 13 & KLO & betteli & $3,4,5,6$ & $\mathbf{x}$ & O-3, P-3 \\
\hline 14 & KLA & batolei & $3,4,6$ & $\mathbf{x}$ & P-4 \\
\hline 15 & KAL & batolei & $3,4,6$ & $\mathbf{x}$ & O-3, P-3 \\
\hline 16 & KBD & batolei & $3,4,5,6$ & $\mathbf{v}$ & O-3, P-3 \\
\hline 17 & KBK & beta'au, beteli & $3,4,5,6$ & $\mathbf{x}$ & O-3, P-3 \\
\hline 18 & PEB & batolei & $3,4,5,6$ & $\mathbf{v}$ & O-3, P-3 \\
\hline
\end{tabular}

Notes : 


\section{Antbocepbalus chinensis (Lamk) Rich ex. Walp. (Rubiaceae) \\ COMMON BUR-FLOWER TREE. JABON, KELEMPAYAN}

\section{DESCRIPTION}

Syn: Neolamarckia cadamba (Roxb.) Bosser

Tree, to $45 \mathrm{~m}$, bole straight cylindrical, stiff outstanding branches; bark smooth to scaly, grey brown; leaves, opposite, elliptic to obovate, 12-30 x 5-16 cm; flowers yellow on globular, terminal head, $2-4 \mathrm{~cm} \mathrm{~d}$; fruiting head orange; two varieties

\section{HABITAT}

Lowland secondary forests, pioneer species

\section{ORIGINS}

India, Malaysia and western Indonesia

\section{USES}

Timber for blowpipes, firewood; leaves for medicine; fruit eaten; hunting location

\section{REFERENCES}

Corner 1988: 624; Kessler and Sidiyasa 1994: 200;

PROSEA Vol. 5(1): 107; TFM Vol. 4: 381

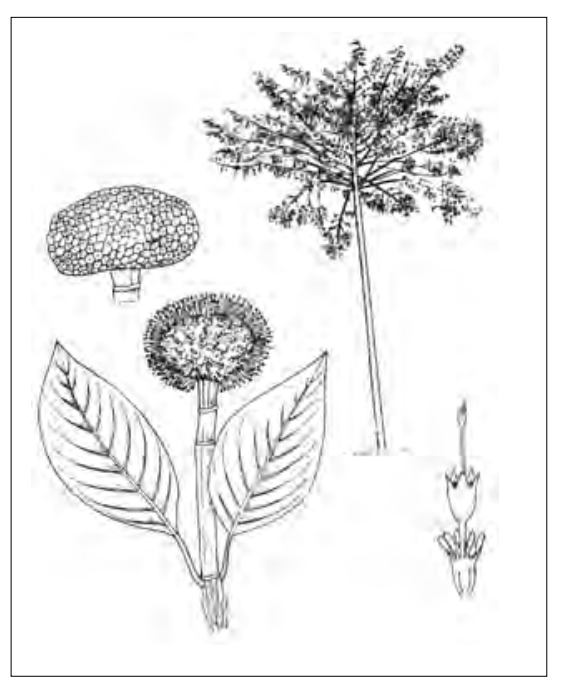

\begin{tabular}{|c|c|l|c|c|c|}
\hline$\#$ & Code & Local Name & Use & RU & Hab.-Ab. \\
\hline 1 & PUT & poung & 6,10 & $\mathbf{x}$ & Y-2, O-3, P-1 \\
\hline 2 & PUB & poung & $1,6,10$ & $\mathbf{x}$ & Y-3, O-4 \\
\hline 3 & PUD & paung & 7 & $\mathbf{X}$ & Y-2, O-3, P-4 \\
\hline 4 & PUM & paung & 1,7 & $\mathbf{v}$ & Y-3, O-3, P-2 \\
\hline 5 & ALS & taun tearn & 6 & $\mathbf{X}$ & Y-2, O-3 \\
\hline 6 & LDY & kayu tangdan & $1,6,7$ & $\mathbf{x}$ & \\
\hline 7 & LIT & & & & $\mathbf{X}$ \\
\hline 8 & BRU & uen tuap & & $\mathbf{X}$ & Y-3, O-4, P-1 \\
\hline 9 & MRP & tuwa' & 6,7 & $\mathbf{X}$ & Y-4, O-4, P-2 \\
\hline 10 & PTN & kayo saong & & & \\
\hline 11 & KLK & & & $\mathbf{X}$ & ALL \\
\hline 12 & KLM & nyaung & & & \\
\hline 13 & KLO & ta' bavang & & & \\
\hline 14 & KLA & & & & \\
\hline 15 & KAL & kazu saong & & & \\
\hline 16 & KBD & kayu sa'ong & & & \\
\hline 17 & KBK & & 6 & $\mathbf{v}$ & Y-2, O-3, P-4 \\
\hline 18 & PEB & betonang beu, betenong & & \\
\hline
\end{tabular}

Notes : 


\section{Antbocepbalus sp. (Rubiaceae)}

\section{(NO ENGLISH/INDONESIAN. NAME)}

\section{DESCRIPTION}

Syn: Neolamarckia sp.

Tree, stem quadrangular dark brown 2-4 $\mathrm{mm}$; petiole $1.5 \mathrm{~cm}$; leaves elliptic to oval, short tipped, cuneate base, 16-17 x 7-8 cm, drying dark brown above, light brown below; tert. veins perpendicular to 10-11 pairs prominent secondary veins; two varieties

\section{HABITAT}

Lowland primary and secondary forest, village lands

\section{ORIGINS}

Unknown

\section{USES}

Leaves for medicine; timber for firewood,

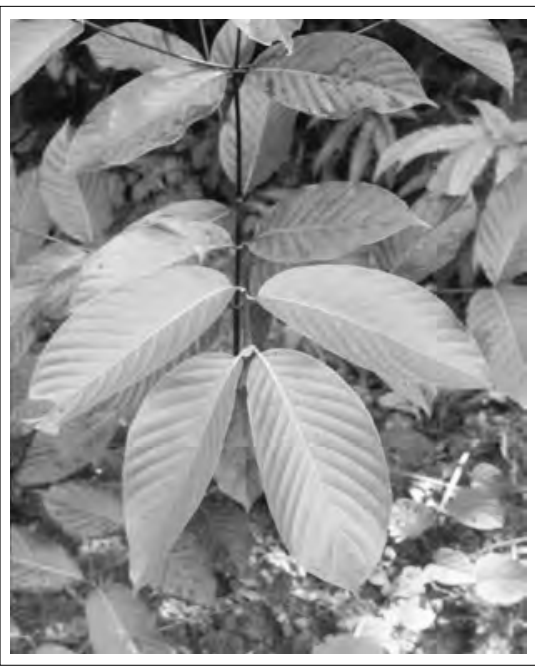
material for tools

\section{REFERENCES}

Kessler and Sidiyasa 1994: 200

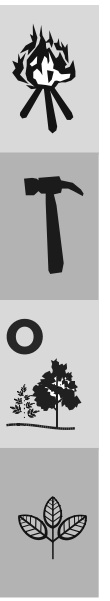

\begin{tabular}{|c|c|l|c|c|c|}
\hline$\#$ & Code & \multicolumn{1}{|c|}{ Local Name } & Use & RU & Hab.-Ab. \\
\hline 1 & PUT & kebalun atang, kebalun & 6,7 & $\mathbf{X}$ & Y-3, O-2, P-3 \\
\hline 2 & PUB & kebalun atang, kebalun & 6,7 & $\mathbf{X}$ & O-2, P-3, M-2 \\
\hline 3 & PUD & kebalun & 6,7 & $\mathbf{X}$ & Y-3, O-2, P-3 \\
\hline 4 & PUM & kebalun atang & 6,7 & $\mathbf{X}$ & Y-3, O-3 \\
\hline 5 & ALS & kemalu logon & 6,7 & $\mathbf{V}$ & Y-3, O-3, P-2 \\
\hline 6 & LDY & kayu atap & $5,6,7$ & $\mathbf{X}$ & Y-2, O-3, P-4 \\
\hline 7 & LIT & tap, tap libu & $2,6,7$ & $\mathbf{X}$ & Y-2, O-3, P-3 \\
\hline 8 & BRU & kemalu lagon, uen tuap & 6,7 & $\mathbf{X}$ & Y-2, O-3 \\
\hline 9 & MRP & kemalaung, kayu latip, kemung & 6,7 & $\mathbf{X}$ & Y-3, O-4, P-2, R \\
\hline 10 & PTN & kevalun usok, kebelun, kebalum & 6,7 & $\mathbf{V}$ & Y-4, O-4, P-1, R \\
\hline 11 & KLK & tebalut & 6,7 & $\mathbf{X}$ & Y-2, O-3, P-4 \\
\hline 12 & KLM & tebalut, tembaut & 6 & $\mathbf{X}$ & O-2 \\
\hline 13 & KLO & tegema, da'eng ta' & 6,7 & $\mathbf{V}$ & S-3, Y-3, O-3 \\
\hline 14 & KLA & ketalut & 6,7 & $\mathbf{V}$ & Y-3, O-3, P-3, R \\
\hline 15 & KAL & tehamput & $2,6,7$ & $\mathbf{v}$ & \\
\hline 16 & KBD & tebalut & 1,8 & $\mathbf{v}$ & Y-3, O-3, P-3 \\
\hline 17 & KBK & ketambalut iut & & & \\
\hline 18 & PEB & tap & 6,7 & $\mathbf{v}$ & Y-2, 0-4, P-2 \\
\hline
\end{tabular}

Notes : 


\section{Coffea robusta L. (Rubiaceae) \\ COFFEE. KOPI

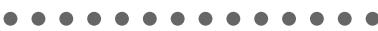

\section{DESCRIPTION}

Tree, to $3 \mathrm{~m}$, long drooping limbs; leaves ovate, strongly ribbed and wrinkled; flowers with 6-8, mostly 7 petals, white fragrant, clustered in leaf axils; fruit ellipsoid, $12 \mathrm{~mm}$ long, red then black; 2 seeds

\section{HABITAT}

Cultivated in villages and swidden gardens; often under bananas or other fruit trees, with cocoa

\section{ORIGINS}

Africa, brough to Indonesia in 1900

\section{USES}

Fruit for coffee; sold locally and to traders for export; medicine

\section{REFERENCES}

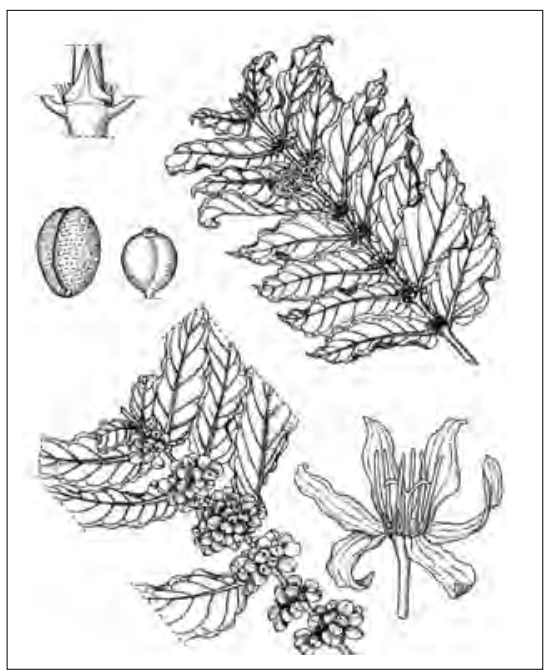

Burkill 1966: 636; Corner 1988: 628;

PROSEA 16: 69

\begin{tabular}{|c|c|c|c|c|c|}
\hline \# & Code & Local Name & Use & RU & Hab.-Ab. \\
\hline 1 & PUT & kawa & 1,11 & $\mathrm{v}$ & $\mathrm{G}-4$ \\
\hline 2 & PUB & kawa & 1,11 & v & $\mathrm{G}-4, \mathrm{~S}-3$ \\
\hline 3 & PUD & kawa & 1,10 & $\mathrm{v}$ & $G-4, S-3$ \\
\hline 4 & PUM & kawa & 1,11 & v & $G-4, S-3$ \\
\hline 5 & ALS & bua' kupi & $1,7,11$ & V & $\mathrm{G}-4, \mathrm{~S}-3$ \\
\hline 6 & LDY & bua kufi, kupi & 1,11 & V & $\mathrm{G}-4, \mathrm{~S}-2$ \\
\hline 7 & LIT & kopi, kupi & 1,11 & v & $\mathrm{G}-4, \mathrm{~S}-3$ \\
\hline 8 & BRU & wi kupi & $1,2,11$ & V & $\mathrm{G}-4, \mathrm{~S}-3$ \\
\hline 9 & MRP & kawa', kawa & 1,11 & V & $G-4, S-3$ \\
\hline 10 & PTN & kupi & 1,11 & v & $\mathrm{G}-4, \mathrm{~S}-3$ \\
\hline 11 & KLK & kupi, kopi & 1,11 & V & $\mathrm{G}-4, \mathrm{~S}-3$ \\
\hline 1,1 & KLM & kupi & 1,11 & v & $G-4, S-3$ \\
\hline 13 & KLO & kopi, kupi & 1 & V & $G-4, S-3$ \\
\hline 14 & KLA & hopi, hupi, kupi & 1 & v & G-4 \\
\hline 15 & KAL & kupi & 1,11 & v & G-4, S-3 \\
\hline 16 & KBD & kupi & 1,11 & v & $\mathrm{G}-4, \mathrm{~S}-3$ \\
\hline 11,1 & KBK & kupi & 1,11 & v & G-4 \\
\hline 18 & PEB & kupi & 1,11 & $\mathrm{v}$ & $\mathrm{G}-4, \mathrm{~S}-3$ \\
\hline
\end{tabular}

Notes : 


\section{Citrus bystrix DC. (Rutaceae)}

\section{LEECH-LIME.JERUK PERUT}

\section{DESCRIPTION}

Tree, 2-12 m, crooked, spiny branches; leaves alternate, ovate to oblong, 3-15 x 2.5-6 cm, with winged stalk as wide as leaf blade, slightly toothed; fruit round, $5-7 \mathrm{~cm} \mathrm{~d}$, wrinkled and bumpy, green turning yellow; sour yellow green pulp

\section{HABITAT}

Cultivated in interior Bulungan villages; wild in abandoned villages

\section{ORIGINS}

Malaysia and western Indonesia, Indochina

\section{USES}

Rind for shampoo; leaves for condiment, medicine

\section{REFERENCES}

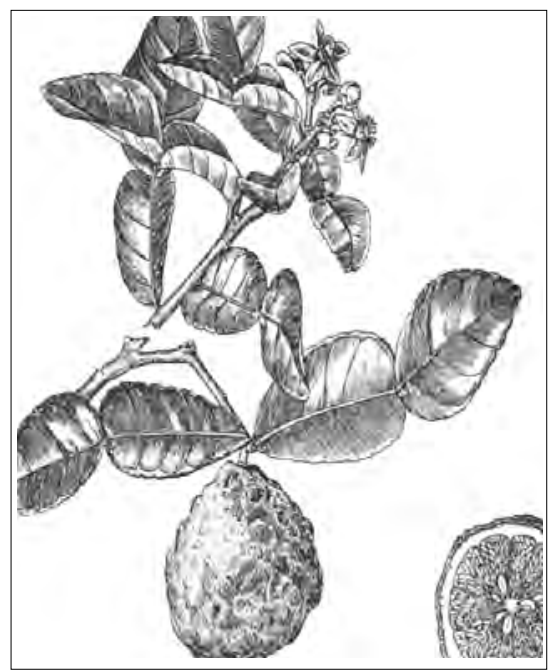

Burkill 1966: 575; Corner 1988: 661;

PROSEA Vol. 2: 326

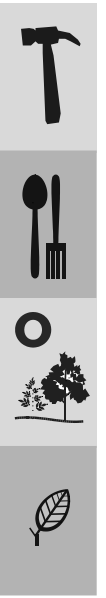

\begin{tabular}{|r|c|l|c|c|c|}
\hline$\#$ & Code & \multicolumn{1}{|c|}{ Local Name } & Use & RU & Hab.-Ab. \\
\hline 1 & PUT & buyau, laben, laben ayo kung & & & \\
\hline 2 & PUB & laben & & & \\
\hline 3 & PUD & laben & & & \\
\hline 4 & PUM & doun buyau & & & \\
\hline 5 & ALS & limau temadur & & & \\
\hline 6 & LDY & boyo rawang, buyo & & & \\
\hline 7 & LIT & buyau lam, buyau raye, buyau rayoh & & & \\
\hline 8 & BRU & wi laban men & & & \\
\hline 9 & MRP & laban, meiu, me'o & & & \\
\hline 10 & PTN & bunyou zangan, bunyo aya & 6 & $\mathbf{x}$ & O-1, P-1, M-1 \\
\hline 11 & KLK & bunyau bio', bunyau kla'ang & 6 & $\mathbf{x}$ & O-1, P-1 \\
\hline 12 & KLM & bunyau bio, bunyau baen & 6 & $\mathbf{x}$ & O-1, P-1 \\
\hline 13 & KLO & bonyo alo & 6 & $\mathbf{x}$ & O-1 \\
\hline 14 & KLA & bunyo & 1,6 & $\mathbf{x}$ & O-1, P-1 \\
\hline 15 & KAL & bunyo late' & 6 & $\mathbf{x}$ & O-1, P-1 \\
\hline 16 & KBD & bonyo alo & 6 & $\mathbf{x}$ & O-1, P-1, M-1 \\
\hline 17 & KBK & bunyo bali & 6 & $\mathbf{x}$ & O-1 \\
\hline 18 & PEB & bunyeu, bunyeu baleu & & \\
\hline
\end{tabular}

\section{Notes :}




\section{Citrus maxima (Burm.) Merr. (Rutaceae)}

\section{Pomelo. Jeruk Besar

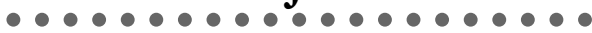

\section{DESCRIPTION}

Tree, 5-10 m, low branching, crooked, spiny; leaves ovate to elliptical $5-10 \times 2-5 \mathrm{~cm}$, toothed edges, winged leaf stalk less than half size of blade; fruits pear-shaped, 10-25 cm d, pale green to yellow; pulp reddish pink or pale yellow, sweet

\section{HABITAT}

Cultivated in villages

\section{ORIGINS}

Malaysia and western Indonesia, Indochina

\section{USES}

Fruit eaten, sold locally; timber for light construction

\section{REFERENCES}

Burkill 1966: 575; Corner 1988: 661; PROSEA Vol. 2: 128

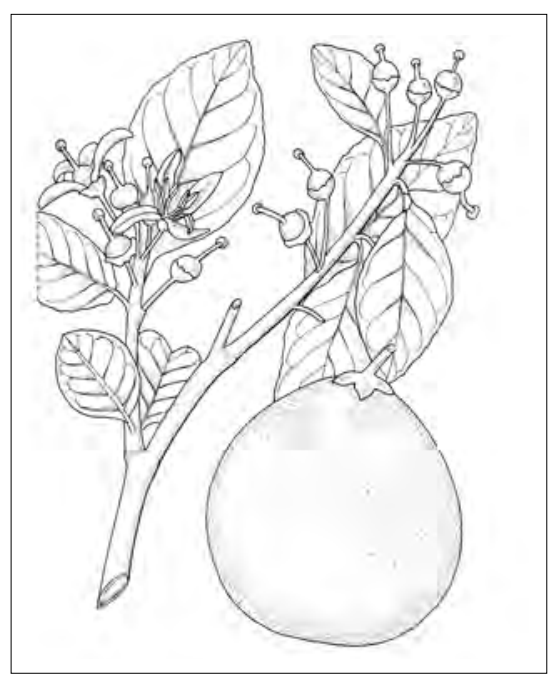

\begin{tabular}{|c|c|c|c|c|c|}
\hline \# & Code & Local Name & Use & RU & Hab.-Ab. \\
\hline 1 & PUT & laben & 1,11 & $\mathrm{v}$ & G-3 \\
\hline 2 & PUB & laben & 1,11 & v & G-3 \\
\hline 3 & PUD & laben & 1,11 & v & G-3 \\
\hline 4 & PUM & laben & 1,11 & v & G-3 \\
\hline 5 & ALS & bua' buyau & 1,11 & v & G-3 \\
\hline 6 & LDY & buyo & 1,11 & v & G-3 \\
\hline 7 & LIT & bua' buyau & 1,11 & v & G-4 \\
\hline 8 & BRU & wi laben gong & 1,11 & v & G-3 \\
\hline 9 & MRP & laban bue, laban bue & 1,11 & v & G-3 \\
\hline 10 & PTN & mieu' & 1,11 & v & G-3 \\
\hline 11 & KLK & miu, bunyau ba'an & $1,2,11$ & v & G-3 \\
\hline 12 & KLM & mieu, mieu bio & 1,11 & v & G-3 \\
\hline 13 & KLO & ke mi & 1 & v & G-2 \\
\hline 14 & KLA & miu & $1,6,11$ & $\mathrm{v}$ & G-4 \\
\hline 15 & KAL & miu & 1,11 & $\mathrm{v}$ & G-3 \\
\hline 16 & KBD & mieu, limau & 1,11 & v & G-2 \\
\hline 17 & KBK & miu & 1,11 & v & G-2 \\
\hline 18 & PEB & miu beledau & 1 & v & G-1 \\
\hline
\end{tabular}

Notes : 


\section{Citrus reticulata Blanco (Rutaceae)}

\section{KING MANDARIN. JERUKJEPUN, JERUKMANIS}

\section{DESCRIPTION}

Tree, 5-10 m, upright spiny, leaves broadly lanceolate, narrowly winged leafstalks, fruits large broad, 12-14 segments, peel thick, orange to vermilion, sweet fruit

\section{HABITAT}

Cultivated in villages

\section{ORIGINS}

Malaysia and western Indonesia, Indochina

\section{USES}

Fruit eaten, medicine, sold locally; timber for construction

\section{REFERENCES}

Burkill 1966: 575; PROSEA Vol. 2: 135;

TFM Vol. 1: 375
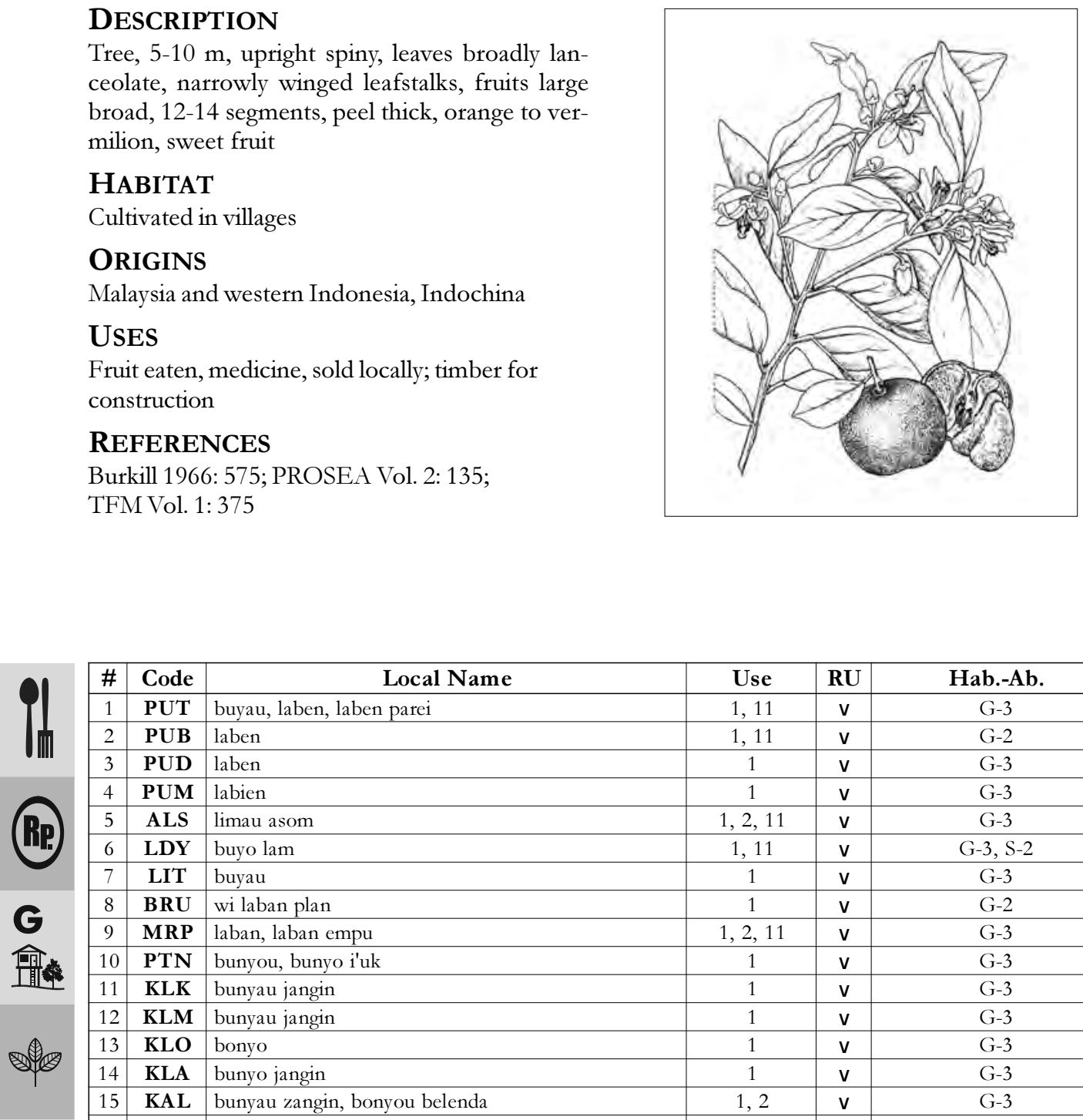

\begin{tabular}{|c|c|l|c|c|c|}
\hline$\#$ & Code & Local Name & Use & RU & Hab.-Ab. \\
\hline 1 & PUT & buyau, laben, laben parei & 1,11 & $\mathbf{v}$ & G-3 \\
\hline 2 & PUB & laben & 1,11 & $\mathbf{v}$ & G-2 \\
\hline 3 & PUD & laben & 1 & $\mathbf{V}$ & G-3 \\
\hline 4 & PUM & labien & 1 & $\mathbf{v}$ & G-3 \\
\hline 5 & ALS & limau asom & $1,2,11$ & $\mathbf{v}$ & G-3 \\
\hline 6 & LDY & buyo lam & 1,11 & $\mathbf{v}$ & G-3, S-2 \\
\hline 7 & LIT & buyau & 1 & $\mathbf{v}$ & G-3 \\
\hline 8 & BRU & wi laban plan & 1 & $\mathbf{v}$ & G-2 \\
\hline 9 & MRP & laban, laban empu & $1,2,11$ & $\mathbf{v}$ & G-3 \\
\hline 10 & PTN & bunyou, bunyo i'uk & 1 & $\mathbf{v}$ & G-3 \\
\hline 11 & KLK & bunyau jangin & 1 & $\mathbf{v}$ & G-3 \\
\hline 12 & KLM & bunyau jangin & 1 & $\mathbf{v}$ & G-3 \\
\hline 13 & KLO & bonyo & 1 & $\mathbf{v}$ & G-3 \\
\hline 14 & KLA & bunyo jangin & 1,2 & $\mathbf{v}$ & G-3 \\
\hline 15 & KAL & bunyau zangin, bonyou belenda & 1 & $\mathbf{v}$ & G-3 \\
\hline 16 & KBD & bunyau jangin & 1 & $\mathbf{v}$ & G-3 \\
\hline 17 & KBK & bunyeu, pungo & 1 & $\mathbf{v}$ & G-3 \\
\hline 18 & PEB & bunyau rumit, bunyo jangin & & G-3 \\
\hline
\end{tabular}

Notes : 


\section{Dimocarpuslongan Lour.ssp. malesianus var.malesiamus Leenh. (Sapindaceae) \\ BROWN CAT'S EYE. MATA KUCING}

\section{DESCRIPTION}

Tree, to $30 \mathrm{~m}$; stems hairy, 5 faint grooves; dark green glossy foliage, leaves pinnate, leaflets 3-5 pairs, elliptical 3-45 x 1.5-20 cm, velvety beneath; fruit round $1-3 \mathrm{~cm}$ diameter, thin, smooth, brown shell with dark flecks; white sweet pulp, shiny brown seed

\section{HABITAT}

Lowland primary and secondary forests to $500 \mathrm{~m}$; riverine; cultivated in village fruit gardens

\section{ORIGINS}

Malaysia and western Indonesia, Indochina

\section{USES}

Fruit eaten, sold to traders; leaves for medicine timber for firewood, light construction; hunting location

\section{REFERENCES}

Corner 1988: 685; PROSEA Vol. 2: 146

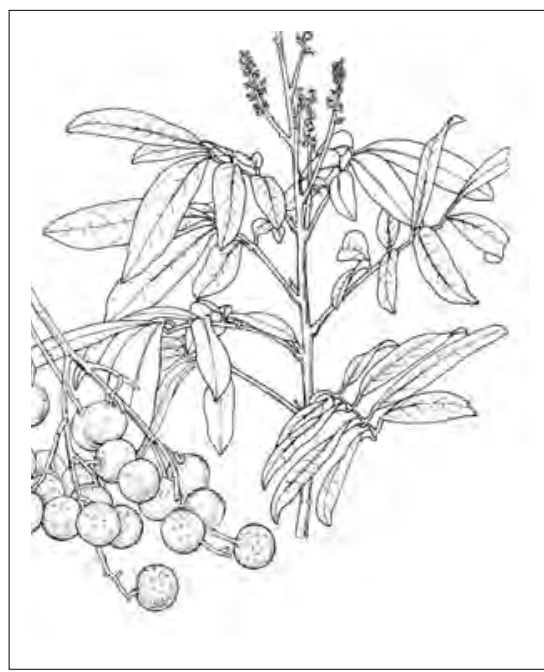

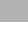




\section{Dimocarpuslongan Lour. ssp. malesianusvar. malesianus Leenh. (Sapindaceae)}

\section{GREEN CAT'S EYE. MATA KUCING \\ ○}

\section{DESCRIPTION}

Tree, to $40 \mathrm{~m}$; stems hairy, 5 faint grooves; dark green glossy foliage, leaves pinnate, leaflets 3-5 pairs, elliptical 3-45 x 1.5-20 cm, velvety beneath; fruit oblong $2 \times 1 \mathrm{~cm}$, thicker, warty green shell, white seed pulp, shiny brown seed

\section{HABITAT}

Lowland and hill primary forests; abandoned villages, hillsides, ridges to $1000 \mathrm{~m}$; cultivated in village fruit gardens

\section{ORIGINS}

Malaysia and western Indonesia, Indochina

\section{USES}

Fruit eaten, sold to traders; leaves for medicine; timber for tools, firewood and construction

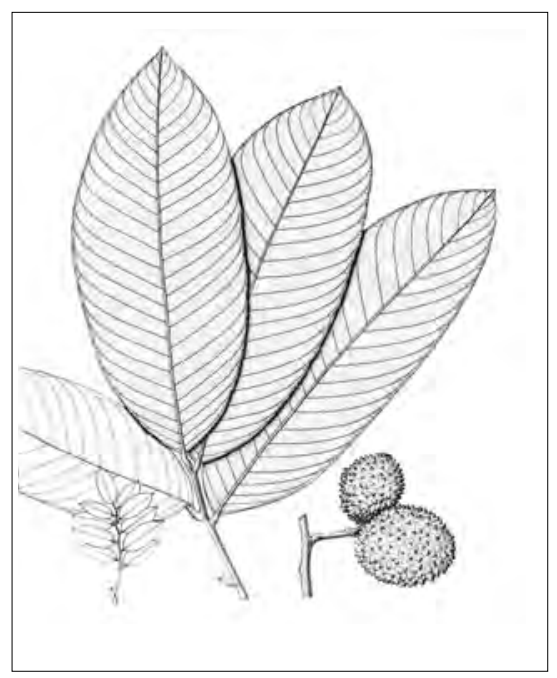

\section{REFERENCES}

Corner 1988: 685; PROSEA Vol. 2: 146;

Valkenburg 1997: 72

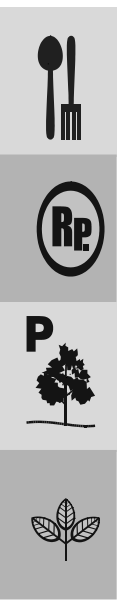

\begin{tabular}{|c|c|l|c|c|c|}
\hline$\#$ & Code & \multicolumn{1}{|c|}{ Local Name } & Use & RU & Hab.-Ab. \\
\hline 1 & PUT & nyau baung & $1,7,11$ & $\mathbf{v}$ & P-3 \\
\hline 2 & PUB & nyau baung & $1,7,11$ & $\mathbf{v}$ & G-2, P-3 \\
\hline 3 & PUD & nyau bung & $1,7,11$ & $\mathbf{v}$ & P-3 \\
\hline 4 & PUM & nyau baung & 1,11 & $\mathbf{v}$ & P-3, M-2 \\
\hline 5 & ALS & mamu bura' & $1,7,11$ & $\mathbf{v}$ & G-3, P-3 \\
\hline 6 & LDY & bua' mapu bata', mepu pulung & $1,6,7,11$ & $\mathbf{v}$ & G-2 \\
\hline 7 & LIT & bua yau, yau & 1,11 & $\mathbf{v}$ & G-3, P-2 \\
\hline 8 & BRU & wi yau ata & $1,11,7$ & $\mathbf{v}$ & G-2 \\
\hline 9 & MRP & heau laue, heyo la'lieng, hio mawe & $1,7,11$ & $\mathbf{v}$ & G-2, P-2 \\
\hline 10 & PTN & iho bileng & $1,7,11$ & $\mathbf{v}$ & G-2, P-3 \\
\hline 11 & KLK & isau bilang, isau bileng & $1,7,11$ & $\mathbf{v}$ & G-3, P-4 \\
\hline 12 & KLM & esau belang, isau belang & 1,11 & $\mathbf{v}$ & G-2 \\
\hline 13 & KLO & iso beleng, esau beleng & 1,7 & $\mathbf{x}$ & G-3, Y-3, O-3, P-3 \\
\hline 14 & KLA & esau bileng, isau bileng, isau vileng & $1,7,11$ & $\mathbf{x}$ & G-3, Y-3, O-3, P-3 \\
\hline 15 & KAL & isau beleng & $1,7,11$ & $\mathbf{v}$ & G-1, P-3 \\
\hline 16 & KBD & esau beleng, isau beleng & $1,7,10,11$ & $\mathbf{v}$ & G-4, O-3, P-3 \\
\hline 17 & KBK & isau beleng & 1,11 & $\mathbf{v}$ & G-1, O-3, P-4, M-4 \\
\hline 18 & PEB & sau, sau marung & 1,11 & $\mathbf{v}$ & G-3, S-2, Y-2, P-2 \\
\hline
\end{tabular}

Notes : 


\section{Nephelium cuspidatum Bl. var. eriopetalum (Miq.)Leenh. (Sapindacea
(NO ENGLISH NAME). DEKET, RAMBUTAN KABUNG}

\section{DESCRIPTION}

Tree, to $25 \mathrm{~m}$; twigs, veins, midrib of leaflets densely hairy; leaflets oblong, 17-28 x 3-12.5 cm, 3-4 pairs, not glossy above, drooping, terminal pair to 25 $\mathrm{cm}$; fruit ellipsoid to globular, 2-4 x 2-3 cm, densely set red appendages to $2 \mathrm{~cm}$ long

\section{HABITAT}

Lowland and hill primary forests; old secondary forest

\section{ORIGINS}

Malaysia and western Indonesia

\section{USES}

Fruit eaten, sold locally; timber for tools, firewood, light construction; hunting location

\section{REFERENCES}

Corner 1988: 684; PROSEA Vol. 2: 233; TFM

Vol. 4: 452

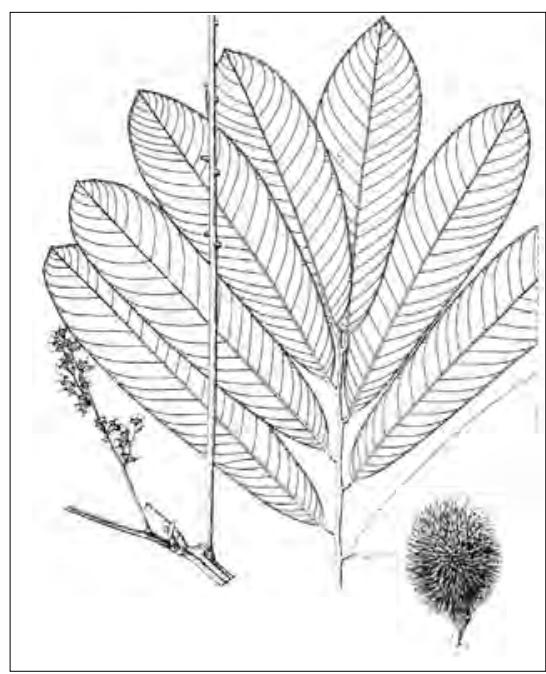

\begin{tabular}{|c|c|l|c|c|c|}
\hline \# & Code & \multicolumn{1}{|c|}{ Local Name } & Use & RU & Hab.-Ab. \\
\hline 1 & PUT & ha'iu & $1,6,7,10,11$ & $\mathbf{v}$ & G-2, O-3, P-3 \\
\hline 2 & PUB & ha'iu & $1,7,10$ & $\mathbf{v}$ & G-3, O-2, P-3 \\
\hline 3 & PUD & ha'iu & 1,10 & $\mathbf{v}$ & G-2, O-2, P-3, M-2 \\
\hline 4 & PUM & h'iu & 1,7 & $\mathbf{v}$ & G-4, P-3, M-4 \\
\hline 5 & ALS & bua' kelemuku & $1,7,11$ & $\mathbf{v}$ & G-2, P-3 \\
\hline 6 & LDY & bua kelemuku & $1,6,7,11$ & $\mathbf{v}$ & G-3, O-2, P-3, M-3 \\
\hline 7 & LIT & kelemuku & 1,7 & $\mathbf{v}$ & G-3, Y-3, P-2 \\
\hline 8 & BRU & wi lemuku & $1,7,11$ & $\mathbf{v}$ & G-3, O-3, P-3 \\
\hline 9 & MRP & ha'ai & $1,7,10,11$ & $\mathbf{v}$ & G-2, O-2, P-3 \\
\hline 10 & PTN & bua kelungo & $1,7,11$ & $\mathbf{v}$ & G-2, O-2, P-4 \\
\hline 11 & KLK & kelemawa, kelamawa & 1,11 & $\mathbf{v}$ & G-2, O-2, P-3 \\
\hline 12 & KLM & sibau & $1,10,11$ & $\mathbf{v}$ & G-3 \\
\hline 13 & KLO & beva levavu & 1 & $\mathbf{x}$ & G-2, Y-2, O-2, M-2 \\
\hline 14 & KLA & kelem mava & 1,10 & $\mathbf{x}$ & P-3 \\
\hline 15 & KAL & kelemavo & & & \\
\hline 16 & KBD & sebau, bua empat, embui luan & $1,2,7$ & $\mathbf{v}$ & G-2, O-3, P-3 \\
\hline 17 & KBK & bua' empat & & & \\
\hline 18 & PEB & kemawa & $1,6,7$ & $\mathbf{v}$ & $0-2$, P-4, M-3 \\
\hline
\end{tabular}

Notes : 


\section{Nepbelium juglandifolium B1. (Sapindaceae)}

\section{(NOENGLISH NAME). LUNGSIR}

\section{DESCRIPTION}

Tree, to $30 \mathrm{~m}$, twigs thick, slightly hairy; leaflets 3-7 pairs, 7.5-32 x 2.5-9.5 cm, apex blunt to round, thin, dark green above, glabrous or sparsely hairy below; fruits flattened 3.5-5 × $2.5 \times 2 \mathrm{~cm}$, appendages blunt triangular and flattened, waxy, green

\section{HABITAT}

Lowland primary forests to $650 \mathrm{~m}$; planted in villages

\section{ORIGINS}

Malaysia and western Indonesia

\section{USES}

Fruit eaten, sold locally; timber for firewood; hunting location when fruiting

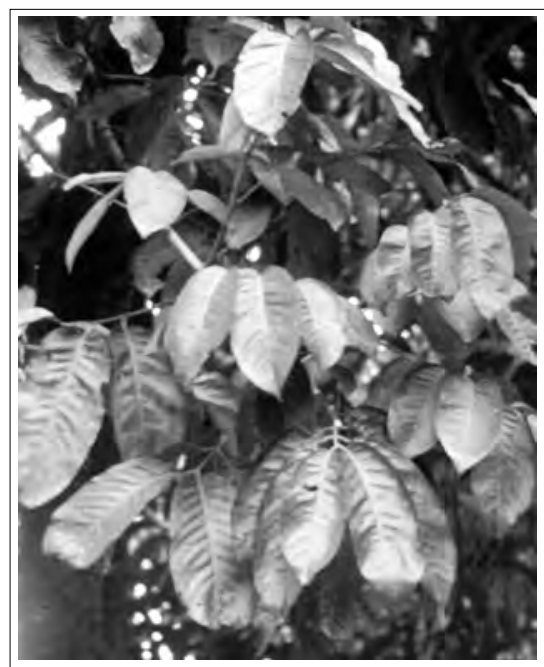

\section{REFERENCES}

PROSEA Vol. 2: 348; TFM Vol. 4: 452

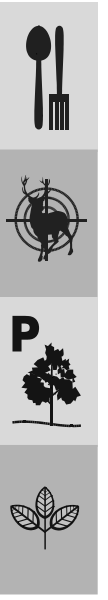

\begin{tabular}{|c|c|c|c|c|c|}
\hline \# & Code & Local Name & Use & RU & Hab.-Ab. \\
\hline 1 & PUT & ivo & $1,7,10$ & $\mathrm{x}$ & $\mathrm{G}-2, \mathrm{O}-2, \mathrm{P}-3, \mathrm{M}-3$ \\
\hline 2 & PUB & ivo & $1,7,10$ & $\mathrm{v}$ & G-3, O-3, P-2 \\
\hline 3 & PUD & & & & \\
\hline 4 & PUM & & & & \\
\hline 5 & ALS & bua rufak & 1,10 & v & G-2, P-3 \\
\hline 6 & LDY & kayu dupar, bua lapak & $1,7,11$ & v & G-3, O-3, P-4, M-2 \\
\hline 7 & LIT & & & & \\
\hline 8 & BRU & uen beeu & 1,7 & v & G-2, P-3 \\
\hline 9 & MRP & & & & \\
\hline 10 & PTN & & & & \\
\hline 11 & KLK & sibau & & & \\
\hline 12 & KLM & sibau & 1,10 & $x$ & ALL \\
\hline 13 & KLO & seletti & 1,10 & $x$ & G-2, P-3 \\
\hline 14 & KLA & sibau & 1,10 & $x$ & G-2, P-2 \\
\hline 15 & KAL & si'bo & 1,10 & $\mathrm{x}$ & G-2, P-2 \\
\hline 16 & KBD & sibau & $1,7,10$ & v & G-3, P-3 \\
\hline 17 & KBK & bua' sibau & $1,7,10$ & v & G-3, O-3, P-2 \\
\hline 18 & PEB & tewi, rupe & 1,7 & $\mathrm{v}$ & G-3, O-2, P-3, M-4 \\
\hline
\end{tabular}

Notes : 


\section{Nepbelium lappaceum L. (Sapindaceae)}

\section{RAMBUTAN. RAMBUTAN}

\section{DESCRIPTION}

Tree, 4-7 m cultivated, to $30 \mathrm{~m}$ wild; buttresses, bark greyish brown, smooth; dense untidy crown, leaves alternate para-pinnate; 3-8 elliptic, blunt, upstanding leaflets, $5-28 \times 2-10.5 \mathrm{~cm}$; fruits to $7 \times 5$ $\mathrm{cm}$, yellow to purple red, spines $0.5-2 \mathrm{~cm}$ long

\section{HABITAT}

Lowland primary and secondary forests; cultivated in village fruit gardens

\section{ORIGINS}

Malaysia and western Indonesia, Indochina

\section{USES}

Fruit eaten, sold locally; medicine; dye; timber for firewood

\section{REFERENCES}

Burkill 1966: 1767; FOJ Vol. 1: 173;

Kessler and Sidiyasa 1994: 208

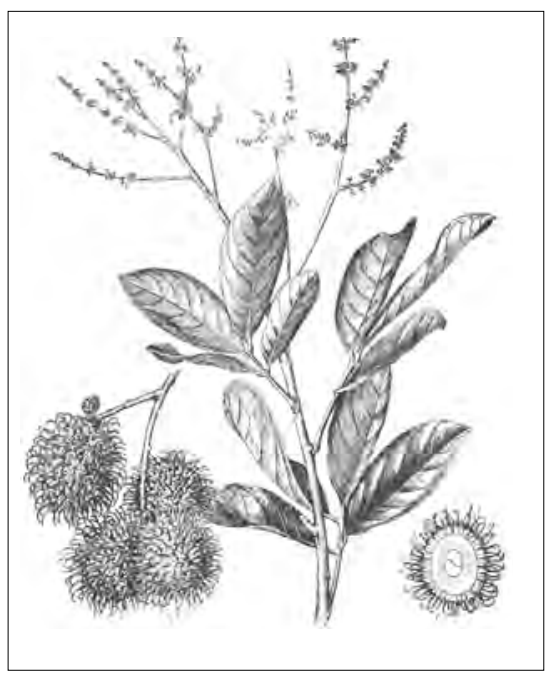

\begin{tabular}{|r|c|l|c|c|c|}
\hline$\#$ & Code & \multicolumn{1}{|c|}{ Local Name } & Use & RU & Hab.-Ab. \\
\hline 1 & PUT & lemati, lemti, lemeti & $1,7,11$ & $\mathbf{v}$ & G-3 \\
\hline 2 & PUB & bua lemeti, lemati & $1,7,11$ & $\mathbf{v}$ & G-3 \\
\hline 3 & PUD & lemati & $1,7,11$ & $\mathbf{v}$ & G-4 \\
\hline 4 & PUM & bua lemati & $1,7,11$ & $\mathbf{v}$ & G-3 \\
\hline 5 & ALS & bua' kelemati & $1,7,11$ & $\mathbf{v}$ & G-3 \\
\hline 6 & LDY & kelemati, bua kelemati & $1,7,11$ & $\mathbf{v}$ & G-3, O-3, P-3, M-2 \\
\hline 7 & LIT & bua sia', kelemati, ruget & $1,7,11$ & $\mathbf{v}$ & G-3 \\
\hline 8 & BRU & wi lamaki & 1,11 & $\mathbf{v}$ & G-2 \\
\hline 9 & MRP & bua matai, bua matae & $1,7,11$ & $\mathbf{v}$ & G-3 \\
\hline 10 & PTN & beleti', beleti & 1,7 & $\mathbf{v}$ & G-4 \\
\hline 11 & KLK & belette, bua' sanggit & $1,7,11$ & $\mathbf{v}$ & G-2 \\
\hline 12 & KLM & betti, bua sanggit & 1,11 & $\mathbf{v}$ & G-3 \\
\hline 13 & KLO & seleti, seletti & 1,7 & $\mathbf{X}$ & G-3 \\
\hline 14 & KLA & belettie, belettia', belette & 1,7 & $\mathbf{X}$ & G-3 \\
\hline 15 & KAL & belettie & 1 & $\mathbf{v}$ & G-3 \\
\hline 16 & KBD & bua beletti & $1,7,11$ & $\mathbf{v}$ & G-4, O-3, P-2 \\
\hline 17 & KBK & bua' belette & $1,7,11$ & $\mathbf{v}$ & G-4, O-2 \\
\hline 18 & PEB & beloti & $1,7,11$ & $\mathbf{v}$ & G-4, O-3, P-4 \\
\hline
\end{tabular}

Notes : 


\section{Nepbelium maingayi Hiern. (Sapindaceae)}

\section{(NOENGLISH NAME). REDAN, BUAH UNIING}

\section{DESCRIPTION}

Tree, to $40 \mathrm{~m}, 90 \mathrm{~cm}$ dbh; bole fluted; bark pinkish brown, pimply; twigs and leaves glabrous; crowns small; leaves alternate, para-pinnate; 2-3 pairs leaflets, upturned, upcurled edges, 6-22 x 3-9 cm; fruit ellipsoid, 2 x $1.6 \mathrm{~cm}$, smooth to warty, red

\section{HABITAT}

Lowland primary forests; riverine and swamps

\section{ORIGINS}

Malaysia and western Indonesia

\section{USES}

Fruit eaten; medicine; timber for firewood, tools; hunting location when fruiting

\section{REFERENCES}

PROSEA Vol. 2: 233; TFM Vol. 4: 454;

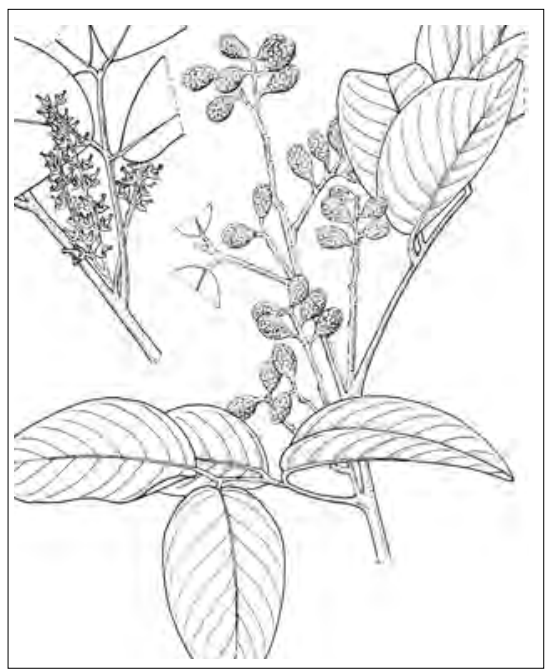

TFSS Vol. 2: 346

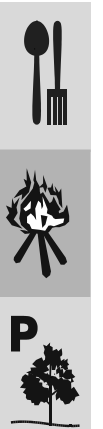

\begin{tabular}{|c|c|l|c|c|c|}
\hline$\#$ & Code & Local Name & Use & RU & Hab.-Ab. \\
\hline 1 & PUT & bua fait, vait & $1,7,10$ & $\mathbf{X}$ & P-3, M-3 \\
\hline 2 & PUB & vait & 1 & $\mathbf{V}$ & P-3 \\
\hline 3 & PUD & vait & 1,6 & $\mathbf{V}$ & P-3 \\
\hline 4 & PUM & & & & \\
\hline 5 & ALS & bua' lavak & 1 & $\mathbf{X}$ & P-3, M-2 \\
\hline 6 & LDY & lafak & $1,2,6$ & $\mathbf{X}$ & P-3, M-1 \\
\hline 7 & LIT & bua' puder & 1 & $\mathbf{X}$ & P-3, M-1 \\
\hline 8 & BRU & wi del & 1,2 & $\mathbf{X}$ & P-3 \\
\hline 9 & MRP & bua ria & 1,2 & $\mathbf{X}$ & P-3 \\
\hline 10 & PTN & bua unjing & 1 & $\mathbf{X}$ & P-3 \\
\hline 11 & KLK & bua' unjing & 1 & $\mathbf{X}$ & P-3, M-3 \\
\hline 12 & KLM & bua' telo, bua' poda & 1 & $\mathbf{X}$ & P-3, M-3 \\
\hline 13 & KLO & va tilo & 1 & $\mathbf{X}$ & P-3, M-3 \\
\hline 14 & KLA & fudo & 1 & $\mathbf{V}$ & P-3, M-3 \\
\hline 15 & KAL & beva' fodo' & 1 & $\mathbf{X}$ & P-3 \\
\hline 16 & KBD & & & & \\
\hline 17 & KBK & & & & \\
\hline 18 & PEB & kupiu & $1,7,10$ & $\mathbf{V}$ & $0-3$, P-3, M-3 \\
\hline
\end{tabular}

Notes : 


\section{Nephelium meduseum Leenh. (Sapindaceae) \\ FOREST RAMBUTAN. RAMBUTANHUTAN}

\section{DESCRIPTION}

Tree, to $27 \mathrm{~m}, 50 \mathrm{~cm}$ dbh; buttresses; leaves pinnate, 2-5 pairs elliptic leaflets, 5-12.5 x 2.5-5 $\mathrm{cm}$, no glands, veinlets finely reticulate and raised above; fruits ellipsoid, 3.25-4 x 2.5-3, dense, curled appendages, $1.5 \mathrm{~cm}$ long, swollen base, red.

\section{HABITAT}

Lowland primary forests to $450 \mathrm{~m}$; hills, slopes

\section{ORIGINS}

Borneo endemic

\section{USES}

Fruit eaten

\section{REFERENCES}

TFSS Vol. 2 1996: 346; Soepadmo and Wong 1995: 346

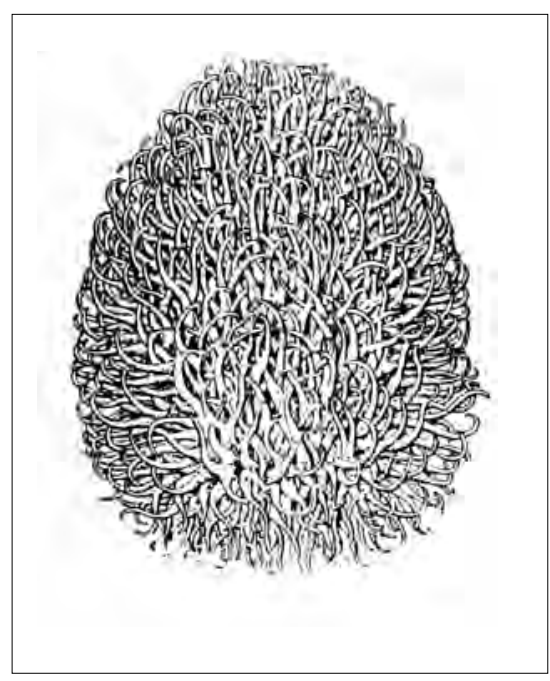

\begin{tabular}{|c|c|l|c|c|c|}
\hline$\#$ & Code & Local Name & Use & RU & Hab.-Ab. \\
\hline 1 & PUT & lengeca' & 1 & $\mathbf{X}$ & P-2 \\
\hline 2 & PUB & & & & \\
\hline 3 & PUD & & & & \\
\hline 4 & PUM & & & & \\
\hline 5 & ALS & & & \\
\hline 6 & LDY & & & \\
\hline 7 & LIT & & & \\
\hline 8 & BRU & & & & \\
\hline 9 & MRP & wa ngan & & & \\
\hline 10 & PTN & & & & \\
\hline 11 & KLK & mbui luan & & & \\
\hline 12 & KLM & & 1 & $\mathbf{x}$ & P-3, M-2 \\
\hline 13 & KLO & mbi levieng & 1 & $\mathbf{x}$ & P-2, M-2 \\
\hline 14 & KLA & mbui levan & 1 & $\mathbf{x}$ & P-3, M-3 \\
\hline 15 & KAL & mpui leven & 1 & $\mathbf{v}$ & P-3, M-3 \\
\hline 16 & KBD & mbui luan & 1 & $\mathbf{x}$ & P-3, M-2 \\
\hline 17 & KBK & mbui luan & 1 & $\mathbf{v}$ & P-3, M-3 \\
\hline 18 & PEB & sagup & & & \\
\hline
\end{tabular}

Notes : 


\section{Nepbelium ramboutan-ake (Labill.) Leenh. (Sapindaceae)}

\section{(NO ENGLISH NAME). KAPULASAN, MERITAM}

\section{DESCRIPTION}

Tree, 10-40 m, $80 \mathrm{~cm}$ dbh; buttresses; bark light brown, cracked; leaflets 1-7 pairs, thin, narrowly elliptic, 4-20 x 1.75-11 cm, drying reddish brown above, curled; fruits ellipsoid, 4-6.5 x 2.5-5 cm, stout woody spines $1.5 \mathrm{~cm}$, red to black

\section{HABITAT}

Lowland and hill primary and secondary forest to $1500 \mathrm{~m}$; cultivated in villages

\section{ORIGINS}

Southeast Asia

\section{USES}

Fruit eaten, sold locally; leaves for medicine; timber for firewood, construction

\section{REFERENCES}

Corner 1988: 686; PROSEA Vol. 2: 233;

TFM Vol. 4: 454; TFSS Vol. 2: 349;

Soepadmo and Wong 1995: 346
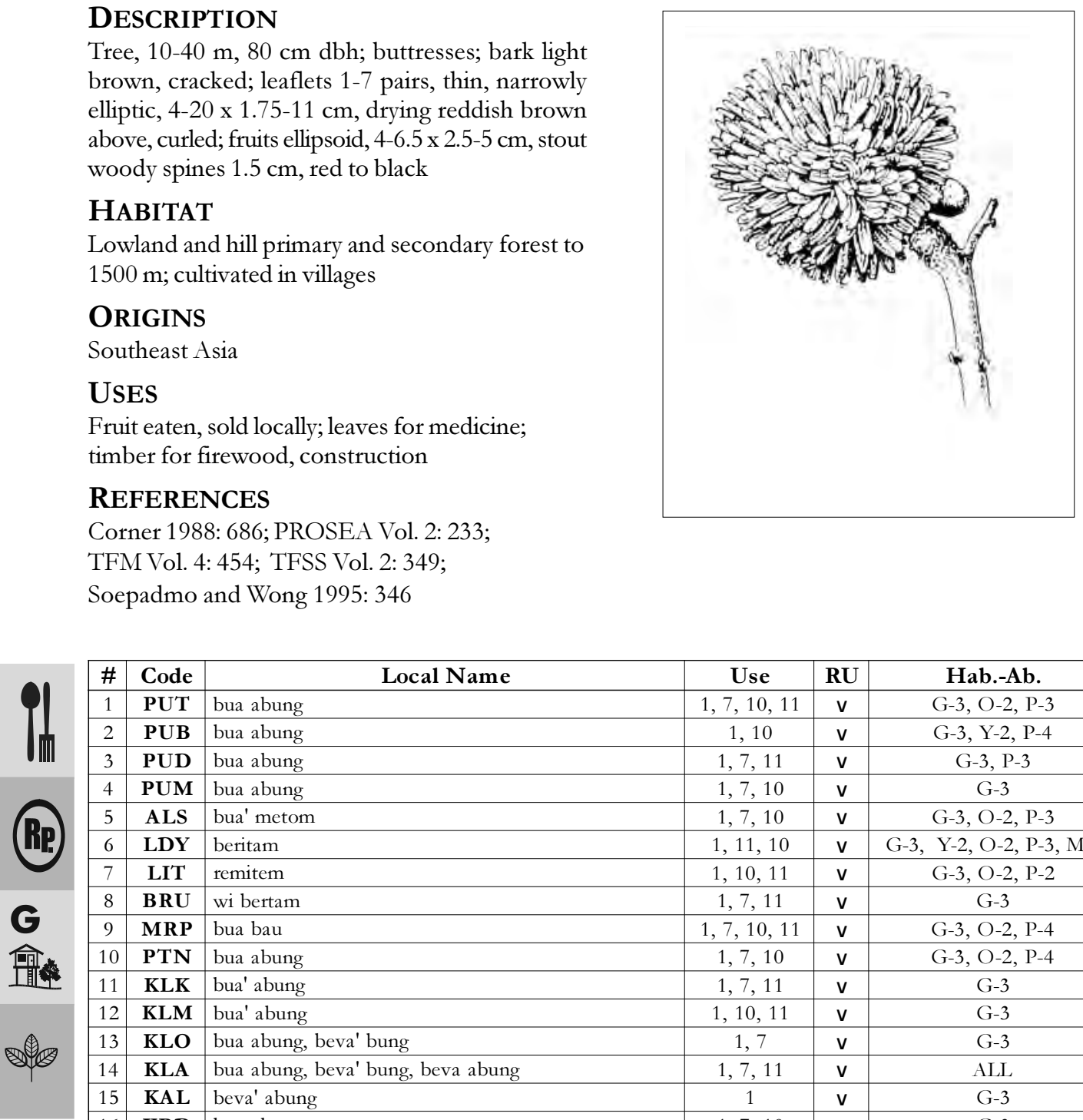

\begin{tabular}{|c|c|l|c|c|c|}
\hline$\#$ & Code & \multicolumn{1}{|c|}{ Local Name } & Use & RU & Hab.-Ab. \\
\hline 1 & PUT & bua abung & $1,7,10,11$ & $\mathbf{v}$ & G-3, O-2, P-3 \\
\hline 2 & PUB & bua abung & 1,10 & $\mathbf{v}$ & G-3, Y-2, P-4 \\
\hline 3 & PUD & bua abung & $1,7,11$ & $\mathbf{v}$ & G-3, P-3 \\
\hline 4 & PUM & bua abung & $1,7,10$ & $\mathbf{v}$ & G-3 \\
\hline 5 & ALS & bua' metom & $1,7,10$ & $\mathbf{v}$ & G-3, O-2, P-3 \\
\hline 6 & LDY & beritam & $1,11,10$ & $\mathbf{v}$ & G-3, Y-2, O-2, P-3, M-1 \\
\hline 7 & LIT & remitem & $1,10,11$ & $\mathbf{v}$ & G-3, O-2, P-2 \\
\hline 8 & BRU & wi bertam & $1,7,11$ & $\mathbf{v}$ & G-3 \\
\hline 9 & MRP & bua bau & $1,7,10,11$ & $\mathbf{v}$ & G-3, O-2, P-4 \\
\hline 10 & PTN & bua abung & $1,7,10$ & $\mathbf{v}$ & G-3, O-2, P-4 \\
\hline 11 & KLK & bua' abung & $1,7,11$ & $\mathbf{v}$ & G-3 \\
\hline 12 & KLM & bua' abung & $1,10,11$ & $\mathbf{v}$ & G-3 \\
\hline 13 & KLO & bua abung, beva' bung & 1,7 & $\mathbf{v}$ & G-3 \\
\hline 14 & KLA & bua abung, beva' bung, beva abung & $1,7,11$ & $\mathbf{v}$ & ALL \\
\hline 15 & KAL & beva' abung & 1 & $\mathbf{v}$ & G-3 \\
\hline 16 & KBD & bua abung & $1,7,10$ & $\mathbf{v}$ & G-3 \\
\hline 17 & KBK & bua' abung, titi da'an & $1,7,11$ & $\mathbf{v}$ & ALL \\
\hline 18 & PEB & meu & 1,7 & $\mathbf{v}$ & G-4, O-3, P-4 \\
\hline
\end{tabular}

\section{Notes :}




\section{Palaquium quercifolium (De Vriese) Burck. (Sapotaceae)}

\section{NYATOH.NYATOH}

\section{DESCRIPTION}

Tree, to $40 \mathrm{~m}, 75 \mathrm{~cm}$ dbh; buttresses; white exudate; bark dark brown, fissured; leaves spirally arranged, petioles 3-7 cm long, blade obovate-oblong 12-30 x 5-10 cm, 9-16 sec. veins, glabrous above, silky below; fruits round $2 \mathrm{~cm}$ diameter, glabrous

\section{HABITAT}

Lowland primary forest; ridges

\section{ORIGINS}

Indonesia

\section{USES}

Latex used for caulking boats, fastening machetes and axes

\section{REFERENCES}

Kessler and Sidiyasa 1994: 216

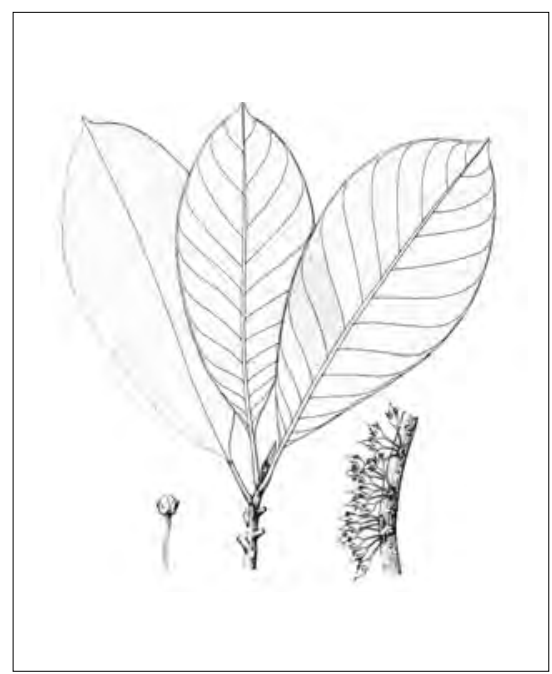

\begin{tabular}{|c|c|c|c|c|c|}
\hline \# & Code & Local Name & Use & $\mathbf{R U}$ & Hab.-Ab. \\
\hline 1 & PUT & ketipai mpou, indut & 5,6 & $x$ & P-3, M-3 \\
\hline 2 & PUB & ketipai bavui, ketipai & 5,6 & $x$ & P-3, M-3 \\
\hline 3 & PUD & intut & 5,6 & $x$ & P-3, M-3 \\
\hline 4 & PUM & & & & \\
\hline 5 & ALS & ula' tavu & 5,6 & $x$ & P-3, M-3 \\
\hline 6 & LDY & ula' karet & 5,6 & $x$ & P-3, M-3 \\
\hline 7 & LIT & & & & \\
\hline 8 & BRU & kayau lafung abiu & 5,6 & $x$ & $\mathrm{P}-3$ \\
\hline 9 & MRP & tepei, tepauo & 6 & $x$ & P-3 \\
\hline 10 & PTN & ketipai & 6 & $x$ & P-3 \\
\hline 11 & KLK & ketipai, nyato ulem & 5,6 & V & $\mathrm{P}-3, \mathrm{M}-3$ \\
\hline 12 & KLM & ketaipai, keteipai potuk & 5,6 & V & $\mathrm{P}-3, \mathrm{M}-3$ \\
\hline 13 & KLO & tereifae & 5,6 & V & $\mathrm{P}-3, \mathrm{M}-3$ \\
\hline 14 & KLA & kettifai & 5,6 & V & P-1, M-1 \\
\hline 15 & KAL & ketaifai & 5,6 & $x$ & P-3 M-2 \\
\hline 16 & KBD & nyato & 5,6 & v & P-3 M-2 \\
\hline 17 & KBK & ketipai putuk, keteipai & 5,6 & $x$ & $\mathrm{P}-3, \mathrm{M}-3$ \\
\hline 18 & PEB & ketipei & 5,6 & $\mathrm{v}$ & P-3, M-3 \\
\hline
\end{tabular}

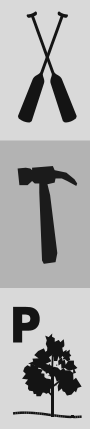

Notes : 


\section{Selaginella plana (Desv.) Hieron (Selaginellaceae)}

\section{(NO ENGLISH NAME).RUTU-RUTU}

\section{DESCRIPTION}

Fern, 10-120 cm long, creeping, copiously branching, feathery, light green leaflets

\section{HABITAT}

Lowland and hill primary and secondary forest, shady, fertile soils

\section{ORIGINS}

Asia

\section{USES}

Indicator of fertile soils for swidden rice agriculture; shoots eaten, for medicine

\section{REFERENCES}

Heyne Vol. I: 78
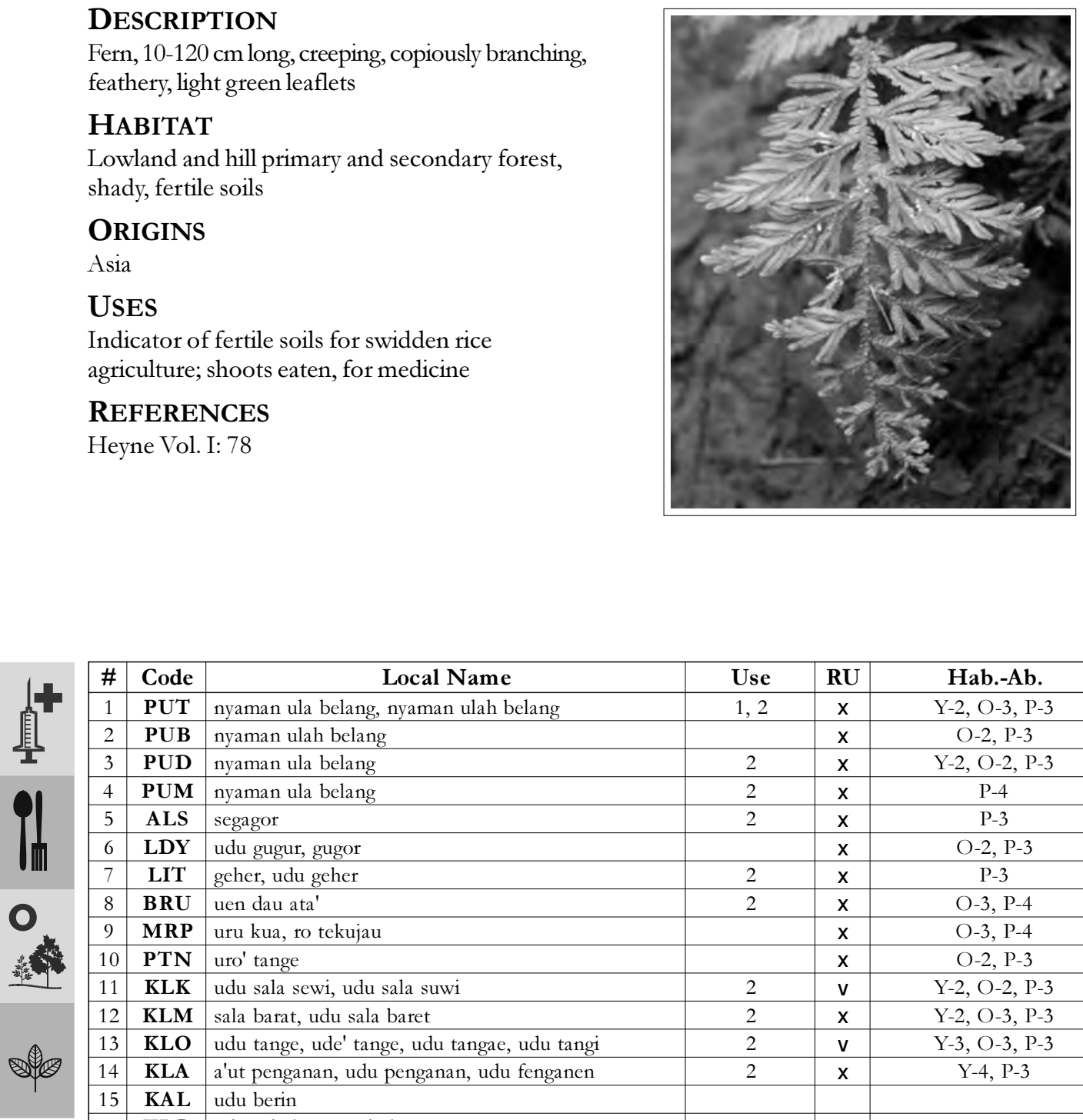

\begin{tabular}{|c|c|l|c|c|c|}
\hline$\#$ & Code & \multicolumn{1}{|c|}{ Local Name } & Use & RU & Hab.-Ab. \\
\hline 1 & PUT & nyaman ula belang, nyaman ulah belang & 1,2 & $\mathbf{X}$ & Y-2, O-3, P-3 \\
\hline 2 & PUB & nyaman ulah belang & & $\mathbf{X}$ & O-2, P-3 \\
\hline 3 & PUD & nyaman ula belang & 2 & $\mathbf{X}$ & Y-2, O-2, P-3 \\
\hline 4 & PUM & nyaman ula belang & 2 & $\mathbf{X}$ & P-4 \\
\hline 5 & ALS & segagor & 2 & $\mathbf{X}$ & P-3 \\
\hline 6 & LDY & udu gugur, gugor & & $\mathbf{X}$ & O-2, P-3 \\
\hline 7 & LIT & geher, udu geher & 2 & $\mathbf{X}$ & P-3 \\
\hline 8 & BRU & uen dau ata' & 2 & $\mathbf{X}$ & O-3, P-4 \\
\hline 9 & MRP & uru kua, ro tekujau & & $\mathbf{X}$ & O-3, P-4 \\
\hline 10 & PTN & uro' tange & & $\mathbf{X}$ & O-2, P-3 \\
\hline 11 & KLK & udu sala sewi, udu sala suwi & 2 & $\mathbf{V}$ & Y-2, O-2, P-3 \\
\hline 12 & KLM & sala barat, udu sala baret & 2 & $\mathbf{X}$ & Y-2, O-3, P-3 \\
\hline 13 & KLO & udu tange, ude' tange, udu tangae, udu tangi & 2 & $\mathbf{V}$ & Y-3, O-3, P-3 \\
\hline 14 & KLA & a'ut penganan, udu penganan, udu fenganen & 2 & $\mathbf{X}$ & Y-4, P-3 \\
\hline 15 & KAL & udu berin & & & \\
\hline 16 & KBD & udu sala barap, sala barap & & $\mathbf{X}$ & P-3 \\
\hline 17 & KBK & udu penganan & & & \\
\hline 18 & PEB & ureu penganan, uru penganan & 2 & $\mathbf{x}$ & Y-2, O-4, M-2 \\
\hline
\end{tabular}

Notes : 
Capsicum annuum L. var. abbreviata Fingerhuth. (Solanaceae)

CHILI PEPPER, CAPSICUM. LOMBOK

\section{DESCRIPTION}

Herb, 1-2 m, perennial, woody base; leaves solitary or 2-3 together, broadly ovate-oblong or ellipticlanceolate, base acute, $1.5-12 \times 0.7-5 \mathrm{~cm}$; berries, long stalk, pendulous, ovoid-globose, 2-3 x 1.5$2.5 \mathrm{~cm}$, yellowish white to green

\section{HABITAT}

Cultivated in home and swidden gardens

\section{ORIGINS}

Tropical America

\section{USES}

Fruit eaten, medicine, sold locally

\section{REFERENCES}

Burkill 1966: 451; Ochse 1980: 668;

PROSEA Vol. 8: 136

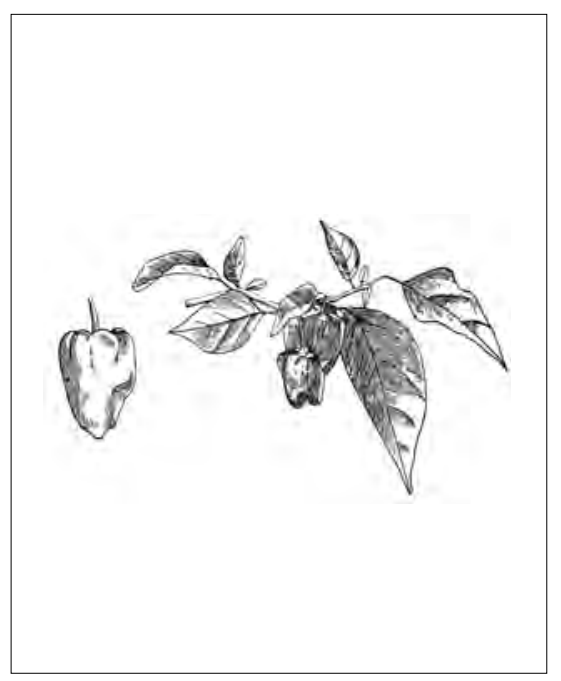

\begin{tabular}{|c|c|c|c|c|c|}
\hline \# & Code & $\begin{array}{l}\text { Local Name } \\
\end{array}$ & Use & $\mathbf{R U}$ & Hab.-Ab. \\
\hline 1 & PUT & serbeh ulom, serbeh & 1,11 & v & $\mathrm{G}-2$ \\
\hline 2 & PUB & serbeh & 1 & v & G-2 \\
\hline 3 & PUD & serbeh & 1,11 & $\mathrm{v}$ & G-2 \\
\hline 4 & PUM & serbeh & 1,11 & $\mathrm{v}$ & G-2 \\
\hline 5 & ALS & libau lowos & 1,11 & V & G-3 \\
\hline 6 & LDY & bua lesepula, bua ladeh & 1,11 & $x$ & G-3 \\
\hline 7 & LIT & bua ladeh, bua lade & 1,11 & $x$ & G-2 \\
\hline 8 & BRU & wi bua adie & 1,11 & V & G-2 \\
\hline 9 & MRP & tempei, tempih, tempe & 1,11 & V & G-2 \\
\hline 10 & PTN & sempe time & 1 & V & G-3 \\
\hline 11 & KLK & sempe, sembe lata & 1,11 & V & G-3 \\
\hline 12 & KLM & sempe lumit, sembe & 1,11 & V & G-2, G-3 \\
\hline 13 & KLO & sa'be kele', se'be, se'bi, se beh gelei & 1 & v & G-2, S-2 \\
\hline 14 & KLA & se'be, se'bei, se'bei dumit, sa'be rumit & 1 & v & G-4 \\
\hline 15 & KAL & se'be rumit & 1 & V & G-3, S-3 \\
\hline 16 & KBD & lia kelai & 1 & $\mathrm{v}$ & G-2 \\
\hline 17 & KBK & lia kelai & 1,11 & $\mathrm{v}$ & G-3, S-3 \\
\hline 18 & PEB & suman & 1 & $x$ & $\mathrm{G}-4, \mathrm{~S}-3, \mathrm{Y}-2, \mathrm{O}-2$ \\
\hline
\end{tabular}

Notes : 


\section{Capsicum frutescens L. (Solanaceae) \\ BIRD PEPPER. CABE BURUNG}

\section{DESCRIPTION}

Herb, 0.5-1.5 m, perennial, woody base; leaves solitary or 2-3 together, broadly ovate or oblonglanceolate, base broad, $1.5-10 \times 0.5-5 \mathrm{~cm}$; berries, long stalk, erect, ovate-oblong, $0.75-2.5 \times 0.3-1 \mathrm{~cm}$, red, orange or yellow white

\section{HABITAT}

Cultivated in home and swidden gardens

\section{ORIGINS}

Tropical America

\section{USES}

Fruit eaten, medicine, sold locally

\section{REFERENCES}

Burkill 1966: 452; Ochse 1980: 671;

PROSEA Vol. 8: 136
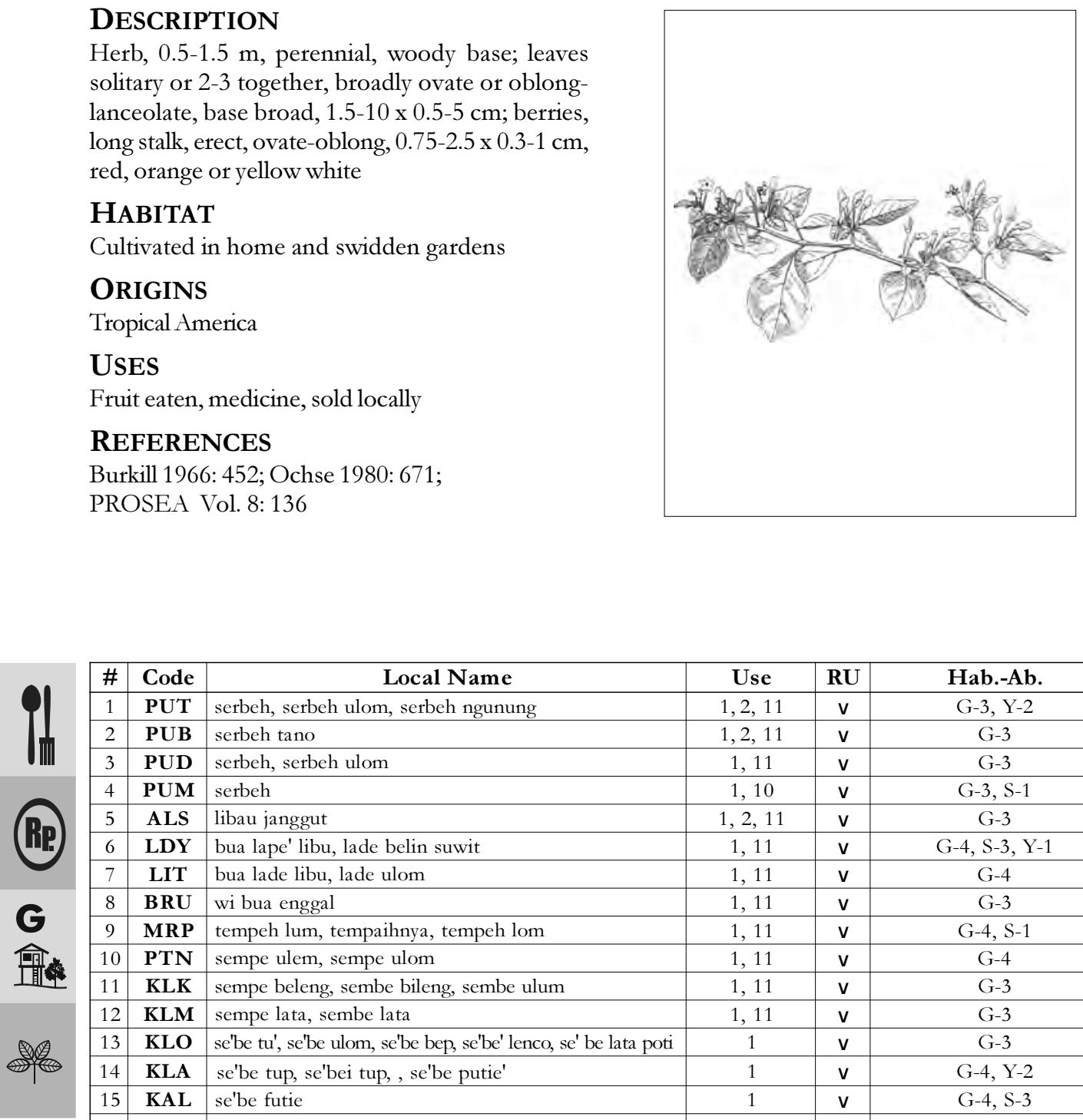

\begin{tabular}{|c|c|l|c|c|c|}
\hline$\#$ & Code & \multicolumn{1}{|c|}{ Local Name } & Use & RU & Hab.-Ab. \\
\hline 1 & PUT & serbeh, serbeh ulom, serbeh ngunung & $1,2,11$ & $\mathbf{v}$ & G-3, Y-2 \\
\hline 2 & PUB & serbeh tano & $1,2,11$ & $\mathbf{v}$ & G-3 \\
\hline 3 & PUD & serbeh, serbeh ulom & 1,11 & $\mathbf{v}$ & G-3 \\
\hline 4 & PUM & serbeh & 1,10 & $\mathbf{v}$ & G-3, S-1 \\
\hline 5 & ALS & libau janggut & $1,2,11$ & $\mathbf{v}$ & G-3 \\
\hline 6 & LDY & bua lape' libu, lade belin suwit & 1,11 & $\mathbf{v}$ & G-4, S-3, Y-1 \\
\hline 7 & LIT & bua lade libu, lade ulom & 1,11 & $\mathbf{v}$ & G-4 \\
\hline 8 & BRU & wi bua enggal & 1,11 & $\mathbf{v}$ & G-3 \\
\hline 9 & MRP & tempeh lum, tempaihnya, tempeh lom & 1,11 & $\mathbf{v}$ & G-4, S-1 \\
\hline 10 & PTN & sempe ulem, sempe ulom & 1,11 & $\mathbf{v}$ & G-4 \\
\hline 11 & KLK & sempe beleng, sembe bileng, sembe ulum & 1,11 & $\mathbf{v}$ & G-3 \\
\hline 12 & KLM & sempe lata, sembe lata & 1,11 & $\mathbf{v}$ & G-3 \\
\hline 13 & KLO & se'be tu', se'be ulom, se'be bep, se'be' lenco, se' be lata poti & 1 & $\mathbf{v}$ & G-3 \\
\hline 14 & KLA & se'be tup, se'bei tup, , se'be putie' & 1 & $\mathbf{v}$ & G-4, Y-2 \\
\hline 15 & KAL & se'be futie & 1 & $\mathbf{v}$ & G-4, S-3 \\
\hline 16 & KBD & lia ulom, lia tup & 1 & $\mathbf{v}$ & G-3 \\
\hline 17 & KBK & lia asu & 1,2 & $\mathbf{v}$ & G-4, S-3, Y-2 \\
\hline 18 & PEB & suman jau belu, suman alo' & & \\
\hline
\end{tabular}

\section{Notes :}




\section{Nicotiana tabacum L. (Solanaceae) \\ TOBACCO. TEMBAKAU}

\section{DESCRIPTION}

Herb, 0.5-1.5 m, erect, annual; flowers rosecoloured

\section{HABITAT}

Cultivated in homegardens; wild in waste areas, fallows

\section{ORIGINS}

Tropical America

\section{USES}

Leaves narcotic, medicinal, sold locally

\section{REFERENCES}

Burkill 1966: 1577; PROSEA Vol. 16

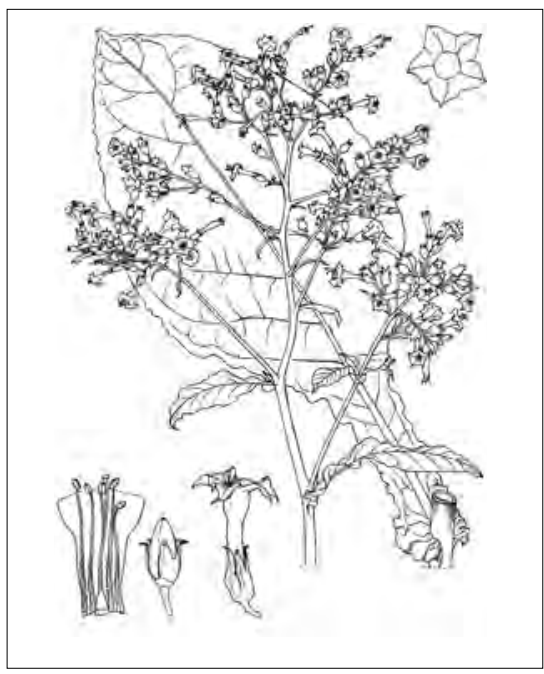

\begin{tabular}{|c|c|c|c|c|c|}
\hline \# & Code & Local Name & Use & RU & Hab.-Ab. \\
\hline 1 & PUT & sigup laung, sigup ong & 1,11 & $\mathrm{v}$ & G-3, S-2 \\
\hline 2 & PUB & sigup, sigup ong & 1,11 & $x$ & G-3 \\
\hline 3 & PUD & sigup ong & 1,11 & $x$ & G-2 \\
\hline 4 & PUM & sigup laung & 1 & $x$ & G-3 \\
\hline 5 & ALS & sigup ong & 1,11 & $x$ & G-1 \\
\hline 6 & LDY & siguk bata', sigug bata & 1,11 & $x$ & G-2 \\
\hline 7 & LIT & siguk, iguk, daun sigup & 1 & V & G-3 \\
\hline 8 & BRU & zeong & 1 & $x$ & G-2 \\
\hline 9 & MRP & tidik, tidiuk, tidip ong & $1,2,11$ & $x$ & G-2 \\
\hline 10 & PTN & luko', luko ong & 1 & $x$ & G-3, S-1 \\
\hline 11 & KLK & jako, jako ung & $1,2,11$ & $x$ & G-3 \\
\hline 12 & KLM & jako ong & 1 & V & G-2 \\
\hline 13 & KLO & & & & \\
\hline 14 & KLA & luko' ung & 1 & $\mathrm{v}$ & G-2, S-1 \\
\hline 15 & KAL & loko' & 1 & v & G-2 \\
\hline 16 & KBD & jako lan & 1 & v & G-2, S-2 \\
\hline 17 & KBK & jako & 1 & v & G-2, S-2 \\
\hline 18 & PEB & sigup kebun & 1 & v & G-4, S-3 \\
\hline
\end{tabular}

Notes : 


\section{Solanum melongena L. (Solanaceae) \\ EGGPLANT. TERONG}

\section{DESCRIPTION}

Herb, 0.5-1.5 m, erect perennial, woody base; branches green and violet, dense grey hairy; leaves alternate, ovate or oblong-ovate, deep lobes, hairy, $7-25 \times 5-15 \mathrm{~cm}$; fruit globose to oblong, white, yellow, green, or violet, $2.5-15 \times 2-5-4 \mathrm{~cm}$, glabrous

\section{HABITAT}

Cultivated in home and swidden gardens, to $1200 \mathrm{~m}$

\section{ORIGINS}

Southeast Asia

\section{USES}

Fruit eaten, medicine, sold locally

\section{REFERENCES}

Burkill 1966: 2081; Ochse 1980: 680;

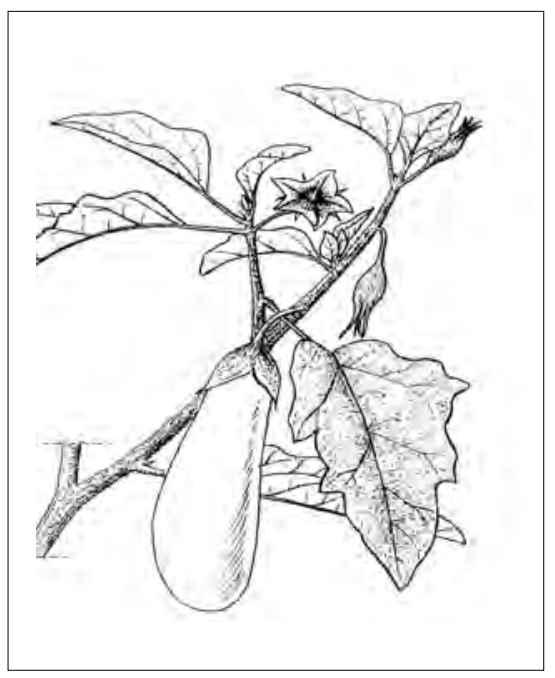

PROSEA Vol. 8: 255

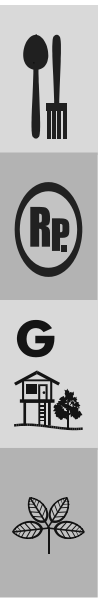

\begin{tabular}{|c|c|c|c|c|c|}
\hline \# & Code & Local Name & Use & RU & Hab.-Ab. \\
\hline 1 & PUT & terong, kelengaung & 1,11 & v & $\mathrm{G}-3, \mathrm{~S}-2$ \\
\hline 2 & PUB & kelenggau & 1,11 & v & G-3, S-2 \\
\hline 3 & PUD & kelengong & 1,11 & v & G-3, S-3 \\
\hline 4 & PUM & & & & \\
\hline 5 & ALS & bua' nawung & 1,11 & v & G-3, S-3 \\
\hline 6 & LDY & bua' betarong & 1,11 & v & G-3, S-3 \\
\hline 7 & LIT & & & & \\
\hline 8 & BRU & wi angong & 1,11 & v & $G-3, S-2$ \\
\hline 9 & MRP & langaue & 1,11 & v & $G-2, S-2$ \\
\hline 10 & PTN & ngelawung & 1,11 & v & G-3, S-3 \\
\hline 11 & KLK & terong & 1,11 & v & $G-3, S-3$ \\
\hline 12 & KLM & terong & 1,11 & v & $G-3, S-3$ \\
\hline 13 & KLO & lengaung & 1 & $\mathrm{v}$ & G-3, S-3 \\
\hline 14 & KLA & lengaung & 1 & $\mathrm{v}$ & $\mathrm{G}-3, \mathrm{~S}-3$ \\
\hline 15 & KAL & langau & 1 & v & G-3, S-3 \\
\hline 16 & KBD & keleng aong & 1 & $\mathrm{v}$ & G-3, S-2 \\
\hline 17 & KBK & terong & 1,11 & $\mathrm{v}$ & G-4, S-2 \\
\hline 18 & PEB & lengaung & 1 & $\mathrm{v}$ & G-2 \\
\hline
\end{tabular}

Notes : 
Solanum torvum Swartz. (Solanaceae)

\section{Plate BRUSH. TERONG PIIIT, TAKOKAK}

\section{DESCRIPTION}

Shrub, 1-6 m, crown broad; branches drooping with prickles, grey hairy; leaves ovate-oblong or broadly elliptic, deep lobes, grass green above, grey green below, softly fuzzy, 5-25 x 5-20 cm; fruit round, yellow, 1-1.5 cm, many seeds

\section{HABITAT}

Cultivated in home and swidden gardens; wild in thickets and secondary forest

\section{ORIGINS}

Antilles Islands

\section{USES}

Fruit eaten; leaves, roots medicinal

\section{REFERENCES}

Burkill 1966: 2083; Ochse 1980: 685;

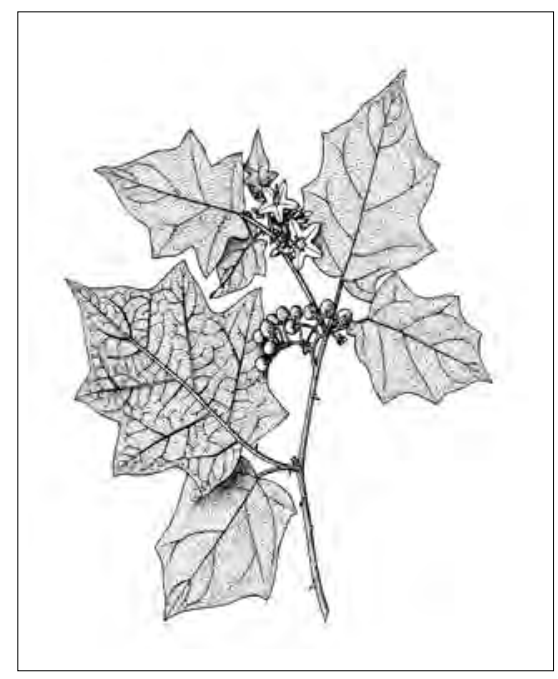

PROSEA Vol. 8: 258

\begin{tabular}{|c|c|c|c|c|c|}
\hline \# & Code & Local Name & Use & RU & Hab.-Ab. \\
\hline 1 & PUT & ulom & 1,2 & V & S-2, Y-3 \\
\hline 2 & PUB & ulom & 1 & V & $\mathrm{S}-3, \mathrm{Y}-3$ \\
\hline 3 & PUD & ulom & 1,2 & v & $\mathrm{S}-3, \mathrm{Y}-3$ \\
\hline 4 & PUM & ulom & 1 & v & $\mathrm{S}-2, \mathrm{Y}-3$ \\
\hline 5 & ALS & bua' olom & 1,2 & v & S-2, Y-3 \\
\hline 6 & LDY & betarung ulom, bua ulom betarung & 1,2 & $\mathrm{v}$ & $\mathrm{G}-3, \mathrm{~S}-2, \mathrm{Y}-3$ \\
\hline 7 & LIT & bua ulom, ulom & 1,2 & v & Y-3 \\
\hline 8 & BRU & wi angong lam & 1 & v & $\mathrm{S}-3, \mathrm{Y}-4$ \\
\hline 9 & MRP & luem, luom & 1,2 & v & G-2, S-3, Y-3 \\
\hline 10 & PTN & ulem, ulom & 1,2 & v & $\mathrm{S}-3, \mathrm{Y}-3$ \\
\hline 11 & KLK & ulem, ulom & 1 & $\mathbf{v}$ & G-1, S-2 \\
\hline 12 & KLM & olom, olem & 1,2 & $\mathrm{v}$ & G-3, S-2, Y-3 \\
\hline 13 & KLO & olem & 1,2 & v & G-3 \\
\hline 14 & KLA & ulem, ulom & 1,2 & $\mathrm{v}$ & G-3, Y-4 \\
\hline 15 & KAL & olem & 1,2 & $\mathrm{v}$ & G-3 \\
\hline 16 & KBD & olom & 1 & $\mathrm{v}$ & G-2 \\
\hline 17 & KBK & ulem & 1,2 & $\mathrm{v}$ & G-3 \\
\hline 18 & PEB & olem, ulom & 1 & $\mathrm{v}$ & G-2 \\
\hline
\end{tabular}

Notes : 
Aquilaria beccariana van Tigh. (Thymelaeaceae)

\section{BORNEAN EAGLEWOOD TREE. GARU TANDUK}

\section{DESCRIPTION}

Tree, to $20 \mathrm{~m}, 110 \mathrm{~cm}$ dbh; bark smooth white, stripping off; leaves, spiral, pronounced tip, 7-27 x 3-9 cm, sec. nerves raised above, drying shiny golden brown below; fruit a green glabrous capsule, 2-3 x $0.7-1.5 \mathrm{~cm}$, two seeds

\section{HABITAT}

Lowland and hill primary forests to $700 \mathrm{~m}$; riverine, swampy areas

\section{ORIGINS}

Borneo, Sumatra, Peninsular Malaysia

\section{USES}

Resin impregnated aromatic wood sold to traders for export; medicine; bark for cordage, cloth; most valuable NTFP in Bulungan area

\section{REFERENCES}

Corner 1988: 729; Kessler and Sidiyasa 1994: 225;

TFM Vol. 2: 385; PROSEA Vol. 19: 173

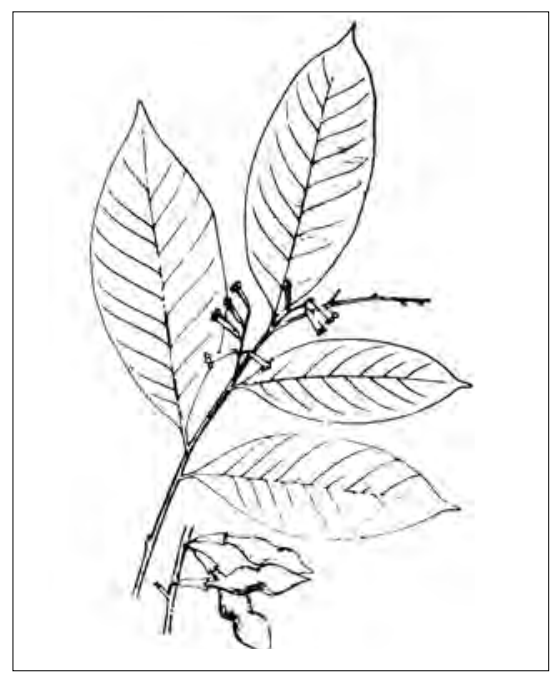

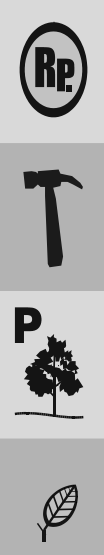

\begin{tabular}{|c|c|c|c|c|c|}
\hline \# & Code & Local Name & Use & RU & Hab.-Ab. \\
\hline 1 & PUT & lelah & $6,8,11$ & $x$ & $\mathrm{M}-3, \mathrm{O}-2, \mathrm{P}-3$ \\
\hline 2 & PUB & lelah, tengon & $6,8,11$ & $x$ & P-1, M-1, R \\
\hline 3 & PUD & lelah & $6,8,11$ & $x$ & P-1, M-1, R \\
\hline 4 & PUM & lelah & $6,8,11$ & $\mathrm{x}$ & P-1, M-1, R \\
\hline 5 & ALS & alas & $6,8,11$ & $x$ & P-1, M-1, R \\
\hline 6 & LDY & alat & $6,8,11$ & $x$ & P-1, M-1, R \\
\hline 7 & LIT & alat & $6,8,11$ & $x$ & P-1, M-1, R \\
\hline 8 & BRU & uen alah langit & $6,8,11$ & $x$ & P-1, M-1, R \\
\hline 9 & MRP & kemela, temalah & $6,8,11$ & $x$ & P-1, M-1, R \\
\hline 10 & PTN & betonong & $6,8,11$ & $x$ & P-1, M-1, R \\
\hline 11 & KLK & sekkau & $2,6,8,11$ & v & P-1, M-1 \\
\hline 12 & KLM & sekkau lalut, dengun & $6,8,11$ & v & P-1, M-1 \\
\hline 13 & KLO & sekko & $6,8,11$ & v & P-1, M-1 \\
\hline 14 & KLA & sekkau & 7,11 & v & $\mathrm{O}-1, \mathrm{P}-4$ \\
\hline 15 & KAL & sekko betonong & $6,8,11$ & v & P-1, M-1 \\
\hline 16 & KBD & sekkau & $6,8,11$ & v & P-1, M-1 \\
\hline 17 & KBK & sekkau, sekkau bai'i & $6,8,11$ & $x$ & $\mathrm{O}-1, \mathrm{P}-4, \mathrm{M}-1$ \\
\hline 18 & PEB & sekau & $6,8,11$ & $\mathrm{v}$ & $\mathrm{O}-1, \mathrm{P}-1, \mathrm{M}-1$ \\
\hline
\end{tabular}

Notes : 


\section{Aquilaria malaccensis Lamk. (Thymelaeaceae) \\ MALAYAN EAGLEWOOD. GAHARU}

\section{DESCRIPTION}

Tree, to $40 \mathrm{~m}, 60 \mathrm{~cm} \mathrm{dbh}$; bark smooth white, stripping off in long pieces; leaves, spiral, ellipticoblong to oblong-lanceolate, 7.5-12 x 2.5-5.5 cm; flowers yellow, bell-shaped; fruit a green obovoid capsule $3-4$ × $2.5 \mathrm{~cm}, 1$ seed

\section{HABITAT}

Lowland and hill primary forests to $1000 \mathrm{~m}$; ridges, slopes, well-drained soils

\section{ORIGINS}

Tropical Asia

\section{USES}

Resin impregnated aromatic wood sold to traders for export; medicine; bark for cordage, cloth; most valuable NTFP in Bulungan area

\section{REFERENCES}

Corner 1988: 729; Kessler and Sidiyasa 1994: 225;

TFM Vol. 2: 386; PROSEA Vol. 19: 64-65

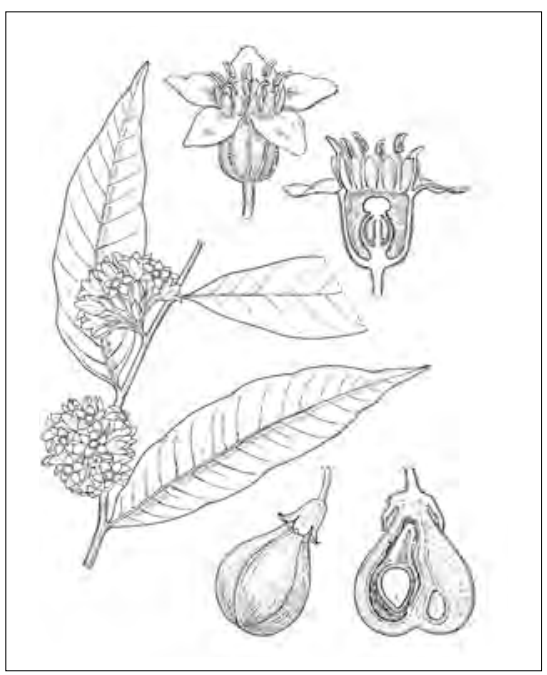

\begin{tabular}{|c|c|c|c|c|c|}
\hline \# & Code & Local Name & Use & RU & Hab.-Ab. \\
\hline 1 & PUT & lelah, tengon & 8,11 & $\mathrm{x}$ & $\mathrm{M}-3, \mathrm{P}-3$ \\
\hline 2 & PUB & tengon, lelah & 8,11 & $\mathrm{x}$ & P-3, M-3 \\
\hline 3 & PUD & tengon tanduk padi & 8,11 & $x$ & P-3, M-3 \\
\hline 4 & PUM & tengon & 8,11 & $x$ & P-3, M-3 \\
\hline 5 & ALS & kapuyoh & 8,11 & $x$ & P-3, M-3 \\
\hline 6 & LDY & kefoyo & 8,11 & $x$ & P-3, M-3 \\
\hline 7 & LIT & alat & 8,11 & $x$ & $\mathrm{P}-3, \mathrm{M}-3$ \\
\hline 8 & BRU & uen alah & 8,11 & $\mathrm{x}$ & P-3, M-3 \\
\hline 9 & MRP & tengon, tenong & 8,11 & $x$ & P-3, M-3 \\
\hline 10 & PTN & tengun & 8,11 & $x$ & P-3, M-3 \\
\hline 11 & KLK & tengon, sekkau & $2,8,11$ & v & P-3, M-3 \\
\hline 12 & KLM & sekkau mudung, sekkau & 8,11 & $\mathrm{v}$ & $\mathrm{P}-3, \mathrm{M}-3$ \\
\hline 13 & KLO & sekko & 8,11 & $\mathbf{v}$ & P-3, M-3 \\
\hline 14 & KLA & sekkau & 7,11 & v & $\mathrm{Y}-1, \mathrm{O}-1, \mathrm{P}-4$ \\
\hline 15 & KAL & sekko & 7,11 & v & $\mathrm{O}-1, \mathrm{P}-3, \mathrm{M}-1$ \\
\hline 16 & KBD & sekkau & 8,11 & v & $\mathrm{O}-1, \mathrm{P}-3, \mathrm{M}-1$ \\
\hline 17 & KBK & sekkau, sekkau modung & 8,11 & $\mathrm{x}$ & $\mathrm{O}-1, \mathrm{P}-4, \mathrm{M}-4$ \\
\hline 18 & PEB & tengon & 8,11 & v & O-1, P-3, M-3 \\
\hline
\end{tabular}

Notes : 


\section{Gonystylus macropbyllus (Miq.) Airy Shaw (Thymelaeaceae)}

\section{(NO ENGLISH NAME). RAMIN}

\section{DESCRIPTION}

Tree, 30-45 m, bark dark brown, fissured; twigs blackish, striate; leaves opposite, stalk rugose, ovaloblong, 7-40 x 4-15 cm, petiole thick 1.5-2.5 cm, prominent tertiary nerves perpendicular to secondary nerves; fruits round to $7 \mathrm{~cm}$, orange brown

\section{HABITAT}

Lowland and hill primary forest to $500 \mathrm{~m}$

\section{ORIGINS}

Western Malesia

\section{USES}

Timber for tools, light construction, firewood; fish poison

\section{REFERENCES}

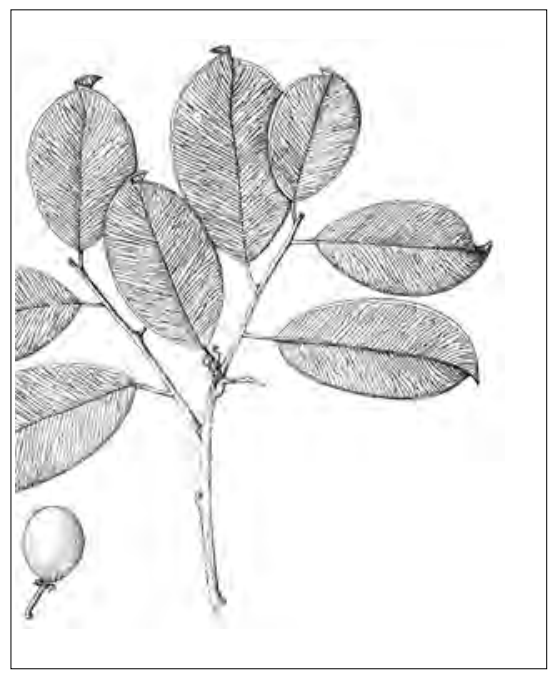

TFM Vol. 2: 387; FOJ Vol. I: 401

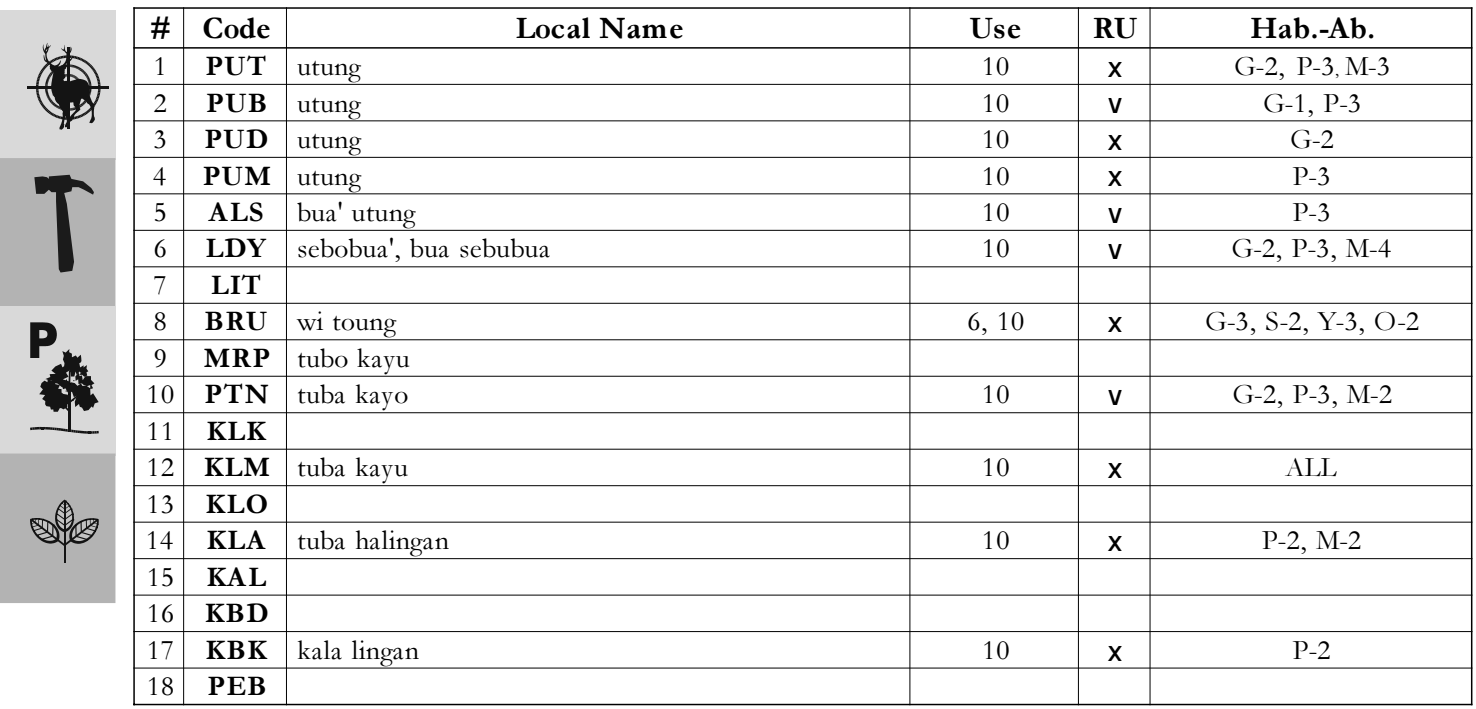

Notes : 


\section{Poikilospermum suaveolens (B1.) Merr. (Urticaceae)}

\section{(NOENGLISH NAME). TENTAWAN, AKAR MURAH}

\section{DESCRIPTION}

Vine, 5-30 m, stout liana from epiphyte, no spines, no latex; twigs thick with leaf scars; leaves spiral, ovate to ovate-oblong, $10-30 \times 8-22 \mathrm{~cm}$, petiole 3.5-27 cm long; basal nerves halfway to apex, sec. nerves 7 pairs; flowers purple, fragrant

\section{HABITAT}

Lowland and hill primary forest; brush wood, open forests

\section{ORIGINS}

Southeast Asia

\section{USES}

Bark for cordage; sap and roots for medicine; water for drinking; dried stem for firewood

\section{REFERENCES}

Burkill 1966: 660; TFM Vol. 4: 472;

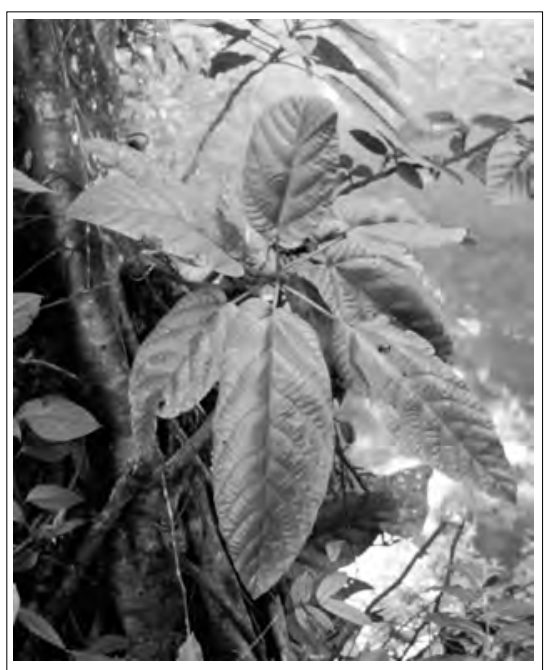

FOJ Vol. 2: 36

\begin{tabular}{|c|c|l|c|c|c|}
\hline$\#$ & Code & \multicolumn{1}{|c|}{ Local Name } & Use & RU & Hab.-Ab. \\
\hline 1 & PUT & kenaten & $1,2,7,11$ & $\mathbf{V}$ & Y-2, O-2, P-3 \\
\hline 2 & PUB & keneten, oka keneten & 1,8 & $\mathbf{X}$ & Y-2, O-2, P-3 \\
\hline 3 & PUD & kenaten & 1 & $\mathbf{X}$ & S-2, Y-3, O-2, P-3 \\
\hline 4 & PUM & naten & 1 & $\mathbf{X}$ & O-2, P-3 \\
\hline 5 & ALS & gemotor & 1 & $\mathbf{X}$ & O-2, P-3 \\
\hline 6 & LDY & riyep pulung, riyep & 1,2 & $\mathbf{v}$ & O-2, P-3, M-4 \\
\hline 7 & LIT & riap, riyap, riyep & 1 & $\mathbf{v}$ & O-3, P-3 \\
\hline 8 & BRU & wi kangtet & 1 & $\mathbf{X}$ & O-2, P-3 \\
\hline 9 & MRP & oka kebau & & & \\
\hline 10 & PTN & kisap bahan & 1 & $\mathbf{X}$ & P-3 \\
\hline 11 & KLK & kelapeso & 1,2 & $\mathbf{X}$ & Y-2, O-3, P-3 \\
\hline 12 & KLM & keten lata & 1 & $\mathbf{X}$ & ALL \\
\hline 13 & KLO & jenaten & & & \\
\hline 14 & KLA & & & & \\
\hline 15 & KAL & & & & \\
\hline 16 & KBD & burung & & & \\
\hline 17 & KBK & & 8 & $\mathbf{X}$ & O-2, P-4 \\
\hline 18 & PEB & lakeu utap & & \\
\hline
\end{tabular}

Notes : 


\section{Callicarpa longifolia Lam. (Verbenaceae)}

\section{WHITE-BERRIED MALAYAN LILAC. TAMPANG BESI PUTIH \\ ○}

\section{DESCRIPTION}

Shrub, to $5 \mathrm{~m}$; young parts brownish or brownish white scurfy-felted; leaves simple, elliptic, 7.5-17.5 $\mathrm{x} 3.5-8.5 \mathrm{~cm}$, pointed, stalk $1-2.5 \mathrm{~cm}$ long, margin toothed, green and scurfy beneath; flowers pink to pale lilac; fruit round $2.5 \mathrm{~cm}$, white

\section{HABITAT}

Wild in village wastelands, fallows and open country

\section{ORIGINS}

Malaysia and western Indonesia, Australia

\section{USES}

Timber for firewood; leaves for medicine, fish poison

\section{REFERENCES}

Burkill 1966: 407; Corner 1988: 743;

TFM Vol. 3: 301

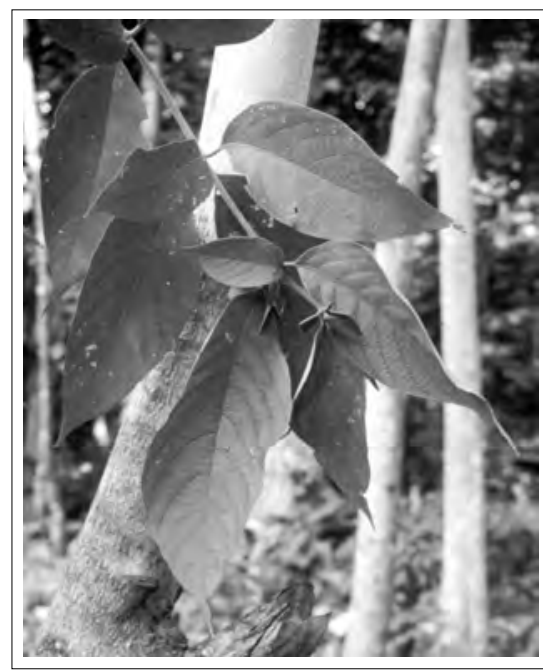

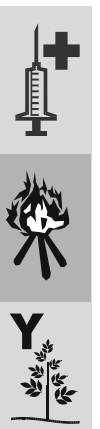

\begin{tabular}{|c|c|c|c|c|c|}
\hline \# & Code & Local Name & Use & RU & Hab.-Ab. \\
\hline 1 & PUT & umbu, turu, puli todok & 2,7 & $\mathrm{x}$ & $\mathrm{Y}-3, \mathrm{O}-3$ \\
\hline 2 & PUB & uta uru, uta uru & 2 & $x$ & $\mathrm{Y}-3, \mathrm{O}-3$ \\
\hline 3 & PUD & metan baco, metan atuk & 2 & $\mathrm{x}$ & $\mathrm{S}-3, \mathrm{Y}-3, \mathrm{O}-2$ \\
\hline 4 & PUM & umbu & 7 & $\mathrm{x}$ & $\mathrm{Y}-2$ \\
\hline 5 & ALS & kutau & 2 & $x$ & $\mathrm{Y}-3, \mathrm{O}-2$ \\
\hline 6 & LDY & kutau buda' & 2 & $\mathrm{x}$ & $\mathrm{Y}-3, \mathrm{O}-3$ \\
\hline 7 & LIT & uduh serawak, udu daya, kayu pirar & 2 & $\mathbf{v}$ & $\mathrm{Y}-3, \mathrm{O}-2$ \\
\hline 8 & BRU & kayau long ata' awit & 2 & $\mathbf{v}$ & $\mathrm{S}-2, \mathrm{Y}-3$ \\
\hline 9 & MRP & terou, torou, tarou & 2 & v & $\mathrm{Y}-3$ \\
\hline 10 & PTN & uro' ba' & 2 & $\mathrm{x}$ & $\mathrm{Y}-3, \mathrm{~S}-2$ \\
\hline 11 & KLK & mata atuk, kayu mata atok & 2 & v & $\mathrm{Y}-3, \mathrm{O}-2$ \\
\hline 12 & KLM & utip ma & 2 & $x$ & ALL \\
\hline 13 & KLO & ncung ace, daeng ubu', ocong ace, ucung ace & 2 & v & $\mathrm{S}-3, \mathrm{Y}-2, \mathrm{O}-3$ \\
\hline 14 & KLA & ucung acai, sak ubu, tung ubu & 1,2 & v & $\mathrm{S}-3, \mathrm{Y}-2$ \\
\hline 15 & KAL & ncung acey & 2 & $x$ & $\mathrm{~S}-2, \mathrm{Y}-3$ \\
\hline 16 & KBD & uti ma', ute ma' & 2 & $x$ & $\mathrm{O}-2, \mathrm{Y}-2$ \\
\hline 17 & KBK & ute ma', & 2 & $x$ & S-2, Y-3 \\
\hline 18 & PEB & ureu uti' ma', tegolem uti' ma' & 2 & $\mathbf{v}$ & $\mathrm{G}-2, \mathrm{Y}-4, \mathrm{O}-2$ \\
\hline
\end{tabular}

Notes : 


\section{Vitex pinnata L. (Verbenaceae)}

\section{MALAYAN TEAK. LABAN, KALAPAPA \\ ○}

\section{DESCRIPTION}

Tree, to $30 \mathrm{~m}$, crooked bole; bark yellowish grey or ashen, fissured, flaky; crown shabby green, twigs, underside of leaves hairy; 3-5 sessile leaflets, elliptic 7.5-27.5 x 3-10 cm; flower bluish white to violet; fruit round, 7-13 $\mathrm{mm}$ purplish black

\section{HABITAT}

Lowland and hill secondary forest; old cultivated lands, riverine

\section{ORIGINS}

Southeast Asia

\section{USES}

Timber for light construction; medicine; highly preferred for firewood

\section{REFERENCES}

Corner 1988: 754; PROSEA Vol. 5(2): 508

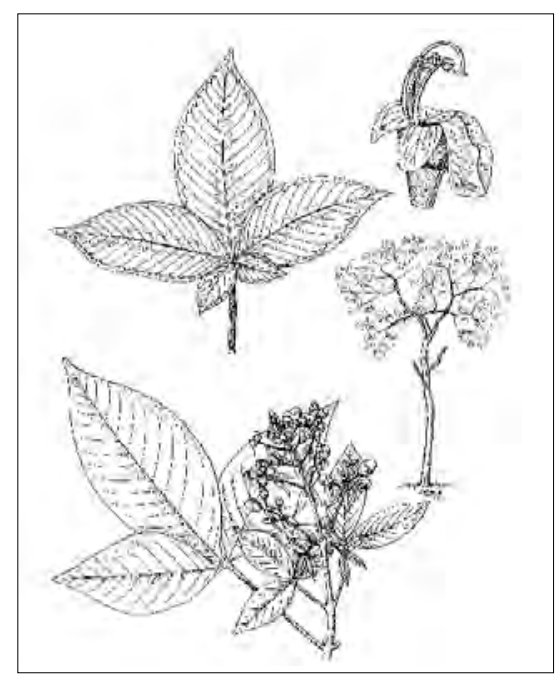

\begin{tabular}{|c|c|l|c|c|c|}
\hline$\#$ & Code & \multicolumn{1}{|c|}{ Local Name } & Use & RU & Hab.-Ab. \\
\hline 1 & PUT & jela kapa, kapa' & 7 & $\mathbf{v}$ & Y-2, O-3 \\
\hline 2 & PUB & kayu jela kapa, jela kapa & 7 & $\mathbf{X}$ & Y-3, O-4 \\
\hline 3 & PUD & kapa', kapa & 7 & $\mathbf{X}$ & S-2, Y-3, O-3, O-4 \\
\hline 4 & PUM & kelopo & 7 & $\mathbf{X}$ & Y-3, O-4 \\
\hline 5 & ALS & levovoh & 7 & $\mathbf{V}$ & Y-2, O-3 \\
\hline 6 & LDY & lefefe, felepo & 7 & $\mathbf{v}$ & S-2, Y-3, O-3, P-2 \\
\hline 7 & LIT & adel & 7 & $\mathbf{v}$ & O-3, P-1 \\
\hline 8 & BRU & lem pa'pa' & 4,7 & $\mathbf{X}$ & Y-3, O-4 \\
\hline 9 & MRP & kayu papa, liu papa' & 7 & $\mathbf{X}$ & Y-4, O-4, P-2 \\
\hline 10 & PTN & kayo jepapa & $2,4,7$ & $\mathbf{v}$ & Y-3, O-3, P-2 \\
\hline 11 & KLK & japa', kayu japa & 4,7 & $\mathbf{X}$ & ALL \\
\hline 12 & KLM & tema'a, japa' & 4,7 & $\mathbf{v}$ & Y-3, O-3, P-2 \\
\hline 13 & KLO & temaa, tema & 4,7 & $\mathbf{v}$ & Y-3, O-3, P-2 \\
\hline 14 & KLA & temma & 4,7 & $\mathbf{v}$ & Y-3, O-3, P-2 \\
\hline 15 & KAL & taman jekkau & 4,7 & $\mathbf{v}$ & Y-3, O-4, P-2 \\
\hline 16 & KBD & tamen jekkau & 3,7 & $\mathbf{x}$ & Y-4, O-4, P-2 \\
\hline 17 & KBK & kayu tema'a, temaha & 7 & $\mathbf{v}$ & Y-2, O-3, P-2 \\
\hline 18 & PEB & tegolem talun & & & \\
\hline
\end{tabular}

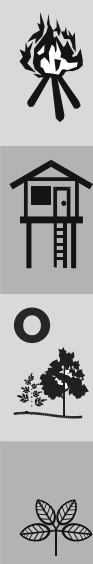

Notes : 


\section{Alpinia galanga (L.) Willd. (Zingiberaceae)}

\section{GREATER GALANGAL. LANGKUAS}

\section{DESCRIPTION}

Herb, 1.5- 3.5 m; robust perennial weed; rhizomes creeping, many branched, light red or pale yellow fragrant, 2-4 cm diameter; leaves alternate, oblonglanceolate, $20-40$ × 7-10 cm, fragrant, white-dotted; flowers white; fruits round yellow, $1-1.5 \mathrm{~cm}$ diameter

\section{HABITAT}

Cultivated in home and swidden gardens; wild in waste areas, fallows

\section{ORIGINS}

Unknown

\section{USES}

Rhizomes for spice, oil, medicine; shoots and flowers eaten

\section{REFERENCES}

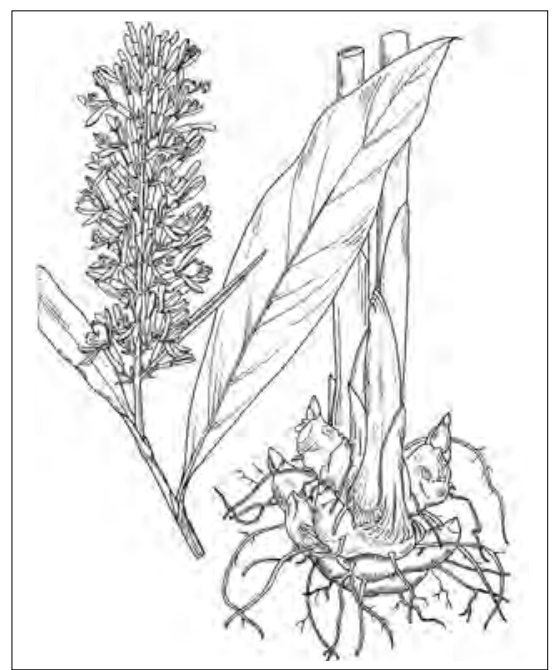

Burkill 1966: 1327; Ochse 1980: 730;

PROSEA Vol. 13: 65

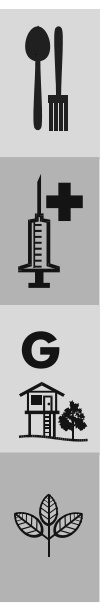

\begin{tabular}{|c|c|l|c|c|c|}
\hline$\#$ & Code & Local Name & Use & RU & Hab.-Ab. \\
\hline 1 & PUT & lekuah & 1,2 & $\mathbf{v}$ & G-2 \\
\hline 2 & PUB & lekuah & 1,2 & $\mathbf{v}$ & G-3, Y-2 \\
\hline 3 & PUD & lekuah & 1,2 & $\mathbf{v}$ & G-2, Y-2 \\
\hline 4 & PUM & da'un lengkuah & 1,2 & $\mathbf{v}$ & G-2 \\
\hline 5 & ALS & longguas & 1,2 & $\mathbf{v}$ & G-3 \\
\hline 6 & LDY & lekua & 1,2 & $\mathbf{v}$ & G-3, S-2, Y-3 \\
\hline 7 & LIT & lekua, kelua & 1,2 & $\mathbf{v}$ & G-3 \\
\hline 8 & BRU & uen legua' & 1,2 & $\mathbf{v}$ & G-2 \\
\hline 9 & MRP & lekuah & 1,2 & $\mathbf{v}$ & G-2 \\
\hline 10 & PTN & lamut & 1,2 & $\mathbf{v}$ & G-3, S-2 \\
\hline 11 & KLK & tung lamut, lia lamut & 1,2 & $\mathbf{v}$ & G-2 \\
\hline 12 & KLM & lia lamut & 1,11 & $\mathbf{v}$ & G-3 \\
\hline 13 & KLO & sin bora', cin bora', sing borak & 1,2 & $\mathbf{v}$ & G-3 \\
\hline 14 & KLA & sin burak, cin burak, burak sin & 1,2 & $\mathbf{v}$ & G-2, S-2, Y-3 \\
\hline 15 & KAL & borak sin & 1,2 & $\mathbf{v}$ & G-3 \\
\hline 16 & KBD & lia lamut, lia lamut & 1,2 & $\mathbf{v}$ & G-2, Y-2, O-2 \\
\hline 17 & KBK & buke borak & 1,2 & $\mathbf{v}$ & G-3 \\
\hline 18 & PEB & lia' lamut & & $\mathbf{v}$ & G-3, S-2 \\
\hline
\end{tabular}

Notes : 
Curcuma aeruginosa Roxb. (Zingiberaceae)

Curcuma. temu hitam

-

\section{DESCRIPTION}

Herb, to $2 \mathrm{~m}$, erect, perennial; rhizomes greenish blue; leaves green with brownish purple clouds along the sides of the midribs; flowers from rhizome, red bracts

\section{HABITAT}

Cultivated in homegardens; wild in waste areas, fallows

\section{ORIGINS}

Burma

\section{USES}

Rhizomes eaten, for medicine; sold locally

\section{REFERENCES}

Burkill 1966: 715; PROSEA Vol. 12(1): 214;

Sastrapradja et al 1977b: 32

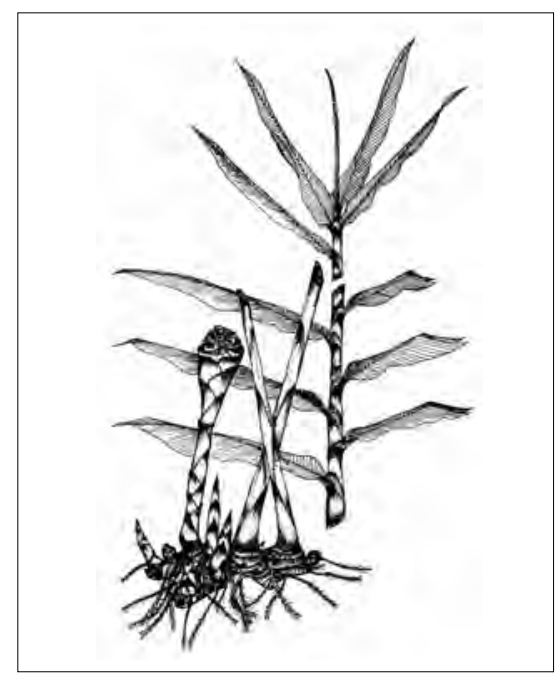

\begin{tabular}{|c|c|l|c|c|c|}
\hline$\#$ & Code & \multicolumn{1}{|c|}{ Local Name } & Use & RU & Hab.-Ab. \\
\hline 1 & PUT & lia mengan, lia pui, lia alu & $1,2,11$ & $\mathbf{v}$ & G-3, S-2 \\
\hline 2 & PUB & lia mengan, lia & $1,2,11$ & $\mathbf{v}$ & G-4 \\
\hline 3 & PUD & lia & 1,2 & $\mathbf{v}$ & G-3 \\
\hline 4 & PUM & lia mengan & 1,2 & $\mathbf{v}$ & G-3, Y-2 \\
\hline 5 & ALS & loyo & 1,2 & $\mathbf{v}$ & G-3 \\
\hline 6 & LDY & lie menangang & $1,2,11$ & $\mathbf{v}$ & G-3 \\
\hline 7 & LIT & lia menangang, lia, liye & 1,2 & $\mathbf{v}$ & G-4, S-2 \\
\hline 8 & BRU & leza menial & 1,11 & $\mathbf{x}$ & G-3 \\
\hline 9 & MRP & ya', ya' bela, yah halomela & $1,2,11$ & $\mathbf{v}$ & G-3 \\
\hline 10 & PTN & lia bela, lia bela & 1,2 & $\mathbf{v}$ & G-4, S-1 \\
\hline 11 & KLK & lia salu bala, lia salu & 1,2 & $\mathbf{v}$ & G-3 \\
\hline 12 & KLM & lia lan, lia upit & 1,2 & $\mathbf{v}$ & G-3, S-2 \\
\hline 13 & KLO & lezo salu, lezo salu bala, lezo salu vala, lezu salu vala & 1,2 & $\mathbf{x}$ & G-3 \\
\hline 14 & KLA & liza tana', liza, liza bala, liza salu bala, iza & 1,2 & $\mathbf{v}$ & G-2 \\
\hline 15 & KAL & salu lezo, salu bala & 1,2 & $\mathbf{v}$ & G-3 \\
\hline 16 & KBD & lia ba'o, lia ba'o & 1,2 & $\mathbf{v}$ & G-3 \\
\hline 17 & KBK & lia mit & 2 & $\mathbf{x}$ & G-2 \\
\hline 18 & PEB & lia' posip, lia' bokeng & & \\
\hline
\end{tabular}

Notes : 


\section{W Curcuma domestica Val.}

\section{DESCRIPTION}

Herb, to $1.5 \mathrm{~m}$, erect, perennial; rhizome tuberous, cylindrical $2.5 \mathrm{~cm}$ diameter, side branching forming stool, bitter, yellow orange color; leaves broadly lanceolate, $40-70 \times 10-14 \mathrm{~cm}$, petiole to $70 \mathrm{~cm}$; flower white or light yellow on false stem

\section{HABITAT}

Cultivated in homegardens, to $2000 \mathrm{~m}$

\section{ORIGINS}

Malaysia and western Indonesia

\section{USES}

Rhizomes for spice, dye, medicine; sold locally

\section{REFERENCES}

Burkill 1966: 716; Ochse 1980: 736;

PROSEA Vol. 12(1): 215
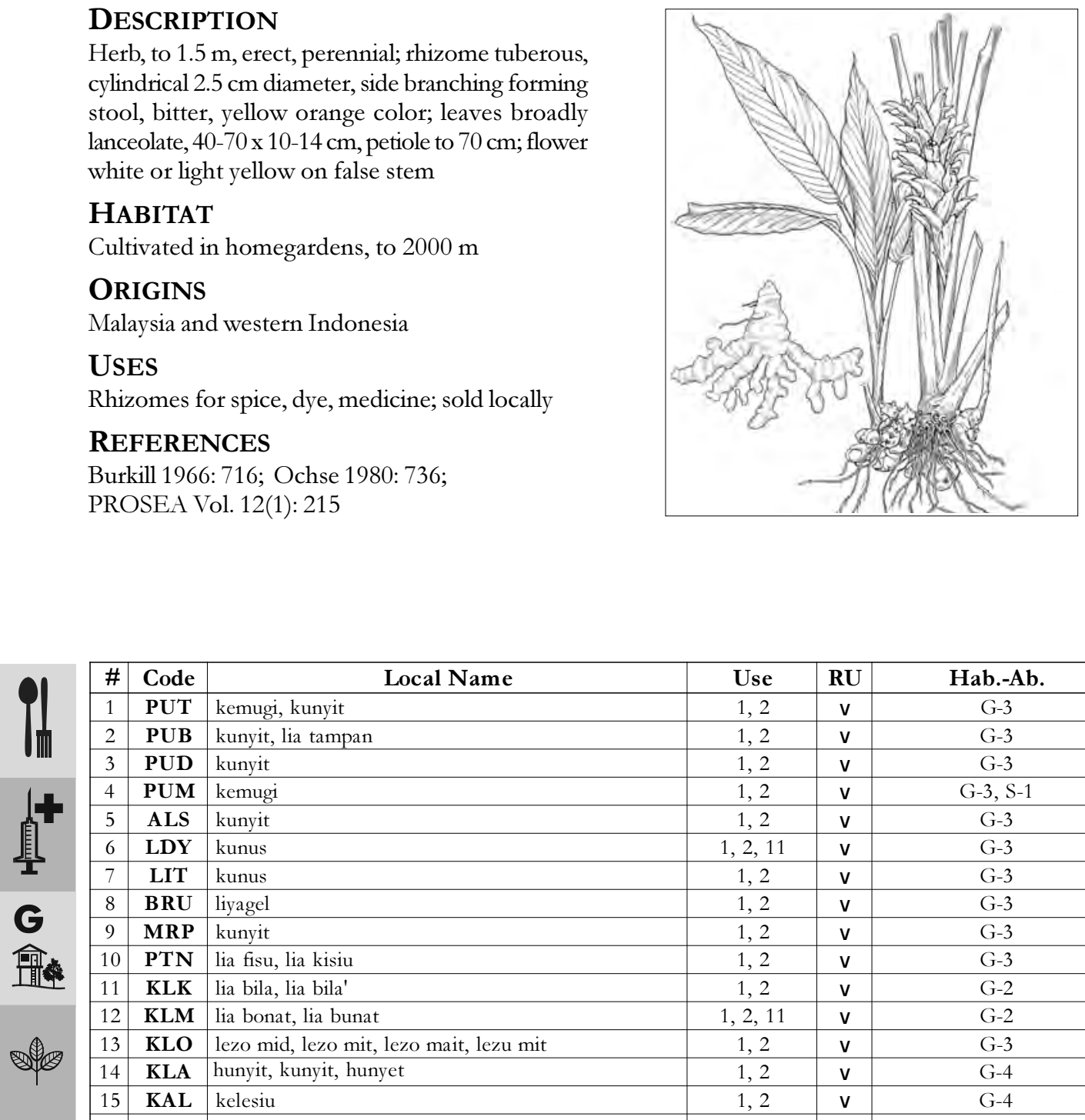

\begin{tabular}{|c|c|l|c|c|c|}
\hline \# & Code & \multicolumn{1}{|c|}{ Local Name } & Use & RU & Hab.-Ab. \\
\hline 1 & PUT & kemugi, kunyit & 1,2 & $\mathbf{v}$ & G-3 \\
\hline 2 & PUB & kunyit, lia tampan & 1,2 & $\mathbf{v}$ & G-3 \\
\hline 3 & PUD & kunyit & 1,2 & $\mathbf{v}$ & G-3 \\
\hline 4 & PUM & kemugi & 1,2 & $\mathbf{v}$ & G-3, S-1 \\
\hline 5 & ALS & kunyit & 1,2 & $\mathbf{v}$ & G-3 \\
\hline 6 & LDY & kunus & $1,2,11$ & $\mathbf{v}$ & G-3 \\
\hline 7 & LIT & kunus & 1,2 & $\mathbf{v}$ & G-3 \\
\hline 8 & BRU & liyagel & 1,2 & $\mathbf{v}$ & G-3 \\
\hline 9 & MRP & kunyit & 1,2 & $\mathbf{v}$ & G-3 \\
\hline 10 & PTN & lia fisu, lia kisiu & 1,2 & $\mathbf{v}$ & G-3 \\
\hline 11 & KLK & lia bila, lia bila' & 1,2 & $\mathbf{v}$ & G-2 \\
\hline 12 & KLM & lia bonat, lia bunat & $1,2,11$ & $\mathbf{v}$ & G-2 \\
\hline 13 & KLO & lezo mid, lezo mit, lezo mait, lezu mit & 1,2 & $\mathbf{v}$ & G-3 \\
\hline 14 & KLA & hunyit, kunyit, hunyet & 1,2 & $\mathbf{v}$ & G-4 \\
\hline 15 & KAL & kelesiu & 1,2 & $\mathbf{v}$ & G-4 \\
\hline 16 & KBD & lia berung, lia berong & 1,2 & $\mathbf{v}$ & G-3 \\
\hline 17 & KBK & lia berong & 1,2 & $\mathbf{v}$ & G-3 \\
\hline 18 & PEB & lia' tuning, lia' tuning & 1,2 & $\mathbf{v}$ & G-3, S-2 \\
\hline
\end{tabular}

Notes : 


\section{Etlingera elatior (Jack) R.M. Smith (Zingiberaceae)}

\section{TORCH GINGER. HONJE. KECOMBRANG}

\section{DESCRIPTION}

Herb, 2.5-5 m, robust perennial; rhizome thick yellowish or red; stem red at base, $2.5-3.5 \mathrm{~cm}$ diameter; leaves 15-30 alternate, elliptic-oblong, abrupt tip, $20-80 \times 10-18 \mathrm{~cm}$, green above, pink below; fruit on round heads, $10-20 \mathrm{~cm} \mathrm{~d}$, red or yellowish green

\section{HABITAT}

Cultivated in home and swidden gardens; wild in secondary forest

\section{ORIGINS}

Southeast Asia

\section{USES}

Leaf buds, flower shoots, seeds eaten; leaves, seeds for medicine; stems for mats

\section{REFERENCES}

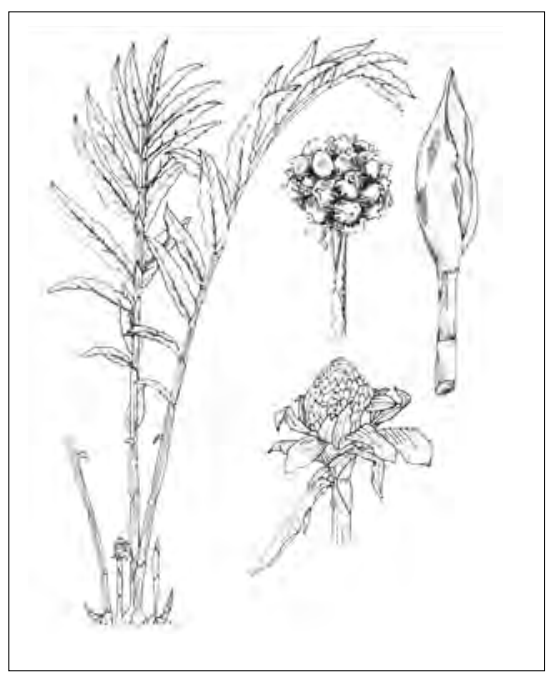

Burkill 1966: 1731; Ochse 1980: 757;

PROSEA Vol. 13: 123

\begin{tabular}{|c|c|l|c|c|c|}
\hline$\#$ & Code & \multicolumn{1}{|c|}{ Local Name } & Use & RU & Hab.-Ab. \\
\hline 1 & PUT & kala, puwang & 1,2 & $\mathbf{x}$ & Y-2, O-3, R \\
\hline 2 & PUB & puhang & 1 & $\mathbf{v}$ & Y-3, O-2, P-1 \\
\hline 3 & PUD & kala & 1 & $\mathbf{v}$ & Y-2, O-3 \\
\hline 4 & PUM & kala & 1,8 & $\mathbf{x}$ & G-1, O-3 \\
\hline 5 & ALS & bua' kalo & 1 & $\mathbf{x}$ & Y-2 \\
\hline 6 & LDY & baku sale', bua teladan & $1,2,8$ & $\mathbf{x}$ & G-2, Y-2, O-3 \\
\hline 7 & LIT & baku udat, bua sale, udad & 1 & $\mathbf{x}$ & Y-4, O-3, R \\
\hline 8 & BRU & wi salai & 1,2 & $\mathbf{x}$ & Y-2, O-3, P-3 \\
\hline 9 & MRP & kala & 1,2 & $\mathbf{x}$ & G-1, Y-2, P-3, M-1, R \\
\hline 10 & PTN & belusut sekala, sekalen & 1 & $\mathbf{x}$ & Y-2, O-3 \\
\hline 11 & KLK & ucu kuang, nyandeng, belusut & 1,2 & $\mathbf{x}$ & R \\
\hline 12 & KLM & ucuk wang, nyandeng & 1 & $\mathbf{v}$ & ALL \\
\hline 13 & KLO & nyatieng & 1 & $\mathbf{v}$ & Y-2, O-2 \\
\hline 14 & KLA & nyating & 1 & $\mathbf{v}$ & Y-3, O-2, P-3 \\
\hline 15 & KAL & lamei & 1 & $\mathbf{v}$ & Y-3, O-3, P-2 \\
\hline 16 & KBD & belusut wang & 1,2 & $\mathbf{v}$ & Y-3, O-3, P-2 \\
\hline 17 & KBK & lame & 1 & $\mathbf{v}$ & G-2, Y-2, O-2, P-2 \\
\hline 18 & PEB & nyaring, nyaring & 1 & $\mathbf{v}$ & O-4, P-2, Y-3 \\
\hline
\end{tabular}

Notes : 
Kaemferia galanga L. (Zingiberaceae)

\section{EAST INDIAN GALANGAL. KENCUR}

\section{DESCRIPTION}

Herb, stemless, perennial; rhizomes white spicy with tubers ovoid yellowish white, aromatic, 1-1.5 cm d; leaves lying on ground, single or 2-3, broadly elliptic or ovate, $7-10 \mathrm{~cm} \times 5-9 \mathrm{~cm}$, pink, reddish brown; flowers white with violet tips

\section{HABITAT}

Cultivated in home and swidden gardens

\section{ORIGINS}

India

\section{USES}

Rhizomes for spice, dye, medicine, oil, poison;

leaves eaten

\section{REFERENCES}

Burkill 1966: 1296; Ochse 1980: 748;

PROSEA Vol. 12(1): 334
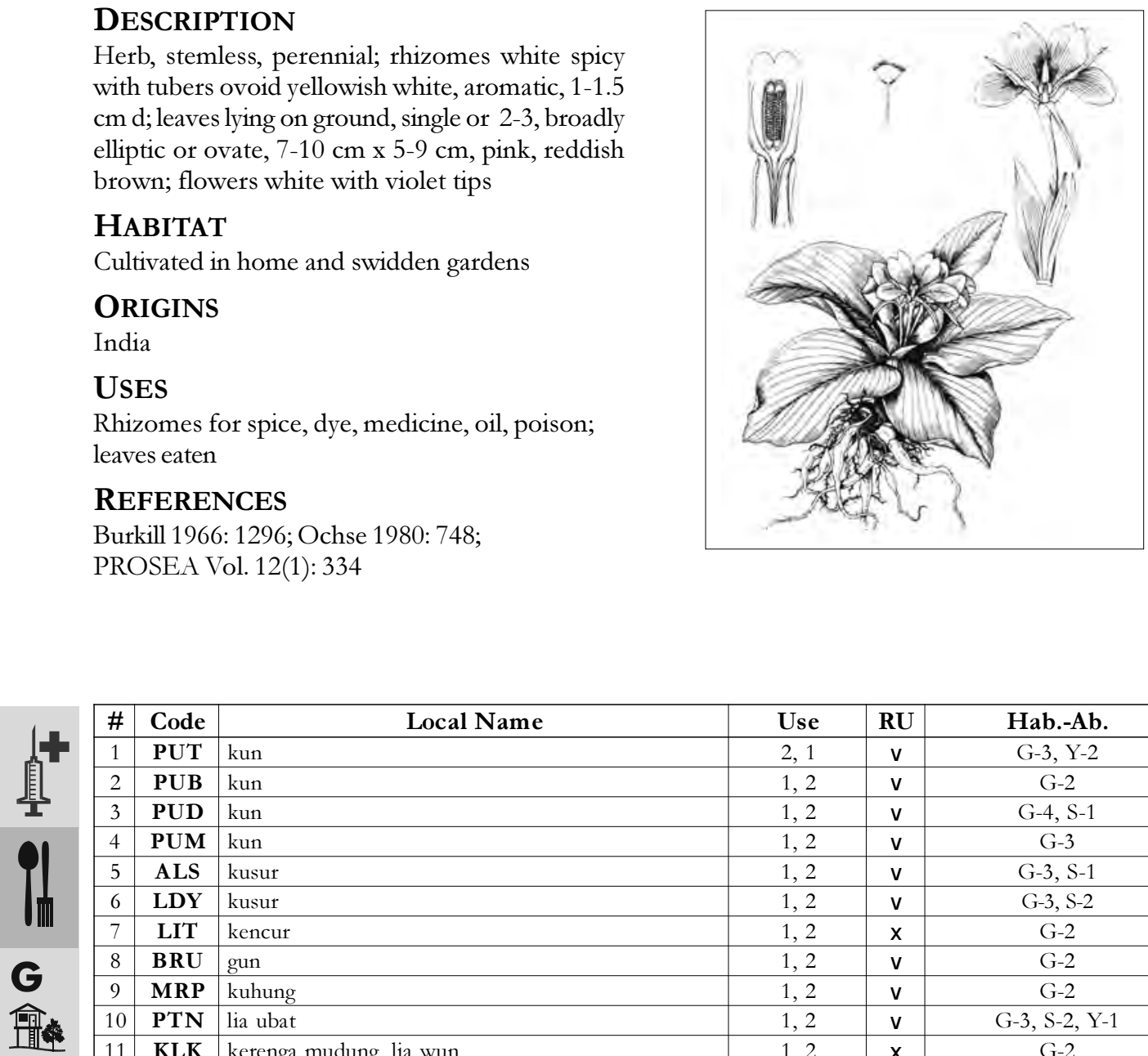

\begin{tabular}{|c|c|c|c|c|c|}
\hline \# & Code & Local Name & Use & RU & Hab.-Ab. \\
\hline 1 & PUT & kun & 2,1 & $v$ & G-3, Y-2 \\
\hline 2 & PUB & kun & 1,2 & v & G-2 \\
\hline 3 & PUD & kun & 1,2 & $\mathrm{v}$ & G-4, S-1 \\
\hline 4 & PUM & kun & 1,2 & v & G-3 \\
\hline 5 & ALS & kusur & 1,2 & v & G-3, S-1 \\
\hline 6 & LDY & kusur & 1,2 & $\mathrm{v}$ & G-3, S-2 \\
\hline 7 & LIT & kencur & 1,2 & $x$ & G-2 \\
\hline 8 & BRU & gun & 1,2 & v & G-2 \\
\hline 9 & MRP & kuhung & 1,2 & v & G-2 \\
\hline 10 & PTN & lia ubat & 1,2 & v & G-3, S-2, Y-1 \\
\hline 11 & KLK & kerenga mudung, lia wun & 1,2 & $x$ & G-2 \\
\hline 12 & KLM & lia indet & 1,2 & $\mathrm{v}$ & G-3 \\
\hline 13 & KLO & lezo obed, lezo obet, lezo bai'i & 1,2 & $\mathrm{v}$ & G-3 \\
\hline 14 & KLA & liza ubat, lia taban, liza ubat & 1,2 & $\mathrm{v}$ & G-2 \\
\hline 15 & KAL & lezo selen tit & 2 & v & G-3 \\
\hline 16 & KBD & lia bo'ut & 1,2 & v & G-2 \\
\hline 17 & KBK & lia tukang & 1,2 & v & G-3 \\
\hline 18 & PEB & lia' poti & 1,2 & v & G-4 \\
\hline
\end{tabular}

Notes : 


\section{Cynocephalus variegatus (Cynocephalidae) \\ MALAYAN FLYING LEMUR. KUBUNG}

\section{DESCRIPTION}

Small herbivorous mammal; nocturnal and arboreal; reddish brown fur beneath, mottled greyish green above, bright red ears; hangs upside-down, gliding membrane encloses tail; lower incisors look like fine-tooth combs

\section{HABITAT}

Lowland forest to $1200 \mathrm{~m}$; secondary forests, plantations and village gardens

\section{ORIGINS/STATUS}

Southeast Asia/RI: Protected

\section{USES}

Eaten; skin for hat, trophy, costume; pet (ALS)

\section{REFERENCES}

Payne et al. 1985: 167; Corbet and Hill 1992: 53; Suyanto et al. 1998

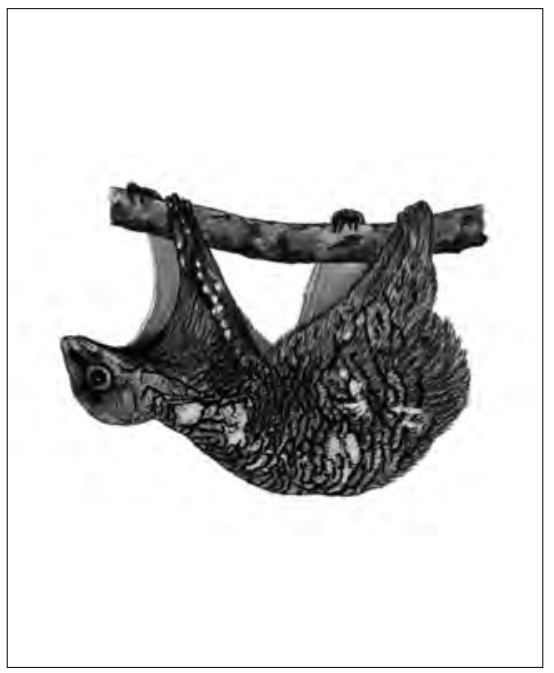

\begin{tabular}{|c|c|c|c|c|c|}
\hline \# & Code & Local Name & Use & RU & Hab.-Ab. \\
\hline 1 & PUT & kuvung, kuvung buli & 1,4 & $x$ & $\mathrm{~S}-2, \mathrm{O}-2, \mathrm{P}-2$ \\
\hline 2 & PUB & kovung, kuvung & 1 & $x$ & S-1, P-1 \\
\hline 3 & PUD & kuvung & 1,4 & v & $\mathrm{O}-4, \mathrm{P}-4$ \\
\hline 4 & PUM & kuvung lumut & 1,4 & $x$ & P-1 \\
\hline 5 & ALS & huyut batang & 1,4 & v & P-1 \\
\hline 6 & LDY & kubong pelanok, kubung lamut & 1,6 & V & $\mathrm{Y}-3, \mathrm{O}-3, \mathrm{P}-4, \mathrm{M}-1$ \\
\hline 7 & LIT & kubung & 1 & $x$ & Y-1, O-1, P-1 \\
\hline 8 & BRU & bweng bli' & 1,4 & $x$ & G-1, Y-1, O-1, P-1 \\
\hline 9 & MRP & kobue pelanau, kubuwe & 1,4 & $x$ & $\mathrm{Y}-3, \mathrm{O}-3, \mathrm{P}-1$ \\
\hline 10 & PTN & kuvung & 1 & $\mathrm{v}$ & $\mathrm{O}-4$ \\
\hline 11 & KLK & kobung bule, kubung pelanok & 1,4 & $x$ & P-1 \\
\hline 12 & KLM & kobong, kobong pelanok & 1,5 & $x$ & P-1 \\
\hline 13 & KLO & kobong & 1,4 & $x$ & P-1 \\
\hline 14 & KLA & kubung, hubong & 1,6 & $x$ & P-1 \\
\hline 15 & KAL & kubung, kubung pelanok & 1 & v & P-1 \\
\hline 16 & KBD & kubung pelanok & 1 & v & $\mathrm{O}-3, \mathrm{P}-1$ \\
\hline 17 & KBK & kubong & 1 & v & P-4 \\
\hline 18 & PEB & kubung & 1,4 & $x$ & $\mathrm{O}-2, \mathrm{P}-2$ \\
\hline
\end{tabular}

Notes : 


\section{Pteropus vampyrus (Pteropodidae) \\ FLYING FOX. KALONG KAPAUK}

\section{DESCRIPTION}

Largest frugivorous and insectivorous bat; nocturnal, roosts in large colonies in trees, often on riverbanks; black back with grey streaking, back of head, neck and shoulders reddish, underparts blackish brown

\section{HABITAT}

Lowland coastal to riverside hill forests; secondary forests and plantations; seasonally abundant due to migrations

\section{ORIGINS/STATUS}

Southeast Asia-Western Pacific/CITES: Appendix II

\section{USES}

Eaten; skin displayed as trophy (PUT)

\section{REFERENCES}

Payne et al. 1985: 172; Corbet and Hill 1992: 64;

Suyanto et al. 1998
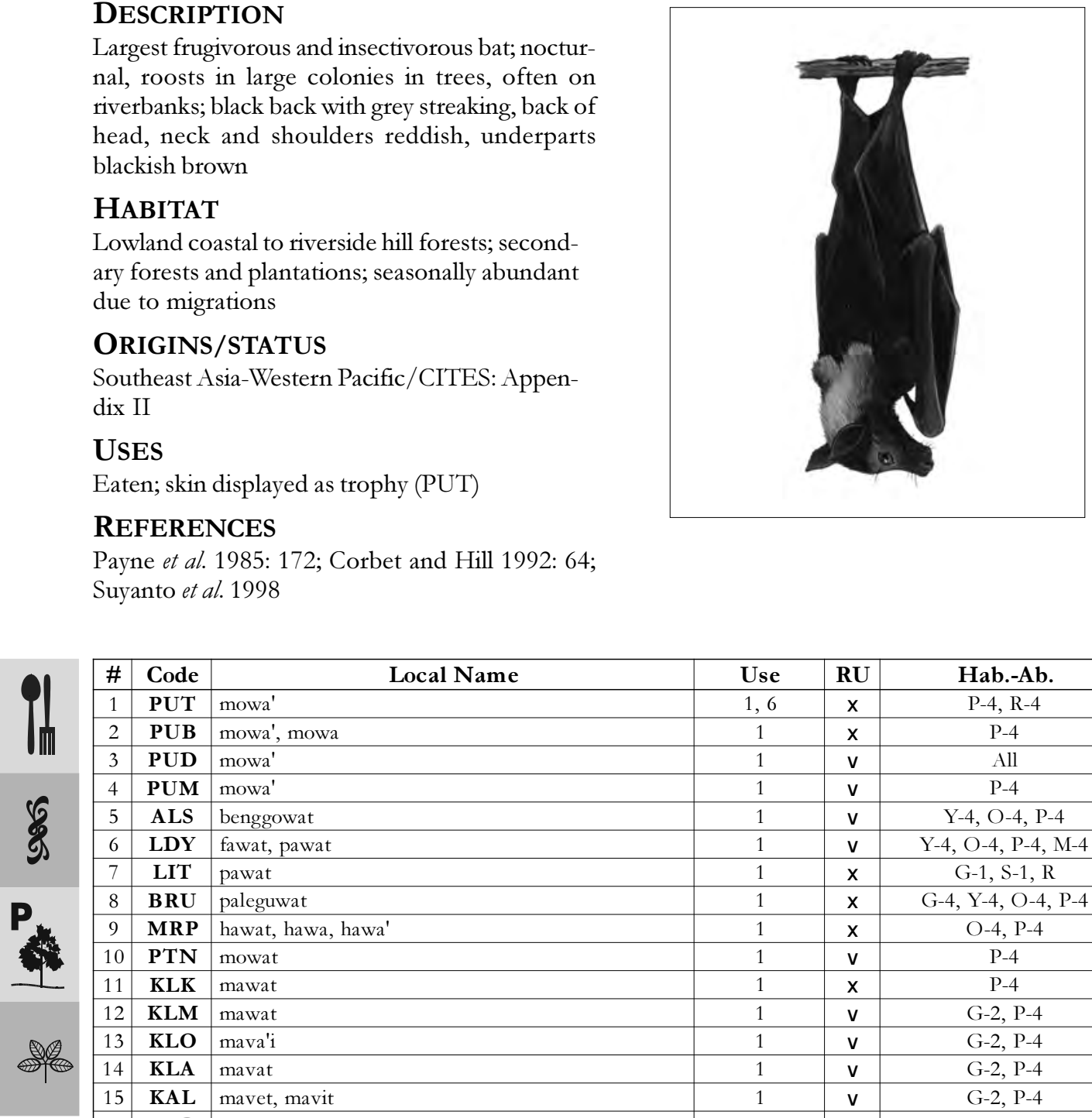

\begin{tabular}{|c|c|c|c|c|c|}
\hline$\#$ & Code & Local Name & Use & $\mathbf{R U}$ & Hab.-Ab. \\
\hline 1 & PUT & mowa' & 1,6 & $x$ & P-4, R-4 \\
\hline 2 & PUB & mowa', mowa & 1 & $x$ & P-4 \\
\hline 3 & PUD & mowa' & 1 & v & All \\
\hline 4 & PUM & mowa' & 1 & v & P-4 \\
\hline 5 & ALS & benggowat & 1 & v & $\mathrm{Y}-4, \mathrm{O}-4, \mathrm{P}-4$ \\
\hline 6 & LDY & fawat, pawat & 1 & $\mathrm{v}$ & $\mathrm{Y}-4, \mathrm{O}-4, \mathrm{P}-4, \mathrm{M}-4$ \\
\hline 7 & LIT & pawat & 1 & $x$ & G-1, S-1, R \\
\hline 8 & BRU & paleguwat & 1 & $x$ & $\mathrm{G}-4, \mathrm{Y}-4, \mathrm{O}-4, \mathrm{P}-4$ \\
\hline 9 & MRP & hawat, hawa, hawa' & 1 & $x$ & $\mathrm{O}-4, \mathrm{P}-4$ \\
\hline 10 & PTN & mowat & 1 & v & P-4 \\
\hline 11 & KLK & mawat & 1 & $x$ & P-4 \\
\hline 12 & KLM & mawat & 1 & v & G-2, P-4 \\
\hline 13 & KLO & mava'i & 1 & v & G-2, P-4 \\
\hline 14 & KLA & mavat & 1 & v & G-2, P-4 \\
\hline 15 & KAL & mavet, mavit & 1 & $\mathrm{v}$ & G-2, P-4 \\
\hline 16 & KBD & mawat & 1 & v & P-4, R-4 \\
\hline 17 & KBK & mawat & 1 & $x$ & R-5 \\
\hline 18 & PEB & mawat & 1 & $\mathrm{v}$ & $\mathrm{R}-4$ \\
\hline
\end{tabular}

Notes : 


\section{Nycticebus coucang (Lorisidae) \\ SLOW LORIS. KUKANG \\ - 0 ○}

\section{DESCRIPTION}

Small frugivorous and insectivorous primate; nocturnal and arboreal, usually solitary; brownish or greyish woolly hair, dark brown stripe from top of head to middle of back, dark brown spectacled mark around eye; very short tail, inconspicuous ears

\section{HABITAT}

Lowland to hill forests; secondary forests, plantations and village gardens

\section{ORIGINS/STATUS}

India and China/CITES: Appendix II; RI:

Protected

\section{USES}

Rarely eaten; women cannot eat (PUT); juveniles sold as pets; omen to move swidden; ghost (MRP); poisonous bites (BRU, MRP, KLM, KBD); skin for hat (PUD, PTN)

\section{REFERENCES}

Payne et al. 1985: 223; Corbet and Hill 1992: 163

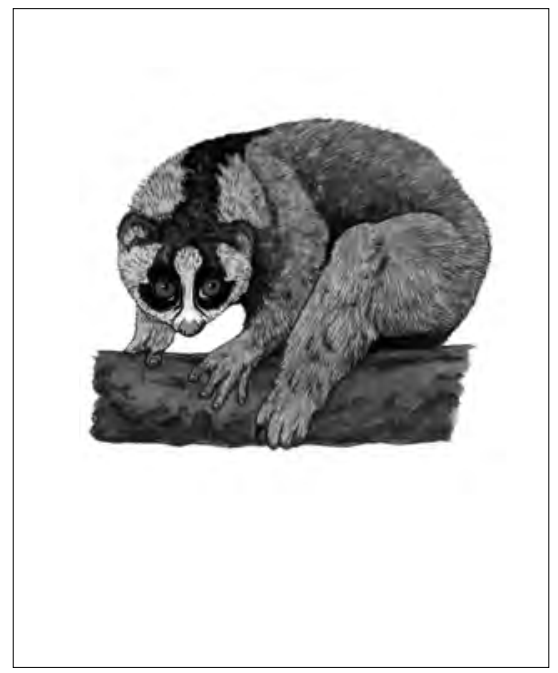

\begin{tabular}{|c|c|l|c|c|c|}
\hline$\#$ & Code & \multicolumn{1}{|c|}{ Local Name } & Use & RU & Hab.-Ab. \\
\hline 1 & PUT & bekikih & 1 & $\mathbf{X}$ & Y-1, O-1 \\
\hline 2 & PUB & bekikih & 5 & $\mathbf{X}$ & O-1 \\
\hline 3 & PUD & duk alun & 1,4 & $\mathbf{V}$ & ALL-1 \\
\hline 4 & PUM & bekikih & 5 & $\mathbf{X}$ & S-4,Y-4, O-4, P-4 \\
\hline 5 & ALS & lotong & 5 & $\mathbf{X}$ & S-1, Y-1, O-1 \\
\hline 6 & LDY & fuge & $1,2,5$ & $\mathbf{V}$ & Y-1, O-1 \\
\hline 7 & LIT & puge & 1 & $\mathbf{X}$ & Y-1, O-1, P-1 \\
\hline 8 & BRU & dio' lun & 1,8 & $\mathbf{v}$ & ALL-1 \\
\hline 9 & MRP & dau' laung & 5 & $\mathbf{X}$ & Y-1, O-1, P-1 \\
\hline 10 & PTN & dok alun & $1,4,6$ & $\mathbf{X}$ & G-1, S-1, Y-1 \\
\hline 11 & KLK & dok talun & 1 & $\mathbf{X}$ & O-1 \\
\hline 12 & KLM & dok kalun & & $\mathbf{X}$ & Y-1, O-1 \\
\hline 13 & KLO & & & & \\
\hline 14 & KLA & dok malun, dok nalun & 1,5 & $\mathbf{X}$ & Y-1, O-1 \\
\hline 15 & KAL & dok maling, dok nalun & 1 & $\mathbf{X}$ & Y-1, O-1 \\
\hline 16 & KBD & dok talun & 5 & $\mathbf{X}$ & Y-1, O-2 \\
\hline 17 & KBK & dok talun & 5 & $\mathbf{x}$ & G-1, S-1, Y-1, O-1, P-1 \\
\hline 18 & PEB & ketuning, ket uning & 5 & $\mathbf{x}$ & S-1, Y-1, O-1 \\
\hline
\end{tabular}

\section{Notes :}




\section{Tarsius bancanus (Tarsiidae) \\ TX WESTERN TARSIER. KRABUKU INGKAT

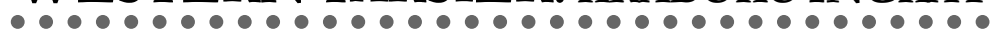

\section{DESCRIPTION}

Small insectivorous primate; nocturnal and mainly arboreal; eyes large; digits long and ending in broad pads and pointed nails; long tailed, leaps from tree to tree; buff coloured tail naked except for tufted tip

\section{HABITAT}

Lowland primary forest to $1200 \mathrm{~m}$; secondary forests

\section{ORIGINS/STATUS}

Sumatra and Borneo/CITES: Appendix II; RI:

Protected

\section{USES}

Usually not eaten; omen to move swidden (PUT, PUM, ALS, LDY, MRP, PTN); descended from humans (PUD); ghost (MRP); killing prohibited (KLM); children's pet (KAL); for medicine (PUM, ALS)

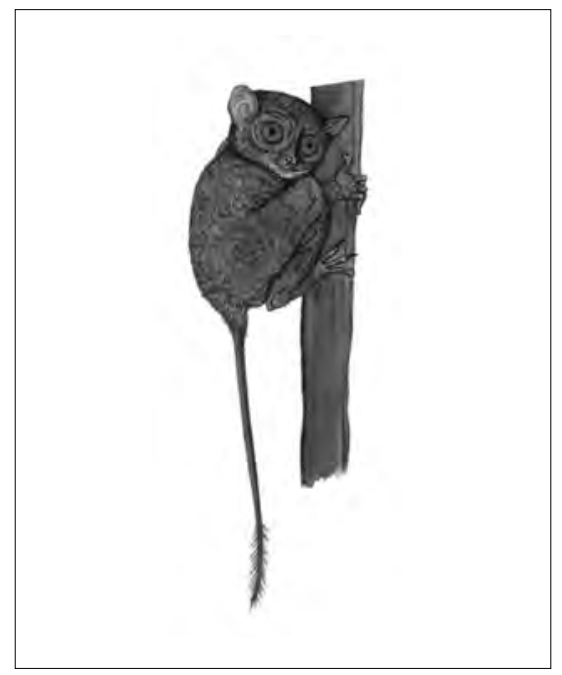

\section{REFERENCES}

Payne et al. 1985: 223; Corbet and Hill 1992: 165;

Suyanto et al. 1998

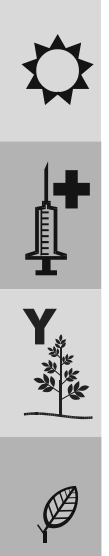

\begin{tabular}{|c|c|l|c|c|c|}
\hline$\#$ & Code & \multicolumn{1}{|c|}{ Local Name } & Use & RU & Hab.-Ab. \\
\hline 1 & PUT & iket & 5 & $\mathbf{X}$ & O-1, P-1 \\
\hline 2 & PUB & iket & & $\mathbf{X}$ & \\
\hline 3 & PUD & iket & & $\mathbf{X}$ & ALL-1 \\
\hline 4 & PUM & iket & 2,5 & $\mathbf{X}$ & ALL-1 \\
\hline 5 & ALS & sembelilih & 2,5 & $\mathbf{X}$ & \\
\hline 6 & LDY & ikau & 1 & $\mathbf{X}$ & G-1, S-1, Y-1, O-1, P-2 \\
\hline 7 & LIT & ikau & & $\mathbf{V}$ & Y-1, O-1, P-1 \\
\hline 8 & BRU & kiau & 5 & $\mathbf{X}$ & G-1, Y-1, O-1, P-1 \\
\hline 9 & MRP & kio, keio & & $\mathbf{X}$ & G-1, S-1, Y-1 \\
\hline 10 & PTN & kuyang laong & & $\mathbf{X}$ & O-1 \\
\hline 11 & KLK & ket & & $\mathbf{X}$ & Y-1, O-1 \\
\hline 12 & KLM & ket & & $\mathbf{X}$ & \\
\hline 13 & KLO & & & $\mathbf{X}$ & \\
\hline 14 & KLA & & 9 & $\mathbf{X}$ & Y-1, O-1 \\
\hline 15 & KAL & toja liget, taho jang la'ong & 5 & $\mathbf{X}$ & Y-1, O-2 \\
\hline 16 & KBD & ket & 5 & $\mathbf{X}$ & Y-1, O-1, P-1 \\
\hline 17 & KBK & jalan & 5 & $\mathbf{X}$ & G-1, S-1, Y-1 \\
\hline 18 & PEB & ket ase & & \\
\hline
\end{tabular}

Notes : 


\section{Presbytis rubicunda (Cercopithecidae) \\ MAROON LEAF MONKEY. LUTUNGMERAH}

\section{DESCRIPTION}

Large folivorous and frugivorous primate; diurnal and arboreal; long tailed; red body, face with bluish tinge

\section{HABITAT}

Lowland to mountain primary forests, to 2000 $\mathrm{m}$; sometimes in secondary forest and plantations

\section{ORIGINS/STATUS}

Borneo endemic/CITES: Appendix II; RI:

Protected

\section{USES}

Eaten; skin for hat (PUT, PUB, PUM, LDY, LIT, PTN, KLK, KAL, KBD); teeth for decoration (LDY)

\section{REFERENCES}

Payne et al. 1985: 226; Corbet and Hill 1992: 172

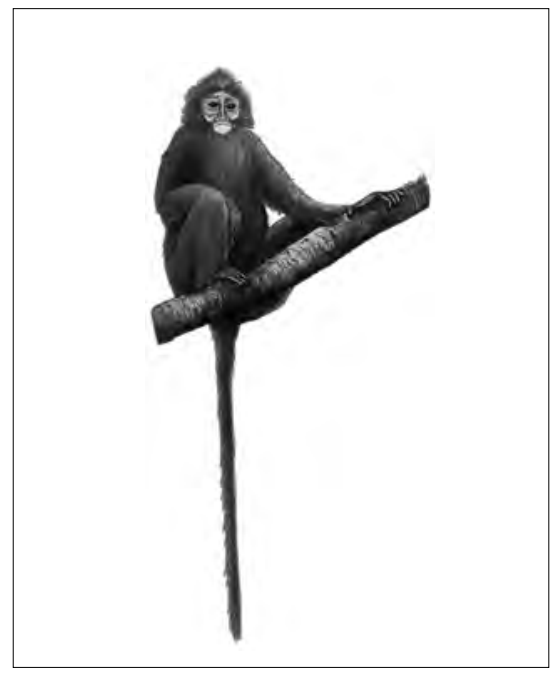

\begin{tabular}{|c|c|c|c|c|c|}
\hline \# & Code & Local Name & Use & RU & Hab.-Ab. \\
\hline 1 & PUT & aci & & $x$ & \\
\hline 2 & PUB & aci' & 1,4 & $x$ & \\
\hline 3 & PUD & acei & 1,4 & $x$ & M-1 \\
\hline 4 & PUM & aci' & 1,4 & $x$ & M-4 \\
\hline 5 & ALS & kelasi & 1,4 & $\mathrm{v}$ & M-1 \\
\hline 6 & LDY & kelasi & $1,4,6$ & v & $\mathrm{P}-3, \mathrm{M}-4$ \\
\hline 7 & LIT & kelasi & 1,4 & $x$ & P-1, M-1 \\
\hline 8 & BRU & pangsih & 1 & $x$ & P-1, M-1 \\
\hline 9 & MRP & hacei & 1 & $x$ & \\
\hline 10 & PTN & kelasih & 1,4 & $x$ & P-1 \\
\hline 11 & KLK & kelasih & 1,4 & $x$ & \\
\hline 12 & KLM & kelasi & 1,4 & $x$ & P-2, M-2 \\
\hline 13 & KLO & kelasi & 1,4 & $x$ & P-2, M-2 \\
\hline 14 & KLA & kelasi & 1 & $x$ & $\mathrm{P}-2, \mathrm{M}-2$ \\
\hline 15 & KAL & kelasi & 1,4 & $x$ & $\mathrm{P}-2, \mathrm{M}-2$ \\
\hline 16 & KBD & kelasi & 1,4 & $x$ & $\mathrm{P}-2, \mathrm{M}-3$ \\
\hline 17 & KВ K & kelasih & 1 & $x$ & M-2 \\
\hline 18 & PEB & kerasi & 1,4 & $x$ & P-2, M-2 \\
\hline
\end{tabular}

Notes : 


\section{Presbytis frontata (Cercopithecidae) WHITE-FRONTED LEAF MONKEY. LUTUNG JIRANGAN}

\section{DESCRIPTION}

Large folivorous and frugivorous primate; diurnal and arboreal; long tailed; grey brown body hair with white star-shaped patch of skin on the forehead

\section{HABITAT}

Lowland and hill primary forests

\section{ORIGINS/STATUS}

Borneo endemic/CITES: Appendix II; RI:

Protected

\section{USES}

Eaten; skin for hat (KAL)

\section{REFERENCES}

Payne et al. 1985: 227; Corbet and Hill 1992: 172;

Suyanto et al. 1998
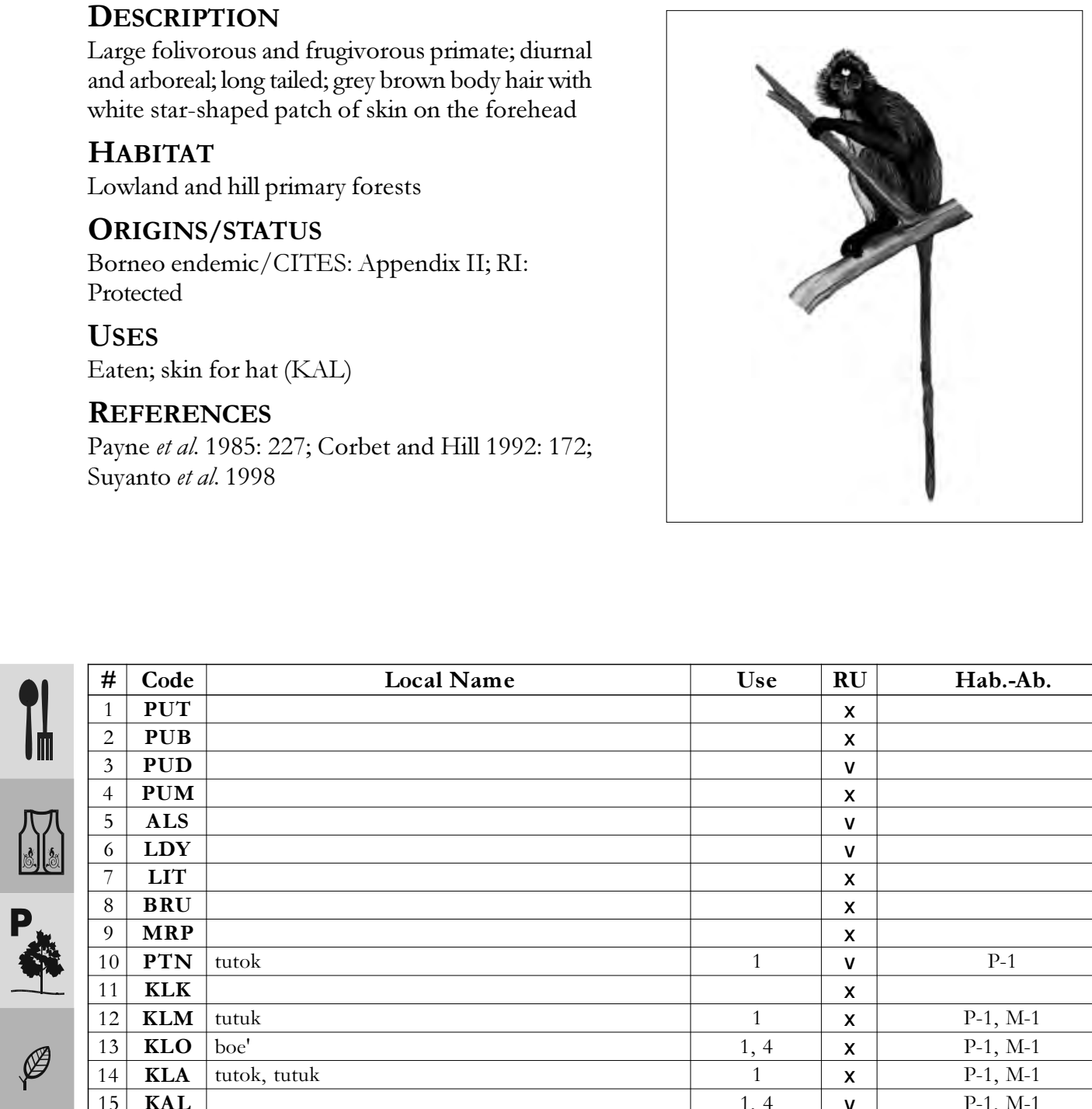

\begin{tabular}{|c|c|l|c|c|c|}
\hline$\#$ & Code & Local Name & Use & RU & Hab.-Ab. \\
\hline 1 & PUT & & & $\mathbf{X}$ & \\
\hline 2 & PUB & & $\mathbf{X}$ & \\
\hline 3 & PUD & & $\mathbf{V}$ & \\
\hline 4 & PUM & & $\mathbf{X}$ & \\
\hline 5 & ALS & & $\mathbf{V}$ & \\
\hline 6 & LDY & & $\mathbf{V}$ & \\
\hline 7 & LIT & & & $\mathbf{X}$ & \\
\hline 8 & BRU & & & $\mathbf{X}$ & \\
\hline 9 & MRP & & & $\mathbf{X}$ & \\
\hline 10 & PTN & tutok & 1 & $\mathbf{V}$ & $\mathrm{P}-1$ \\
\hline 11 & KLK & & & $\mathbf{X}$ & \\
\hline 12 & KLM & tutuk & 1 & $\mathbf{X}$ & $\mathrm{P}-1, \mathrm{M}-1$ \\
\hline 13 & KLO & boe' & 1,4 & $\mathbf{X}$ & $\mathrm{P}-1, \mathrm{M}-1$ \\
\hline 14 & KLA & tutok, tutuk & 1 & $\mathbf{X}$ & $\mathrm{P}-1, \mathrm{M}-1$ \\
\hline 15 & KAL & & 1,4 & $\mathbf{V}$ & $\mathrm{P}-1, \mathrm{M}-1$ \\
\hline 16 & KBD & tutuk & 1,4 & $\mathbf{V}$ & $\mathrm{P}-1, \mathrm{M}-2$ \\
\hline 17 & KBK & tutok & 1 & $\mathbf{V}$ & $\mathrm{M}-2$ \\
\hline 18 & PEB & buwi & 1,4 & $\mathbf{X}$ & $\mathrm{P}-1, \mathrm{M}-1$ \\
\hline
\end{tabular}

Notes : 


\section{Presbytis bosei (Cercopithecidae)}

\section{GREY LEAF MONKEY. LUTUNG BANGGAT}

\section{DESCRIPTION}

Large folivorous and frugivorous primate; diurnal and arboreal; long tailed; upper parts grey, underparts white, with blackish hands and feet, face pinkish with distinct black band across each cheek; white fur completely or partially encircling face

\section{HABITAT}

Lowland and hill forests; sometimes secondary forest; visits salt/mineral springs

\section{ORIGINS/STATUS}

Borneo endemic/CITES: Appendix II

\section{USES}

Eaten; skin for hat; teeth for decoration(LDY); prohibited to use real name (PUD); bezoar stone sold to traders for export (ALS, LDY, BRU, PTN, KLK, KLM, KAL, KBD); pet (ALS)

\section{REFERENCES}

Payne et al. 1985: 226; Corbet and Hill 1992: 172

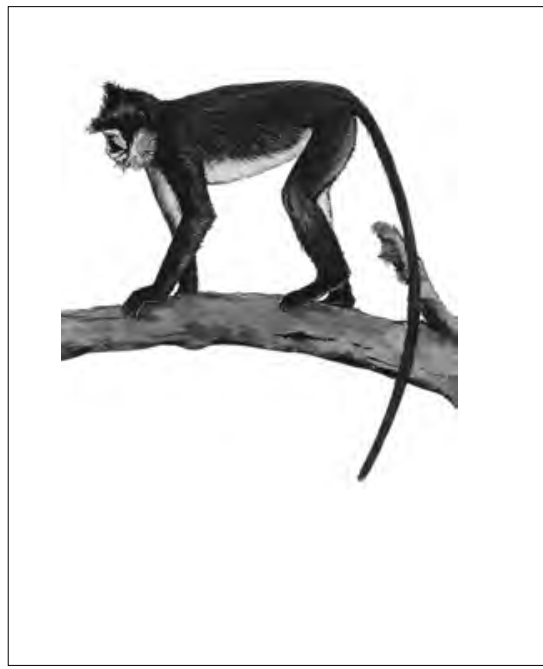

\begin{tabular}{|c|c|c|c|c|c|}
\hline \# & Code & Local Name & Use & RU & Hab.-Ab. \\
\hline 1 & PUT & eciu, oi & 1 & $v$ & $\mathrm{O}-3, \mathrm{P}-4, \mathrm{M}-2$ \\
\hline 2 & PUB & eciu, wei & 1,4 & v & $\mathrm{O}-4, \mathrm{P}-4$ \\
\hline 3 & PUD & eciu & $1,5,8$ & $x$ & $\mathrm{O}-4, \mathrm{P}-4$ \\
\hline 4 & PUM & $a i^{\prime}$ & 1,8 & v & P-4 \\
\hline 5 & ALS & menosop & $1,4,8,9$ & $\mathrm{v}$ & P-4 \\
\hline 6 & LDY & berangan, berangat & $1,4,6$ & v & $\mathrm{Y}-4, \mathrm{P}-4, \mathrm{M}-4$ \\
\hline 7 & LIT & berangat & 1,4 & v & P-4 \\
\hline 8 & BRU & belangat & $1,4,8$ & v & $\mathrm{P}-4, \mathrm{M}-4$ \\
\hline 9 & MRP & nyakait, nyakai' & 1,4 & $x$ & $\mathrm{O}-4, \mathrm{P}-4$ \\
\hline 10 & PTN & bangat & $1,4,5,8$ & $\mathrm{v}$ & $\mathrm{P}-4$ \\
\hline 11 & KLK & bangat & $1,4,8$ & $\mathrm{v}$ & P-4 \\
\hline 12 & KLM & bangat & $1,4,8$ & $\mathrm{v}$ & $\mathrm{O}-4, \mathrm{P}-4$ \\
\hline 13 & KLO & bangat & 1,8 & $\mathrm{v}$ & $\mathrm{O}-4, \mathrm{P}-4$ \\
\hline 14 & KLA & bangat & 1,8 & v & $\mathrm{O}-4, \mathrm{P}-4$ \\
\hline 15 & KAL & bangit & 1,8 & v & $\mathrm{O}-4, \mathrm{P}-4$ \\
\hline 16 & KBD & bangat & $1,4,8$ & v & $\mathrm{O}-4, \mathrm{P}-4, \mathrm{M}-2$ \\
\hline 17 & KBK & bangat & 1,8 & v & $\mathrm{P}-3$ \\
\hline 18 & PEB & bangat & $1,4,8$ & v & $\mathrm{O}-4, \mathrm{P}-4, \mathrm{M}-2$ \\
\hline
\end{tabular}

Notes : 


\section{Nasalis larvatus (Cercopithecidae)}

\section{Proboscis Monkey. BeKantan Kahau}

\section{DESCRIPTION}

Large folivorous and frugivorous primate; diurnal and arboreal, but will swim rivers or walk across areas to reach isolated forest; long tailed, large nose; reddish brown with cap of red hair and red skin on face; tail and rump whitish

\section{HABITAT}

Coastal riverine, peat swamp and mangrove forests; rare or locally absent inland, one pair observed on Bahau River, 1991

\section{ORIGINS/STATUS}

Borneo endemic/CITES: Appendix I; RI:

Protected

\section{USES}

Eaten; skin for hat (ALS, KLM)

\section{REFERENCES}

Payne et al. 1985: 228; Corbet and Hill 1992: 171;

Suyanto et al. 1998

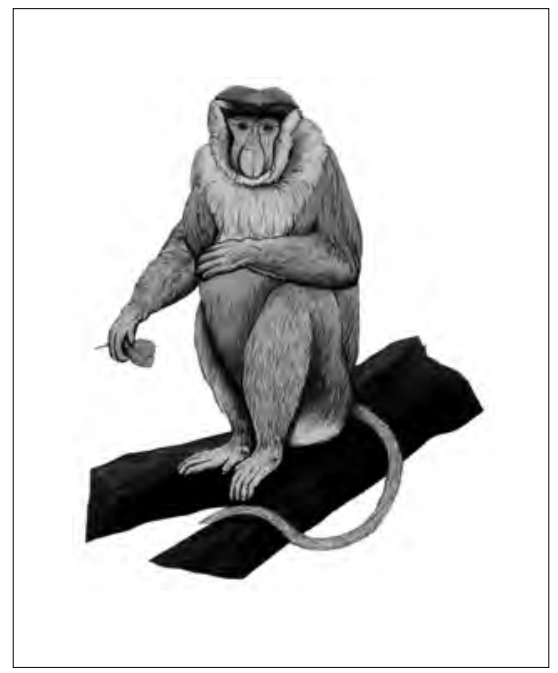

\begin{tabular}{|c|c|c|c|c|c|}
\hline$\#$ & Code & Local Name & Use & RU & Hab.-Ab. \\
\hline 1 & PUT & bekaro & & $\mathrm{x}$ & $\mathrm{R}$ \\
\hline 2 & PUB & belekaro, bekaro & & $\mathrm{x}$ & $\mathrm{R}$ \\
\hline 3 & PUD & bekaro & 1 & $x$ & $\mathrm{R}$ \\
\hline 4 & PUM & bekaro & 1 & $x$ & $\mathrm{R}$ \\
\hline 5 & ALS & bakaro & 1,4 & $x$ & $\mathrm{R}$ \\
\hline 6 & LDY & bungkaro, bengkaro & 1 & $\mathrm{x}$ & $\mathrm{R}, \mathrm{M}-1$ \\
\hline 7 & LIT & kuyu risung, koyo resung & 1 & $x$ & $\mathrm{R}$ \\
\hline 8 & BRU & bekaroh & 1 & $\mathrm{x}$ & $\mathrm{R}$ \\
\hline 9 & MRP & bekerau, kerau, bakerau & 1 & $x$ & $\mathrm{R}$ \\
\hline 10 & PTN & bekaro & 1 & $\mathrm{x}$ & $\mathrm{R}$ \\
\hline 11 & KLK & bekaroh & & $x$ & $\mathrm{R}$ \\
\hline 12 & KLM & bekaro, bekarau & 1,4 & $x$ & $\mathrm{R}$ \\
\hline 13 & KLO & kodei balah & & $x$ & $\mathrm{R}$ \\
\hline 14 & KLA & kero, kerang kero' & & $x$ & $\mathrm{R}$ \\
\hline 15 & KAL & keru', kerang kero & & $x$ & $\mathrm{R}$ \\
\hline 16 & KBD & bekaro & & $x$ & $\mathrm{R}$ \\
\hline 17 & KBK & bekaro & & $x$ & $\mathrm{R}$ \\
\hline 18 & PEB & kikeu & & $\mathrm{x}$ & $\mathrm{R}$ \\
\hline
\end{tabular}

Notes : 


\section{Macaca fascicularis (Cercopithecidae) \\ LONG TAIL MACAQUE. MONYETEKOR PANANG}

\section{DESCRIPTION}

Medium sized omnivorous primate, diurnal, partly terrestrial, sleeps in trees along rivers; long tailed, with cheek pouches and simple stomach; grey brown body, paler on underside; old males have grey cheek whiskers

\section{HABITAT}

Coastal to mountain forests; secondary forests, plantations, village gardens and rice swiddens

\section{ORIGINS/STATUS}

South-Southeast Asia/CITES: Appendix II

\section{USES}

Eaten; for medicine (KAL); juveniles sold as pets (PUT, PUM, ALS, LDY, BRU, KBD); skin for hat (PUD, LIT)

\section{REFERENCES}

Payne et al. 1985: 228; Corbet and Hill 1992: 170;

Suyanto et al. 1998

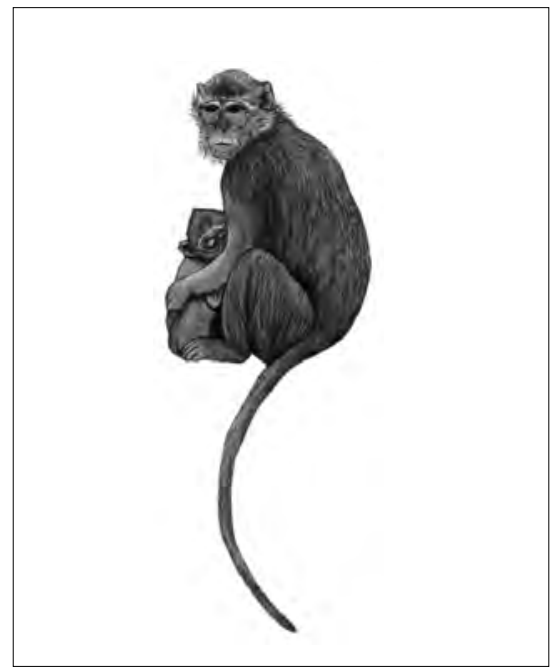

\begin{tabular}{|c|c|c|c|c|c|}
\hline \# & Code & Local Name & Use & RU & Hab.-Ab. \\
\hline 1 & PUT & kuyad, koyat & 1 & $x$ & ALL-4 \\
\hline 2 & PUB & koyat & 1 & $\mathrm{v}$ & ALL-4 \\
\hline 3 & PUD & kuyat & 1,4 & $\mathrm{v}$ & $\mathrm{S}-4, \mathrm{Y}-4, \mathrm{O}-4, \mathrm{P}-4$ \\
\hline 4 & PUM & kuyat & 1,8 & $\mathrm{v}$ & $\mathrm{S}-4, \mathrm{Y}-4, \mathrm{O}-4, \mathrm{P}-4$ \\
\hline 5 & ALS & kara' & $1,4,8$ & $\mathrm{v}$ & ALL-4 \\
\hline 6 & LDY & kuyad, kuyat & $1,2,8$ & v & ALL-4 \\
\hline 7 & LIT & kuyad & 1,4 & v & ALL-4 \\
\hline 8 & BRU & suwat & 1,8 & $\mathrm{v}$ & ALL-4 \\
\hline 9 & MRP & kuyang, koyang, kuya' & 1 & $x$ & $\mathrm{Y}-4, \mathrm{O}-4$ \\
\hline 10 & PTN & kuyan & 1 & $x$ & $\mathrm{G}-4, \mathrm{~S}-4, \mathrm{Y}-4, \mathrm{O}-4$ \\
\hline 11 & KLK & kuyat & 1 & $x$ & $\mathrm{~S}-4, \mathrm{Y}-4, \mathrm{O}-4, \mathrm{P}-4$ \\
\hline 12 & KLM & koyat & $1,4,8$ & v & $\mathrm{S}-4, \mathrm{Y}-4, \mathrm{O}-4, \mathrm{P}-4, \mathrm{M}-4$ \\
\hline 13 & KLO & kodei & 1 & v & $\mathrm{S}-4, \mathrm{Y}-4, \mathrm{O}-4, \mathrm{P}-4, \mathrm{M}-4$ \\
\hline 14 & KLA & kuzat & 1,2 & v & $\mathrm{S}-4, \mathrm{Y}-4, \mathrm{O}-4, \mathrm{P}-4, \mathrm{M}-4$ \\
\hline 15 & KAL & kozet, kozit & 1,2 & v & $\mathrm{S}-4, \mathrm{Y}-4, \mathrm{O}-4, \mathrm{P}-4, \mathrm{M}-4$ \\
\hline 16 & KBD & koyat & 1,9 & v & S-4, Y-4, O-4, P-4, M-4 \\
\hline 17 & KB K & kuyat & 1,9 & v & $\mathrm{S}-2, \mathrm{Y}-3, \mathrm{O}-3, \mathrm{P}-3$ \\
\hline 18 & PEB & kuyat & $1,4,8$ & v & $\mathrm{S}-4, \mathrm{Y}-4, \mathrm{O}-4, \mathrm{P}-4, \mathrm{M}-4$ \\
\hline
\end{tabular}

Notes : 


\section{Macaca nemestrina (Cercopithecidae) \\ Pig Tail MaCaQUe. MONYET Beruk}

\section{DESCRIPTION}

Medium sized omnivorous primate, diurnal, often terrestrial, sleeps in trees along rivers; shorttailed, with cheek pouches and simple stomach; olive brown body, white underparts, dark brown on top of head. Old males may be reddish

\section{HABITAT}

Lowland to hill forests; secondary forests, plantations and village gardens

\section{ORIGINS/STATUS}

South-Southeast Asia/CITES: Appendix II

\section{USES}

Eaten (ALS); meat sold (KLM, PEB); juveniles sold as pets; skin for hat (PUD, LIT, KLK, KBD, $\mathrm{PEB})$; teeth for decoration (ALS); women cannot eat (PUT)

\section{REFERENCES}

Payne et al. 1985: 229; Corbet and Hill 1992: 166

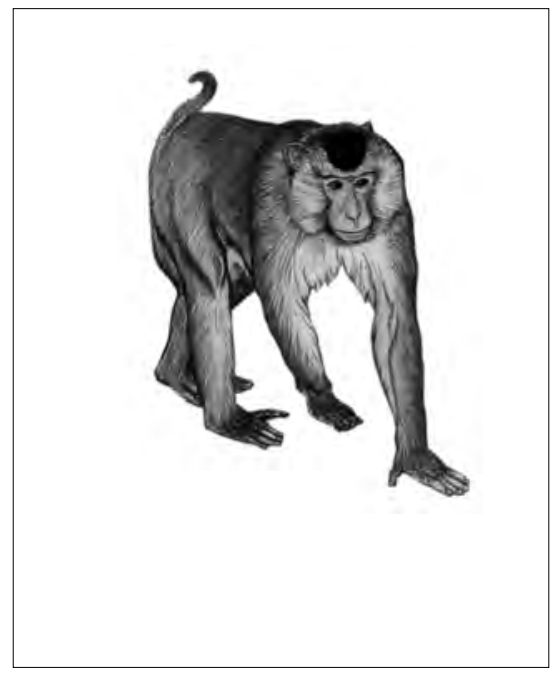

\begin{tabular}{|c|c|l|c|c|c|}
\hline \# & Code & Local Name & Use & RU & Hab.-Ab. \\
\hline 1 & PUT & beruk & 1,8 & $\mathbf{X}$ & ALL-1 \\
\hline 2 & PUB & beruk & 1 & $\mathbf{v}$ & ALL-1 \\
\hline 3 & PUD & beruk & 1,4 & $\mathbf{v}$ & O-4, P-4 \\
\hline 4 & PUM & beruk & 1,8 & $\mathbf{v}$ & S-4, Y-4, O-4, P-4 \\
\hline 5 & ALS & gabok & $1,4,6,8$ & $\mathbf{x}$ & ALL-4 \\
\hline 6 & LDY & becuk, becok & 1,8 & $\mathbf{v}$ & ALL-4 \\
\hline 7 & LIT & besok & 1,4 & $\mathbf{v}$ & ALL-4 \\
\hline 8 & BRU & ce' & 1,8 & $\mathbf{x}$ & ALL-4 \\
\hline 9 & MRP & dau', dao we & 1 & $\mathbf{x}$ & Y-4, O-4, P-4 \\
\hline 10 & PTN & beruk utun & 1,8 & $\mathbf{x}$ & O-4, P-4 \\
\hline 11 & KLK & dok & 1,4 & $\mathbf{v}$ & S-4, Y-4, O-4, P-4 \\
\hline 12 & KLM & dok & $1,4,8$ & $\mathbf{v}$ & S-4, Y-4, O-4, P-4, M-4 \\
\hline 13 & KLO & do' & 1 & $\mathbf{v}$ & S-4, Y-4, O-4, P-4, M-4 \\
\hline 14 & KLA & dok & 1 & $\mathbf{v}$ & S-4, Y-4, O-4, P-4, M-4 \\
\hline 15 & KAL & dok & 1 & $\mathbf{x}$ & S-4, Y-4, O-4, P-4, M-4 \\
\hline 16 & KBD & dok & 1,8 & $\mathbf{x}$ & S-4, Y-4, O-4, P-4, M-4 \\
\hline 17 & KBK & dok & 1 & $\mathbf{x}$ & G-1, S-3, Y-3, O-3, P-3 \\
\hline 18 & PEB & modok & $1,4,8$ & $\mathbf{v}$ & G-1, S-2, Y-2, O-2, P-4 \\
\hline
\end{tabular}

Notes : 


\section{Hylobates muelleri (Hylobatidae)}

\section{BORNEAN GIBBON. OWA KALAWAT}

\section{DESCRIPTION}

Large, mostly frugivorous primate, tail less lesser ape, diurnal and arboreal, moves by brachiation; territorial with couples uttering loud calls in early morning; uniformly brown or greyish brown, grey hair around face, feet and hands tend to be paler

\section{HABITAT}

Lowland to hill forests to $1500 \mathrm{~m}$

\section{ORIGINS/STATUS}

Borneo endemic/CITES: Appendix I;

RI: Protected

\section{USES}

Eaten; juveniles sold as pets; skin for hat; teeth for decoration (LIT)

\section{REFERENCES}

Payne et al. 1985: 229; Corbet and Hill 1992: 181; Suyanto et al. 1998

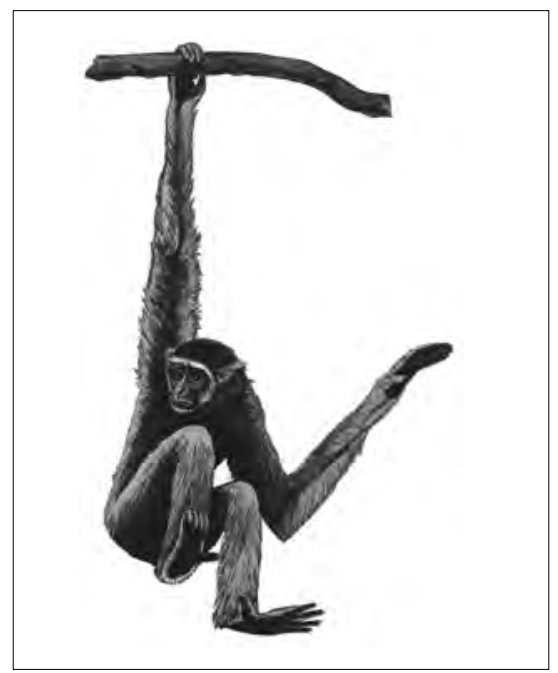

\begin{tabular}{|c|c|c|c|c|c|}
\hline \# & Code & Local Name & Use & RU & Hab.-Ab. \\
\hline 1 & PUT & kelavet & $1,4,8$ & $x$ & $\mathrm{O}-1, \mathrm{P}-3, \mathrm{M}-3$ \\
\hline 2 & PUB & klavet, kelavet & $1,4,8$ & v & $\mathrm{P}-4$ \\
\hline 3 & PUD & kelavet & $1,4,8$ & $x$ & P-4 \\
\hline 4 & PUM & kelavet & $1,4,8$ & v & $\mathrm{P}-4, \mathrm{M}-4$ \\
\hline 5 & ALS & kelowat & $1,4,8$ & $\mathrm{v}$ & $\mathrm{P}-4, \mathrm{M}-4$ \\
\hline 6 & LDY & kelabet & $1,4,8$ & $\mathrm{v}$ & $\mathrm{O}-1, \mathrm{P}-3, \mathrm{M}-4$ \\
\hline 7 & LIT & kelabet & $1,4,6,8$ & $\mathrm{v}$ & P-4, M-4 \\
\hline 8 & BRU & kelebet & $1,4,8$ & $x$ & P-1, M-1 \\
\hline 9 & MRP & habat & $1,4,8$ & $x$ & P-4 \\
\hline 10 & PTN & kelabbet & $1,4,8$ & $x$ & P-2 \\
\hline 11 & KLK & kelabet & $1,4,8$ & $x$ & P-4 \\
\hline 12 & KLM & kelabet, kerabet & $1,4,8$ & $\mathrm{v}$ & P-4 \\
\hline 13 & KLO & kelabit & $1,4,8$ & $\mathrm{v}$ & P-4 \\
\hline 14 & KLA & kelabet, kelabit & $1,4,8$ & $\mathrm{v}$ & P-4 \\
\hline 15 & KAL & kelabet & $1,4,8$ & $x$ & P-4 \\
\hline 16 & KBD & kelabet & 1,8 & $x$ & $\mathrm{P}-4, \mathrm{M}-3$ \\
\hline 17 & KBK & kelabet & 1,8 & $\mathrm{v}$ & $\mathrm{P}-4, \mathrm{M}-3$ \\
\hline 18 & PEB & kelavet & $1,4,8$ & $\mathrm{v}$ & P-3, M-3 \\
\hline
\end{tabular}

Notes : 


\section{Pongo pygmaeus (Pongidae)

\section{DESCRIPTION}

Large herbivorous and insectivorous primate, tail less great ape to $1.4 \mathrm{~m}$ in height; diurnal, partly terrestrial, builds sleeping nest in trees; reddish brown long haired, with large facial skin flaps in adult males

\section{HABITAT}

Lowland, coastal forests; sometimes in hill forests, plantations and village gardens; presently rare or locally absent in Bulungan

\section{ORIGINS/STATUS}

Borneo and Sumatra/CITES: Appendix I; RI:

Protected

\section{USES}

Traditionally avoided or killed out of fear

\section{REFERENCES}

Payne et al. 1985: 230; Corbet and Hill 1992: 185
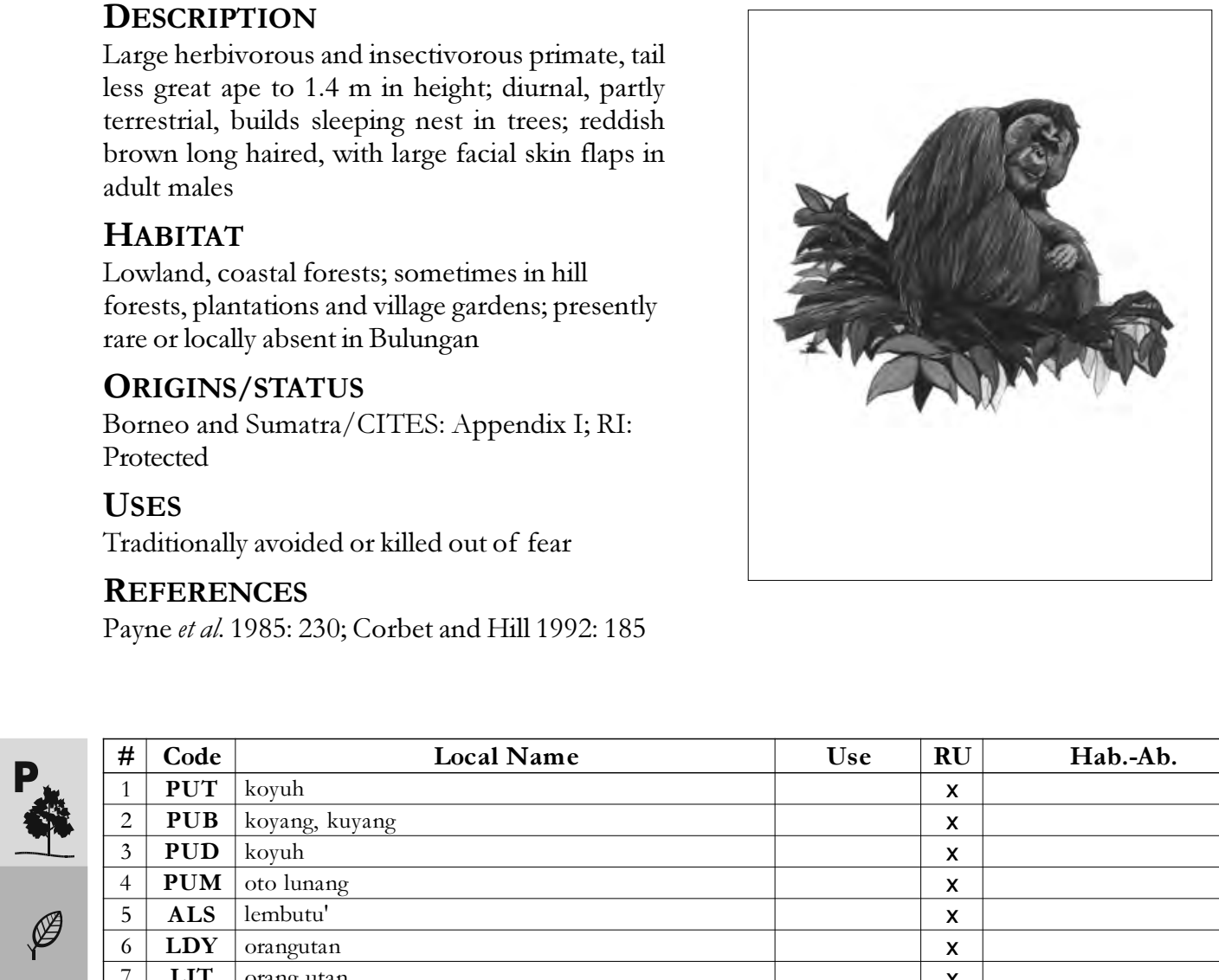

\begin{tabular}{|c|c|l|c|c|c|}
\hline$\#$ & Code & Local Name & Use & RU & Hab.-Ab. \\
\hline 1 & PUT & koyuh & & $\mathbf{X}$ & \\
\hline 2 & PUB & koyang, kuyang & & $\mathbf{X}$ & \\
\hline 3 & PUD & koyuh & & $\mathbf{X}$ & \\
\hline 4 & PUM & oto lunang & $\mathbf{X}$ & \\
\hline 5 & ALS & lembutu' & $\mathbf{X}$ & \\
\hline 6 & LDY & orangutan & $\mathbf{X}$ & \\
\hline 7 & LIT & orang utan & & $\mathbf{X}$ & \\
\hline 8 & BRU & diau & & $\mathbf{X}$ & \\
\hline 9 & MRP & kuyaie, kuae, kuyehe & $\mathbf{X}$ & \\
\hline 10 & PTN & kuyung & & $\mathbf{X}$ & \\
\hline 11 & KLK & kuyang & & $\mathbf{X}$ & \\
\hline 12 & KLM & koyang & & $\mathbf{X}$ & \\
\hline 13 & KLO & kojang & & $\mathbf{X}$ & \\
\hline 14 & KLA & kuzang, kuyang & & $\mathbf{X}$ & \\
\hline 15 & KAL & kozang & & $\mathbf{X}$ & \\
\hline 16 & KBD & oyang & & $\mathbf{X}$ & \\
\hline 17 & KBK & koyang & & $\mathbf{X}$ & \\
\hline 18 & PEB & kuyang & & $\mathbf{X}$ & \\
\hline
\end{tabular}

Notes : 


\section{Manisjavanica (Manidae)}

\section{MALAYAN PANGOLIN.TRENGGLING, TENGGILING}

\section{DESCRIPTION}

Medium sized insectivorous mammal; nocturnal, terrestrial, sleeps in burrows; brownish orange, sometimes whitish, large epidermal scales of agglutinated hairs; no teeth, a strong prehensile tail and long claws on forefeet; two varieties reported

\section{HABITAT}

Lowland to hill forests to $1700 \mathrm{~m}$; secondary forests, plantations and village gardens

\section{ORIGINS/STATUS}

India to Southeast Asia/CITES: Appendix II; RI: Protected

\section{USES}

Eaten, meat for medicine (KAL); scales for trophy or medicine (PUM, ALS, KBD) or sold (LDY, BRU, KLK); scales keep pests from swidden (KBD); sold as pet (ALS)

\section{REFERENCES}

Payne et al. 1985: 232; Corbet and Hill 1992: 19

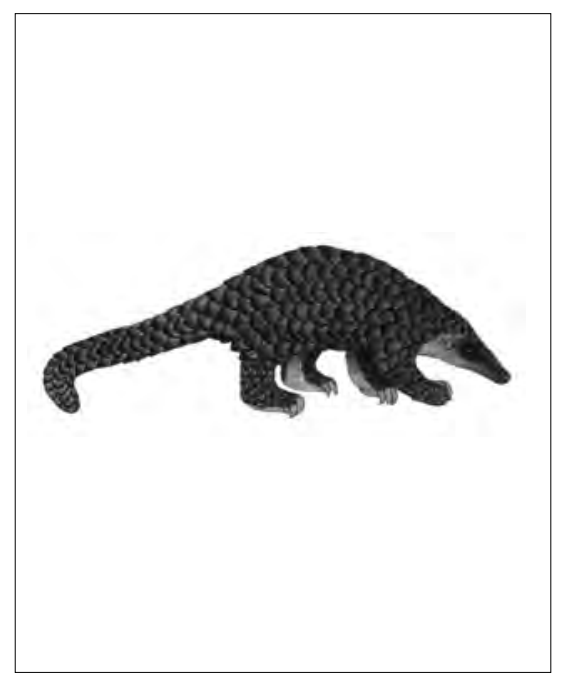

\begin{tabular}{|r|l|l|c|c|c|}
\hline \# & Code & Local Name & Use & RU & Hab.-Ab. \\
\hline 1 & PUT & am & $1,3,6$ & X & Y-1, O-1, P-1 \\
\hline 2 & PUB & am & 1,6 & v & O-1, P-1 \\
\hline 3 & PUD & am & 1,6 & v & Y-2, O-2, P-2 \\
\hline 4 & PUM & am & $1,2,6$ & v & Y-4, O-4, P-4 \\
\hline 5 & ALS & belekun & $1,2,6,8,9$ & v & G-2, S-2, Y-4, O-4, P-4 \\
\hline 6 & LDY & arem & $1,6,8$ & v & Y-2, O-2, P-3 \\
\hline 7 & LIT & arem & 1,6 & X & Y-2, O-2, P-2 \\
\hline 8 & BRU & agam ngget & $1,6,8$ & v & Y-3, O-3, P-3 \\
\hline 9 & MRP & ngang, hangang, nga' & 1,6 & X & Y-4, O-4 \\
\hline 10 & PTN & am & 1,6 & v & Y-4, O-4, P-4 \\
\hline 11 & KLK & am & $1,6,8$ & X & O-4, P-4 \\
\hline 12 & KLM & am & $1,2,8$ & $\mathbf{v}$ & Y-4, P-1 \\
\hline 13 & KLO & am & $1,2,6$ & $\mathbf{v}$ & Y-4, P-1 \\
\hline 14 & KLA & am & 1 & $\mathbf{v}$ & G-1, O-1, P-1 \\
\hline 15 & KAL & am, aam & $1,2,6$ & $\mathbf{v}$ & O-1, P-1 \\
\hline 16 & KBD & am & $1,2,5$ & $\mathbf{v}$ & Y-4, P-1 \\
\hline 17 & KBK & am & 1,2 & $\mathbf{v}$ & G-2, S-2, Y-3, O-3, P-3 \\
\hline 18 & PEB & am & 1 & $\mathbf{v}$ & G-1, S-1, Y-2, O-2, P-3 \\
\hline
\end{tabular}

Notes : 


\section{Ratufa affinis (Sciuridae)

\section{DESCRIPTION}

Large squirrel, diurnal and arboreal, upper parts dark brown in midline, sometimes reddish in tinge, sides speckled buff, underparts almost white, thighs pale, grizzled or not; distinctive call, harsh chatter; several varieties reported in Bulungan

\section{HABITAT}

Lowland primary to lower montane forest; older secondary forests

\section{ORIGINS/STATUS}

Malaysia, Borneo, Sumatra/CITES: Appendix II

\section{USES}

Eaten; meat for medicine (KBD, KBK); skin for hat or trophy

\section{REFERENCES}

Payne et al. 1985: 233
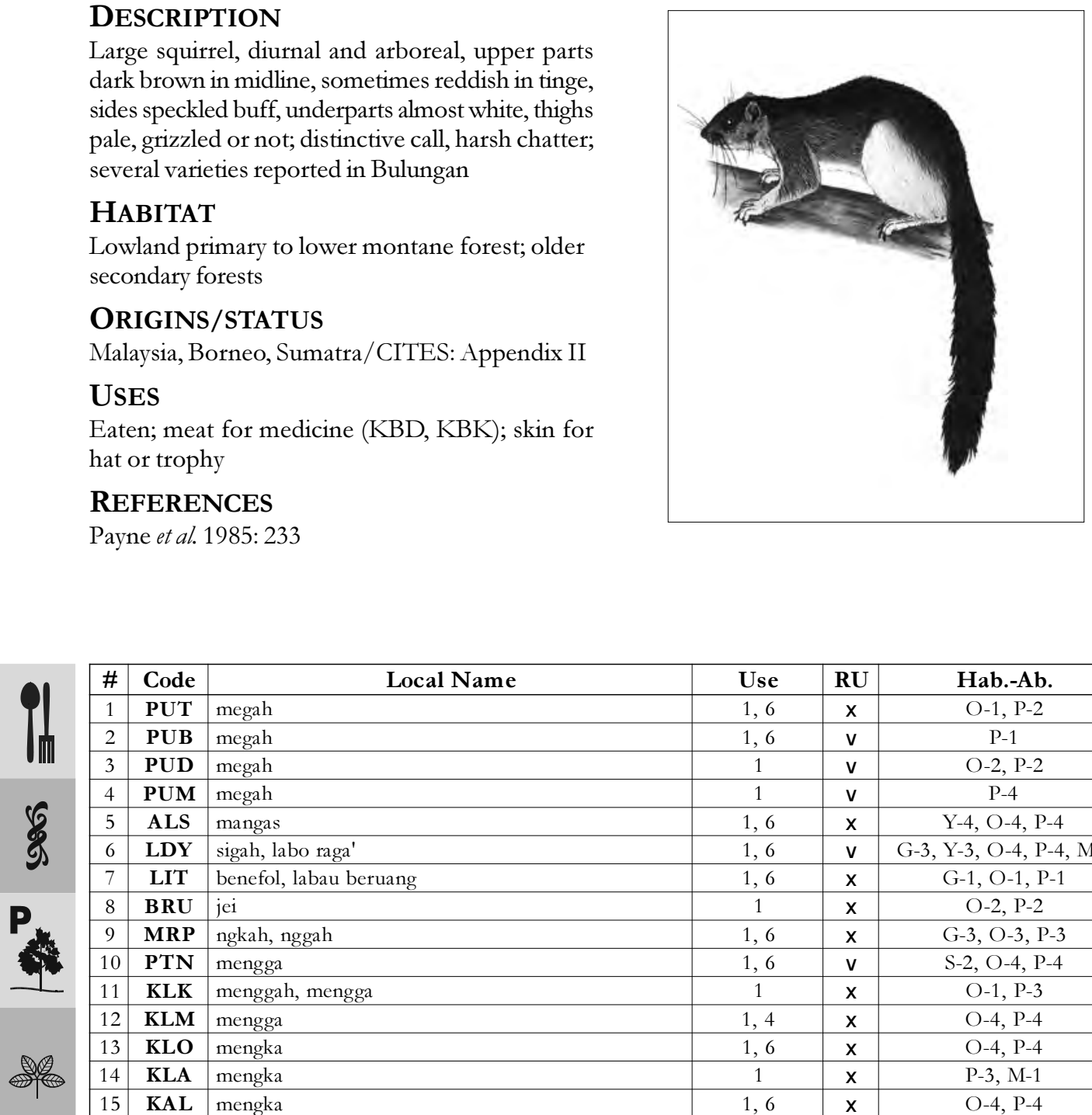

\begin{tabular}{|c|c|c|c|c|c|}
\hline \# & Code & Local Name & Use & RU & Hab.-Ab. \\
\hline 1 & PUT & megah & 1,6 & $x$ & $\mathrm{O}-1, \mathrm{P}-2$ \\
\hline 2 & PUB & megah & 1,6 & v & P-1 \\
\hline 3 & PUD & megah & 1 & v & $\mathrm{O}-2, \mathrm{P}-2$ \\
\hline 4 & PUM & megah & 1 & v & P-4 \\
\hline 5 & ALS & mangas & 1,6 & $x$ & $\mathrm{Y}-4, \mathrm{O}-4, \mathrm{P}-4$ \\
\hline 6 & LDY & sigah, labo raga' & 1,6 & v & $\mathrm{G}-3, \mathrm{Y}-3, \mathrm{O}-4, \mathrm{P}-4, \mathrm{M}-3$ \\
\hline 7 & LIT & benefol, labau beruang & 1,6 & $x$ & $\mathrm{G}-1, \mathrm{O}-1, \mathrm{P}-1$ \\
\hline 8 & BRU & jei & 1 & $x$ & $\mathrm{O}-2, \mathrm{P}-2$ \\
\hline 9 & MRP & ngkah, nggah & 1,6 & $x$ & $\mathrm{G}-3, \mathrm{O}-3, \mathrm{P}-3$ \\
\hline 10 & PTN & mengga & 1,6 & v & $\mathrm{S}-2, \mathrm{O}-4, \mathrm{P}-4$ \\
\hline 11 & KLK & menggah, mengga & 1 & $x$ & $\mathrm{O}-1, \mathrm{P}-3$ \\
\hline 12 & KLM & mengga & 1,4 & $x$ & $\mathrm{O}-4, \mathrm{P}-4$ \\
\hline 13 & KLO & mengka & 1,6 & $x$ & $\mathrm{O}-4, \mathrm{P}-4$ \\
\hline 14 & KLA & mengka & 1 & $x$ & P-3, M-1 \\
\hline 15 & KAL & mengka & 1,6 & $x$ & $\mathrm{O}-4, \mathrm{P}-4$ \\
\hline 16 & KBD & mengga & 1,2 & $x$ & $\mathrm{O}-2, \mathrm{P}-4, \mathrm{M}-1$ \\
\hline 17 & KBK & mengga & 1,2 & $x$ & $\mathrm{O}-4, \mathrm{P}-4, \mathrm{M}-2$ \\
\hline 18 & PEB & mege & 1 & v & $\mathrm{P}-4, \mathrm{M}-2$ \\
\hline
\end{tabular}

Notes : 


\section{Rattus tiomanicus (Muridae)}

\section{MALAYSIAN FIELD RAT. TIKUS BELUKAR}

\section{DESCRIPTION}

Medium sized rat, nocturnal, terrestrial but good climber; upperparts finely grizzled, olive-brown, slightly darker in midline; black moderate length guard hairs; underparts pure white or dull white, tail entirely dark brownish; ridges on underside of foot pads

\section{HABITAT}

Secondary and coastal forests; plantations, village gardens, scrub and grasslands

\section{ORIGINS/STATUS}

Thailand, Malaysia, Indonesia/Unprotected

\section{USES}

Rarely eaten; rice and palm oil pest

\section{REFERENCES}

Payne et al. 1985: 252; Suyanto et al. 1998

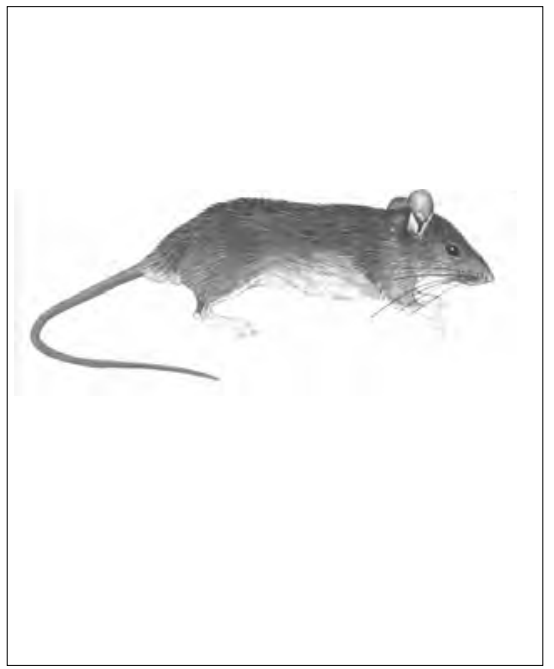

\begin{tabular}{|c|c|c|c|c|c|}
\hline \# & Code & Local Name & Use & RU & Hab.-Ab. \\
\hline 1 & PUT & labau, labau teian, labau te'an & 1 & $x$ & G-4, S-4 \\
\hline 2 & PUB & labau, labau te'an & 1 & v & G-4, S-4 \\
\hline 3 & PUD & labau te'an & 1 & v & S-4 \\
\hline 4 & PUM & labau tei'an & 1 & v & S-5 \\
\hline 5 & ALS & tikus tana' & 1 & v & G-5 \\
\hline 6 & LDY & labo tana' & 1 & $\mathrm{v}$ & $\mathrm{S}-4, \mathrm{Y}-3, \mathrm{P}-3, \mathrm{M}-4$ \\
\hline 7 & LIT & labau tana' & 1 & v & $\mathrm{S}-5$ \\
\hline 8 & BRU & abio tana' & 1 & v & G-4 \\
\hline 9 & MRP & labau kwa', labau moe, labau moa' & 1 & v & $\mathrm{G}-4, \mathrm{~S}-4$ \\
\hline 10 & PTN & labao ba'e & 1 & $\mathrm{v}$ & S-4 \\
\hline 11 & KLK & belabau jami, belabo jami & 1 & $x$ & S-4 \\
\hline 12 & KLM & belabau jami & 1 & v & G-4, S-4 \\
\hline 13 & KLO & belabo jami & 1 & $\mathrm{v}$ & G-4, S-4 \\
\hline 14 & KLA & belabo jami, belabo & 1 & V & G-4, S-4, Y-2 \\
\hline 15 & KAL & belabo to'e, belabo jami & 1 & V & G-3, S-4 \\
\hline 16 & KBD & belabo jami & & $x$ & G-3, S-3 \\
\hline 17 & KBK & belabau jami & & $x$ & G-4, S-4 \\
\hline 18 & PEB & belavau tana' & & $x$ & $G-4, S-4$ \\
\hline
\end{tabular}

Notes : 


\section{Rattus rattus (Muridae)}

\section{HOUSE RAT. TIKUS RUMAH}

\section{DESCRIPTION}

Medium sized rat, nocturnal and arboreal, confined to human settlements and nearby vegetation; upperparts finely grizzled, olive brown, with long black guard hairs on rump, underparts olive brown or greyish brown, tail entirely dark brown

\section{HABITAT}

Human settlements, plantations and village gardens

\section{ORIGINS/STATUS}

Global/Unprotected

\section{USES}

Rarely eaten; rice pest

\section{REFERENCES}

Payne et al. 1985: 252
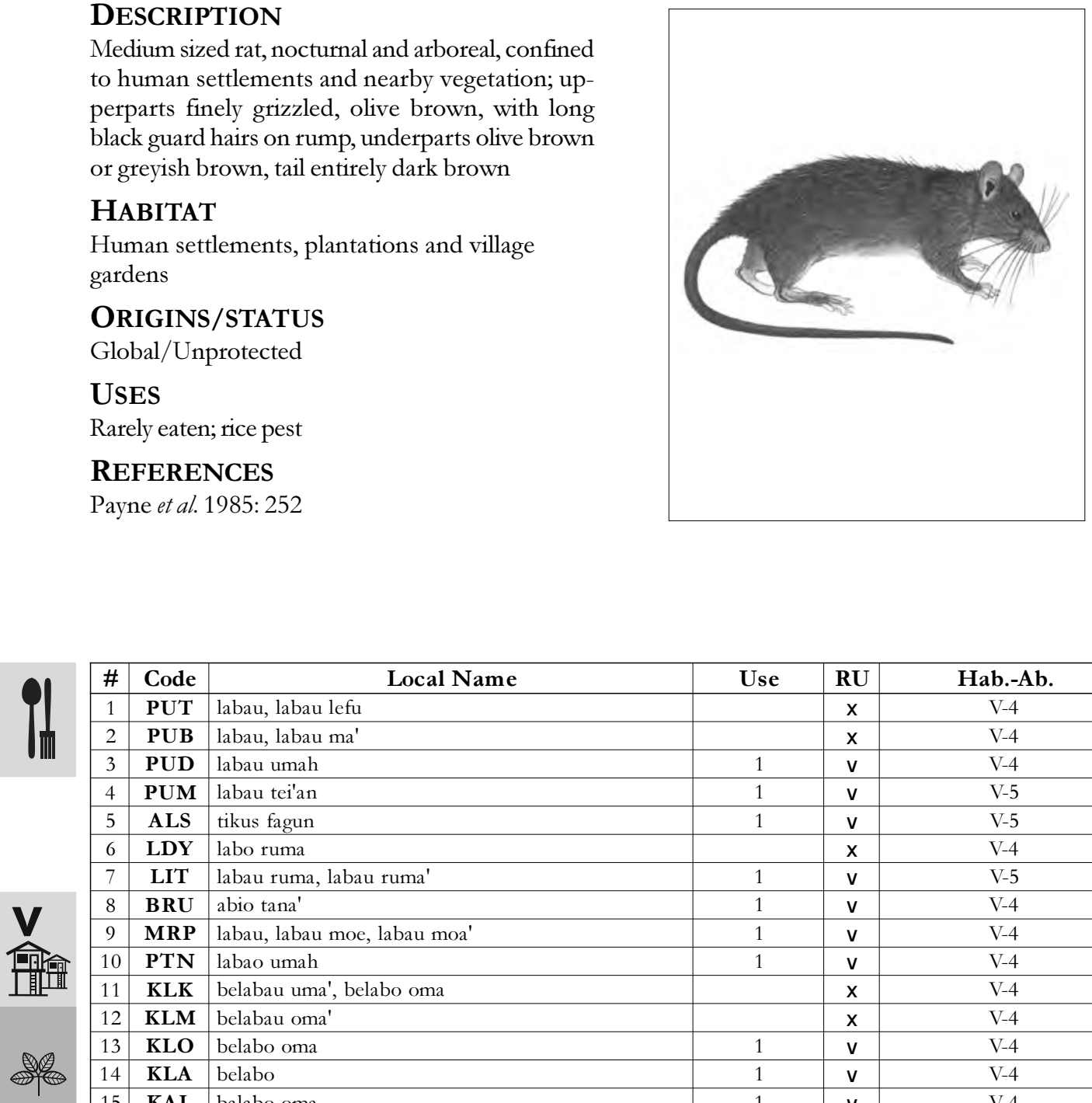

\begin{tabular}{|c|c|l|c|c|c|}
\hline$\#$ & Code & \multicolumn{1}{|c|}{ Local Name } & Use & RU & Hab.-Ab. \\
\hline 1 & PUT & labau, labau lefu & & $\mathbf{X}$ & V-4 \\
\hline 2 & PUB & labau, labau ma' & & $\mathbf{X}$ & V-4 \\
\hline 3 & PUD & labau umah & 1 & $\mathbf{V}$ & V-4 \\
\hline 4 & PUM & labau tei'an & 1 & $\mathbf{V}$ & V-5 \\
\hline 5 & ALS & tikus fagun & & $\mathbf{V}$ & V-5 \\
\hline 6 & LDY & labo ruma & 1 & $\mathbf{V}$ & V-4 \\
\hline 7 & LIT & labau ruma, labau ruma' & 1 & $\mathbf{V}$ & V-5 \\
\hline 8 & BRU & abio tana' & 1 & $\mathbf{V}$ & V-4 \\
\hline 9 & MRP & labau, labau moe, labau moa' & 1 & $\mathbf{V}$ & V-4 \\
\hline 10 & PTN & labao umah & & $\mathbf{X}$ & V-4 \\
\hline 11 & KLK & belabau uma', belabo oma & & $\mathbf{X}$ & V-4 \\
\hline 12 & KLM & belabau oma' & 1 & $\mathbf{V}$ & V-4 \\
\hline 13 & KLO & belabo oma & 1 & $\mathbf{V}$ & V-4 \\
\hline 14 & KLA & belabo & 1 & $\mathbf{V}$ & V-4 \\
\hline 15 & KAL & balabo oma & & $\mathbf{X}$ & V-4 \\
\hline 16 & KBD & belabo oma & & $\mathbf{X}$ & V-4 \\
\hline 17 & KBK & belabau uma' & & $\mathbf{X}$ & V-4 \\
\hline 18 & PEB & belavau padeng & & & \\
\hline
\end{tabular}

Notes : 


\section{Tricbys fasciculata (Hystricidae) \\ LONG-TAILED PORCUPINE. LANDAKEKOR PANJANG}

\section{DESCRIPTION}

Small porcupine, nocturnal and terrestrial; upperparts brown, spines short and flattened, dark brown at tips, whitish at base; tail scaly, brown with tuft of hollow bristles at end

\section{HABITAT}

Lowland to hill primary forests; cultivated areas; rare

\section{ORIGINS/STATUS}

Malaysia, Borneo, Sumatra/RI: Protected

\section{USES}

Eaten; gallstone sold locally or to traders (ALS, LDY, LIT, MRP, PTN, KLK, KLM, KAL, KLM, $\mathrm{KBD}, \mathrm{PEB}$ )

\section{REFERENCES}

Payne et al. 1985: 261

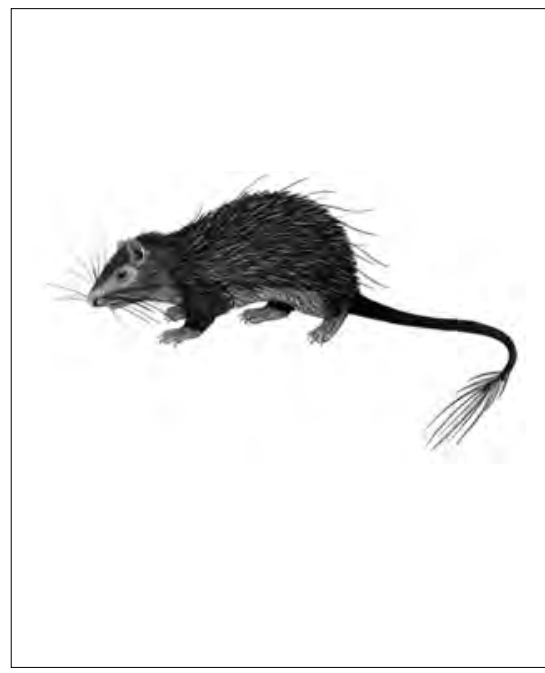

\begin{tabular}{|c|c|c|c|c|c|}
\hline \# & Code & Local Name & Use & RU & Hab.-Ab. \\
\hline 1 & PUT & tai'an, teian, te'an & 1 & $x$ & P-2, M-1 \\
\hline 2 & PUB & te'an & 1 & v & P-1 \\
\hline 3 & PUD & te'an & 1 & $x$ & ALL-4 \\
\hline 4 & PUM & tei'an & 1,8 & v & P-4 \\
\hline 5 & ALS & litis & 1,8 & v & P-4 \\
\hline 6 & LDY & afan, apan & 1,8 & $x$ & ALL-4 \\
\hline 7 & LIT & apan & 1,8 & v & $\mathrm{O}-2, \mathrm{P}-4$ \\
\hline 8 & BRU & apan & 1 & v & $\mathrm{Y}-4, \mathrm{O}-4, \mathrm{P}-4$ \\
\hline 9 & MRP & kwa', kuwa', kwae' & 1,8 & $x$ & $\mathrm{G}-4, \mathrm{~S}-4, \mathrm{Y}-4, \mathrm{O}-3, \mathrm{P}-3$ \\
\hline 10 & PTN & bekia & 1,8 & $x$ & $\mathrm{Y}-3, \mathrm{O}-3, \mathrm{P}-3$ \\
\hline 11 & KLK & bekia & 1,8 & $x$ & $\mathrm{O}-1, \mathrm{P}-2$ \\
\hline 12 & KLM & bekia & 1,8 & $x$ & $\mathrm{O}-1, \mathrm{P}-1$ \\
\hline 13 & KLO & begheza & 1,8 & $x$ & $\mathrm{Y}-1, \mathrm{O}-1, \mathrm{P}-1$ \\
\hline 14 & KLA & behiza, behija & 1,8 & $x$ & $\mathrm{O}-1, \mathrm{P}-1$ \\
\hline 15 & KAL & behezu, beheza & 1,8 & $x$ & $\mathrm{O}-1, \mathrm{P}-1$ \\
\hline 16 & KBD & bekia & 1,8 & $x$ & $\mathrm{O}-1, \mathrm{P}-1$ \\
\hline 17 & KBK & bekia & 1,8 & $x$ & $\mathrm{G}-2, \mathrm{~S}-2, \mathrm{Y}-3, \mathrm{O}-3, \mathrm{P}-4$ \\
\hline 18 & PEB & borek & 1,8 & v & $\mathrm{O}-1, \mathrm{P}-1$ \\
\hline
\end{tabular}

Notes : 


\section{Hystrix bracbyura (Hystricidae)

\section{DESCRIPTION}

Large common porcupine; nocturnal and terrestrial; black body, long spines white with black band toward tips; shorter mostly blackish quills on front parts; short hollow quills on tail

\section{HABITAT}

Lowland to hill primary forests; cultivated areas

\section{ORIGINS/STATUS}

India, China, Southeast Asia/RI: Protected

\section{USES}

Eaten; blood, liver and quills for medicine; gallstone sold locally or to traders (ALS, LDY, LIT, BRU, MRP, PTN, KLK, KAL, KBD, PEB); quills for needles (PUD); skin for clothing (PUT), and trophies (PUM, ALS)

\section{REFERENCES}

Payne et al. 1985: 261; Suyanto et al. 1998

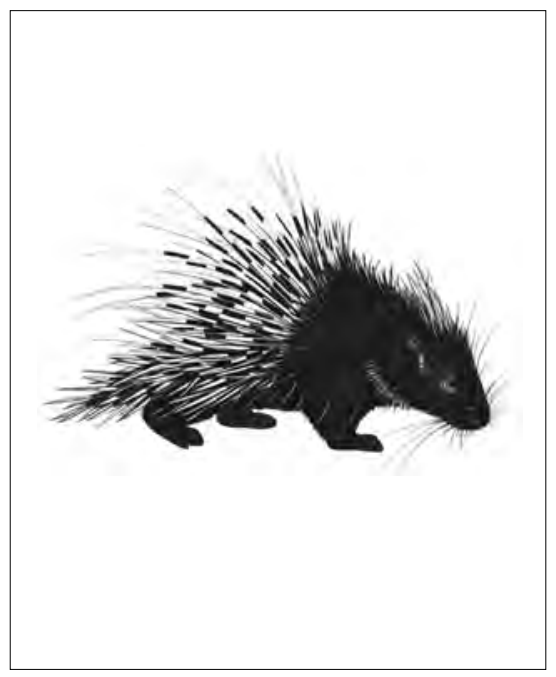

\begin{tabular}{|c|c|l|c|c|c|}
\hline$\#$ & Code & \multicolumn{1}{|c|}{ Local Name } & Use & RU & Hab.-Ab. \\
\hline 1 & PUT & totong, totung kelien & $1,4,8$ & $\mathbf{X}$ & G-1, S-1, Y-3, O-3 \\
\hline 2 & PUB & totung & 1 & $\mathbf{v}$ & G-1, P-3 \\
\hline 3 & PUD & totung & $1,3,8$ & $\mathbf{v}$ & ALL-4 \\
\hline 4 & PUM & totung & $1,2,6,8$ & $\mathbf{v}$ & G-4, Y-4, O-4, P-4 \\
\hline 5 & ALS & butun & $1,2,6,8$ & $\mathbf{v}$ & G-4, Y-4, O-4, P-4 \\
\hline 6 & LDY & terutung & 1,8 & $\mathbf{v}$ & ALL-4 \\
\hline 7 & LIT & teretong, teretung & 1,8 & $\mathbf{v}$ & O-2, P-4 \\
\hline 8 & BRU & teltong & 1,8 & $\mathbf{v}$ & Y-4, O-4, P-4 \\
\hline 9 & MRP & taue, ketawe, tawe & $1,2,8$ & $\mathbf{X}$ & G-3, S-3, Y-4, P-4 \\
\hline 10 & PTN & kettong & $1,2,8$ & $\mathbf{X}$ & S-2, Y-2, O-2, P-2 \\
\hline 11 & KLK & settung lumeng, settung umung & $1,2,8$ & $\mathbf{X}$ & G-4, S-4 \\
\hline 12 & KLM & settung & 1,8 & $\mathbf{v}$ & G-4, S-4, Y-4, O-4 \\
\hline 13 & KLO & tettung & $1,2,8$ & $\mathbf{v}$ & G-4, S-4, Y-4, O-4 \\
\hline 14 & KLA & tettong & $1,2,8$ & $\mathbf{v}$ & G-4, S-4, Y-4, O-4 \\
\hline 15 & KAL & tettuong, tettong & $1,2,8$ & $\mathbf{v}$ & G-4, S-4, Y-4, O-4 \\
\hline 16 & KBD & settung & $1,2,8$ & $\mathbf{v}$ & G-4, S-4, Y-4, O-4 \\
\hline 17 & KBK & setung & $1,2,8$ & $\mathbf{x}$ & G-2, S-2, Y-3, O-3, P-4 \\
\hline 18 & PEB & larak & 1,8 & $\mathbf{v}$ & G-1, S-2, Y-2, O-3, P-3 \\
\hline
\end{tabular}

Notes : 


\section{Canisfamiliaris (Canidae) \\ DOG. ANJINGLOKAL.}

\section{DESCRIPTION}

Small domesticated hunting dog associated with humans and villages; often brownish orange red, but can be black, buff whitish or pied. Often suffering epidemics; rarely feral

\section{HABITAT}

Human settlements

\section{ORIGINS/STATUS}

Global/Unprotected

\section{USES}

Pet; hunting companion; rarely eaten; juveniles sold or traded locally

\section{REFERENCES}

Payne et al. 1985: 275; Corbet and Hill 1992: 188

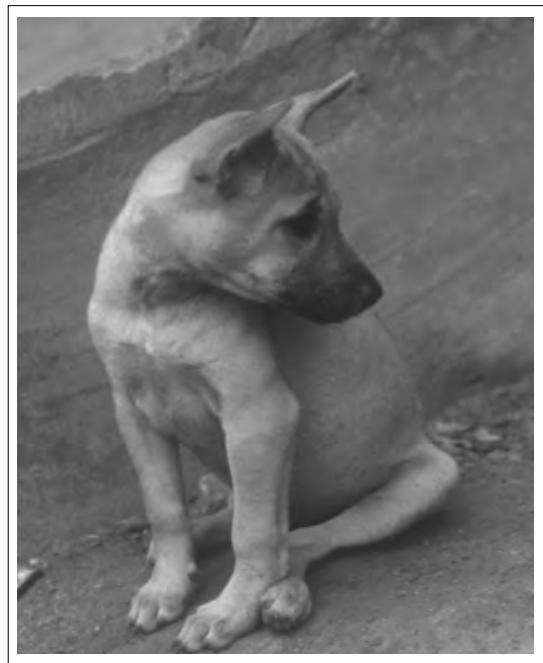

\begin{tabular}{|c|c|c|c|c|c|}
\hline \# & Code & Local Name & Use & RU & Hab.-Ab. \\
\hline 1 & PUT & aoh, auh & 9 & $\mathrm{v}$ & $\mathrm{V}$ \\
\hline 2 & PUB & auh & 9 & v & $\mathrm{V}$ \\
\hline 3 & PUD & auh & 9 & v & $\mathrm{V}$ \\
\hline 4 & PUM & auh & 9 & $\mathrm{v}$ & $\mathrm{V}$ \\
\hline 5 & ALS & asu & 9 & v & $\mathrm{V}$ \\
\hline 6 & LDY & huko', uko' & 9 & v & $\mathrm{V}$ \\
\hline 7 & LIT & \begin{tabular}{|l|l|} 
uko' \\
\end{tabular} & 9 & v & $\mathrm{V}$ \\
\hline 8 & BRU & ko' & 9 & $\mathrm{v}$ & $\mathrm{V}$ \\
\hline 9 & MRP & hau & 9 & v & $\mathrm{V}$ \\
\hline 10 & PTN & ho & 9 & $\mathrm{v}$ & $\mathrm{V}$ \\
\hline 11 & KLK & asu & 9 & v & $\mathrm{V}$ \\
\hline 12 & KLM & asu & $1,8,9$ & v & $\mathrm{V}$ \\
\hline 13 & KLO & asu & $1,8,9$ & v & $\mathrm{V}$ \\
\hline 14 & KLA & asu & $1,8,9$ & v & $\mathrm{V}$ \\
\hline 15 & KAL & asu & $1,8,9$ & v & $\mathrm{V}$ \\
\hline 16 & KBD & asu & $1,8,9$ & v & $\mathrm{V}$ \\
\hline 17 & KBK & asu & $1,8,9$ & $v$ & $\mathrm{~V}$ \\
\hline 18 & PEB & aso & 8,9 & $\mathrm{v}$ & $\mathrm{V}$ \\
\hline
\end{tabular}

Notes : 


\section{Ursus malayanus (Ursidae)}

\section{MALAYAN SUN BEAR. BERUANG MADU \\ -}

\section{DESCRIPTION}

Small but powerful, omnivorous bear; diurnal and nocturnal, arboreal and terrestrial; ill-tempered and unpredictable; body black, grey muzzle, white or yellow ' $\mathrm{V}$ ' shaped mark on upper chest. Reports of a reddish variety without chest marks

\section{HABITAT}

Lowland to hill primary and secondary forests; sometimes plantations and village gardens

\section{ORIGINS/STATUS}

Southeast Asia/CITES: Appendix I; RI: Protected

\section{USES}

Sometimes eaten; teeth and claws for jewellery, decoration, charms, sold locally and to traders; skin for costumes, trophy; gall bladder for medicine, sold locally and to traders; juveniles sold as pets to traders (PUT, ALS, BRU, KBD, PEB)

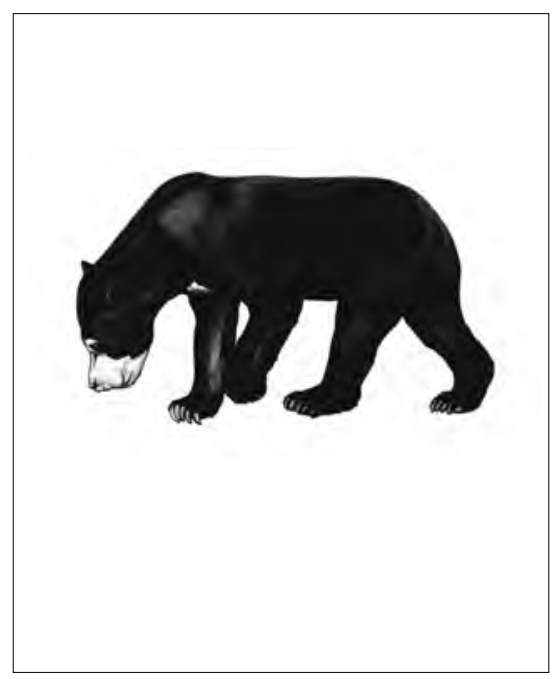

\section{REFERENCES}

Payne et al. 1985: 275; Corbet and Hill 1992: 192

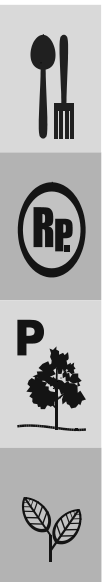

\begin{tabular}{|c|c|l|c|c|c|}
\hline$\#$ & Code & Local Name & Use & RU & Hab.-Ab. \\
\hline 1 & PUT & bowang & $1,2,5,6,8$ & $\mathbf{X}$ & Y-1, O-1, P-1 \\
\hline 2 & PUB & bowang & $1,2,4,6,8$ & $\mathbf{v}$ & O-1, P-1 \\
\hline 3 & PUD & bowang & $1,2,4,5,8$ & $\mathbf{X}$ & ALL-4 \\
\hline 4 & PUM & bowang & $1,2,3,4,6,8$ & $\mathbf{v}$ & Y-4, O-4, P-4 \\
\hline 5 & ALS & bawang & $1,2,4,6,8$ & $\mathbf{v}$ & G-4, Y-4, O-4, P-4 \\
\hline 6 & LDY & beruang & $1,2,4,5,6,8$ & $\mathbf{v}$ & G-4, Y-4, O-4, P-4, M-4 \\
\hline 7 & LIT & beruang & $1,2,4,8$ & $\mathbf{x}$ & O-1, P-1, P-4 \\
\hline 8 & BRU & belwiang & $1,2,4,5,6,8$ & $\mathbf{v}$ & ALL-4 \\
\hline 9 & MRP & buwe, buwie, buwaye & $1,2,5,6$ & $\mathbf{x}$ & ALL-4 \\
\hline 10 & PTN & buang & $1,2,4,5,6,8$ & $\mathbf{x}$ & ALL-1 \\
\hline 11 & KLK & buwang & $1,2,4,6,8$ & $\mathbf{x}$ & G-4, Y-4, O-4, P-4 \\
\hline 12 & KLM & buang & $1,2,4,5$ & $\mathbf{v}$ & G-2, S-2, P-2, M-2 \\
\hline 13 & KLO & bevang & $1,2,6,8$ & $\mathbf{v}$ & G-2, S-2, P-2, M-2 \\
\hline 14 & KLA & buvang, bevang & $1,2,6,8$ & $\mathbf{v}$ & G-2, S-2, P-2, M-2 \\
\hline 15 & KAL & bevang & $1,2,6,8$ & $\mathbf{x}$ & G-2, S-2, P-2, M-2 \\
\hline 16 & KBD & buang & $1,2,5,6,8$ & $\mathbf{x}$ & G-2, S-2, P-2, M-2 \\
\hline 17 & KBK & buwang & $1,2,5,6,8$ & $\mathbf{x}$ & O-1, P-4, M-1 \\
\hline 18 & PEB & buang & $1,2,4,8$ & $\mathbf{v}$ & G-2, O-2, P-3, M-2 \\
\hline
\end{tabular}

Notes : 


\section{Mustela nudipes (Mustelidae)}

\section{MALAYAN WEASEL. PULUSAN CHUK-PUTEH \\ ○}

\section{DESCRIPTION}

Small carnivorous mammal; diurnal and nocturnal, terrestrial, sleeps in ground holes; head white, body orange to golden brown except bushy tail which is paler near tip

\section{HABITAT}

Lowland to mountain primary and secondary forests, to $1700 \mathrm{~m}$

\section{ORIGINS/STATUS}

Southeast Asia/Unprotected

\section{USES}

Eaten; fur burned for exorcising ghosts (PUT, MRP, PTN, KLK, KAL, KBD); medicine for children (BRU); skin for hat (ALS, MRP), trophy (BRU)

\section{REFERENCES}

Payne et al. 1985: 277; Corbet and Hill 1992: 194;

Suyanto et al. 1998

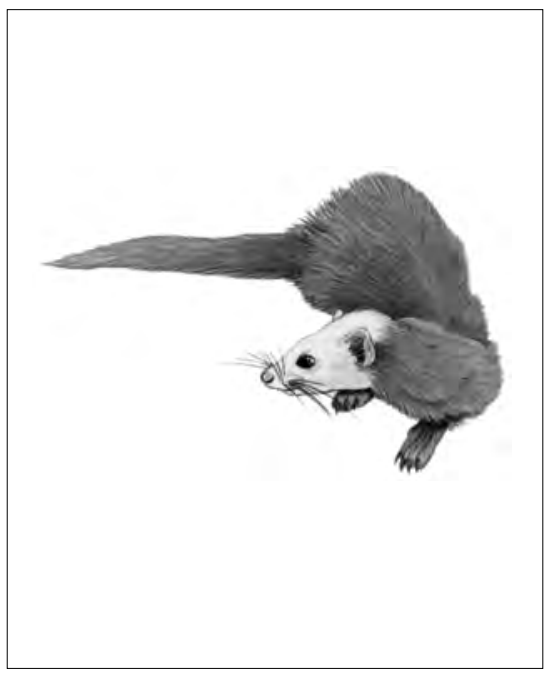

\begin{tabular}{|c|c|c|c|c|c|}
\hline \# & Code & Local Name & Use & RU & Hab.-Ab. \\
\hline 1 & PUT & derenggang, derengang & 1,2 & $x$ & $\mathrm{O}-1, \mathrm{P}-1$ \\
\hline 2 & PUB & derengang & 2 & v & P-1 \\
\hline 3 & PUD & kedurau & 1 & $x$ & $\mathrm{Y}-1, \mathrm{O}-1, \mathrm{P}-1$ \\
\hline 4 & PUM & derengang & 1 & $x$ & P-1 \\
\hline 5 & ALS & kedurau & 1,4 & v & $\mathrm{Y}-4, \mathrm{O}-4, \mathrm{P}-4$ \\
\hline 6 & LDY & labo buda', aeu & 1,2 & V & G-4, Y-4, O-1, P-1, M-1 \\
\hline 7 & LIT & & & $x$ & \\
\hline 8 & BRU & sau & $1,2,6$ & $x$ & $\mathrm{~S}-1, \mathrm{Y}-1, \mathrm{O}-1, \mathrm{P}-1$ \\
\hline 9 & MRP & langai, halang aya & $1,2,4$ & $x$ & $\mathrm{Y}-3, \mathrm{O}-3, \mathrm{P}-3$ \\
\hline 10 & PTN & sengangang & 2 & $x$ & $\mathrm{Y}-1, \mathrm{O}-1$ \\
\hline 11 & KLK & sengangang & 2,5 & $x$ & $\mathrm{Y}-1, \mathrm{O}-1, \mathrm{P}-1$ \\
\hline 12 & KLM & sengangang & 1,2 & $x$ & $\mathrm{Y}-1, \mathrm{O}-1, \mathrm{P}-2$ \\
\hline 13 & KLO & sengangang & 2 & $x$ & $\mathrm{O}-3, \mathrm{P}-3$ \\
\hline 14 & KLA & atui, sengangang & 2 & $x$ & $\mathrm{P}-3, \mathrm{M}-1$ \\
\hline 15 & KAL & sengangang & 2 & V & $\mathrm{Y}-1, \mathrm{P}-3$ \\
\hline 16 & KBD & sengangang & 1,2 & v & $\mathrm{Y}-1, \mathrm{O}-2, \mathrm{P}-2$ \\
\hline 17 & KBK & serangang & 1,2 & $x$ & $\mathrm{G}-1, \mathrm{~S}-1, \mathrm{Y}-4, \mathrm{O}-4, \mathrm{P}-4$ \\
\hline 18 & PEB & sengangang & 1,2 & $x$ & $\mathrm{Y}-1, \mathrm{O}-2, \mathrm{P}-3$ \\
\hline
\end{tabular}

Notes : 


\section{Aonyx cinerea (Mustelidae)

\section{DESCRIPTION}

Small carnivorous river otter; diurnal and semiaquatic, but prefers tree cover; upperparts dark brown or greyish brown; underparts slightly paler. Chin, throat, cheeks and sides of neck buffcoloured; digits partially webbed, short claws

\section{HABITAT}

Large rivers to small streams in the hills, ponds and lakes, to $1300 \mathrm{~m}$

\section{ORIGINS/STATUS}

India to Southeast Asia/CITES: Appendix II

\section{USES}

Eaten; gallstone medicinal (LDY); skin for hat (ALS)

\section{REFERENCES}

Payne et al. 1985: 280; Corbet and Hill 1992: 201; Suyanto et al. 1998

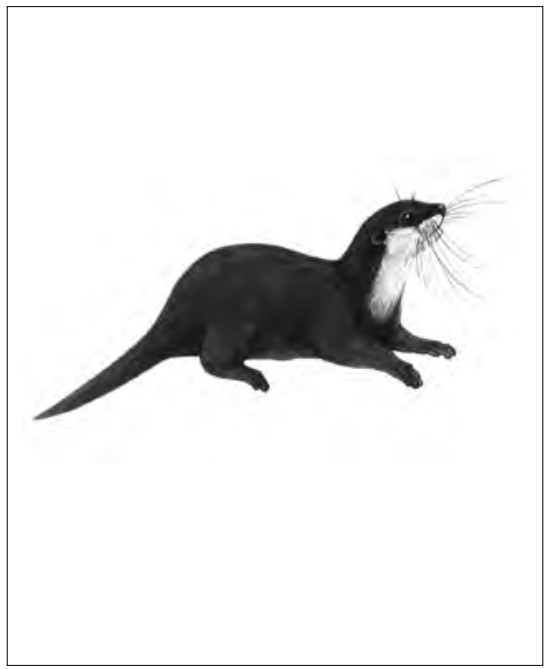

\begin{tabular}{|c|c|l|c|c|c|}
\hline \# & Code & \multicolumn{1}{|c|}{ Local Name } & Use & RU & Hab.-Ab. \\
\hline 1 & PUT & dengon, dengon cilik & 1 & $\mathbf{X}$ & $\mathrm{R}-2$ \\
\hline 2 & PUB & dengon & 1 & $\mathbf{V}$ & $\mathrm{R}-3$ \\
\hline 3 & PUD & dengon cilik & 1 & $\mathbf{X}$ & $\mathrm{R}-4$ \\
\hline 4 & PUM & dengon silik & 1 & $\mathbf{X}$ & $\mathrm{R}-4$ \\
\hline 5 & ALS & tilong silik & 1,4 & $\mathbf{V}$ & $\mathrm{R}-4$ \\
\hline 6 & LDY & dengen & 1,2 & $\mathbf{V}$ & $\mathrm{R}-3$ \\
\hline 7 & LIT & dengen & 1 & $\mathbf{X}$ & $\mathrm{R}-3$ \\
\hline 8 & BRU & langan ta'o & 1 & $\mathbf{X}$ & $\mathrm{R}$ \\
\hline 9 & MRP & ngan & 1 & $\mathbf{X}$ & $\mathrm{R}-4$ \\
\hline 10 & PTN & dengen & 1 & $\mathbf{X}$ & $\mathrm{R}-4$ \\
\hline 11 & KLK & dengen turing, dengen & 1 & $\mathbf{X}$ & $\mathrm{R}-1$ \\
\hline 12 & KLM & dengen, dengen lalut & 1 & $\mathbf{X}$ & $\mathrm{R}-3$ \\
\hline 13 & KLO & dengen & 1 & $\mathbf{X}$ & $\mathrm{R}-3$ \\
\hline 14 & KLA & dengen & 1 & $\mathbf{X}$ & $\mathrm{R}-2$ \\
\hline 15 & KAL & dengen dumit & 1 & $\mathbf{V}$ & $\mathrm{R}-2$ \\
\hline 16 & KBD & dengen & 1 & $\mathbf{V}$ & $\mathrm{R}-3$ \\
\hline 17 & KBK & dengen & 1 & $\mathbf{X}$ & $\mathrm{R}-2$ \\
\hline 18 & PEB & dengen sik & 1 & $\mathbf{X}$ & $\mathrm{R}-3$ \\
\hline
\end{tabular}

Notes : 


\section{Paradoxurus bermaphroditus (Viverridae) \\ COMMON PALM CIVET. MUSANGLUUWAK}

\section{DESCRIPTION}

Medium sized omnivorous mammal; nocturnal, arboreal and terrestrial, raids gardens, fruit orchards; upperparts olive brown to dark grey-brown, underparts paler; face, limbs and tail dark brown or black; 3 indistinct broken dark stripes along midline

\section{HABITAT}

Lowland secondary forests, plantations and village gardens

\section{ORIGINS/STATUS}

Asia/CITES: Appendix III

\section{USES}

Eaten; pet (ALS, KBD); skin for hat (ALS, PTN, KLM), trophy (PUT, BRU); teeth for charms (LBD, $\mathrm{KBK})$

\section{REFERENCES}

Payne et al. 1985: 283; Corbet and Hill 1992: 209; Suyanto et al. 1998

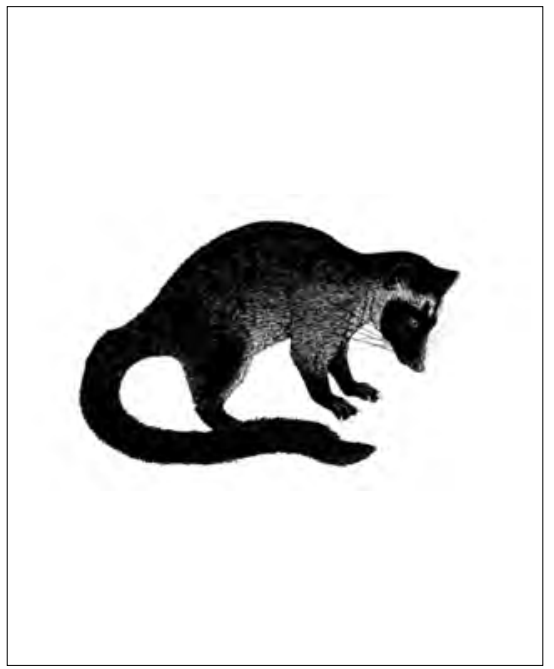

\begin{tabular}{|r|r|l|c|c|c|}
\hline \# & Code & Local Name & Use & RU & Hab.-Ab. \\
\hline 1 & PUT & pugoh, pugo & 1,6 & $\mathbf{x}$ & G-2, O-3, P-3 \\
\hline 2 & PUB & munin & 1 & $\mathbf{v}$ & O-3, P-3 \\
\hline 3 & PUD & pugoh & 1 & $\mathbf{v}$ & S-4, Y-4, O-4, P-4 \\
\hline 4 & PUM & pugo & 1,2 & $\mathbf{v}$ & P-4 \\
\hline 5 & ALS & munin & $1,4,9$ & $\mathbf{v}$ & G-4, Y-4, O-4, P-4 \\
\hline 6 & LDY & badan, libuwan & 1 & $\mathbf{v}$ & G-3, Y-3, O-4, P-4 \\
\hline 7 & LIT & badan & 1 & $\mathbf{v}$ & ALL-4 \\
\hline 8 & BRU & pangwa' & 1,6 & $\mathbf{x}$ & Y-4, O-4, P-4 \\
\hline 9 & MRP & punggowe, nuing, ponggowa' & 1 & $\mathbf{x}$ & G-4, Y-4, O-4, P-4 \\
\hline 10 & PTN & labao pun & 1,4 & $\mathbf{x}$ & O-1, P-1 \\
\hline 11 & KLK & kitan teppu, bunin & $\mathbf{x}$ & O-1, P-1 \\
\hline 12 & KLM & bunin & 1,4 & $\mathbf{v}$ & G-4, S-4 \\
\hline 13 & KLO & bevulu & 1 & $\mathbf{v}$ & O-1, P-1 \\
\hline 14 & KLA & bunin & 1 & $\mathbf{v}$ & G-3, S-3, P-3 \\
\hline 15 & KAL & bunin & 1 & $\mathbf{x}$ & G-3, S-3, P-2 \\
\hline 16 & KBD & munin & $1,5,9$ & $\mathbf{x}$ & G-2, S-2, Y-3, O-3, P-4 \\
\hline 17 & KBK & munin & 1,5 & $\mathbf{x}$ & G-2, S-2, Y-3, O-3, P-4 \\
\hline 18 & PEB & bunin & 1 & $\mathbf{v}$ & G-2, O-2, P-3, M-1 \\
\hline
\end{tabular}

Notes : 


\section{Paguma larvata (Viverridae) \\ MASKED PALM CIVET. MUSANG GALING \\ -}

\section{DESCRIPTION}

Medium sized omnivorous mammal; nocturnal, arboreal, raids gardens, fruit orchards; upperparts pale to dark reddish brown, yellow-grey underfur, no stripes; face yellowish white with black mask around eyes; white-tipped tail

\section{HABITAT}

Lowland and hill primary and secondary forests; plantations and village gardens

\section{ORIGINS/STATUS}

Asia/CITES: Appendix III

\section{USES}

Eaten; skin for hat (ALS, PTN) and trophy

(ALS); preys on chickens; omen to move swidden (PUT, BRU); pet (ALS, KBD)

\section{REFERENCES}

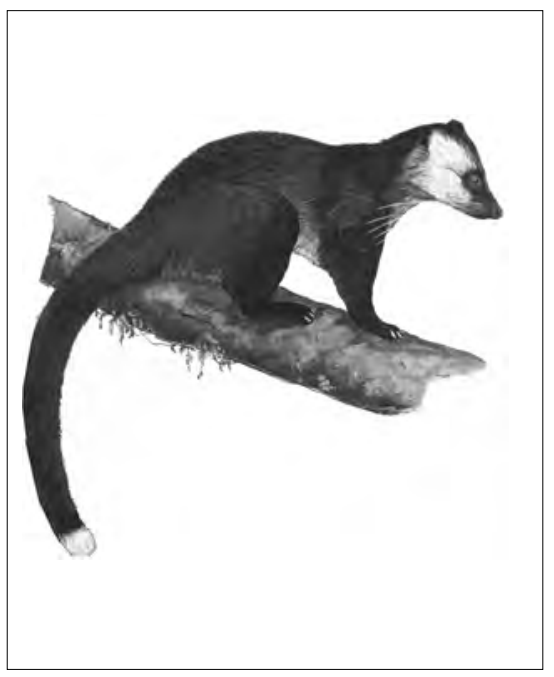

Payne et al. 1985: 284; Corbet and Hill 1992: 210;

Suyanto et al. 1998

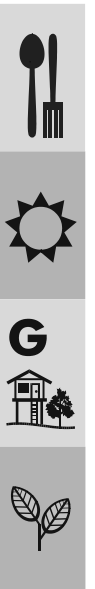

\begin{tabular}{|c|c|l|c|c|c|}
\hline$\#$ & Code & Local Name & Use & RU & Hab.-Ab. \\
\hline 1 & PUT & angan, angan kiring, yamuh & 1,5 & $\mathbf{X}$ & S-2, O-2, P-2 \\
\hline 2 & PUB & angan & 1 & $\mathbf{V}$ & O-2, P-2 \\
\hline 3 & PUD & angan & 1 & $\mathbf{V}$ & ALL-4 \\
\hline 4 & PUM & angan & 1 & $\mathbf{X}$ & G-4, P-4 \\
\hline 5 & ALS & busan & $1,4,6,9$ & $\mathbf{v}$ & G-4, Y-4, O-4, P-4 \\
\hline 6 & LDY & bung aleh, pugup & 1,2 & $\mathbf{v}$ & G-2, Y-1, O-2, P-2 \\
\hline 7 & LIT & rebuan & 1 & $\mathbf{x}$ & Y-2, O-2, P-2 \\
\hline 8 & BRU & luin & 1,5 & $\mathbf{x}$ & S-1, Y-1, O-1, P-1 \\
\hline 9 & MRP & meheung, bohowe & 1 & $\mathbf{X}$ & G-4, S-4, Y-4, O-4 \\
\hline 10 & PTN & buan & 1,4 & $\mathbf{x}$ & S-1, Y-1, O-1 \\
\hline 11 & KLK & basen, pingat & 1 & $\mathbf{x}$ & Y-1, O-1, P-1 \\
\hline 12 & KLM & belabau puti eko & 1 & $\mathbf{v}$ & G-4, S-4 \\
\hline 13 & KLO & belabo timeng & 1 & $\mathbf{v}$ & O-1, P-1 \\
\hline 14 & KLA & belabo putih eko, busan & 1 & $\mathbf{v}$ & G-3, S-3, P-3 \\
\hline 15 & KAL & bosen, belabo puti aiho & 1 & $\mathbf{v}$ & G-3, S-3, P-2 \\
\hline 16 & KBD & busan & $1,5,9$ & $\mathbf{v}$ & G-4, S-4 \\
\hline 17 & KBK & busan & 1,5 & $\mathbf{x}$ & G-2, S-2, Y-3, O-3, P-4 \\
\hline 18 & PEB & basen & 1 & $\mathbf{v}$ & G-2, O-2, P-3 \\
\hline
\end{tabular}

\section{Notes :}




\section{Arctictis binturong (Viverridae) \\ BEARCAT. BINTURUNG}

\section{DESCRIPTION}

Large omnivorous mammal; mainly nocturnal and arboreal; drops from trees, sometimes attacking humans; mostly black fur, grizzled with yellow, red and brown; older males reddish; long bushy prehensile tail, ears with tufts of long hair

\section{HABITAT}

Lowland and hill primary and secondary forests

\section{ORIGINS/STATUS}

India, China, Southeast Asia/CITES: Appendix III; RI: Protected

\section{USES}

Sometimes eaten; skin for hat, trophy; gall bladder for medicine, sold locally (ALS, LDY, LIT, MRP, PTN, KLK, KAL, KBD, PEB)

\section{REFERENCES}

Payne et al. 1985: 285; Corbet and Hill 1992: 211

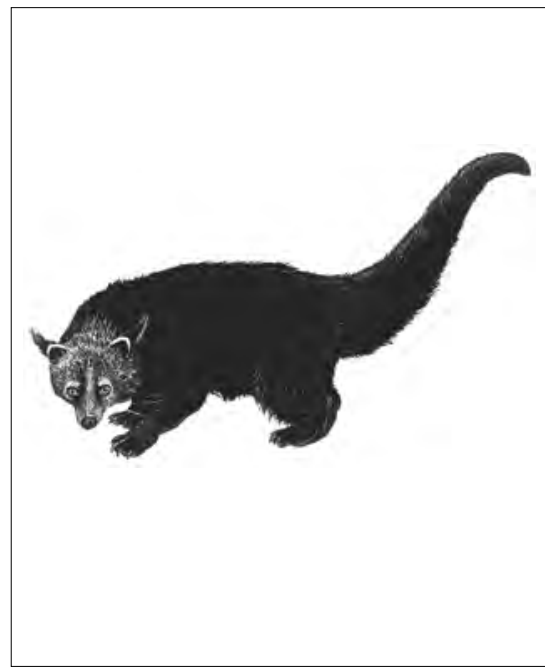

\begin{tabular}{|c|c|c|c|c|c|}
\hline \# & Code & Local Name & Use & RU & Hab.-Ab. \\
\hline 1 & PUT & kaitan, keitan ibu, ketan & $1,4,6$ & $x$ & $\mathrm{O}-1, \mathrm{P}-2, \mathrm{M}-2$ \\
\hline 2 & PUB & ketan & 1 & v & O-1, P-1 \\
\hline 3 & PUD & jamuh & 1 & $x$ & P-4 \\
\hline 4 & PUM & keitan & $1,2,6$ & $x$ & P-4 \\
\hline 5 & ALS & pasui & $1,2,6,8$ & $\mathrm{v}$ & $\mathrm{O}-4, \mathrm{P}-4$ \\
\hline 6 & LDY & fayu, payu & $1,2,8$ & v & $\mathrm{O}-4, \mathrm{P}-4$ \\
\hline 7 & LIT & payu & $1,2,8$ & $x$ & $\mathrm{O}-1, \mathrm{P}-1$ \\
\hline 8 & BRU & peo & 1,2 & v & $\mathrm{O}-1, \mathrm{P}-1$ \\
\hline 9 & MRP & tean, tehan, tea & $1,2,6$ & $x$ & $\mathrm{O}-3, \mathrm{P}-3$ \\
\hline 10 & PTN & kitan & $1,2,4$ & $x$ & P-1 \\
\hline 11 & KLK & kitan, ketan & $1,2,4$ & $x$ & $\mathrm{O}-1, \mathrm{P}-1$ \\
\hline 12 & KLM & ketan & $1,2,8$ & $x$ & $\mathrm{O}-1, \mathrm{P}-1$ \\
\hline 13 & KLO & ketan & $1,2,6$ & $x$ & $\mathrm{O}-1, \mathrm{P}-1$ \\
\hline 14 & KLA & ketan & $1,2,6$ & $x$ & P-3, M-1 \\
\hline 15 & KAL & kettan & $1,2,6$ & $x$ & $\mathrm{O}-1, \mathrm{P}-2$ \\
\hline 16 & KBD & ketan & $1,2,8$ & $x$ & $\mathrm{O}-1, \mathrm{P}-1$ \\
\hline 17 & KBK & kitan & $1,2,8$ & $x$ & P-3 \\
\hline 18 & PEB & pasui & $1,2,8$ & v & P-1, M-1 \\
\hline
\end{tabular}

Notes : 


\section{Herpestes brachyurus (Viverridae)

\section{DESCRIPTION}

Small carnivorous mammal; diurnal and terrestrial; foul smelling; body blackish brown with orange speckling, long narrow muzzle with chin and throat pale brown, tail bushy with base hairs longer than at tip

\section{HABITAT}

Lowland and hill primary and secondary forests to $1500 \mathrm{~m}$; sometimes in gardens, plantations

\section{ORIGINS/STATUS}

India and Southeast Asia/Unprotected

\section{USES}

Eaten; for medicine (LDY, MRP); skin for hat (LDY)

\section{REFERENCES}

Payne et al. 1985: 287; Corbet and Hill 1992: 214; Suyanto et al. 1998

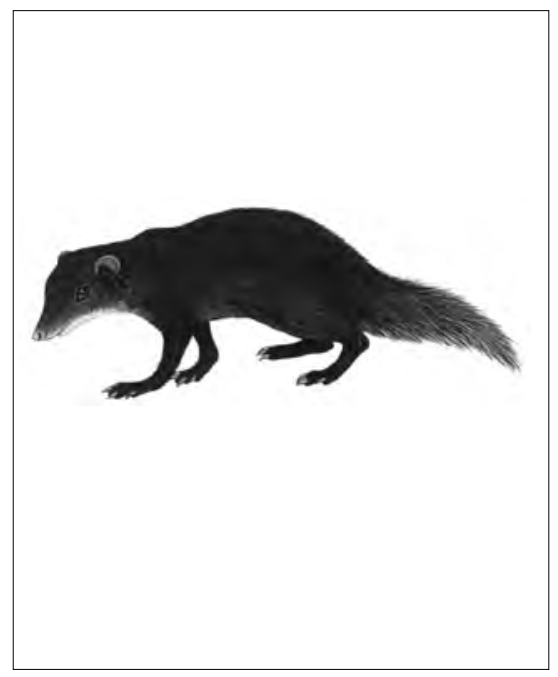

\begin{tabular}{|c|c|c|c|c|c|}
\hline$\#$ & Code & Local Name & Use & RU & Hab.-Ab. \\
\hline 1 & PUT & tuvang & 1 & $x$ & $\mathrm{O}-1, \mathrm{P}-3$ \\
\hline 2 & PUB & tuvang & & $\mathrm{v}$ & P-3 \\
\hline 3 & PUD & tuvang & 1 & v & $\mathrm{Y}-4, \mathrm{O}-4, \mathrm{P}-4$ \\
\hline 4 & PUM & tuvang & 1 & $x$ & P-4 \\
\hline 5 & ALS & simong & 1 & v & $\mathrm{O}-4, \mathrm{P}-4$ \\
\hline 6 & LDY & sibang, sibeng & $1,2,4$ & $\mathrm{v}$ & $\mathrm{Y}-4, \mathrm{O}-4, \mathrm{P}-4$ \\
\hline 7 & LIT & sibung & & $x$ & $\mathrm{O}-4$ \\
\hline 8 & BRU & bang & 1 & $x$ & P-1 \\
\hline 9 & MRP & tomboye, mohohowe & 1,2 & $x$ & G-1, Y-1, P-3 \\
\hline 10 & PTN & juwik & 1 & $x$ & $\mathrm{O}-2, \mathrm{P}-2$ \\
\hline 11 & KLK & tumbang & 1 & $x$ & $\mathrm{O}-1, \mathrm{P}-1$ \\
\hline 12 & KLM & tombang & 1 & $x$ & $\mathrm{O}-3, \mathrm{P}-3$ \\
\hline 13 & KLO & jembo & 1 & $x$ & $\mathrm{O}-3, \mathrm{P}-3$ \\
\hline 14 & KLA & sengangan, atui & 1 & $x$ & $\mathrm{O}-3, \mathrm{P}-3$ \\
\hline 15 & KAL & jevuk & 1 & $x$ & $\mathrm{O}-3, \mathrm{P}-3$ \\
\hline 16 & KBD & jembok saleng & & $x$ & $\mathrm{O}-3, \mathrm{P}-3$ \\
\hline 17 & KBK & & & $x$ & P-2 \\
\hline 18 & PEB & posu padeng & 1 & $x$ & $\mathrm{O}-3, \mathrm{P}-3$ \\
\hline
\end{tabular}

Notes : 


\section{Pardofelis nebulosa (Felidae)}

\section{Clouded leopard. MACANDAHAN}

\section{DESCRIPTION}

Large carnivorous cat, to $1.5 \mathrm{~m}$ in length from nose to tip of tail; mainly nocturnal and arboreal; very pale sandy brown to dark brown with dark cloudlike markings on side of body; large upper canines relative to skull; tail as long as body

\section{HABITAT}

Lowland and hill primary and secondary forests

\section{ORIGINS/STATUS}

South-Southeast Asia/CITES: Appendix I; RI:

Protected

\section{USES}

Eaten, meat for medicine; teeth and claws for jewellery, costumes, charms; skin for trophy; teeth and skin for ritual, sold locally and to traders; traditionally eaten by aristocrats only (Kenyah groups)

\section{REFERENCES}

Payne et al. 1985: 288; Corbet and Hill 1992: 226

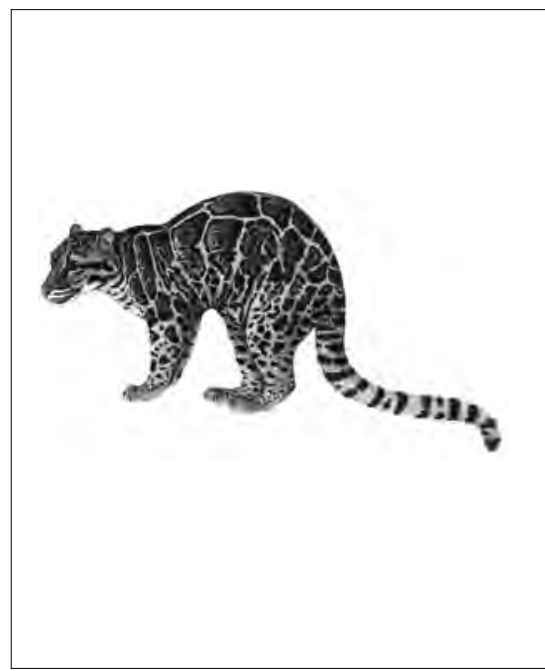

\begin{tabular}{|c|c|c|c|c|c|}
\hline \# & Code & Local Name & Use & RU & Hab.-Ab. \\
\hline 1 & PUT & kuli bovong, kuli avo & $1,6,8$ & $x$ & P-1, M-1 \\
\hline 2 & PUB & kuli bovong & $1,4,6,8$ & v & P-1 \\
\hline 3 & PUD & kuli bovong & $1,6,8$ & $x$ & P-1 \\
\hline 4 & PUM & kuli ayo' & $1,2,5,6$ & $\mathrm{x}$ & $\mathrm{P}-1, \mathrm{M}-1$ \\
\hline 5 & ALS & nekinon & $1,2,5,6,8$ & $x$ & P-1, M-1 \\
\hline 6 & LDY & kuwir, kuwer bilung & $1,4,6,8$ & v & $\mathrm{Y}-2, \mathrm{O}-2, \mathrm{P}-2, \mathrm{M}-4$ \\
\hline 7 & LIT & kuwir, kuwer & $1,4,5,6$ & $x$ & P-1 \\
\hline 8 & BRU & kuwer & $1,6,8$ & $x$ & P-1 \\
\hline 9 & MRP & keloi, keloai & 5,6 & $x$ & P-1 \\
\hline 10 & PTN & lenjo & $1,4,5,6,8$ & $x$ & P-1, M-1 \\
\hline 11 & KLK & kole lenjau & $1,6,8$ & $x$ & P-1 \\
\hline 12 & KLM & kole lenjau, kole taum & $1,4,5,8$ & $x$ & P-1 \\
\hline 13 & KLO & koli empuh & $1,5,6,8$ & $x$ & P-1 \\
\hline 14 & KLA & kule, lenjau & $1,5,6,8$ & $x$ & $\mathrm{Y}-1, \mathrm{O}-1, \mathrm{P}-1, \mathrm{M}-1$ \\
\hline 15 & KAL & kuli, kole lenjau & $1,5,6,8$ & $x$ & P-1, M-1 \\
\hline 16 & KBD & kole lenjau & $1,2,4,6,8$ & $x$ & P-1 \\
\hline 17 & KBK & kole bora' & $1,2,4,6,8$ & $x$ & $\mathrm{P}-1, \mathrm{M}-1$ \\
\hline 18 & PEB & dura' & $1,2,4,5,8$ & $x$ & $\mathrm{P}-3, \mathrm{M}-2$ \\
\hline
\end{tabular}

Notes : 


\section{Pardofelis marmorata (Felidae) \\ MARBLED CAT. KUCING BATU}

\section{DESCRIPTION}

Medium sized carnivorous cat, to $1.1 \mathrm{~m}$ in length; mainly nocturnal, arboreal and terrestrial; brown with smaller, less distinctive cloudlike markings on side of body; white spot behind ears, dark black spots on legs, tail as long as body

\section{HABITAT}

Lowland and hill primary and secondary forests

\section{ORIGINS/STATUS}

South-Southeast Asia/CITES: Appendix I;

RI: Protected

\section{USES}

Eaten, meat for medicine; teeth and skin for jewellery, costumes, charms, trophies, sold locally and to traders; traditionally eaten by aristocrats only (Kenyah groups)

\section{REFERENCES}

Payne et al. 1985: 284; Corbet and Hill 1992: 226
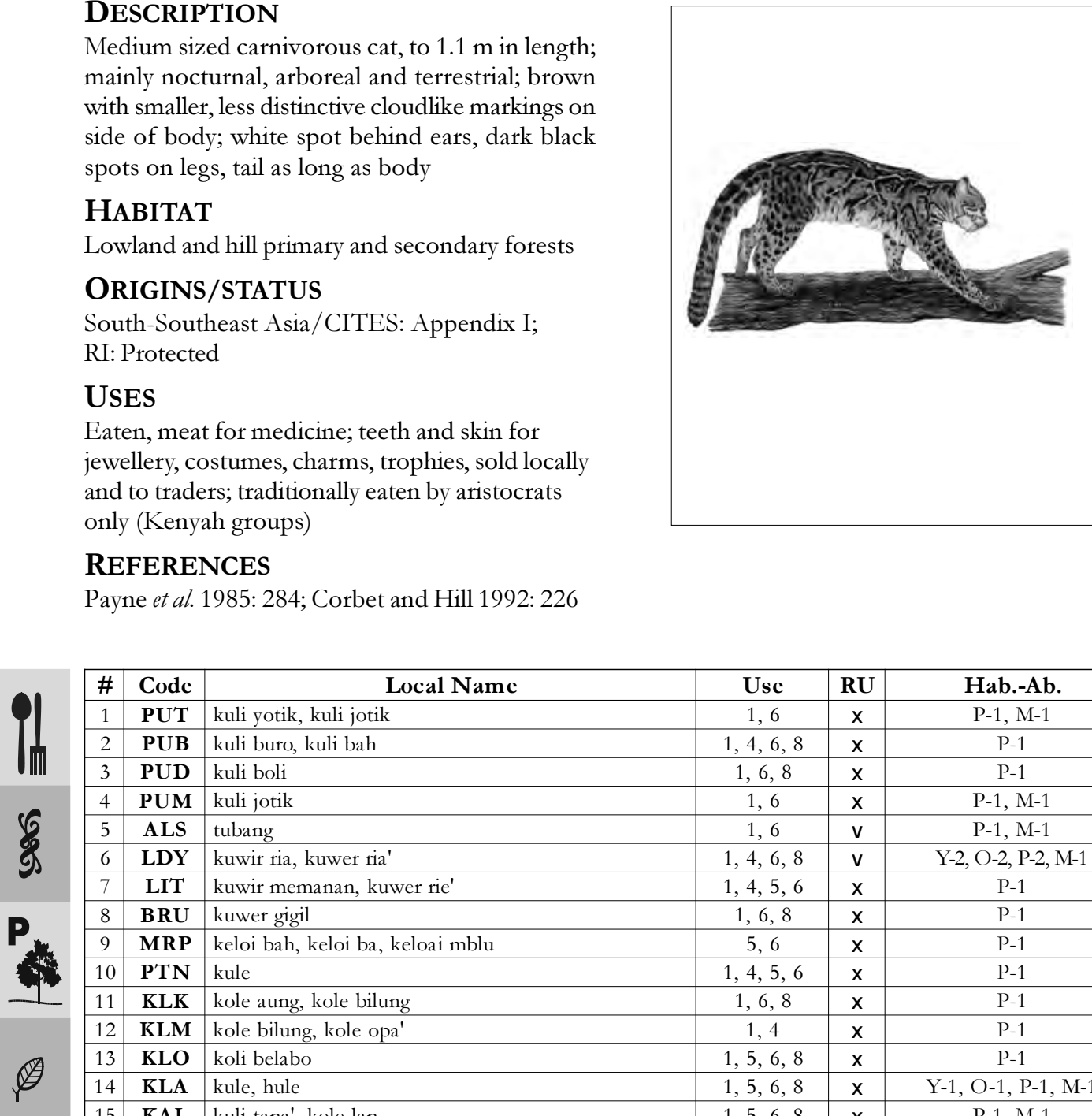

\begin{tabular}{|c|c|l|c|c|c|}
\hline$\#$ & Code & \multicolumn{1}{|c|}{ Local Name } & Use & RU & Hab.-Ab. \\
\hline 1 & PUT & kuli yotik, kuli jotik & 1,6 & $\mathbf{X}$ & P-1, M-1 \\
\hline 2 & PUB & kuli buro, kuli bah & $1,4,6,8$ & $\mathbf{X}$ & P-1 \\
\hline 3 & PUD & kuli boli & $1,6,8$ & $\mathbf{X}$ & P-1 \\
\hline 4 & PUM & kuli jotik & 1,6 & $\mathbf{X}$ & P-1, M-1 \\
\hline 5 & ALS & tubang & 1,6 & $\mathbf{V}$ & P-1, M-1 \\
\hline 6 & LDY & kuwir ria, kuwer ria' & $1,4,6,8$ & $\mathbf{V}$ & Y-2, O-2, P-2, M-1 \\
\hline 7 & LIT & kuwir memanan, kuwer rie' & $1,4,5,6$ & $\mathbf{X}$ & P-1 \\
\hline 8 & BRU & kuwer gigil & $1,6,8$ & $\mathbf{X}$ & P-1 \\
\hline 9 & MRP & keloi bah, keloi ba, keloai mblu & 5,6 & $\mathbf{X}$ & P-1 \\
\hline 10 & PTN & kule & $1,4,5,6$ & $\mathbf{X}$ & P-1 \\
\hline 11 & KLK & kole aung, kole bilung & $1,6,8$ & $\mathbf{X}$ & P-1 \\
\hline 12 & KLM & kole bilung, kole opa' & 1,4 & $\mathbf{X}$ & P-1 \\
\hline 13 & KLO & koli belabo & $1,5,6,8$ & $\mathbf{X}$ & P-1 \\
\hline 14 & KLA & kule, hule & $1,5,6,8$ & $\mathbf{X}$ & Y-1, O-1, P-1, M-1 \\
\hline 15 & KAL & kuli tana', kole lan & $1,5,6,8$ & $\mathbf{X}$ & P-1, M-1 \\
\hline 16 & KBD & kole lenjau & $1,2,4,6,8$ & $\mathbf{X}$ & P-1 \\
\hline 17 & KBK & koleh bilung & $1,2,4,6,8$ & $\mathbf{X}$ & P-1, M-1 \\
\hline 18 & PEB & dura' selian & $1,2,4,5,8$ & $\mathbf{X}$ & P-3, M-2 \\
\hline
\end{tabular}

Notes : 


\section{Prionailurusbengalensis (Felidae) \\ LEOPARD CAT. KUCING KUWUK}

\section{DESCRIPTION}

Small carnivorous cat, to $0.8 \mathrm{~m}$, tail half as long as body; nocturnal and terrestrial; tawny yellow coat with black spots over upperparts; stripes on face and down back, white spot behind ears which are set on side of head and rounded at top

\section{HABITAT}

Lowland and hill primary and secondary forests; plantations and village gardens

\section{ORIGINS/STATUS}

Asia/CITES: Appendix II

\section{USES}

Rarely eaten; preys on chickens; skin for trophy, teeth for ornamentation; skin for hat (PUD, PTN); medicine for children (BRU)

\section{REFERENCES}

Payne et al. 1985: 290; Corbet and Hill 1992: 222;

Suyanto et al. 1998

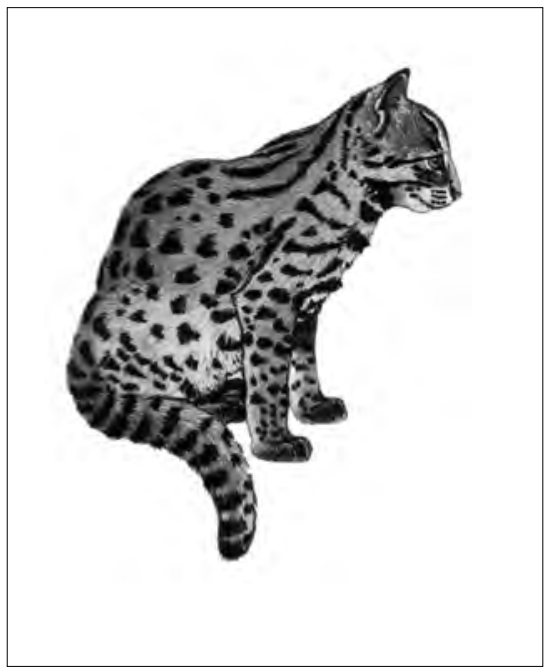

\begin{tabular}{|c|c|l|c|c|c|}
\hline \# & Code & Local Name & Use & RU & Hab.-Ab. \\
\hline 1 & PUT & kuli ukih, bekulu & 1,6 & $\mathbf{X}$ & G-1, S-1, O-3, P-3 \\
\hline 2 & PUB & bekulu & 1 & $\mathbf{X}$ & G-1, P-1 \\
\hline 3 & PUD & bekulu & 1,4 & $\mathbf{V}$ & ALL-3 \\
\hline 4 & PUM & bekulu & 1 & $\mathbf{X}$ & G-1, P-1 \\
\hline 5 & ALS & gisan & $1,4,6$ & $\mathbf{V}$ & G-2, Y-2, O-2, P-2 \\
\hline 6 & LDY & tubang borit, tubang & 1,6 & $\mathbf{V}$ & G-4, S-4, Y-4, O-3, P-3 \\
\hline 7 & LIT & tubang & 1 & $\mathbf{X}$ & G-3, Y-3, O-1, P-2 \\
\hline 8 & BRU & buyang al & 1,2 & $\mathbf{X}$ & ALL-1 \\
\hline 9 & MRP & betelo', botelo & 1,6 & $\mathbf{X}$ & G-3, Y-3, O-3, P-3 \\
\hline 10 & PTN & kule ba & $1,4,6,8$ & $\mathbf{X}$ & Y-1, O-1, P-1 \\
\hline 11 & KLK & bekulu & 1 & $\mathbf{X}$ & G-1, Y-1, O-1, P-1 \\
\hline 12 & KLM & bekulu & 1,4 & $\mathbf{X}$ & G-1, S-1, O-3, P-3 \\
\hline 13 & KLO & beghole lung & 1 & $\mathbf{X}$ & G-1, O-1, P-1 \\
\hline 14 & KLA & behulu mpe', behulu & 1 & $\mathbf{X}$ & G-1, S-1, Y-3, O-3, P-3 \\
\hline 15 & KAL & behulu mpe, behulu zap & 1 & $\mathbf{X}$ & G-1, O-3, P-3 \\
\hline 16 & KBD & bekulu & $1,2,6,8$ & $\mathbf{x}$ & G-2, O-3 \\
\hline 17 & KBK & bekulu & $1,2,6,8$ & $\mathbf{x}$ & G-1, S-1, O-2, P-2 \\
\hline 18 & PEB & bekuleu & 1 & $\mathbf{x}$ & G-1, S-1, Y-2, O-2, P-3 \\
\hline
\end{tabular}

Notes : 


\section{Felis catus (Felidae)}

\section{DOMESTIC CAT. KUCING PIARA}

\section{DESCRIPTION}

Small carnivorous domesticated cat, to $0.8 \mathrm{~m}$, tail half as long as body, often short and bent; diurnal and terrestrial; variably colored, from black to white with orange patches; ears are set on top of head and pointed at top

\section{HABITAT}

Human settlements

\section{ORIGINS/STATUS}

Global/Unprotected

\section{USES}

Pet, primarily for catching mice; occasionally eaten; occasionally traded

\section{REFERENCES}

Payne et al. 1985: 290; Corbet and Hill 1992: 221; Suyanto et al. 1998

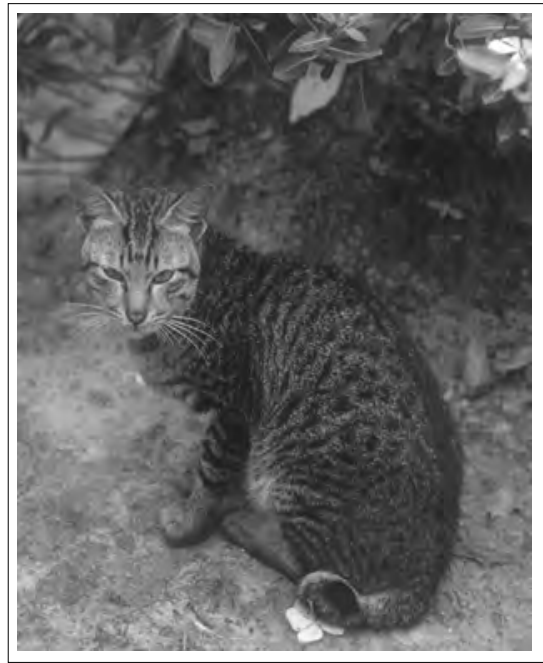

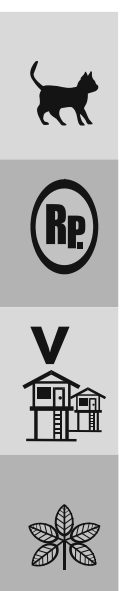

\begin{tabular}{|c|c|c|c|c|c|}
\hline \# & Code & Local Name & Use & RU & Hab.-Ab. \\
\hline 1 & PUT & ucih, uci, ngau & 9 & $\mathrm{v}$ & $\mathrm{V}$ \\
\hline 2 & PUB & ngau & 9 & v & $\mathrm{V}$ \\
\hline 3 & PUD & ngau & 9 & v & $\mathrm{V}$ \\
\hline 4 & PUM & usih & 9 & v & $\mathrm{V}$ \\
\hline 5 & ALS & usi' & 9 & v & V \\
\hline 6 & LDY & using & 9 & v & $\mathrm{V}$ \\
\hline 7 & LIT & using, ngau & 9 & v & V \\
\hline 8 & BRU & ko' ngau & 9 & v & $\mathrm{V}$ \\
\hline 9 & MRP & ngau & 9 & v & $\mathrm{V}$ \\
\hline 10 & PTN & sing ngiyu & 9 & v & $\mathrm{V}$ \\
\hline 11 & KLK & sing & 9 & v & $\mathrm{V}$ \\
\hline 12 & KLM & sieng & $1,8,9$ & v & $\mathrm{V}$ \\
\hline 13 & KLO & sieng & $1,8,9$ & v & $\mathrm{V}$ \\
\hline 14 & KLA & sieng hulong & $1,8,9$ & v & $\mathrm{V}$ \\
\hline 15 & KAL & sieng & $1,8,9$ & $\mathrm{v}$ & $\mathrm{V}$ \\
\hline 16 & KBD & ngieu & $1,8,9$ & v & $\mathrm{V}$ \\
\hline 17 & KBK & ngieu & $1,8,9$ & $\mathrm{v}$ & $\mathrm{V}$ \\
\hline 18 & PEB & olang & 9 & v & $\mathrm{V}$ \\
\hline
\end{tabular}

Notes : 


\section{Dicerorbinus sumatrensis (Rhinocerotidae) SUMATRAN RHINOCEROS. BADAK SUMATRA}

\section{DESCRIPTION}

Large herbivorous mammal, to $2.5 \mathrm{~m}$ long, 1.2-1.3 $\mathrm{m}$ at shoulder height; diurnal and terrestrial; dark brown hairy body, front horn c. $30 \mathrm{~cm}$, second horn very short; 3 -toed footprints $18.5-23.5 \mathrm{~cm}$ across

\section{HABITAT}

Lowland to hill primary and secondary forests; visits salt/mineral springs and mud wallows; presently rare or locally absent

\section{ORIGINS/STATUS}

Thailand, Malaysia, Sumatra, Borneo/

CITES: Appendix I; RI: Protected

\section{USES}

Prior to 1940: hunted for food; meat for medicine; horn sold to traders for export

\section{REFERENCES}

Payne et al. 1985: 293; Corbet and Hill 1992: 243

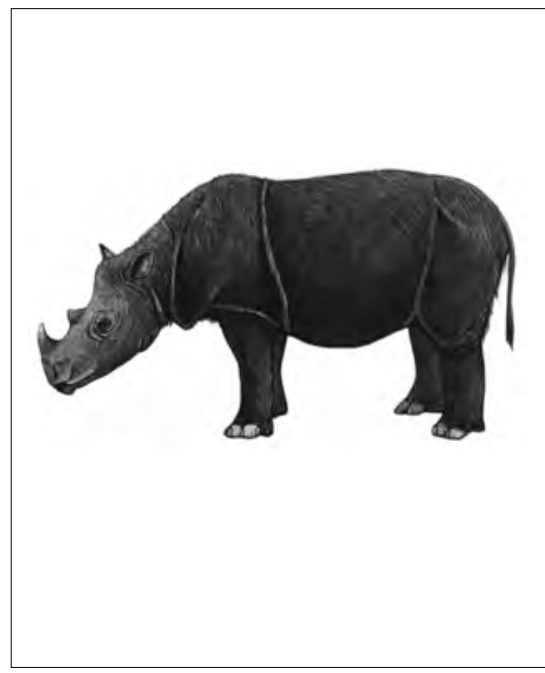

\begin{tabular}{|r|l|l|r|r|l|}
\hline \# & Code & \multicolumn{1}{|c|}{ Local Name } & Use & RU & Hab.-Ab. \\
\hline 1 & PUT & temeru & $1,2,8$ & $\mathbf{X}$ & \\
\hline 2 & PUB & temeru, temaro bun & $1,2,8$ & $\mathbf{X}$ & \\
\hline 3 & PUD & temeru & $1,2,8$ & $\mathbf{X}$ & \\
\hline 4 & PUM & temeru & $1,2,8$ & $\mathbf{X}$ & \\
\hline 5 & ALS & badak & $1,2,8$ & $\mathbf{X}$ & \\
\hline 6 & LDY & temasur, temesur & $1,2,8$ & $\mathbf{X}$ & \\
\hline 7 & LIT & temesor, temesur & $1,2,8$ & $\mathbf{X}$ & \\
\hline 8 & BRU & kamsul & $1,2,8$ & $\mathbf{X}$ & \\
\hline 9 & $\mathbf{M R P}$ & temberau, tamberau & $1,2,8$ & $\mathbf{X}$ & \\
\hline 10 & PTN & temetto & $1,2,8$ & $\mathbf{X}$ & \\
\hline 11 & KLK & temetto & $1,2,8$ & $\mathbf{X}$ & \\
\hline 12 & KLM & temetto & $1,2,8$ & $\mathbf{X}$ & \\
\hline 13 & KLO & temettu & $1,2,8$ & $\mathbf{X}$ & \\
\hline 14 & KLA & temetto & $1,2,8$ & $\mathbf{X}$ & \\
\hline 15 & KAL & temetto & $1,2,8$ & $\mathbf{X}$ & \\
\hline 16 & KBD & pemetto & $1,2,8$ & $\mathbf{X}$ & \\
\hline 17 & KBK & temetto & $1,2,8$ & $\mathbf{X}$ & \\
\hline 18 & PEB & temeto & $1,2,8$ & $\mathbf{X}$ & \\
\hline
\end{tabular}

Notes : 


\section{Sus barbatus (Suidae)}

\section{BEARDED PIG. BABI NANGUI, BABI JANGGUT}

\section{DESCRIPTION}

Large omnivorous mammal, to $120 \mathrm{~kg}$; diurnal, nocturnal, terrestrial; builds nests of undergrowth for birthing; infants brown with black stripes, juveniles black, adults white buff; lower jaw with bristles

\section{HABITAT}

Lowland to lower montane forests; plantations, village gardens; visits salt/mineral springs; sleeps in mud-wallows or in thickets during day; seasonally abundant due to migrations

\section{ORIGINS/STATUS}

Sumatra, Borneo and Philippines/Unprotected

\section{USES}

Meat and fat eaten; rendered fat traded or sold locally and to traders; jaws and tusks kept as trophies

\section{REFERENCES}

Payne et al. 1985: 296; Corbet and Hill 1992: 247

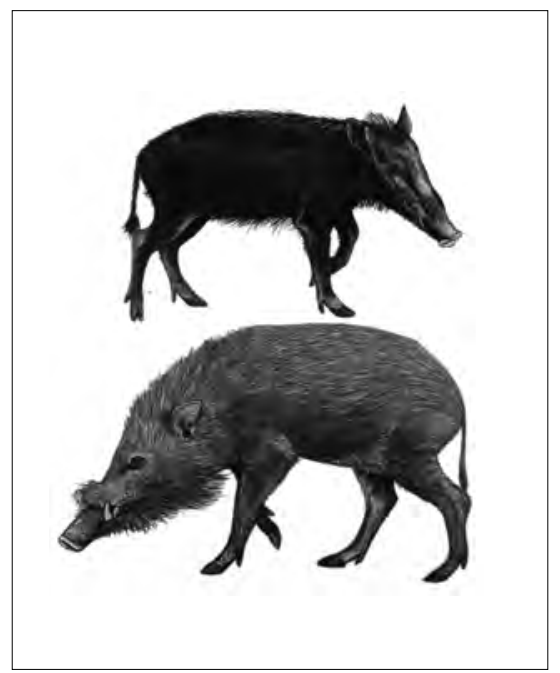

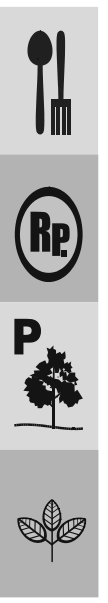

\begin{tabular}{|c|c|l|c|c|c|}
\hline$\#$ & Code & Local Name & Use & RU & Hab.-Ab. \\
\hline 1 & PUT & bavui & $1,6,8$ & $\mathbf{v}$ & ALL-4 \\
\hline 2 & PUB & bavui & 1 & $\mathbf{v}$ & ALL \\
\hline 3 & PUD & bavui & 1,8 & $\mathbf{v}$ & G-2, S-2, Y-4, O-4, P-4 \\
\hline 4 & PUM & bavui & 1,8 & $\mathbf{v}$ & ALL \\
\hline 5 & ALS & kana' & $1,6,8$ & $\mathbf{v}$ & ALL \\
\hline 6 & LDY & baka & $1,6,8$ & $\mathbf{v}$ & ALL \\
\hline 7 & LIT & baka & 1 & $\mathbf{v}$ & ALL \\
\hline 8 & BRU & aka & $1,2,8$ & $\mathbf{v}$ & ALL-4 \\
\hline 9 & MRP & mabai & 1,8 & $\mathbf{v}$ & ALL-4 \\
\hline 10 & PTN & babui & 1,8 & $\mathbf{v}$ & ALL-4 \\
\hline 11 & KLK & babui & 1,8 & $\mathbf{v}$ & G-1, S-1, Y-3, O-3, P-4 \\
\hline 12 & KLM & babui & 1,8 & $\mathbf{v}$ & S-1, Y-3, O-4, P-4, M-1 \\
\hline 13 & KLO & babi & 1,6 & $\mathbf{v}$ & G-1, S-1, Y-3, O-3, P-4, M-3 \\
\hline 14 & KLA & babui & 1,6 & $\mathbf{v}$ & G-1, S-1, Y-3, O-4, P-4, M-2 \\
\hline 15 & KAL & babei, ba'bui & 1,6 & $\mathbf{v}$ & S-2, Y-3, O-3, P-4, M-2 \\
\hline 16 & KBD & babui & 1,8 & $\mathbf{v}$ & ALL-4 \\
\hline 17 & KBK & babui & 1,8 & $\mathbf{v}$ & G-1, S-1, Y-3, O-4, P-4, M-3 \\
\hline 18 & PEB & kan, mabui & 1,8 & $\mathbf{v}$ & S-1, Y-4, O-4, P-4, M-2, R-4 \\
\hline
\end{tabular}

Notes : 


\section{Sus domesticus (Suidae)}

\section{DOMESTIC PIG. BABITERNAK, BABIPUTII}

\section{DESCRIPTION}

Large omnivorous domesticated pig, descended from Sus scrofa; traditionally free ranging in Dayak longhouse villages; fed rice husks, cooked rice and waste materials

\section{HABITAT}

Human settlements; rare or locally absent (PUT, PUB, PUD, PUM, PEB)

\section{ORIGINS/STATUS}

Global/Unprotected

\section{USES}

Meat and fat eaten; traditionally exchanged or slaughtered for ritual or ceremonial occasions; raised for coastal city markets

\section{REFERENCES}

Payne et al. 1985: 296; Corbet and Hill 1992: 246

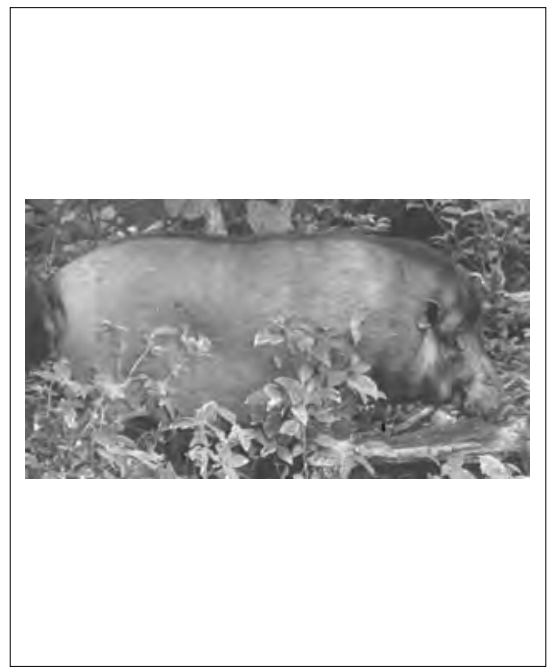

\begin{tabular}{|c|c|l|c|c|c|}
\hline$\#$ & Code & Local Name & Use & RU & Hab.-Ab. \\
\hline 1 & PUT & uting & $1,5,8,9$ & $\mathbf{X}$ & $\mathrm{V}$ \\
\hline 2 & PUB & uting & $1,5,8,9$ & $\mathbf{X}$ & $\mathrm{V}$ \\
\hline 3 & PUD & uting & $1,5,8,9$ & $\mathbf{X}$ & $\mathrm{V}$ \\
\hline 4 & PUM & uting & $1,5,8,9$ & $\mathbf{X}$ & $\mathrm{V}$ \\
\hline 5 & ALS & borok & $1,5,8,9$ & $\mathbf{V}$ & $\mathrm{V}-2$ \\
\hline 6 & LDY & berak, berek vanin & $1,5,8,9$ & $\mathbf{V}$ & $\mathrm{V}-1$ \\
\hline 7 & LIT & berek & $1,5,8,9$ & $\mathbf{V}$ & $\mathrm{V}-1$ \\
\hline 8 & BRU & gia' & $1,5,8,9$ & $\mathbf{V}$ & $\mathrm{V}-1$ \\
\hline 9 & MRP & yang, nyahe & $1,5,8,9$ & $\mathbf{V}$ & $\mathrm{V}$ \\
\hline 10 & PTN & ayam & $1,5,8,9$ & $\mathbf{V}$ & $\mathrm{V}$ \\
\hline 11 & KLK & buin & $1,5,8,9$ & $\mathbf{X}$ & $\mathrm{V}$ \\
\hline 12 & KLM & bu'in & $1,5,8,9$ & $\mathbf{V}$ & $\mathrm{V}$ \\
\hline 13 & KLO & buin & $1,5,8,9$ & $\mathbf{V}$ & $\mathrm{V}$ \\
\hline 14 & KLA & buin & $1,5,8,9$ & $\mathbf{V}$ & $\mathrm{V}$ \\
\hline 15 & KAL & buin & $1,5,8,9$ & $\mathbf{X}$ & $\mathrm{V}$ \\
\hline 16 & KBD & buin & $1,5,8,9$ & $\mathbf{X}$ & $\mathrm{V}$ \\
\hline 17 & KBK & bu'in & $1,5,8,9$ & $\mathbf{X}$ & $\mathrm{V}$ \\
\hline 18 & PEB & boin & $1,5,8,9$ & $\mathbf{X}$ & $\mathrm{V}$ \\
\hline
\end{tabular}

Notes : 


\section{Tragulus javanicus (Tragulidae) \\ LESSER MOUSEDEER. PELANDUK KANCIL}

\section{DESCRIPTION}

Small herbivorous mammal; nocturnal and terrestrial; upperparts plain reddish brown, underparts white with pale brown tinge in the middle, in profile, single white bar from chin to chest; slender legs, no horns, protruding upper canines

\section{HABITAT}

Lowland primary and secondary forests to $600 \mathrm{~m}$; sometimes in cultivated areas

\section{ORIGINS/STATUS}

Southeast Asia/RI: Protected

\section{USES}

Eaten; meat sold (ALS, BRU); skin for hat (PTN), for trophy (ALS, LDY); 'antlers' of female for good luck charm (LDY, MRP, KBD); 'antlers' for medicine (KAL); uterine 'stone' for medicine for easing child birth (KLK, KBD, KAL)

\section{REFERENCES}

Payne et al. 1985: 297; Corbet and Hill 1992: 250
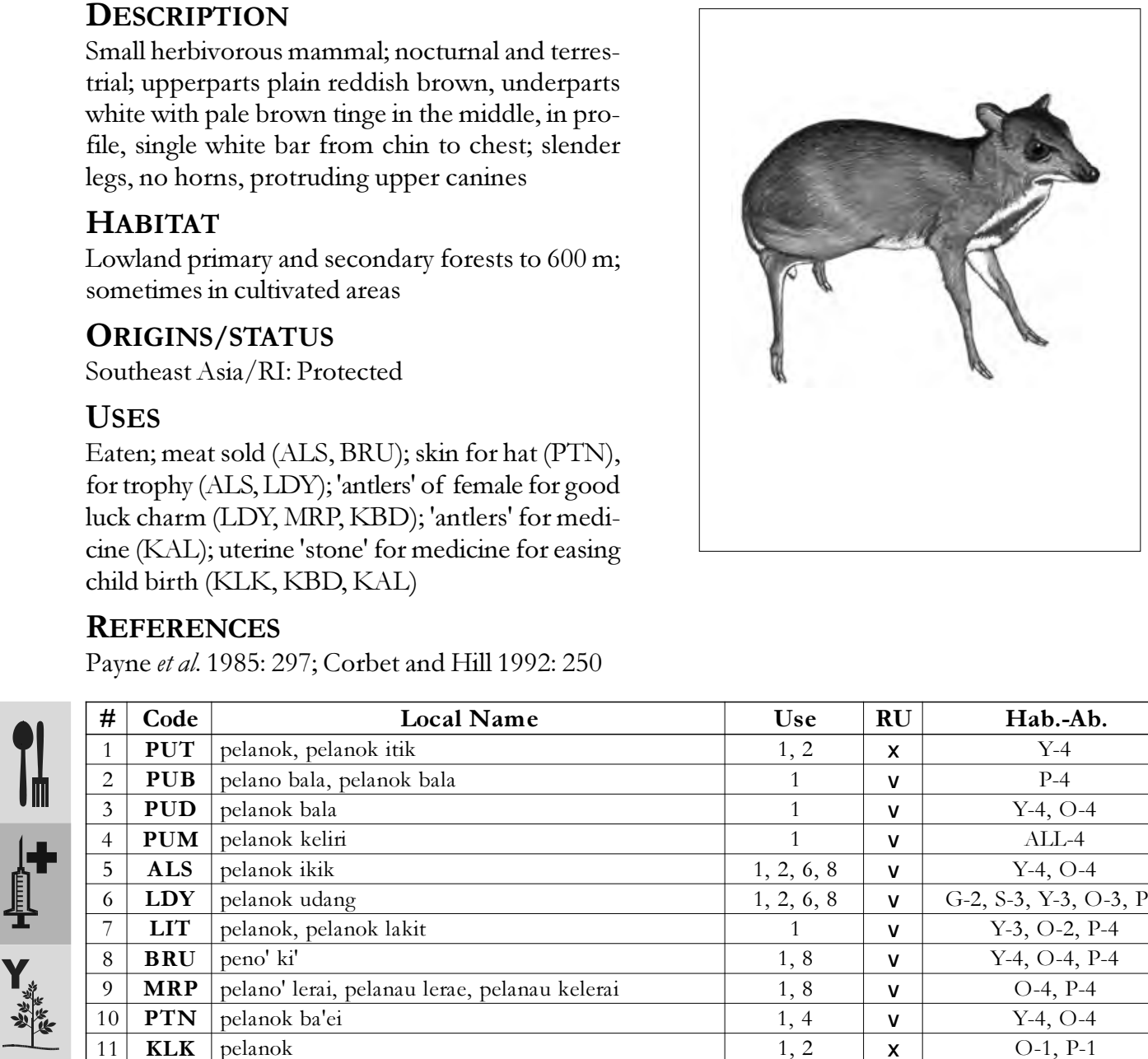

\begin{tabular}{|c|c|l|c|c|c|}
\hline$\#$ & Code & \multicolumn{1}{|c|}{ Local Name } & Use & RU & Hab.-Ab. \\
\hline 1 & PUT & pelanok, pelanok itik & 1,2 & $\mathbf{X}$ & Y-4 \\
\hline 2 & PUB & pelano bala, pelanok bala & 1 & $\mathbf{v}$ & P-4 \\
\hline 3 & PUD & pelanok bala & 1 & $\mathbf{V}$ & Y-4, O-4 \\
\hline 4 & PUM & pelanok keliri & 1 & $\mathbf{v}$ & ALL-4 \\
\hline 5 & ALS & pelanok ikik & $1,2,6,8$ & $\mathbf{V}$ & Y-4, O-4 \\
\hline 6 & LDY & pelanok udang & $1,2,6,8$ & $\mathbf{v}$ & G-2, S-3, Y-3, O-3, P-3 \\
\hline 7 & LIT & pelanok, pelanok lakit & 1 & $\mathbf{v}$ & Y-3, O-2, P-4 \\
\hline 8 & BRU & peno' ki' & 1,8 & $\mathbf{v}$ & Y-4, O-4, P-4 \\
\hline 9 & MRP & pelano' lerai, pelanau lerae, pelanau kelerai & 1,8 & $\mathbf{v}$ & O-4, P-4 \\
\hline 10 & PTN & pelanok ba'ei & 1,4 & $\mathbf{v}$ & Y-4, O-4 \\
\hline 11 & KLK & pelanok & 1,2 & $\mathbf{X}$ & O-1, P-1 \\
\hline 12 & KLM & pelanok andun, pelanok ano & $1,2,8$ & $\mathbf{x}$ & S-2, Y-3, O-3, P-3 \\
\hline 13 & KLO & pelano' & $1,2,8$ & $\mathbf{X}$ & S-4, Y-4, O-4, P-4 \\
\hline 14 & KLA & pelanok, pelanduk & $1,2,8$ & $\mathbf{X}$ & Y-3, O-3, P-3, M-2 \\
\hline 15 & KAL & pelano', pelanok & $1,2,8$ & $\mathbf{v}$ & S-2, Y-3, O-3, P-3 \\
\hline 16 & KBD & pelanok & $1,2,5,8$ & $\mathbf{v}$ & S-4, Y-4, O-4, P-4 \\
\hline 17 & KBK & pelanok uduk & $1,2,5,8$ & $\mathbf{x}$ & S-2, Y-2, O-4, P-4, M-2 \\
\hline 18 & PEB & pelanok ase & 1,2 & $\mathbf{v}$ & S-4, Y-4, O-4, P-4 \\
\hline
\end{tabular}

\section{Notes :}




\section{Tragulus napu (Tragulidae)}

\section{GREATER MALAY MOUSEDEER. PELANDUKNAPU \\ ○}

\section{DESCRIPTION}

Small herbivorous mammal; nocturnal and terrestrial; larger body, thicker legs than T.javanicus; upperparts coarsely flecked, grey buff to orange buff hairs with black tips; in profile, white on neck appears as two bars

\section{HABITAT}

Lowland to hill primary and secondary forests; sometimes in cultivated areas

\section{ORIGINS/STATUS}

Southeast Asia/RI: Protected

\section{USES}

Eaten; meat sold (ALS, BRU); skin for hat (PTN), for trophy (ALS, LDY); 'antlers' of female good luck charm (LDY, MRP, KBD); 'antlers' for medicine (KAL); uterine 'stone' for medicine for easing child birth (MRP, PTN, KLK, KBD, KAL)

\section{REFERENCES}

Payne et al. 1985: 297; Corbet and Hill 1992: 252

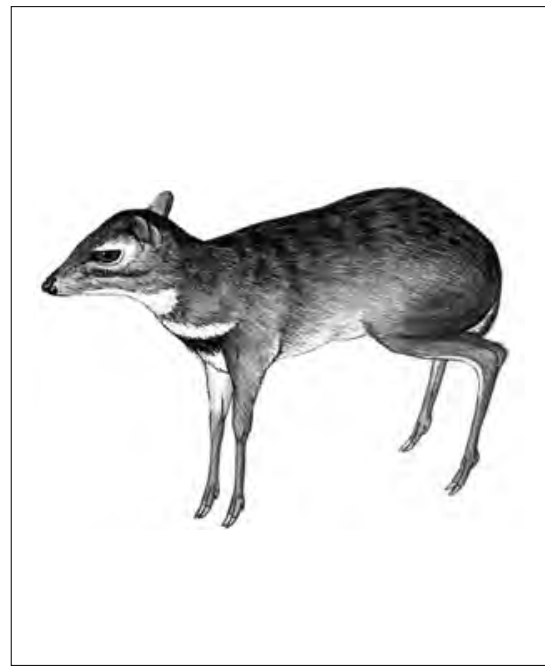

\begin{tabular}{|c|c|c|c|c|c|}
\hline \# & Code & Local Name & Use & $\mathbf{R U}$ & Hab.-Ab. \\
\hline 1 & PUT & pelanok, pelanok tu'an & 1,2 & $x$ & P-4 \\
\hline 2 & PUB & pelano tu'an, pelanok tu'an & 1 & $\mathrm{v}$ & $\mathrm{O}-4$ \\
\hline 3 & PUD & pelanok tu'an & 1 & $\mathrm{v}$ & P-4 \\
\hline 4 & PUM & pelanok tu'an & 1 & v & ALL-4 \\
\hline 5 & ALS & pelanok batang & $1,2,6,8$ & v & $\mathrm{P}-4, \mathrm{M}-4$ \\
\hline 6 & LDY & pelanok batang, pelanok itan & $1,2,6,8$ & V & G-2, S-3, Y-3, O-3, P-3 \\
\hline 7 & LIT & pelanok, pelanok batang & 1 & v & $\mathrm{Y}-2, \mathrm{O}-2, \mathrm{P}-4$ \\
\hline 8 & BRU & peno' kalut & 1,8 & $x$ & $\mathrm{Y}-1, \mathrm{O}-1, \mathrm{P}-1$ \\
\hline 9 & MRP & pelano keloi, pelanau keloi, pelanu keloai & 1,8 & v & $\mathrm{O}-4, \mathrm{P}-4$ \\
\hline 10 & PTN & pelanok mba' & $1,2,4$ & $\mathrm{v}$ & P-4 \\
\hline 11 & KLK & pelanok ano & 1,2 & $x$ & $\mathrm{O}-1, \mathrm{P}-1$ \\
\hline 12 & KLM & pelanok bala & $1,2,8$ & $x$ & $\mathrm{~S}-2, \mathrm{Y}-3, \mathrm{O}-3, \mathrm{P}-3$ \\
\hline 13 & KLO & pelano' & $1,2,8$ & $x$ & $\mathrm{~S}-4, \mathrm{Y}-4, \mathrm{O}-4, \mathrm{P}-4$ \\
\hline 14 & KLA & pelanok, pelanduk & $1,2,8$ & $x$ & $\mathrm{Y}-3, \mathrm{O}-3, \mathrm{P}-3, \mathrm{M}-2$ \\
\hline 15 & KAL & pelano', pelanok & $1,2,8$ & V & $\mathrm{S}-2, \mathrm{Y}-2, \mathrm{O}-3, \mathrm{P}-3$ \\
\hline 16 & KBD & pelanok & $1,2,5,8$ & v & $\mathrm{S}-4, \mathrm{Y}-4, \mathrm{O}-4, \mathrm{P}-4$ \\
\hline 17 & KBK & pelanok & $1,2,5,8$ & $x$ & $\mathrm{~S}-2, \mathrm{Y}-2, \mathrm{O}-4, \mathrm{P}-4, \mathrm{M}-2$ \\
\hline 18 & PEB & pelanok date & 1,2 & v & $\mathrm{S}-4, \mathrm{Y}-4, \mathrm{O}-4, \mathrm{P}-4$ \\
\hline
\end{tabular}

Notes : 


\section{Muntiacus muntjak (Cervidae)

\section{DESCRIPTION}

Medium sized herbivorous mammal; diurnal and terrestrial; upperparts reddish brown, darker along midline, underparts whitish; tail brown above, white below; antlers to $13 \mathrm{~cm}$ long; loud barking call when startled

\section{HABITAT}

Lowland to lower montane forests; plantations, visits salt/mineral springs

\section{ORIGINS/STATUS}

India to Southeast Asia/RI: Protected

\section{USES}

Eaten, meat sold locally and to traders (ALS); skin for clothes (PUT, ALS, KLK); antlers for tools, kept as trophies or sold to traders (KLK, KLM, KAL, KBD, PEB); omen animal (KBD, $\mathrm{KBK})$

\section{REFERENCES}

Payne et al. 1985: 298; Corbet and Hill 1992: 259

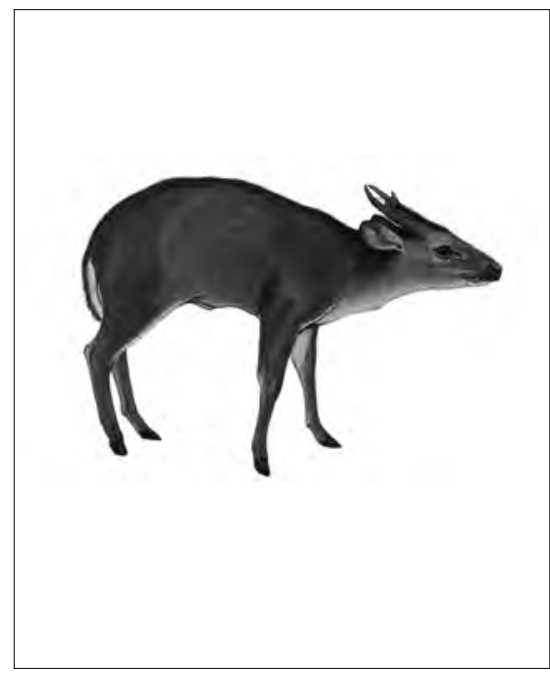

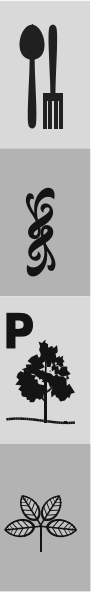

\begin{tabular}{|c|c|l|c|c|c|}
\hline$\#$ & Code & \multicolumn{1}{|c|}{ Local Name } & Use & RU & Hab.-Ab. \\
\hline 1 & PUT & telau, telo pawen, telo' pawen & $1,4,6$ & $\mathbf{X}$ & P-1 \\
\hline 2 & PUB & telo rau & $1,6,8$ & $\mathbf{V}$ & P-1 \\
\hline 3 & PUD & telo' tu'an & 1,6 & $\mathbf{V}$ & P-4 \\
\hline 4 & PUM & telo kunyo & 1,6 & $\mathbf{V}$ & G-1, S-1, Y-4, O-4, P-4 \\
\hline 5 & ALS & faus itom & $1,4,6,8$ & $\mathbf{X}$ & Y-4, O-4, P-4 \\
\hline 6 & LDY & bingo, talao bingo & $1,6,8$ & $\mathbf{V}$ & S-3, Y-3, O-3, P-3, Y-1, O-1, P-1 \\
\hline 7 & LIT & bingo, tela'u hitam & $1,4,6$ & $\mathbf{X}$ & P-4 \\
\hline 8 & BRU & telau agon & 1,6 & $\mathbf{X}$ & Y-1, O-1, P-1 \\
\hline 9 & MRP & telau toung, telau to'ong, tela' au & $1,6,8$ & $\mathbf{X}$ & O-1, P-1 \\
\hline 10 & PTN & usa' & 1,6 & $\mathbf{X}$ & S-1, Y-1, O-1 \\
\hline 11 & KLK & uca' ano, uca & $1,4,6,8$ & $\mathbf{X}$ & S-1, O-1, P-1 \\
\hline 12 & KLM & oca' mandun, oca' andun & $1,6,8$ & $\mathbf{v}$ & Y-4, O-4, P-4 \\
\hline 13 & KLO & tela'o & $1,6,8$ & $\mathbf{V}$ & S-1, O-1, P-1, M-1 \\
\hline 14 & KLA & tela'o & $1,6,8$ & $\mathbf{v}$ & Y-3, O-3, P-3 \\
\hline 15 & KAL & tela'o saleng, tela'o saben & $1,6,8$ & $\mathbf{X}$ & Y-2, O-2, P-3, M-2 \\
\hline 16 & KBD & osa' & $1,5,6,8$ & $\mathbf{X}$ & Y-4, O-4, P-4 \\
\hline 17 & KBK & tela'o saleng & $1,5,6,8$ & $\mathbf{X}$ & S-2, Y-2, O-4, P-4, M-2 \\
\hline 18 & PEB & telau sau & $1,3,8$ & $\mathbf{v}$ & Y-4, O-4, P-4 \\
\hline
\end{tabular}

Notes : 


\section{Muntiacus atberodes (Cervidae) \\ BORNEAN BARKING DEER, YELLOW MUNTJAC. KJANG KUNING}

\section{DESCRIPTION}

Medium sized herbivorous mammal; diurnal and terrestrial; smaller than $M$. muntjak; upperparts yellowish red, brown along midline, underparts yellow whitish; tiny unbranched antlers; loud barking call

\section{HABITAT}

Coastal lowland to hill forests; plantations

\section{ORIGINS/STATUS}

Borneo endemic/RI: Protected

\section{USES}

Eaten, meat sold locally or to traders (ALS, KLK, KLM, KLO, KLA, KAL, KBD, KBK, PEB); antlers kept as trophies or sold to traders; skin for hats (PUT, ALS, LIT, KLK); omen animal (KBD, KBK)

\section{REFERENCES}

Payne et al. 1985: 299; Corbet and Hill 1992: 261

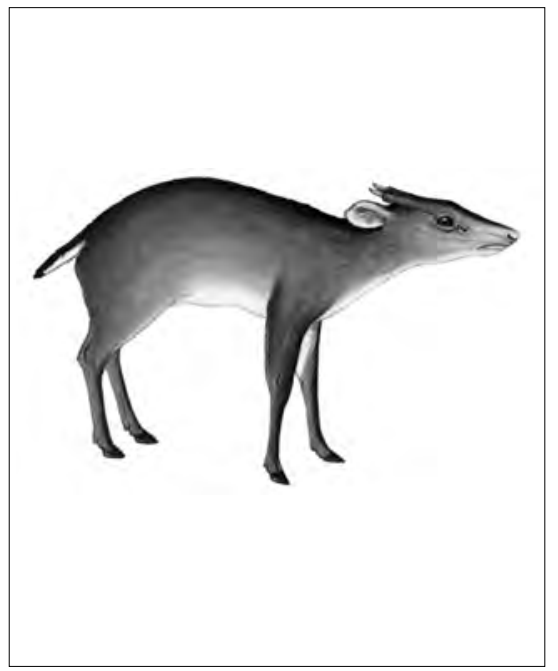

\begin{tabular}{|c|c|c|c|c|c|}
\hline \# & Code & Local Name & Use & RU & Hab.-Ab. \\
\hline 1 & PUT & telau, telo' beli' & $1,4,6$ & $\mathrm{v}$ & P-1 \\
\hline 2 & PUB & telo tawin, telo jemit, tela'o jemit & 1,6 & $\mathrm{v}$ & P-1 \\
\hline 3 & PUD & telo' bala & 1,6 & $\mathrm{v}$ & $\mathrm{Y}-4, \mathrm{O}-4$ \\
\hline 4 & PUM & telo pawen & 1,6 & $\mathrm{v}$ & G-1, S-1, Y-4, O-4, P-4 \\
\hline 5 & ALS & faus ria' & $1,4,6,8$ & $\mathrm{v}$ & $\mathrm{Y}-4, \mathrm{O}-4, \mathrm{P}-4$ \\
\hline 6 & LDY & talau, talao & $1,6,8$ & v & $\mathrm{S}-3, \mathrm{Y}-4, \mathrm{O}-4, \mathrm{P}-4$ \\
\hline 7 & LIT & tela'u, tela'u sia & $1,4,6$ & $x$ & $\mathrm{P}-4$ \\
\hline 8 & BRU & telau ngian & 1,6 & v & $\mathrm{Y}-4, \mathrm{O}-4, \mathrm{P}-4$ \\
\hline 9 & MRP & telau mela, tela' au mela & $1,6,8$ & $x$ & $\mathrm{O}-1, \mathrm{P}-1$ \\
\hline 10 & PTN & usa' & 1,6 & $x$ & $\mathrm{O}-1, \mathrm{P}-1$ \\
\hline 11 & KLK & uca' ano & 1,4 & $\mathrm{v}$ & $\mathrm{S}-3, \mathrm{O}-3, \mathrm{P}-3, \mathrm{M}-1$ \\
\hline 12 & KLM & oca' bala & $1,6,8$ & $x$ & $\mathrm{~S}-3, \mathrm{Y}-3, \mathrm{O}-3, \mathrm{P}-2$ \\
\hline 13 & KLO & tela'o & 1 & $x$ & $\mathrm{~S}-2, \mathrm{Y}-4, \mathrm{O}-4$ \\
\hline 14 & KLA & tela'o & 1 & $x$ & $\mathrm{~S}-2, \mathrm{Y}-2, \mathrm{O}-3$ \\
\hline 15 & KAL & tela'o bala & 1 & $x$ & $\mathrm{~S}-3, \mathrm{Y}-3, \mathrm{O}-4$ \\
\hline 16 & KBD & osa' & $1,5,6,8$ & $x$ & $\mathrm{~S}-4, \mathrm{Y}-4, \mathrm{O}-4$ \\
\hline 17 & KBK & uca' & $1,5,6,8$ & $x$ & P-4, M-2 \\
\hline 18 & PEB & telau loti & $1,3,8$ & $x$ & P-2, M-2 \\
\hline
\end{tabular}

Notes : 


\section{Cervus unicolor (Cervidae)}

\section{DESCRIPTION}

Large herbivorous mammal, to $2 \mathrm{~m}$ long; nocturnal, terrestrial; upperparts grey brown, some red, darker along midline; underparts pale brown to white; tail bushy, black; antlers to $60 \mathrm{~cm}$; call is a yelp or shrill

\section{HABITAT}

Lowland to hill forests; plantations, swiddens and village gardens; visits salt/mineral springs; increasingly rare (ALS, BRU, MRP, KLK)

\section{ORIGINS/STATUS}

India to Southeast Asia/RI: Protected

\section{USES}

Eaten, meat sold locally or to traders; antlers for tools (PUT, PTN, KLK, PEB), medicine (MRP, KBD, $\mathrm{KBK}$ ), trophies, or sold; skin for clothes (LIT, KBD, $\mathrm{KLK})$; traditionally a prohibited food (PEB); antlers used to protect against lightening strikes and fires (MRP)

\section{REFERENCES}

Payne et al. 1985: 299; Corbet and Hill 1992: 256

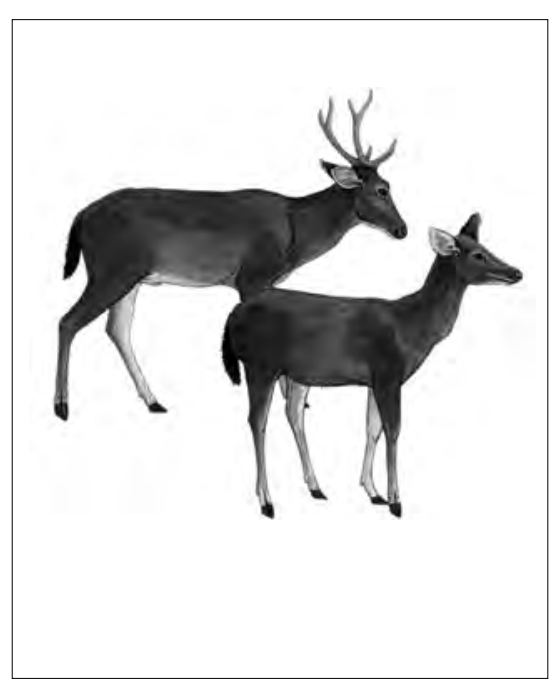

\begin{tabular}{|c|c|c|c|c|c|c|}
\hline & \# & Code & Local Name & Use & RU & Hab.-Ab. \\
\hline & 1 & PUT & payo & $1,3,6,8$ & $x$ & $\mathrm{G}-1, \mathrm{~S}-3, \mathrm{Y}-31 \mathrm{O}-3, \mathrm{P}-3, \mathrm{M}-$ \\
\hline & 2 & PUB & payo & $1,6,8$ & v & $\mathrm{S}-3, \mathrm{Y}-3, \mathrm{O}-3, \mathrm{P}-3$ \\
\hline & 3 & PUD & payo & $1,5,6,8$ & $\mathbf{v}$ & ALL-4 \\
\hline & 4 & PUM & payo & $1,2,6,8$ & v & ALL-4 \\
\hline & 5 & ALS & temang & $1,6,8$ & v & ALL-4 \\
\hline & 6 & LDY & fayo & $1,6,8$ & v & $\mathrm{S}-3, \mathrm{G}-4, \mathrm{O}-4, \mathrm{P}-4$ \\
\hline & 7 & LIT & payau & $1,4,6$ & V & $\mathrm{G}-1, \mathrm{~S}-1, \mathrm{P}-3, \mathrm{O}-4, \mathrm{P}-4$ \\
\hline & 8 & BRU & payau agon & 1,6 & v & - \\
\hline & 9 & MRP & payau & $1,2,6,8$ & $\mathbf{v}$ & $\mathrm{S}-4, \mathrm{Y}-4, \mathrm{O}-4, \mathrm{P}-4$ \\
\hline & 10 & PTN & payo & $1,3,5,6$ & $\mathrm{~V}$ & $\mathrm{Y}-2, \mathrm{O}-2, \mathrm{P}-2$ \\
\hline & 11 & KLK & payau, payo & $1,3,5,6,8$ & v & $\mathrm{S}-3, \mathrm{Y}-2, \mathrm{Y}-2, \mathrm{P}-1$ \\
\hline \multirow{7}{*}{10} & 12 & KLM & payau & $1,6,8$ & v & S-4, Y-4, O-4, P-4 \\
\hline & 13 & KLO & fazo & $1,6,8$ & v & G-2, S-2, Y-3, O-4, P-4 \\
\hline & 14 & KLA & fazo, payo & $1,6,8$ & v & $\mathrm{S}-3, \mathrm{Y}-4, \mathrm{O}-4, \mathrm{P}-4, \mathrm{M}-2$ \\
\hline & 15 & KAL & fazo, pazo & $1,6,8$ & V & G-1, S-4, Y-4, O-4, P-4, M-1 \\
\hline & 16 & KBD & payou & $1,2,4,5,6,8$ & v & $\mathrm{S}-4, \mathrm{Y}-4, \mathrm{O}-4, \mathrm{P}-4$ \\
\hline & 17 & KBK & payau & $1,2,4,5,6,8$ & $x$ & G-1, S-2, Y-2, O-3, P-4, M-2 \\
\hline & 18 & PEB & lage & $1,3,8$ & v & $\mathrm{G}-1, \mathrm{~S}-4, \mathrm{Y}-4, \mathrm{O}-4, \mathrm{P}-4$ \\
\hline
\end{tabular}

Notes : 


\section{Bosjavanicus (Bovidae)}

\section{BANTENG. SAPI BANTENG}

\section{DESCRIPTION}

Large herbivorous mammal, males to $1.7 \mathrm{~m}$ at shoulder height; diurnal, terrestrial; males blackish, adult females and young males reddish brown, both sexes with white buttocks and white lower part of legs; large horns

\section{HABITAT}

Lowland to hill primary forests; swampy areas, grasslands, decidous forest; visits salt springs; rare or locally absent in most of Bulungan

\section{ORIGINS/STATUS}

Southeast Asia/RI: Protected

\section{USES}

Eaten, meat sold locally (ALS, LDY, KLK, KLM); horns kepts as trophies or sold (ALS, LDY, KLK, KLM); skin for clothes (KLM) and ritual costumes $(\mathrm{KBD}, \mathrm{KBK})$

\section{REFERENCES}

Payne et al. 1985: 300; Corbet and Hill 1992: 264

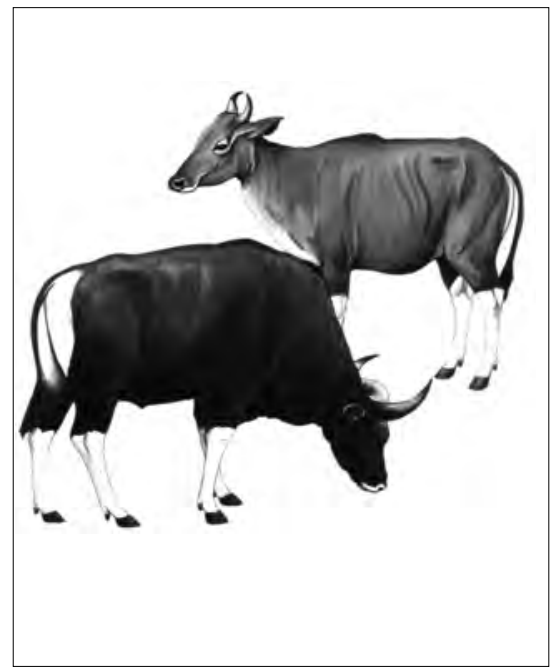

\begin{tabular}{|c|c|l|c|c|c|}
\hline$\#$ & Code & \multicolumn{1}{|c|}{ Local Name } & Use & RU & Hab.-Ab. \\
\hline 1 & PUT & kelesiau, kelecau & & $\mathbf{X}$ & \\
\hline 2 & PUB & kelecau, kelesau & & $\mathbf{X}$ & \\
\hline 3 & PUD & kelecau & & $\mathbf{X}$ & \\
\hline 4 & PUM & lekiau & $\mathbf{X}$ & \\
\hline 5 & ALS & kelenjiu & $1,6,8$ & $\mathbf{X}$ & \\
\hline 6 & LDY & kalio & 1,6 & $\mathbf{X}$ & P-1, M-1 \\
\hline 7 & LIT & keliau & 1,6 & $\mathbf{X}$ & P-1 \\
\hline 8 & BRU & keliau & 1,6 & $\mathbf{X}$ & \\
\hline 9 & MRP & kelehio, kelehiao, kelehiau & 1,6 & $\mathbf{X}$ & P-1 \\
\hline 10 & PTN & kelesio & $1,6,8$ & $\mathbf{X}$ & \\
\hline 11 & KLK & kelesiau & $1,4,6,8$ & $\mathbf{X}$ & \\
\hline 12 & KLM & kelesiau & 1,6 & $\mathbf{X}$ & \\
\hline 13 & KLO & kelesezo & 1,6 & $\mathbf{X}$ & \\
\hline 14 & KLA & lesizau, kelesizau & 1,6 & $\mathbf{X}$ & \\
\hline 15 & KAL & lesezo, kelesezau & $1,5,6,8$ & $\mathbf{X}$ & \\
\hline 16 & KBD & kelesiau & $1,5,6,8$ & $\mathbf{X}$ & \\
\hline 17 & KBK & kelesiau & & $\mathbf{X}$ & \\
\hline 18 & PEB & kelio & & \\
\hline
\end{tabular}

Notes : 


\section{Bos taurus (Bovidae) \\ DOMESTIC CATTLE. SAPI TERNAK}

\section{DESCRIPTION}

Large herbivorous domesticated cattle; confined to village areas; hump above shoulders; no white rump and horns also shorter, thicker and straighter than B. javanicus

\section{HABITAT}

Human settlements; rare or locally absent

\section{ORIGINS/STATUS}

Asia/Unprotected

\section{USES}

Eaten and sold locally (PUT, PUM, ALS, LDY, LIT, BRU, MRP)

\section{REFERENCES}

Payne et al. 1985: 301; Corbet and Hill 1992: 263

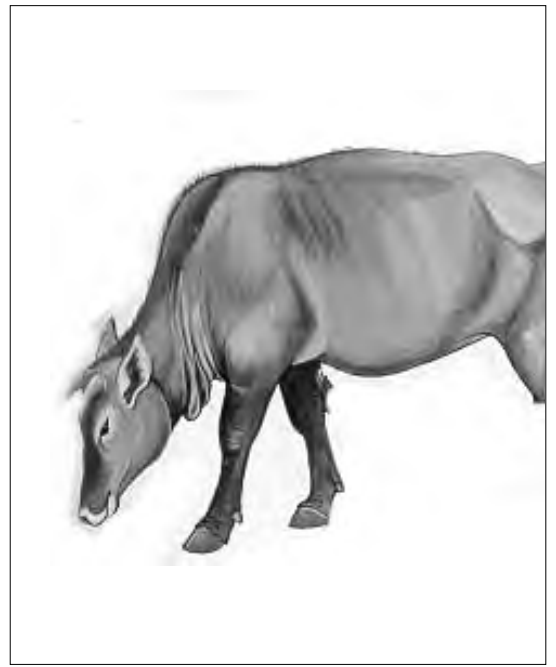

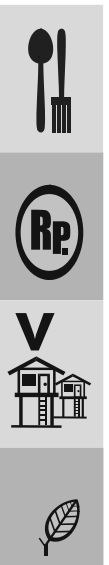

\begin{tabular}{|c|c|c|c|c|c|}
\hline \# & Code & Local Name & Use & RU & Hab.-Ab. \\
\hline 1 & PUT & sapi', sapi & 1 & $x$ & \\
\hline 2 & PUB & sapi & 1 & $x$ & \\
\hline 3 & PUD & sapi' & & $x$ & \\
\hline 4 & PUM & sapi' & 1 & $x$ & \\
\hline 5 & ALS & savi' & $1,8,9$ & $x$ & \\
\hline 6 & LDY & safi', safi & $1,8,9$ & $x$ & $\mathrm{~V}-1$ \\
\hline 7 & LIT & sapi', sapei' & $1,8,9$ & $x$ & $\mathrm{~V}-1$ \\
\hline 8 & BRU & sapei & $1,8,9$ & $x$ & \\
\hline 9 & MRP & sapei, sapi & $1,8,9$ & $x$ & \\
\hline 10 & PTN & sapi' & $1,8,9$ & $\mathrm{x}$ & \\
\hline 11 & KLK & sapei, sapi, pebaki uma & & $x$ & \\
\hline 12 & KLM & sapi & & $x$ & \\
\hline 13 & KLO & sapi & & $x$ & \\
\hline 14 & KLA & sapi & & $x$ & \\
\hline 15 & KAL & sapi & & $x$ & \\
\hline 16 & KBD & sapi & & $x$ & \\
\hline 17 & KBK & sapi & & $x$ & \\
\hline 18 & PEB & sapi & & $x$ & \\
\hline
\end{tabular}

Notes : 


\section{Capra bircus (Bovidae)}

\section{DOMESTIC GOAT. KAMBING}

\section{DESCRIPTION}

Medium sized herbivorous domesticated mammal, confined to village areas, coastal and inland; body uneven pattern of brown and white; horns curved backwards

\section{HABITAT}

Human settlements; rare or locally absent in the interior villages, but more common in coastal towns and cities

\section{ORIGINS/STATUS}

Turkey, Middle East to Central Asia/Unprotected

\section{USES}

Occasionally raised and sold to traders; skin for ritual clothing (KLO, KLA, KAL, KBD, KBK)

\section{REFERENCES}

Payne et al. 1985: 155, 301; Corbet and Hill 1992: 273

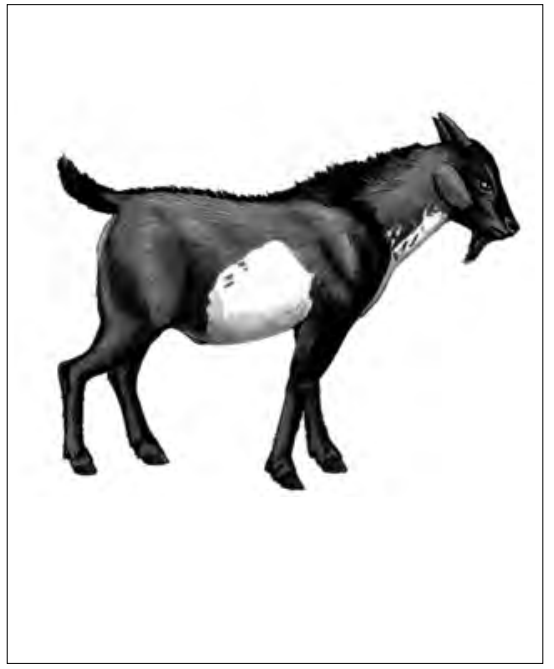

\begin{tabular}{|c|c|l|c|c|c|}
\hline$\#$ & Code & Local Name & Use & RU & Hab.-Ab. \\
\hline 1 & PUT & kambing & & $\mathbf{X}$ & \\
\hline 2 & PUB & kambing, kabing & & $\mathbf{X}$ & \\
\hline 3 & PUD & kading & & $\mathbf{X}$ & \\
\hline 4 & PUM & kambing & & $\mathbf{X}$ & \\
\hline 5 & ALS & kambing & & $\mathbf{X}$ & \\
\hline 6 & LDY & amek, mek & & $\mathbf{X}$ & \\
\hline 7 & LIT & mek & & $\mathbf{X}$ & \\
\hline 8 & BRU & meak & & $\mathbf{X}$ & \\
\hline 9 & MRP & kadaie & & $\mathbf{X}$ & \\
\hline 10 & PTN & ka'ding & & $\mathbf{X}$ & \\
\hline 11 & KLK & kading & $1,8,9,4$ & $\mathbf{X}$ & \\
\hline 12 & KLM & kadieng & $1,8,9,4$ & $\mathbf{X}$ & \\
\hline 13 & KLO & kadieng & $1,8,9,4$ & $\mathbf{X}$ & $\mathrm{V}$ \\
\hline 14 & KLA & kadieng & $1,8,9,4$ & $\mathbf{X}$ & \\
\hline 15 & KAL & kadieng & $1,8,9,4$ & $\mathbf{X}$ & $\mathrm{V}$ \\
\hline 16 & KBD & kadeng & $1,8,9,4$ & $\mathbf{X}$ & $\mathrm{V}$ \\
\hline 17 & KBK & kadeng & & $\mathbf{X}$ & $\mathrm{V}$ \\
\hline 18 & PEB & kadeng & & \\
\hline
\end{tabular}

Notes : 


\section{Haliastur indus (Accipitridae) \\ BRAHMINY KITE. ELANG BONDOL}

\section{DESCRIPTION}

Medium sized raptor, $45 \mathrm{~cm}$, adult reddish chestnut with white

head; long rounded tail; call shrill, a catlike mew

HABITAT

Coastal to hill forests; large rivers and swamps; often near

human settlements; to $3000 \mathrm{~m}$

\section{ORIGINS/STATUS}

Asia-Pacific/CITES: Appendix II; RI: Protected

UsES

Traditional omen bird (ALS, BRU, Kenyah groups); preys on chickens

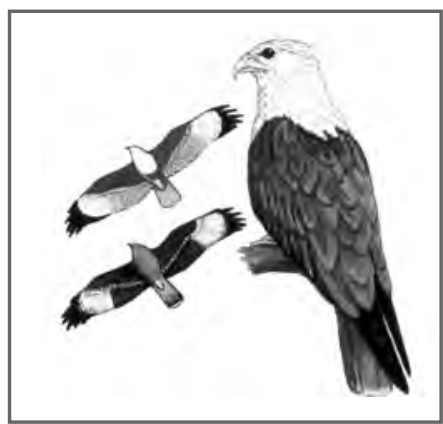

REFERENCES

Smythies 1981: 43; MacKinnon and Phillipps 1993: 85

\section{Lopbura bulweri (Phasianidae)}

BULWER'S PHEASANT. SEMPIDAN KALIMANTAN

\section{DESCRIPTION}

Endemic pheasant, $50-75 \mathrm{~cm}$; male is black with pure white tail and blue head, female brown with blue facial skin; red ring around red eye, reddish legs; call a loud 'bek kia!'

\section{Habitat}

Primary and old secondary forest

ORIGINS/STATUS

Borneo endemic/RI: Protected

\section{USES}

Eaten; feathers sold; indicator of bearded pigs

\section{REFERENCES}

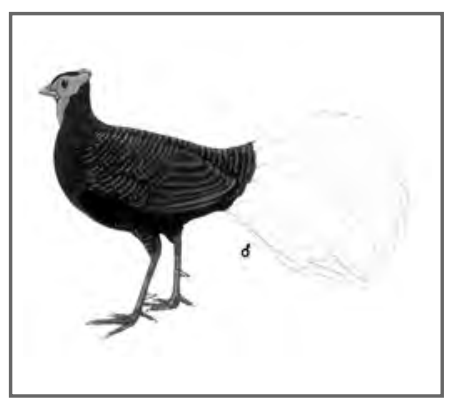

Smythies 1981: 69; MacKinnon and Phillipps 1993: 107

\begin{tabular}{|r|l|l|l|}
\hline$\#$ & Code & \multicolumn{1}{|c|}{ Haliastur indus } & \multicolumn{1}{|c|}{ Lophura bulweri } \\
\hline 1 & PUT & nyau & belengyu, titiau, belengiu \\
\hline 2 & PUB & nyau & belengiu, caium \\
\hline 3 & PUD & nyau & belengiu \\
\hline 4 & PUM & nyau & belengyu \\
\hline 5 & ALS & kanui & fiak \\
\hline 6 & LDY & kanui balang, kanwi balang & kepiak, kefiak \\
\hline 7 & LIT & keniu, kenieu & tepia' \\
\hline 8 & BRU & neo & bongai \\
\hline 9 & MRP & nyeou, kunyau mbla, nyau & buai, boai, buwai \\
\hline 10 & PTN & pelaki & pengeng \\
\hline 11 & KLK & pelaki & bengiau, ubang i'au \\
\hline 12 & KLM & pelaki & bengai'i \\
\hline 13 & KLO & felaghi & obang a'ieng \\
\hline 14 & KLA & felahi, pelahi abung & ubang a'ieng \\
\hline 15 & KAL & kabung & obang aieng \\
\hline 16 & KBD & pelaki & bengau \\
\hline 17 & KBK & pelaki & bangiyeu \\
\hline 18 & PEB & pelakei atom & belengiu \\
\hline
\end{tabular}


Gallus sp. (Phasianidae)

\section{DOMESTICATED CHICGEN. AYAM KAMPUNG}

\section{DESCRIPTION}

Medium sized domesticated fowl, variable colorings from

black, white to reddish brown and mixed, males with red wattles; yellow legs

\section{HABITAT}

Human settlements

\section{ORIGINS/STATUS}

Southeast Asia/RI: Unprotected

\section{USES}

Eaten, often for ceremonies; used for compensation, settling disputes; sold locally and in coastal markets

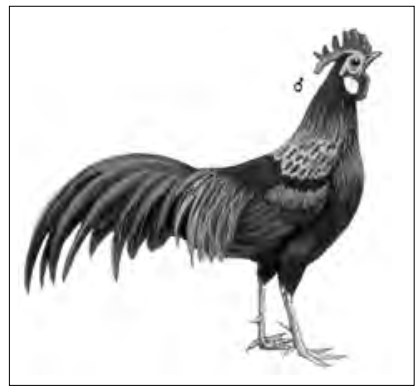

\section{REFERENCES}

MacKinnon and Phillipps 1993: 108

\section{Argusianus argus (Phasianidae) \\ GREAT ARGUS PHEASANT. KUAU RAJA}

\section{DESCRIPTION}

Large pheasant, 75-190 cm; dark brown; chestnut breast, dark tail, bare blue skin on face, neck; male elongated wing, tail feathers, eye-spot pattern; small crest; call a loud 'ku wow!'

\section{HABITAT}

Lowland and hill primary forests; display clearing on ridge tops

\section{ORIGINS/STATUS}

Southeast Asia/CITES: Appendix II; RI: Protected

\section{USES}

Eaten, sold locally; feathers sold (KAL, KBD, PEB)

\section{REFERENCES}

Smythies 1981: 72; MacKinnon and Phillipps 1993: 110

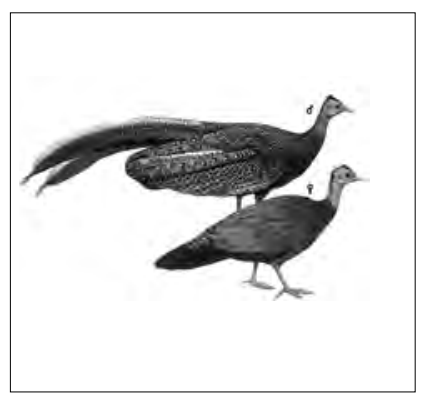

\begin{tabular}{|c|l|l|l}
\hline$\#$ & Code & \multicolumn{1}{c|}{ Gallus sp. } & \multicolumn{1}{c}{ Argusianus argus } \\
\hline 1 & PUT & yoh, yo & owae, owei \\
\hline 2 & PUB & yo & owei, owai \\
\hline 3 & PUD & yo & owai \\
\hline 4 & PUM & yoh & owie \\
\hline 5 & ALS & manok & tuwoh \\
\hline 6 & LDY & lal & tuwau \\
\hline 7 & LIT & la'al & tuwau \\
\hline 8 & BRU & al & kuwau \\
\hline 9 & MRP & he'a, hea, hihe & kwai, kuwai \\
\hline 10 & PTN & iyap & kuwe \\
\hline 11 & KLK & iyap & kuwei, kuwai \\
\hline 12 & KLM & iyap & kuwai, kuwei \\
\hline 13 & KLO & za' & kuvei \\
\hline 14 & KLA & izap & huvai, kuvai \\
\hline 15 & KAL & izap & kevai \\
\hline 16 & KBD & iyap & kuwai \\
\hline 17 & KBK & iyap & kuwae \\
\hline 18 & PEB & dek & kuwai \\
\hline
\end{tabular}




\section{Lophura ignita (Phasianidae)}

CRESTED FIREBACK. SEMPIDAN BIRU

\section{DESCRIPTION}

Large pheasant, $55 \mathrm{~cm}$; male deep shiny blue black, buff tail feathers; red abdomen, blue facial skin; female bright chestnut brown, scaled whitish below, dull blue facial skin; both with whitish flesh colored legs and bobble crest

\section{HABITAT}

Lowland and hill primary forest; riverine

\section{ORIGINS/STATUS}

Southeast Asia/CITES: Appendix III (Malaysia)

USES

Eaten; feathers and juveniles sold locally

REFERENCES

Smythies 1981: 68; MacKinnon and Phillipps 1993: 107

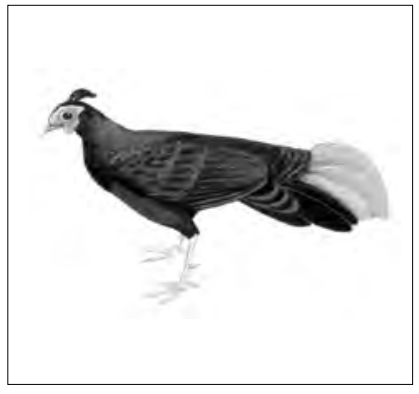

\section{Treron curvirostra (Columbidae)}

\section{THICK-BILLED GREEN PIGEON. PUNAI LENGGUAK}

\section{DESCRIPTION}

Medium sized flocking pigeon, $27 \mathrm{~cm}$, bright thick, yellow green bill with red base, blue green skin around eye, red feet, grey cap; male maroon wings and back, female with green wings and back

\section{HABITAT}

Mangrove to lower montane forest; visits salt/mineral springs

\section{ORIGINS/STATUS}

Southeast Asia/Unprotected

\section{USES}

Eaten (PUM, KLK, KLM, KBD, PEB)

\section{REFERENCES}

Smythies 1981: 126; MacKinnon and Phillipps 1993: 159

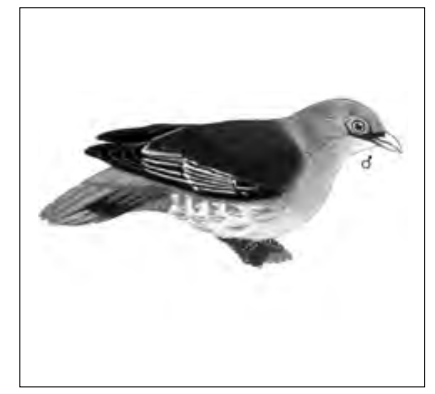

\begin{tabular}{|c|l|l|l}
\hline$\#$ & Code & \multicolumn{1}{|c}{ Lophura ignita } & \multicolumn{1}{c}{ Treron curvirostra } \\
\hline 1 & PUT & landu, lando, lantu & kunae, bangko' \\
\hline 2 & PUB & manok lung & terekuku, belekuken \\
\hline 3 & PUD & lantu & balut \\
\hline 4 & PUM & landu & kunei \\
\hline 5 & ALS & bolun & tawan \\
\hline 6 & LDY & baulun & limun \\
\hline 7 & LIT & bung iyau, tepiak & uwit main, suwit main \\
\hline 8 & BRU & mano' long & mano' lemun \\
\hline 9 & MRP & manau lawe, manau laue & lekun, tenggem, manau tengai \\
\hline 10 & PTN & manok long & tok tu \\
\hline 11 & KLK & manok lung & punai mba' \\
\hline 12 & KLM & manok long & punai mba' \\
\hline 13 & KLO & manok lung & funai tano \\
\hline 14 & KLA & manok lung & funai \\
\hline 15 & KAL & manok lung & pekkem \\
\hline 16 & KBD & manok lung & punai \\
\hline 17 & KBK & manok payau & punai \\
\hline 18 & PEB & data & kuni \\
\hline
\end{tabular}


Loriculus galgulus (Psittacidae)

BLUE-CROWNED HANGING PARROT. SERINDIT MELAYU

DESCRIPTION

Small hanging parrot, to $14 \mathrm{~cm}$, brilliant green with scarlet breast and rump patches, orange yellow back and black bill, blue crown is inconspicuous; female is green with black bill and red rump

\section{HABITAT}

Lowland to hill primary forests to $1100 \mathrm{~m}$; high canopy

\section{ORIGINS/STATUS}

Malaysia and western Indonesia/CITES: Appendix II

\section{USES}

Pet; sold locally and to traders

\section{REFERENCES}

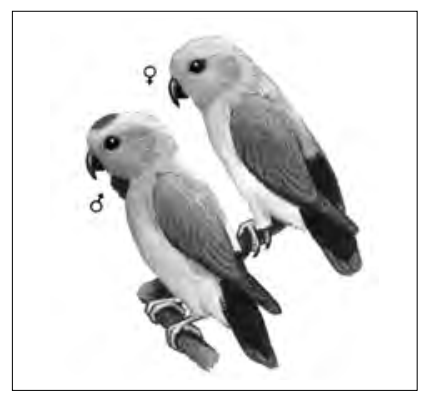

Smythies 1981: 145; MacKinnon and Phillipps 1993: 174

\section{Collocalia fuciphaga subsp. vestita (Apodidae) BROWN-RUMPED SWIFTLET. WALET SARANG PUTIH}

\section{DESCRIPTION}

Smallish cave swiflet, to $12 \mathrm{~cm}$; tail slightly forked; upperparts blackish brown with dark bown rump; underparts brown; iris dark brown; bill black; feet black; high pitched tscheerrr, produces valuable 'white' nests from saliva

\section{HABITAT}

Limestone cave dwellings in coastal to hill forest, to $2800 \mathrm{~m}$

\section{ORIGINS/STATUS}

Sumatra and Borneo/Unprotected

\section{USES}

Nests sold to traders for export (KBD, MRP, PUD, PUT)

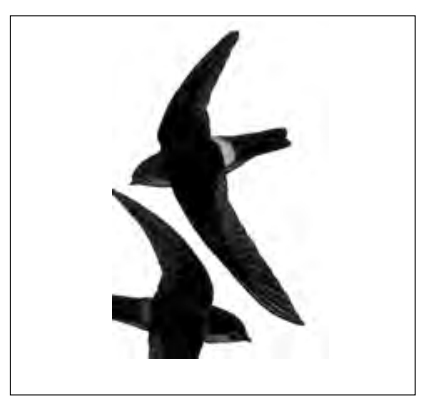

\section{REFERENCES}

Smythies 1981: 189; MacKinnon and Phillipps 1993: 202

\begin{tabular}{|c|c|l|l}
\hline$\#$ & Code & \multicolumn{1}{|c|}{ Loriculus galgulus } & \multicolumn{1}{c}{ Collocalia fuciphaga subsp. vestita } \\
\hline 1 & PUT & tenggih, tengkih & tefilih, tepilih \\
\hline 2 & PUB & tengkikih, tengkih & tepilih \\
\hline 3 & PUD & janae tengkih & tepilih \\
\hline 4 & PUM & tenggih & tefili \\
\hline 5 & ALS & fiakir & selangan \\
\hline 6 & LDY & piakir, fiakir & selalangan, tetayan \\
\hline 7 & LIT & serindit & tetayan, pele lua \\
\hline 8 & BRU & mano' piau & longawie \\
\hline 9 & MRP & tengkai, tenggai, tanggai & teplai, tepili, tepli' \\
\hline 10 & PTN & kikeng & ule batat \\
\hline 11 & KLK & kikeng & uleh bilung, uleh \\
\hline 12 & KLM & kekieng & ole \\
\hline 13 & KLO & keghieng & \\
\hline 14 & KLA & kehieng & \\
\hline 15 & KAL & kehieng & ole vilung \\
\hline 16 & KBD & kekeng & ole \\
\hline 17 & KBK & tiking & ule \\
\hline 18 & PEB & kiking & tevilih \\
\hline
\end{tabular}




\section{Harpactes duvaucelii (Trogonidae) \\ SCARLET-RUMPED TROGON. LUNTUR PUTRI}

\section{DESCRIPTION}

Medium sized trogon, $24 \mathrm{~cm}$; male has black head, scarlet breast and abdomen, cinnamon brown back, scarlet patch on rump, bright blue skin above eye, deeper blue bill; female mostly pink

\section{Habitat}

Lowland and hill primary forest to $600 \mathrm{~m}$; low to mid storey

\section{ORIGINS/STATUS}

Malyasia and western Indonesia/Unprotected

USES

Eaten (KLM); omen bird (ALS, KAL, KBD)

REFERENCES

Smythies 1981: 199; MacKinnon and Phillipps 1993: 211

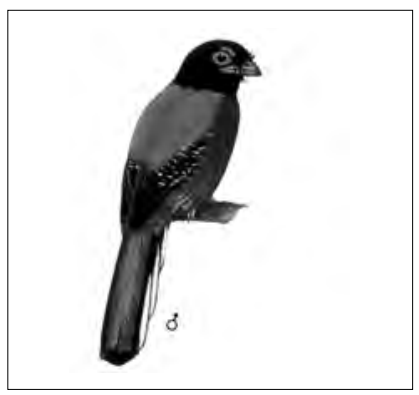

\section{Pelargopsis capensis (Alcedinidae) STORK-BILLED KINGFISHER. PEKAKA EMAS}

\section{DESCRIPTION}

Large kingfisher, $37 \mathrm{~cm}$; large red bill, head brown, yellow brown breast and abdomen, blue wings with bright blue stripe on back; longish tail; call a loud 'kow-koo'

\section{HABITAT}

Lowland and hill primary forest; human settlements; large rivers

\section{ORIGINS/STATUS}

South-Southeast Asia/RI: Protected

\section{USES}

Eaten (KLM); pest, raids fish ponds (KLK, KLM, KBD)

\section{REFERENCES}

Smythies 1981: 206; MacKinnon and Phillipps 1993: 215

\begin{tabular}{|c|l|l|l}
\hline \# & Code & \multicolumn{1}{|c}{ Harpactes duvaucelii } & \multicolumn{1}{|c}{ Pelargopsis capensis } \\
\hline 1 & PUT & upau meroh, upau & tekuan hah \\
\hline 2 & PUB & upau & tekuan hah \\
\hline 3 & PUD & upau & tekuan hah \\
\hline 4 & PUM & upau & tekuan hah \\
\hline 5 & ALS & koringan & sangang gasa' \\
\hline 6 & LDY & bayur, banyur & menangan ra' \\
\hline 7 & LIT & suwit apoi & menangan ra', meno 'ok \\
\hline 8 & BRU & sigo & mengang la' \\
\hline 9 & MRP & belera', manau hopoe & tekewa hah, tokuan aha, tekwang ha \\
\hline 10 & PTN & mba' & temengang asa' \\
\hline 11 & KLK & upau, upou & temengang asa' \\
\hline 12 & KLM & pengolong, opau & temengang asa' \\
\hline 13 & KLO & & \\
\hline 14 & KLA & & \\
\hline 15 & KAL & ofao & temengang asa' \\
\hline 16 & KBD & upau & temengang asa' \\
\hline 17 & KBK & upau & temengang asa' \\
\hline 18 & PEB & epau & belangang asa' \\
\hline
\end{tabular}


Buceros rbinoceros (Bucerotidae)

RHINOCEROS HORNBILL. RANGKONG PAPAN

DESCRIPTION

Large territorial hornbill, 90-120 cm; black body, white abdomen; tail with wide black band; wings black; bill yellow with red base, casque with yellow upturned tip; call in flight a male-female duet of 'kronk krank'

HABITAT

Lowlands to lower montane primary forests; high canopy

ORIGINS/STATUS

Southeast Asia/CITES: Appendix II; RI: Protected

USES

Eaten; feathers, casque for ritual, decoration, sold locally; juveniles sold locally

REFERENCES

Smythies 1981: 222; MacKinnon and Phillipps 1993: 225

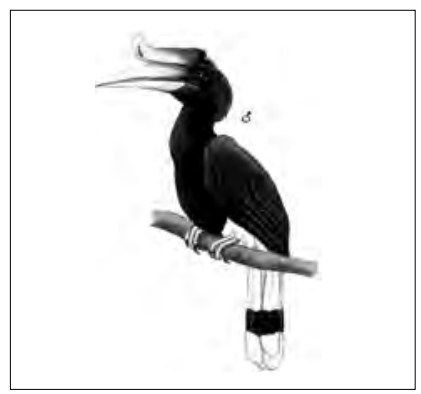

\section{Buceros vigil (Bucerotidae)}

HELMETED HORNBILL. RANGKONG GADING

\section{DESCRIPTION}

Large territorial hornbill, $125 \mathrm{~cm}$; two central tail feathers to $70 \mathrm{~cm}$; body brown, neck red; short red casque yellow end; tail white with black band toward end; call 'took' leading to climax laugh of 4-6 notes HABITAT

Lowlands to lower montane primary forests; high canopy

ORIGINS/STATUS

Malaysia and western Indonesia/CITES: Appendix I; RI: Protected

USES

Eaten; feathers, casque for ritual, decoration; juveniles sold locally REFERENCES

Smythies 1981: 223; MacKinnon and Phillipps 1993: 227

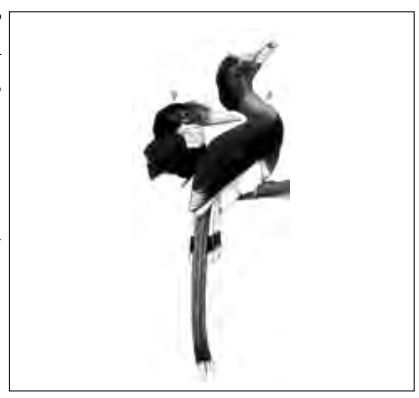

\begin{tabular}{|c|c|l|l}
\hline$\#$ & Code & \multicolumn{1}{|c|}{ Buceros rbinoceros } & \multicolumn{1}{c}{ Buceros vigil } \\
\hline 1 & PUT & tekuan & pecaku \\
\hline 2 & PUB & tekuan & pecaku, pesaku \\
\hline 3 & PUD & tekuan & pesaku \\
\hline 4 & PUM & tekuan & pesaku \\
\hline 5 & ALS & sangang & tendudun \\
\hline 6 & LDY & menangan & menudun \\
\hline 7 & LIT & menangan, menengang & menedun, menudun \\
\hline 8 & BRU & mano' mengang & mano' mendun \\
\hline 9 & MRP & tekuang & tolo', tolu', talo' \\
\hline 10 & PTN & temengang & teva'un \\
\hline 11 & KLK & temengang & teba'un \\
\hline 12 & KLM & temengang & teba'un \\
\hline 13 & KLO & temengang & teva'ung \\
\hline 14 & KLA & temengang & tebaun, teva'un \\
\hline 15 & KAL & temengang & teva'un \\
\hline 16 & KBD & temengang & tebun \\
\hline 17 & KBK & temengang & tebun \\
\hline 18 & PEB & belangang & tobun \\
\hline
\end{tabular}




\section{Anorrbinus galeritus (Bucerotidae) \\ BUSHY-CRESTED HORNBILL. ENGGANG KLIHINGAN}

\section{DESCRIPTION}

Smallish gregarious hornbill, $70 \mathrm{~cm}$; body greyish black, tail paler grey, broad black tip, face and throat bare bluish skin, bill and feet blackish; call a continuous seagull-like yapping

\section{Habitat}

Lowland and hill primary and secondary forests; middle storey

ORIGINS/STATUS

Malaysia and western Indonesia/CITES: Appendix II; RI: Protected

USES

Eaten; feathers for ritual, decoration (KLM); sold locally

REFERENCES

Smythies 1981: 219; MacKinnon and Phillipps 1993: 223

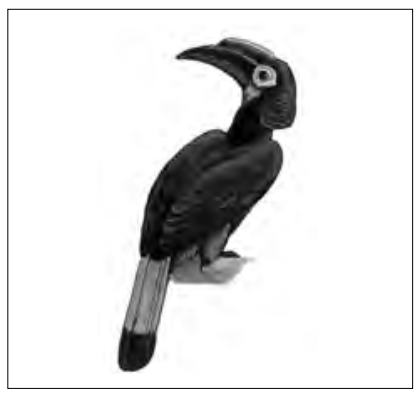

\section{Anthracoceros malayanus (Bucerotidae) \\ BLACK HORNBILL. KANGKARENG HITAM}

\section{DESCRIPTION}

Small territorial hornbill, $75 \mathrm{~cm}$; body black, tail with white corners; male bill and casque large and whitish; female bill black with some reddish facial skin; call a rasping, retching sound

HABITAT

Lowland primary and secondary forest; fragments; middle storey ORIGINS/STATUS

Malaysia and western Indonesia/CITES: Appendix II; RI: Protected USES

Eaten; feathers for ritual, decoration (KLM); sold locally

REFERENCES

Smythies 1981: 221; MacKinnon and Phillipps 1993: 225

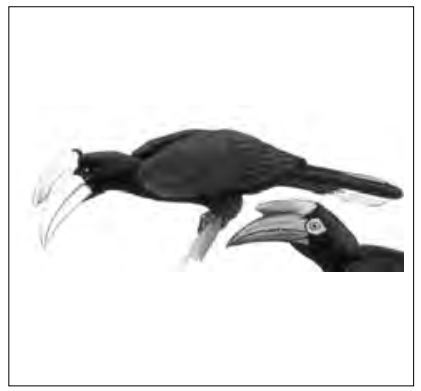

\begin{tabular}{|c|c|l|l}
\hline$\#$ & Code & \multicolumn{1}{|c}{ Anorrbinus galeritus } & \multicolumn{1}{c}{ Anthracoceros malayanus } \\
\hline 1 & PUT & lokap, lukap & berenat \\
\hline 2 & PUB & lukap & merenat \\
\hline 3 & PUD & lukap & berenat \\
\hline 4 & PUM & lukap & berenat \\
\hline 5 & ALS & lukap & beranas \\
\hline 6 & LDY & lukap & beranas, agung \\
\hline 7 & LIT & lukap & ruwek, wit baka \\
\hline 8 & BRU & mano' kehang & belenyet \\
\hline 9 & MRP & lokoa, lokua, lukua & nah \\
\hline 10 & PTN & lukap & manok uek \\
\hline 11 & KLK & lukap & manok wek \\
\hline 12 & KLM & lokap & manok wiek, manok wek \\
\hline 13 & KLO & logha' & manik vei' \\
\hline 14 & KLA & luhap & manok viek, tui \\
\hline 15 & KAL & lohap & manok uiek \\
\hline 16 & KBD & lokap & manok wek \\
\hline 17 & KBK & lukap & manok wek \\
\hline 18 & PEB & lukap & belanat \\
\hline
\end{tabular}




\section{Aceros corrugatus (Bucerotidae) \\ WRINKLED HORNBILL}

\section{DESCRIPTION}

Medium sized, $85 \mathrm{~cm}$, body black; tail white yellow, black base; male head, throat-pouch white; casque short, red; bill yellow, reddish base, lower bill raised wrinkles; female head black, throat-pouch blue

\section{HaBitat}

Coastal to lowland and hill primary forests; upper storey

Origins/STATUS

Southeast Asia/CITES: Appendix II; RI: Protected

UsES

Eaten; feathers for ritual and decoration; sold locally

REFERENCES

Smythies 1981: 219; MacKinnon and Phillipps 1993: 223

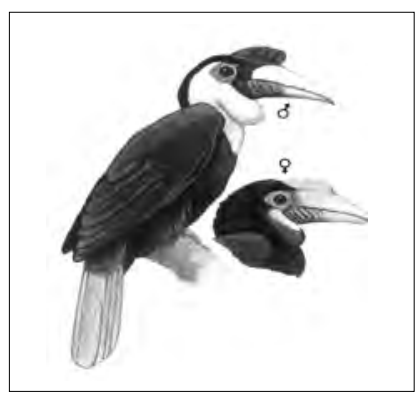

Sasia abnormis (Picidae)

RUFOUS PICULET: TUKIK TIKUS

\section{DESCRIPTION}

Tiny woodpecker, $9 \mathrm{~cm}$; upperparts olive green, underparts yellowish brown, red breast; iris red, eye-ring red; upper mandible black, lower yellow; feet orange yellow, claws yellow; call a single sharp note

\section{HABITAT}

Lowland and hill secondary forest; low-middle storey; decaying trunks

\section{ORIGINS/STATUS}

Southeast Asia/Unprotected

USES

Traditional omen bird (BRU, KLK, KLM, KLO, KLA, KAL, KBD)

\section{REFERENCES}

Smythies 1981: 232; MacKinnon and Phillipps 1993: 235

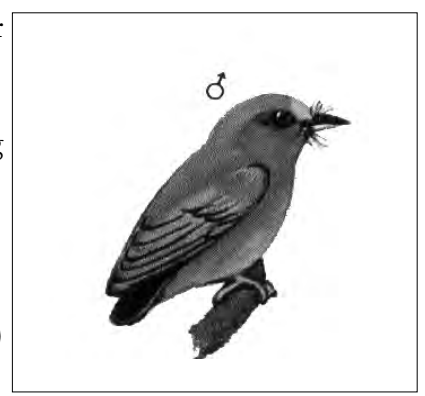

\begin{tabular}{|c|c|l|l}
\hline$\#$ & Code & \multicolumn{1}{|c|}{ Aceros corrugatus } & \multicolumn{1}{c}{ Sasia abnormis } \\
\hline 1 & PUT & metui & bukong \\
\hline 2 & PUB & metui & \\
\hline 3 & PUD & metui & \\
\hline 4 & PUM & metui & bukong \\
\hline 5 & ALS & man dui & femunod \\
\hline 6 & LDY & rod & tik badan \\
\hline 7 & LIT & metoi, rengut & belini \\
\hline 8 & BRU & mentoi & paet kwang \\
\hline 9 & MRP & tokehong, tai & tekwong, tukwung \\
\hline 10 & PTN & belok & ukeng \\
\hline 11 & KLK & toi & okeng, ukeng \\
\hline 12 & KLM & tui & okeng \\
\hline 13 & KLO & tei' & oghung \\
\hline 14 & KLA & tui, manok viek & okung \\
\hline 15 & KAL & tui & ohung \\
\hline 16 & KBD & tui & okung \\
\hline 17 & KBK & tui & okung \\
\hline 18 & PEB & motui & \\
\hline
\end{tabular}




\section{Blytbipicus rubiginosus (Picidae) MAROON WOODPECKER. PELATUK PANGKAS}

\section{DESCRIPTION}

Medium sized woodpecker, $22 \mathrm{~cm}$; body maroon brown, bill short ivory yellow, male neck with scarlet patch on back and sides; call a repetitive squeak or descending trill

\section{HABITAT}

Lowland primary forests; understorey; amongst bamboo

\section{ORIGINS/STATUS}

Malaysia and western Indonesia/Unprotected

\section{USES}

Traditional omen bird (ALS, BRU, KLM, KAL, KBD)

\section{REFERENCES}

Smythies 1981: 243; MacKinnon and Phillipps 1993: 243

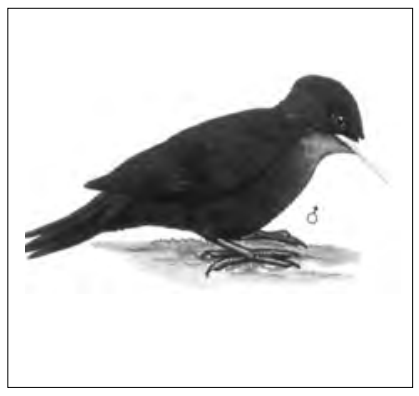

\section{Pycnonotus zeylanicus (Pycnonotidae) STRAW-HEADED BULBUL: CUCAK RAWA}

\section{DESCRIPTION}

Large bulbul, $28 \mathrm{~cm}$, body olive green and brown with light streaks, crown rufous buff with streaks, blackish moustache and mark through eye; pale throat and underparts; call a lilting rich melodious song

HABITAT

Lowland to hill forest; riverine; rare or locally absent in Bulungan ORIGINS/STATUS

Southeast Asia/CITES: Appendix II; RI: Protected

USES

Pet; sold locally and to traders

REFERENCES

Smythies 1981: 289; MacKinnon and Phillipps 1993: 268

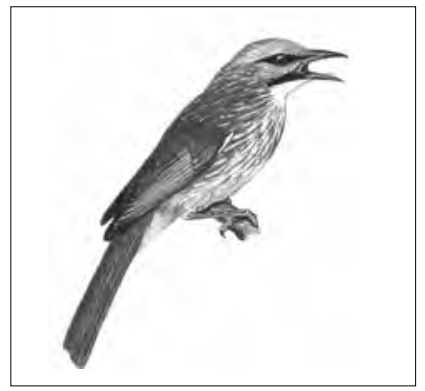

\begin{tabular}{|c|c|l|l}
\hline$\#$ & Code & \multicolumn{1}{|c|}{ Blythipicus rubiginos $\boldsymbol{~}$ S } & \multicolumn{1}{c}{ Pycnonotus zeylanicus } \\
\hline 1 & PUT & tekali, belarik, tekalih & kubikabau \\
\hline 2 & PUB & tekalih, bukong & kubikabau, janae kabau \\
\hline 3 & PUD & tekalih & kubikabau \\
\hline 4 & PUM & takali & kubik kabau \\
\hline 5 & ALS & wit & kelegiau \\
\hline 6 & LDY & fiet, tekali & kelekiau, belekiau \\
\hline 7 & LIT & tetuding, pele & bung karofa', bung karo' \\
\hline 8 & BRU & paettou & kang gagau \\
\hline 9 & MRP & pe'ik, pi'ik & manau kabau, kaibai kabau \\
\hline 10 & PTN & tunduk la'an & manok uto \\
\hline 11 & KLK & keing & belakubik \\
\hline 12 & KLM & keing, keieng & belakotau \\
\hline 13 & KLO & amet & sevi ato' \\
\hline 14 & KLA & heing, keing & helutau bai'i, lutau bai'i \\
\hline 15 & KAL & amet & ubiek ibuk \\
\hline 16 & KBD & keing & bela' kotau \\
\hline 17 & KBK & keing & bangbau \\
\hline 18 & PEB & tekewit & lebokak \\
\hline
\end{tabular}


Platylophus galericulatus (Corvidae)

CRESTED.JAY. TANGKAR ONGKLET

\section{DESCRIPTION}

Large jay, $32 \mathrm{~cm}$; body dark brown with darker head, crest standing upright, white patch on back of neck; rufous wing tips, belly paler, crest shorter in juveniles; call a long harsh machine-gun chatter

HABITAT

Lowland primary forest; middle storey

ORIGINS/STATUS

Southeast Asia/Unprotected

USES

Omen bird (ALS, BRU, KLM, KLO, KLA, KAL, KBD, KBK)

REFERENCES

Smythies 1981: 426; MacKinnon and Phillipps 1993: 286

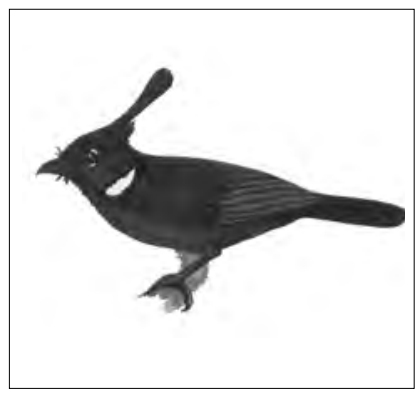

Copsychus malabaricus (Turdidae) WHITE-RUMPED SHAMA THRUSH. KUCICA

\section{DESCRIPTION}

Endemic, small territorial thrush, $20-27 \mathrm{~cm}$; head, breast and wings glossy black; abdomen rufous orange; song melodious, call while perching a single angry 'chack'

\section{HaBiTAT}

Lowland to hill forests; tall secondary forests; lower and middle storey

\section{ORIGINS/STATUS}

Borneo endemic/Unprotected

USES

Cagebird pet, sold locally and to traders (PUT, PUM, LIT, KAL)

REFERENCES

MacKinnon and Phillips 1993: 320

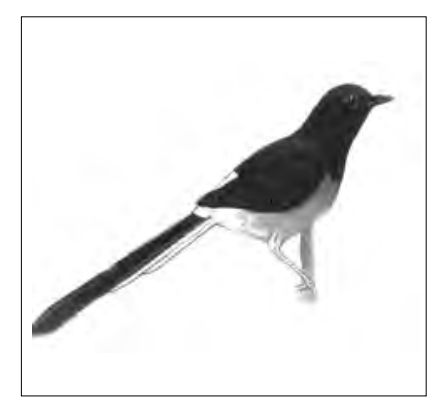

\begin{tabular}{|c|c|l|l}
\hline$\#$ & Code & \multicolumn{1}{|c}{ Platylophus galericulatus } & \multicolumn{1}{c}{ Copsychus malabaricus } \\
\hline 1 & PUT & kecang, belara, belara' & lepu'un, kilan \\
\hline 2 & PUB & telecan, telesan & kilan betelu, kilan \\
\hline 3 & PUD & telesan & kilan \\
\hline 4 & PUM & teresan & kilan \\
\hline 5 & ALS & kisit & virak folod \\
\hline 6 & LDY & belini, fier & berikara aror, belikara alor \\
\hline 7 & LIT & & bungiran aror, bungeran batu \\
\hline 8 & BRU & belnei & long kien agol \\
\hline 9 & MRP & telanjang, telajang, telanja & berai talaya, belerai telaye, berai telaya \\
\hline 10 & PTN & pi'et & manok talang \\
\hline 11 & KLK & telajan & berep talang, nya' kilang \\
\hline 12 & KLM & telajan & manok elang \\
\hline 13 & KLO & telajing & manok elang \\
\hline 14 & KLA & telajan, telajen & manok elang \\
\hline 15 & KAL & telajan & manok elang \\
\hline 16 & KBD & telajan & manok elang \\
\hline 17 & KBK & telajan & mano' kilang \\
\hline 18 & PEB & telajan & manok elang \\
\hline
\end{tabular}




\section{Gracula religiosa (Sturnidae) \\ HILL MYNA. TIONG EMAS}

DESCRIPTION

Heavy-set, large myna, $30 \mathrm{~cm}$; body black, purplish gloss; white base of primary feathers; bill orange to yellow, feet yellow, flaps of bare yellow skin on face and nape; call a ringing 'tiong'

HaBITAT

Tall lowland primary forest; forest edges; high canopy

ORIGINS/STATUS

Southeast Asia/CITES: Appendix II; RI: Protected

UsES

Traditional omen bird, sold locally and to traders (PUM, KLM, KAL, KBD, PEB); indicator of pig migration (LIT, PEB)

REFERENCES

Smythies 1981: 407; MacKinnon and Phillipps 1993: 376

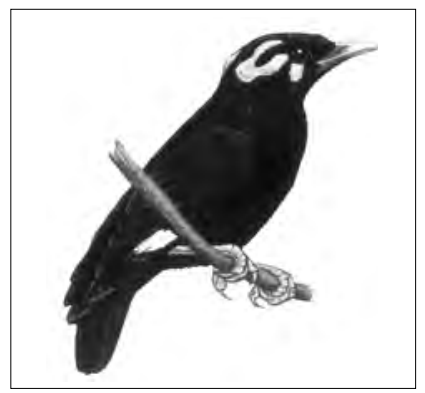

\section{Arachnotbera longirostra (Nectariniidae) \\ LITTLE SPIDERHUNTER. PIJANTUNG KEC!L}

\section{DESCRIPTION}

Tiny nectivorous spiderhunter, $16 \mathrm{~cm}$ with long curved bill; body olive green above, yellow abdomen, head grey, throat white breast olive; call a loud 'tchek' during rapid flight

HABITAT

Lowland to hill primary forest; disturbed forests; banana gardens ORIGINS/STATUS

Southeast Asia/RI: Protected

USES

Traditional omen bird (ALS, BRU, KLM, KLO, KLA, KAL, KBD, $\mathrm{KBK}, \mathrm{PEB}$ )

\section{REFERENCES}

Smythies 1981: 394; MacKinnon and Phillipps 1993: 383

\begin{tabular}{|c|c|l|l}
\hline$\#$ & Code & \multicolumn{1}{|c|}{ Gracula religiosa } & \multicolumn{1}{c}{ Arachnothera longirostra } \\
\hline 1 & PUT & kiong & ticit \\
\hline 2 & PUB & kiong & ticit \\
\hline 3 & PUD & kiung & ticit \\
\hline 4 & PUM & kiou & tisit \\
\hline 5 & ALS & kiou & sasat \\
\hline 6 & LDY & siuk, siok & mengai \\
\hline 7 & LIT & sio' & bungai \\
\hline 8 & BRU & yong & ngai' \\
\hline 9 & MRP & ki au, kiue, kiowe & ci', cik \\
\hline 10 & PTN & kiong & isit \\
\hline 11 & KLK & kiong & isit \\
\hline 12 & KLM & kiong & isit \\
\hline 13 & KLO & kezung & kisit \\
\hline 14 & KLA & kiong & isit \\
\hline 15 & KAL & kiong & isit \\
\hline 16 & KBD & kiong & isit \\
\hline 17 & KBK & kio & isit \\
\hline 18 & PEB & manok kiong & sit \\
\hline
\end{tabular}


Lonchura malacca (Ploceidae)

BLACK-HEADED MUNIA. BONDOL RAWA

DESCRIPTION

Small, granivorous, flocking, $11 \mathrm{~cm}$; head and upper breast

black; body, wings and tail rich brown; bill thick conical,

bluish grey

HabitaT

Rice swiddens, scrub, swamp areas and secondary forests

ORIGINS/STATUS

Southeast Asia/Unprotected

USES

Rice pest; eggs eaten; whole bird eaten (KLM)

REFERENCES

Smythies 1981: 416; MacKinnon and Phillipps 1993: 404

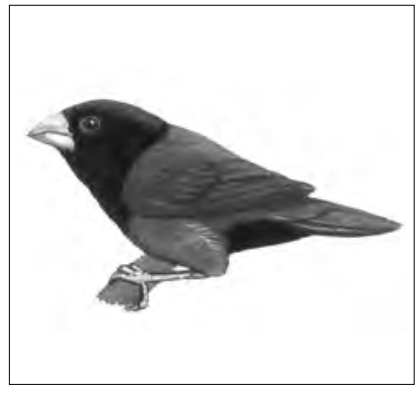

\section{Crocodylus porosus (Crocodylidae) SALT-WATER CROCODILE. BUAYA}

\section{DESCRIPTION}

Large predatory reptile, to $4 \mathrm{~m}$; terrestrial and aquatic; armored by sheets of bony plates; front feet smaller, unwebbed with five digits; hindfeet webbed with four digits; digs nests in soils

HABitat

Larger rivers, coastal, mangroves; but also reported far inland ORIGINS/STATUS

Asia; western Pacific/CITES: Appendix II

\section{USES}

Eaten (ALS, LDY); skin sold (LDY); taboo since descended from humans (KLK, KLM, KBD)

\section{REFERENCES}

Zug 1993; Inger and Tan 1996

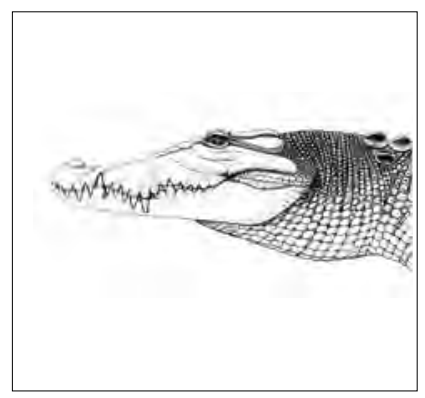

\begin{tabular}{|c|c|l|l}
\hline$\#$ & Code & \multicolumn{1}{|c}{ Lonchura malacca } & \multicolumn{1}{c}{ Crocodylus poros $u$ s } \\
\hline 1 & PUT & lugok, lugom & bowai \\
\hline 2 & PUB & pirit & bowai \\
\hline 3 & PUD & pirit & bowai \\
\hline 4 & PUM & lugom & bowai \\
\hline 5 & ALS & firit & boyo \\
\hline 6 & LDY & firit, firit itam & buaye, bewaya \\
\hline 7 & LIT & pirit & baye \\
\hline 8 & BRU & fiet tam & biah \\
\hline 9 & MRP & tei'o, te'a te'un, te'o & maya, meya' \\
\hline 10 & PTN & pito bela & baya' \\
\hline 11 & KLK & upit bala, upit saleng & baya \\
\hline 12 & KLM & upit saleng & baya' \\
\hline 13 & KLO & & baze \\
\hline 14 & KLA & ufit & baze \\
\hline 15 & KAL & upit caleng & baze \\
\hline 16 & KBD & upit saleng & baya \\
\hline 17 & KBK & upit saleng & baya \\
\hline 18 & PEB & upit & baya \\
\hline
\end{tabular}




\section{Varanus salvator (Varanidae) MALAYAN. WATER MONITOR. BIAWAK AIR}

DESCRIPTION

Largest of monitors in Borneo, to $2 \mathrm{~m}$; terrestrial-arborealaquatic predator; upperparts shiny dark green scaly skin mixed with yellow, spots on legs; underparts solid yellow brown

\section{HabitaT}

Lowland and hill forest, riverine; scavenges in campsites, human settlements

\section{ORIGINS/STATUS}

Asia/CITES: Appendix II

\section{USES}

Eaten

\section{REFERENCES}

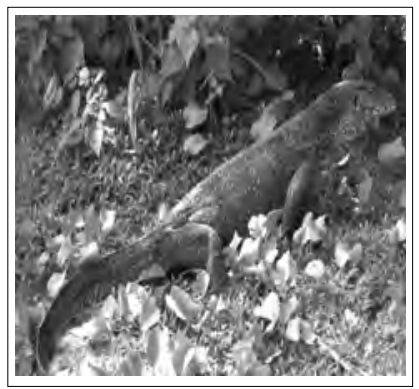

Zug 1993; Inger and Tan 1996

\section{Varanus rudicollis (Varanidae) \\ ROUGH-NECKED MONITOR. BIAWAK LEHER KASAR}

\section{DESCRIPTION}

Medium sized monitor, to $1 \mathrm{~m}$; terrestrial-arboreal predator; black skin with raised nonoverlapping round scales on head and body; underparts grey; erect spiny scales on back and tail HABITAT

Lowland and hill primary forests

ORIGINS/STATUS

Southeast Asia/CITES: Appendix II

USES

Eaten

REFERENCES

Zug 1993; Inger and Tan 1996

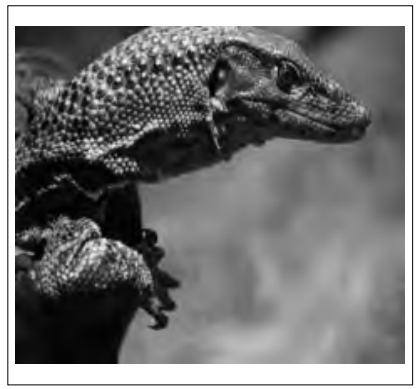

\begin{tabular}{|c|c|l|l}
\hline$\#$ & Code & \multicolumn{1}{|c|}{ Varanus salvator } & \multicolumn{1}{c}{ Varanus rudicollis } \\
\hline 1 & PUT & parang & aluh vung, alu, aluh \\
\hline 2 & PUB & & alu \\
\hline 3 & PUD & parang & aluh \\
\hline 4 & PUM & parang & aluh \\
\hline 5 & ALS & tedayu & pilos \\
\hline 6 & LDY & kabaren & kabuk, kabok item \\
\hline 7 & LIT & kebaren & kabuk \\
\hline 8 & BRU & pagang & kabe akan \\
\hline 9 & MRP & parai & kabau, kabawe, kabau labaya \\
\hline 10 & PTN & & kabok \\
\hline 11 & KLK & padang, pa'dang & kabok, kabok mba' \\
\hline 12 & KLM & kabok padang & kabok tana' \\
\hline 13 & KLO & - & kabuk mudung \\
\hline 14 & KLA & kabok bai & kabuk, kabok modung \\
\hline 15 & KAL & kabok bai & kabok \\
\hline 16 & KBD & padang & kabok \\
\hline 17 & KBK & padang & kabok \\
\hline 18 & PEB & sawe & kovok \\
\hline
\end{tabular}




\section{Varanus dumerilii (Varanidae)}

DUMERIL'S MONITOR. BIAWAK IKAN

\section{DESCRIPTION}

Small monitor lizard, to $50 \mathrm{~cm}$; black grey scaly skin, with paler undersides; erect spines on back and tail

\section{HABITAT}

Lowland and hill forests

\section{ORIGINS/STATUS}

Southeast Asia/CITES: Appendix II

\section{USES}

Eaten (PUD, PUM, ALS, LDY, BRU, KBD, KBK, PEB); good omen for pig hunters (PEB)

REFERENCES

Zug 1993; Inger and Tan 1996

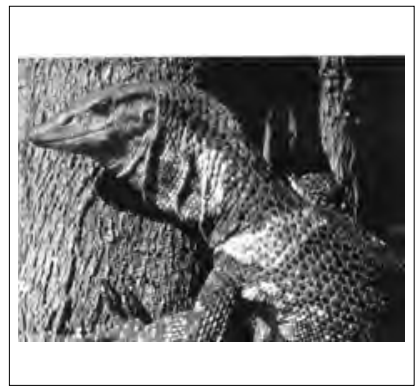

\section{Gonocephalus doriae (Agamidae)}

DORIA'S ANGLEHEAD AGAMID. BUNGLON BERSISIR PENDEK

\section{DESCRIPTION}

Small, slender, long tailed, long legged lizard, to $15 \mathrm{~cm}$; orange green mix; underparts redder with dark bands on tail; spine of short erect scales; yellow orange throat flap

\section{HABITAT}

Small trees in hill forests

\section{ORIGINS/STATUS}

Asia/Unprotected

\section{USES}

Eaten (PUM, BRM, PEB)

\section{REFERENCES}

Zug 1993; Inger and Tan 1996

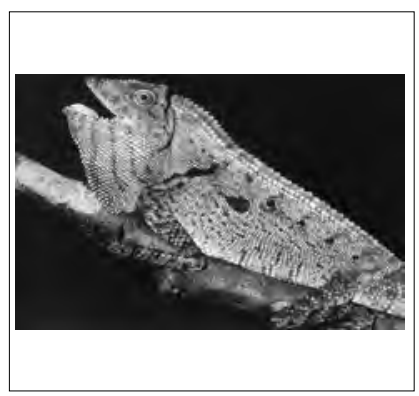

\begin{tabular}{|r|l|l|l}
\hline$\#$ & Code & \multicolumn{1}{c|}{ Varanus dumerilii } & \multicolumn{1}{c}{ Gonocephalus doriae } \\
\hline 1 & PUT & bucoh & vuan, vuan laui, vuan la'ui \\
\hline 2 & PUB & & takang, nyun puan \\
\hline 3 & PUD & bucoh & vaun nyun \\
\hline 4 & PUM & bucoh & vuan \\
\hline 5 & ALS & kamuk & garang surut \\
\hline 6 & LDY & kabuk kara & garang \\
\hline 7 & LIT & & garang \\
\hline 8 & BRU & kabe so & pu'un keve \\
\hline 9 & MRP & kabau mbuei & kelakaya, kotakaya, hakhah \\
\hline 10 & PTN & & takang \\
\hline 11 & KLK & kabok & takang, takang batu \\
\hline 12 & KLM & & takang \\
\hline 13 & KLO & & kuk kuvo' \\
\hline 14 & KLA & & tahang \\
\hline 15 & KAL & kabok bato & tahang \\
\hline 16 & KBD & & takang \\
\hline 17 & KBK & & takang \\
\hline 18 & PEB & bu & takang surut \\
\hline
\end{tabular}




\section{Gonocephalus liogaster (Agamidae) COMB-CRESTED AGAMID. BUNGLON SISIR PANJANG}

\section{DESCRIPTION}

Small, slender, long tailed, long limbed lizard, to 15 $\mathrm{cm}$; brown body with mid dorsal crest of erect scales; underparts and throat flap light brown mixed with white; white bands on tail

\section{HABITAT}

Lowland and hill primary forest; small trees

ORIGINS/STATUS

Asia/Unprotected

USES

Eaten (PUM)

\section{REFERENCES}

Inger and Tan 1996

\section{Mabuya rudis (Scincidae) ROUGH SCALED BROWN SKINK. KADAL SISIR KASAR}

\section{DESCRIPTION}

Medium sized skink lizard, to $20 \mathrm{~cm}$; cylindrical body, brown, darker bands running from the eye down to the tail on the body sides; head conical; rough shiny scales

HABITAT

Hill primary forest; ground dwelling

ORIGINS/STATUS

Asia/Unprotected

\section{USES}

Eaten (PUM, LDY, PTN)

\section{REFERENCES}

Inger and Tan 1996

\begin{tabular}{|c|c|l|l}
\hline$\#$ & Code & \multicolumn{1}{|c|}{ Gonocephalus liogaster } & \multicolumn{1}{c}{ Mabuya rudis } \\
\hline 1 & PUT & takang, vuan nyun & belang lunang, belang paku, belang boeng \\
\hline 2 & PUB & kevan, nyun puan & beleng, beleng alih \\
\hline 3 & PUD & takang & beleng \\
\hline 4 & PUM & vuan & beleng \\
\hline 5 & ALS & garang & alai \\
\hline 6 & LDY & garang tefila', garang & aleh \\
\hline 7 & LIT & garang & alai \\
\hline 8 & BRU & pu'un keve & alai \\
\hline 9 & MRP & kelakaya, hakhah, telakaya & keleia, telaya, teli'ie \\
\hline 10 & PTN & takang & teliap \\
\hline 11 & KLK & takang batu & keliap \\
\hline 12 & KLM & takang & bodut \\
\hline 13 & KLO & taghang & teliak \\
\hline 14 & KLA & tahang & teliap \\
\hline 15 & KAL & tahang & talei'ap \\
\hline 16 & KBD & umpit tulit & teliap \\
\hline 17 & KBK & takang & kataleiap \\
\hline 18 & PEB & takang surut & keliyap \\
\hline
\end{tabular}




\section{Python curtus (Boidae) BLOOD PYTHON. ULAR DARAH}

\section{DESCRIPTION}

Large terrestrial python snake; to $1.5 \mathrm{~m}$; body yellow with brown patches above grey white and black patches below HABITAT

Lowland and hill primary forests; river banks

ORIGINS/STATUS

Southeast Asia/CITES: Appendix II

USES

Eaten

REFERENCES

Zug 1993; Inger and Tan 1996

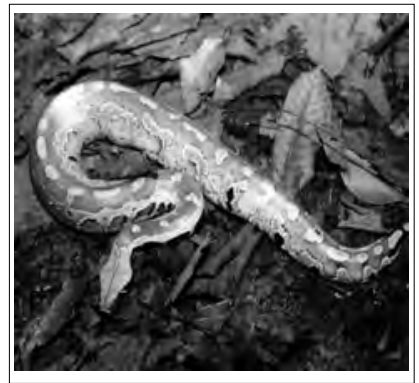

\section{Python reticulatus (Boidae) RETICGULATED.PYTTHON. ULAR SAWAG}

\section{DESCRIPTION}

Largest terrestrial-arboreal-aquatic python, to $6 \mathrm{~m}$; olive green brown with black and yellow reticulated markings and grey patches below

\section{HABITAT}

Lowland, hill primary forests, riverine, sometimes in cultivated areas

\section{ORIGINS/STATUS}

Southeast Asia/CITES: Appendix II

\section{USES}

\section{Eaten}

\section{REFERENCES}

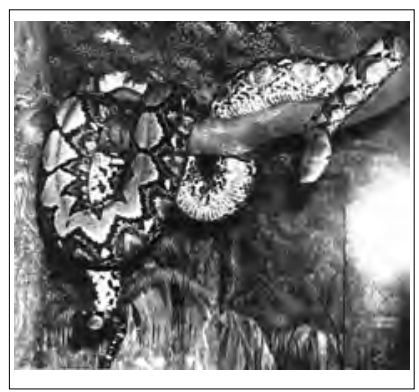

Zug 1993; Inger and Tan 1996

\begin{tabular}{|c|c|l|l}
\hline$\#$ & Code & \multicolumn{1}{|c}{ Python curtus } & \multicolumn{1}{c}{ Python reticulatus } \\
\hline 1 & PUT & cai bongih, bongih & cai penganan, penganen \\
\hline 2 & PUB & bongih, bongi & penganan \\
\hline 3 & PUD & cai bungih & penganan \\
\hline 4 & PUM & bongih & penganan \\
\hline 5 & ALS & tafung & menolon \\
\hline 6 & LDY & dafung & menelan \\
\hline 7 & LIT & depung & menelen \\
\hline 8 & BRU & belefwa' lapung & belefwa' melan \\
\hline 9 & MRP & tolatang, pie ngai, ngai' & penganan, pie penganan, panganan \\
\hline 10 & PTN & lepung ba'ek & nyipa aya' \\
\hline 11 & KLK & tepung boet & penganan \\
\hline 12 & KLM & pengirau & penganan \\
\hline 13 & KLO & lepung boet & lepung \\
\hline 14 & KLA & lepung boet & lepung \\
\hline 15 & KAL & & lepung \\
\hline 16 & KBD & lepung enang & penganan \\
\hline 17 & KBK & lepung & penganan \\
\hline 18 & PEB & dopung & penganan \\
\hline
\end{tabular}




\section{Ophiophagus hannab (Elapidae) KING COBRA. ULAR TEDUNG}

\section{DESCRIPTION}

Large erect-fanged venomous snake; terrestrial, to $4 \mathrm{~m}$; brown scales, narrow yellow bands; underside black, light brown below head; head slightly hooded; brown (1) and black varieties (2) reported in Bulungan area

\section{HABITAT}

Lowland and hill primary and secondary forests, riverine

\section{ORIGINS/STATUS}

Asia/CITES: Appendix II

\section{USES}

Eaten (ALS, LDY, BRU, KBD, PEB); venom for dart poison (PUD, MRP, KBD, PEB)

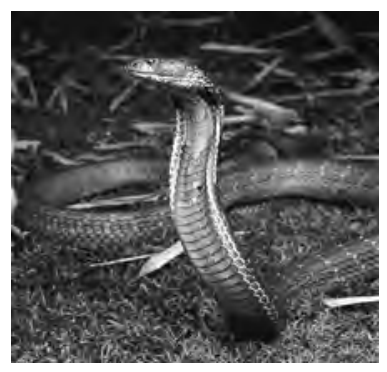

\section{REFERENCES}

Zug 1993; Inger and Tan 1996; Puri 1997a

\section{Tropidolaemus wagleri (Viperidae)}

KEELED MALAYSIAN PIT-VIPER. ULAR WAGLER

\section{DESCRIPTION}

Medium hinged-fanged venomous pit-viper snake; to $70 \mathrm{~cm}$; arboreal-terrestrial; light green above with darker green bands, whitish below; broad triangular head

\section{HABITAT}

Lowland and hill primary forests

\section{ORIGINS/STATUS}

Southeast Asia/Unprotected

\section{USES}

Eaten (BRU); venom for dart poison (KBD, $\mathrm{PEB}$ )

\section{REFERENCES}

Zug 1993; Inger and Tan 1996; Puri 1997a

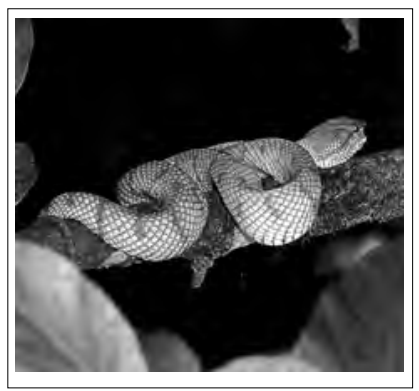

\begin{tabular}{|c|c|c|c|}
\hline \# & Code & Ophiophagus bannab (Elapidae) & Tropidolaemus wagleri \\
\hline 1 & PUT & kelimut (1); cai kelimut (2), punyuh (2) & cai baluh, cai balu \\
\hline 2 & PUB & cai mungoh (1), motu (1); punyoh (2), punyo'(2) & cai balu \\
\hline 3 & PUD & cai mungoh (1); kelimut (2) & cai balu \\
\hline 4 & PUM & mungoh (1); kelimut (2) & sai baluh \\
\hline 5 & ALS & aloi (1); mendahan (2) & temegasan \\
\hline 6 & LDY & rangboh (1); seruwang (2) & gergasan, urau \\
\hline 7 & LIT & seruang bule (1), merin putung (1); seruang (2) & ulau \\
\hline 8 & BRU & belefwa' laguan (1); belefwa' luang tang (2) & belefwa' luan \\
\hline 9 & MRP & pie' tebuweng (1), nibie (1); pie' tiem (2) pie netihem (2) & piet lorau, pie loh, pie' loh \\
\hline 10 & PTN & nyipa bua (1); bo'am pitam (2) & nyipa urem \\
\hline 11 & KLK & buah (1), njauliban (1); buah (2) & ipo' dau, joleh ma' \\
\hline 12 & KLM & njau leban toban (1); njau leban (2) & polau \\
\hline 13 & KLO & njo' leibeng (1); ncung olei liban (2) & sulo bileng \\
\hline 14 & KLA & njau liban tuban (1) & pulao, pulau bileng \\
\hline 15 & KAL & njau olei bin (1) & polau \\
\hline 16 & KBD & njau leban kameng (1); njau leban toban (2) & orem peppa \\
\hline 17 & KB K & njung ole liban (1); njung ole nyateng (2) & ipa' ulo \\
\hline 18 & PEB & buwa (1); silei (2) & urem \\
\hline
\end{tabular}




\section{Abaetulla prasina (Colubridae) ORIENTAL WHIP SNAKE. ULAR PUCUU,}

\section{DESCRIPTION}

Small rear-fanged venomous tree snake, to $80 \mathrm{~cm}$; slender pale green or white, larger head

\section{HabitaT}

Lowland and hill primary forests

\section{UsES}

Mildly poisonous tree snake

\section{REFERENCES}

Zug 1993; Inger and Tan 1996; Puri 1997a

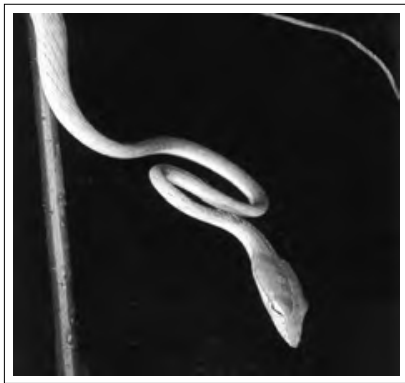

\section{Maticora bivirgata (Elapidae) STRIPED ORIENTAAL COQRAL SNAKE. ULAR CABE BESAR}

\section{DESCRIPTION}

Small erect-fanged venomous snake; slender to $80 \mathrm{~cm}$; terrestrial; red head and tip of tail; body black above with white, blue, green stripes on sides

\section{HABITAT}

Lowland and hill forest; cultivated areas, human settlements

\section{ORIGINS/STATUS}

Southeast Asia/Unprotected

\section{USES}

Bad omen, could cause abandonment of village in past; eaten for medicine (KBD, MRP)

\section{REFERENCES}

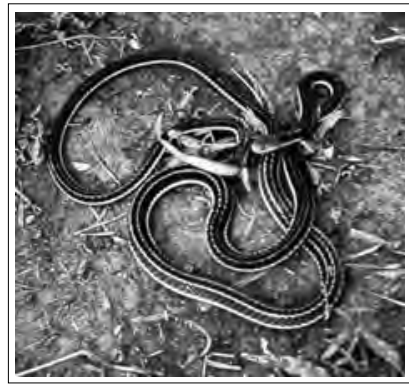

Zug 1993; Inger and Tan 1996; Puri 1997a

\begin{tabular}{|c|c|l|l}
\hline$\#$ & Code & \multicolumn{1}{|c}{ Ahaetulla prasina } & \multicolumn{1}{c}{ Maticora bivirgata } \\
\hline 1 & PUT & cai angoh, nawan, cai nawan & cai lawing, lawing \\
\hline 2 & PUB & cai, nawan & jerung, lawing \\
\hline 3 & PUD & cai angau & cai lawing \\
\hline 4 & PUM & sai ango & sai lawing \\
\hline 5 & ALS & nawan & sunjuwari \\
\hline 6 & LDY & rarui & aya' fadeh \\
\hline 7 & LIT & & aya' padai, tesak padai \\
\hline 8 & BRU & belefwa' gwan un & belefwa' sia' \\
\hline 9 & MRP & leru icam, pie loh, pia' & lua', pie luya, pia' luya \\
\hline 10 & PTN & nyipa ulo & nyipa malei \\
\hline 11 & KLK & nawan, ipu olau & unjung uley paren, ulet paren \\
\hline 12 & KLM & & todok paren \\
\hline 13 & KLO & olet & ncung ole osang \\
\hline 14 & KLA & pulau & ncung olei usang \\
\hline 15 & KAL & polau & njau olei osang \\
\hline 16 & KBD & toduk sungai & toduk tega \\
\hline 17 & KBK & nawan adeng & njung ole usang \\
\hline 18 & PEB & nawan ileng & semuwei \\
\hline
\end{tabular}




\section{Dogania subplana (Trionychidae)}

MALAYAN SOOFT-SHELLED TUURTLE. LABI-LABI BATU

\section{DESCRIPTION}

Small semi-aquatic turtle; oval, very flat shell to $35 \mathrm{~cm}$, black to olive or dark brown with a black medial stripe; head brown to olive with yellow and black spots with bony snout

HABITAT

Lowland and hill forest, rivers and streams

ORIGINS/STATUS

Southeast Asia/Unprotected

UsES

Eaten

REFERENCES

Ernst and Barbour 1989; Inger and Tan 1996

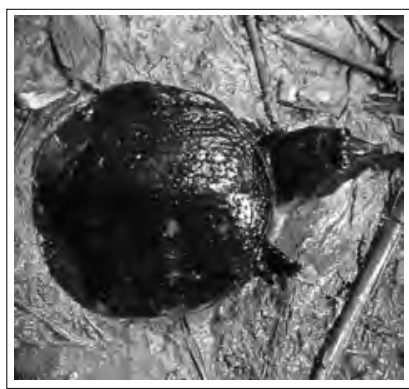

\section{Cyclemys dentata (Emydidae)}

\section{ASIAN LEAF TURTLE. KURA-KURA COKLAT}

\section{DESCRIPTION}

Small semi-aquatic turtle; oval shell to $24 \mathrm{~cm}$, arched, one medial keel; brown, narrow black radiations; yellow to light brown underneath; head reddish brown on top, sides and lower jaws darker brown; slightly projected snout

\section{HABITAT}

Small shallow rocky streams, lowland and hill forests

\section{ORIGINS/STATUS}

Asia/Unprotected

USES

Eaten

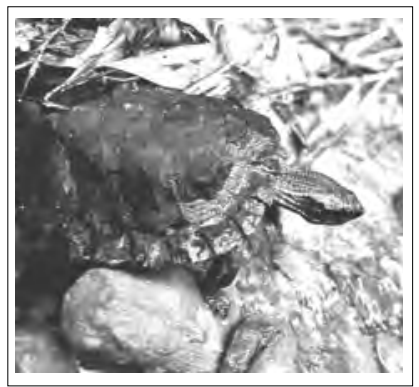

\section{REFERENCES}

Ernst and Barbour 1989; Inger and Tan 1996

\begin{tabular}{|c|c|l|l}
\hline$\#$ & Code & \multicolumn{1}{|c|}{ Dogania subplana } & \\
\hline 1 & PUT & kapih, kapi & kelop \\
\hline 2 & PUB & kapi, kelavang & kelop \\
\hline 3 & PUD & kapih & kelop \\
\hline 4 & PUM & kapi & kelop \\
\hline 5 & ALS & sisik & buh \\
\hline 6 & LDY & beladan & abu, abuh \\
\hline 7 & LIT & beladan & bu'u \\
\hline 8 & BRU & bladin tabil & beredin tigiah \\
\hline 9 & MRP & labai, kelabaia & kelap \\
\hline 10 & PTN & labei & kellap \\
\hline 11 & KLK & labi & kelep \\
\hline 12 & KLM & labi & kellep \\
\hline 13 & KLO & la'bi' & kellep \\
\hline 14 & KLA & la'bi, labi & kellep \\
\hline 15 & KAL & labi & kellep \\
\hline 16 & KBD & sungit & kellep \\
\hline 17 & KBK & lembet lamin & kelep luran \\
\hline 18 & PEB & siap parai & dia \\
\hline
\end{tabular}




\section{DESCRIPTION}

Small semi-aquatic turtle, oval to elongated shell to $32 \mathrm{~cm}$, flattened top, 6-7 plates; green brown to yellow brown, with dark spots; yellow to orange below; retracts with hiss and defecates on handler

\section{HABITAT}

Ground dwelling, hill forests

ORIGINS/STATUS

Southeast Asia/Unprotected

\section{USES}

Eaten

\section{REFERENCES}

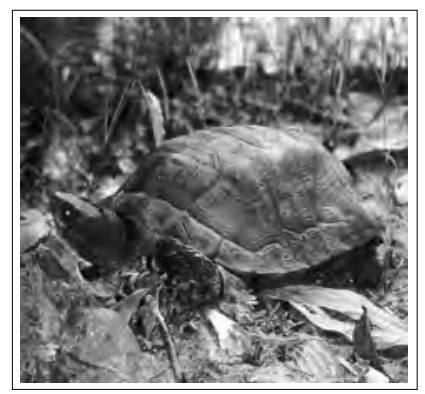

Ernst and Barbour 1989; Inger and Tan 1996

\section{Rbacopborus reinwardti (Rhacophoridae) REINWARDT'S FLYING FROG. KATAK POHON REINWART}

\section{DESCRIPTION}

Small tree frog; lime green backside, blue and orange spotted markings below; with skinflaps on front forearms; tadpoles (j:) brown and green

\section{HABITAT}

Lowland primary forest; trees; breeds in mud-wallows

\section{ORIGINS/STATUS}

Asia/Unprotected

\section{USES}

Eaten (PUD, PUM, ALS, LDY)

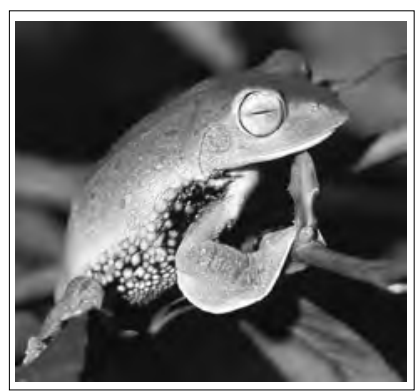

\section{REFERENCES}

Zug 1993; Inger and Tan 1996

\begin{tabular}{|c|l|l|l}
\hline$\#$ & Code & \multicolumn{1}{|c|}{ Notochelys platynota } & \multicolumn{1}{c}{ Rhacophorus reinwardti (j: juvenile) } \\
\hline 1 & PUT & kelop, unyan & becih iluh, ca'ai mali, malih (j: baung, nal ca'ai, baong) \\
\hline 2 & PUB & unyan & ca'ai, bunong (j: nak ca'ai, baung) \\
\hline 3 & PUD & unyan & ca'ai (j: baong) \\
\hline 4 & PUM & unyan & sa'ai (j: bo'ung) \\
\hline 5 & ALS & suyan & sai (j: anak rumut) \\
\hline 6 & LDY & bung kakung & telaka, lemango (j: libatu) \\
\hline 7 & LIT & tuyan, bung kakung & teleka (j: lebetuh) \\
\hline 8 & BRU & yuan & set (j: tam uwe) \\
\hline 9 & MRP & kelap, nyang, nyah & nuweng, nong, noeng (j: tai', mawe, teh') \\
\hline 10 & PTN & sian & kerotek (j: telo nei betak) \\
\hline 11 & KLK & sian & keretek (j: baong, ba'ong) \\
\hline 12 & KLM & sian & kerettuk (j: ba'ong) \\
\hline 13 & KLO & sezing & utung bazeng (j: ba'ong) \\
\hline 14 & KLA & sian & sa'ai (j: ba'ong) \\
\hline 15 & KAL & sezin & sa'ai (j: ba'ong) \\
\hline 16 & KBD & sian & sa'ai bileng (j: ba'ong lentek) \\
\hline 17 & KBK & sian & sa'ai cip (j: ba'ung) \\
\hline 18 & PEB & sian & sai daun kayu (j: atut) \\
\hline
\end{tabular}




\section{Rhacopborus nigropalmatus (Phasianidae) WALLACE'S FLYING FROG. KATAK POHON WALLACE}

\section{DESCRIPTION}

Small tree frog; green backside, white underparts; blackish webbing; skinflaps on front forearms

\section{HABITAT}

Lowland primary forest; trees; breeds in mud-wallows

\section{ORIGINS/STATUS}

Asia/Unprotected

\section{USES}

Eaten (PUD, ALS, LDY)

\section{REFERENCES}

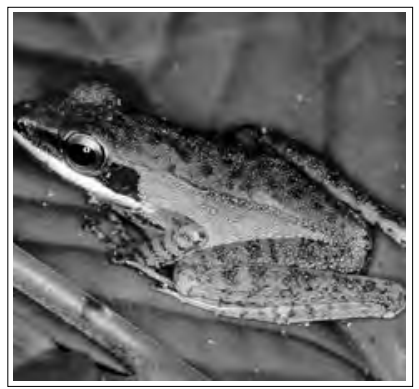

Zug 1993; Inger and Tan 1996

\section{Polypedates macrotis (Rhacophoridae) MASKED TREE FROG. KATAK POHON PERAK}

\section{DESCRIPTION}

Small tree frog; silvery grey with black streak behind eyes

HABITAT

Lowland primary forest; trees; breeds in mud-wallows

\section{ORIGINS/STATUS}

Asia/Unprotected

\section{USES}

Eaten (ALS, BRU, PTN)

\section{REFERENCES}

Zug 1993; Inger and Tan 1996

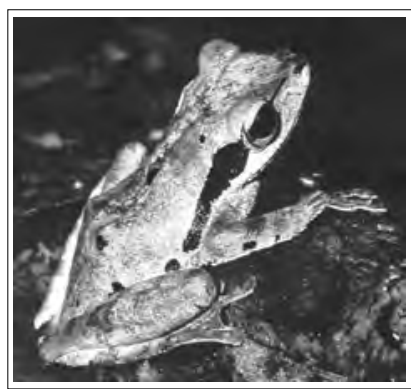

\begin{tabular}{|c|c|l|l}
\hline$\#$ & Code & \multicolumn{1}{|c}{ Rhacophorus nigropalmatus } & \multicolumn{1}{c}{ Polypedates macrotis } \\
\hline 1 & PUT & malih, mali, becih & ca'ai, bunong \\
\hline 2 & PUB & cai, bunong & ca'ai, na'ang \\
\hline 3 & PUD & cai & becih \\
\hline 4 & PUM & bunong & becih \\
\hline 5 & ALS & sai & kiak \\
\hline 6 & LDY & atit, atit bata & atit \\
\hline 7 & LIT & ang, tet bata' & sengangau \\
\hline 8 & BRU & set ata' & set adia' \\
\hline 9 & MRP & nuweng, nong, noeng nya'ai & nuweng, nong, noeng \\
\hline 10 & PTN & kerotek & \\
\hline 11 & KLK & sa'ai bileng, keretak & ketikak, sa'ae \\
\hline 12 & KLM & sa'ai & sa'ai \\
\hline 13 & KLO & kerettek & sa'ae \\
\hline 14 & KLA & kerettuk & s'ai \\
\hline 15 & KAL & sa'ai beleng & kerettuk \\
\hline 16 & KBD & sa'ai kerettek & sa'ai \\
\hline 17 & KBK & sa'ai lali & sa'ai \\
\hline 18 & PEB & sai sip & sai \\
\hline
\end{tabular}




\section{Kaloula baleata (Microhylidae) \\ BROWN BULLFROG. KATAK GEMUK COKLAT \\ DESCRIPTION}

Large terrestrial bullfrog; muddy brown with paler underparts, snub nosed with characteristic loud call 'wang kok'

\section{HABiTAT}

Lowland primary forest; swamps, mud-wallows

\section{ORIGINS/STATUS}

Asia/Unprotected

\section{USES}

Eaten in famine times only; fishbait

\section{REFERENCES}

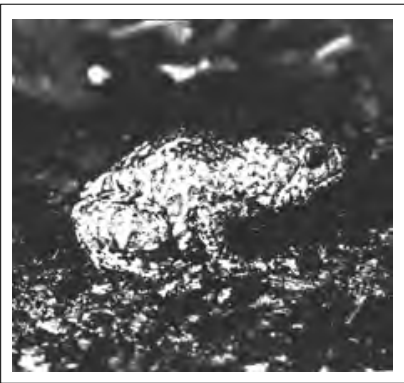

Zug 1993; Inger and Tan 1996

\section{Rana ingeri (Ranidae) \\ GREATER SWAMP FROG. KATAK RAWA \\ DESCRIPTION}

Large heavy bodied terrestrial frog, feeds on crabs and other frogs

\section{HabitaT}

Lowland to hill primary and disturbed forest; shallow marshes

\section{ORIGINS/STATUS}

Asia/Unprotected

\section{USES}

Eaten (PUM, ALS, LDY, LIT, BRU, PTN, KLK, KBD, PEB)

\section{REFERENCES}

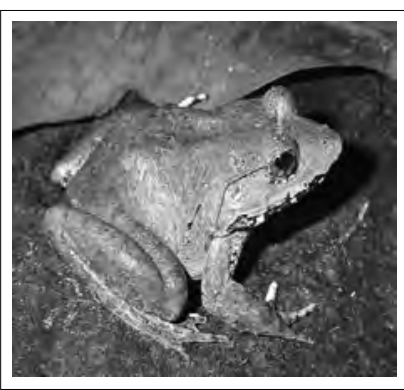

Zug 1993; Inger and Tan 1996

\begin{tabular}{|c|l|l|l|}
\hline$\#$ & Code & \multicolumn{1}{|c|}{ Kaloula baleata } & \multicolumn{1}{c|}{ Rana ingeri } \\
\hline 1 & PUT & la'ak, ngang & ca'ai \\
\hline 2 & PUB & & ca'ai \\
\hline 3 & PUD & ngang & jaui \\
\hline 4 & PUM & na'ang & bunong \\
\hline 5 & ALS & tahang & sai \\
\hline 6 & LDY & atit, takang & ai \\
\hline 7 & LIT & a'ang & ya'i \\
\hline 8 & BRU & set tana' & set ai \\
\hline 9 & MRP & ai, ayah & nuweng tung, nong tung, noeng tung \\
\hline 10 & PTN & ang & ha'ae \\
\hline 11 & KLK & ang, sa'ae ang & sa'ai tu'uh, sa'ae tu'u \\
\hline 12 & KLM & ang & sa'ai tu'u \\
\hline 13 & KLO & ang & kerettek \\
\hline 14 & KLA & vak, ang & sa'ai, bunong \\
\hline 15 & KAL & vak & sa'ai lanyo \\
\hline 16 & KBD & la'ak & sa'ai su'u \\
\hline 17 & KBK & wangkok & sa'ai lali \\
\hline 18 & PEB & kup & sai \\
\hline
\end{tabular}




\section{1 \\ Hemibagrus wyckii (Bagridae) \\ BAGGID COATFISH. IKAN BAUNGG}

\section{DESCRIPTION}

Large brown catfish, to $50 \mathrm{~cm}$; upper surface of head smooth; margin or pectoral fin, dorsal fin tips of adipose fat; upper and lower margins of caudal fin white

\section{HabitaT}

Large to small rivers, coastal to mountain areas

\section{ORIGINS/STATUS}

Borneo, Sumatra, Java/Unprotected

\section{USES}

Eaten

\section{REFERENCES}

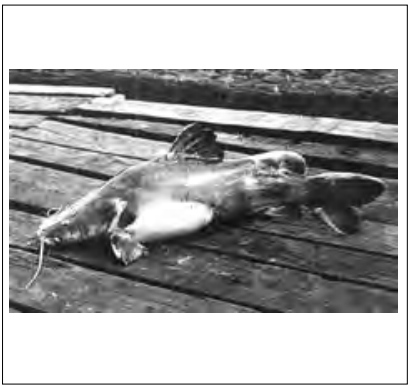

Inger and Chin 1962; Kottelat et al. 1993

\section{Barbodes balleroides (Cyprinidae) BARB. LAMPAM}

\section{DESCRIPTION}

Small to medium sized silvery freshwater fish, to $20 \mathrm{~cm}$; lateral line; red orange tint on anterior part of dorsal fin; local category may also include Barbodes schwanenfeldii

\section{HABITAT}

Large to medium, clear, fast flowing rivers and streams

\section{ORIGINS/STATUS}

Southeast Asia/Unprotected

\section{USES}

\section{Eaten}

\section{REFERENCES}

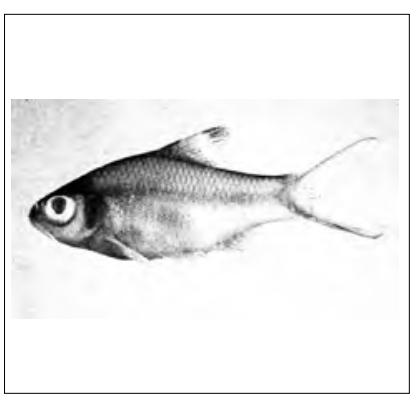

Inger and Chin 1962; Kottelat et al. 1993

\begin{tabular}{|c|c|l|l|}
\hline$\#$ & Code & \multicolumn{1}{|c|}{ Hemibagrus reyckii } & \multicolumn{1}{c|}{ Barbodes balleroides } \\
\hline 1 & PUT & cikai & alap \\
\hline 2 & PUB & cikai, sikai & halap, alap \\
\hline 3 & PUD & cikai & alap \\
\hline 4 & PUM & sikai & salap \\
\hline 5 & ALS & sovong & salap \\
\hline 6 & LDY & tinien, tiniyen & salap \\
\hline 7 & LIT & seniyen, siniyen & salap \\
\hline 8 & BRU & awit talyan & awit alap \\
\hline 9 & MRP & teikien, taiken, tikien & hala \\
\hline 10 & PTN & telikan & jupet \\
\hline 11 & KLK & teliken, telikan & salap \\
\hline 12 & KLM & tenneiken & salap \\
\hline 13 & KLO & tennai ghen & sala' \\
\hline 14 & KLA & tennihan & salap \\
\hline 15 & KAL & tennai hen & salap \\
\hline 16 & KBD & tenneiken & salap \\
\hline 17 & KBK & tenikan & salap \\
\hline 18 & PEB & ta'ok & salap \\
\hline
\end{tabular}




\section{Lobocheilos sp. (Cyprinidae) \\ BARB.}

\section{DESCRIPTION}

Medium to large sized scaly freshwater fish, to $35 \mathrm{~cm}$; squarish nose with many rough wartlike tubercles on the lower jaw

\section{HabitaT}

Inland; large to medium rivers; fast running water

\section{ORIGINS/STATUS}

Asia/Unprotected

\section{USES}

Eaten

\section{REFERENCES}

Inger and Chin 1962; Kottelat et al. 1993

\section{Tor tambra (Cyprinidae)}

\section{CARP. IKAN BELIAN}

\section{DESCRIPTION}

Medium to large freshwater fish, to $50 \mathrm{~cm}$; dark olive green to black backside, paler below; large scales with yellow to gold edges; local category includes Tor tambroides

\section{Habitat}

Inland; large to medium rivers and streams; fast water

\section{ORIGINS/STATUS}

Asia/Unprotected

\section{USES}

Eaten; sold locally, to traders, and city markets

\section{REFERENCES}

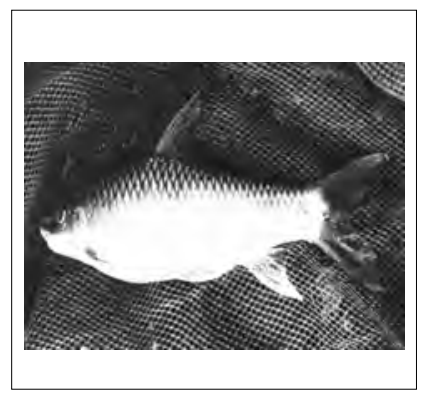

Inger and Chin 1962; Kottelat et al. 1993

\begin{tabular}{|c|c|l|l}
\hline$\#$ & Code & \multicolumn{1}{|c}{ Lobocheilos sp. } & \multicolumn{1}{c}{ Tor tambra } \\
\hline 1 & PUT & pa', paha' $^{\prime}$ & tengoh \\
\hline 2 & PUB & naha, ngaha & tengoh \\
\hline 3 & PUD & ngaha & tengoh \\
\hline 4 & PUM & purut & tengoh \\
\hline 5 & ALS & furud & felian \\
\hline 6 & LDY & furud & luang, luwang \\
\hline 7 & LIT & & luang \\
\hline 8 & BRU & awit paha' & awit leguom \\
\hline 9 & MRP & paha, can paha' & pai, can pai, paih \\
\hline 10 & PTN & pasa' $^{\prime}$ & sinaya' \\
\hline 11 & KLK & pasa' & padek \\
\hline 12 & KLM & pasa' $^{\prime}$ & padek \\
\hline 13 & KLO & pase' & ato' \\
\hline 14 & KLA & pase' $^{\prime}$ & padek \\
\hline 15 & KAL & fase' $^{\prime}$ & faduk \\
\hline 16 & KBD & atok pasa' & padek \\
\hline 17 & KBK & pasa' & padek \\
\hline 18 & PEB & pasa' $^{\prime}$ & goga \\
\hline
\end{tabular}


Pangasius niewenbuisi (Pangasidae)

BORNEO RIVER CATFISH. PATIN

DESCRIPTION

Large catfish-like freshwater fish; stinging poisonous frontal rays

\section{HaBitaT}

Coastal to inland mountain areas; larger deep rivers

\section{ORIGINS/STATUS}

East Kalimantan endemic/Unprotected

\section{USES}

Eaten; sold locally, to traders, and city markets

\section{REFERENCES}

Inger and Chin 1962; Kottelat et al. 1993

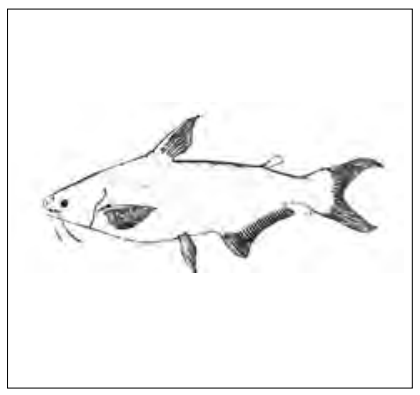

\section{Potamidae}

\section{SMALL RIVER CRABS. KEPITING}

\section{DESCRIPTION}

General term for a variety of freshwater crabs

\section{HABITAT}

Coastal, lowland and hill areas; rivers and streams

\section{USES}

Eaten

\section{REFERENCES}

$\mathrm{Ng}$ 1988, Holthuis 1979

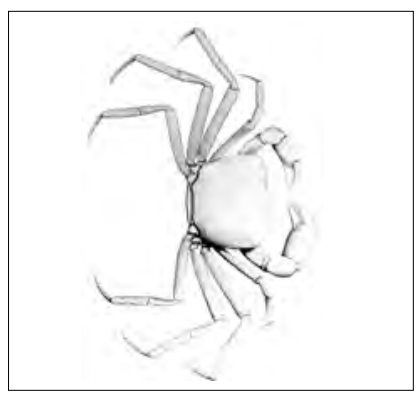

\begin{tabular}{|c|l|l|l|}
\hline$\#$ & Code & \multicolumn{1}{|c|}{ Pangasius niewenhuisi } & \multicolumn{1}{c|}{ Potamidae } \\
\hline 1 & PUT & larong & kang, tuyu \\
\hline 2 & PUB & larong & kang, tuyu \\
\hline 3 & PUD & larong & kang, tuyu \\
\hline 4 & PUM & larong & tuyu' \\
\hline 5 & ALS & selodong & fua' \\
\hline 6 & LDY & seladang, seladeng & kara \\
\hline 7 & LIT & seladang, lawit patin & kera' \\
\hline 8 & BRU & awit sodong & gia' \\
\hline 9 & MRP & klaran, can laran, larang & tuyo, toyoh, tuyo' \\
\hline 10 & PTN & selareng & tuyo' \\
\hline 11 & KLK & selarang & iyu, yu \\
\hline 12 & KLM & selareng & yu \\
\hline 13 & KLO & selarang & zu' \\
\hline 14 & KLA & selarang & izu \\
\hline 15 & KAL & selarang & zu \\
\hline 16 & KBD & selarang & yu \\
\hline 17 & KBK & selarang & yu \\
\hline 18 & PEB & selareng & iye \\
\hline
\end{tabular}




\section{Macbrobrachium sp. (Palaemonidae) RIVERR SHRIṂPP: UDANG}

\section{DESCRIPTION}

General term for several species of freshwater shrimp; may also include species from the genus Atya

\section{HabitaT}

Coastal, lowland and hill forest, rivers and streams; edge of flood waters and inundated grasses and river banks

USES

Eaten

REFERENCES

Chin 1985

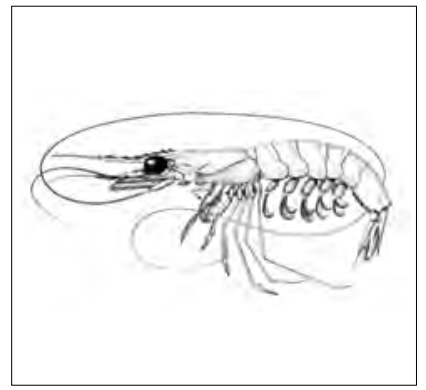

Cipangopaludina sp. (Viviparidae)

RIVER SNAILS. SIPUT SUNGAI

\section{DESCRIPTION}

General term for several species of freshwater snails

\section{HABITAT}

Coastal, lowland and hill areas; rocky areas in shallow rivers and streams; collected from exposed river rocks during drought periods

\section{USES}

Eaten; shells used as decoration, ornamentation; larger species hung from baby carriers to scare off ghosts and sickness

\section{REFERENCES}

Chin 1985

\begin{tabular}{|c|l|l|l|}
\hline$\#$ & Code & \multicolumn{1}{|c|}{ Machrobrachium sp. } & \multicolumn{1}{c|}{ Cipangopaludina sp. } \\
\hline 1 & PUT & urang & hih \\
\hline 2 & PUB & urang & hih \\
\hline 3 & PUD & urang & hih \\
\hline 4 & PUM & urang & hih \\
\hline 5 & ALS & udang & tundul \\
\hline 6 & LDY & udang & girig \\
\hline 7 & LIT & udang & akeb, akep \\
\hline 8 & BRU & diang & kebatau \\
\hline 9 & MRP & roya, roye, eroya & hei \\
\hline 10 & PTN & urang & hi \\
\hline 11 & KLK & udang & sei' \\
\hline 12 & KLM & odang & si' \\
\hline 13 & KLO & o'dang & si' \\
\hline 14 & KLA & udang & si' \\
\hline 15 & KAL & odang & sie' \\
\hline 16 & KBD & odang & si' olung \\
\hline 17 & KBK & udang & sei' \\
\hline 18 & PEB & urang & siek \\
\hline
\end{tabular}




\section{Buthidae \\ SCORPION. KALAJENGKING}

\section{DESCRIPTION}

Large scorpion, to $12 \mathrm{~cm}$, black

HABITAT

Human settlements and cultivated areas

USES

Dangerous poisonous sting; for medicine (KLM)

REFERENCES

Elzinga 1997; Pinhey 1974

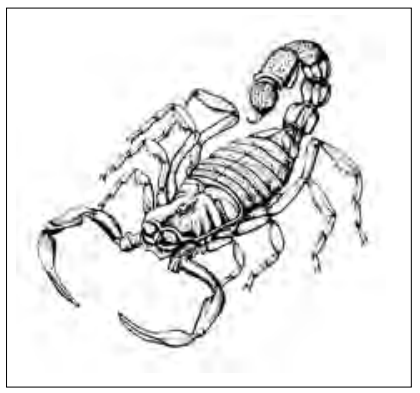

\section{Lycosida \\ SPIDER. LABA-LABA}

\section{DESCRIPTION}

General term for a variety of species

\section{HABITAT}

Human settlements and cultivated areas

\section{USES}

Sometimes dangerous, poisonous sting

\section{REFERENCES}

Elzinga 1997

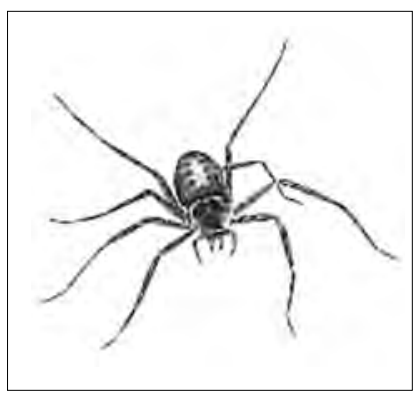

\begin{tabular}{|r|l|l|l|}
\hline \# & Code & \multicolumn{1}{|c|}{ Buthidae } & \multicolumn{1}{|c|}{ Lycosida } \\
\hline 1 & PUT & katip baieu, katip beieu & teluvaja, televaya \\
\hline 2 & PUB & katip behieu, katip beheu & tevaia bulun, tegowok \\
\hline 3 & PUD & katip beheu & tevaya \\
\hline 4 & PUM & katip behiu & tevaya' \\
\hline 5 & ALS & sengangait & langut \\
\hline 6 & LDY & rufang kara, lufnag kara' & tekelawa besuk, tekelawa becuk \\
\hline 7 & LIT & menipal kera', menipal kera & anai, kelawa' \\
\hline 8 & BRU & longong kabiet & kang agong kabiet \\
\hline 9 & MRP & kelepie tait, telepie tai', telipia' tai' & kelawah kuyang, kelawah, tie ngoa \\
\hline 10 & PTN & telipan busong & telawa \\
\hline 11 & KLK & lelipan bosung, lelipan busung & kelaba \\
\hline 12 & KLM & lipan busung, lipan bosung & mbui bong, telawa bodieng \\
\hline 13 & KLO & pang bofung & telava \\
\hline 14 & KLA & lempa busung & tehelava \\
\hline 15 & KAL & leppung busung & tahalava \\
\hline 16 & KBD & leppa busung & telawa' \\
\hline 17 & KBK & leppang busung & katalawa ba'i \\
\hline 18 & PEB & duyung & sanam kebuvu \\
\hline
\end{tabular}


Cordulegastridae

\section{DRAGONFLYY. CAPUNG}

\section{DESCRIPTION}

General term for a variety of species

\section{HabitaT}

Lowland and hill swamps, ponds and still water

USES

Subject of myths (KBD)

\section{REFERENCES}

Elzinga 1997; Tweedie and Harrison 1954

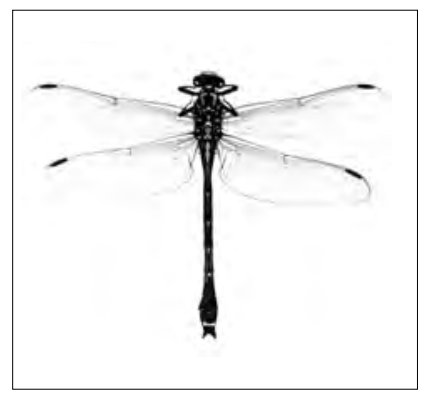

\section{Mantis sp. (Mantidae) \\ PREYING MANTIS. BELALANG SEMBAH}

\section{DESCRIPTION}

General term for a variety of species

\section{HABITAT}

Human settlements and cultivated areas

\section{USES}

Eaten (P'TN, KLM)

\section{REFERENCES}

Elzinga 1997; Little 1963

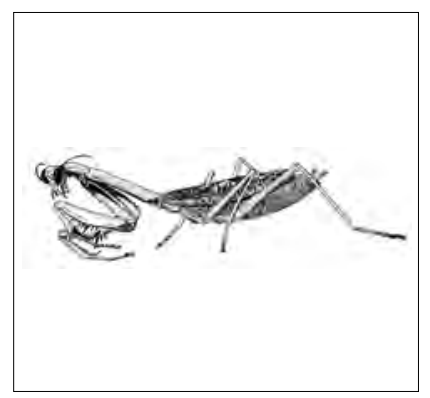

\begin{tabular}{|r|l|l|l|}
\hline$\#$ & Code & \multicolumn{1}{|c|}{ Cordulegastridae } & \multicolumn{1}{c|}{ Mantis sp. } \\
\hline 1 & PUT & capuk telang, capok telang & ungin parei \\
\hline 2 & PUB & apuk telang & ungen, ungin \\
\hline 3 & PUD & apuk telang & ungin padi \\
\hline 4 & PUM & sapok telang & ungin \\
\hline 5 & ALS & segading & moyot \\
\hline 6 & LDY & sawa' bah, sawa' ba & kato bayu, kato bayo \\
\hline 7 & LIT & dut ba', dot ba' & katau mek \\
\hline 8 & BRU & atau kong & ayang \\
\hline 9 & MRP & linggaye, lenggaye, langgaya & pau ngun, pau nguon, pau nguang \\
\hline 10 & PTN & kesibet & pau langgau \\
\hline 11 & KLK & kelesibet & pau padei \\
\hline 12 & KLM & kese'bet, kelese'bet & kadei padai \\
\hline 13 & KLO & sekeibet liung & fae radu \\
\hline 14 & KLA & sehibet ulo', sehibet & pau dadu, pau radu \\
\hline 15 & KAL & sehaibet & fau radu \\
\hline 16 & KBD & sekeibet & pau dado \\
\hline 17 & KBK & sibet & pau dado \\
\hline 18 & PEB & tekonyit & pau pagau \\
\hline
\end{tabular}




\section{DESCRIPTION}

General term for a variety of species

\section{HABITAT}

Human settlements and cultivated areas

\section{USES}

Eaten (PUM, ALS, LDY, BRU, PTN, KLM, KBD); fish-

ing bait

\section{REFERENCES}

Elzinga 1997; Pinhey 1974

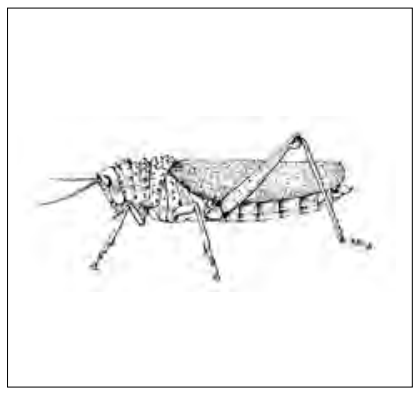

\section{Phyllium sp. (Phylliidae) LEAF GRASSHOPPER. BELALANG DAUN}

\section{DESCRIPTION}

Grasshopper, body flat, 3-10 cm length; green with mottled brown edges as in a dead leaf

\section{HabitaT}

Lowland and hill primary forests; cultivated areas and human settlements

\section{USES}

Exudes noxious blinding fluid (KBD)

\section{REFERENCES}

Elzinga 1997; Imms 1957

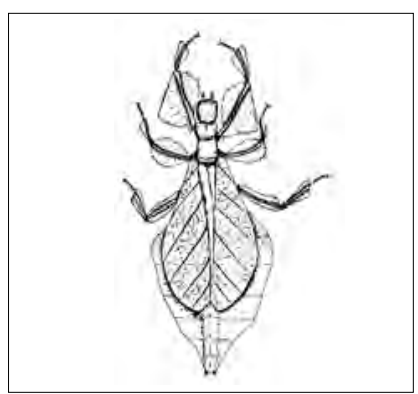

\begin{tabular}{|c|c|l|l|}
\hline$\#$ & Code & \multicolumn{1}{|c|}{ Ariophantidae } & \multicolumn{1}{c|}{ Phyllium sp. } \\
\hline 1 & PUT & po jangin & ungin lo', ungin lunang \\
\hline 2 & PUB & po kalap, pao & ungen \\
\hline 3 & PUD & pau jangin & ungin daun \\
\hline 4 & PUM & poh & ungin \\
\hline 5 & ALS & kavoh & tembuyung \\
\hline 6 & LDY & kato, kato mapue & kato, kato bayo \\
\hline 7 & LIT & ketau, katau & \\
\hline 8 & BRU & atau piau & ayang un \\
\hline 9 & MRP & pau ngangaing, pau yangain, pau & pau kai, ngau melai, pau \\
\hline 10 & PTN & pau & sap \\
\hline 11 & KLK & pau & pau \\
\hline 12 & KLM & kadei uwai & kameng singit, cap \\
\hline 13 & KLO & vae mpung & vae nyarei \\
\hline 14 & KLA & pau & pau, pau radu \\
\hline 15 & KAL & fau fadei & fau \\
\hline 16 & KBD & pau padei & cap \\
\hline 17 & KBK & pau mbung & pau lideh \\
\hline 18 & PEB & pau parai & pau daun kayo \\
\hline
\end{tabular}


Gerris remigis (Gerridae)

WATER STRIDERR: KEPIK AIR.

\section{DESCRIPTION}

Small grasshopper-like insect to $3 \mathrm{~cm}$, with long spindly legs for resting on surface of water, antenna present, wings absent

\section{Habitat}

Lowland and hill swamps, ponds, still water

USES

Medicine for poor hunting dogs (PUM)

REFERENCES

Elzinga 1997; Tweedie and Harrison 1954

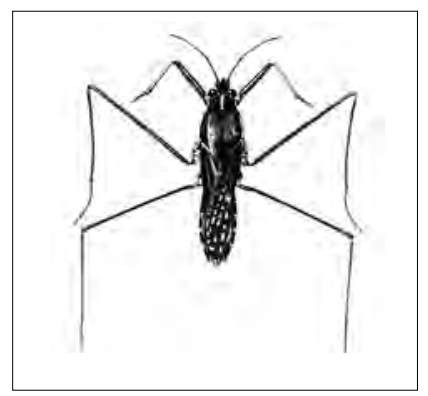

\section{Tibicen sp. (Cicadidae) \\ CICADA. TONGGERET}

\section{DESCRIPTION}

Large herbivorous arboreal insects, from 2 to $12 \mathrm{~cm}$; metallic blue, green, red; large compound eyes; two pairs transparent wings; nymph with large digging front legs; species-specific calls, a loud repetitive chirp, buzz or trill

\section{Habitat}

Lowland, hill and montane forests, old secondary forests

\section{USES}

Omen animal (PEB, PUD); ecological, climatic indicators

\section{REFERENCES}

Elzinga 1997; Little 1963

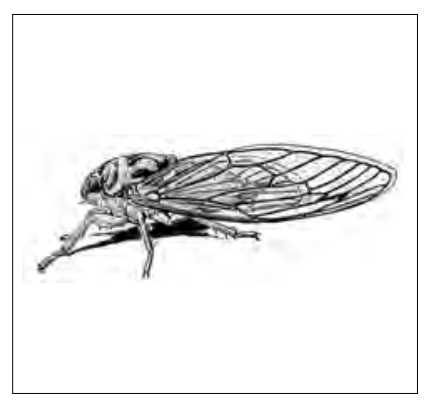

\begin{tabular}{|c|c|l|l|}
\hline \# & Code & \multicolumn{1}{|c|}{ Gerris remigis } & \multicolumn{1}{c|}{ Tibicen sp. } \\
\hline 1 & PUT & sebakau, cebakau & netnet, netnet \\
\hline 2 & PUB & sebakau & tereging, torok loli \\
\hline 3 & PUD & sebakau & netnet \\
\hline 4 & PUM & ceriong & nowet \\
\hline 5 & ALS & temakang & lolong \\
\hline 6 & LDY & ato' apa', kato' apa' & berebidang, berbidang \\
\hline 7 & LIT & akang & berebidang \\
\hline 8 & BRU & kang apai pi' & melawet \\
\hline 9 & MRP & tamekau, tamakau, tamakau & telie, kelie \\
\hline 10 & PTN & dong bule' & tiling \\
\hline 11 & KLK & jang jago, jang jatong & tileng tana, tileng \\
\hline 12 & KLM & cang jakau & tilieng, telieng \\
\hline 13 & KLO & ca' sa'a & tilieng se' beri \\
\hline 14 & KLA & ka'ang sa'ai, cang sa'ai & tilieng \\
\hline 15 & KAL & tozang atok & tilieng \\
\hline 16 & KBD & dak dakau & tiling \\
\hline 17 & KBK & palang likau & tiling \\
\hline 18 & PEB & sekupang beu & ngit ase \\
\hline
\end{tabular}




\section{Lucanus sp. (Lucanidae) \\ STAG BEETLE. KUMBANG RUSA}

\section{DESCRIPTION}

Large herbivorous terrestrial beetle, to $8 \mathrm{~cm}$, brown or black body, large pincer-like mandibles on males; antenna elbowed

\section{HABITAT}

Lowland and hill forests; under logs and debris

USES

Eaten (LDY, KLM)

REFERENCES

Elzinga 1997; Imms 1957: 784

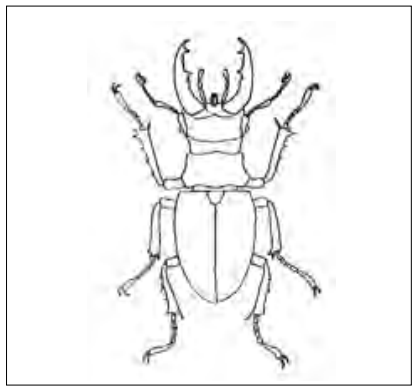

\section{Rbynchopborus ferrugineus (Curculionidae)}

RED PALM WEEVIL(SAGO GRUB). KUMBANG SAGU (ULAT SAGU)

\section{DESCRIPTION}

Terrestrial palm weevil, to $5 \mathrm{~cm}$; brown or orange red with black spots on pronatum; elbowed antenna; juvenile (j:) large, to $4 \mathrm{~cm}$, fat, legless grub, pale cream to light brown, found decaying tree palm trunks, such as Arenga

\section{HabitaT}

Lowland and hill forests; under logs and debris

\section{USES}

\section{Eaten}

\section{REFERENCES}

Chin 1985; Elzinga 1997; Tweedie and Harrison 1954

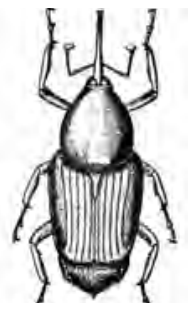

\begin{tabular}{|r|r|l|l|}
\hline$\#$ & Code & \multicolumn{1}{|c|}{ Lucanus sp. } & \multicolumn{1}{|c|}{ Rhynchophorus ferrugineus (j: juvenile) } \\
\hline 1 & PUT & kara, tebiluk & vung kovang, wung kovang (j: si'et) \\
\hline 2 & PUB & ki'ik & vung \\
\hline 3 & PUD & uwung & vung \\
\hline 4 & PUM & kara & vung (j: si'et) \\
\hline 5 & ALS & buong batang & butot (j: butot) \\
\hline 6 & LDY & bueng kik, bueng & bueng kurad, bueng fulod (j: wat, uwet) \\
\hline 7 & LIT & & bueng kenangan, bueng pulud \\
\hline 8 & BRU & longong & longong saleng (j: wat) \\
\hline 9 & MRP & kelohu, kuluhua, kungaue & kelohu telaie, kelohuwe, kelehuwe \\
\hline 10 & PTN & unga & kelehong (j: hi'et) \\
\hline 11 & KLK & ketarip & kelesunga (j: soet) \\
\hline 12 & KLM & kameng singit, keti'et & kelasunga, kelesunga (j: soet) \\
\hline 13 & KLO & lesukung & lesungu (j: so'ut) \\
\hline 14 & KLA & lesukung, kelesunga & lesunga (j: su'et, so'ut) \\
\hline 15 & KAL & kutu jaong & halasungou \\
\hline 16 & KBD & ke'ek & tekepip talang (j: so'et) \\
\hline 17 & KBK & tekepip isau & tekepip (j: su'et) \\
\hline 18 & PEB & punga modok & besunga (j: so'et) \\
\hline
\end{tabular}




\section{Tenebroides sp. (Tenebrionidae) \\ DARKLING BEETLE. KUMBANG GELAP}

\section{DESCRIPTION}

Large scavenging terestrial beetle, to $3 \mathrm{~cm}$, dull black colour, lengthwise ridges on rigid dorsal abdominal covering; antennae straight

\section{HabitaT}

Lowland and hill forests; soils, under rocks, stored products, wood

\section{USES}

Eaten (KAL); cagebird food

REFERENCES

Elzinga 1997; Imms 1957

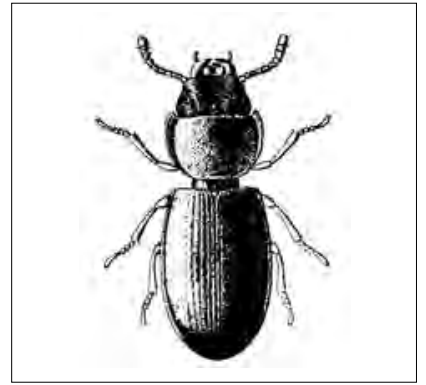

\section{Apis dorsata (Apidae) GIANT HONEY BEE. LEBAH MADU \\ DESCRIPTION}

Large honey producing bees, dusky coloured wings; drones as large as workers; produce large hanging combs up to $2 \mathrm{~m}$. in length, $1 \mathrm{~m}$. in depth; migratory; local 'bee' categories may include other species, such as Apis indica

\section{HABITAT}

Lowland and hill forests; huge nests either hanging from branches (e.g., Koompassia excelsa), the undersides of overhanging rocks, or the eaves of tall buildings

\section{USES}

Honey eaten, sold locally, to traders; made into alcoholic drink

\section{REFERENCES}

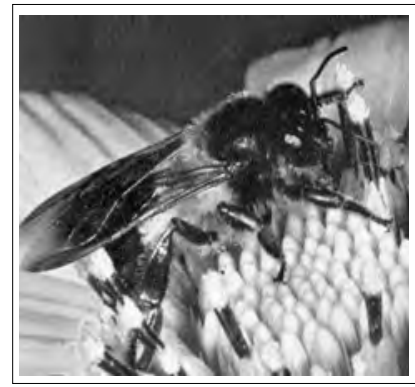

Elzinga 1997; Butler 1962

\begin{tabular}{|r|l|l|l}
\hline$\#$ & Code & \multicolumn{1}{|c}{ Tenebroides sp. } & \multicolumn{1}{c}{ Apis dors ata } \\
\hline 1 & PUT & kara, kara' & fanih, kanyih \\
\hline 2 & PUB & kara', korong & tegering, fanih \\
\hline 3 & PUD & kara & vanyih \\
\hline 4 & PUM & kara & vanyih \\
\hline 5 & ALS & buong & binjai \\
\hline 7 & LIT & bueng talal, bueng ilang & tebikan \\
\hline 8 & BRU & longong fet & sebikan \\
\hline 9 & MRP & kara', kara & nyet kian \\
\hline 10 & PTN & unga luheng & nyet tenyet, nyet, nyet tanye \\
\hline 11 & KLK & akuk & layuk \\
\hline 12 & KLM & uwang bang & layuk lalo, layuk \\
\hline 13 & KLO & jeti & layuk \\
\hline 14 & KLA & kelesunga & nyefieng \\
\hline 15 & KAL & ahuk & lazuk \\
\hline 16 & KBD & ayang beng & lazuk \\
\hline 17 & KBK & jait & nyewan \\
\hline 18 & PEB & punga' o'ok & layuk \\
\hline
\end{tabular}




\section{Sphecidae}

WASP. TAWON KEMIT

\section{DESCRIPTION}

Stinging wasp, to $2 \mathrm{~cm}$; black with yellow slender waist and yellow legs, large compound eyes; antenna of 10 segments

\section{HABITAT}

Human settlements and cultivated areas

\section{USES}

Pest; parasite

\section{REFERENCES}

Imms 1957: 734; Elzinga 1997

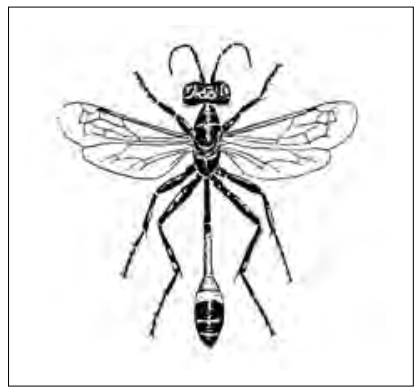

\section{Formicidae}

\section{ANTS. SEMUT}

\section{DESCRIPTION}

General term for a variety of species of terrestrial ants

\section{HABITAT}

Lowland and hill forests; human settlements and cultivated areas

\section{USES}

Medicinal (KLM)

\section{REFERENCES}

Elzinga 1997

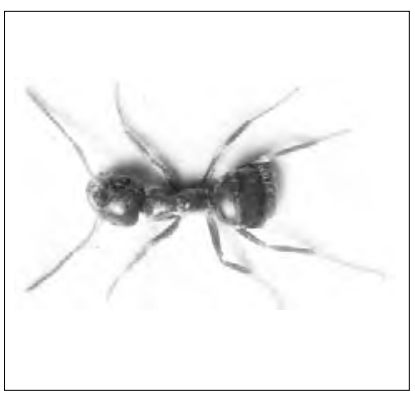

\begin{tabular}{|r|l|l|l|}
\hline$\#$ & Code & \multicolumn{1}{|c|}{ Sphecidae } & \multicolumn{1}{c|}{ Formicidae } \\
\hline 1 & PUT & wong, wung & ulah beleng, ulah belang \\
\hline 2 & PUB & lowah, wong & ulah belang \\
\hline 3 & PUD & tekawang & ulah beleng \\
\hline 4 & PUM & vung & ulah \\
\hline 5 & ALS & nyatod & kilau \\
\hline 6 & LDY & sulun, ated & tetungal, dera' tetungal \\
\hline 7 & LIT & seb & petungar \\
\hline 8 & BRU & lenyah & gia' toal \\
\hline 9 & MRP & nyet, nguong, nguang & tie', tie nguong, tie \\
\hline 10 & PTN & & bitek ukong \\
\hline 11 & KLK & adek, sengit ikeng & sanem, sanam \\
\hline 12 & KLM & singet ekeng, singet aikeng & sanam, sanam jago \\
\hline 13 & KLO & & sanam bezu \\
\hline 14 & KLA & & sanem \\
\hline 15 & KAL & senget adek & sanem \\
\hline 16 & KBD & & sanem \\
\hline 17 & KBK & katan bali & sanam \\
\hline 18 & PEB & kejawang & ketungan \\
\hline
\end{tabular}




\section{Sphingidae \\ SPHINX MOTH. NGENGAT}

\section{DESCRIPTION}

General term for a variety of species of nocturnal moths; juvenile (j:) a dark caterpillar with reddish stripes

\section{HabitaT}

Lowland and hill forests; human settlements and cultivated areas

\section{USES}

Caterpillar may be used as bait; no known uses for moth in Bulungan

\section{REFERENCES}

Elzinga 1997; Imms 1957

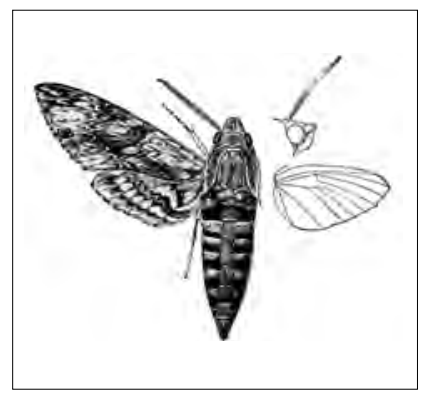

\section{Attacus atlas (Saturniidae) \\ ELLPPHANT MOTH. KUPU GAIAH}

\section{DESCRIPTION}

General term for a variety of species of diurnal butterflies

\section{HABITAT}

Lowland and hill forests; human settlements and culti-

vated areas

\section{ORIGINS/STATUS}

India to Southeast Asia/Unprotected

USES

Reared for sale in Thailand; cocoons for Fagara silk; no known uses in Bulungan

\section{REFERENCES}

Elzinga 1997; Imms 1957

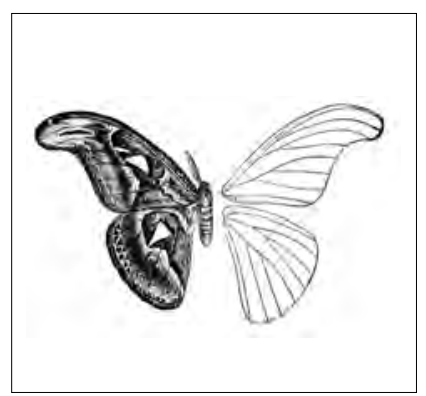

\begin{tabular}{|r|l|l|l|}
\hline \# & Code & \multicolumn{1}{|c|}{ Sphingidae (j: juvenile) } & \multicolumn{1}{c|}{ Attacus atlas } \\
\hline 1 & PUT & mai'apei, apei (j: telaroh, telaruh) & televang \\
\hline 2 & PUB & televang (j: opung) & me'ape' \\
\hline 3 & PUD & televang (j: opung) & mei apei \\
\hline 4 & PUM & amei apei' (j: telaru') & amei apei' \\
\hline 5 & ALS & tengelayat (j: tendaru) & tengelayat \\
\hline 6 & LDY & belelapang, belapang (j: tetadu) & belelapang, belapang \\
\hline 7 & LIT & berefang (j: tedadu, tetadu) & berefang \\
\hline 8 & BRU & long piang lagit nuop (j: liat oua') & long piang \\
\hline 9 & MRP & kubu hapau, tau' hea, tau' hia (j: lun taterau, luen) & taung hie, tau' hea, tau' hia \\
\hline 10 & PTN & baret (j: ulen tang taro') & tauhiap \\
\hline 11 & KLK & beret, mata bali (j: ulet, ulet tadu) & mata bali \\
\hline 12 & KLM & bambet, ilun duk (j: ketadu, olet tetadu) & bambet, bambet uwai \\
\hline 13 & KLO & baret (j: betade) & felaghi bali \\
\hline 14 & KLA & baret (j: ulet) & pelahi bali \\
\hline 15 & KAL & baret (j: betadu) & pelahi vali \\
\hline 16 & KBD & beret (j: olet) & ulut \\
\hline 17 & KBK & tekuyong (j: ulet luwei) & katan bali \\
\hline 18 & PEB & kebava' suvut (j: lengat gaten) & kebava' \\
\hline
\end{tabular}




\section{Anopheles sp. (Culicidae)

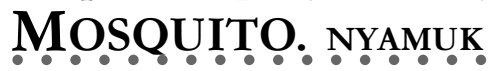

\section{DESCRIPTION}

Small bloodsucking flying insect, $1 \mathrm{~cm}$; slender pale brown bodies and long legs; long brown proboscis with white bands and white tip; long filiform antenna; active at dusk and in evening

\section{Habitat}

Human settlements and cultivated areas

USES

Pest, carries malaria

\section{REFERENCES}

Elzinga 1997; Pinhey 1974

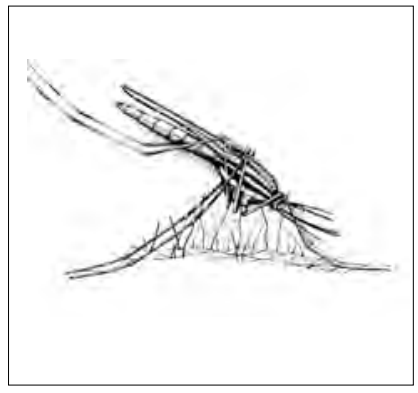

\section{Musca domestica (Muscidae) \\ HOUSE FLY. LALAT RUMAH}

\section{DESCRIPTION}

Small scavenging flying insect, less than $1 \mathrm{~cm}$; diurnal; black body and large compound eyes, short antenna

\section{Habitat}

Human settlements and cultivated areas

\section{USES}

Pest, believed to carry disease (KBD, PEB)

\section{REFERENCES}

Elzinga 1997; Pinhey 1974

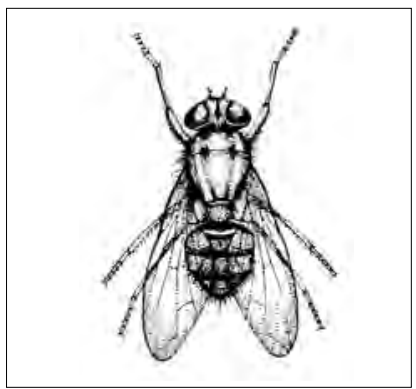

\begin{tabular}{|c|l|l|l}
\hline$\#$ & Code & \multicolumn{1}{|c|}{ Anopheles sp. } & \multicolumn{1}{c}{ Musca domestica } \\
\hline 1 & PUT & terukuk, terukuku & telegan, pokot \\
\hline 2 & PUB & nyanok kayo, teroko' kayo & pokot, terebik \\
\hline 3 & PUD & terukuk & lango \\
\hline 4 & PUM & telukuk & ketembuh \\
\hline 5 & ALS & tokung & menggurup \\
\hline 6 & LDY & tokung, tukong & lango \\
\hline 7 & LIT & tukong ragong, tokong & tukong, pitak \\
\hline 8 & BRU & kewong malaria & langau \\
\hline 9 & MRP & lekue, kelukuwe, nyamaue & langau, langga tau \\
\hline 10 & PTN & iyang & lango pilo \\
\hline 11 & KLK & jamok malaria, iyeng & langau pilau, langau \\
\hline 12 & KLM & iyeng malaria, iyeng jago & langau \\
\hline 13 & KLO & zeng & lango \\
\hline 14 & KLA & izeng & langau \\
\hline 15 & KAL & ezeng & langgau \\
\hline 16 & KBD & iyeng & langau \\
\hline 17 & KBK & iyeng & langau bileng \\
\hline 18 & PEB & yeng & piket \\
\hline
\end{tabular}




\section{ENVIRONMENTAL GLOSSARY}

\author{
Go upstream (in a boat) \\ Mudik \\ PUT : murik \\ PUD : murik \\ PUM : murik \\ ALS : nyundi \\ LDY : semonoy \\ LIT : mengaror mey daye \\ BRU : kwat, kuat \\ MRP : ndia, eniei ye', temti \\ PRT : mundik \\ KLK : metik \\ KLM : tai k'aieng \\ KLO : meti' \\ KLA : tei razah \\ KAL : tae razo \\ KBD : metik \\ KBK : metik \\ PEB : ke daya
}

\section{Downriver (location) \\ Di hilir}

PUT : an li va', anlifah, anlifa'

PUD : liva

PUM : anlifa

ALS : bugus go'

LDY : ilod, daei ilod

LIT : la'ud

BRU : duang bei', bey ngai

MRP : ka deya, nian teya, kentia teh hire

PRT : ka bai

KLK : kaba

KLM : kaba'

KLO : ka' ba' te'

KLA : hoh ha' bah te'

KAL : habah

KBD : kaba'

KBK : ka aba'

PEB : la' bai

Go to the river's edge Ke pinggir sungai PUT : demei lirin ungei, mey lirin
ungei, demeylirin ungei

PUD : ba'liren ungae

PUM : kah mey lirin ungei

ALS : kingtimug

LDY : ameh apa, ame iking apa'

LIT : mi tengub fah

BRU : taigon mai sien pi', mai kul giang agol

MRP : keliring, keliring hungai, tekelirin hungai

PRT : ka'beng ai tae

KLK : sada bai

KLM : sada bai

KLO : kesado bai'i

KLA : ke sada bai sungai

KAL : ke sado bay

KBD : ke bai

KBK : tai ka sada bay

PEB : la' bai

\section{Go downstream (in a boat)}

Melir (Menghilir)

PUT : pelabu, dedefah

PUD : nabu'

PUM : nabuh

ALS : bugus

LDY : tupa, ne lod

LIT : mengaror mey la'ud

BRU : pweih, puwe

MRP : hahau, haou, teh hao, kentia

PRT : ha'o

KLK : sao

KLM : sa'O

KLO : sa'O

KLA : sa'o

KAL : tae habah

KBD : sa'o

KBK : sao

PEB : ke bai

Across the river

Di seberang sungai

PUT : ri pah, anripa, anriva, anrifa

PUD : ripah

PUM : an lida

ALS : lipag

LDY : di far, dai' difar

LIT : paring ai

BRU : phi ngai

MRP : kapia, nian peyah, kapeh

PRT : kadipah

KLK : ka dipa

KLM : ka' depah

KLO : ke hiva' tei te'

KLA : hoh rifah te'

KAL : ha rayfah

KBD : ke depa

KBK : ka dipa

PEB : la' dipeu

\section{Village}

Kampung

PUT : tukung

PUD : tukong

PUM : tukung

ALS : pagun

LDY : bawang

LIT : bawang

BRU : awiang

MRP : tekuwe, tukuwa', tuku

PRT : leppo'

KLK : lepo

KLM : lepu'

KLO : lepu

KLA : lepua

KAL : lepu'a

KBD : leppo'

KBK : leppo

PEB : lepu'

\section{Upriver (location) \\ Di bulu}

PUT : ri cu', anricu, anrisu, anrisu'

PUD : ricu'

PUM : anlicu

ALS : golayo

LDY : i daya, daye

LIT : sai'e

BRU : duang ta', tangai

MRP : kawei, nian waei, kewaith'

PRT : ka' bueh ale'

KLK : ke daya

KLM : k'aieng

KLO : ke razo te'

KLA : hoh razah te'

KAL : ha razo

KBD : ke' daya

KBK : ka daija

PEB : la' daya

\section{Go inland}

Ke darat

PUT : dae rai, dederai, derai, matekon, dederai

PUD : lalom

PUM : kaderai

ALS : mud

LDY : ameh lun, ame lun

LIT : mi naklon

BRU : mai won quet, mai engkuet

MRP : ka lam, kelam, kalam,

PRT : uhuntana

KLK : ke usun

KLM : k'usun

KLO : kusun

KLA : k'usun tana'

KAL : tae husun

KBD : kusun

KBK : tai ka' usun

PEB : la' bui

\section{Forest shelter}

Pondok

PUT : leppo', levou

PUD : leppo

PUM : levou

ALS : lubung

LDY : lafo, levo

LIT : lepau

BRU : mien, pau

MRP : bua, keliap, bui

PRT : lepoh

KLK : lepau

KLM : lepan

KLO : lepo

KLA : lepou

KAL : lepau

KBD : lepau

KBK : lepau

PEB : lamin 
Abandoned village Bekas kampung

PUT : lepu'un, kelekan tukung, lepuun, levu'un

PUD : lekang tukong

PUM : kelekan tukung

ALS : tokonpagun

LDY : ruma'mon, bawang tican

LIT : ruma' tisan, lun sepai

BRU : mesien awiang, bekas pau

MRP : lapu'ong, lopu'ung, lepu'ung tuku

PRT : leppu'un lepo'

KLK : uban lepo'

KLM : oban lapu'un lepu'

KLO : levu'un

KLA : lepu'un lepua

KAL : lefu'un

KBD : lepu'un leppo'

KBK : lepu'un lepo

PEB : -

Upland

Tanah di darat

PUT : tano bota', tandan ridai, tano'an ridai, tanah an ridai

PUD: -

PUM: tano an lidai

ALS : tanamud

LDY : tana' lun, tana' daei ilung

LIT : lun sepai

BRU: quet, tana engkuet

MRP: tana kala'am, tana kalam

PRT : ke uhun tanah

KLK : tana' ke usun

KLM: tana' k'usun tai

KLO : -

KLA : tana' kusun

KAL : tana husun

KBD: tana' kusun

KBK: tana ka' usun

PEB: la' daya

\section{Plateau (terrace)}

Apau, daratan

PUT: tano ri cuh, la'au, kentat, lau', kendat

PUD: apaureh

PUM: lau'u

ALS : gana'

LDY : raked, rakadiring tangap

LIT : lun sapai lun surut

BRU: batul, patal

MRP: latah, tana latah

PRT : apoh

KLK : apau

KLM: apo

KLO: afoh

KLA : apau, tana' leka'

KAL : afo

KBD : apo

KBK : apau leken

PEB : data

\section{Abandoned camp}

Bekas tempat pondok

PUT : kelekan la'ah, kelekan levou, lea'aa, kelekan la'a

PUD : la'ah

PUM : kelekan la'a'

ALS : gaidlubung

LDY : ruma'mon, levotican

LIT : lepau tisan

BRU : nan pau lemdiau, bekas nan pau

MRP : laung bua, lopu'ung keliap, lepu'ung bu

PRT : leppu'un leppo'

KLK : uban lepau

KLM : oban duku lepan

KLO : levu'un lepo

KLA : uban lepou, uvan lepou

KAL : lefu'un lepau

KBD : lepu'un lepau

KBK : lepu'un lepau

PEB : -

Riverine (riparian) land

Tanah di pinggir sungai

PUT: tano bengai, tandan lirih ungei, tano' lirin ungei, tano

PUD: anlirin ungei

PUM: tano an lirin ungei

ALS : tana king timug

LDY : tengub apa', tana' iring apa'

LIT : tana' merekan

BRU: tana kul giang agol

MRP: tana' liring hungoai, tana keliring huei,

PRT : tanah pengai

KLK : tana' sada bai

KLM: tana' sada bai

KLO : -

KLA : tana' sada bai sungai

KAL : tana' sadobai

KBD: tana ka' sada bai

KBK : tana ka' sada bai

PEB : la' bai

\section{Hill}

Bukit

PUT: tokuk icit

PUD: tokuk icit

PUM: tukuk icit

ALS : tidong lumot

LDY : surud, foug isud

LIT : surut

BRU: lajau, kuet kie

MRP: nggang uwa, tukou auh', nggungu' wo

PRT : murung iuk

KLK : mudung iut

KLM: mudung iut

KLO: ngemudung

KLA : mudung iut

KAL : mudong iut

KBD : modung

KBK : mudung iut

PEB : morong
Land

Tanah

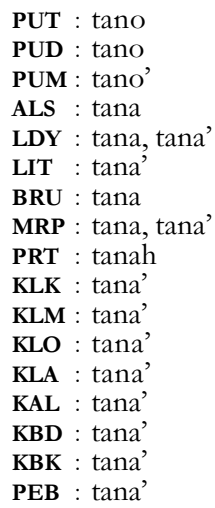

PUT : $\operatorname{tano}$

PUD : $\operatorname{tano}$

PUM : tano'

ALS : $\tan a$

LDY : tana, tana'

LIT : tana'

BRU : tana

MRP : tana, tana'

PRT : tanah

KLK : tana'

KLM : tana'

KLO : tana'

KLA : tana'

KAL : tana'

KBD : tana'

KBK : tana'

PEB : tana'

\section{Riverside plain}

Dataran pinggir sungai

PUT: tano' lo', lau' ulirin ungei, lau'u lirin ungei, lau'lirin ungei

PUD: tano lo'

PUM: lauu lirin ungei

ALS : ganaking siang

LDY : raked apa', rakadiring apa

LIT : rekut

BRU: patal, engkuet kulgian agol

MRP: latah, latah liring hungoai, lata liring hungai,

PRT : tana ratah (datah)

KLK : leka'

KLM: leka'

KLO: leka'

KLA : leka' d'empo bai'

KAL : leka' sado bai

KBD: leka'

KBK : leka' sada' bai

PEB : data

\section{Mountain}

Gunung

PUT: tokuk, tukuk

PUD: tukok

PUM: tukuk

ALS : juwau

LDY : fagung, surud

LIT : pun

BRU: quet, engkuet

MRP: tukuwa, tukou, nggaung

PRT : murung

KLK : mudung

KLM: modung

KLO : mudung

KLA : mudung

KAL : mudong

KBD: tokung

KBK : mudung

PEB : tokong 


\section{Peak}

Puncak

PUT: unjut, uncut tukuk, bota, bota'

PUD: kendat tokuk

PUM: bota

ALS : tembelulu juwau

LDY: lun fagung, fefed, budug pog

LIT : buduk

BRU: suan doek, engguan kuank

MRP: kehung tokuwe, ke mpau, kahung izuk

PRT : usuk murung

KLK : unjut empo

KLM: lunjut

KLO: tuseng mudung

KLA : leporut

KAL : lepurut

KBD: unyuk

KBK : uncut

PEB : peugi

Cliff

Tebing

PUT: ngelin, o'ung, belfingam, tikup, timbang

PUD: tambang

PUM: tekecang, lingou

ALS : lagam

LDY : ungub, balungob

LIT : toran

BRU: langap, puel

MRP: marai, lering, marah

PRT : bei berreh

KLK : bila

KLM: beilah

KLO : borung

KLA : bila

KAL : baelah

KBD: bila

KBK : bila

PEB: bila, ngup

\section{Mountain pass (saddle)}

Antara gunung

PUT : ting, owa', aking, layuk bota, tangtukuk

PUD: ting

PUM: aking, petulong

ALS : lolot juwau

LDY : ruwan, arang fuog

LIT : ra'an

BRU: bueng la'ang, enggang kuank

MRP: hawa, ngaiyatukou, kangei nggung

PRT : hawah

KLK : tenjalon, kalebek, sawa'

KLM: sawa'

KLO : savah

KLA : sava

KAL : savah

KBD : sawa'

KBK : nenga ang mudung

PEB : aweu
Outcrop

Batu di puncak

PUT: tokek bato', atu an bota, uncut tukuk, batuan bota',

PUD: batuhan bota'

PUM: batuan bota

ALS : batu tembelulu juwau

LDY : batunan, budug pog

LIT : -

BRU: atau engguan kuank

MRP: matounian kempau, matau kanjuk

PRT : -

KLK : unjut batu

KLM: -

KLO : tuseng batu

KLA : leporut batu

KAL : lepurut batu

KBD : unyuk bato

KBK : batu ka uncut

PEB : peugi bato

Steep hillside

Tanah miring

PUT: ti'ang, tano nekeling/tiang

PUD: tano ntai

PUM: tano nekeling

ALS : tana temeging

LDY : tana tebiring

LIT : -

BRU: ngiang, tebigieng tana

MRP: baing gaun, lettelaiyoh, lotelai tana

PRT : pekenoh

KLK : tana' miang, tai la bata'

KLM: tana' mehang

KLO : meyang

KLA : miang tana'

KAL : tana' miang

KBD : iyang keno?

KBK : miang tana'

PEB: selono-lono

Goa

PUT: lovang bato', lufang, luvan batuh, lulang batuh

PUD: luvang

PUM: lufang batu

ALS : luang

LDY : lubang batu, lubang

LIT : lubang batu

BRU: buyang, goa

MRP: loh boya, loboya, lubuai matoa

PRT : lobang bato'

KLK : lubang batu

KLM: -

KLO: lepo' vato

KLA : lubang batu

KAL : lobang batu

KBD : lobang bato

KBK : lubang batu

PEB : lovang bato
Ridge

Punggung, Pematang gunung

PUT: bota', bota'a, latbota', betang bota'

PUD: wat tano, bota'

PUM: bota

ALS : tido'ong

LDY : surud, batang tana'

LIT : surut

BRU: lajau

MRP: matae inggung, pon nggaing, nyawo', matai' nggaung

PRT : bei murung

KLK : modang ang

KLM: urut mudung

KLO: mudung ang

KLA : fuun mudung

KAL : jomin mudong

KBD: pu'un modung

KBK : pu'un mudung

PEB : pun morong

\section{Landslide}

Tanah longsor

PUT: tuan, tano tuan, kenyut, lonjon, kenyut, tano' tuan

PUD: tuwan

PUM: tano tuan

ALS : tana luyu

LDY : meturan, tanatukan

LIT : tana' netoran

BRU: tiguwan, tana tiguan

MRP: tuang, tanatuan, tanatuwang

PRT : tuwan

KLK : tuwan

KLM: tuwan tana

KLO: to'ieng

KLA : tana' tuwan, tana' leroh

KAL : towen

KBD : tuan

KBK : tana' tuan

PEB : tuan

\section{Rocky bank}

Pinggir sungai berbatu

PUT: lingah, lirin ungei nahbatu, lirin ungei fi' batuh, lirin ungei bebatuh

PUD: batu rejang

PUM: lirin ungei fih batuh

ALS : king siang sino batu

LDY : tengub nara, iring tangab inanbatu

LIT : mesura'

BRU: tebliyah-tebliyah, tebblia

MRP: na paiau, liraing hungoai ma-

PRT : paro'-paro'

KLK : nah

KLM: paro-paro

KLO: varu

KLA : jelekay

KAL : nah

KBD: nah

KBK : nah

PEB : nah 


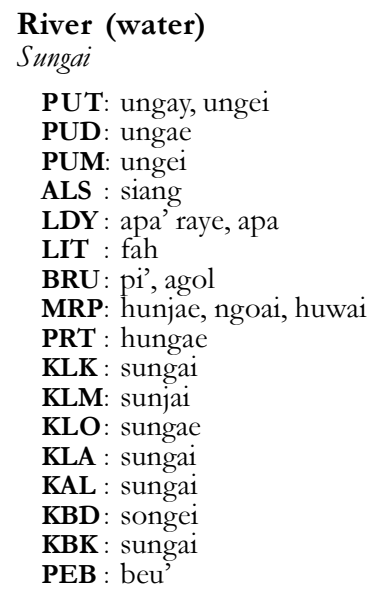

\section{Fork}

Cabang

PUT: pangat, angat

PUD: juman

PUM: angat

ALS : gulungan

LDY : rangat apa', rangat

LIT : ranggat

BRU: angat agol, pangat

MRP: pahanggah, hangeat, hanga'

PRT : hangat

KLK : sangat

KLM: sangat

KLO: sangait sunjae

KLA : pesangat

KAL : sangit

KBD : ebang

KBK : songat

PEB : ibang

\section{Shallows (white water) \\ Andras}

PUT: enga', lingah

PUD: ringah

PUM: lingah

ALS : nagas

LDY : nara

LIT : nerah

BRU: lagiea, legea

MRP: anah, nah, hunah

PRT : nggah

KLK : kah

KLM: nah

KLO: kah

KLA : kah

KAL : kah

KBD: kah

KBK : kah

PEB : kah
Stream

Sungai kecil

PUT: leruk, ungei leruk, ungei icit, leruk, lalut

PUD: leruk

PUM: ungei leruk

ALS : siang lumot

LDY : apa' aror, apa isutd

LIT : fah aror

BRU: agol, agol kie

MRP: hu'ai uwh, ngoai a'uh', huwai uwo'

PRT : hungae iuk

KLK : lalut

KLM: lalut

KLO: lalet

KLA : lalut

KAL : lalut

KBD: lalut

KBK : lalut

PEB : bawang

Waterfall

Air terjun

PUT: o'ung, ungei o'ung, ungei beceri

PUD: becerih

PUM: o'ung

ALS : bosoy

LDY : rueb, apa maruwob

LIT : ru'ub

BRU: owap, pie pun

MRP: mbiah, kata henjah, huwai biah

PRT : u'ong

KLK : u'ong, enbau, libah

KLM: o'ong

KLO: u'ang

KLA : pu'ong

KAL : u'ong

KBD : o'ong

KBK : o'ong

PEB : ong

Island

Pulau

PUT: poru', liyu, puru'

PUD: liu'

PUM: liyu

ALS : pulung

LDY : pulau, puru'

LIT : lio

BRU: lai, long

MRP: ayau, yau, yauu'

PRT : luloh

KLK : lio

KLM: lio

KLO: uli

KLA : musang

KAL : bosang

KBD: lio

KBK : musang

PEB : lio

\section{Feeder stream}

Mata air sungai

PUT: pai leruk, lufang ungei, luvang-ungei

PUD: no'ong

PUM: lufang ungei

ALS : luang timug

LDY : bang bued apa', buwod apa

LIT : bued fah

BRU: ata' hagol, atah kie

MRP: loh boya katah, luboyah ngoai, matang huwei

PRT : ubung hungae

KLK : lumbut

KLM: lobang lumbat sungai

KLO : tavong

KLA : ketoput sungai

KAL : ketumput sungai

KBD : asi lalut

KBK : lubang but

PEB : awuk

\section{Rapids}

Giram

PUT: bawat, nggahlakun, bawad

PUD: lakun

PUM: paru'

ALS : palu, bawad

LDY : faru, bawad

LIT : paro'

BRU: pagap

MRP: lakaum, lakoum, lakung

PRT : jiham

KLK : niam

KLM: giam

KLO: nyiam

KLA : siam

KAL : je' han

KBD : diam

KBK : giam

PEB : diam

\section{Pond/lake (oxbow)}

Danau

PUT: poru' bukung, tabau

PUD: lengetong

PUM: tabau

ALS : takung

LDY : kolam, takung, takunga

LIT : takung

BRU: takong

MRP: takauwe, tabaud, takau, tabau

PRT : belauh

KLK : takung

KLM: landung

KLO: tagong

KLA : empeng

KAL : lenteng

KBD : peng, takung

KBK: takung

PEB : peng, lua' 


\section{Stillwater}

Air tenang

PUT: lulou, jayung, ungei lenung, lolau, ungei buning-buning, ibou

PUD: kilang lulau aru

PUM: ungei lotong, lenung

ALS : timuk lonong timuk dayung

LDY : riberu, apa matugong

LIT : tang

BRU: twap

MRP: lanawe, lolo, tenang ngoai, liriuu', lolau rau

PRT : pekeno'

KLK : lerae', lulan

KLM: lolan

KLO: belaq, lolo

KLA : lojau, belau

KAL : lolau, belau

KBD : lolau

KBK : lulau, belau

PEB : lelau

\section{Salt spring}

Tempat air asin PUT: pan, lun ungei pan, ungei
lunpan

PUD: pan

PUM: lun ungei pan

ALS : tohon savon, tokon sopon

LDY : rufan, inan rufan

LIT : rupan

BRU: lepuan, nan pie men

MRP: nah, laung kata maeih, huainah

PRT : hungan

KLK : sungan

KLM: sunjan

KLO : sungian, ading

KLA : lehua sunjai ari

KAL : sungen

KBD: songan

KBK : sungan

PEB: sungan

Fruit grove

Kelompok pohon buah di butan

PUT: laran buah, lenta bua'an lunang, lida bua'an lunang,

PUD: vi' lenta buaan lunang

PUM: larung buah

ALS : fagangan bua, bua kai yak

LDY : tala, tala' bua bang pulung

LIT : pulong bua

BRU: legiah wi', wie tala

MRP: lindia, po'kou embua' nian luhuiya, muvui bua luhui

PRT : lindah

KLK : pulung bua'

KLM: polong bua'

KLO: solung bavah

KLA : kelempun udip bava'

KAL : pulung bevah

KBD: lenta' bua'

KBK: lindah buah

PEB: pulung bue

\section{Whirpool}

Putaran air

PUT: kelikat, kelikat ungei, kalikat ungei

PUD: kelikut

PUM: kelikat, nawog

ALS : liuk, olot timug

LDY : ribuerr apa', orot

LIT : benner

BRU: lebueng pi', lebuang kie

MRP: kley keya, keliket ngoai, klika huwei

PRT : lirung

KLK : lidung

KLM: belilieng sendek

KLO : lidung

KLA : belirang

KAL : lidung

KBD : lidung, belau

KBK : lidung

PEB: livung, belau

Wallow (pig or rhino)

Tempat mandi babi

PUT: kenawon, tabaupom bafui, kenowan, tabau'u, kenowan bafui

PUD: kenowan

PUM: kenowan, tabau

ALS : lutan kana', lugon bakas

LDY : lutan baka, lutan

LIT : takuaq baka

BRU: takong akah, takong aka

MRP: tengaang, tabau laun mabai ntaoh, tauh tanga ang

PRT : kenae'an

KLK : ketina' babui

KLM: ketayna

KLO: tegeyna

KLA : hetina

KAL : ketayna'

KBD : tekena

KBK : tekena

PEB: tekena

Wet rice paddy

Sawah

PUT: sawah, paton

PUD: sawah

PUM: paton

ALS : ba, sawa

LDY : ba, ba'a

LIT : ba'

BRU: patowan, ba'

MRP: suwah, sawah, muwo panggah

PRT : bawang, luma' bawang

KLK : mpeng

KLM: peng

KLO: savah

KLA : savah

KAL : bavang

KBD: sawah

KBK: sawah

PEB: sawah

\section{Swamp}

Rawa

PUT: tano' udap, pangah, paton,

PUD: panggah

PUM: tano kudek

ALS : dagal

LDY : baba', tanamaba

LIT : ragar

BRU: tana' ledua', lepieh

MRP: panggah

PRT : bawang-bawang

KLK : bawang

KLM: bawang

KLO: kempasa'

KLA : bavang

KAL : bavang (pencim)

KBD: bawang

KBK : bawang

PEB: rapeu

\section{Garden}

Kebun

PUT: lidah, lida, lida'

PUD: lidah

PUM: lida

ALS : kabun

LDY : kabun, rara

LIT : kabun

BRU: lagiah, kebun

MRP: lindia, linteya, linteaa

PRT : lindah tubo'

KLK : pulah

KLM: pola

KLO: kebun

KLA : hu'bun

KAL : kebun

KBD: kebun

KBK : banit

PEB: kebun

\section{Dry rice field (swidden) \\ Ladang}

PUT: umoh

PUD: umoh

PUM: umoh

ALS : umoh, umo

LDY : lati', lati

LIT : latey'

BRU: muah

MRP: moa', muwao, muwo

PRT : luma'

KLK : umah, ba'i

KLM: omah

KLO: umo

KLA : ketua'

KAL : Omo

KBD: oma

KBK : uma

PEB: umah 
Secondary forest (swidden fallow) New fallow (1-2 yrs.)

Hutan sekunder (belukar)

PUT: balah

PUD: baloh

PUM: -

ALS : talun, talun

LDY : amug

LIT : amub

BRU : amup, amub

MRP: melah, malah

PRT : ba'eh

KLK : jekkau

KLM: jekkau

KLO: jekkoh

KLA : jekkau

KAL : jekkau

KBD : juwe

KBK : jekkau

PEB: bokken

\section{Old secondary forest}

Hutan sekunder yang sudab tua

PUT: balah tu'an, balah uron, balah kelu'u, uron

PUD: tu'an

PUM: balah kelu

ALS : talun salag, talun laid

LDY : bawi ripa, amug mon

LIT : -

BRU: amup kemlot, amub adin

MRP: to' uang, to' ueng, malah tarai

PRT : kelawan aya', tu'an

KLK : jekkau mukun, mba'

KLM: mba' mukun mba' pu'un

KLO: laveng, bali, jekkoh bali

KLA : jekkau mba'

KAL : jekkau mpa'

KBD: jekkau mba'

KBK : ba'i jekkau

PEB : bokken jau

\section{Riverine forest}

Hutan di pinggir sungai

PUT: lunang bungae, lunang anlirin, buangtang, ulung, lu-

PUD: nang tang, tano

PUM: lunang an lirin

ALS : gimaking

LDY : fulung, pulung iring

LIT : -

BRU: kulgiang deau

MRP: luhuiyanian ngoai, luhui keliring

PRT : pulung nggang

KLK : a'ut ya sada bai

KLM: -

KLO : -

KLA : a'ut ke sada bai

KAL : a'ut sado bai

KBD : -

KBK : -

PEB : -

\section{Belukar baru}

PUT: balah ovah, baladekin tukung, balah dekin tukung

PUD: ba'i iva'

PUM: balah dekin tukung

ALS : atar, tun ma'at paguh

LDY : amug bawi, amug muneng bawang

LIT : amub beru

BRU: ahal, amub nuang awiang

MRP: maai, malah liang tukadu, malahlingtuku

PRT : ba'eh iuk, ba'eh

KLK : bekanjani

KLM:

KLO: jekkoh duwit

KLA : bekun

KAL : jekkau ceng alem

KBD: bekkun kalam

KBK : -

PEB : bokken jami parei

Primary forest

Hutan rimba

PUT: tu'an, tuan

PUD: tu'an

PUM: tuan

ALS : gima', gima

LDY : fulung, pulung kara

LIT : pulong

BRU: lepi', lepih

MRP: to' uweng tengan, tuoan, tu'ung

PRT : tuan aya', pulung tu'an

KLK : mba' lawen

KLM: -

KLO: mba' lata

KLA : mba'

KAL : mpa'

KBD: mba' mawen

KBK : mba'

PEB : ba' lelum

\section{Upriver forest}

Hutan di bulu

PUT: lunang melang, lunang anrisu, lunang vai ungei, melang,

PUD: larap

PUM: lunang an risu

ALS : gimalayo

LDY : fulung, pulung daig daye

LIT : -

BRU: lheam deau segai

MRP: luhuiyanan kewai, luhui kewea

PRT : -

KLK : a'ut k' usun

KLM: -

KLO : -

KLA : a'ut kerazah

KAL : -

KBD : -

KBK : -

PEB : -
Young secondary forest

Hutan sekunder yang masib muda

PUT: balah tokan, balahtokan, tuan

PUD: balah voh

PUM: balah tokan

ALS : talun rumut, taluntuwo

LDY : amug mon, amug kara

LIT : amub ma'un

BRU: amup, amub lengit

MRP: melah mbau, malah cat laou, malah dau

PRT : ba'eh aya', kelawan

KLK : jekkau bo'et

KLM: -

KLO : laveng

KLA : jekkau lata'

KAL : jekkau lavin

KBD: juwe latah

KBK : ba’i jekkau

PEB : bokken sik

\section{Forest}

Hutan

PUT: lunang

PUD: tu'an

PUM: lunang

ALS : fagangan, pugangan

LDY : fulung, pulung

LIT : pulong

BRU: lem diau, lheam deau

MRP: lamhoya, luhuiya, luhui

PRT : pulung aya'

KLK : a'ut

KLM: niba'

KLO : mba'

KLA : empa'

KAL : a'ut

KBD: mba', ba'i

KBK : ba'i

PEB : ba', tana', pulung

\section{Mountain forest}

Hutan gunung

PUT: bota' ayo', lunang tukuk, lungtukuk

PUD: tu'an tukok

PUM: lunang tukuk

ALS : fagangan tidong, gimati dong

LDY : pulung pegung

LIT : pulong, punraye

BRU: lepi' unquet

MRP: toko lomat, luhuiya tukou,

PRT : tu'ung nggaung

KLK : mba' ka mudong

KLM: mba' mudung

KLO : -

KLA : mba' ka' mudung

KAL : mpa' mudong

KBD : mba' paya

KBK : ba'i mudung

PEB : ba' paya 


\section{Moss forest}

Hutan lumut

PUT: lunang lumut, melang, melang lumut

PUD: melang (atos ari halo')

PUM: lunang lumut

ALS : fagangan lumut, gima kelumut

LDY : faye, pulung melamut

LIT : pulong, di pun raye

BRU: lepi' lemut

MRP: luhiya lumug, luhuia lumuk

PRT : tu'an lumut

KLK : modung butuh

KLM: mba' paya'

KLO: mba' mugun

KLA : mba' pazah

KAL : mpa' butuh

KBD : mba' paya

KBK : ba'i mudung

PEB : ba' paya

Grove, clump of trees

Kelompok pohon

PUT : tampang, lenta kayu, lida kayuh, buang kayuh lida' akayuh, lenta kayuh

PUD: vi'

PUM: larung kayu

ALS : fagangan taun, oyo kai yak

LDY : tala, tala-tala kayu

LIT : perepokok, ye' talatala

BRU: legiah kayau, kayau tala

MRP: lindia, po'kou tengan kayou, muvuiia' kayao

PRT : pekelindah

KLK : pulung

KLM: polong

KLO: volung, solung

KLA : kelempun udip kazu

KAL : pulung

KBD: lenta”

KBK: lindah

PEB: lida'

\section{Cultivated plant}

Tanaman

PUT: tuvuh
PUD: tuvuh
PUM: -
ALS : tonem-tonem
LDY : tibu-tibu
LIT : tibu
BRU: bi'au,
MRP: toboh, tobouh
PRT : tubo'
KLK: -
KLM: -
KLO: fuluh
KLA : fulah
KAL : udip, polo
KBD: pola
KBK: pula
PEB : pola

Gap vegetation

Tumbuhan di tempat kayu rebah

PUT: uca' ovo', lunang anovoh,

PUD: uca' lu-nang an ofo', ucak ovo'

PUM: tana ofo'

ALS : rorop, gaba

LDY : pulung nerapa

LIT : amub balien

BRU: legiah don kayau, kayau kuwa

MRP: blabah, luhuiya kayadu ngeba, lunui blakba

PRT : kayu ndimurip

KLK : pepa'

KLM: pepa'

KLO: la veng

KLA : jekkau

KAL : ekkau asieng-zeng

KBD : pe'pah

KBK : re'pa'

PEB: kenalai

Grasslands (Imperata sp.)

Padang alang

PUT: tano hae, lena ha'ei, ukan hai'i, lendaha'iy

PUD: -

PUM: lenta hai'i

ALS : tana butit, butid

LDY : tala budud, tala budut

LIT : tana budud

BRU: pubal duet

MRP: liting, luhuiya ngai, muvoei ngai

PRT : tan uni ai'i

KLK : a'ut ai

KLM: -

KLO : teng ai'i

KLA : aie

KAL : ten ai' ya

KBD: tana' metan

KBK: apau, aie'

PEB: tana' moten

Tree

Pohon

PUT: pu'un kayu

PUD: pu'un kayu

PUM: -

ALS : taun,

LDY : kayu,

LIT : kayu

BRU: po'on kayau,

MRP: poun kayan, nyauh kayou

PRT : kenjo'

KLK : po'un

KLM: -

KLO : ayneng

KLA : pu'un

KAL : pu'un kazu

KBD: pu'un kayu

KBK : pu'un kayu

PEB: pun kayo

\section{Forests on landslip}

Hutan di tanah longsor

PUT: uca' tuan, lunang antang tuan, tano' kenyut, tuan, ucak tuan

PUD: uca'

PUM: lung tuan

ALS : rorop, gimaluyu

LDY: pulung naturan

LIT : amub balien

BRU: daum non tegiaun, lham deau kulgian tiguan

MRP: nai mbroit, luhuiya tana tuan,

PRT: luhui tuwang

KLK : jekkau ke tuwan

KLM: -

KLO: la veng

KLA : a'ut teleoh tana'

KAL : jekkau towen

KBD: pe'pah tuan

KBK: pe'pa' tuan

PEB: kenalai

\section{Plants}

Tumbuh-tumbuhan

PUT: uru

PUD: -

PUM: -

ALS : taun-taun,

LDY : -

LIT : -

BRU: -

MRP: ma'mauwe, mpruik mpuik

PRT : lindah ara' ara' kayo'

KLK : udip

KLM: -

KLO: a'uk

KLA : udip-udip

KAL : udip ja'udip

KBD: udip ja' udip

KBK : mudip-udip kayo

PEB : kayo

\section{Shrubs}

Semak-semak

PUT: pu'un dero

PUD: deroh

PUM: -

ALS : fun iti'

LDY : nevu

LIT : deser

BRU: bukut

MRP: mu kuk, kayou u'u

PRT : -

KLK : -

KLM: -

KLO: udek kazeu

KLA : pu'un iut

KAL : -

KBD : -

KBK : -

PEB : - 
Vine

Akar

PUT: oka

PUD: okah

PUM: -

ALS : baghag

LDY : uwat

LIT : war

BRU: guel

MRP: paka, kah

PRT : akah

KLK : akah

KLM: -

KLO : ayah

KLA : aha

KAL : aha

KBD: akah

KBK : akah

PEB : lakeu kayo

\section{Moss}

Lumut

PUT: lumut

PUD: lumut

PUM: -

ALS : lumut

LDY : lamut

LIT : lumut

BRU: lemat

MRP: lomu, lumuk

PRT : himut

KLK : butuh

KLM: -

KLO: buteu

KLA : butuh

KAL : butuh

KBD : butu

KBK: salem bawae

PEB: butu

\section{Mushroom \\ Jamur}

PUT: kulat

PUD: kulat

PUM: -

ALS : kulat

LDY : kurat,

LIT : kulat

BRU: hluwat

MRP: kloa lawe, levau'

PRT : ya'ap

KLK : kolat

KLM: -

KLO: golay

KLA : hulat

KAL : kolet

KBD : kolet

KBK : kulat lung

PEB: kolet
Herb

Tumbuban bawab

PUT: uru'

PUD: uru'

PUM:-

ALS : dikut,

LDY : udu,

LIT : udu

BRU: dian (dow),

MRP: ro, rou

PRT : uro'

KLK : a'ut

KLM:

KLO: a'euk

KLA : a'ut

KAL : a'ut

KBD : udu

KBK : udu

PEB: uru'

Lichen

Lumut di batu (bijau)

PUT: litak

PUD: rap

PUM: -

ALS : kemavit taun

LDY : kurat batu

LIT : lumut

BRU: aluat kayau

MRP: kloa, ngelaid matau mie

PRT : litak-litak kayo'

KLK : litak

KLM: -

KLO: gokey

KLA : lumut

KAL : -

KBD :

KBK : kulat

PEB : -

\section{Rattan}

Rotan

PUT: wey

PUD: wei

PUM: -

ALS : awey

LDY : uwey

LIT : wai

BRU: gueh,

MRP: nguwey, ngoi'

PRT : uwey

KLK : uwai

KLM: -

KLO: vey

KLA : vai

KAL : vai

KBD: uwai

KBK : uwei

PEB: lakeu
Grass

Rumput

PUT: uru'

PUD: uru'

PUM: -

ALS : dikut

LDY : udu,

LIT : udu

BRU: dian (dow),

MRP: ro, raou

PRT : uro'

KLK : a'ut

KLM: -

KLO: a'euk

KLA : a'ut

KAL : a'ut

KBD: a'ut

KBK: ao'ut

PEB: ai, ma'ut

\section{Fruit}

Buah

PUT: bua'
PUD: bua'
PUM: -
ALS : bua',
LDY : bua,
LIT : buwa'
BRU: wi',
MRP: ngwah, m'bua
PRT: buah
KLK: bua'
KLM: -
KLO : bevah
KLA : beva
KAL : bevah
KBD: bua
KBK : bua
PEB : bue




\section{Flower}

Bunga

PUT: lavun

PUD: lavun

PUM: -

ALS : busak

LDY : bunga

LIT : busek, bunja'

BRU: siek (sie')

MRP: pendeye, penteya

PRT : pindang

KLK : sapok

KLM: -

KLO: pih

KLA : piu

KAL : piu

KBD: bonga

KBK : bunga

PEB : bunga

\section{Branch}

Dahan

PUT: da'an

PUD: da'an

PUM: -

ALS : daan

LDY : angat

LIT : angat

BRU: la'in,

MRP: la'ang, la'aen

PRT : da'an

KLK : da'an

KLM: -

KLO : da'eng

KLA : da'an

KAL : da'en

KBD: da'an

KBK : da'an

PEB: dan
Trunk

Batang kayu

PUT: betang

PUD: betang kayu

PUM: -

ALS : batang taun

LDY : lawa

LIT : lawan kayu

BRU: lagoa

MRP: po'ung, pu'ung kayao

PRT : pu'un kayo'

KLK : batang kayu

KLM: -

KLO : batang

KLA : fu'un hazu

KAL : fu'un kazu

KBD : pu'un

KBK: pu'un

PEB: pun

\section{Resin exudate}

Sap

Getah

PUT: pulut

PUD: pulut

PUM: -

ALS : hulut taun, fulut taun

LDY : fara'

LIT : para'

BRU: pagah

MRP: pluk

PRT: pulut

KLK : pulut

KLM: -

KLO: feloteng

KLA : fulut

KAL : pulut

KBD: pulut

KBK: pulut

PEB: pulut

Root

Akar tanah

PUT: pakat

PUD: pakat

PUM: -

ALS : berahat daltui butak

LDY : watfutung

LIT : wat

BRU: -

MRP: paka kalam tone, paka tana

PRT : pakat

KLK : pakat

KLM:

KLO : farae' kazeu

KLA : pahat

KAL : pahet

KBD: pakat

KBK: pakat

PEB: pakat
Damar

PUT: ilu'

PUD: nyatong

PUM:

ALS : solong

LDY : ilu

LIT : ilo'

BRU: liau

MRP: latang

PRT: nyateng

KLK : nyabang

KLM: -

KLO: nyatung

KLA : nyateng

KAL :

KBD: nyatung

KBK : nyateng

PEB : -

\section{Leaf}

Daun

PUT: doun

PUD: da'un

PUM: -

ALS : daun

LDY : don

LIT : da'un

BRU: un, iin

MRP: tium, la'aung

PRT : da'un

KLK : tong

KLM: -

KLO : da'ung

KLA : tong

KAL : da'un

KBD: tong

KBK : da'un

PEB: daun 


\section{Wood}

Kayu

PUT: kayuh

PUD: tauh

PUM: -

ALS : fafan

LDY : bangar

LIT : bengar

BRU: ngal

MRP: kayau, kayao

PRT : kayo'

KLK : batang (u'u : papan)

KLM:

KLO: kazeu

KLA : hazu

KAL : kazu

KBD: kayu

KBK : kayu

PEB: kayo
Seed

Bibit

PUT: beni

PUD: benih

PUM: -

ALS : subon

LDY : ilung

LIT : ilung

BRU: pwiah

MRP: naih, po'o, m'nai

PRT : benih

KLK : benei (padi)

KLM: -

KLO : benih

KLA : benia'

KAL : benie

KBD: benih

KBK : benae'

PEB: benih
Animal

Binatang

PUT: aih layau

PUD: ai layau

PUM:-

ALS : kium

LDY : fung bang fulung

LIT : pung

BRU: puang

MRP: kelueng, tlu'un

PRT : cin

KLK : cen

KLM: cin

KLO : cin

KLA : cin

KAL : cen

KBD: cen

KBK : cen, pun

PEB : kan mesenah

\section{Caterpillars/larvae \\ Ulat}

PUT: jeleh

PUD: jelae'

PUM: -

ALS : lengguwang

LDY : kelatih (latih)

LIT : kelatih

BRU: kelemuah

MRP: kunya, kunya'

PRT : haleng

KLK : latih

KLM: latih

KLO: latih

KLA : latih

KAL : latih

KBD: latih

KBK : lati

PEB: latey

PUT: ulet

PUD: ulet

PUM: -

ALS : ulod

LDY : uled

LIT : uled

BRU: liat

MRP: lun, lu'uun

PRT : tekunyoh

KLK : ulet

KLM: olet

KLO : olet

KLA : ulet

KAL : olet

KBD : ulet

KBK : ulet

PEB: olet

Fish

Ikan

\section{Semut/ rayap}

PUT: sevut

PUD: cerut

PUM:-

PUT: ula

PUD: ulah

PUM: -

ALS : -

LDY : dara

LIT : lera'

BRU: -

MRP: tiya'a

PRT : bitek

KLK : sanam

KLM: -

KLO: sanem

KLA : sanem

KAL : sanam

KBD: sanam

KBK : sanam

PEB : -
PUT: bacou

PUD: bacoh

PUM: -

ALS : fait

LDY : lawit

LIT : lawid

BRU: awit

MRP: can

PRT: $\sin$

KLK : atok

KLO : ata'

KAL : atok

KBD: atok

KBK: atok

PEB: betolu
KLM: atok

KLA : atuk 


\section{Snakes}

Ular

PUT: cai

PUD: cai

PUM: -

ALS : nyivoh

LDY : menife

LIT : menipe

BRU: behwa'

MRP: pia'a

PRT : nyipa'

KLK : ulet

KLM: -

KLO : olet

KLA : uncung ulai

KAL : olet

KBD: tuduk

KBK : ulet

PEB: torok
Birds

Burung

PUT: janei

PUD: janae

PUM:

ALS : manok

LDY : suwit

LIT : suwit

BRU: manae'

MRP: manauk

PRT : manok

KLK : suwi

KLM:

KLO: se'vih

KLA : sevi'

KAL : sevi

KBD: suwi

KBK: suwi, upit

PEB: manok

\section{Mammals}

Mamalia

PUT: -

PUD: -

PUM: -

ALS : -

LDY : fung

LIT : pang

BRU: -

MRP: -

PRT : cin

KLK : bunin, belabo

KLM: -

KLO : cin tana' cinjaket

KLA : -

KAL : cin

KBD: pun

KBK : -

PEB: kan

\section{Feather}

Bulu burung

PUT: telu

PUD: telu'

PUM: -

ALS : talo'

LDY : terur

LIT : teror

BRU: tigol

MRP: klau, telaoh

PRT : teloh

KLK : tilo

KLM: -

KLO : teyloh

KLA : tilo

KAL : telo

KBD: telo

KBK: tilo

PEB: iluh

PUT: bulun tangi

PUD: bulun janae

PUM: -

ALS : bulu manok

LDY : bulu suwit

LIT : bulu

BRU: blau mano'

MRP: mlum, blung manaou

PRT : bulo' manok

KLK : bulu suwi

KLM: -

KLO: bula', buleu

KLA : bulu sevi

KAL : bulu sevi

KBD : bulu suwi

KBK : bulu suwi/upit

PEB : bulun manok

Claws
Kuku

Gigi

PUT: nyipen

PUD: nyipen

PUM:

ALS : difon

LDY : lifen

LIT : lipe

BRU: pan

MRP: tonggo, tenggou

PRT : nyipen

KLK : jipen

KLM:

KLO: seyfen

KLA : jifen

KAL : jehfen

KBD: jepan

KBK : jipen

PEB : zipen

PUT: ilun
PUD: ilun
PUM: -
ALS : senggiluh
LDY: lisun
LIT : lisun
BRU: len
MRP: hloh, h'lung
PRT : hulo
KLK: silu
KLM: -
KLO: sileu
KLA : silu
KAL : siluh
KBD: silu
KBK: silu
PEB : silu




\section{Horn}

Tanduk

PUT: wong

PUD: wung

PUM: -

ALS : sangau

LDY : unga

LIT : unga

BRU: ka'ah,

MRP: ngwung, nguong

PRT : wung

KLK : uwung

KLM: -

KLO: owung

KLA : wung

KAL : uwung

KBD: wung

KBK : wung

PEB : uwung
Gall bladder

Empedu

PUT: perun

PUD: perun

PUM:

ALS : lembaduh

LDY : fesu

LIT : pesu

BRU: cauh

MRP: peram, praung

PRT : perro'

KLK : petuh

KLM:

KLO : feh'tu'

KLA : fetu

KAL : petuh

KBD: empedu

KBK : petu

PEB : pedu

\section{Bezoar stone (gall stone)}

Batu guliga

PUT: betia ai'

PUD: batu neticiu

PUM: -

ALS : betia'

LDY :

LIT : betia

BRU: atauh belenggat

MRP: mataum, matuo tlu'un

PRT : bato' banget

KLK : batu bangat

KLM: -

KLO: bateu vang'ai, bangai'

KLA : batu banjat

KAL : batu bangit

KBD : batu bangat

KBK : batu bangat

PEB : batu bangat

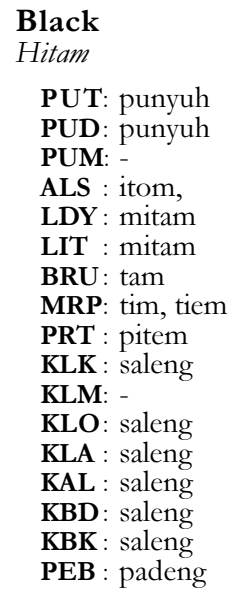

Green

Hijan

PUT: menyemu

PUD: mbuh

PUM: -

ALS : mebata'

LDY : mebata

LIT : mebata

BRU: hatah

MRP: mik la'aum, mie

PRT : bileng

KLK : bileng

KLM:

KLO: beyleng

KLA : bileng

KAL : be' leng

KBD : beleng

KBK : bileng

PEB : bileng

\section{White}

Putih

PUT: seroh

PUD: sero

PUM: -

ALS : hurak

LDY : mebuda

LIT : mebuda

BRU: nuap

MRP: toi, tui'i

PRT : puti

KLK : puta

KLM: -

KLO: futih

KLA : futiah

KAL : futiah

KBD: pute'

KBK : pute

PEB : poti'

Blue

Biru

PUT : embuh

PUD: menyemuh

PUM: -

ALS : u

LDY : mebata

LIT : mebata

BRU: hatah,

MRP: mik lamau

PRT : silem

KLK : aing langit

KLM:

KLO : seylem

KLA : silem

KAL : se' lem

KBD: selem

KBK : silem

PEB : bileng
Red

Merah

PUT: mengan

PUD: mengan

PUM: -

ALS : ria'

LDY : mesia'

LIT : mesia

BRU: menial

MRP: melah, m'bla'

PRT : belah

KLK : balah

KLM: -

KLO: balah

KLA : bala

KAL : balah

KBD : bala

KBK : balah

PEB : baleu

\section{Yellow}

Kuning

PUT: jemit
PUD: jemit
PUM: -
ALS : siloh
LDY: mebirar
LIT : mebira
BRU: gijil
MRP: mik kunyit, mie kunyik
PRT : memit
KLK: bilah
KLM: -
KLO: meumit
KLA : memit
KAL : memit
KBD: kuning
KBK: tuning
PEB : tuning




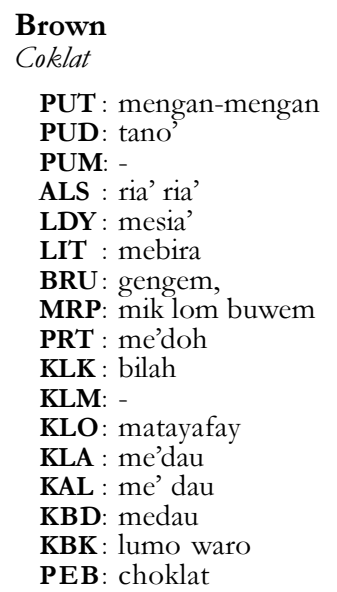

\section{Old (age)}

Tua

PUT: tokan

PUD: tokan

PUM: -

ALS : tu'ow, metuwok

LDY : tua, metaray

LIT : remerar

BRU : -

MRP: mokoh, mokou

PRT : -

KLK : mukun

KLM: -

KLO : mugun

KLA : muhun

KAL : muhun

KBD : pu'un

KBK : mukun

PEB : mokun

\section{Gray}

Abu-abu

PUT: avuh-avu

PUD: avuavuh

PUM: -

ALS : dau-dau

LDY : abu-abu

LIT : mabu

BRU: abian-abian

MRP: bau, bauk-bauk

PRT : poh-poh

KLK : me' poh

KLM:

KLO: ma'puh

KLA : me'poh

KAL : abuh

KBD: mepoh

KBK : mepoh

PEB : abu-abu

\section{Indigenous/true type}

Asli/benar

PUT: tu'uh

PUD: -

PUM: -

ALS : bonjoy

LDY : -

LIT : -

BRU:

MRP: pu'un nah

PRT : atek pu'un

KLK : (true = lan)

KLM: -

KLO : leng

KLA : tulen

KAL : len

KBD : lan

KBK : iya' lan

PEB : tonang, elan
Young (age)

Muda

PUT: tenuh/uyung

PUD: tuyung

PUM:

ALS : mulok, lemulok

LDY : mangud

LIT : mere

BRU: -

MRP: nau, waiy

PRT : pawang

KLK : mengut

KLM: -

KLO : meng'at

KLA : mu'wat

KAL : mo'et

KBD : -

KBK : mengut

PEB : -

Introduced/foreign

Asing

PUT: aji' (lain)

PUD:

PUM: -

ALS : sakai, sahai

LDY :

LIT : sakai (guest)

BRU: -

MRP: kuih

PRT : halo

KLK : alo'

KLM: -

KLO: alo'

KLA : alo'

KAL : ava'

KBD : alo

KBK : sakai (alo)

PEB : alo

\section{Short}

Pendek

PUT: ayo

PUD: ayo

PUM: -

ALS : mayo

LDY : raye

LIT : raiye

BRU: langit

MRP: aya, nya'

PRT : aya'

KLK : bio

KLM: -

KLO : bezu'

KLA : biu

KAL : beguh

KBD : biyo'

KBK : bio

PEB : jau

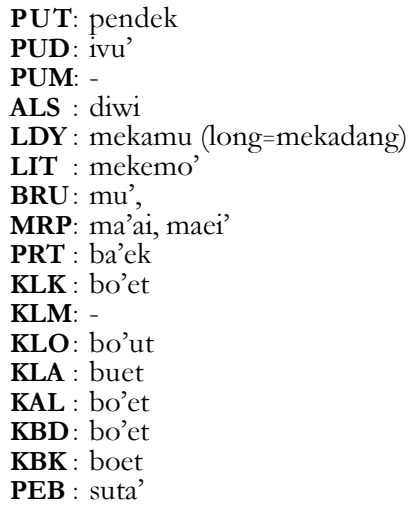


Tall

Tinggi

PUT: vou

PUD: voh, aru (long)

PUM: -

ALS : sawat (buwat $=$ long)

LDY : medita

LIT : medita

BRU: belta'

MRP: mbau, mpau

PRT : mboh, aru (long)

KLK : embau, (long = da'do)

KLM: -

KLO: kohsae

KLA : mpau

KAL : empau

KBD : mbou

KBK : emboh

PEB : bawe

\section{Smooth}

Halus

PUT: ninut

PUD: temai

PUM: -

ALS : lumis

LDY : meligan

LIT : meselud

BRU: naunau

MRP: ngahan, temai

PRT : memut

KLK : timai

KLM:

KLO: feyloh

KLA : lanyah

KAL : lanyon

KBD : momut

KBK : memut, menuk

PEB : beramo

\section{Rough}

Kasar

PUT: -

PUD: jet

PUM: -

ALS : kabulu, kasisi'

LDY : -

LIT : melaba

BRU: am niauniau

MRP: lu'uwe, lu'ueah

PRT : kai'eh

KLK : membut

KLM: -

KLO : kerih

KLA : harae

KAL : kerey

KBD: kerae

KBK : kerae

PEB : belerang

\section{Smelly \\ Berbau}

PUT : bun

PUD: bun

PUM: -

ALS : awoh (awou)

LDY : mebuen

LIT : mebuen

BRU: win

MRP: mbau, mpau'

PRT : mbuh

KLK : ba'o

KLM: -

KLO: ba'u

KLA : ba'u

KAL : ba'ua

KBD : ba'o

KBK : ba'o

PEB : bau

\section{Sweet}

Manis

PUT: mi

PUD: mi'

PUM: -

ALS : linus

LDY : mevesit

LIT : mepesey'

BRU: men

MRP: mai, maih

PRT : meh

KLK : mei, tiga

KLM: -

KLO : mei

KLA : mei

KAL : mey

KBD: mey

KBK : mey

PEB : mi:'
Sour

Asam

PUT: mom

PUD: $m o m$

PUM: -

ALS : asom

LDY : malam

LIT : melam

BRU: plam

MRP: ham, ham

PRT : ham

KLK : mesem

KLM: -

KLO : mesem

KLA : masem

KAL : $\operatorname{sim}$

KBD: mesem

KBK : mesam

PEB : petem
Salty

PUT: pe'ityoh

PUD: miyoh

PUM: -

ALS : asin

LDY : mevesit

LIT : mepesey'

BRU: ci'

MRP: mai hie, maeh

PRT : la'it

KLK : la'it

KLM: -

KLO la'it

KLA : la'it

KAL : la'it

KBD: la'it

KBK : mey osen

PEB : paten
Bland

Hambar

PUT: beleh

PUD: beleh belae'

PUM: -

ALS : foloh

LDY : metalang

LIT : metelang

BRU: klan

MRP: mlai, mayan nayan bong

PRT : beleh

KLK : jeling

KLM: -

KLO : jelieng

KLA : jelieng

KAL : jelieng

KBD: jeleng

KBK : jeling

PEB : jeleng
Bitter

Pahit

PUT: pe'it

PUD: pehit

PUM: -

ALS : afait

LDY : mefet

LIT : mepa'it

BRU: pa'et

MRP: paai, pa'ai

PRT : pa'ih

KLK : pa'it

KLM: -

KLO: fait

KLA : pait

KAL : pa'it

KBD: pa'it

KBK : pa'it

PEB : petem 
Intended as a resource for researchers working in the Bulungan area in northern East Kalimantan, Indonesian Borneo, this handbook serves as both a field guide to common plants and animals, and a dictionary of their vernacular names from 18 different linguistic and cultural groups. The book profiles 164 plant species and $\mathbf{1 1 1}$ animal taxa likely to be encountered in villages or markets or to be subjects for scientific investigation, economic development, conservation initiatives or government policy. The book also includes a gl ossary of terms for geological, hydrological and ecological features and descriptors that often appear in the local names of plants and animals. The book should facilitate communication about familiar flora and fauna in the area and provide the basis for future analyses of regional patterns of resource use, ethnobiological classification and comparative linguistics.
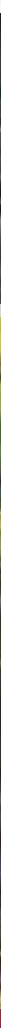

ISBN 979-8764-45-5 